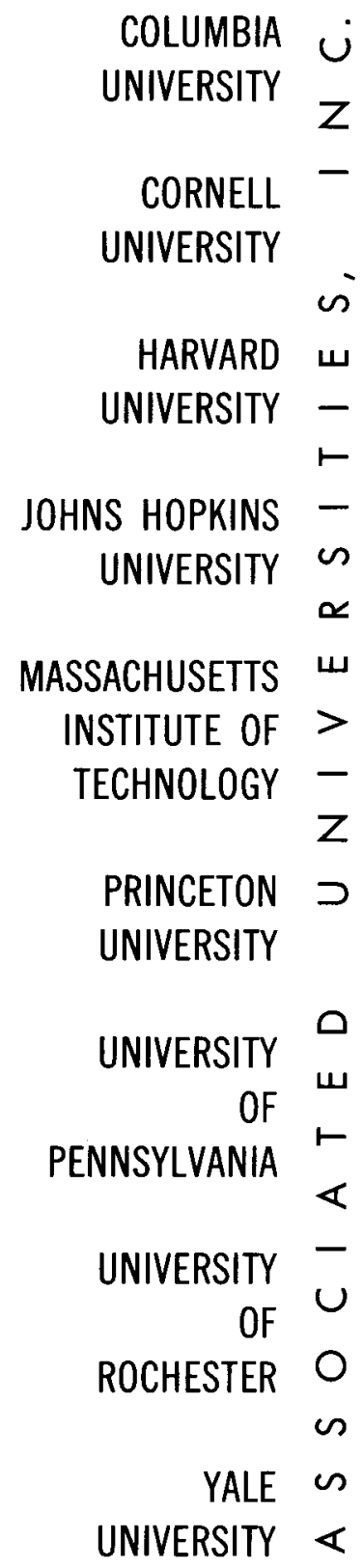


Brookhaven National Laboratory is operated under a contract between the United States Atomic Energy Commission and Associated Universities, Inc. This, the twentieth in a series of unclassified Annual Reports, gives an account of the progress of the Laboratory during the period July 1, 1968 - June 30, 1969, and its plans for the future. It is submitted under the terms of Contract No. AT-30-2-GEN-16 between Associated Universities, Inc., and the Atomic Energy Commission.

Previous reports in this series are

BNL 74 (AS-4) July 1, 1950

BNL 131 (AS-5) July 1, 1951

BNL 196 (AS-6) July 1, 1952

BNL 246 (AS-7) July 1, 1953

BNL 303 (AS-8) July 1, 1954

BNL 364 (AS-9) July 1, 1955

BNL 426 (AS-10) July 1, 1956

BNL 462 (AS-11) July 1, 1957

BNL 523 (AS-12) July 1, 1958

BNL 560 (AS-13) July 1, 1959

BNL 632 (AS-14) July 1, 1960

BNL 682 (AS-15) July 1, 1961

BNL 742 (AS-16) July 1, 1962

BNL 806 (AS-17) July 1, 1963

BNL 867 (AS-18) July 1, 1964

BNL 929 (AS-19) July 1, 1965

BNL 994 (AS-20) July 1, 1966

BNL 50057 (AS-21) July 1, 1967

BNL 50106 (AS-22) July 1, 1968

\section{E G A L NOT I G E}

This report was prepared as an account of Government sponsored work. Neither the United States, nor the Commission, nor any person acting on behalf of the Commission:

A. Makes any warranty or representation, expressed or implied, with respect to the accuracy, completeness, or usefulness of the information contained in this report, or that the use of any information, apparatus, method, or process disclosed in this report may not infringe privately owned rights; or

B. Assumes any liabilities with respect to the use of, or for damages resulting from the use of any information, apparatus, method, or process disclosed in this report.

As used in the above, "person acting on behalf of the Commission" includes any employee or contractor of the Commission, or employee of such contractor, to the extent that such employee or contractor of the Commission, or employee of such contractor prepares, disseminates, or provides access to, any information pursuant to his employment or contract with the Commission, or his employment with such contractor.

Printed in the United States of America Available from

Clearinghouse for Federal Scientific and Technical Information National Bureau of Standards, U.S. Department of Commerce Springfield, Virginia 22151

Price: Printed Copy $\$ 3.00$; Microfiche $\$ 0.65$ 


\section{CONTENTS}

INTRODUCTION

ix

\section{Physical Sciences and Engineering}

Physics

High Energy Accelerators

INSTRUMENTATION

Chemistry

Applied Science

Applied Mathematics

\section{Life Sciences}

BIOLOGY

Medical Research

\section{Supporting Activities}

Technical Operations and Services 155

Administration and Operations

Appendix A. Publications

Appendix B. Officers and Scientific and Professional Staff 
Brookhaven National Laboratory is operated by Associated Universities, Inc., under contract with the United States Atomic Energy Commission. Nine northeastern universities sponsor Associated Universities, Inc. The Board of Trustees consists of two individuals from each university, one a principal administrative or corporate officer and the other a scientist, and not more than six Trustees-atLarge selected by the Board. The purpose of the latter is to insure adequate representation of any scientific discipline in which the corporation may undertake work and to provide general flexibility in methods of operation. The composition of the Board as of June 1969 is given below. The officers of AUI are shown on the organization chart for the Laboratory. AUI has appointed five visiting committees to Brookhaven National Laboratory to provide a continuing independent evaluation of the research program. The committees cover the fields of applied science, biology, chemistry, medicine, and physics and report annually to the Board of Trustees.

\section{BOARD OF TRUSTEES \\ OF}

\section{ASSOCIATED UNIVERSITIES, INC.} AS OF JUNE 30, 1969

\section{UNIVERSITY TRUSTEES}

RalPh S. Halford

Columbia University

I.I. RABI

Franklin A. Lovg, Chairman

Cornell University

Boyce D. McDaniel

William H. Sweet

Harvard University

L. GARD WigGINS

Allyn W. Kimball

Johns Hopkins University

Bruce J. Partridge

Peter T. Demos

Massachusetts Institute of Technology

Carl F. Floe

Milton G. White

Princeton University

Joseph C. Elgin

Righard H. Chamberlain

University of Pennsylvania

Carl C. Chambers

Louis H. HempelmanN

University of Rochester

LaRoy B. THOMPSON

Vernon W. Hughes

Yale University

Gharles H. Taylor, Jr.

TRUSTEES-AT-LARGE

Bart J. BoK, University of Arizona Thomas Gold, Cornell University

Herbert Friedman, Naval Research Laboratory Francis E. Low, Massachusetts Institute of Technology

Trustee ex Officio

Gerald F. TAPE, President 


\title{
VISITING COMMITTEES
}

Edward C. Creutz

General Dynamics Corporation

WARren C. Johnson, Chairman

University of Chicago

Theos J. Thompson*

Massachusetts Institute of Technology

JoHN TURKevich

Princeton University

\section{Applied Science}

\author{
Robert C. Axtmann
}

Princeton University

Arthur Kantrowitz

Avco-Everett Research Laboratory

Shelby A. Miller

Argonne National Laboratory

Frankin K. Moore

Cornell University

Trustee Member: Mitton G. White, Princeton-Pennsylvania Accelerator
Frederic M. Richards, Chairman

Yale University

Adrian M. SRb

Cornell University

FrankLin Hutchinson

Yale University

LAWRENCE B. SLOBODKIN

State University of New York at Stony Brook
Biology

EDWARD Novitski

University of Oregon

William H. Stein

Rockefeller University

ANDRÉ T. JagendorF

Cornell University

Folke Skoog

University of Wisconsin

Chemistry

William von E. Doering, Chairman

Harvard University

WaLter H. STOCKMAYER

Dartmouth College

HARRISON SHULL

Indiana University

\author{
S.I. WEISSMAN \\ Washington University \\ N. Bruge Hannay \\ Bell Telephone Laboratories \\ JoHn O. Rasmussen, JR. \\ Yale University
}

Trustee Member: Josph C. Elgin, Princeton University

Medical

Stanley E. Bradley, Chairman

Columbia University

Joseph F. Ross

University of California at Los Angeles

JaCOB FurTh

Columbia University

JoHN C. KENDREW

Cambridge University

Trustee Member: Louis H. HempelmanN, University of Rochester

\section{Henry George Kunkel Rockefeller University \\ W.T.S. THORP University of Minnesota \\ John M. Buchanan} Massachusetts Institute of Technology

Charles L. Dunham National Research Council

\section{Physics}

Nicolaas Bloembergen, Chairman

E. J. LofGren University of California at Berkeley

Harvard University

T.D. LEE

Columbia University

WALTER KoHN

University of California at San Diego

Thomas Lauritsen

California Institute of Technology
Trustee Member: Vernon W. Hughes, Yale University

WolfGang K.H. PANOFSKY

Stanford Linear Accelerator Center

Stephen L. Adler

Institute for Advanced Study

LeON MADANSKy

Stanford Linear Accelerator Center

*Resignation became effective June 12, 1969, upon appointment to Atomic Energy Commission. 
ASSOCIATED UNIVERSITIES, INC.

ORGANIZATION CHART

July 1,1969

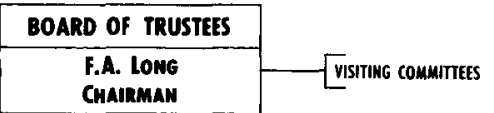

F.A. LOMG
CHAIRMAN

PRESIDENT

PRESIDENT

Tom

CONTROLIER - L.R. BUNCHILL
TREASURER - L. GARO WIGGINS

SPECIAL ASSISTANT - L.J. HAWOOH,

\section{BROOKHAVEN NATIONAL LABORATORY}

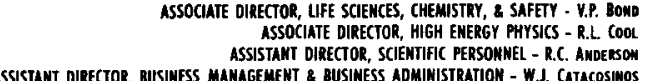

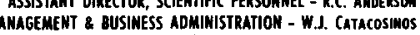

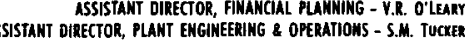

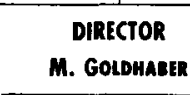

M. GOLDHABER

COATROLLER - LR. BURCHHI

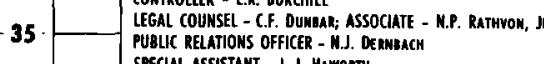

G.H. Vimrand

SPECIAL ASSISTANT - L.S. HAWOOTH

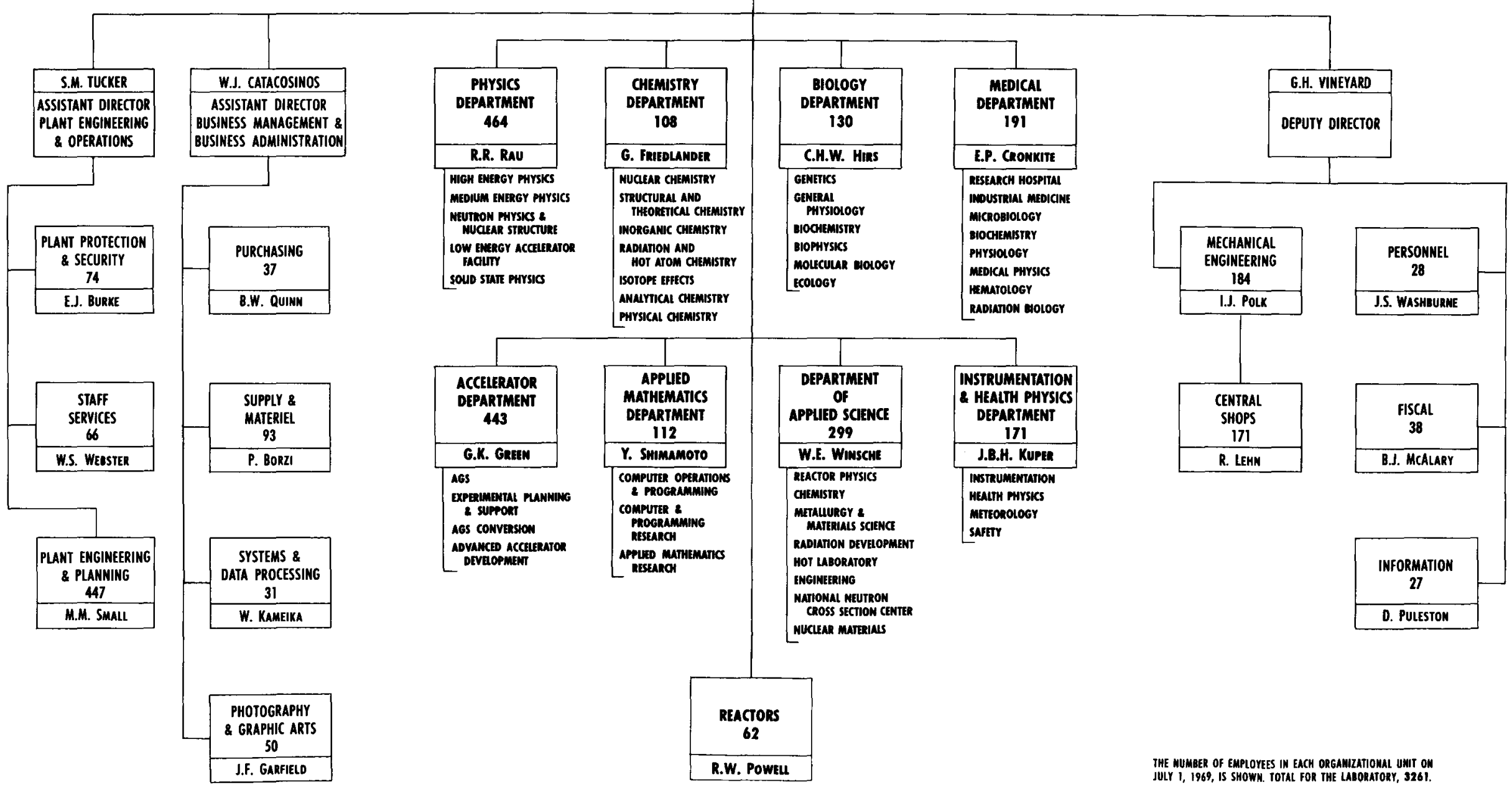




\section{ros}

Aerial view of progress on the AGS conversion project,

May 1969. At the top is the housing for the $200-\mathrm{MeV}$

linear accelerator, which will replace the present $50-\mathrm{MeV}$

injector. At the lower right of the magnet ring, construc

tion is under way on an addition to the Target Building.

To the right of this is an extension to the East Experimen-

tal Building. Directly below the magnet ring an addition

to the Service Building is being constructed.

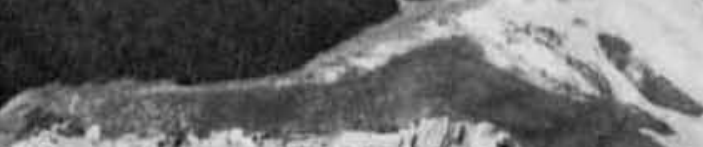

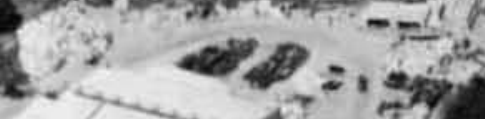

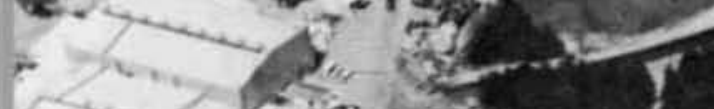

4

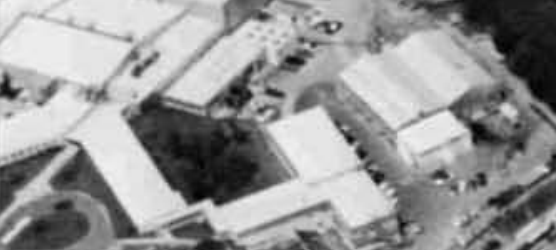

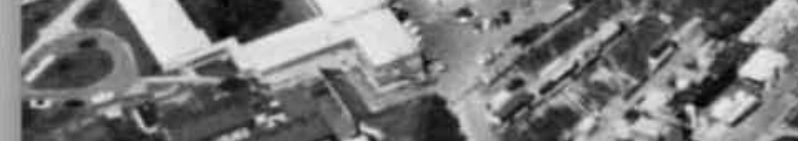
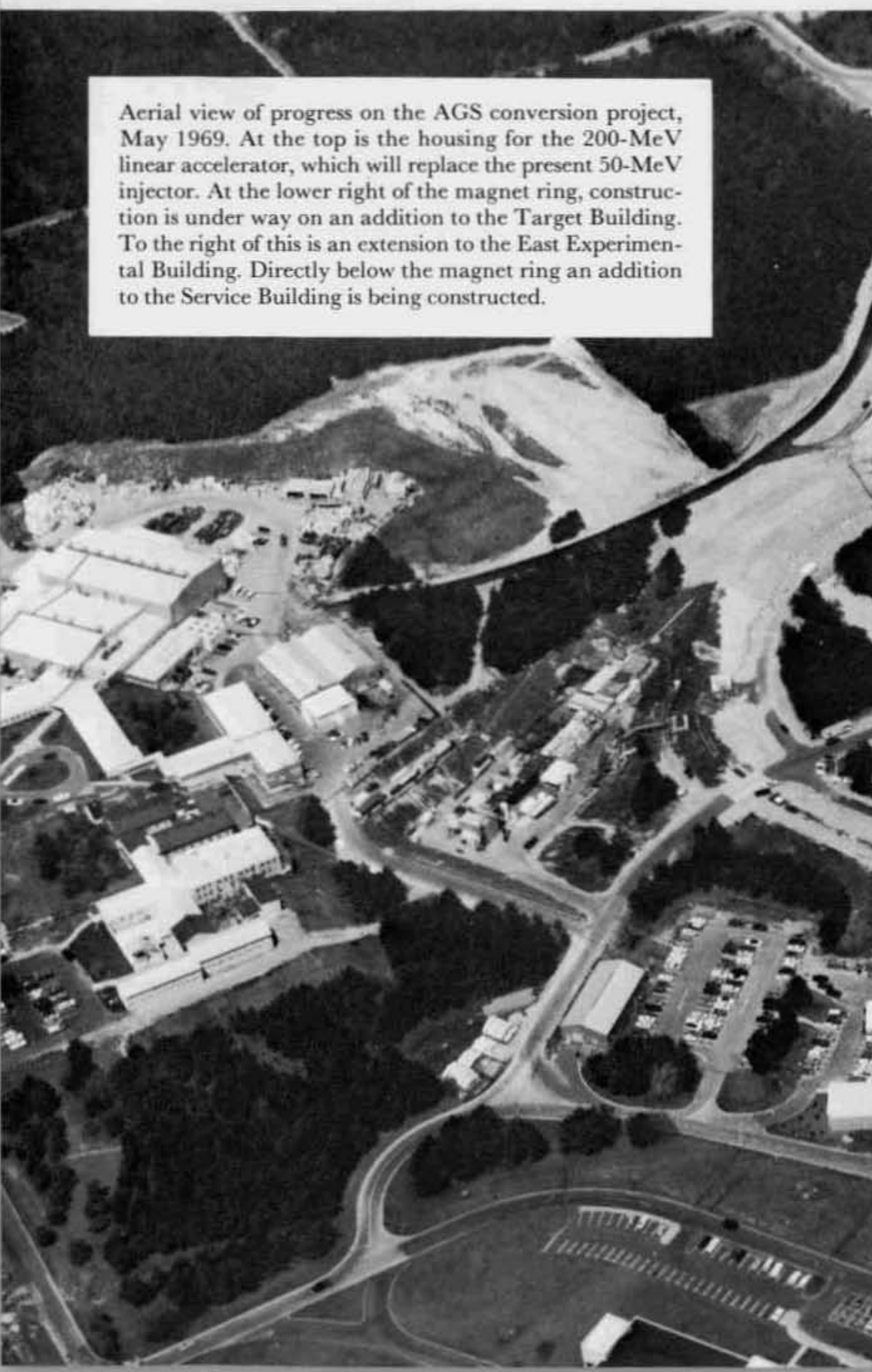


\section{Introduction}

The program and activities of Brookhaven $\mathrm{Na}$ tional Laboratory during the fiscal year 1969 are described in this annual report. The progress and trends of the research program are presented together with a description of the operational, service, and administrative activities of the Laboratory. The scientific and technical details of the many research and development activities are covered more fully in scientific and technical periodicals and special reports of the Laboratory. A list of all publications published or submitted for publication during the year may be found in Appendix A.

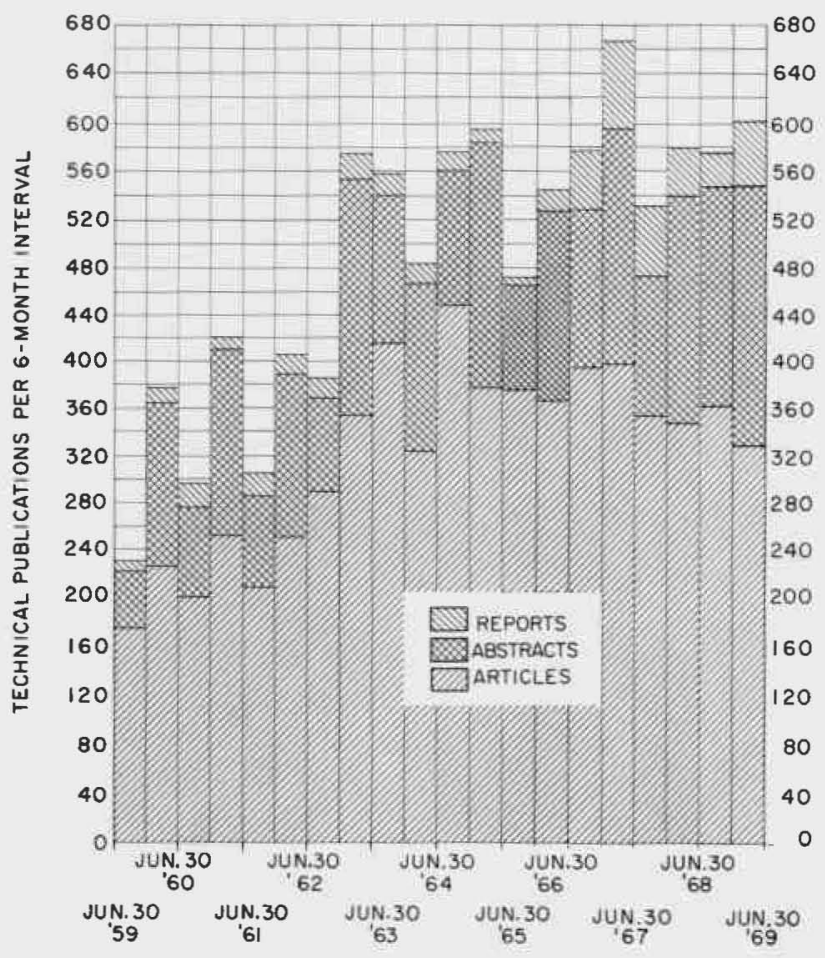

Technical publications.

\section{RESEARCH PROGRAM}

The Brookhaven research program, which covers a wide range of subjects in the physical and biological sciences and in engineering, has as its central motif the development and exploitation of nuclear science and technology. It can be broadly described under five main headings:

1. Fundamental studies of atomic nUClei, THE PARTICLES THAT CONSTITUTE THEM, AND THE FORCES INVOLVED IN THEIR STRUGTURE. These studies, which are basic to all nuclear science and technology, involve the use of all the major machines of the Laboratory. They range from measurements of the properties of undisturbed nuclei to study of the violent disruptions resulting from nuclear fission or from bombardment with high energy protons from the Laboratory's $33-\mathrm{BeV}$ Alternating Gradient Synchrotron (AGS).

2. Studies of the physical, chemical, and BIOLOGIGAL EFFECTS OF NUCLEAR RADIATION. The effects of radiation are utilized to determine the characteristics of physical and chemical structures and to elucidate the more complex properties of living systems and the changes they undergo in their life and reproductive cycles. Of direct practical interest are the possible deleterious effects of radiation upon biological systems and upon various materials, for example, those involved in reactor construction. Of equal interest are the potentially beneficial effects of radiation in the treatment of diseases, in the induction of genetic changes in plants or animals, and in the improvement of manufacturing processes.

3. The USE OF NuGlear tools, SUCh AS NEUTRONS, CHARGED PARTICLES, GAMMA RAYS, AND ISOTOPIC TRACERS, IN ALL BRANCHES OF SCIENTIFIC RESEARCH. In this broad and diversified field, which overlaps the one just mentioned, nuclear particles and radiations are used as tools in studying physical, chemical, and biological systems in their undisturbed states. Neutrons and other particles are used as probes: by observing their penetration and scattering, minute details of physical and chemical structure can be studied. Wide use is made of isotopic tracers in many fields to yield information on such matters as the mechanisms and rates of chemical reactions and biological processes.

4. Research and DEVElopment, NOt NECESSARILY ITSELF OF A NUCLEAR NATURE, DIRECTED 
In studies on the treatment of Parkinsonism and related diseases, a medical scientist at the BNL Medical Research Center evaluates the improvement induced by L-dopa in a young patient suffering from dystonia muscularum deformans.
Radioactive sample of dopamine labeled with carbon-11, for use by BNL's medical scientists in studies of the drug's effectiveness in the treatment of Parkinson's disease. BNL chemists produced this labeled compound by nuclear recoil techniques in the 60 -in. cyclotron.

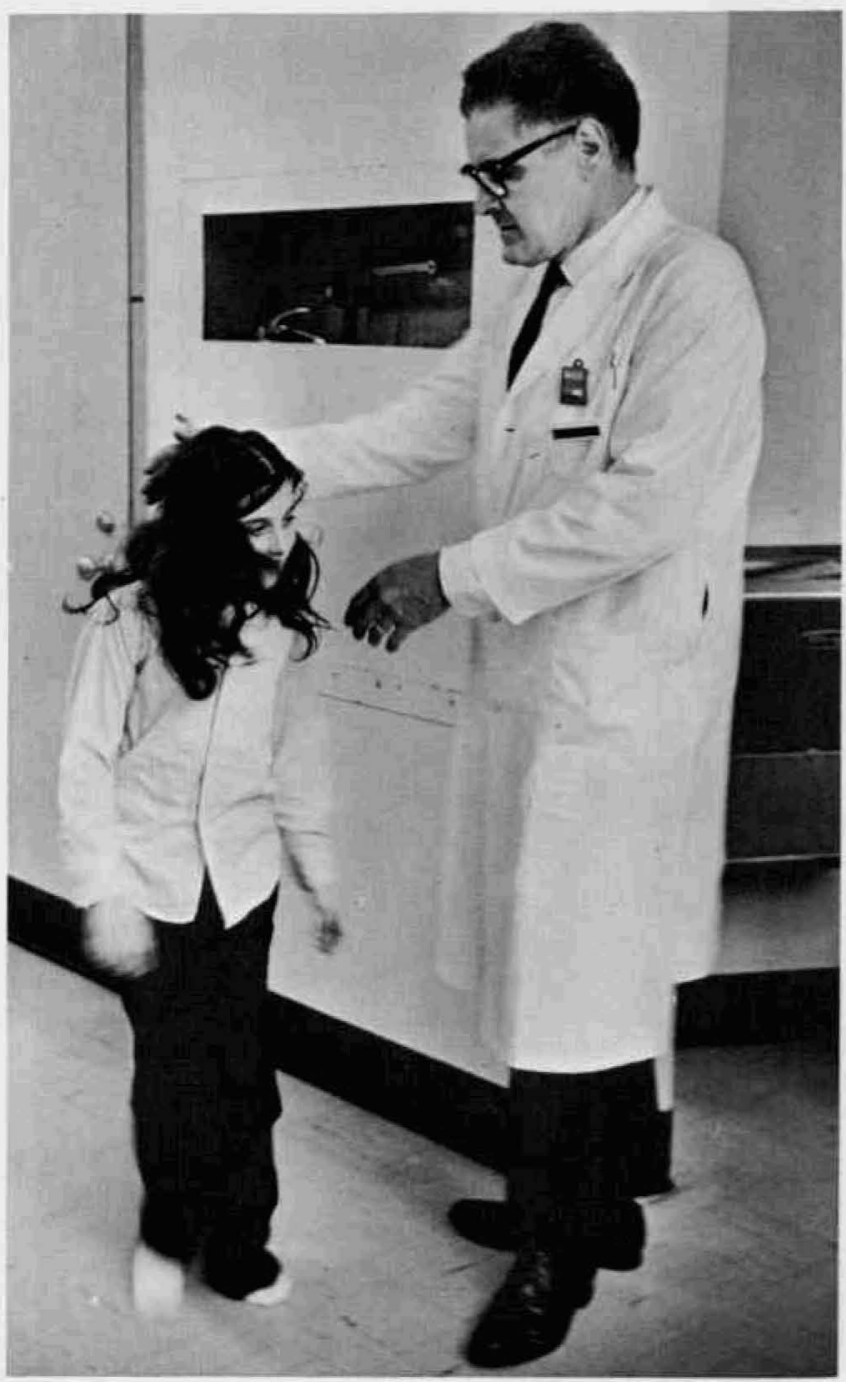

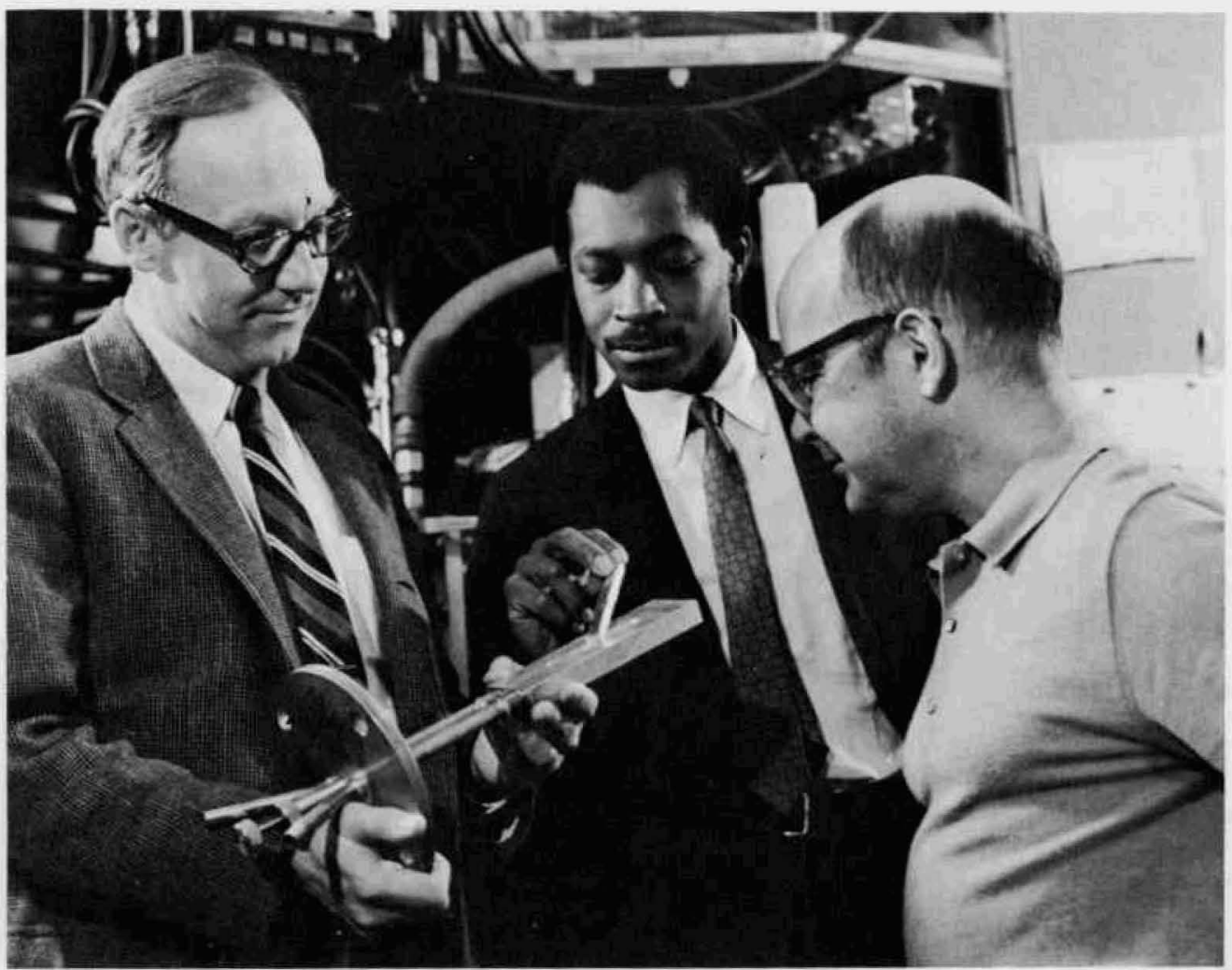




\section{Alternating Gradient Synchrotron}

The AGS, Brookhaven's 33-BeV synchrotron, continues to be one of the major centers for research in high energy physics. Its performance improved significantly during the year, largely as a result of improvements in the injector and the linear accelerator $\mathrm{rf}$ structure. A peak intensity of $3.1 \times 10^{12}$ protons/pulse has been achieved, considerably above the $2.6 \times 10^{12}$ intensity attained last year.

In order to make efficient use of the increased beam intensities at the AGS, studies are under way to expand the external beam system into a beam "switchyard."

The slow external beam facility has been used successfully for three experiments. The improved reliability of the machine is indicated by a $10 \%$ increase in the scheduled time available to experimenters.

At the end of May a five-month shutdown was begun to allow shielding to be removed from the magnet enclosure as part of the AGS conversion project. This conversion calls for a $200-\mathrm{MeV}$ linear accelerator to replace the present $50-\mathrm{MeV}$ injector, additions to the Target, East Experimental, and Service Buildings, and various other improvements designed to meet the future research requirements of the AGS.

Injection of $200-\mathrm{MeV}$ protons into the AGS is expected to commence in the last quarter of 1971. A complete report on the AGS and the conversion project will be found in the section on High Energy Accelerators.

\section{Low Energy Accelerator Facility}

Upon completion of the new $30-\mathrm{MeV}$ tandem Van de Graaff accelerator, the principal research efforts of the accelerator center will begin to be transferred to that complex. Assembly of the two accelerators has begun, and as soon as the second of the two machines has undergone acceptance tests it will immediately be used to activate a research program in the second target room at energies corresponding to two-stage operation of the second accelerator, or 20-MeV maximum. This program will be useful for testing various new pieces of experimental apparatus and data-processing equipment, in addition to initiating tandem nuclear structure research. Many of the experiments will be continued to the maximum three-stage energy of $30 \mathrm{MeV}$ as soon as three-stage interconnection and operation have been achieved, which should be by July 1970 .
The SDS Sigma-7 computer, initially installed in the Cyclotron Building, has been moved to the electrical equipment room of the Tandem Van de Graaff Building and is being prepared to support the new tandem research program. The new location can easily accommodate the complete computer facility. The cyclotron and the small Van de Graaff can continue to use the computer via remote data stations.

The three low energy accelerators at BNL, the small Van de Graaff, the 60-in. sector-focused cyclotron, and the three-stage tandem Van de Graaff will all be operated and used for research in a coordinated and complementary fashion as one large low energy accelerator facility (LEAF) rather than as separate accelerators with independent programs and operations. This concept is now gradually being implemented as the tandem accelerators are being constructed. By the time threestage operation is achieved, the research programs and operation of the various accelerators will be completely coordinated, with the smaller machines in supporting roles to the tandem accelerators.

\section{High Flux Beam Research Reactor}

The High Flux Beam Research Reactor (HFBR), which achieved initial criticality in Oct. 1965 and attained its full design power of $40 \mathrm{MW}$ in Feb. 1966 , produced a total of $10,147 \mathrm{MWd}$ of energy during the year, compared with $9742 \mathrm{MWd}$ during fiscal 1968. The experimental program with this reactor continued to grow, and all experimental facilities were used during the year. Two new sample irradiation systems were placed in service. No major operating difficulties arose during the year.

Additional information on the HFBR may be found in the section on Technical Operations and Services.

\section{Graphite Research Reactor}

The Brookhaven Graphite Research Reactor (BGRR), which has been in almost continuous operation since its initial start-up in 1950, was placed on standby at the end of last fiscal year. This year it operated on 27 days only. In standby condition it could be operated, with appropriate advance notice, for as many as five days during periods of scheduled HFBR shutdown. HFBR operating crews were used on such occasions.

The total energy produced by the BGRR to date is $99,857.8 \mathrm{MWd}$. 


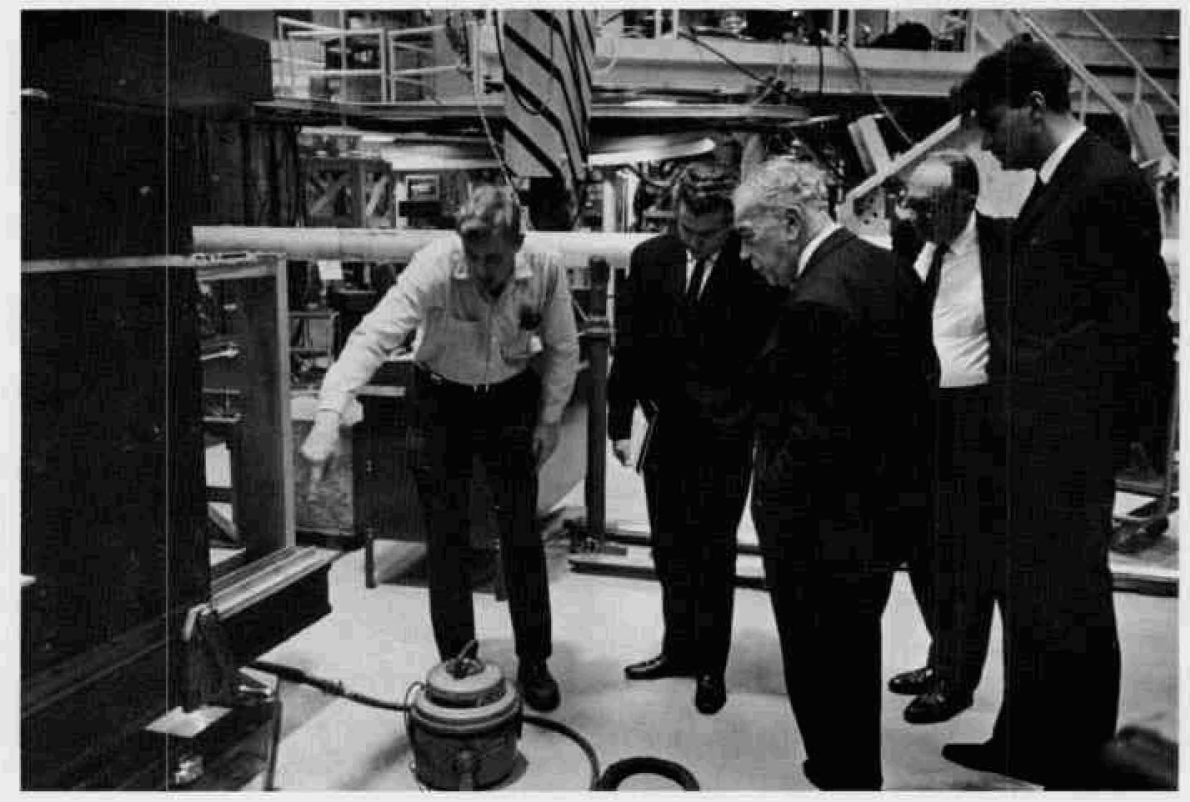

A delegation from Rumania, headed by Professor Horia Hulubei (right foreground), President of the Rumanian Nuclear Energy Committee, inspecting the High Flux Beam Research Reactor. The delegation visited the Laboratory on Nov. 26 and 27.

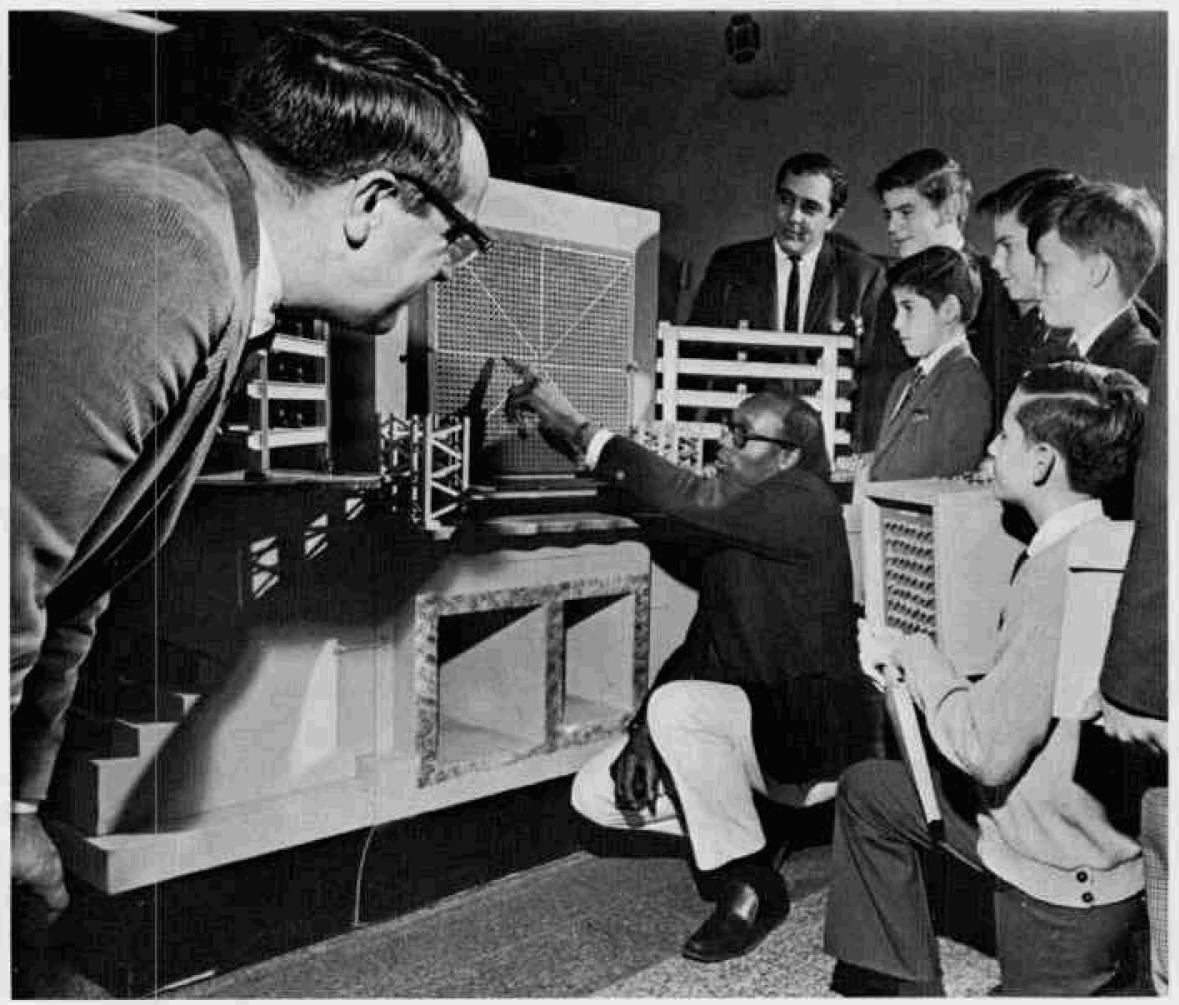

BNL scientist explaining the model of the Graphite Research Reactor to a group of visiting high school students. 


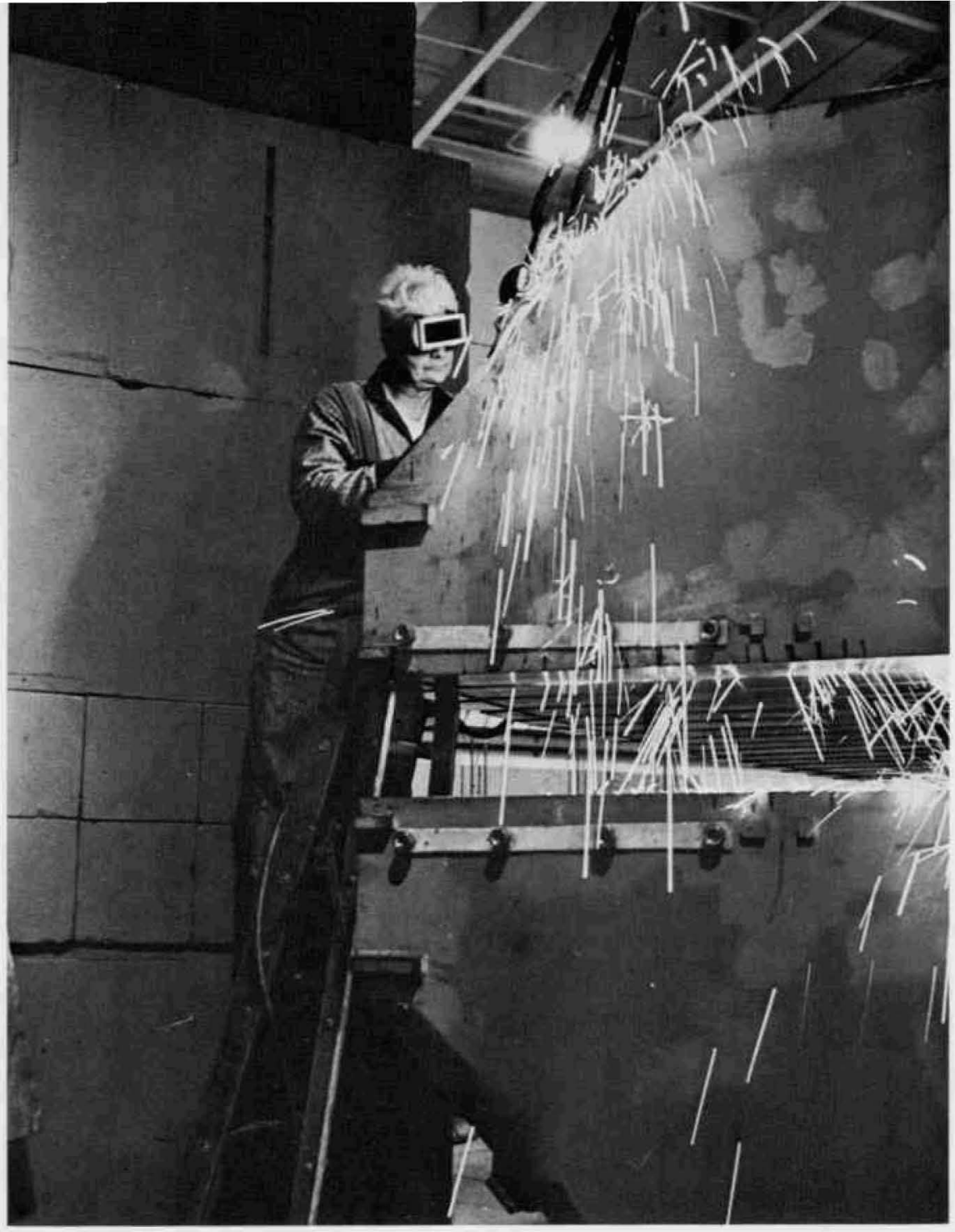

Cosmotron magnet sections being cut apart by acetylene torch prior to removal for storage.

Final stages in the disassembly of the Cosmotron in May 1969. The last magnet section is being removed from the ring for placement in a waste storage area while residual radiation is dissipated.

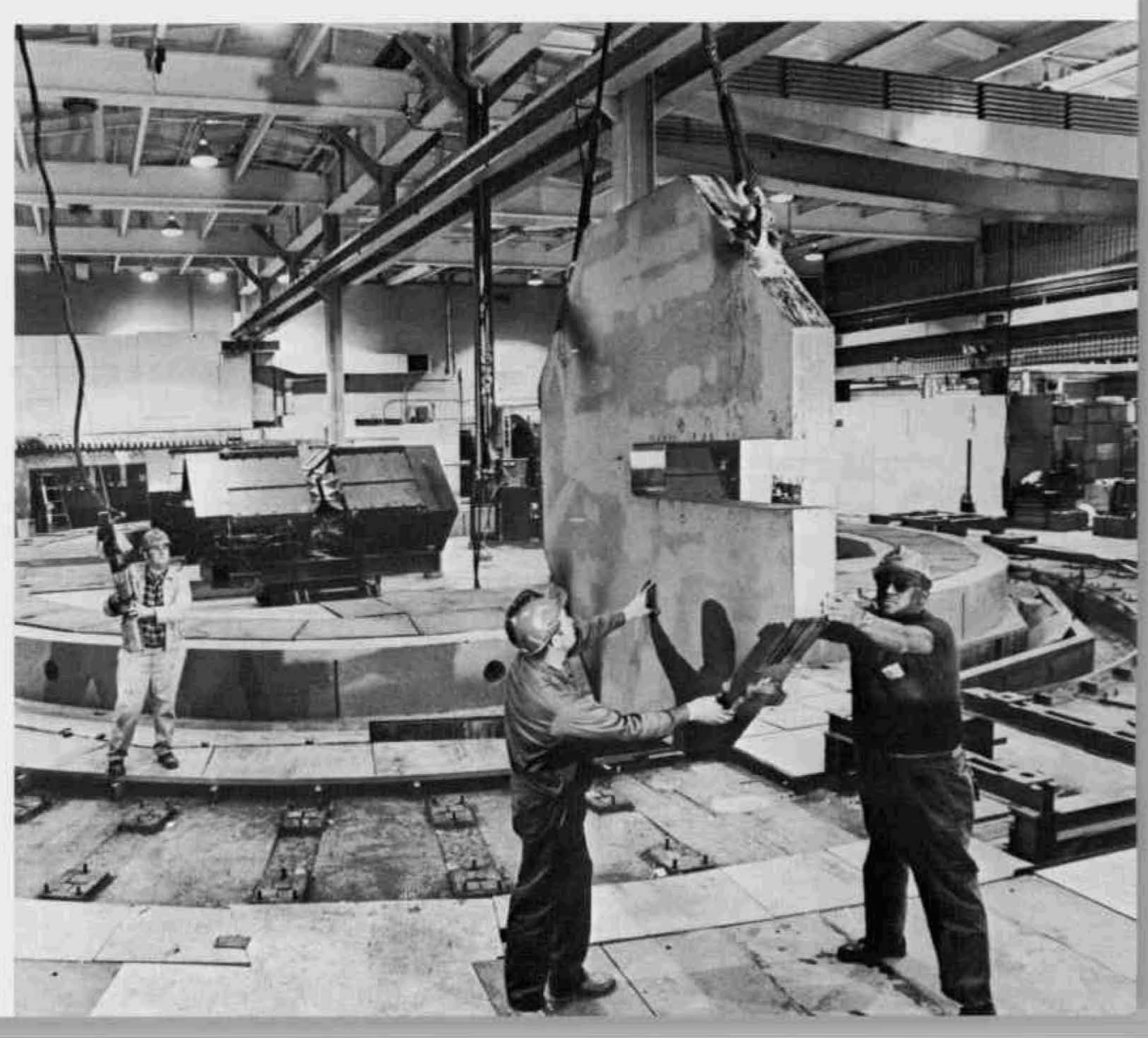




\section{Medical Research Reactor}

The Medical Research Reactor (MRR), which achieved criticality in March 1959, was designed for the purpose of exploring the possible applications of nuclear reactors to the study of man and his diseases. During the past year it was operated on 203 days and 1,764.997 MWh of operation were accumulated. A total of 965 irradiations were made; 45 of these were for off-site users.

\section{Hot Laboratory}

The Hot Laboratory, which is adjacent to the BGRR, contains extensive facilities for processing, handling, and analyzing highly radioactive materials. It includes three hot cells in which chemical operations can be performed remotely while being observed by periscope, a large hot cell for complete physical examination of radioactive metals and other materials, and several totally sealed systems, some of which are shielded, for working with alpha emitters alone or accompanied by gamma radiation. The Hot Laboratory Division provides to others certain services such as chemical analysis and radiography. Since 1950 it has been developing special radioisotopes, in particular, those useful in medical research.

\section{Critical Assembly Laboratory}

The Critical Assembly Laboratory provides specialized facilities for research in reactor physics and for reactor development studies. These facilities include five assembly cells and their associated control areas, a counting room, two uranium storage vaults, and an analogue computer and two digital computers used for on-line processing as well as for standard data reduction. Two of the cells contain neutron source reactors, and a fast source reactor (FS-1) is available for experimental use in another cell. This latter assembly, containing $\approx 150 \mathrm{~kg}$ of $\mathrm{U}^{235}$, is currently being used to study heterogeneity and spectral effects in fast reactors.

\section{High Intensity Radiation Development Laboratory}

The primary function of the High Intensity Radiation Development Laboratory (HIRDL) is to obtain engineering data on a variety of radiation sources in the million-curie range and to develop more efficient techniques for handling largescale radiation sources. This information is essential for the design of future irradiation facilities needed for a wide range of applications of radiation energy. During the fiscal year source inventories included more than 2.5 million curies of $\mathrm{Co}^{60}, 400,000$ curies of $\mathrm{Cs}^{137}$, and 5000 curies of $\mathrm{Sr}^{90}$. A number of irradiation units, including portable cesium irradiators and a blood irradiator, were loaded under remote-control conditions. An underwater gamma facility capable of delivering doses from 5000 to 10 million rads/hr was constructed. One BNLdesigned irradiator was received from the vendor, modified, and made available to Argentina; a second and a third will be ready soon for shipment to Chile and India.

\section{0-in. Liquid Hydrogen Bubble Chamber}

During the year the 80 -in. liquid hydrogen bubble chamber, located in the North Experimental Area of the AGS, produced a total of 1.6 million photographs distributed among 14 experiments. All these experiments were run with use of the high energy rf separated beam; for 13 of them hydrogen was used in the chamber, and for one, deuterium.

A new, hydraulic-powered expansion system was installed on the chamber to minimize the possible loss of deuterium. Work has begun on the design of a bright-field illumination system and a change from 70 to $35-\mathrm{mm}$ film. More information on the 80 -in. chamber may be found in the section on Physics.

\section{Central Scientific Computing Facility}

The operations of the Central Scientific Computing Facility (CSCF) are supported by two large-scale and one small-scale digital computer: two CDC 6600's and a CDC 924, which serves principally as peripheral support for the two largescale machines. An IBM 7094 computer, which is devoted almost entirely to bubble-chamber dataprocessing, was transferred to the Physics Department in July 1968.

Throughout the entire year the CSCF computer system was in operation seven days a week for three shifts a day; it is utilized for most of the Laboratory's general-purpose computation. The two CDC 6600 's were coupled directly to a one-millionword extended core storage during the year. Information on CSCF procedures and systems is disseminated to computer users through the publication of the Computer Newsletter. Further details on the Laboratory's computer program are contained in the section on Applied Mathematics. 


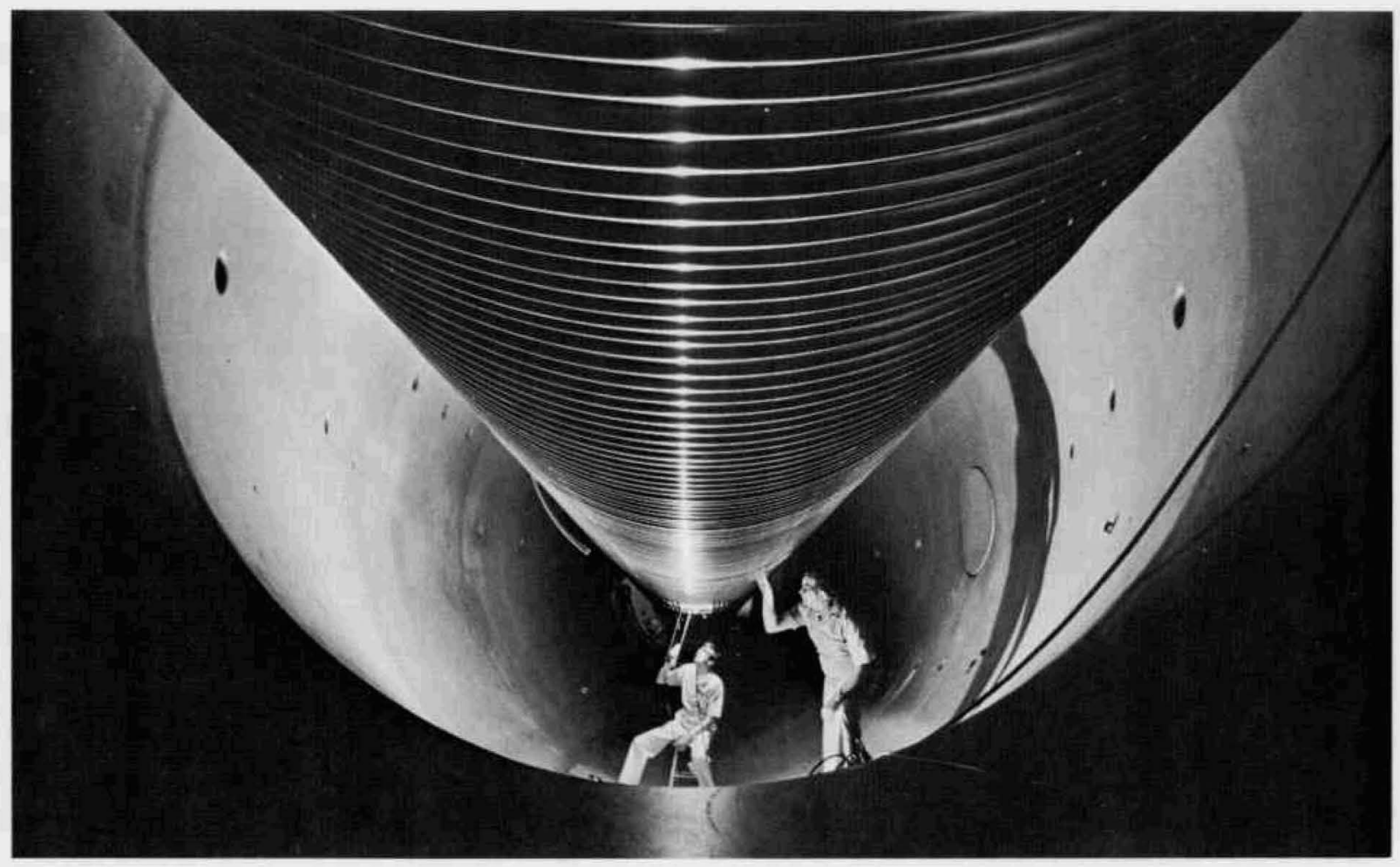

Inspection during construction of the Tandem Van de Graaff system of accelerator components inside one of the accelerator pressure vessels .

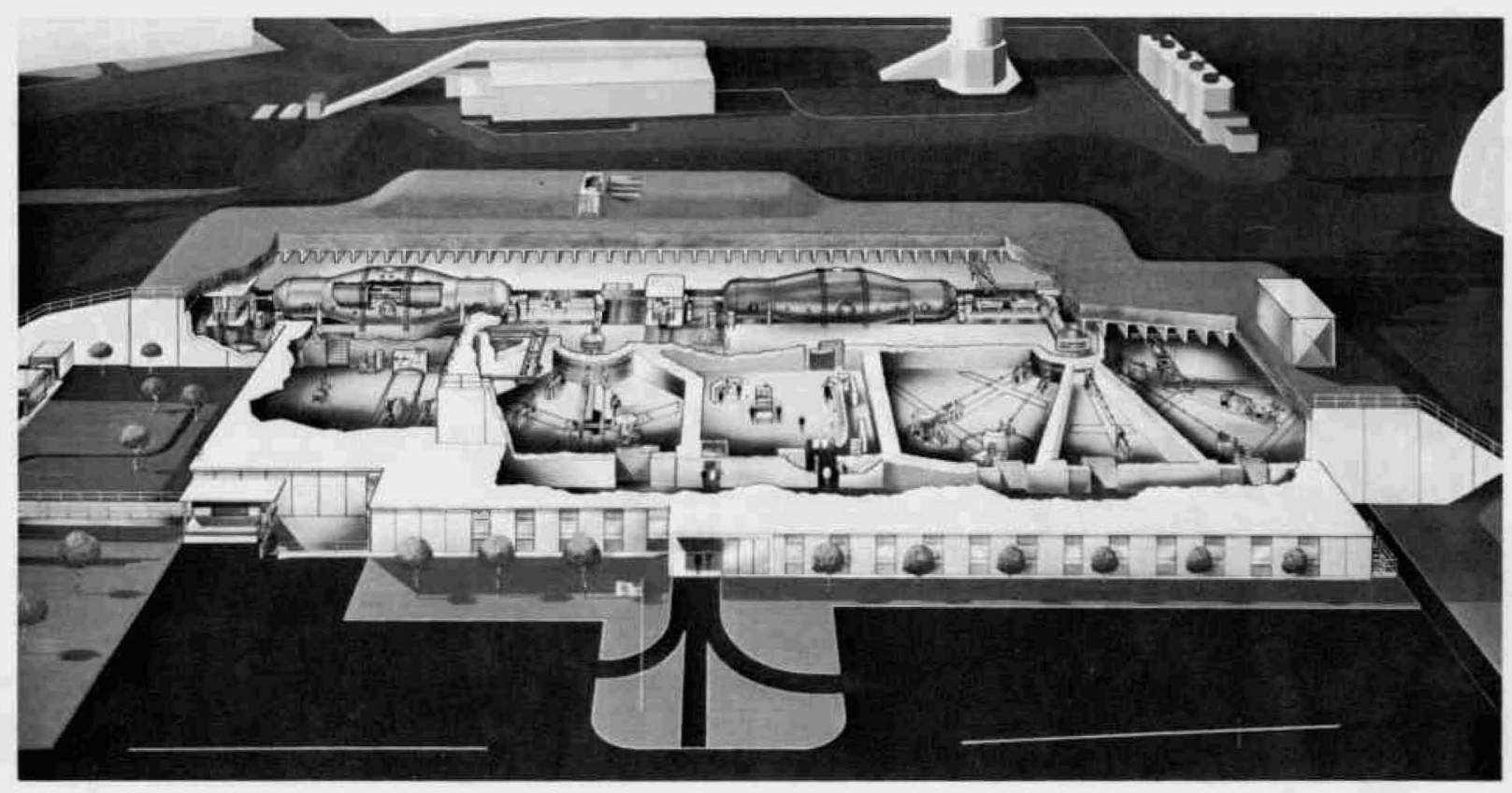

Cutaway drawing of the Tandem Van de Graaff Accelerator Facility as it will appear when construction and assembly are completed. 


\section{MAJOR RESEARCH FACILITIES UNDER DESIGN AND CONSTRUCTION}

\section{Tandem Van de Graaff Accelerator}

The Tandem Van de Graaff Facility currently under construction will consist of two High Voltage Engineering Corp. model MP Van de Graaff accelerators capable of being operated independently or in conjunction. Independently, each accelerator will provide protons with energies up to $20 \mathrm{MeV}$ and a large variety of heavier ions at higher energies. The two machines operating as a three-stage accelerator will provide protons with energies up to $30 \mathrm{MeV}$ and some heavier ions at higher energies.

The building for this facility is now almost finished, although the air-conditioning system, as well as certain portions of other systems, is yet to be completed. The building was occupied in January, and the assembly of the two accelerators began in February. At the end of March the second of the two accelerators was $15 \%$ complete. The present schedule calls for completion of the second accelerator and acceptance tests early in the fall, and of the first by mid-winter.

\section{GENERAL CONSTRUCTION PROGRAM}

During the year, construction was completed on the Lecture Hall-Cafeteria Building, and it was first available for staff activities in September. This facility has a gross area of $51,000 \mathrm{sq} \mathrm{ft}$ and includes a 750-seat cafeteria, a 450-seat lecture hall, and three meeting rooms for small groups. The lecture hall has been named Berkner Hall in honor of the late Lloyd V. Berkner, who served as President of Associated Universities, Inc., from 1951 to 1960.

In the AGS conversion program, for which $>\$ 35.2$ million has been allocated to date, almost all building and facility construction work is under contract, and several of the buildings are essentially completed. Four major new buildings became available for occupancy during the year. The $50,000-\mathrm{ft}^{2}$ addition to the East Experimental Building was turned over to the Accelerator Department; the Magnet Power Supply Building and the Rf System Building were completed to the extent that installation of equipment could commence; and the complex of buildings for the $200-\mathrm{MeV}$ linac was also completed, except for the final commissioning of the electrical and mechanical services. All major machine components for the conversion are now on order, and injection of 200 $\mathrm{MeV}$ protons into the AGS is expected to commence late in 1971.

\section{PERSONNEL}

The total number of employees at the Laboratory on June 30,1969, excluding temporary appointees, Research Collaborators, and guests, was 3261 , a net decrease of 76 from the 1968 figure.

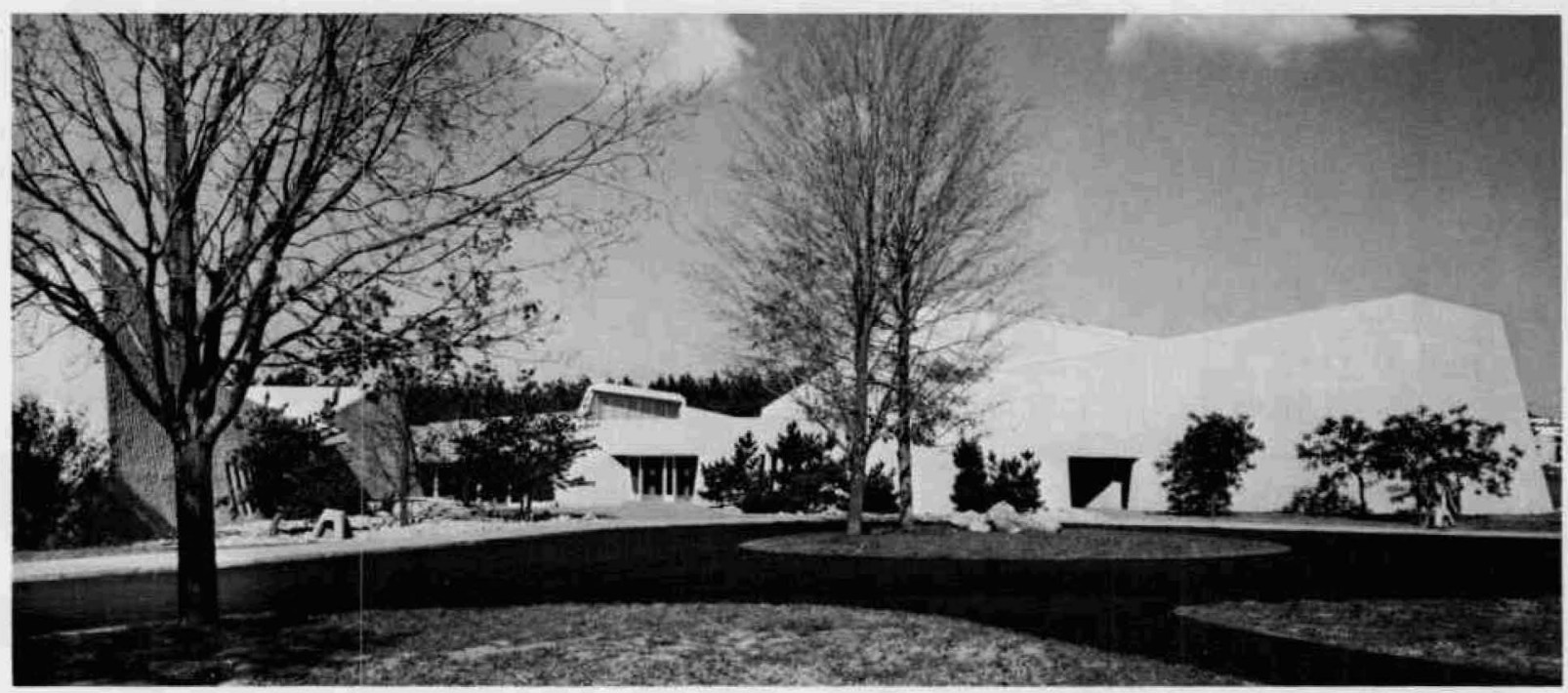

Exterior of the new Lecture Hall-Cafeteria Building, which was first available for Laboratory activities in September. 
During the year the regular scientific staff (Ph.D. or equivalent) decreased from 476 to 464 . Research Associates* decreased from 72 to 67 , and salaried visitors* increased from 28 to 29 , for a net decrease in salaried staff of 16 , from 576 to 560 . The nonsalaried scientific staff increased from 830 in

*These categories include students, For further details, see the section on Administration and Operations.
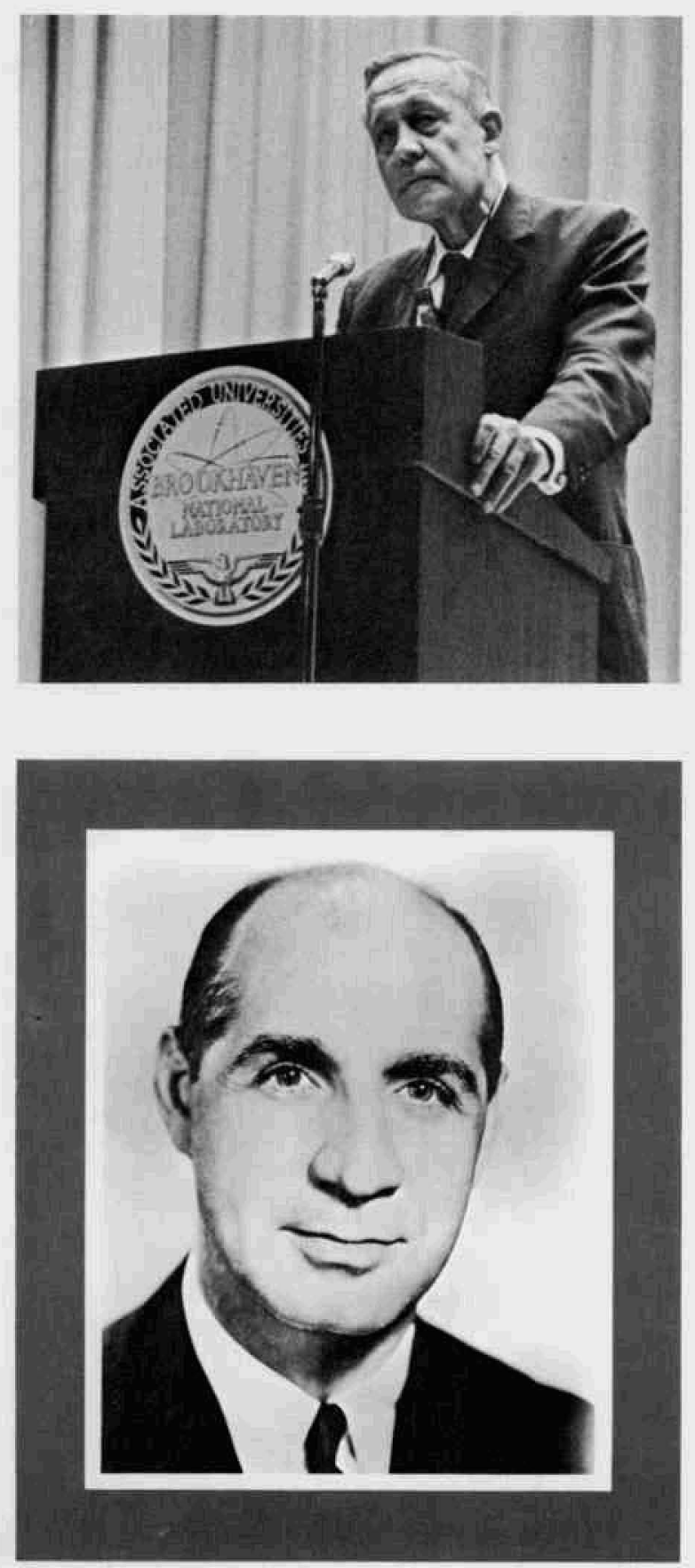

1968 to 922 in 1969 as of May 31 of these years. The latter visitors are not at Brookhaven on a fulltime basis. About one-fourth to one-third of them are on site at any one time. A total of 633 , as compared with 644 in 1968, visiting scientists and students worked at the Laboratory during the year; this number does not include Research Associates.

Summer visitors continue to play a significant role in the Laboratory's program. Arrangements have been made for 300 visiting scientists and students to work at Brookhaven during the summer of 1969 . Of this number, 133 are staff members from various educational and research institutions, and 167 are students.

\section{ADMINISTRATION}

The organization of the Laboratory and the corporate structure, both as of July 1, 1969, are shown on the Organization Chart and in other

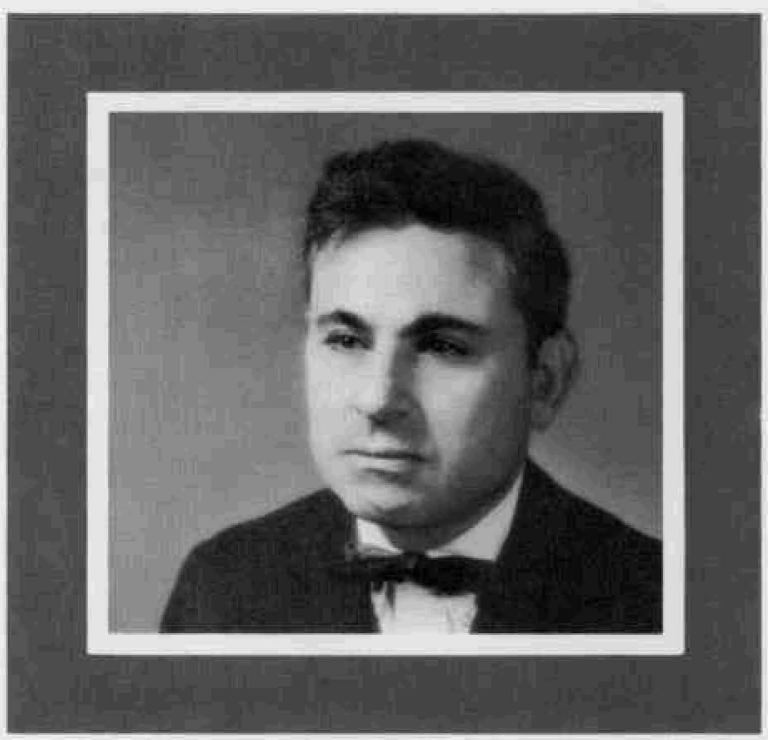

Lecturers at BNL during fiscal 1969. Professor Roger Revelle (top left), Director of the Harvard Center for Population Studies, gave the tenth annual series of George B. Pegram lectures on April 17, 22, and 24. The title of the series was "The Population Problem and What Can Be Done About It." The seventh AUI Distinguished Lecture was delivered on Nov, 7 by Professor Adam Yarmolinsky (top right), Professor of Law at Harvard University. His subject was "The Limits of Violence." The eighth AUI Distinguished Lecture, entitled "Shaping the Environment of Federal Laboratories," was given on March 20 by the Honorable Emilio Q. Daddario (left), member of the Congress of the United States and also of the Joint Committee on Atomic Energy. 


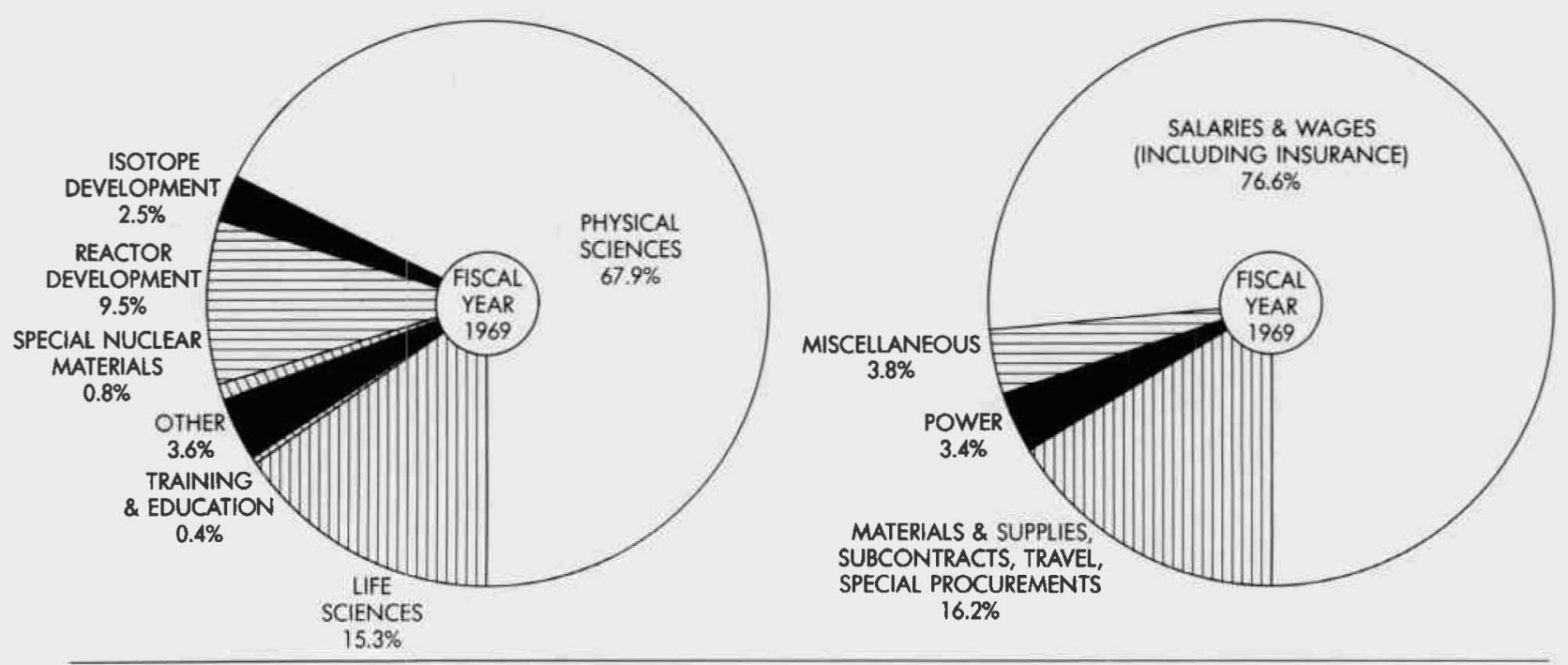

\begin{tabular}{|c|c|c|c|c|c|c|c|c|}
\hline \multirow[b]{2}{*}{$\begin{array}{l}\text { FISCAL } \\
\text { YEAR }\end{array}$} & \multirow[b]{2}{*}{$\begin{array}{l}\text { PHYSICAL } \\
\text { SCIENCES }\end{array}$} & \multirow[b]{2}{*}{$\begin{array}{c}\text { REACTOR } \\
\text { DEVELOPMENT }\end{array}$} & \multicolumn{3}{|c|}{ OPERATING COST DISTRIBUTION BY PROGRAMS } & \multirow[b]{2}{*}{$\begin{array}{c}\text { SPECIAL NUCLEAR } \\
\text { MATERIALS }\end{array}$} & \multirow[b]{2}{*}{$\begin{array}{l}\text { OTHER } \\
\text { (NET) }\end{array}$} & \multirow[b]{2}{*}{$\begin{array}{l}\text { TOTAL } \\
\text { COST }\end{array}$} \\
\hline & & & $\begin{array}{c}\text { LIFE } \\
\text { SCIENCES }\end{array}$ & $\begin{array}{c}\text { ISOTOPE } \\
\text { DEVELOPMENT }\end{array}$ & $\begin{array}{l}\text { TRAINING \& } \\
\text { EDUCATION }\end{array}$ & & & \\
\hline 1969 & $34,283,899$ & $4,771,541$ & $7,745,345$ & $1,257,277$ & 214,137 & 385,498 & $1,834,281$ & $50,491,978$ \\
\hline 1968 & $33,737,789$ & $5,098,856$ & $7,605,319$ & $1,209,620$ & 227,390 & - & $1,553,662$ & $49,432,636$ \\
\hline 1967 & $33,584,030$ & $5,947,334$ & $7,258,843$ & $1,212,747$ & 288,148 & - & $1,226,859$ & $49,517,961$ \\
\hline
\end{tabular}

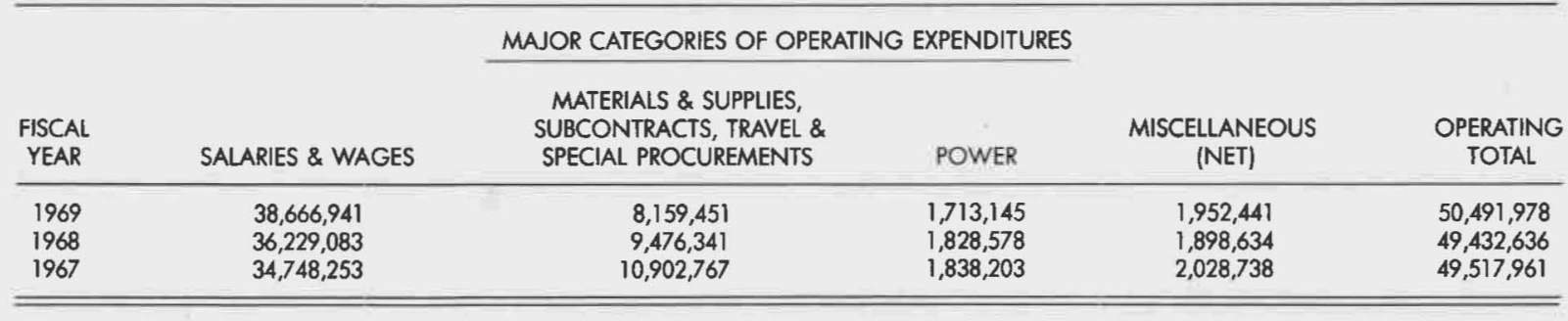

\section{BROOKHAVEN NATIONAL LABORATORY}

COMPARATIVE BALANCE SHEET

\section{Assets}

Cash

Accounts receivable

Advances, deposits, and prepaid expenses

Property, plant, and equipment (less reserves of $\$ 99,473,625$

at June 30,1969 , and $\$ 87,502,666$ at June 30,1968 )

Research materials and supplies

Total assets

Liabilities

Accounts payable

Accrued vacation

Accrued payroll

Atomic Energy Commission

Total liabilities
June 30, 1969

$\$ 74,070$

186,514

137,342

$181,627,014$

$12,961,990$

$\$ 194,986,930$

$\$ 5,307,053$

$3,443,037$

297,715

$185,939,125$

$\$ 194,986,930$
June 30, 1968

$\$ \quad 129,158$

191,602

68,042

$161,286,582$

$18,558,445$

$\$ 180,233,829$
$\$ 4,780,094$ $3,225,824$

271,750

$171,956,161$

$\$ 180,233,829$

NotE: Although the Laboratory has custody and use of the assets, title remains vested in the United States Government. 


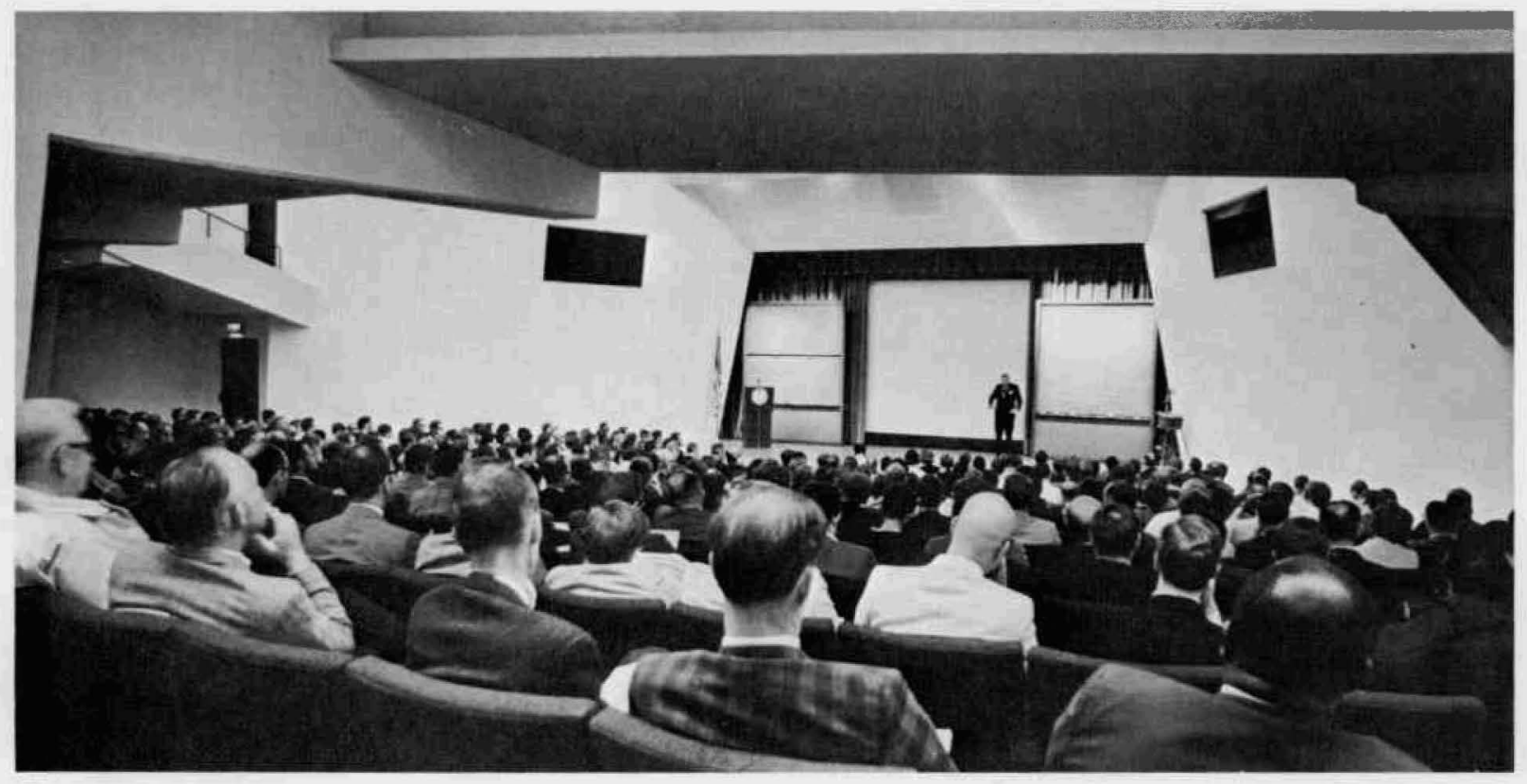

Session of the annual Biology Symposium, held May 26-28 in Berkner Hall in the newly opened Lecture Hall-Cafeteria Building.

material at the front of this report. At its annual meeting in October 1968 the AUI Board of Trustees re-elected the Controller, the Secretary, and the Assistant Secretary. Mr. L. Gard Wiggins was elected Treasurer to succeed Mr. Joseph F. Lord, who requested that his name not be proposed for re-election. Dr. Franklin A. Long, in accordance with the action at the 1967 annual meeting, continued as Chairman of the Board for a second year and in addition, as previously authorized by the Board, continued to exercise the powers of the President pending the selection of a new incumbent for that office. Some changes were made in the composition of the Board itself. Dr. Louis $\mathrm{H}$. Hempelmann was chosen for a three-year term as a University Trustee from the University of Rochester, succeeding Dr. Ernst W. Caspari, whose term expired at the annual meeting. Among the Trustees-at-Large, Dr. Bart J. Bok of the University of Arizona was elected for a three-year term, succeeding Dr. Fred T. Haddock, whose term expired at the annual meeting. In March 1969 Dr. Francis E. Low of Massachusetts Institute of Technology was elected a Trustee-at-Large for a term ending in October 1971. At the same time, Dr. Gerald F. Tape was elected President of AUI; he entered on the duties of this office on May 1, 1969.
Effective January 23, the name of the Nuclear Engineering Department at BNL was changed to Department of Applied Science, the more accurately to describe the current work of the Department, which is applied science research rather than engineering.

\section{FINANCE}

AEC-supported research at the Laboratory continued to be financed by the AEC Divisions of Reactor Development and Technology, Research (Physical Sciences), Biology and Medicine (Life Sciences), Training and Education, and Isotope Development. Operating costs for the work in each program and the major categories of operating expenditures are shown in the accompanying figure.

\section{CONFERENCES}

Ten major conferences were held at Brookhaven during fiscal 1969: International Conference on Lymphocytes, Sept. 12-13; Seminar on Medical Planning and Care in Radiation Accidents( sponsored by the US AEC), Oct. 21-23; IAEA Panel on Neutron Data Compilation, Feb. 10-14; Seminar on Radiation Chemical Processing, March 3-4; 
Subcommittee on Radiobiology to the Committee on Nuclear Science of the National Academy of Sciences, March 15-17; Lidar Uses in Atmospheric Research, April 15-16; High-Temperature Superconductors, May 1-2; Biology Symposium No. 22, entitled "Diversity and Stability in Ecological Systems," May 26-28; IAEA International Nuclear Data Committee, June 2-6, and Planning and Care for Radiation Accidents (sponsored by the US AEC), June 15-18. 


\author{
PHYSICAL \\ SCIENCES \\ AND \\ ENGINEERING
}




\section{Physics}

The physics research program consists of experimental and theoretical studies of the structure and fundamental properties of matter. These characteristics of matter can most effectively be studied by observing the interactions of charged particles, neutral particles, and radiation with individual atoms, atomic nuclei, or nucleons, and conglomerates of atoms and molecules in bulk matter. The Alternating Gradient Synchrotron, 60-inch cyclotron, Van de Graaff Accelerator, Graphite Research Reactor, and the High Flux Beam Research Reactor at Brookhaven provide many types of particles and/or radiation as well as a wide range of particle energies with which to carry out experimental investigations. The researches reported here are described under the categories of particle physics, medium energy physics, nuclear structure, neutron physics, solid state physics, and theoretical physics. The theoretical studies are pursued in conjunction with the experimental research and provide stimuli for development of new approaches to the problems.

The work of Brookhaven scientists is augmented by that of a number of visiting and guest scientists on leave from other institutions in this country and abroad. These visitors and guests are attracted to Brookhaven not only by the availability of its facilities but also by the opportunity to collaborate with Brookhaven scientists. The interchange of ideas and experience resulting from the presence of these visiting scientists is very important to the active research program.

\section{PARTICLE PHYSICS}

Most scientists believe that all phenomena of nature are based on laws that do not change with time and position and can eventually be understood. Quantum mechanics, which has been so successful in explaining atomic processes, is an outstanding example of this orderliness of nature. For phenomena involving nucleons no such complete understanding exists, despite the fact that these particles have been studied intensively for 20 years or more. Although a satisfactory explanation of particle physics still seems quite remote, the field continues to be of great interest because it is a frontier in the understanding of one of the most fundamental aspects of nature.

This section of the annual report describes the work done here by theoretical physicists on this problem and the work of experimental physicists who have used Brookhaven's remarkable resources for exploration of this field. A great many facts are known about the nature and behavior of the many fundamental particles that have been identified, and some simplification of these facts has been achieved through the formulation of conservation laws and invariance principles. Much of the work reported here has been directed toward testing the validity and extent of these concepts, which may provide the basis for an essentially complete understanding of particle physics.

The number of universities and laboratories sharing in the particle physics research at BNL clearly demonstrates Brookhaven's national nature. Over the years about two-thirds of the work at the Alternating Gradient Synchrotron (AGS) has been done by groups from these organizations. However, during calendar 1968 efficient scheduling of the AGS required that an unusually large fraction of the beam time (about $90 \%$ ) be allotted to the visiting university groups. More than 318 guest scientists participated in the program, including representatives of all the AUI institutions:

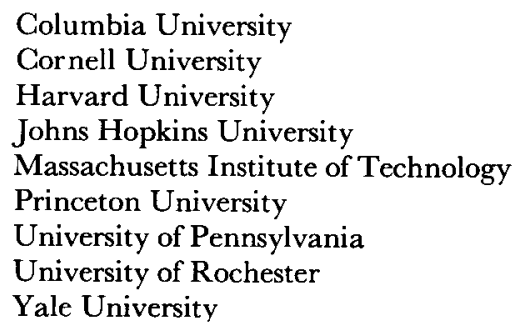

Scientists from the following academic centers also participated in the research:

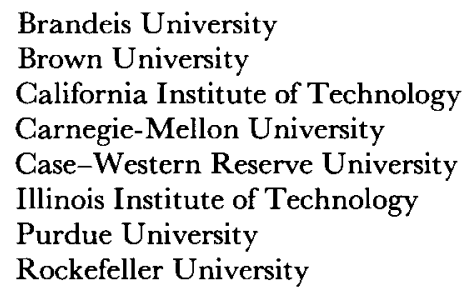


Rutgers University

Stanford University

State University of New York at Stony Brook

Syracuse University

Tufts University

University of California at Berkeley

University of California at Davis

University of California at La Jolla

University of California at Los Angeles

University of Chicago

University of Colorado

University of Kansas

University of Maryland

University of Massachusetts

University of Michigan

University of Notre Dame

University of Wisconsin

Vanderbilt University

Virginia Polytechnic Institute

Guest scientists also included representatives of the following foreign laboratories and universities:

University of Melbourne, Australia

McGill University, Montreal, Canada

University College, London, England

University of Bologna, Italy

University of Padua, Italy

CERN, Geneva, Switzerland

No attempt has been made to cover all the work in particle physics completed at BNL during the past fiscal year. However, a list of publications describing this work is included in Appendix A. The material that follows presents the highlights and a typical cross section of the research activity for this period.

\section{Introduction to AGS Research}

The goal of research at the AGS is comprehension of the nature of matter in terms of its fundamental building blocks and their properties. This requires a continuing search for new particles and antiparticles, as well as investigations designed to gain understanding of the forces that give rise to interactions among particles. The primary proton beam of the AGS is available at energies up to 33 $\mathrm{BeV}$. When allowed to strike various internal targets, this beam provides many beams of secondary particles, including high energy neutrinos. The primary beam itself is also ejected from the accelerator to provide, in short bursts, beams of very high energy for bubble chambers. In the "flat-top" mode it is ejected as a "slow" extracted beam (lasting about $0.4 \mathrm{sec}$ during each AGS pulse) that provides spark chamber-counter experiments with a high flux of protons at the highest possible energy and permits experimental setups at $0^{\circ}$ or at very small angles where the flux of secondary particles is maximum. The various beams available are used in experiments designed to search for new particles, resonances, and other phenomena. Studies are made of elastic and inelastic collisions of various particles with nucleons and nuclei in order to gain insight into strong, weak, and electromagnetic forces and interaction mechanisms. Observations made of the production, scattering, and decay processes of the baryons, mesons, and leptons lead to a determination of the intrinsic properties of the particles, such as charge, rest mass, spin, lifetime, decay modes, isospin, strangeness, magnetic moment, and parity, as well as the properties of their interactions with other particles.

AGS research has led to significant progress in the field of elementary particle physics during the past year. The following paragraphs describe only partially the progress made.

\section{AGS Research Activity}

Counter-Spark-Chamber Experiments. 1. Weak interactions. The study of weak interactions was the objective of many of the experiments performed at the AGS.

The charge asymmetry $\delta=\left(\Gamma_{+}-\Gamma_{-}\right) /\left(\Gamma_{+}+\right.$ $\left.\Gamma_{-}\right)$, where $\Gamma_{+}$and $\Gamma_{-}$are the partial decay rates of the long-lived neutral $K$ meson (kaon) to $e^{+} \pi^{-} \nu$ and $e^{-} \pi^{+} \nu$, was measured by a Columbia University group in a counter experiment performed in the $-20^{\circ}$ neutral beam at the AGS. The charge asymmetry $\delta$ is related to the other $C P$ (charge conjugation, parity) violating parameters, $\eta_{+-}$and $\eta_{00}$, through the parameters $\varepsilon$ and $\varepsilon^{\prime}$ : $\eta_{+-}=\varepsilon+\varepsilon^{\prime}, \quad \eta_{00}=\varepsilon-2 \varepsilon^{\prime}$, and $\delta \cong 2 \operatorname{Re} \varepsilon$. The knowledge of $\varepsilon$ can aid in selecting the correct theory of $C P$ violation and provides a consistency check on experimental values of $\eta_{+-}$and $\eta_{00}$.

The electric charge signs of the pion and electron were determined by hodoscope arrays, while electron identification was provided by a large gas Cerenkov counter. This technique is an extension of previous work at the AGS but was improved as follows: (1) Interactions of the decay products that would confuse identification were reduced by decreasing the mass of the detector, and (2) a special electronic arrangement provided two distinct resolution times for each event. The latter technique permits an accurate measurement of the rate and charge asymmetry of accidental coincidences (all 
possible combinations). This arrangement also allows the separation of time-correlated extra counts such as delta rays and pion interactions from uncorrelated extra counts. The corrections to $\delta$ due to these effects can be made with good accuracy.

Data from the experiment were continually analyzed by the BNL On-Line Data-Processing Facility (OLDF). Use of the computer made it feasible to collect the necessarily large number of events and to perform the required careful search for possible asymmetries in the apparatus which might have obscured the results. Preliminary results give $\delta=(3.15 \pm 0.6) \times 10^{-3}$, consistent with models such as the superweak model giving $\varepsilon^{\prime}=0$.

The analysis of an experiment carried out by a University of Maryland-BNL team in the previous year is nearly complete. One part of the experiment provided a new experimental technique to check previous work on $K_{e 3}$ decays which had given results inconsistent with the $|\Delta I|=1 / 2$ rule. The second part sought to determine whether charge asymmetry existed in the Dalitz plot of $K_{\pi 3}$ decays, which would be evidence for a theory that attributes the $C P$ violation in $K_{2}{ }^{0}$ decay to the decay amplitude $(\Delta I=1 / 2) . K_{2}{ }^{0}$ decays were recorded in a double wide-gap spark chamber with a lead radiator in its center, located in a magnetic field. $K_{\pi 3}$ and $K_{e 3}$ decays were identified by showers created in the radiator by $\gamma$ rays and electrons, respectively. Analysis involving Monte Carlo techniques of about one-half of the events (1080, after cuts) gave, for the coefficient of the energy dependence of the $K_{\mathrm{e} 3}$ form factor, $\lambda=0.043_{-0.035}^{+0.040}$, in good agreement with previous results. The result for the charge asymmetry in $K_{\pi 3}$ (from 1340 events) was $0.15 \pm 0.027$.

A University of Rochester group completed the analysis of a measurement made last year of the interference in the decay of the $K_{S}{ }^{0}$ and $K_{L}{ }^{0}$ into two neutral pions. The aim was to provide further information for the study of the $K^{0}$ meson. It was shown in a direct manner that the quantity $\varepsilon^{\prime}\left[\equiv\left\langle K_{L}{ }^{0}|T| \pi^{0} \pi^{0}(I=2)\right\rangle\right]$ is $\left\langle 1 \times 10^{-3}\right.$ in magnitude at the $90 \%$ confidence level. Alternatively, the result was expressed by stating that a third quadrant solution for the phase of

$$
\eta_{00}\left(\equiv\left\langle K_{L}^{0}|T| \pi^{0} \pi^{0}\right\rangle\right)
$$

is excluded by two standard deviations; a first quadrant solution is acceptable.

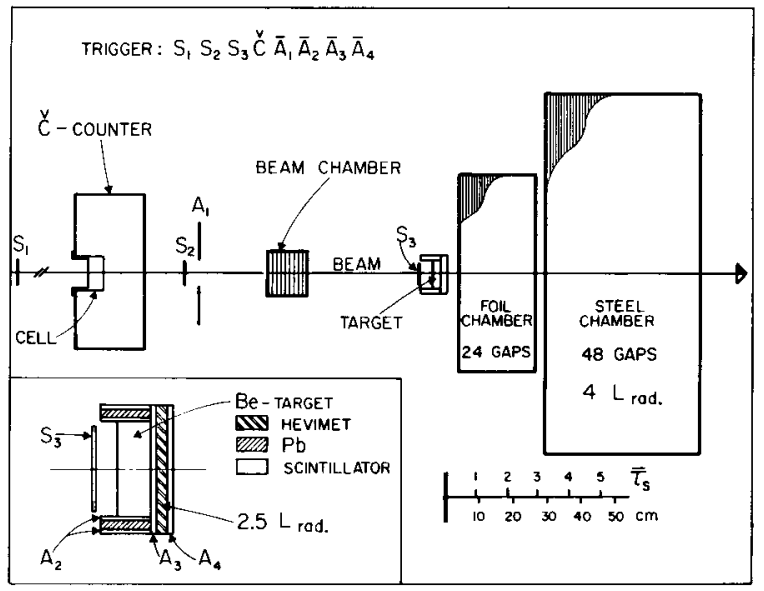

Figure 1. Schematic layout of experiment to measure the charged-to-neutral branching ratio of the $K_{s}{ }^{0}$ decay. $S_{1}, S_{2}$, and $S_{3}$ are scintillation counter telescopes. $A_{1}$ to $A_{4}$ are veto counters. $\mathrm{C}$ is the Cerenkov counter.

Using the equipment and insight gained during this work, the same group moved into the AGS test beam six months later and performed a precision measurement of the charged-to-neutral branching ratio of the $K_{S^{0}}$, an important parameter in the phenomenology of kaon physics. Such a measurement provides useful information on the nature of the violation of the $|\Delta I|=1 / 2$ rule in the decay $K \rightarrow \pi \pi$.

A schematic layout of the experiment is shown in Figure 1. An unseparated beam of $2-\mathrm{BeV} / c K^{+}$ mesons was detected by a counter telescope and a liquid-cell differential Gerenkov counter which rejected $\pi^{+}, p$, and $\mu^{+}$. A source of $K_{S}{ }^{0}$ 's was obtained by the charge exchange $K+n \rightarrow p K^{0}$ in a $2.5-\mathrm{cm}$ thick beryllium target surrounded by an arrangement of veto counters, lead, and tungsten $\gamma$ converters. This served to define the charge-exchange trigger and effectively suppressed large momentum transfers and inelastic charge exchanges. Decays were studied in a well-defined fiducial volume $27 \mathrm{~cm}\left(=2.8 K_{1}{ }^{0}\right.$ mean decay lengths) in extent. This region contained a 24 -gap aluminumfoil spark chamber which registered charged vee decays. Neutral events were detected by $\gamma$ conversion in a 2.0-radiation-length 49-gap steel spark chamber. No detection counters except the target vetoes and the $K+$ defining telescope were used. A 6-gap beam spark chamber registered the entry point of the $K^{+}$into the beryllium target. This information permits the kinematical reconstruction 


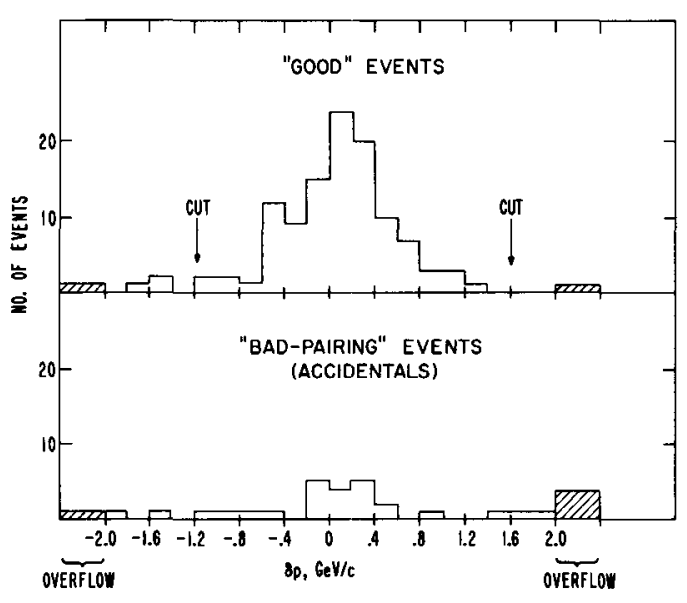

Figure 2. Number of events vs difference between total momentum of the final three particles and incident momentum.

of the vee decays and yields angular and momentum distributions for the $K_{1}{ }^{0}$. The corrected branching ratio was found to be $C / \mathcal{N}=2.285 \pm$ 0.055 and is to be compared with the $|\Delta I|=1 / 2$ rule prediction of 2.00 .

A search for the $W$ boson, presumed to mediate weak interactions, was conducted by a Yale University-BNL team. Measurements were made of the polarization and flux of high energy muons produced through the interaction of $28-\mathrm{BeV}$ protons with uranium. Protons from the slow external beams of the AGS were focused on a target constructed of sections that could be separated to vary the effective target density. Forward muons passed through a magnet which directed the positive muons through a thick steel shield into a rangepolarization detector consisting of 234 -in. aluminum slabs separated by scintillation counters. The stopped muons precessed in a magnetic field imposed on the detector. The muon range, the decay time, and the direction of the decay electron were recorded. The variation of the forward-backward ratio with time determined the muon polarization indeperident of many biases. The muon flux from the decay of mesons varies inversely with the target density, and the polarization direction is opposite to the beam direction. In contrast, the muon flux from $W$ decays should be independent of the density, and the polarization would be aligned with the beam. A preliminary analysis, based on an extrapolation to infinite target density, suggests that the cross section for the production of $W$ 's by proton-nucleon $(p-\mathcal{N})$ interactions is $<5$

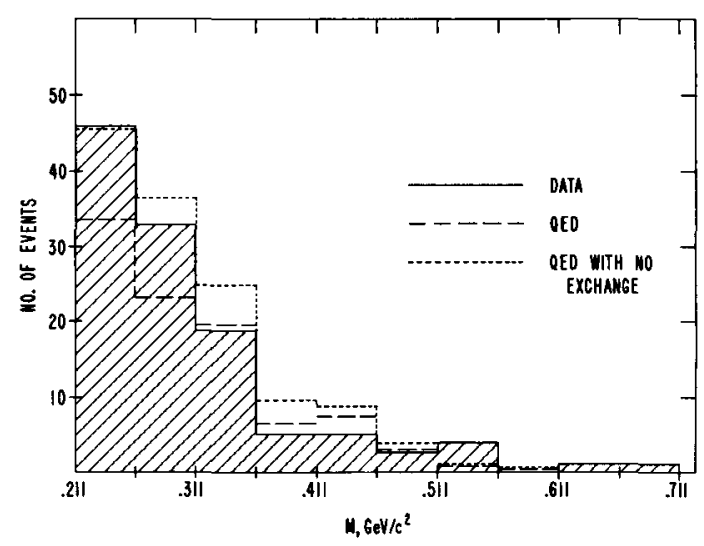

Figure 3. Number of events vs invariant mass of oppositely charged muons. Data show rough agreement with quantum electrodynamics $(Q E D)$.

$\times 10^{-36} \mathrm{~cm}^{2}$ if the $W$ decays to a muon about $25 \%$ of the time.

A team from Harvard, Massachusetts, and McGill Universities observed the direct production of muon pairs by muons in the field of a lead nucleus, a process whose cross section is sensitive to muon statistics because there are two identical muons in the final state. A $12-\mathrm{BeV} / c$ muon beam traversed a spectrometer before striking a $10-\mathrm{cm}-$ thick lead target. Optical spark chambers were triggered when at least two of the three final-state muons (tridents) passed through a large-aperture magnet, shower spark chambers, a 1-m-thick iron absorber, and an array of triggering counters. The vector momenta of all the initial and final-state energies equaled the incident energy. A preliminary analysis of the data has yielded 85 good events.

Configurations in which the two identical fermions overlap in phase space should be suppressed. This shows up as a suppression of low mass states in the invariant mass distribution of the two like particles. In this experiment with $12-\mathrm{BeV}$ incident muons, the requirement that the three final particles each have energy $>1.5 \mathrm{BeV}$ constrains the phase space so that all the particles tend to overlap. This greatly enhances the sensitivity so that the observed cross section should be reduced by $25 \%$ because of the exchange effect.

The experiment was designed to provide the first measurement of the exclusion principle for identical muons. The pictures were scanned, and those having at least two tracks in the upstream 
spark chamber and three in the downstream chamber were selected. In all, 215 candidates were found and measured in an attempt to reconstruct the momenta of all three particles. There were 117 good fits (with acceptable $\chi^{2}$ 's). There were $69 \mathrm{bad}$ fits from accidental events. For all the events giving an acceptable $\chi^{2}$ for the momentum analysis the total final momentum was computed and compared with the measured momentum of the incident muon. Figure 2 shows a nice peak about $\delta p=0$ for the good events but no peak for the accidentals. In this beautiful evidence of tridents, one muon is incident and three particles are outgoing. The latter have properly balanced momentum and in addition penetrate a 1-m-thick steel wall. The comparison with theory is shown in Figure 3. Because of some unfitted events and the approximate way in which the beam spectrum correction was made, it is not possible at this stage of the analysis to say more than that muon tridents were observed with a cross section in agreement with quantum electrodynamics (QED). It is possible to observe clearly the exclusion principle with these data, but first a precise study must be made of the detection efficiency vs incident momentum.

The analysis of a previously performed AGS experiment to observe the inelastic scattering of 12$\mathrm{BeV}$ muons was completed by a team from Harvard, Cornell, McGill, and Case-Western Reserve Universities. The first part of the experiment was a test of quantum electrodynamics for the muon by observing muon bremsstrahlung. The muon propagator was tested to an invariant fourmomentum of $600 \mathrm{MeV} / c$, and the measurement agrees with the predictions of the theory. A lower limit for the $\Lambda$ parameter is measured to be 0.73 $\mathrm{BeV} / \mathrm{c}$ at the $95 \%$ confidence limit. In the second part, the total photoproduction cross section for $\gamma$ rays incident on the carbon nucleus was measured by extrapolating the inelastic muon cross section to zero four-momentum transfer. The result was that $\sigma_{\gamma c}=1.62 \pm 0.19 \mathrm{mb}$.

A search for heavy muon pairs produced in 30$\mathrm{BeV}$ proton interactions was conducted by physicists from Columbia and CERN, with use of the slow external beam at the AGS. The experiment is sensitive to pairs having effective masses between 1 and $6 \mathrm{BeV} / c^{2}$ and production cross sections as small as $10^{-37} \mathrm{~cm}^{2}$.

The muon pairs are produced when the high energy protons strike a thick uranium target. A massive steel wall absorbs all strongly interacting products ( $\pi$ 's, $p$ 's, etc.) but allows the muons to pass through to the detection apparatus. A large array of scintillation counters serves to measure the vector momentum of each of the two muons by determining both the angle of the trajectory and its momentum by range. These measurements allow a determination of the di-muon mass to about $10 \%$, which furnishes the primary event signature. A large background results from accidental coincidences of random muons from those pions that decayed before interacting in the absorber. By simultaneously monitoring real and delayed coincidences, the true di-muon signal may be extracted by subtraction. The PDP- 6 computer of the OLDF is used on-line to reduce the enormous bulk of raw data to a compact useful form and to monitor the performance of the experiment. To date, the analysis is half completed and shows that $\approx 1 \%$ of the events are indeed "real" muon pairs, exponentially distributed over the mass range of interest. Whether or not there is any structure in the mass spectrum, indicative perhaps of the existence of a new massive vector meson, is an open question awaiting further data and an improved understanding of the experiment.

A group from the University of California at La Jolla completed an experiment to study the decay $K^{+} \rightarrow \pi^{+} \pi^{0} \gamma$ and the corresponding decay $K^{-} \rightarrow$ $\pi^{-} \pi^{0} \gamma$. The purpose of the experiment was to find out whether there is a charge asymmetry between these two types of decay. They should be completely symmetric except for violation of $C P$ symmetry.

In order to observe any effect of $C P$ violation, the final decay state of the kaons must be a superposition of two different isospin eigenstates. This could happen in this particular decay because two channels are available to reach the final state, namely, electromagnetic bremsstrahlung and direct photon emission. It is not definitely known whether both such states are actually present in the final product. However, with an adequate number of events (even of one sign only), it should be possible to make a study of the energy spectra of the $\gamma$ rays and of the pions in order to conclude for or against the presence of two states. If the answer is affirmative, a comparison of the rate for positive charges with that for negative charges would make sense, and any discrepancy, if filtered through a severe critique of the functioning of the apparatus, would be an evidence of $C P$ violation in such decays. 


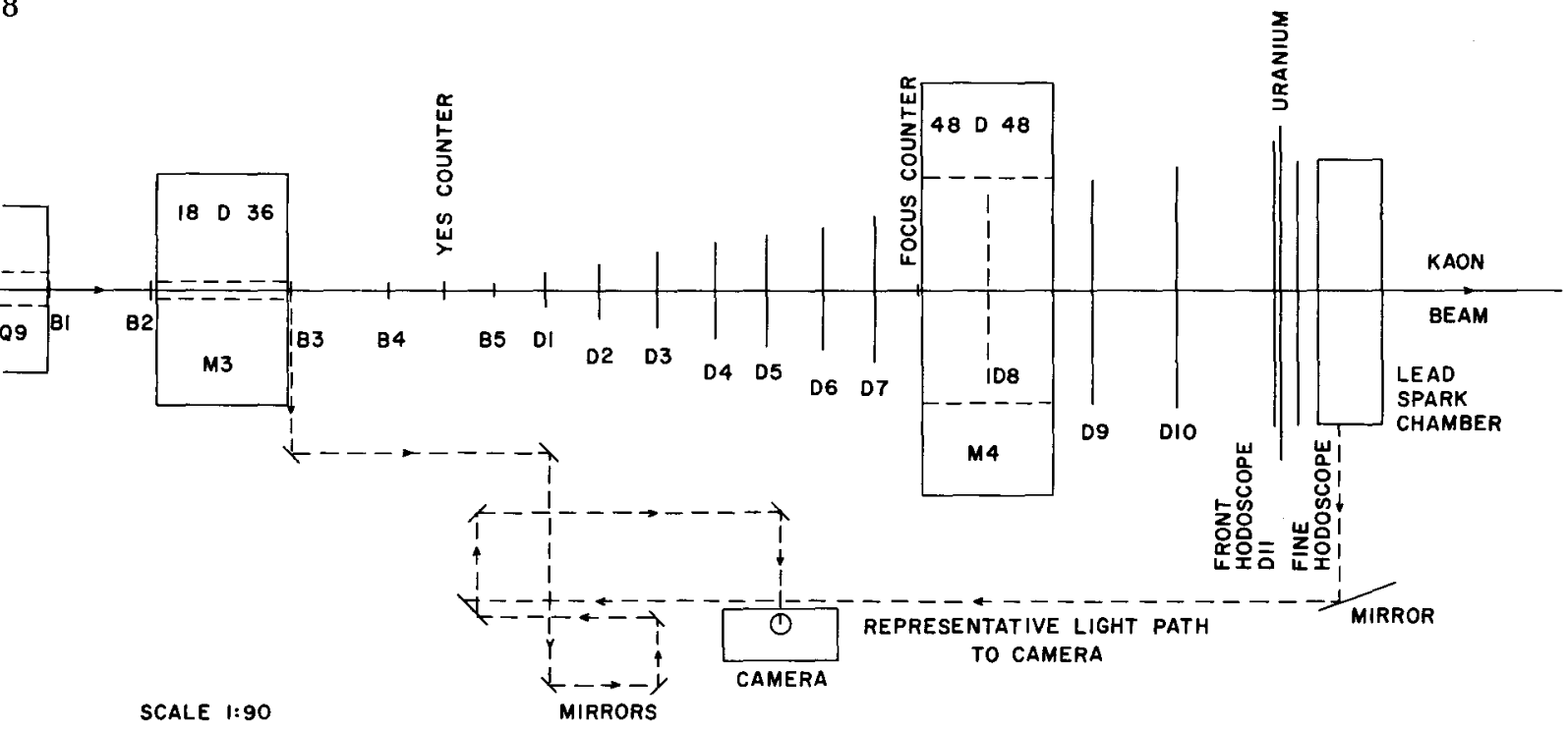

Figure 4. Plan view of the arrangement for the experiment performed by the group from the University of California at La Jolla. Only a few typical mirrors are shown.

The reason for suspecting an asymmetry lies in the suggestion made, mainly by T.D. Lee, that the electromagnetic interaction of particles having strong interactions, such as kaons, pions, and nucleons, may indeed show a $C P$ violation when $C$ and $P$ are defined, as is commonly done, on the basis of strongly interacting particles.

Figure 4 is a schematic representation of the experiment. A nonseparated beam of mesons, kaons, and protons of $6 \mathrm{BeV} / c$ enters the apparatus from the left, after passing through a Cerenkov counter (not shown) which distinguishes kaons from the rest of the beam. A series of spark chambers $\left(D_{1}\right.$ through $D_{7}$ ) then extends longitudinally, along the direction of the beam, for a distance of some $40 \mathrm{ft}$. The spark chambers are of small size near the beam entrance and of progressively larger size toward the exit in order to accept the products of the decay of the kaons. The definition of a decay event consists in requiring that the kaon pass through the yes counter and not through the focus counter, which is located in the focal point of the beam. Some $5 \%$ of the particles decay between the yes and the focus counters. The charged pion passes through the large magnet (M4) and its trajectory is determined by the sparks in chambers $D-8, D-9$, and $D-10$. The two $\gamma$ rays from the $\pi^{0}$, together with the extra $\gamma$ ray produced in the decay, will generate showers in the large $(8 \times 8$ - $\mathrm{ft})$ uranium plate, which is followed by a hodoscope of 240 counters. The electronic logic connected to the hodoscope demands that three independent showers be formed. A large lead chamber, made of 20 single spark chambers separated by $1-\mathrm{mm}$ lead plates, is located after the hodoscope. All the other chambers, $B-1$ through $D-11$, are at a distance from each other considerably larger than their own gap dimensions. A complex system of mirrors makes the spark chambers appear to the camera as if they were adjacent to one another, and the top view adjacent to the side view (see Figure 5). In this fashion, the film image size is only $0.75 \mathrm{in}$. long. Yet the maximum possible transverse dimension of the film, about $32 \mathrm{~mm}$, is entirely used for the largest chamber, for best resolution. Thus it was possible to take pictures at a high rate of occurrence during a machine pulse. The camera constructed by the group was capable of taking a picture every $10 \mathrm{msec}$, even though the recovery of the spark chambers and the intensity of the background were such as to preclude taking pictures at such a rate.

The experiment ran for $\approx 250 \mathrm{hr}$, about equally divided between positive and negative kaons, and the $\approx 750,000$ pictures taken should yield a few thousand events. The analysis is in progress.

Preparations by a BNL group to repeat this important measurement with use of a different technique have been completed. The experiment was planned so that $10^{4} \pi^{ \pm}+\pi^{0}+\gamma$ events could be detected. The equipment for the experiment consists, in part, of a wire spark-chamber spectrom- 


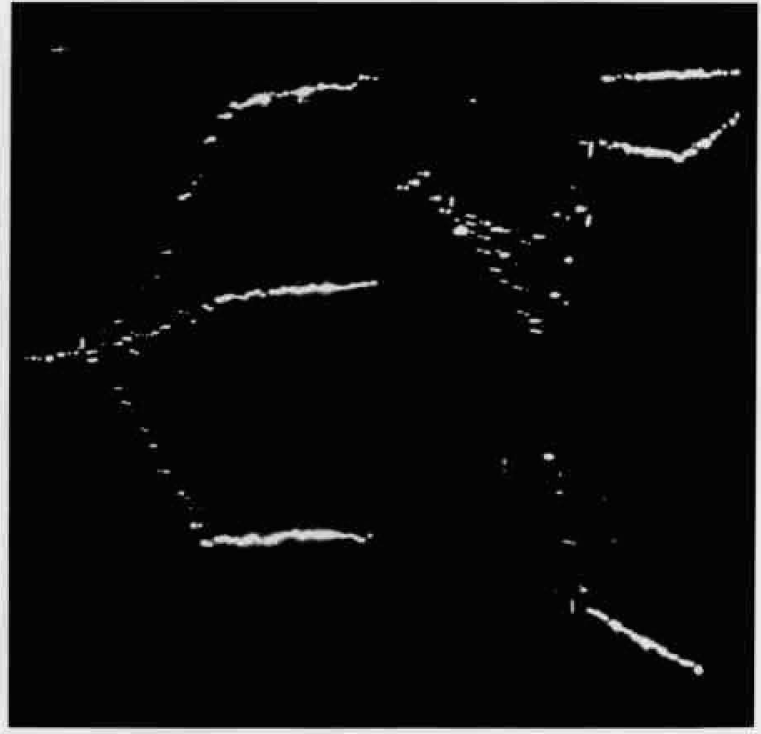

Figure 5. Photograph of a tau decay $\left(\pi^{+} \rightarrow \pi^{+} \pi^{+} \pi^{-}\right)$in the spark chambers used in the University of California experiment to study $\pi^{ \pm} \rightarrow \pi^{ \pm} \pi \mp \gamma$. The mirror system has projected both a top and a side view of the event onto one frame of the film.

eter which is used to locate the incoming $K^{ \pm}$ mesons and outgoing $\pi^{ \pm}$mesons. All tests carried out on the spectrometer so far indicate a time and space resolution corresponding to that planned originally. The information from the wire chambers is fed directly to the OLDF at the AGS. The two photons from the $\pi^{0}$-meson decay and the third decay photon are observed by means of a shower detector consisting of eight layers of lead, scintillators, and optical chambers. The $\gamma$ ray detector has also successfully passed all stages of testing and has been incorporated with the spectrometer. Some preliminary $\pi^{ \pm}+\pi^{0}+\gamma$ data were taken before the AGS was shut down in May. Information from the on-line wire spark-chamber spectrometer is used to prescan all the data and indicate the likely events. For these the photon showers, photographed in the optical chambers, will be hand measured. Columbia University's neutrino chambers have been erected behind the wire spark-chamber spectrometer for simultaneous study of $K_{\mu 3}$ decay and polarization. They have sufficient stopping power to bring muons from $K_{\mu 3}$ decay to rest and to allow the decay electrons to be observed and photographed. A sample of several thousand $K_{\mu 3}$ decay events has been recorded and is being analyzed.
A group from Princeton University has studied at the AGS the decay of the $K^{+}$and $K^{-}$mesons into the $\tau$ mode $\left(K^{ \pm} \rightarrow \pi^{ \pm} \pi^{ \pm} \pi^{\mp}\right)$. A sample of $>1$ million $\tau$ decays of each sign has been obtained. The comparison between the two matrix elements characterizing these decays constitutes a test of $C P$ invariance. For example, any difference between the energy spectra of the odd pions (i.e., between the $\pi^{-}$from $\tau^{+}$and the $\pi^{+}$from $\tau^{-}$) would constitute a violation of $C P$ invariance.

The experimental method was based on the detection of $\tau$ decays in flight and on the recording, in wire spark chambers, of the paths of the decay pions through a magnetic spectrometer. Figure 6 shows the experimental arrangement around the spectrometer magnet. The partially separated 3.0$\mathrm{BeV} / c K$-meson beam enters the 418-cm-long decay region (He bag) from the left. The three decay pions, detected in an array of counters mounted on a 5 -ft wheel, traverse five $1 \times 1-\mathrm{m}$ wire spark chambers before entering the magnet. More spark chambers and an array of scintillation counters, located on the other side of the magnet, serve to record the path of the odd decay pion.

The experiment was monitored on-line by two computers, a PDP-9 belonging to the experimenters, and the BNL OLDF's PDP- 6 computer. The main tasks of the PDP-9 (8K, 18-bit word memory) were to perform continuous checks on the apparatus and to transfer raw data to magnetic tapes. The PDP-6 (82K, 36-bit word memory) performed the complete analysis of a fraction of the events (20 to $40 \%$, depending on the processor time available). The experiment proceeded very successfully, and $\approx 2$ million events are in the process of analysis.

An MIT group completed an experiment to test the selection rule $\Delta S=\Delta Q$ in the decay $K^{0} \rightarrow$ $\pi^{ \pm} e^{\mp} \nu$. This selection rule states that the reaction $K^{0} \rightarrow \pi^{-}+e^{+}+\nu$ does occur, but that the charge conjugate reaction $K^{0} \rightarrow \pi^{+}+e^{-}+\nu$ does not.

$K^{+}$mesons with a momentum of $3 \mathrm{BeV} / c$ produced at the AGS's G-10 target were specially selected in the $10^{\circ}$ external separated beam. They were then allowed to charge-exchange in a $2-\mathrm{cm}$ thick platinum target in the apparatus to produce about 20 usable $K^{0}$ 's per machine pulse. The subsequent decays of these $K^{0}$ 's were observed in a system of spark chambers immersed in a $14.5-\mathrm{kG}$ magnetic field. This provided a measurement of the momentum and direction of both the electron and the pion. A lead-plate spark chamber was used 

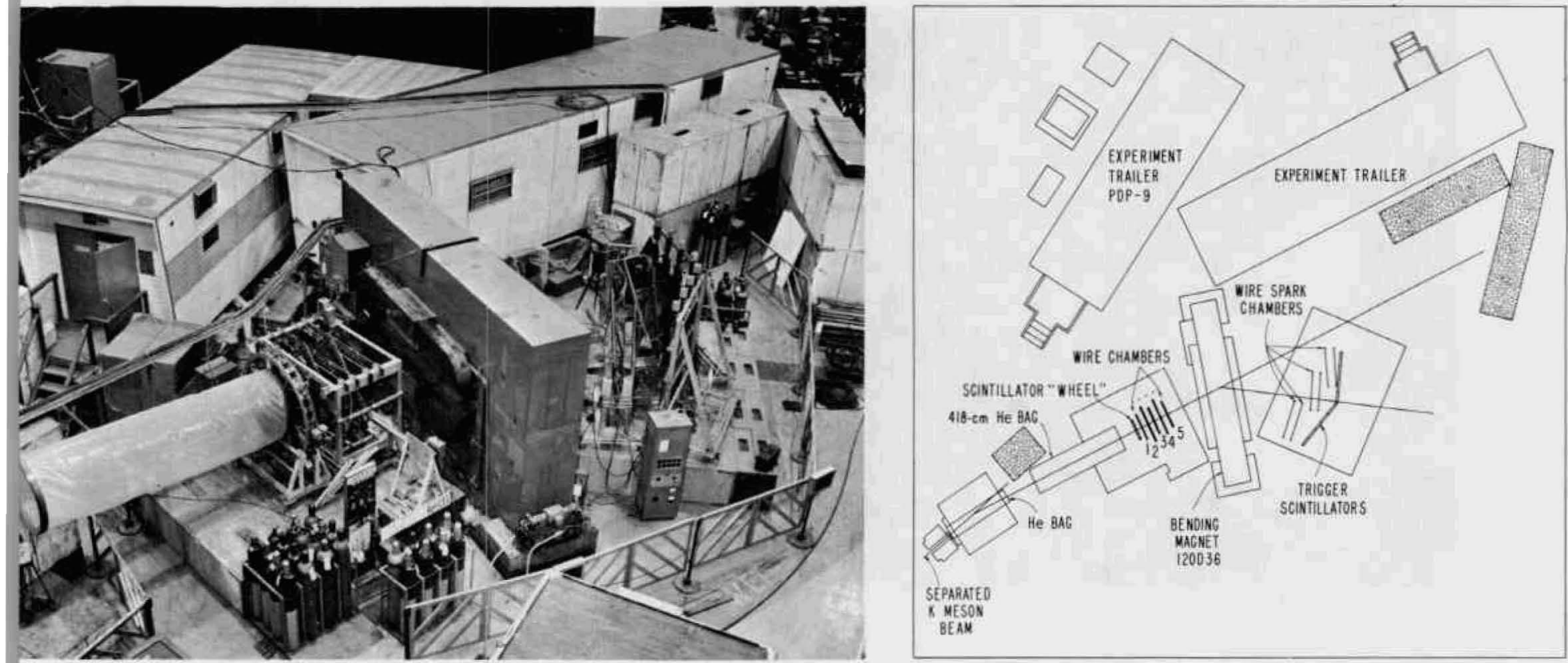

Figure 6. Photograph and schematic of the Princeton group's experimental arrangement for testing CP violation in $\tau$ decay.

to observe the characteristic electron shower signature, and a selective trigger was provided by various scintillation counters and a lead-Lucite Cerenkov counter. (See Figure 7.)

During the experiment $>2$ million spark-chamber pictures were taken, of which about $25 \%$ have been analyzed to date. Although final results must await analysis of the remaining pictures and a more detailed background study, it is already clear that about $700 K^{0} \rightarrow \pi+\ell+\nu$ events in the first two $K_{1}{ }^{0}$ lifetimes should be observed and that the major objectives in suppressing backgrounds have been met.

2. Strong interactions. The study of strong interactions occupied the attention of many of the experimentalists working at the AGS. The work on strong interactions involves searching for new particles or resonances and determining their properties. Usually, a resonance is detected by plotting the mass spectrum derived from experimental data; if a sudden peak or "bump" appears in the mass spectrum it is often due to a resonance. For a system of particles comprising such a bump to be classified as a resonance, it must be describable in terms of a well-defined set of numbers (quantum numbers) which include spin, parity, isospin $(I)$,
$G$-parity, strangeness, charge conjugation, and baryon number. The experimenter determines as many of these quantum numbers as possible by investigating in detail how the resonances are formed and subsequently decay into other particles or resonances. It is important to determine the quantum numbers of the elementary particles, so that the particles can be classified into families or groups whose members have certain common properties. For example, the pi mesons $\left(\pi^{+}, \pi^{-}, \pi^{0}\right)$, $K$ mesons $\left(K^{+}, K^{-}, K^{0}, \bar{K}^{0}\right)$, and the eta meson $\left(\eta^{0}\right)$ make up such a group. These eight particles have the same spin and parity quantum numbers but different isospin and/or strangeness quantum numbers and, according to the group theoretical symmetry scheme $S U(3)$, belong to the same family. Categorizing particles in such a way enables theorists to predict the masses of undiscovered particles and helps them to make other important calculations.

The experimenter is also concerned with the detailed mechanisms by which particles are produced. The collision process can be loosely described as either "peripheral" or "central." As the names imply, a central collision refers to the case in which the beam particle hits the target particle 


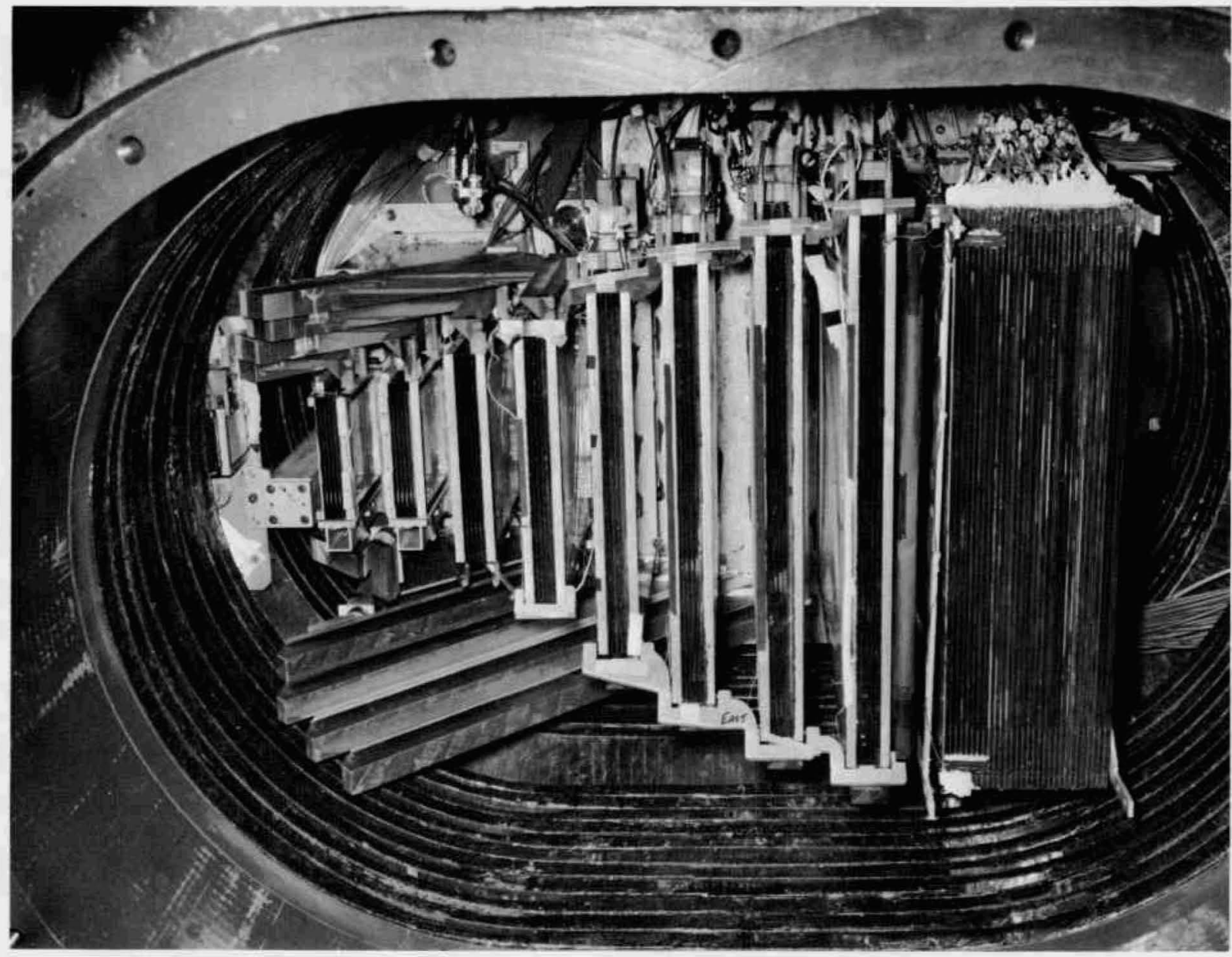

Figure 7. Photograph of the nine sets of spark chambers installed in the gap of the large magnet ("Argo") brought to Brookhaven by the MIT group studying $K^{0}$ decay. The whole spark-chamber system was thus immersed in a 14.5-kG magnetic field. $K^{+}$mesons enter from the left and strike the platinum target placed just inside the magnet coils.

head on, whereas a peripheral collision occurs when the two particles hit each other a glancing blow. One way of visualizing what happens in a peripheral interaction is to picture the beam particle transferring a small amount of momentum to the target particle while the beam and/or target particle becomes excited to a higher mass state and subsequently decays into two or more particles. Thus, the result of a peripheral interaction is the production of a "beamlike" set of particles whose total momentum is nearly that of the beam, and/or a "targetlike" set of particles whose total laboratory momentum is very small. A central collision is thought to occur when many relatively uncorre- lated particles are produced. Such reactions are analyzed in terms of statistical or thermodynamical models. Experimentally, most reactions observed are peripheral in nature.

To obtain a quantitative measure of the degree to which a reaction is peripheral, the variable $t$ (four-momentum transfer squared) is used. Continuing in the vein described above, $t$ depends on the change in momentum and angle between a colliding particle and the higher mass state into which it transforms as a result of the collision. For a given final state, the more peripheral the reaction, the smaller the magnitude of $t$.

Data obtained last year by a BNL-CarnegieMellon University team, using a wire spark-chamber spectrometer, have been partially analyzed. An extensive run at the AGS has established the effectiveness of a particle spectrometer system of this type, which has proved to have important advantages. 


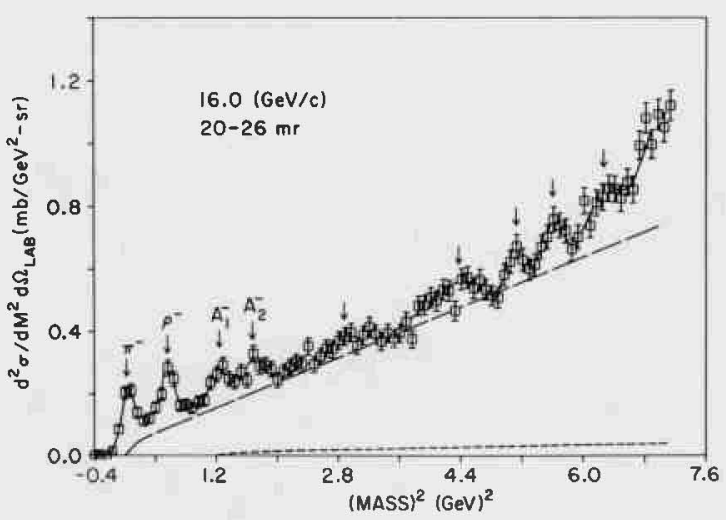

Figure 8. Cross section for the reaction $\pi^{-}+p \rightarrow p+X^{-}$, where $X$ - represents the missing mass produced backward in the center-of-mass system. The cross section is plotted as a function of the missing mass. The peaks in the curve, indicated by arrows, represent boson resonances that have emerged from the head-on collision.

The detecting elements were wire spark chambers that recorded the unanalyzed data on magnetic tape for study on completion of the run. The system in addition operated on-line to a computer, so that the course of the experiment could be followed by analyzing fully a sample of the data. The information about each event was recorded on tape. The event-by-event information on the incoming particles was essential, since it allowed acceptance of a wide range of angles and momenta for the incident particles, which permitted the use of high incident intensities to accelerate data accumulation. Since the precise angle and momentum were recorded event by event, the result was high precision, together with high intensities. Incident and final particles were identified with $\mathrm{Ce}$ renkov counters as either $\pi^{-}, K^{-}$, or antiprotons (complete identification of the final particle was not always possible), and the identifications were recorded on the tape. The incident beam consisted of a mixture of these particles, and thus data on several distinct reactions were recorded simultaneously during the run and separated later, by magnetic-tape tagging, for separate analysis. Examples of the dozen or so reactions for which data were obtained are $\pi^{-}+p \rightarrow \pi^{-}+p, \pi^{-}+p \rightarrow \pi^{-}+\mathcal{N}^{*}$, $K^{-}+p \rightarrow K^{-}+p \rightarrow K^{-}+\mathcal{N}^{*}$, and $\pi+p \rightarrow K^{-}+Y^{*+}$.

Wire chambers can record data at a high rate ( 1 event every $2 \mathrm{msec}$ ) and the spectrometer system had a large angular $\left(10^{-4} \mathrm{sr}\right)$ and momentum $(\Delta p / p \approx 50 \%)$ acceptance. The result was a very fast accumulation $\left(10^{7}\right.$ events in $\left.500 \mathrm{hr}\right)$ of precise data (momentum resolution, $\approx 0.5 \%$ ) incorporating elastic and inelastic scattering in both the forward and backward directions. The mass of data accumulated has brought to light a number of interesting features of the strong interactions.

The $\pi^{-}-p$ backward inelastic scattering at 16$\mathrm{BeV} / c$ incident $\pi^{-}$momenta were analyzed first. Figure 8 shows the production cross section as a function of the missing mass $X-$ where $X$ - is produced backward in the center-of-mass system according to the reaction $\pi^{-}+p \rightarrow p+X^{-}$. It can be seen that many of the peaks $\left(\rho, A_{2}\right.$, etc.) correspond to resonances well established from forward scattering processes and that, in contrast to their forward production in the center-of-mass system, their cross sections here are all comparable with the backward elastic cross section and in general have similar angular dependences.

A second new finding of interest was isobar production by $K-$ and $\bar{p}$ incident particles, as well as more extensive data on $\pi^{-}$-produced isobars. New structures in the $t$ dependence of the production of the various isobars were observed. A third result was the setting of new upper limits for reactions that are forbidden by $S U(3)$ theory and the quark model. In particular, a forbidden excited state of the baryon with strangeness of $+1,\left(Z^{*}\right)$, postulated by others, was shown not to be produced at cross sections above the low level of $1.4 \mu \mathrm{b}$. Other reactions, which are still being analyzed, include new high energy data on elastic $p$ - $p$ scattering, $\gamma^{*}$ production by $\pi^{-}-p$ interactions as a function of four-momentum transfer, and annihilation of high energy $\bar{p}-p$ interactions into two $\pi$ mesons.

New evidence for the existence of baryon-exchange forces among particles was provided by an experiment performed by a BNL-Rochester team at the AGS. A digitized wire spark-chamber array was used to measure the differential cross section for elastic $K^{+}-p$ scattering for a wide range of backward angles and momenta from 1.0 to $2.5 \mathrm{BeV} / c$. The measurements were made with use of the electrostatically separated beam from the G-10 target. The spark-chamber array was connected on line to a small computer and precisely measured the particle tracks. In this way the selection of the elastic events from the more numerous inelastic reactions was made.

The on-line computer recorded data on magnetic tape and performed simple checks on the quality of the data. Complete analysis of the data 
was made every few hours by using the more extensive facilities of the Central Scientific Computing Facility of the Applied Mathematics Department. Results of the complete analysis were used to guide the course of the experiment and to provide precision checks on the performance of the apparatus.

A preliminary analysis of the data showed that the distributions are relatively flat until about 1.8 $\mathrm{BeV} / c$, when a backward peak begins to develop that becomes more prominent at higher momenta, a behavior indicative of baryon exchange (in this case a $\Lambda^{0}, r^{0}$, etc.). The data will provide constraints on the parameters of the Reggeized baryonexchange model.

A group from California Institute of Technology, Rochester University, and BNL completed a combined experiment on the annihilation of antiprotons and protons into the charged pions or two kaons near $0^{\circ}$ and $180^{\circ}$ and on the backward elastic scattering of antiprotons by protons. The experiment used essentially the same apparatus previously used by the BNL-Rochester team to measure the backward scattering of $\pi$ 's and $K$ 's from protons, which saved months of setup and testing time for the high energy physics program. All these experiments were done in the partially separated counter beam (G-10) at the AGS in the momentum range of 1.0 to $2.5 \mathrm{BeV} / c$ in $\approx 0.1$ $\mathrm{BeV} / \mathrm{c}$ intervals. Two results of particular interest emerged from the initial analysis of the data: (1) a very close correlation between the backward elastic scattering of $\pi$ 's and $K$ 's and the corresponding $\bar{p} p$ annihilation which had been predicted theoretically was verified, and (2) structure was observed at $\approx 2000$ and $2400 \mathrm{MeV}$, which is strong evidence for resonance behavior.

In last year's annual report the Cornell-Brookhaven group reported preliminary results of backward peaks in $\pi^{+}-p$ and $\pi^{-}-p$ elastic scattering at energies from 6 to $17 \mathrm{BeV}$. At that time additional data at intermediate scattering angles from $20^{\circ}$ to $130^{\circ}$ were under analysis. This analysis of about $10^{5}$ spark-chamber pictures has now been completed. Figure 9 shows the $\pi^{-}-p$ angular distribution at $\approx 10 \mathrm{BeV} / c$. The forward cross section drops by $>7$ orders of magnitude until the backward peak takes over (it rises by $>3$ orders of magnitude).

Figures 10 and 11 show similar large-angle results for $K^{--} p$ and $\bar{p}-p$ elastic scattering, respectively. In each case new structures in the form of

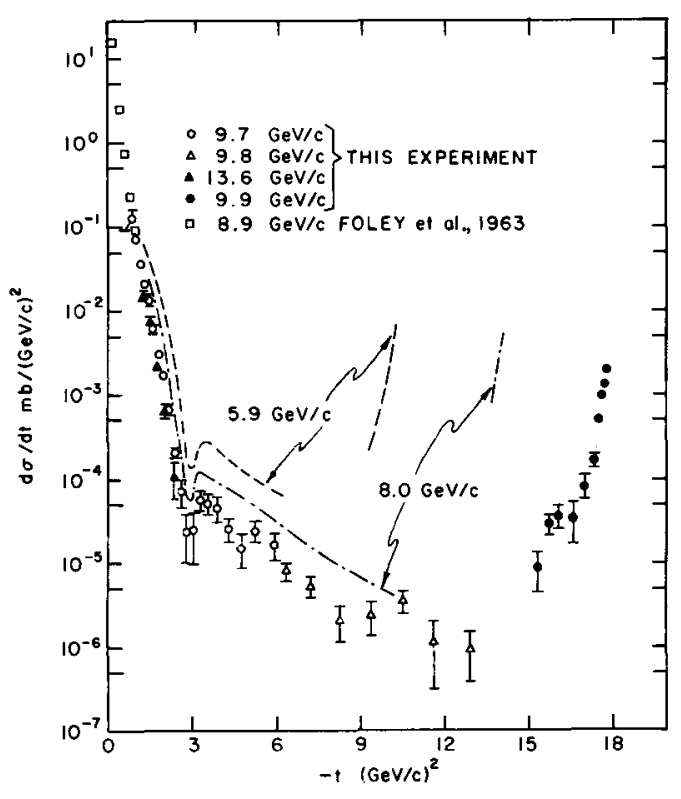

Figure 9. The angular distribution of the $\pi^{-}-p$ elastic scattering cross section for incident $\pi^{-}$mesons of $\approx 10$ $\mathrm{BeV} / c$. The broken curves show the fit to data at $\approx 6$ and $8 \mathrm{BeV} / c$ for comparison. A few of the experimental points at $13.6 \mathrm{BeV} / c$ are also shown.

dips or shoulders have been seen. Such structures are predicted by diffraction theories, Regge-pole theories, and the new multiple scattering theories. However, the rapid decrease in cross section with increasing energy at these large angles is not explained in most of these approaches. Further, it is surprising to see most of the dips fading away with increasing energy.

The Cornell group completed a new experiment this year in which the study of the backward pionproton scattering was continued by measuring the reaction $\pi^{-}+p \rightarrow n+\pi^{0}$ for backward neutral pions. The formalism of Reggeized baryon exchange, which fits the backward $\pi^{+}$and $\pi^{-}$results so well, gives a fairly definite prediction for the backward $\pi^{0}$ angular distribution. It also predicts that backward $\eta^{0}$ from $\pi^{-}+p \rightarrow n+\eta^{0}$ should have the same sharp dip seen in the backward $\pi^{+}$angular distribution.

The backward $\pi^{0}$ 's and $\eta^{0}$ 's are "seen" by detecting the two decay $\gamma$ rays $\left(\pi^{0} \rightarrow 2 \gamma\right.$ and $\eta^{0} \rightarrow 2 \gamma$ ) in lead-plate spark chambers surrounding a liquid hydrogen target. These are the chambers on the left in Figure 12. The chambers in the center are used to detect the forward-going neutron. The analysis is now in progress. 


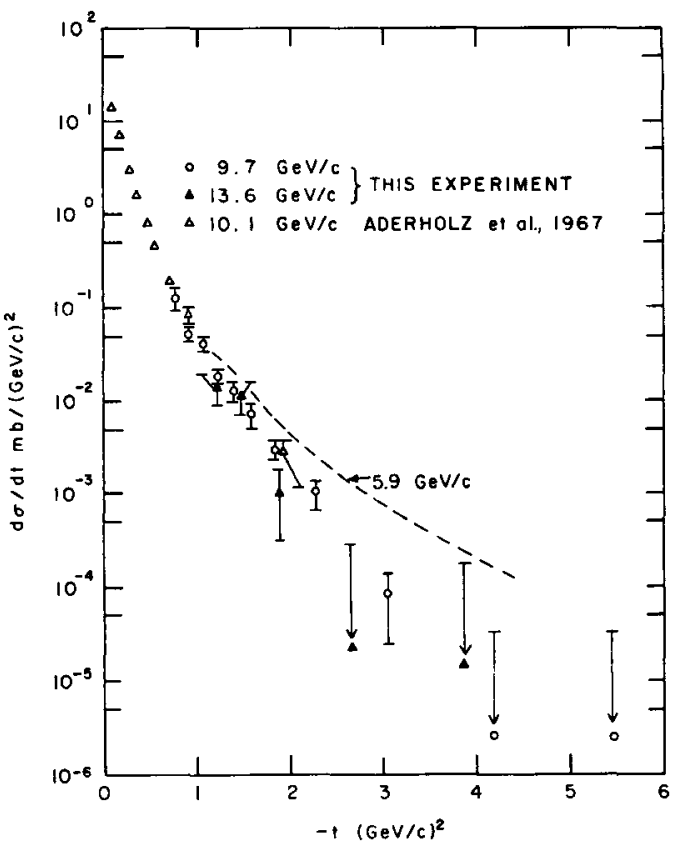

Figure 10. The angular distribution of the $K--p$ elastic scattering cross section for various incident momenta.

Another group from the University of Bari (Italy), Brown University, and Massachusetts Institute of Technology were doing the same experiment, but with a modified experimental technique suited to the lower momentum region (2 to $5 \mathrm{BeV} / c$ ) being studied. The reactions $\pi^{-}+p \rightarrow \mathcal{N}+\pi^{0}$ and $\pi^{-}+p \rightarrow \mathcal{N}+\eta^{0}$ are of great interest as a tool in baryon spectroscopy because of the possible appearance of resonant states above a background amplitude. In the backward direction it is hoped that the background amplitude is not grossly dominant over any single resonant partial wave contribution, as is certainly the case in the forward direction in this momentum region. This region is abundantly supplied with resonant states, and it should be possible to deduce the partial widths and quantum numbers of these states. In addition, this is the region of transition between low and high energy phenomena and is an important region for testing the Regge phenomenology. In these reactions the exchanged objects are baryon trajectories, and again there is, from a different point of view than above, an opportunity for insight into baryon spectroscopy.

Scintillation counters and visual spark chambers are used as the detectors. The method of the experiment is to require a $\pi^{-}$to disappear in a liquid hydrogen target unaccompanied by any $\pi^{0}$

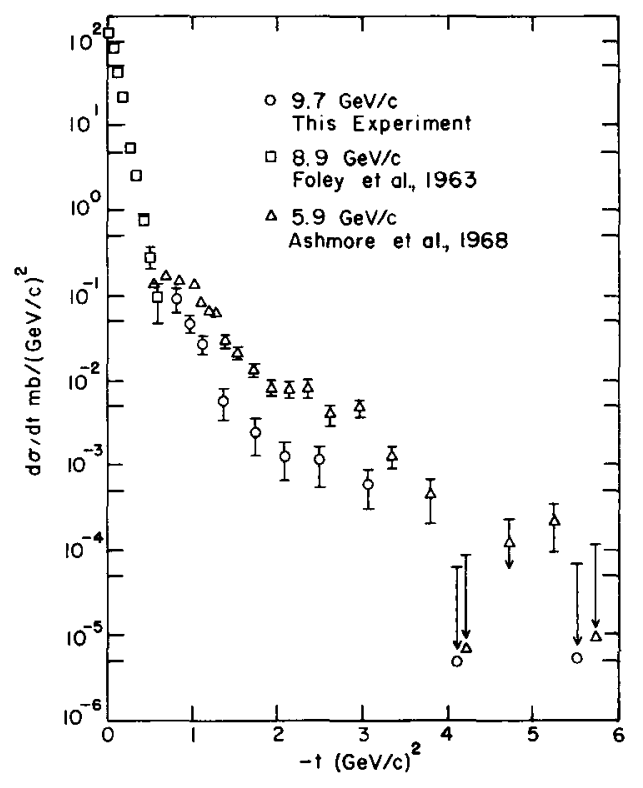

Figure 11. The angular distribution of the $\bar{p}$ - $p$ elastic scattering cross section for incident $\bar{p}$ of $9.7 \mathrm{BeV} / c$. Other work at $\approx \mathbf{6}$ and $9 \mathrm{BeV} / c$ is shown for comparison.

decay $\gamma$ rays in most of the forward hemisphere via a combination of scintillation counters and leadscintillator shower counters. In addition, a neutron detector is located in the forward direction and a detected neutron is required to complete the trigger requirements for the spark chambers. The spark chambers are $\gamma$-ray detecting devices and detect the $\pi^{0}$ and $\eta^{0}$ through two $\gamma$-ray decay modes. The analysis of these data is also in progress.

An experiment performed by a group from the State University of New York at Stony Brook had as its primary purpose the study of the reactions $\pi^{ \pm}+p \rightarrow K^{ \pm}+\Sigma^{ \pm}$at small momentum transfer in the energy region 2 to $16 \mathrm{BeV} / c$. Because of the presence of $K$ 's and protons in the beam and identification of incident and secondary particles by the use of Cerenkov counters, other reactions, such as $\pi^{ \pm}+p \rightarrow p+\pi^{ \pm}, \bar{p}+p \rightarrow p+\bar{p}, \bar{p}+p \rightarrow \pi^{+}+\pi^{-}$, and $K^{ \pm}+p \rightarrow p+K^{ \pm}$, can also be studied.

The forward $K+-\Sigma+$ cross section is believed to be dominated by exchange of both vector and tensor $K^{*}$ 's. Accurate differential cross-section and polarization data will be valuable in ascertaining to what extent the Regge-pole hypothesis coupled with $S U(3)$ theory can fit the experimental data. For this purpose the high energy region (6 to 10 $\mathrm{BeV} / \mathrm{c}$ ) is most useful. 


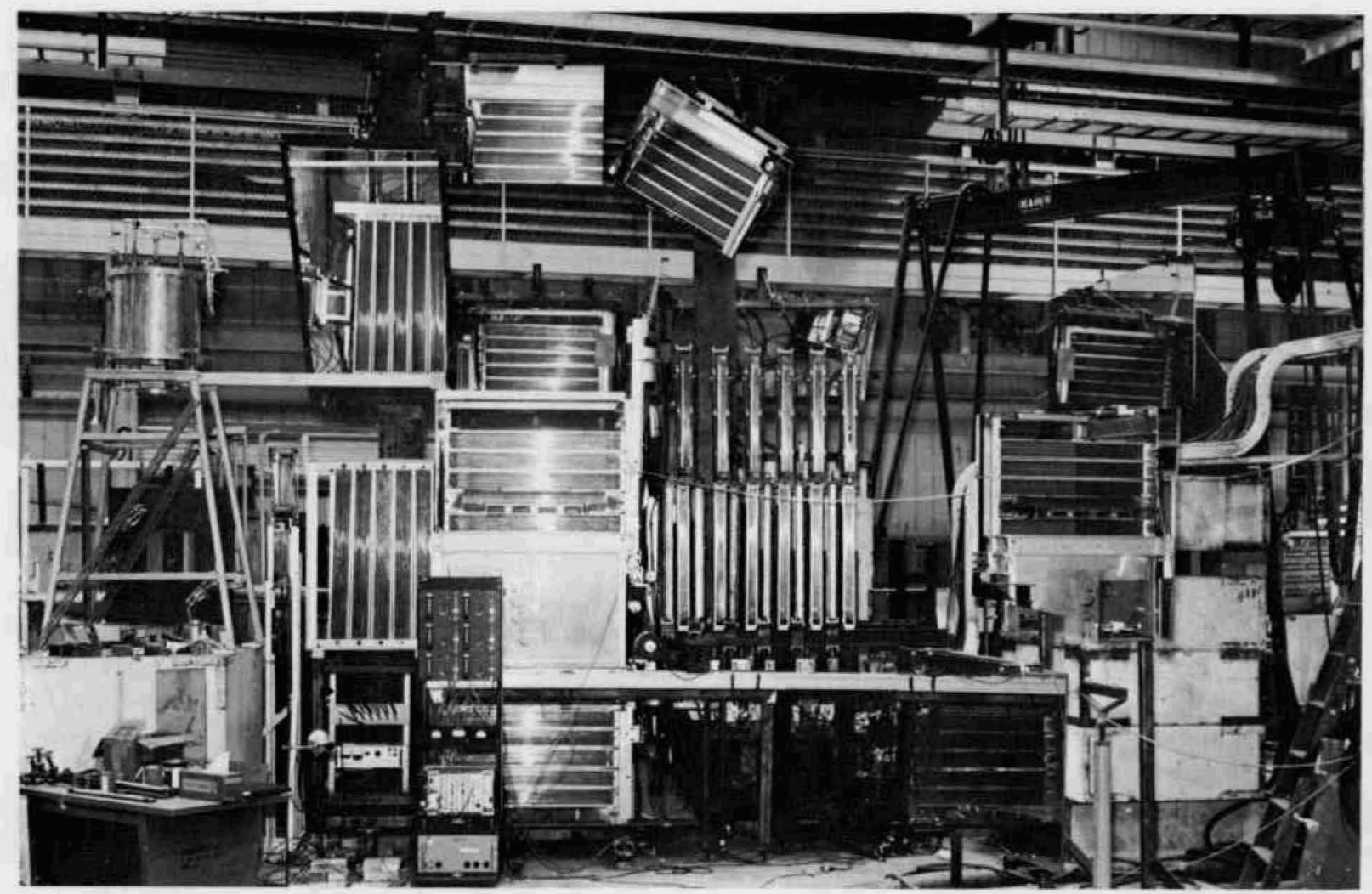

Figure 12. The arrangement for the backward $\pi-p$ scattering experiment performed by the Cornell group.

At lower energies (2 to $6 \mathrm{BeV} / c$ ) it is expected that in the $K+\Sigma+$ channel, the $I=3 / 2 \mathcal{N}^{*}$ amplitudes will interfere with the large $K^{*}$ exchange amplitude in a way that is sensitive to details of the interaction. At the lower energies in the $K+\Sigma-$ channel, where there is no known $K^{*}$ to exchange, it is hoped to find the $I=1 / 2$ and $I=3 / 2 \mathcal{N}^{*}$ contributions standing alone. This also is of considerable interest.

The apparatus used was a single-arm spectrometer with wire-plane spark chambers to define the scattering angle and magnetic deflection angle of the forward-going secondary particle. A simple detector examined the position and particle identity of the recoil particle or its decay products. As employed for the reaction $\pi^{+}+p \rightarrow K^{+}+\Sigma^{+}$, this recoil detector allowed measurement of the polarization parameter. The trigger requirement was varied according to the momentum and sign of the beam to include different combinations of incident $\pi, K$, or $p$ and outgoing $\pi, K$, or $p$. Events were processed on line with a PDP-8 computer and stored on magnetic tape.
Data analysis of the experiment is in progress but some of the preliminary results are available. In the reaction $\pi^{+}+p \rightarrow K^{+}+\Sigma+$, the finite differential cross section $d \sigma / d t$ shows essentially no shrinking in the momentum range studied. The polarization is consistent with 0 for $|t| \leqslant 0.3$ and becomes positive for larger $|t|$. The reactions $\pi^{-}+p \rightarrow K+$ $+\Sigma-$ and $\pi^{-}+p \rightarrow K^{+}+r^{*-}(1385)$ were also studied at 6 and $10 \mathrm{BeV} / c$ for momentum transfers $0 \leqslant|t| \leqslant 0.5$. These reactions are forbidden in single-particle exchange models unless doubly charged strange meson states exist. In the case of $\pi^{-}+p \rightarrow K^{+}+\Sigma^{-}$, no events are seen, which allows an upper limit at $t=0$ of $d \sigma / d t \leqslant 0.1 \mu \mathrm{b} /(\mathrm{BeV} / c)^{2}$ to be set. In the case of $\pi^{-}+p \rightarrow K^{+}+r^{*-}$ (1385), a clear signal is seen at both beam momenta.

No $\alpha$ particles were detected in the early beam surveys at the CERN Proton Synchrotron and in a recent experiment at the Argonne Zero Gradient Synchrotron. A BNL scientist carried out an experiment at the AGS to determine whether $\alpha$ particles are produced by high energy proton-nucleus collisions and to explore the possibility of obtain- 
ing useful secondary $\alpha$ beams for various experiments, in particular those relevant to the study of the origin and propagation of cosmic rays. The result shows that sizable numbers of $\alpha$ particles are indeed produced at the AGS.

The measurement was carried out in the AGS test beam. Secondary particles produced at $18.8^{\circ}$ by the $28.5-\mathrm{BeV}$ internal protons incident on the $\mathrm{BeO}$ target were momentum-analyzed by a bending magnet and then classified according to (1) their time-of-flight (TOF) between two scintillators, and (2) their energy loss in a third scintillator. The $d E / d x$ analysis is essential for the identification of alpha particles because $\mathrm{He}^{4++}$ has twice the momentum of the deuteron and hence the same TOF. The TOF vs $d E / d x$ technique is rather conventional, except that some different electronic logic was employed. The joint ( $d$ and $\alpha$ ) peak in the TOF spectrum was decomposed into two peaks after the $d E / d x$ analysis. To confirm that the second peak in the $d E / d x$ spectrum is really due to $\alpha$ particles, the system was calibrated by the proton and $\mathrm{He}^{3++}$ of corresponding momenta. Thus, the relative energy loss of $p$ vs $\mathrm{He}^{3}$ and $d$ vs $\mathrm{He}^{4}$ establishes an unambiguous identification of the $\alpha$ particles. The $\alpha$ to $d$ ratio is found to be of the order of $1 \%$ for secondary momenta below $1000 \mathrm{MeV} / c$ per nucleon, an intensity sufficient to warrant consideration of the feasibility of using $\alpha$ beams from high energy accelerators.

An interesting application of the $\alpha$ beam is related to a problem of fundamental importance in astrophysics, namely the origin and the distance of propagation of primary cosmic rays in interstellar space. It is known that $\mathrm{He}^{3}$ and $d$ are rare in a large majority of astronomical bodies, and therefore their abundance in cosmic rays can be attributed to secondary origins, namely production by nuclear interactions of primary cosmic rays with interstellar gas. Thus the isotopic composition of the helium and hydrogen nuclei in cosmic rays provides an important clue to the understanding of the origin and propagation of these rays. $\mathrm{Re}$ cently, more data have become available through the flights of satellites and balloons, but their interpretation depends heavily on the cross sections of relevant nuclear reactions and the modulation of galactic cosmic rays in the interplanetary magnetic field. By comparing the data with their calculations on the $\mathrm{He}^{3}$ and $d$ production by cosmicray interactions in the galaxy, Ramaty and Lingenfelter have suggested an independent determi- nation of the cosmic-ray path-length as well as the total residual interplanetary field modulation. However, among the various reactions contributing to the production of $\mathrm{He}^{3}$ and $d$, cross sections are completely unknown for $\alpha-\alpha$ interactions, which may account for a large fraction (up to $40 \%$ ) of the $\mathrm{He}^{3}$ and $d$ in cosmic rays. It is therefore very valuable to measure the various breakup cross sections in the $\alpha$ - $\alpha$ collision below $1000 \mathrm{MeV} /$ nucleon. Such experiments are now feasible with a helium bubble chamber and the possible secondary $\alpha$ beams at high energy accelerators.

A reanalysis was carried out of world data on $K+$-nucleon partial and total cross sections in the $I=0$ state by a BNL group. A new peak was discovered in the $I=0$ total cross section at 790 $\mathrm{MeV} / c$. After subtracting the inelastic cross section, a very clear peak is seen in the total elastic cross section. From a fitting of the data to a modified Breit-Wigner expression, it was found that the peak can be due to an almost elastic resonance with a mass of $1780 \mathrm{MeV}$ and a width of $565 \mathrm{MeV}$. Should this turn out to be a resonance, it would seriously complicate the quark model in which all known particles and resonance can be formed by a combination of no more than three quarks. An $S=+1$ baryon resonance would require five quarks.

The three BNL bubble chambers continue to support a wide variety of research activities both

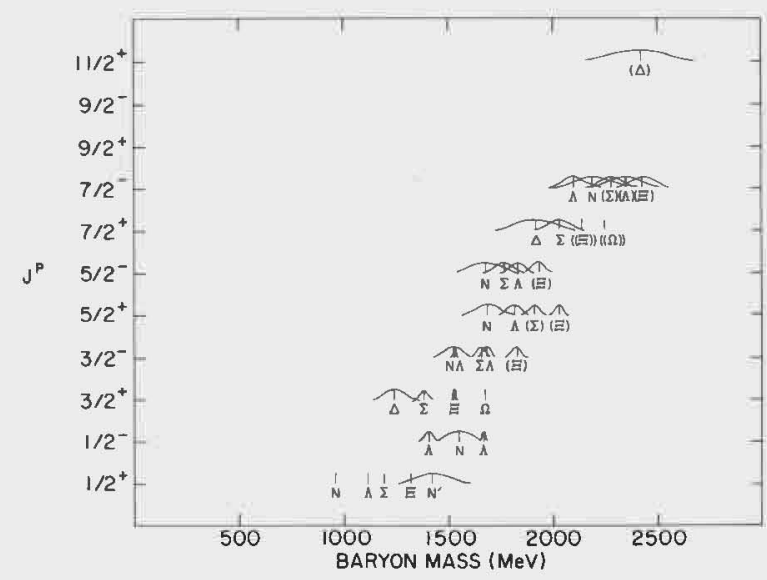

Figure 13. A plot of the masses and widths of known hadron states versus their spin-parity $\left(J^{P}\right)$. Parentheses indicate that the spin-parities have not yet been determined; those in double parentheses correspond to predicted states. The $\Xi$ resonances described in the text are those that complete the $3 / 2-, 5 / 2+, 5 / 2-$, and $7 / 2-S U(3)$ families of particles. 
at Brookhaven and at more than 30 other institutions. Experiments were directed toward obtaining a fuller understanding of the nature of elementary particles, with emphasis on exploring their strong and weak interactions.

Bubble-Chamber Experiments. 1. Strong interactions. A partial summary follows of results of bubble-chamber experiments performed during the past year.

The study of $K^{-}-p$ interactions at $3.9,4.6$, and 5.0 BeV/c in the BNL 80-in. hydrogen bubble chamber by the Brookhaven Bubble Chamber and Nuclear Interactions Groups and Syracuse University was fruitfully continued. A total of 600,000 pictures has been analyzed. The major new results were the discovery of two new $\Xi$ reso-

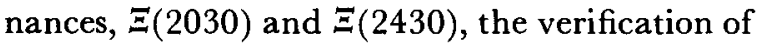
the previously questionable $\Xi(1930)$, and the confirmation of the $\Xi$ (1815).

These findings have a direct bearing on the $S U(3)$ formulation of the classification of the hadrons, or strongly interacting particles. It is well known that all octet (8) and decuplet (10) representations must contain among their constituents a $\Xi$ particle. The discovery of the above particles served to complete many octet families of hadrons, namely those with spin-parity $\left(J^{P}\right) 5 / 2-, 5 / 2+$, and $7 / 2-$. These families are shown in Figure 13, in which the masses of known hadron states are plotted as a function of their spin-parity. Previously only the $1 / 2^{+}$and $3 / 2-$ octets and the $3 / 2+$ decuplet were well established. The widths and decay branching fractions of the $\Xi$ resonances measured in this experiment, coupled with values for the previously known $\mathcal{N}$ (nucleon) and $\Lambda, \Sigma$ hyperon states, give further credence to the validity of unbroken $S U(3)$. In the $S U(3)$ formulation 2 parameters serve to fit the 9 to 10 different decay rates of the particles belonging to a given octet family. In a total of about 40 predicted decay rates in 4 octet families there was previously 1 discrepancy with experiment, namely the nonobservation of the $\Sigma(1940) \rightarrow \Sigma \pi$, which was expected to be dominant. Evidence for this decay has now been found in this experiment. The same data also confirmed the existence of the $\Sigma(2240)$ resonance previously observed in the $K--p$ total cross-section measurements. Evidence was obtained for a meson-like enhancement at a mass of $970 \mathrm{MeV}$ decaying into $\eta^{0} \pi^{-}$and $\pi^{-} \pi^{0} \gamma$.

The analysis by the BNL Bubble Chamber Group of 280,000 pictures taken in the 80 -in.

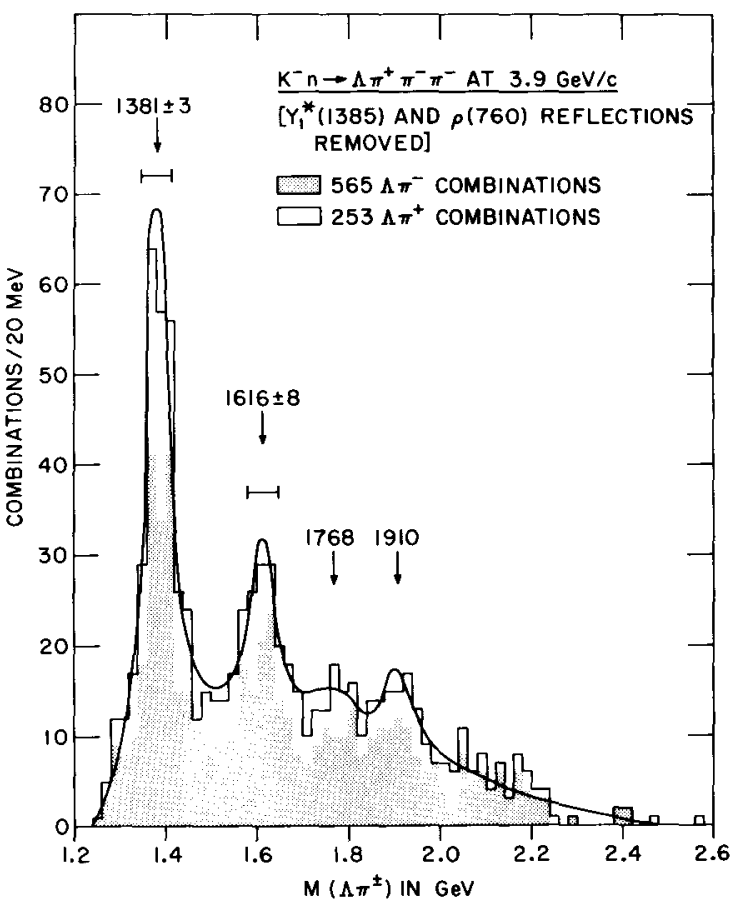

Figure 14. Effective-mass distributions of the $\Lambda \pi$ systems, showing a peak at $1.61 \mathrm{BeV}$ corresponding to a newly discovered hyperon resonance.

chamber filled with deuterium and exposed to 3.6 and $3.9-\mathrm{BeV} / c K^{-}$mesons had led to several interesting results. A new isospin-1 hyperon resonance has been observed with mass $1616 \pm 8 \mathrm{MeV}$ and width $66 \pm 16 \mathrm{MeV}$, which decays copiously into the $\Lambda \pi$ state but is also seen in the $r_{1}{ }^{*}(1385) \pi$ state (see Figure 14).

The data were searched for evidence of a $\left(\Xi_{3 / 2}{ }^{*-}\right)$ state in the $\Xi-\pi^{-}, \Xi-\pi^{-} \pi^{0}$, and $\Lambda \pi^{-} K^{-}$ systems. The simple nonrelativistic quark model for baryon ( $q q q)$ states predicts that all the wellestablished baryon states belong to singlets, octets, or decuplets, and to no other representation. The only available experimental observations that might contradict this picture are the isospin $I=0$ and $I=1$ enhancements previously observed by counters in the $K^{+}$-nucleon total cross sections: such states (hypercharge $r=2$ ) must belong to higher $S U(3)$ multiplets ( $\overline{10}$ for $I=0$ and 27 for $I=1)$. The large peak $(\approx 6 \mathrm{mb})$ in the $I=0$ cross section, if interpreted as a resonance, implies the existence of a 10-multiplet of baryon resonances which would also contain an $I=3 / 2, r=-1$ member with the quantum numbers of a $\Xi^{*}$ system. No strong evidence was found for such a 


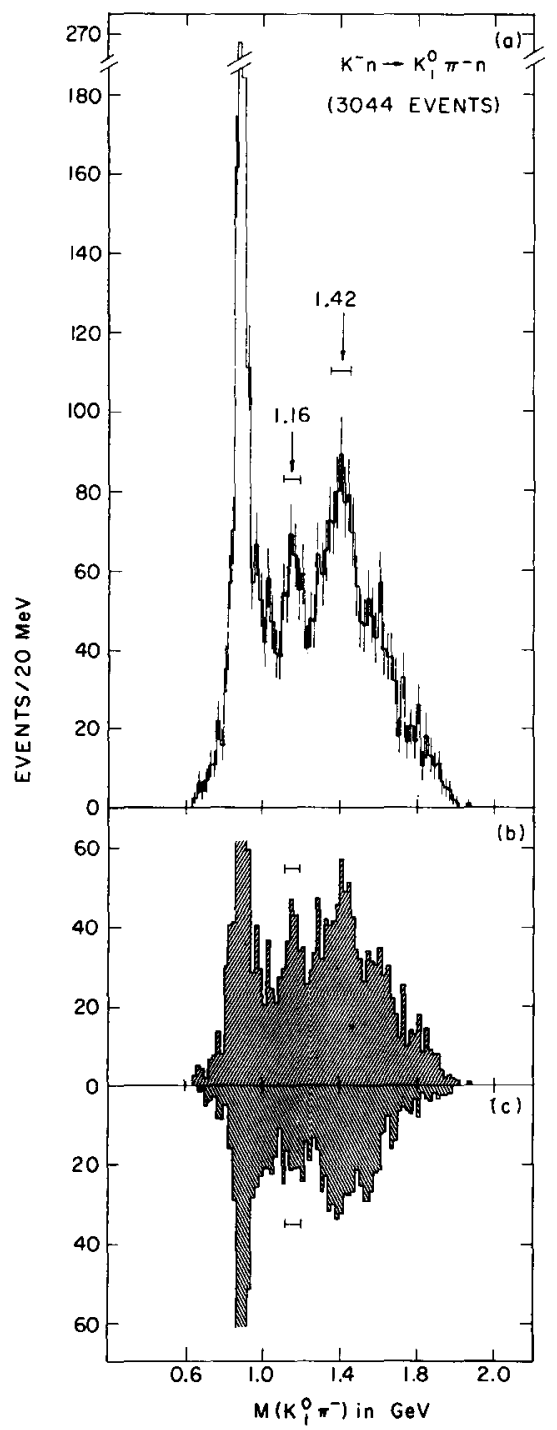

Figure 15. Effective-mass plots showing a new $K \pi$ resonance at $1.16 \mathrm{BeV}$.

$\left(\Xi_{3 / 2}{ }^{*--}\right)$ state in this experiment. The upper limit for the production of any $\left(\Xi_{3 / 2}{ }^{*-}\right)$ resonance is estimated at $\approx 1.5 \mu \mathrm{b}$, assuming a width $\leqslant 80 \mathrm{MeV}$ and a mass $<2.2 \mathrm{BeV}$, with a $99 \%$ confidence level. This negative result is consistent with the quark model.

An isospin-1/2, $K \pi$ enhancement (see Figure 15) was also observed in the $K-d$ experiment at a mass of $1160 \pm 10 \mathrm{MeV}$ and a width of $90 \pm 30 \mathrm{MeV}$ from the reaction $K^{-}+n \rightarrow K_{1}{ }^{0}+\pi+n$ at 3.9 $\mathrm{BeV} / c$. However, no evidence was found for the previously reported $K \pi$ effects at 1080 and 1200 $\mathrm{MeV}$.
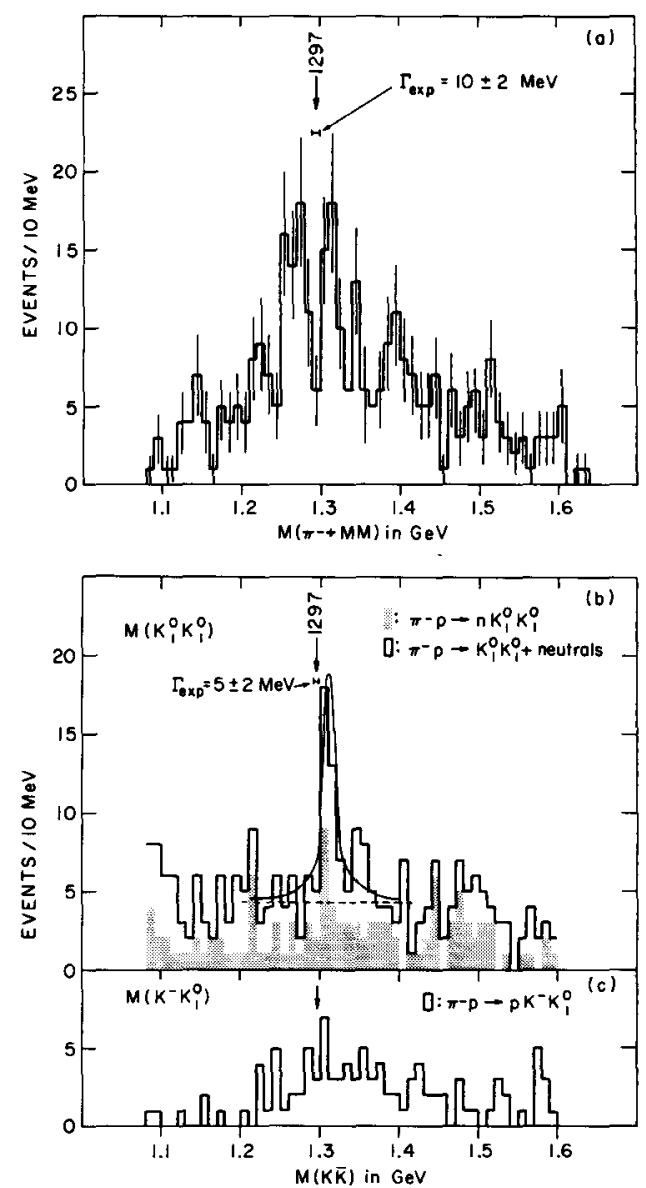

Figure 16. (a) Effective mass of $\pi^{-}$plus missing mass, showing both peaks of the "split $A_{2}$." (b) Effective mass of $K_{1}{ }^{0} K_{1}{ }^{0}$, showing a peak at the position of the " $A_{2}$ high." (c) Effective mass of $K^{-} K^{0}$.

This experiment, together with the $K-p$ experiment previously described has yielded further information on the $K_{1}{ }^{0} K_{1}{ }^{0}$ enhancement near threshold. Previous experimental evidence for an isosinglet, scalar $K_{1}{ }^{0} K_{1}{ }^{0}$ enhancement $\left(I^{G}=0^{+}\right.$, $J^{P}=0^{+}$) comes mainly from a study of the reaction $\pi^{-}+p \rightarrow K_{1}{ }^{0}+K_{1}{ }^{0}+n$ at pion momenta from 2 to $12 \mathrm{BeV} / c$. Because the enhancement is near the $\bar{K} K$ threshold, the available data could be interpreted either in terms of an $s$-wave resonance or a simple $s$-wave complex scattering length (a nonresonant threshold phenomenon). In the present $K-d$ experiment a $K_{1}^{0} K_{1}^{0}$ enhancement is produced which has mass $1030 \pm 10 \mathrm{MeV}$, width $40_{-15}^{+35} \mathrm{MeV}$, and spin, parity, and charge conjugation $\vec{J}^{P C}=0^{++}$. To the extent that the simple scattering-length procedures carried out are valid, the data presented 
here give strong support for the existence of a $J^{P C}=0^{++}$boson resonance at a mass of $1030 \pm 10$ $\mathrm{MeV}$. This resonance is associated with the $S^{*}(1069)$, since both enhancements have the same $J^{P C}$ and, within errors, similar mass values.

About 30,000 interactions of $K$ - mesons in the 30-in. chamber were analyzed from photographs taken in a prior year by the Brookhaven Bubble Chamber Group. An extremely well-defined beam of $725 \pm 2 \mathrm{MeV} / c$ was utilized in order to obtain a clearer understanding of the nature of the $\Lambda-\eta$ interaction at the $\Lambda \eta$ threshold. Results indicate that previous experiments may have underestimated the contribution of a large $S_{1 / 2}$ scattering length in this channel. Cross sections, angular distributions, and polarizations of the two-body final states $K p, K^{0} \mathcal{N}, \Lambda \pi$, and $\Sigma \pi$ were studied in an effort to better understand the large number of resonances with masses near $1660 \mathrm{MeV}$. Analysis of the three-body states $\Lambda \pi \pi$ and $\Sigma \pi \pi$ indicates that they are produced almost exclusively via the quasitwo-body states $\Sigma(1385) \pi$ and $\Lambda(1405) \pi$.

The reactions $K-+p \rightarrow V^{0}+$ neutrals and $K-+p \rightarrow K^{-}+p$ obtained in the 30 -in. chamber have been studied by a group at $Y$ ale University. The $K^{-}$- momentum region covered was 590 to 820 $\mathrm{MeV} / c$. A partial-wave fit to the $\bar{K} \mathcal{N}$ channels revealed the presence of the $D_{3 / 2}$ resonance at around $1700 \mathrm{MeV}$. The mass and width of this resonance seem significantly different from the values obtained in the $\Sigma \pi$ channel, a fact that may only indicate an inadequate parametrization of the amplitudes, but may also point to the presence of additional structure in this region.

The reactions $K^{-}+p \rightarrow \Sigma^{ \pm}+\pi^{\mp}\left(\pi^{0}\right)$ have been studied in the same exposure. A partial-wave analysis of the two-body channels shows the presence of resonances in the $S_{01}, D_{03}$, and $D_{13}$ states. The first is probably to be identified with the $\Lambda \eta$ resonance discovered earlier in this experiment. The cross section for $K^{-}+p \rightarrow \Lambda(1405)+\pi^{0}$ does not show any significant peaking at the $\Sigma(1600)$ mass, even though a substantial signal is expected on the basis of some production-experiment branching-ratio measurements. This result adds to the evidence that more than one " $\Sigma(1660)$ " resonance is involved.

Continuing analysis at BNL of an exposure of the 80-in. chamber filled with hydrogen to 6$\mathrm{BeV} / c \pi^{-}$mesons has yielded new characteristics of the $A_{2}(1300)$ and the $g(1640)$ mesons.

In this experiment, a splitting of the $A_{2}$ meson into two peaks, $A_{2}{ }^{\mathrm{H}}$ ( $\mathrm{H}$ for high) and $A_{2} \mathrm{~L}$ (L for
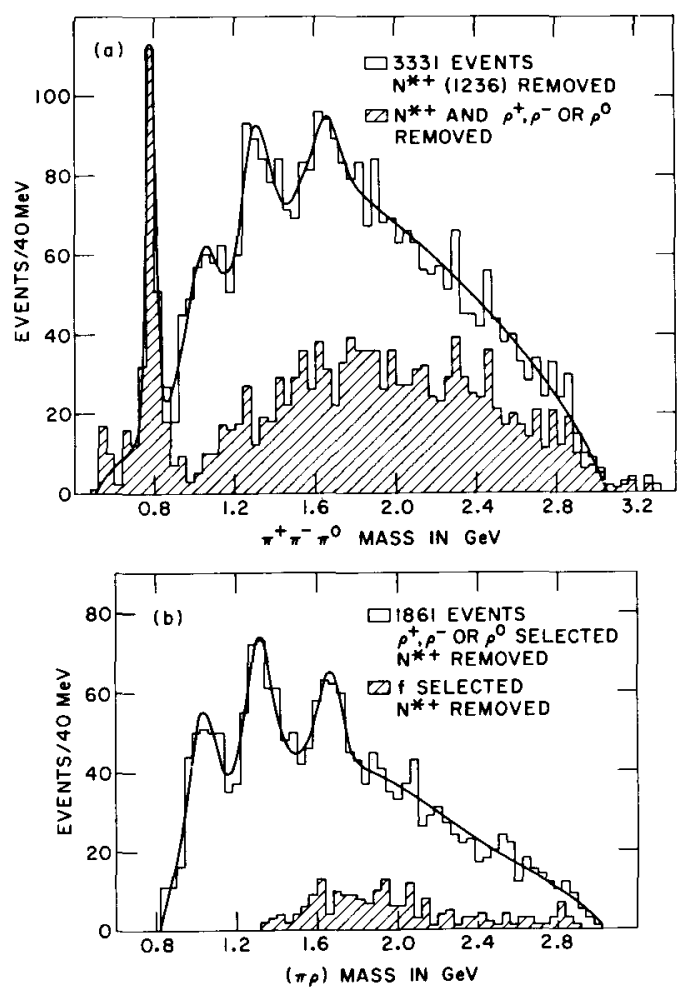

Figure 17. (a) Effective-mass distribution of $3 \pi$ combinations from the reaction $\pi^{+}+n \rightarrow p+\pi^{+}+\pi^{-}+\pi^{0}$. The prominent peak on the left is the $\omega$ meson. (b) The same plot as in $(a)$ after selection of those events in which a $\rho$ meson is produced. The three peaks are the $A_{1}, A_{2}$, and $A_{3}$ mesons.

low), was observed in the reaction $\pi^{-}+p \rightarrow p+\pi^{-}+$ missing mass, similar to that seen in the CERN missing-mass spectrometer experiment. In addition, investigation of the $K \bar{K}$ mass spectrum in the $A_{2}$ region reveals a narrow $K_{1}{ }^{0} K_{1}{ }^{0}$ enhancement centered at the mass of the $A_{2}{ }^{\mathrm{H}}$. This observation suggests that there are two resonances with different spin-parity in the $A_{2}$ mass region. These results are shown in Figure 16.

From the same $\pi^{-} p$ exposure a detailed spinparity analysis of the $g(1640)$ meson was made from its dipion decay mode. It was found that a spin-3 assignment is strongly favored for this resonance, the highest spin yet found for a meson resonance. An isospin-1 $K \bar{K}$ state at a mass of 1640 $\mathrm{MeV}$ was also observed; this could be a new decay mode of the $g$ meson.

The analysis at BNL of 65,000 events produced in $8-\mathrm{BeV} / c \pi^{+}-d$ interactions in the 80 -in. chamber was brought near completion. A study of $\pi^{0} \rho^{0}$ systems produced in these interactions confirmed the 


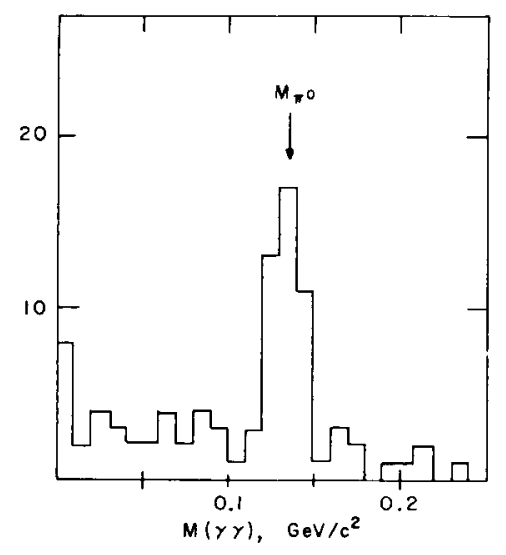

Figure 18. $\gamma$ Rays from the decay of neutral pions are converted into electron pairs by collision with nuclei. Measurements on the electron tracks seen in the 31-in. bubble chamber permit calculation of the mass of the parent particle, plotted here.

existence of both an isoscalar resonance (the $A_{3}$ meson) at a mass of $1.660 \mathrm{BeV}$ and a neutral threepion resonance of mass $1.679 \mathrm{BeV}$ decaying primarily into $\pi \rho$. Since no evidence was observed for a $\pi f$ decay mode (Figure 17), it appears that this resonance has different characteristics from the charged resonance of similar masses previously observed. A preliminary analysis suggests that the spin is $\geqslant 1$ and confirms isospin-0.

Both Harvard and University of Rochester groups have observed the $A_{3}$ enhancement at 1.64 $\mathrm{BeV}$. The state decays into $\rho^{0} \pi^{+}$and $f^{0} \pi^{+}$and has isospin-1 and negative $G$-parity. The Rochester group has observed another $I=1$ peak at 1.72 $\mathrm{BeV}$, which has positive $G$-parity and decays into $\omega^{0} \pi^{+}$and $A_{2}{ }^{0} \pi^{+}$. A Maryland group has found evidence for a meson resonance of mass $1.67 \mathrm{BeV}$ decaying into $\omega \pi \pi$.

The University of Illinois has continued to study the decays of the $B$ - mesons in the $\omega^{0} \pi^{-}$decay modes. The results at $7.5 \mathrm{BeV} / c$ are in essential agreement with their work at $5 \mathrm{BeV} / c$. A clean peak is seen, and the earlier angular distributions are roughly confirmed. In the $7.5-\mathrm{BeV} / c$ data, however, a significant component with helicity-0 appears to be produced. Assuming that both samples come from the same particle, the combined results strengthen the probability for spin-parity $1^{+}$and rule against the previous possibilities of $2^{+}$, $3^{-}$, etc.

The University of Wisconsin group has observed a strong backward peak in the differential cross sections of backward hemisphere $\bar{p}-p$ elastic scat- tering in the momentum range 300 to $700 \mathrm{MeV} / c$. The differential cross section near $180^{\circ}$ and near $90^{\circ}$ shows energy-dependent fluctuations. A consistent interpretation of these fluctuations can be made in terms of direct-channel boson resonances with masses (widths) of 1.925 (0.010) and 1.945 $(0.020)$, and a state with mass $>1.975$ and width $>0.020 \mathrm{BeV}$.

A Brookhaven experiment in the 31 -in. chamber started in 1967 was continued in order to study the nature of the enhancement found by a counter experiment in the $I=1 \bar{p}-p$ total cross section at 1.3 $\mathrm{BeV} / c$. A total of 64,000 pictures containing 88,000 $\bar{p}-p$ interactions was obtained at $1.11,1.31,1.33$, 1.35 , and $1.52 \mathrm{BeV} / c$. Measurement of the fourpronged events revealed than an $I=1$ state, $\pi(2190)$, decaying to $\rho^{0} \rho^{0} \pi^{0}$ is responsible for a major part of the total cross-section enhancement.

In an experiment using a neon-hydrogen mixture in the 80 -in. bubble chamber, $K^{-} \pi^{+} \pi^{-}$states produced by $K$ - mesons scattering coherently on neon have been analyzed at BNL. In addition to a strong $J^{P}=1^{+}, K^{*}(890) \pi$ enhancement $(Q)$ at $1.3 \mathrm{BeV}$, these data show a strong interference between a $K \rho$ state and the $K^{*} \pi$ for $M_{k^{*} \pi}>1.25$. This effect is being studied further by looking at the state $K-\pi^{0} \pi^{0}$, in which only $K^{*} \pi$ can be produced. The $\pi^{0}$ 's are "measured" by analyzing the electron pairs produced by their decay $\gamma$ 's in the neon. Figure 18, a sample plot of the mass obtained from these measurements, shows a clean separation of the $\pi^{0}$ from the background.

The Yale group has continued the investigation of diffraction-produced effects in the reactions $K^{-}+p \rightarrow \bar{K}^{0}+\pi^{+}+\pi^{-}+p$ at $12.6 \mathrm{BeV}$. This investigation included a study of the $K \pi \pi$ threshold $Q$-enhancement in terms of a possible interpretation as one or more resonant states with energyor momentum-transfer-dependent interference effects, as well as a detailed spin-parity analysis. No evidence is found for multiple resonances; the data are consistent with a single $J^{P}=1^{+}$or $2-$ resonant state decaying wholly to $K^{*}(890) \pi$ or $K \rho$. A comparison of these results with those of other high energy $K+p$ experiments does suggest the presence of energy-dependent interference effects. The subreactions $K^{-}+p \rightarrow \bar{K}^{* 0}(890)+\pi p$ and $K^{-}+p \rightarrow K^{-}+$ $\Delta^{++}+\pi^{-}$have been analyzed in the light of a nonresonant, double-peripheral production model involving exchange of pion and pomeron Regge trajectories. The model gives good agreement with the data in many respects for both the $K^{*} \pi$ and 
$\Delta \pi$ samples; it thus strengthens earlier results suggesting similar production mechanisms (diffraction-production) for the two systems and allows a quantitative evaluation of the Dolen-HornSchmid duality of recent Regge-pole theory.

The $K \pi \pi$ enhancement at $1.3 \mathrm{BeV}$ has also been investigated in $K+-p$ interactions by groups at Rochester, UCLA, and Johns Hopkins. Attempts to fit the data with a diffraction-dissociation model were unsuccessful; rather, the enhancement region appears to be dominated by resonance effects with spin-parity $1^{+}$and with production and decay characteristics similar to those of the $A_{1}$ meson. The quark predicts the existence of two boson resonances, with which the data are consistent if it is assumed that the resonances overlap in this mass region. The Johns Hopkins group also reports finding a weaker $K \pi \pi$ enhancement at $1.7 \mathrm{BeV}$, the $L$ meson, which appears to be a $T=1 / 2,2^{-}$ system.

In a continuing study of $28.5-\mathrm{BeV} / c p-p$ interactions, various techniques to explain the variety of experimental distributions observed in high energy scattering are being investigated. Unlike most bubble-chamber experiments at lower energies, in which the primary aim is to search for resonances, at this high energy the main objective is to understand the mechanism for the production of particles. A model that has achieved considerable success in fitting the data was applied to the three-body final states $p n \pi^{+}$and $\Delta^{++} p \pi^{-}$by E.L. Berger, a visiting theorist at BNL. This doubleRegge-pole-exchange model is an extension of the original single-Regge model that works well in explaining two-body final states. Fits to the four independent kinematic variables in the final state $\Delta^{++} p \pi^{-}$are shown in Figure 19. Although agreement with the data is not perfect, the model works quite well for those values of the variables to which it is expected to apply. Only two parameters are varied to obtain these fits; with a slight increase in the number of parameters, even better fits can be obtained.

A number of other groups have had success in fitting three-body processes at high energy with the double-Regge model. The reaction $\pi^{-}+$ $p \rightarrow \pi^{-}+X+p$ at $25 \mathrm{BeV}$, where $X$ is composed of a $\pi^{+}$and $\pi^{-}$, was examined at Wisconsin and the California Institute of Technology. It was found that momentum-transfer and invariant-mass distributions were in reasonable agreement with the predictions of the double-Regge model in the kine-
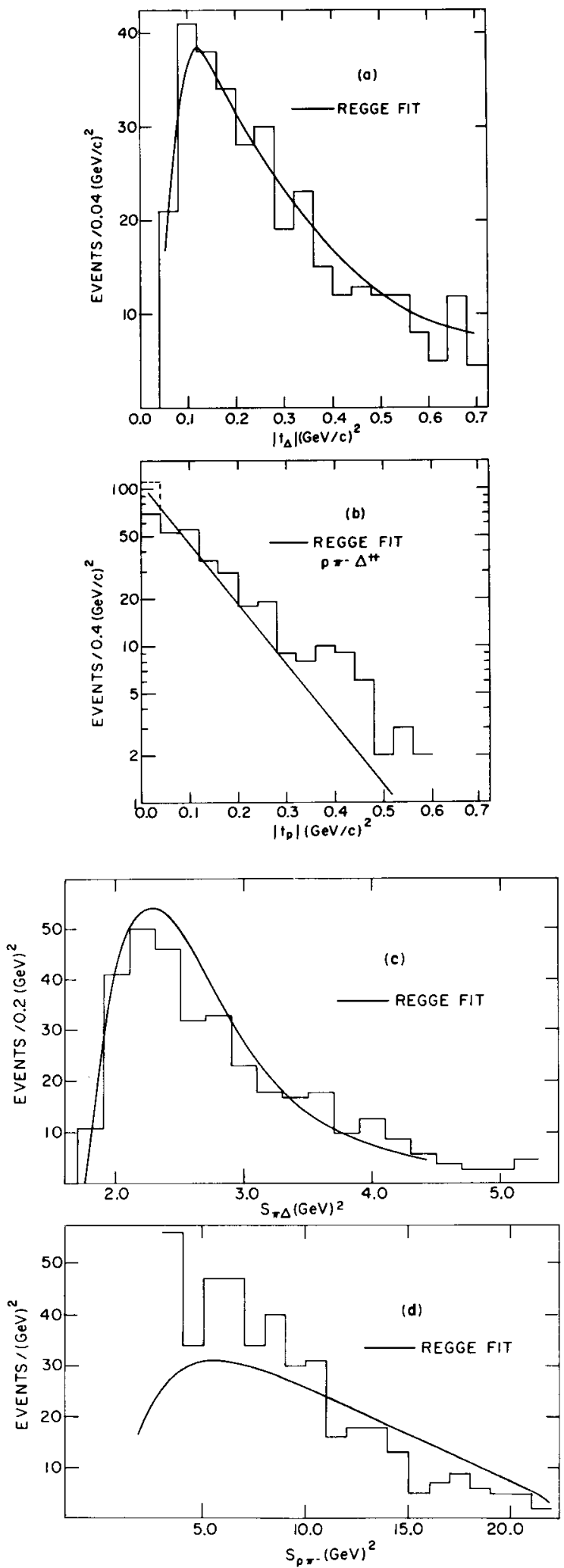

Figure 19. Momentum-transfer- $(a, b)$ and effective-mass$(c, d)$ squared distributions from the reaction $p+p \rightarrow p+$ $\pi^{-}+\Delta^{++}$. The curves show the predictions of the doubleRegge-pole model. 
matic regions in which the model is supposed to apply. $\rho$-Meson and pomeron exchange were assumed, and the parameters $\alpha_{\rho}(t=0) \approx^{1 / 2}$ and $\alpha_{\mathrm{p}}(t=0) \approx 1$ used in the model were consistent with those obtained in previous single-Reggemodel calculations.

A group at Harvard has studied the $3 \pi$ spectrum in the $A$-meson region ( 1.07 to $1.3 \mathrm{BeV}$ ) in the reaction $\pi^{-}+p \rightarrow p+\pi^{+}+\pi^{-}+\pi^{-}$at 13 and $20 \mathrm{BeV} / c$. Individual peaks are not resolved, but a broad enhancement is found in this region which decays into $\rho^{0} \pi^{-}$, as do the $A$ mesons. Attempts to fit the subsample of $p+\pi^{-}+\rho^{0}$ events to one-pionexchange and diffraction-dissociation models were conspicuously unsuccessful. However, results of a double-Regge-model calculation were in good agreement with the shapes of the $\pi^{-} \rho^{0}$ mass distributions, the momentum-transfer distribution, and the Treiman-Yang angle. The Regge interpretation is not necessarily incompatible with resonance production in the same mass region.

Several groups have investigated the production of nucleon isobars ( $\mathcal{N}^{*}$ 's) at high energy. Peaks at $\approx 1.4$ to $1.45 \mathrm{BeV}$ and $\approx 1.7 \mathrm{BeV}$ have been variously interpreted as kinematic effects or identified with resonances previously found by phaseshift analysis of $\pi$-p cross sections. The BNL Bubble Chamber Group has found an enhancement at 1.4 BeV occurring in two-prong events which accounts for most of the cross section previously observed in counter missing-mass experiments. This enhancement decays almost entirely into $n \pi^{+}$or $p \pi^{0}$ in the ratio $2: 1$, which confirms the isospin value $1 / 2$. Another peak at $1.65 \mathrm{BeV}$ may be the same as the $I=1 / 2$ resonance observed by counters at $1.69 \mathrm{BeV}$.

Peaks have been seen in the $p \pi^{+} \pi^{-}$final state in the following reactions:

$$
\begin{array}{ll}
p+p \rightarrow p+p+\pi^{+}+\pi^{-} & (22 \mathrm{BeV}, \text { Iowa State; } \\
& 25 \mathrm{BeV}, \text { Columbia- } \\
& \text { Rutgers; 28 BeV } \\
& \text { Brookhaven }) \\
& \\
K^{+}+p \rightarrow \mathrm{K}^{+}+\pi^{+}+\pi^{-}+p & (5.5 \mathrm{BeV} / c, \text { Johns } \\
& \text { Hopkins }) \\
\pi^{+}+p \rightarrow p+\pi^{+}+\pi^{+}+\pi^{-} & (1.6 \mathrm{BeV} / c, \text { Stanford } \\
& \text { Linear Accelerator } \\
& \text { Center) }
\end{array}
$$

Not all experiments agree in detail; however, in general the data seem to be consistent with an enhancement occurring at $\approx 1.45 \mathrm{BeV}$ whose cross section has a steep $t$ dependence $\left(\approx^{-b t}\right.$, with $\left.b \gtrsim 10 \mathrm{BeV}^{-2}\right)$, together with another peak at $\approx 1.69 \mathrm{BeV}$ with a much smaller $t$ dependence. The Brookhaven experiment at $28 \mathrm{BeV}$ does not resolve two peaks, although the cross section in the region of the peaks exhibits roughly the $t$ dependence just described. The Johns Hopkins group finds that the $1.45-\mathrm{BeV}$ enhancement in the data is formed principally of a $\Delta^{++} \pi^{-}$system whose decay asymmetry is incompatible with a pure-resonance interpretation. Dissociation-type models are found to fit the data reasonably well when cuts are made on the $K+\pi^{-}$mass; this is interpreted as evidence for diffractive $K^{+-} \pi^{-}$virtual scattering in the $K^{+} \pi^{-}$mass region.

Pion-deuteron elastic scattering at $3.65 \mathrm{BeV} / c$ was studied by a University of Michigan group using a sample of about 25,000 events from 230,000 photographs from the BNL 20-in. chamber (since converted to the 31 -in. chamber). The differential cross section is presented out to $-t \approx 1$ $(\mathrm{BeV} / c)^{2}$, and results are compared with theoretical predictions of the Glauber formula, which takes into account the scattering of the pions by the constituent proton and neutron in the deuterium. Agreement between experiment and theory can be achieved by hypothesizing various forms for the $t$ dependence on the pion-nucleon phases.

2. Weak interactions. Results in the area of weak interactions during the past year all come from experiments using the 30 -in. chamber. A Columbia-Stony Brook collaboration obtained forty-six $\Sigma-\rightarrow \Lambda^{0} e^{-} \nu$ and six $\Sigma+\rightarrow \Lambda^{0} e^{+} \nu$ decays. The ratios of these decay modes to the corresponding nonleptonic modes are, respectively, $(0.52 \pm$ $0.09) \times 10^{-4}$ and $(0.16 \pm 0.07) \times 10^{-4}$. A study of the momentum and angle correlations of the decay products yields the ratio of the vector to the axialvector coupling constants $G_{v} / G_{a}=0.7 \pm 0.4$ for the combined sample of $\Sigma^{ \pm} \rightarrow \Lambda^{0} e^{ \pm} \nu$. This result can be compared with the prediction of the hypothesis of conserved vector current for these decays, which says that $G_{v}=0$. A study of the strangeness-changing, hyperon-leptonic decays by a group from the University of Maryland gave the ratios $\left|G_{a} / G_{v}\right|=0.76$ \pm 0.24 for the decay $\Lambda \rightarrow p e^{-\nu}$ and $\left|G_{a} / G_{v}\right|=0.3 \pm$ 0.3 for $\Sigma-\rightarrow n e^{-} \nu$. These numbers are important for a deeper understanding of the $\beta$ decay interaction. It is possible to tie together various hyperon decay rates, viz., those of $\Lambda^{0}$ 's, $\Sigma \pm$, and $\Xi-$ hyperons, since these objects are all members of the same $S U(3)$ family of particles. 
The Maryland group also measured the ratio

$$
\frac{\Gamma\left(\Sigma^{-} \rightarrow n \mu^{-} \nu\right)}{\Gamma\left(\Sigma^{-} \rightarrow \text { all }\right)}=(4.1 \pm 0.8) \times 10^{-4},
$$

which is in excellent agreement with $(\mu \nu),(e \nu)$ universality for the weak interactions. In other words, except for mass, muons and electrons have identical properties.

A Rutgers University group analyzed $\approx 150,000$ pictures of stopping $K^{+}$particles in the 30 -in. chamber. About 140 events consistent with $K+\rightarrow$ $\pi^{+}+\pi^{0}+\pi^{0}$ were obtained by requiring that two or more converted $\gamma$ rays be associated with the $K+$ decay vertex. The dependence of the matrix element on the $\pi^{0}$ energy has been measured, and a linear extrapolation has been made to zero fourmomentum. The result is consistent with the softpion prediction of current algebra.

The lifetime of the short-lived $K^{0}$ meson was measured by the Brookhaven Nuclear Interactions Group using 20,000 $K_{S} \rightarrow \pi^{+} \pi^{-}$decays observed in the 30 -in. chamber filled with deuterium. An extensive study was made of the systematic errors and a best value of $\tau=(0.865 \pm 0.009) 10^{-10} \mathrm{sec}$ was obtained. This result can be compared with the latest value of $(0.874 \pm 0.011) 10^{-10} \mathrm{sec}$ compiled by Rosenfeld et al. This measurement was a by-product of the search for leptonic decays of the $K^{0}$ meson. About 600 such events have been found to date, and their analysis suggests the existence of $\Delta S=-\Delta Q$ events at the 2 to $2 \frac{1}{2}$ standard deviation level. This experiment was a collaboration with Carnegie-Mellon and Case-Western Reserve Universities.

A BNL group working in collaboration with groups at Yale and the University of Massachusetts studied $K^{0}$ decay to test $C P$ violation. If the long-lived and short-lived $K^{0}$ 's $\left(K_{L}\right.$ and $\left.K_{S}\right)$ have a common decay mode (in the same quantum state) then the decay into that mode violates the principle of $C P$ invariance. The long-lived $K^{0}$ is known to decay into three pions. Therefore the detection of the decay of the short-lived $K^{0}, K_{S} \rightarrow \pi^{+} \pi^{-} \pi^{0}$, and its interference with the decay products from $K_{L}$ decay would demonstrate a $C P$ violation. The initial phase of this experiment has been completed. A coherent sample of $K_{L}$ and $K_{S}$ was produced in the charge exchange reaction $K^{-}+p \rightarrow$ $\mathcal{N}+K^{0}$ in the 30 -in. bubble chamber at the AGS. From 80,000 measurements $(40,000$ at BNL), a sample of 30 three-pion decays was obtained within the first six $K_{S}$ lifetimes. The data reveal no
$K_{S}-K_{L}$ interference effects and show no evidence for the three-pion decay of $K_{\mathrm{S}}$, which rules out any large $C P$ violation.

\section{Cosmic-Ray Research}

A search by BNL-Yale collaborators for heavy stable particles in cosmic rays was concluded. While the experiment was expressly designed to detect heavy triplets that might form the bases of the $S U(3)$ symmetries observed in elementary particles, the results also concern particles of other possible origins. Particle detection was accomplished by means of a mass spectrograph which incorporated fast time-of-flight techniques and momentum analysis for the measurement of particle mass. The spectrograph had an aperture of $10^{-2} \mathrm{~m}^{2} \mathrm{sr}$, a time-of-flight resolution of $2.5 \mathrm{nsec}$, and a maximum detectable momentum $>300$ $\mathrm{BeV} / \mathrm{c}$. Measurements were made at an angle of $75^{\circ}$ with respect to the zenith. Four possible heavy particles were found in $700 \mathrm{hr}$ of running, whereas estimates indicate that five background events were to be expected for the run. The results set an upper limit of $2.4 \times 10^{-8}\left(\mathrm{~cm}^{2} \mathrm{sr} \mathrm{sec}\right)^{-1}$ to the flux of particles with a velocity of between 0.5 and $0.75 c$. Calculations based on plausible assumptions for triplet production in nucleon-nucleon interactions and based on the results of this experiment give an upper limit of about $2 \mu \mathrm{b}$ for the production cross section of triplets of mass $<15 \mathrm{BeV} / c^{2}$.

The energy spectrum of high energy cosmic-ray muons was measured at an angle of $75^{\circ}$ from the vertical. The results show that the direct muon production inferred from underground measurements of muons with energies $>1000 \mathrm{BeV}$ is not observed in these spectrograph measurements at energies up to $300 \mathrm{BeV}$.

An upper limit for the flux of heavy stable fundamental particles in the primary cosmic radiation is presented which is about two orders of magnitude less than that suggested to explain the anomalous cosmic-ray muon spectrum.

\section{Theory}

The high energy theory group has continued to pursue a number of topics in the major areas of current particle physics research.

When particles collide at high energies the most important effects taking place are described in terms of the exchange of virtual particles. One of the colliding particles emits a virtual particle which is observed by the other after possibly itself 
emitting one or more real particles. In the past few years it has been increasingly recognized that the fundamental process is not the exchange of a specific virtual particle, but the combined effect of the exchange of a whole family of particles, their individual contributions being inextricably mixed. The development of the techniques for dealing directly with these families, or "Regge trajectories," has been actively pursued. As a step toward the description of the emission of a real particle by such a Regge trajectory, an invariant amplitude expansion has been obtained for vertex functions of fields corresponding to arbitrary representations of the Lorentz group between states of arbitrary spins. Other work has dealt with the effect of multiparticle production on elastic scattering. By assuming a multiperipheral Regge exchange form for the multiparticle production, an integral equation had previously been obtained. Considerable progress has now been made in reducing the 6 dimensional equation to a 1 -dimensional one by group theoretical techniques, to give a possible approach to a solvable model for nonforward scattering, not previously tractable.

An important current problem is to find descriptions of scattering amplitudes that are consistent with both the high energy Regge exchange description and the low energy resonance dominance models. An elegant mathematical model due to Veneziano exhibits such a connection but only allows resonances of zero width, and thus violates unitarity. An approximate unitary solution for the $\pi-\pi$ scattering amplitude, for which the Veneziano model is the leading term, has been developed, incorporating finite width resonances.

The formalism of quantum electrodynamics has long been well developed. The difficulties in this field lie in the complicated calculations needed to extract results. Several problems in this area were studied with extensive use of the computer as an algebraic aid. Results were obtained on the twophoton exchange contribution to electron scattering from a proton. It was found that radiative corrections from this source differed from those in electron-electron scattering and that a contribution to the difference between electron and positron scattering from protons, expressible in terms of a sum over photoproduction cross sections, grows with unexpected rapidity at high energies and may affect the analysis of form factor measurements. Other problems under investigation in this area include a calculation of the radiative correc- tions to the decay process $\pi^{0} \rightarrow e^{+} e^{-} \gamma$ and the calculation of the slope of the electron form factor in fourth order, involving sixth-order electrodynamic contributions.

A number of phenomenological studies were undertaken. An analysis was given of the possible angular correlations that can occur when a boson decays into two spin- $1 / 2$ particles, which then undergo weak parity-violating decays, and measurements were suggested that would assist in determining the spin and parity of the parent boson. A double-baryon exchange model was used to investigate the high energy backward $A_{1}$ production in the reaction $\pi+p \rightarrow p+\pi+\rho$. In a simple form in which $\Delta$ and $\mathcal{N}$ trajectories are exchanged, this model shows a "kinematic" enhancement in the $A_{1}$ region. However, the detailed agreement is not sufficient to regard this alone as the explanation of the $A_{1}$ meson.

Other work of the group has been concerned with problems of uniqueness in the so-called hardpion formalism of current algebra, in regard to both sum rules and the Ward identities for the three point functions.

\section{0-in. Bubble Chamber}

The 80 -in. bubble chamber operated during most of the year. From April 1, 1968, to March 31, 1969 , a total of 1.5 million pictures, distributed among 14 experiments, were produced. All experiments used the high energy rf separated beam; the chamber was filled with hydrogen for 13 experiments and with deuterium for one.

A new hydraulic-powered expansion system was installed on the chamber to minimize the loss of deuterium. This system enables the chamber to be filled with deuterium and then sealed, which eliminates all losses except those due to external gas handling. The hydraulic system is also expected to reduce the rate of dirt accumulation in the chamber, improve pulse-to-pulse stability of the chamber minimum pressure, and provide greater long-term reliability than did the old pneumatic system. Installation of the new system introduced numerous operational problems, whose solution resulted in a considerable loss of operating time. Work has started on design of a bright-field illumination system and a change from 70 to $35-\mathrm{mm}$ film.

\section{0- and 31 -in. Bubble Chambers}

An alternating mode of operation of the 30 and 31 -in. bubble chambers was begun in July 1968 . 
In this mode one chamber is scheduled for high energy physics research while the other is maintained in a standby condition or is used to investigate new developments and techniques. Staffing these two chambers with a combined crew has released some operating personnel for work in other areas.
In the 30 -in. chamber, 886,000 pictures were taken for six experiments using either hydrogen or deuterium.

\section{Particle Physics Apparatus}

The use of liquid neon-hydrogen mixtures in cryogenic bubble chambers permits the detection

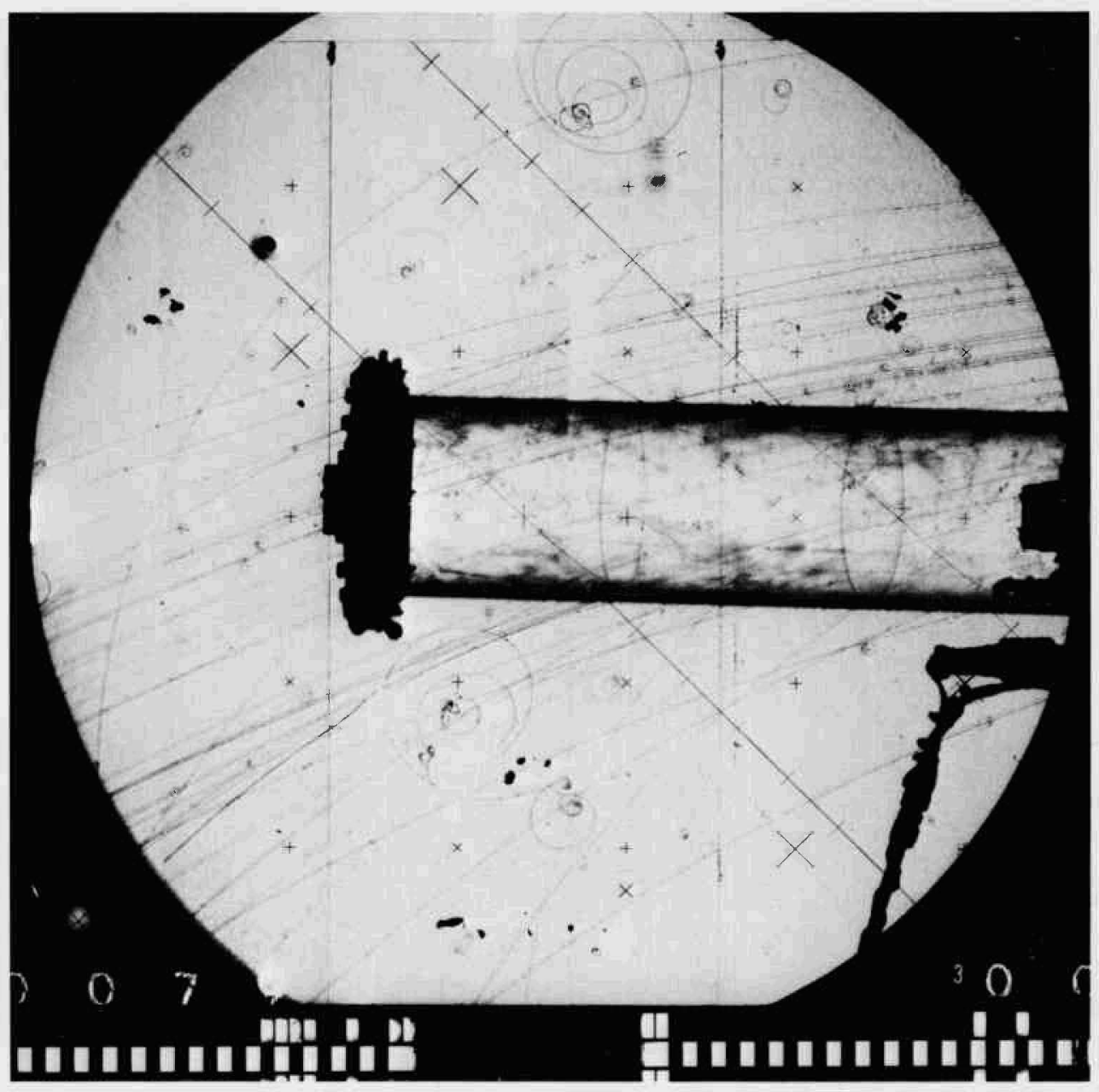

Figure 20. Photograph of the track-sensitive target mounted in the 30 -in. bubble chamber. The target contained pure hydrogen; the bubble chamber, $20 \%$ neon-80\% hydrogen (mole $\%$ ).

During the year 1.2 million pictures for five experiments were taken in hydrogen, deuterium, and liquid neon-liquid hydrogen mixtures in the 31-in. bubble chamber. Modifications of the cameras, data-box system, and illumination system for double-pulse operation were completed; it is now possible to expand the chamber twice during each AGS pulse and thereby double the rate at which pictures are taken. of $\gamma$ rays produced in certain interactions. However, the neon causes a reduction in measurement accuracy and in the number of interactions with hydrogen. These difficulties have prompted the development of track-sensitive targets in order to combine the advantages of hydrogen (or deuterium) and of hydrogen-neon mixtures. A tracksensitive target is a small, transparent chamber within the bubble chamber, both chambers being 


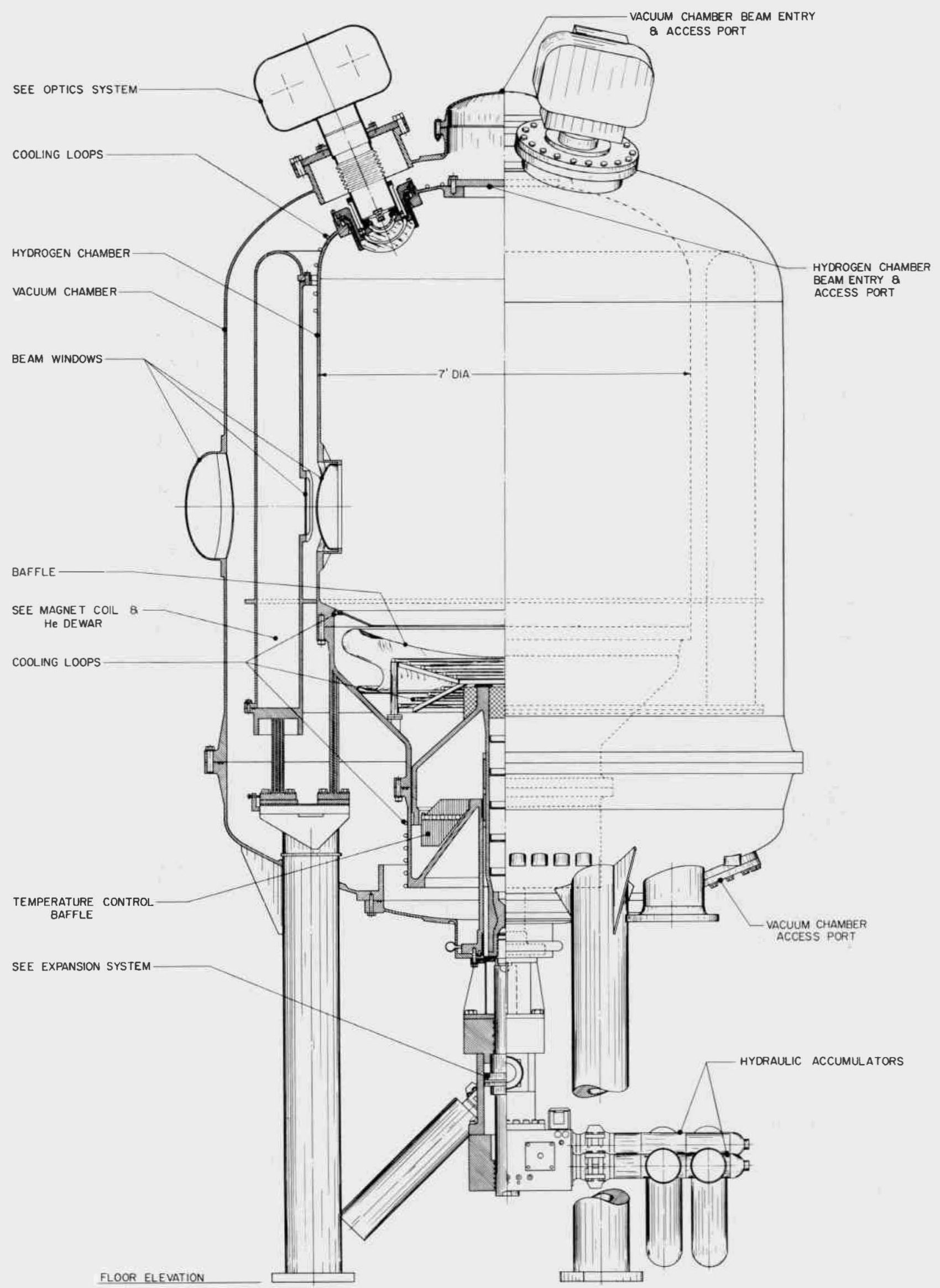

Figure 21. Cross-section drawing of the 7-ft hydrogen bubble chamber. The common vacuum tank for the chamber and helium Dewar is $10^{1 / 2} \mathrm{ft}$ in diameter, stands $18 \mathrm{ft}$ high, and serves as a protective enclosure in case of component failure. 
made sensitive and photographed simultaneously. A test has been successfully completed in which a 5-in.-diam Plexiglas cylinder 20 in. long was used in the 30-in. bubble chamber (see Figure 20). The inner chamber contained pure hydrogen, and the outer chamber, $20 \%$ neon- $80 \%$ hydrogen (mole \%). An experiment with such a device has been proposed for the 30 -in. bubble chamber. It is clear that track-sensitive targets will be extremely useful in large chambers such as the 7-ft chamber described below.

Assembly and testing of a small streamer chamber borrowed from the Stanford Linear Accelerator Center was begun in order to gain experience with this type of track detector. The streamer chamber is an instrument with high detection efficiency and with space resolution approaching that of a bubble chamber, which can be triggered in response to external counter signals. By triggering on particular configurations of emerging particles, adequate data can be acquired on certain types of rare events occurring in the presence of large background. An attempt will be made to install a small liquid hydrogen target inside the chamber.

The 7-ft bubble chamber has been set up in a neutrino beam at the AGS. The chamber will he filled with about 9000 liters of hydrogen and tested. Ultimately about 1 million pictures will be taken with a deuterium filling to produce about 2000 neutrino interactions if the present full AGS intensity of $2 \times 10^{12}$ protons/pulse is used and if a threeelement magnetic-finger focusing device is effective in maximizing the neutrino flux.

As long ago as 1964, large-volume hydrogen chambers were designed at BNL. However, no funds were approved for constructing devices similar to Argonne's 12-ft chamber or CERN's 3.7-m chamber. Consequently in 1966 the 7 - $\mathrm{ft}$ model chamber program was started for research and development of new concepts involved in the new generation of hydrogen bubble chambers. These techniques include wide-angle fisheye-window optics, magnets employing superconducting materials, and plastic expansion pistons. When in later years there were still no funds for a large chamber, Brookhaven decided to operate the 7-ft model for a neutrino experiment while continuing the research and development work.

A cross-section view of the $7-\mathrm{ft}$ chamber is shown in Figure 21. The chamber vessel has a total volume of 9400 liters and is constructed of 1/2-in.-thick stainless steel. A Scotchlite retrodirective coating will be mounted on the interior surface. Two of three camera ports located at the top of the chamber can be seen in the figure. Images of the track bubbles appear on film as dark spots on the bright background provided by the Scotchlite, which is illuminated by a light source located near each camera.

The expansion system is at the bottom of the chamber; this location is one of the new design concepts under consideration for most currently proposed large-volume chambers. Several novel devices are used in the 7-ft expansion system, e.g., the 42-in.-diam Fiberglas-reinforced plastic piston, which must move 4 in. in about 25 msec. The piston is driven by a fast-response hydraulic unit, which will allow several expansions on each accelerator pulse.

Perhaps the most important feature of the new chamber is the use of superconducting coils in the magnet. In May 1968 the magnet was tested at $60 \%$ of the design current of $6000 \mathrm{~A}$, which will produce a field of $30,000 \mathrm{G}$ in the center of the chamber.

Figure 22 shows the last piece of the vacuum tank assembly being moved into position on March 24, 1969. Figure 23 is an aerial photograph of the neutrino experimental area where the $7-\mathrm{ft}$ chamber will be operated. Further information about the 7-ft chamber can be found in the 1968 Annual Report.

The neutrinos are provided by directing a proton beam from the AGS onto an external target. Mesons produced in the target are focused in the direction of the chamber by a "magnetic finger" device. A drift space provides time for the mesons to decay, after which all particles except the resulting neutrinos are absorbed in a filter consisting of $110 \mathrm{ft}$ of steel. All components of the beam have been installed and tested. The magnetic finger was successfully pulsed 100,000 times to full power. A simple charged-particle test beam has also been provided in this area.

During the past year a major activity of the BNL Bubble Chamber Group has been the preparation of a proposal for construction of a 25-ft cryogenic bubble chamber which is to be a joint project between BNL and the National Accelerator Laboratory. This bubble chamber, to be operated at the 200-BeV accelerator at Batavia, Ill., will be $25 \mathrm{ft}$ long, will contain about 100,000 liters of liquid, and will be provided with a magnetic 

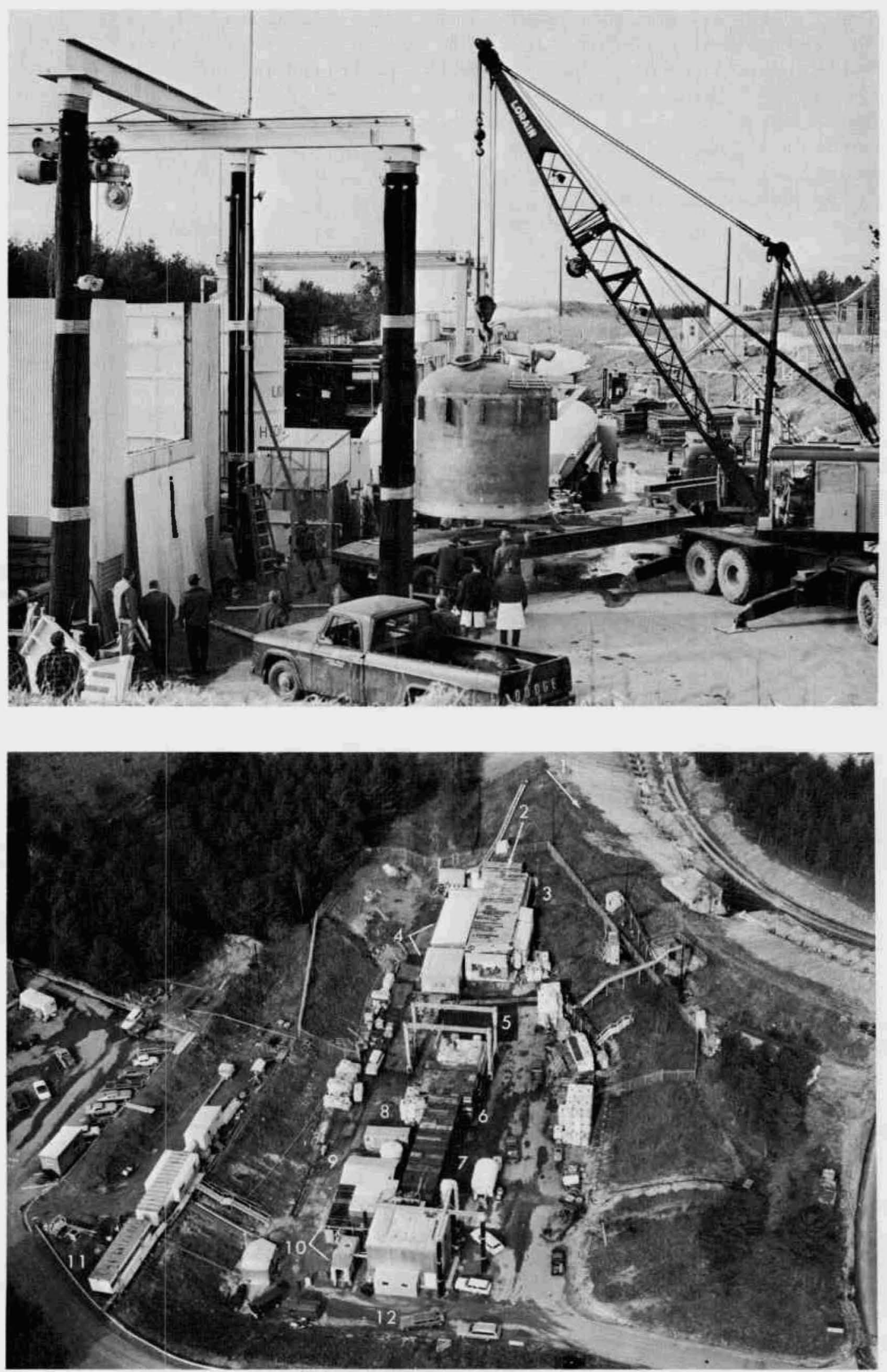
$\leftarrow$ Figure 22. The vacuum tank for the 7-ft bubble chamber being moved into position for completion of the chamber assembly. The chamber is in the building to the left, which has a removable roof and an outdoor crane suspended on 35 -ft-high telephone poles.

field of $40 \mathrm{kG}$. Since the proposal was not accepted for funding in fiscal 1970, it will be resubmitted for fiscal 1971. A research and development program is continuing on wide-angle optics and hollow superconductor-normal conductor composites for possible use in such a project.

Another BNL group is developing a double-vee magnetic spectrometer system, part of which has been assembled in the AGS slow external beam area. A special beam system allows negative and positive momentum-analyzed secondary-beam particles to be incident on a hydrogen target. Part of the setup of the forward vee detecting apparatus at the AGS, which includes a set of spark chambers with a sensitive area as large as $4 \times 13 \mathrm{ft}$, is shown in Figure 24. This setup has been used to test the functioning of the new large-scale sparkchamber system. The three sets of chambers before the magnet each contain sets of $x, y$, and $w$ coordinate detector hodoscopes and thus allow the reconstruction of each individual track from a vertex resulting from a forwardly emitted decaying vee or some other collection of particles. The sparkchamber windows are transparent so that the sparks obtained in actual test operation are visible. However, no photography is used in this entirely automatically digitized system. Each spark location in the various $x, y$, and $w$ planes is read out by a magnetostrictive readout wire, digitized, and automatically stored on tape, and a sample of the

$\leftarrow$ Figure 23. Aerial photograph taken in November 1968 of the equipment being installed for the neutrino experiment at the AGS with the 7-ft hydrogen bubble chamber. (1) Indicated direction of protons in the main ring tunnel. (2) Fast external proton beam direction. (3) Concrete shielding house for magnetic-finger focusing device and target. (4) Power supply houses. (5) Large gantry crane used for installing shielding. (6) Iron filter (12,000 ton, $110 \mathrm{ft}$ long), which prevents all particles except neutrinos from entering the chamber. (7) Large hydrogen and deuterium Dewars. (8) Hydraulic power supply trailer for chamber's expansion system. (9) House for helium equipment needed for superconducting chamber magnet. (10) Vacuum pump trailers. (11) Control trailer. (12) House for 7 -ft bubble chamber. data is simultaneously transmitted to the on-line computer system of the On-Line Data-Processing Facility (OLDF). Although the tests have only recently commenced, the results are encouraging and imply that track resolutions of $0.25 \mathrm{~mm}$ can be attained (as predicted) in this large-scale system (up to $13 \mathrm{ft}$ sensitive length will be installed in the forward vee system, and up to $23 \mathrm{ft}$ in the wide-angle or recoil vee system).

The readout system, neon-helium gas circulation and purification system, and the elementary track recognition software were all found to function reasonably well in these first preliminary tests.

A large counter hodoscope, $13 \mathrm{ft}$ wide at the downstream end of the forward vee detecting leg, triggers the spark chambers when two or more charged particles pass through it. The large number of tracks shown in Figure 24 represent the slow camera's integration of repeated trigger events (perhaps ten or more). This system is capable of triggering and measuring one million events per day and is expected to increase the statistics obtainable in many classes of double vertex events, such as

$$
\begin{aligned}
\pi^{ \pm}+p & \rightarrow \Upsilon\left(\text { or } \Upsilon^{*}\right)+K\left(\text { or } K^{*}\right), \\
& \rightarrow \mathcal{N}\left(\text { or } \mathcal{N}^{*}\right)+(\rho \text { or } \phi, \text { etc. }),
\end{aligned}
$$

by more than two orders of magnitude compared with those previously available in visual techniques. The precision of angular and momentum

Figure 24. The three sets of digitized chamber hodoscopes that detect and reconstruct a forwardly emitted vee. The hydrogen target is located outside the photograph to the right, and the magnet for measuring the momenta of the particles, to the left. The actual sparks that mark the tracks of charged particles can be seen as short, bright dashes in the three spark chambers shown.

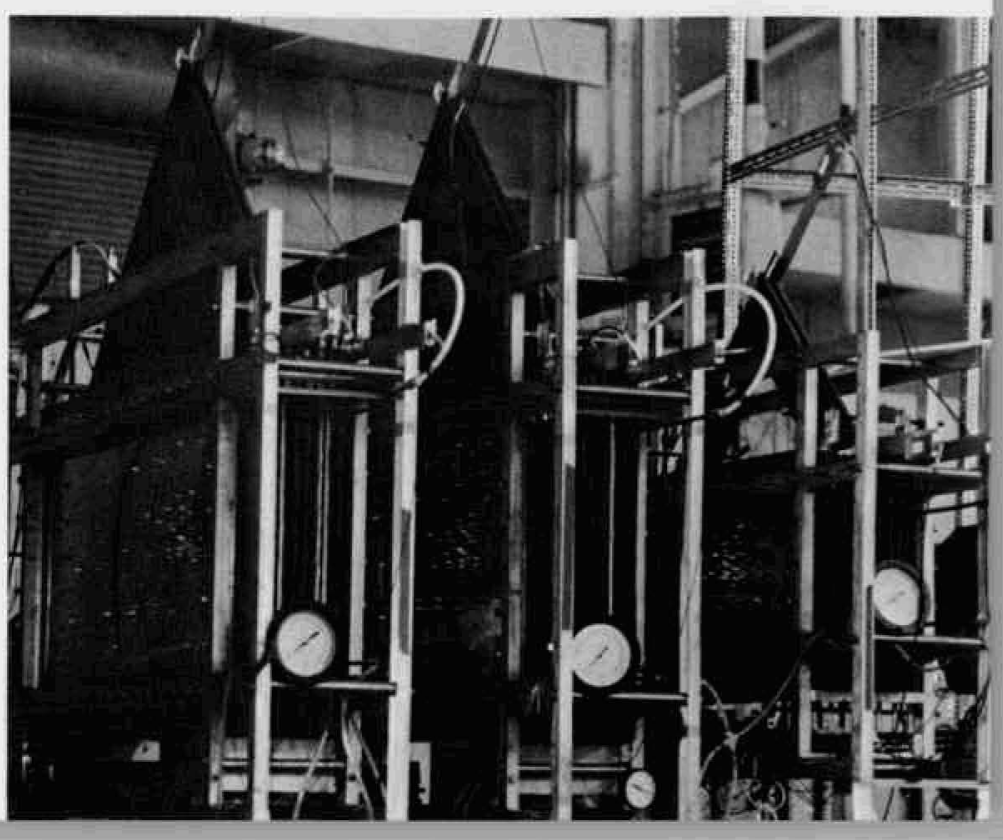


measurements will also be greatly increased over that obtained in previous investigations.

During this primary test period, the on-line computer program was successfully used for kinematical reconstruction of forward-going $K$ particles and the associated recoil mass from the $\pi^{-}-p$ interaction.

\section{Data Analysis}

The data-processing capability of the Bubble Chamber Group has improved to the extent that the group now ranks third (after two groups at the Lawrence Radiation Laboratory) in the world in number of events measured. The latter totaled 310,000 , of which 290,000 were measured by the flying-spot digitizer (FSD). Two FSD's were in use, both on-line to an IBM 7094 computer. The scanning and road-guidance measurements were done on 11 scanning machines. By road guidance is meant the rough measurement of three points on each track to guide the FSD system to the tracks to be measured.

The data analysis system of the Nuclear Interaction Group now has 12 high-precision image plane digitizers, each with a $2-\mu$ least-count on film, all connected on line to an SDS 920 computer. The system produced $\approx 100,000$ events last year involving two experiments, one mainly a scanning experiment with a measurement rate of $\approx 1$ event/ 100 pictures, and the second a high-measurement experiment with a measuring rate of $\approx 1$ event/ 2 pictures. The on-line features of the system provide constant monitoring of scanning and measuring, including the individual rates and efficiencies of the personnel.

Improvement in the bubble-chamber dataprocessing is occurring in two areas: (1) A satellite computer system, the Data Terminal Network (DTN), using a Sigma-7 computer has been set up to provide data collection for, and data distribution by, the CDC 6600 computers of the Laboratory's central computing facility. The DTN is rapidly becoming the control center of the entire bubble-chamber data-processing operation. An attempt is under way to increase the scanning rate by eliminating the need to make roads for FSD measurement. "Minimum guidance" (MG) FSD measurement (where only vertices are digitized at the scan table) has been shown to be feasible. A production MG system capable of about twice the present 500,000 events/year (without increased operating staff) is under construction. Figure 25 shows a DTN television display of the track-finding performance of the main MG program for a particular event. A prototype of a similar display which an operator can use for correction and completion of MG program results is shown in Figure 26.

During the past year the OLDF has been employed for the majority ( $75 \%$ ) of the counter-sparkchamber experiments performed at the AGS. The percentage would have been even larger had there been adequate facilities to meet all requests.

Recently a disk system was made operational, and this, with the $80 \mathrm{~K}$ of core, made it possible to regularly put two users simultaneously on line while two off-line user groups were preparing and modifying programs. These off-line users changed places with the on-line users every week or two. Thus, four or five groups overlapping in AGS schedule were accommodated two at a time on line. The groups included physicists from CERN, from Columbia, Carnegie-Mellon, Case-Western Reserve, Harvard, McGill, Rochester, Yale, and Princeton Universities, and from BNL.

In the post-AGS shutdown period (from November 1969), it is estimated from surveys made by members of the OLDF and the High Energy Advisory Committee that it will be necessary to provide for four on-line and four off-line user groups simultaneously, which will require a doubling of the facility's capability. To effect this, a basic PDP-10 system (Phase Ia) has been ordered and delivered. The completion of this system will be pursued, to the extent that funds are available, in fiscal 1970.

\section{MEDIUM ENERGY PHYSICS}

One of the interesting facets of the work performed by the Brookhaven group at the Cosmotron was the observation of structure in the high energy spectrum of deuterons. These deuterons are produced in the forward direction by high energy protons incident on deuterium and complex nuclei. This was one of several pieces of information that led to the conjecture that 2-nucleon-pion resonance states might occur in nature. For the last year the group has been involved in preparing and carrying out an experiment in which one of the aims is to check the above hypothesis. A trailer was outfitted with the necessary fast logic and counting electronics, the required hodoscopes were assembled, and all the equipment was shipped to 


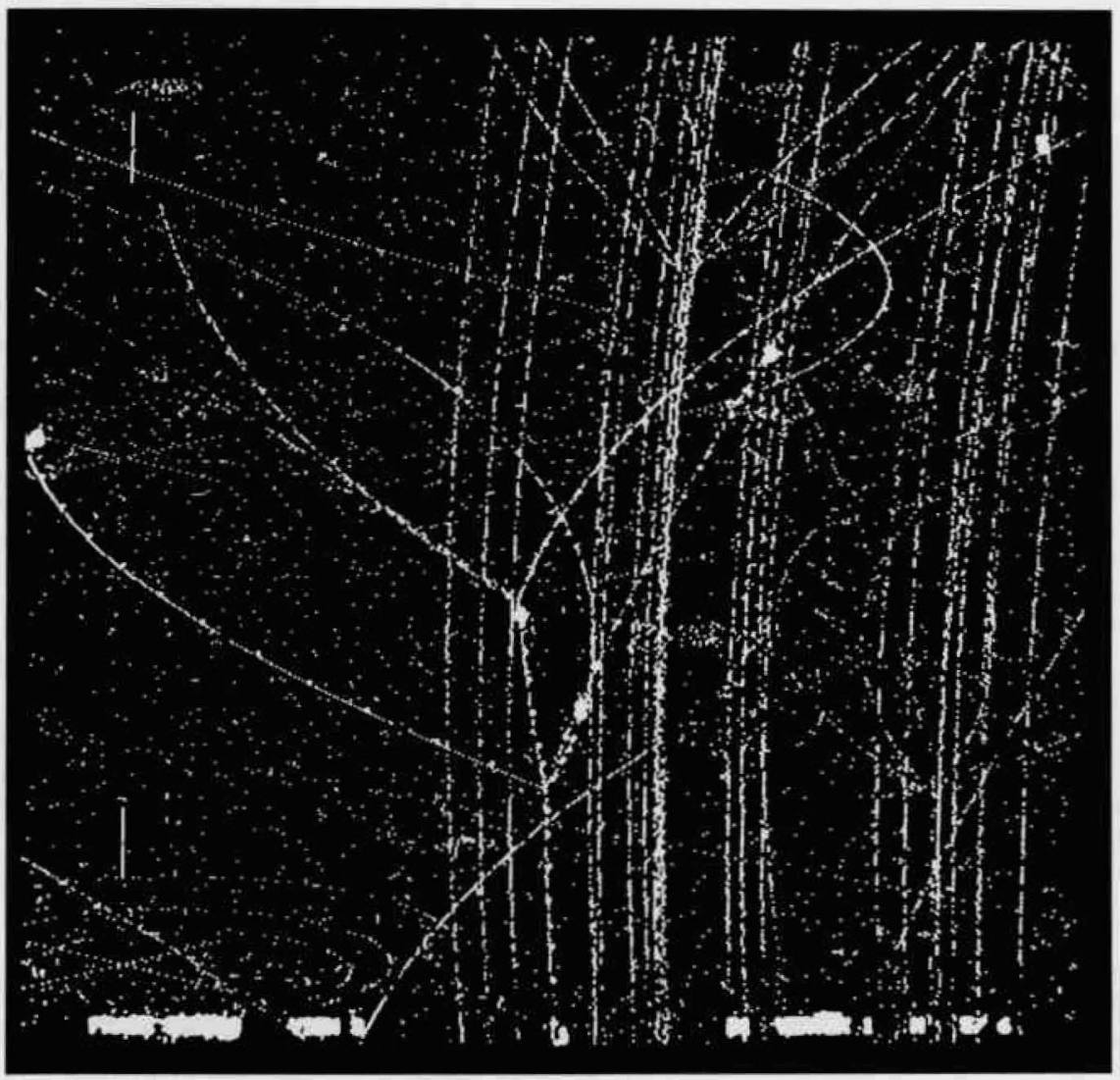

Figure 25. Data terminal television display showing the flying-spot-digitizer measurements of a bubble-chamber picture. Bright spots superimposed on the picture show the tracks found by a track-recognition program operating on the measurements.
Figure 26. A "fix-up" station at which an operator, by referring to the original bubble-chamber photograph, can correct failures of the track-recognition system.

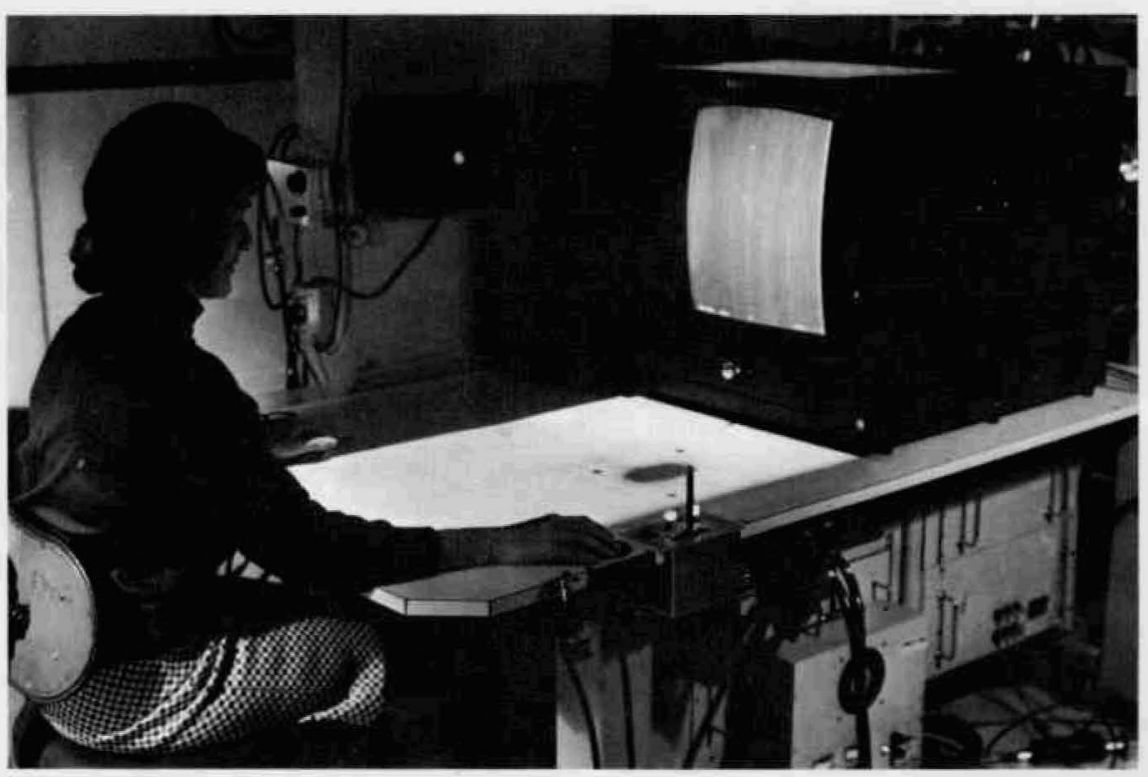


the University of California at Berkeley early in 1969. The experiment was set up on the Bevatron floor. The numerous counting elements of the scintillation counter hodoscopes were adjusted, the background rates were investigated, and the modifications required to keep these rates from being troublesome were made. Data-taking started about the middle of April, and the measurement was completed by the middle of June. The data are now being analyzed.

\section{NUCLEAR STRUCTURE}

The study of nuclear ground and excited states continues to play an essential role in providing the accurate information without which nuclear theories and models cannot evolve. Properties of such states that are amenable to precise measurement and to which attention has been devoted include energies, spins, parities, lifetimes, and electromagnetic moments. In addition, those properties of individual transitions that may provide significant nuclear structure information have been intensively investigated in several instances. These properties include multipolarity mixing ratios, internal conversion coefficients, and related factors, such as particle parameters. It is important to emphasize the need for detailed knowledge of all these properties for individual transitions, for it is precisely such information that often makes it possible to design and execute tests of physical principles and ideas of general interest in physics, not merely in nuclear structure physics. A few selected examples of recent research efforts are briefly described in the following paragraphs.

The neutron capture reaction is ordinarily regarded as the classic case of the compound nucleus or resonance reaction, in which complex nuclear states, whose properties usually obey statistical laws, are formed. Alternatively, neutron capture may also proceed as a direct reaction, with a much smaller cross section. A confirmation of the existence of the direct capture process was reported earlier by the Neutron Physics Group: interference between direct and resonance capture was observed for several target nuclei. Independent evidence for direct capture has also existed for some time in the form of a correspondence between the $(n, \gamma)$ and $(d, p)$ reactions in a few light nuclei $(A \approx 60)$. Evidence of the latter type has now been found for heavier nuclei $(A \approx 140)$. A strong correlation is observed between the $(n, \gamma)$ and $(d, p)$ re- actions for three nuclei with filled $\mathcal{N}=82$ neutron shells: $\mathrm{Ba}^{138}, \mathrm{Ce}^{140}$, and $\mathrm{Nd}^{142}$. For $\mathrm{Ba}^{138}$, for which the most detailed $(d, p)$ reaction data exist, the coefficient of the correlation between $(d, p)$ and $(n, \gamma)$ strengths attains the high value 0.95 . For such closed neutron shell nuclei, this effect may be understood in terms of the common unique parent assumption of Lane and Wilkinson. In this formulation a nucleus containing $A$ nucleons is described in terms of a complete set of parent states containing $A-1$ nucleons coupled to a single nucleon. The initial state in the $(d, p)$ reaction then consists naturally of a single parent state, the ground state of the target nucleus, and a single odd neutron. If the initial state formed in the $(n, \gamma)$ reaction contains a large admixture of the same parent state, then a correlation between the strengths of the two reactions will arise. Similar arguments apply to the final states in the reactions. The strong correlations observed for $\mathrm{Ba}^{138}$ and $\mathrm{Ce}^{140}$ are believed to be attributable to this process. For $\mathrm{Nd}^{142}$, however, the thermal neutron cross section $(\approx 17 \mathrm{~b})$ is believed to originate largely with a bound level which should lead to a complicated configuration for the capture state. The observed correlation with the $(d, p)$ reaction may thus arise from the predominance of the parent state in only the final state. The channel resonance capture process postulated by Lane and Lynn may also be important for $\mathrm{Nd}^{142}$.

Experiments have been performed to study the Coulomb excitation of the odd-mass molybdenum isotopes, $\mathrm{Mo}^{95}$ and $\mathrm{Mo}^{97}$. Thick separated isotope targets of $\mathrm{Mo}^{95}$ and $\mathrm{Mo}^{97}$ were bombarded with $\alpha$ particles of up to 7.3-MeV energy. Observation of the de-excitation $\gamma$ rays with high-resolution $\mathrm{Ge}(\mathrm{Li})$ detectors has revealed the excitation of five levels below about $1.1 \mathrm{MeV}$ in each of the two isotopes. Relatively large $B(E 2)$ values ranging from 5 to 30 single-particle units were deduced for each of these states.

It is thought that states with large $B(E 2)$ to the ground state are exhibiting collective effects of excitation of the core nucleons to a $2+$ configuration similar to the $2+$ states of the even nuclei. The simple description of core excitation multiplets by de Shalit characterizes these states as resulting from the coupling of a single odd nucleon to the excited $2+$ core. If such a description, with small mixing of single-particle excitation, applies, then these states would be only weakly excited in stripping reactions on the neighboring even nuclei. 
It was found, in fact, that for both molybdenum isotopes the states that exhibit large $E 2$ transition speeds are poorly excited in $(d, p)$ stripping reactions, and vice versa. Indeed, some of the levels with large $B(E 2)$ strengths are not observed at all in the stripping experiments. This is in contrast to what is observed for several medium-mass nuclei ( $\mathrm{Cu}^{63}$, for example), which show large spectroscopic factors for states with large $B(E 2)$ values. It appears, therefore, that the core excitation states in the molybdenum isotopes are particularly pure.

The strongly deformed nucleus $\mathrm{Er}^{166}$ exhibits a well-developed rotational band built on the $0+$ ground state and a well-developed rotational band (up to spin 8) built on the $\gamma$-vibrational state at $787 \mathrm{keV}$. The $I \rightarrow I-1$ transitions within the $\gamma$ band are expected to be of the $E 2$ type, with intensities relative to the crossover $E 2$ originating from the same level by an expression of the form

$$
\frac{I_{\gamma}(E 2 ; \text { stopover })}{I_{\gamma}(E 2 ; \text { crossover })}=\left(\frac{E_{s o}}{E_{c o}}\right)^{5} \cdot \frac{\left|C_{2}^{I_{i} 2 I_{i}-1}\right|^{2}}{\left|C_{2}^{I_{i} 2 I_{i} I_{i}-2}\right|}
$$

To the extent that rotational states correspond to the rotation of a uniform nuclear fluid, $M 1$ intraband transition rates are zero. A departure from such behavior should manifest itself by the introduction of an $M 1$ admixture into the intraband $\Delta I=-1$ transitions. Although this $M 1$ rate can be expected to be small, its measurement may provide an additional way to determine departures from pure rotation within the band and may suggest the mechanism responsible for the departure. A search was made for these $\Delta I=-1$ transitions within the $\gamma$ band with a $\mathrm{Ge}(\mathrm{Li})$ detector. The $7+\rightarrow 6+, 6+\rightarrow 5+$, and $5+\rightarrow 4+\gamma$-band transitions were observed and their intensities measured relative to the $E 2$ crossover transitions which originate from the same state. The intensities of the $I \rightarrow I-1$ transitions exceed the prediction derived from Eq. (1). The excess intensity is attributed to an $M 1$ admixture into the transition and allows a determination of $\delta^{2} \equiv E 2 / M 1$ for each transition.

To provide a comparison of the ratio of the $E 2 / M 1$ matrix elements, the $\delta^{2}$ values have been manipulated in the following manner.

$$
\begin{aligned}
& \frac{I(E 2, s o)}{I(M 1, s o)} \equiv \delta^{2} \frac{\left|M_{E 2}\right|^{2}}{\left|M_{M 1}\right|^{2}} \cdot \frac{E_{s o}^{5}}{E_{s o}^{3}} \cdot \frac{\left|C_{2}^{I_{i}} \mathbf{2}_{2}^{I_{i}-1}\right|^{2}}{\left|C_{2}^{I_{i}} \mathbf{1}_{2}^{I_{i}-1}\right|^{2}}
\end{aligned}
$$

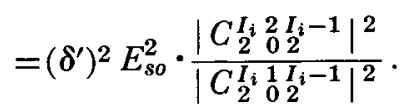

The reduced mixing ratio

$$
\left(\delta^{\prime}\right)^{2} \equiv \frac{\left|M_{E 2}\right|^{2}}{\left|M_{M 1}\right|^{2}}
$$

is the quantity of interest. The experimentally observed branching ratios, mixing ratios $\left(\delta^{2}\right)$, and reduced mixing ratios $\left[\left(\delta^{\prime}\right)^{2}\right]$ are listed in Table 1 , where the $E^{2}$ correction has been made by normalizing to unity for the $118.9-\mathrm{keV} 5+\rightarrow 4+$ transition. The branching ratio data are summarized in Figure 27. Although the precision available is not high, the values of $\left(\delta^{\prime}\right)^{2}$ decrease on going up the band. The $\left(\delta^{\prime}\right)^{2}$ values are most consistent with an $I^{2}(I+1)^{2}$ dependence of $\left|M_{M 1}\right|^{2}$, as illustrated in Table 1, although a dependence proportional to either $I(I+1)$ or $I^{3}(I+1)^{3}$ cannot be ruled out by these data.

Table 1

\begin{tabular}{|c|c|c|c|c|c|c|}
\hline$I_{i}$ & $\lambda_{\exp }$ & $I_{\gamma}(E 2)$ & $I_{\gamma}(M 1)$ & $\delta^{2}$ & $\left(\delta^{\prime}\right)^{2}$ & $f$ \\
\hline 7 & $0.032 \pm 0.004$ & 0.0176 & $0.0144 \pm 0.0040$ & $1.22+0.47$ & $2.7 \pm 0.6$ & $8 \pm \pm_{-1.9}^{3.1}$ \\
\hline 6 & $0.0372 \pm 0.0084$ & 0.0281 & $0.0091 \pm 0.0084$ & $\leqslant 1.6$ & $\leqslant 3.3$ & $\leqslant 5.8$ \\
\hline 5 & $0.063 \pm 0.005$ & 0.0514 & $0.0116 \pm 0.0050$ & $4.43_{-1.33}^{+3.36}$ & $8.9_{-2.7}^{+6.7}$ & $8.0_{-2.4}^{+6.0}$ \\
\hline \multicolumn{4}{|c|}{$I_{i}=$ initial spin. } & & \multicolumn{2}{|c|}{$I_{\gamma}(M 1)=I_{\gamma}\left(I_{i} \rightarrow I_{i}-1\right)-I_{\gamma}(E 2)$} \\
\hline \multicolumn{3}{|c|}{$\lambda_{\exp }=\frac{I_{\gamma}\left(I_{i} \rightarrow I_{i}-1\right)}{I_{\gamma}\left(I_{i} \rightarrow I_{i}-2\right)}}$. & & & \multicolumn{2}{|c|}{$\left(\delta^{\prime}\right)^{2}=\frac{\left|M_{E 2}\right|^{2}}{\left|M_{M 1}\right|^{2}}$; see Eq. (2). } \\
\hline \multicolumn{4}{|c|}{$I_{\gamma}(E 2)=$ calculated $E 2 \gamma$-ray intensity for stopover; from Eq. (1). } & & \multicolumn{2}{|c|}{$f=10^{-3}\left(\delta^{\prime}\right)^{2} \cdot I_{i}^{2}\left(I_{i}+1\right)^{2}$} \\
\hline
\end{tabular}

Summary of Branching Ratio Data, Calculated Stopover $\gamma$-Ray Ratios, Reduced Mixing Ratios (see text), and Reduced Mixing Ratios Multiplied by $I_{i}^{2}\left(I_{i}+1\right)^{2}$ 


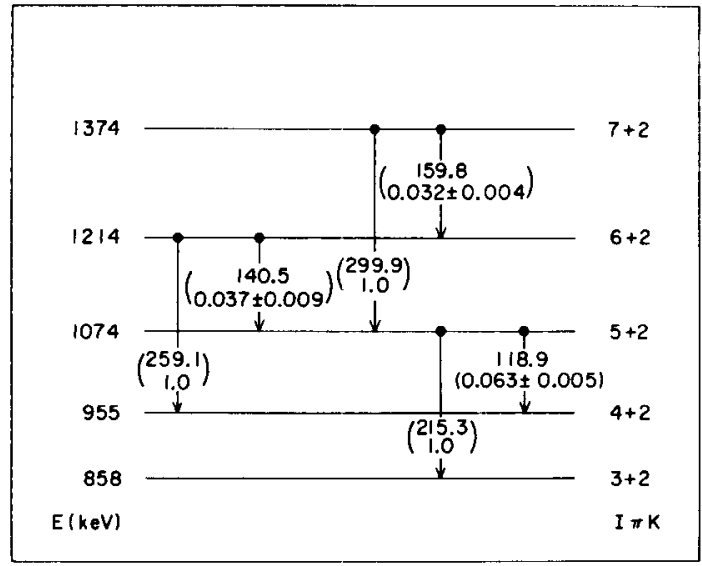

Figure 27. Summary of measured $\Delta I=-2$ and $\Delta I=-1$ intraband $\gamma$-ray branching intensities within the $\gamma$ band of $\mathrm{Er}^{166}$. Interband transitions into the ground-state band are not shown.

The absolute $M 1$ transition probabilities for these intraband transitions, although comparable to the enhanced $E 2$ rates, are nevertheless still small, of the order of 0.01 Weisskopf units. With a reasonable assumption about the intrinsic quadrupole moment $Q_{0}$ for the $\gamma$ band, the $M 1$ rates in the $\Delta I=-1$ transitions imply a $g$-factor difference which is not zero but rather is a significant fraction $\left(\approx^{1 / 3}\right.$ to $\left.1 / 2\right)$ of the rotational $g$ factor.

By assuming that the nuclear moment of inertia is a function of nuclear spin, a model for even-even nuclei has been developed that successfully predicts level spacing in ground-state bands, which are level sequences with spin and parity $I^{P}=2^{+}, 4^{+}$, $6^{+}, \ldots$. Such bands are found both in deformed and in "spherical" or "vibrational" nuclei.

To a first approximation well-deformed nuclei have energy levels proportional to $[I(I+1)] / J$ which occupy a rotational band ( $\mathcal{J}$ denotes the moment of inertia of the nucleus). In spherical nuclei, ground-state band levels are approximately equidistant (that is, proportional to $I$ ) and are therefore usually thought to be members of a vibrational band. Energy-level patterns intermediate between the rotational and vibrational types are found, for example, in the regions of the osmium, platinum, and xenon nuclei. Figure 28 presents 5 examples of level patterns found in ground-state bands of even-even nuclei.

An analysis of these level patterns showed that spherical nuclei can also be interpreted as rotational nuclei. The proposed model is based on the idea that the moment of inertia increases "elas-

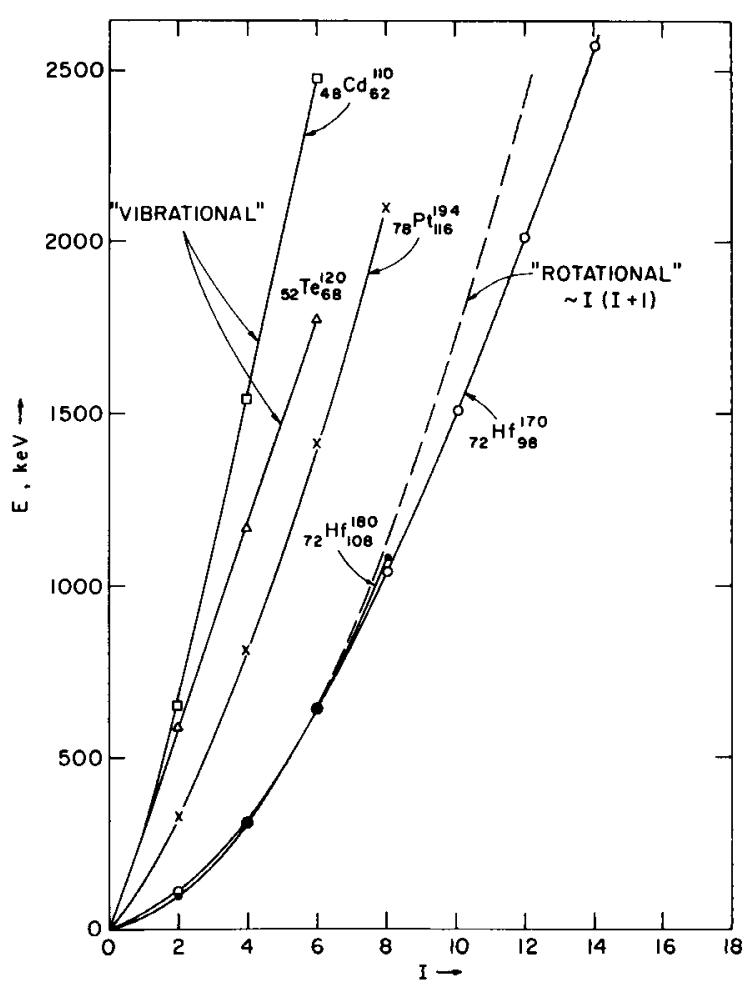

Figure 28. The level energies for the ground-state bands of five representative nuclei are plotted versus the angular momentum $I$. The curve for $\mathbf{H f}{ }^{180}$ is closest to that of an ideal rotor (broken line), while the curve for $\mathrm{Hf}^{170}$, a neutron-deficient, unstable nucleus, deviates appreciably from the $I(I+1)$ dependence. $\mathrm{Pt}^{194}$ typifies the transition nuclei, while $\mathrm{Cd}^{110}$ and $\mathrm{Te}^{120}$ have apparently "vibrational" level schemes.

tically" when the nucleus goes from a lower to a higher rotational state, according to the formula $E_{J}=(C / 2)\left(J-J_{0}\right)^{2}+(1 / 2) I(I+1) / J$. It is further assumed that the moment of inertia varies in such a way that the energy for each state attains the smallest possible value. $C$ (the stiffness parameter) and $J_{0}$ (the "ground-state moment of inertia") are, for each nucleus, obtained by a least-squares fit of measured energy levels of the ground-state band, which may include states up to $I=16$.

Figure 29 demonstrates the smooth variation obtained for $\mathcal{J}_{0}$ as a function of neutron number $(\mathcal{N})$ and atomic number $(Z)$. It can be seen that $J_{0}$ reaches its highest value midway between closed shells, for either neutrons or protons. $C$ is highest for nuclei along the stability line and falls off as the binding energy decreases.

For strongly deformed stable nuclei, $\mathcal{J}$ is almost constant as a function of $I$. In spherical nuclei, 
Figure 29. Ground-state moment of inertia $\mathcal{J}_{0}$ plotted against the neutron $(\mathcal{N})$ and proton $(Z)$ numbers in the nucleus. The heavier double lines indicate closed shells. It can be seen that $J_{0}$ is highest midway between the shells. The oblique line indicates the region of stable nuclei.

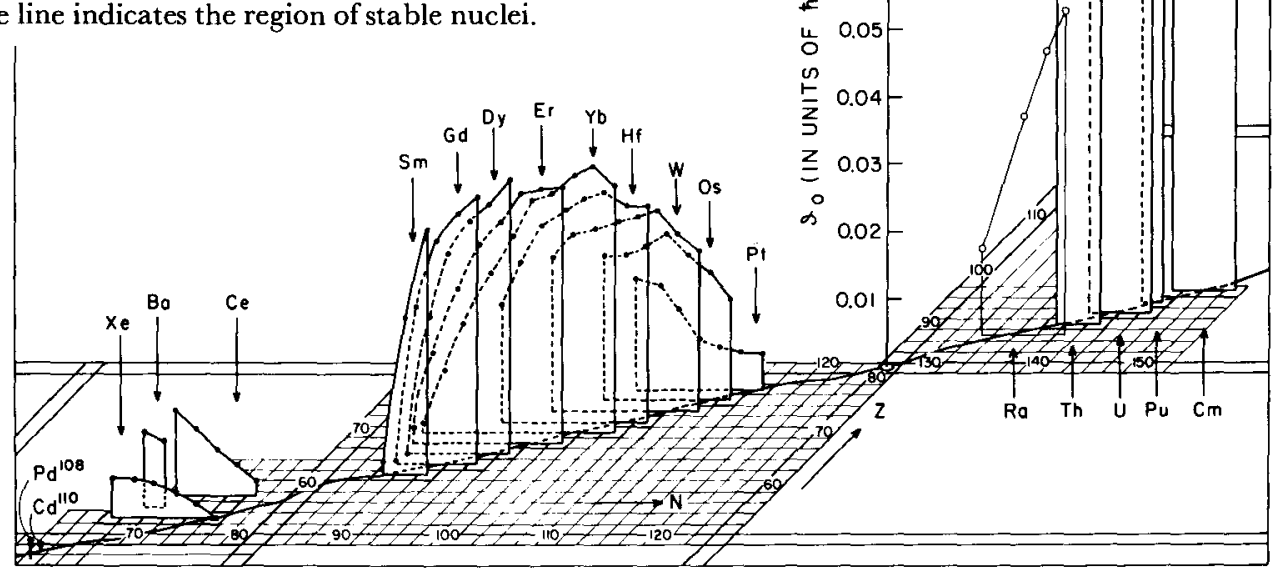

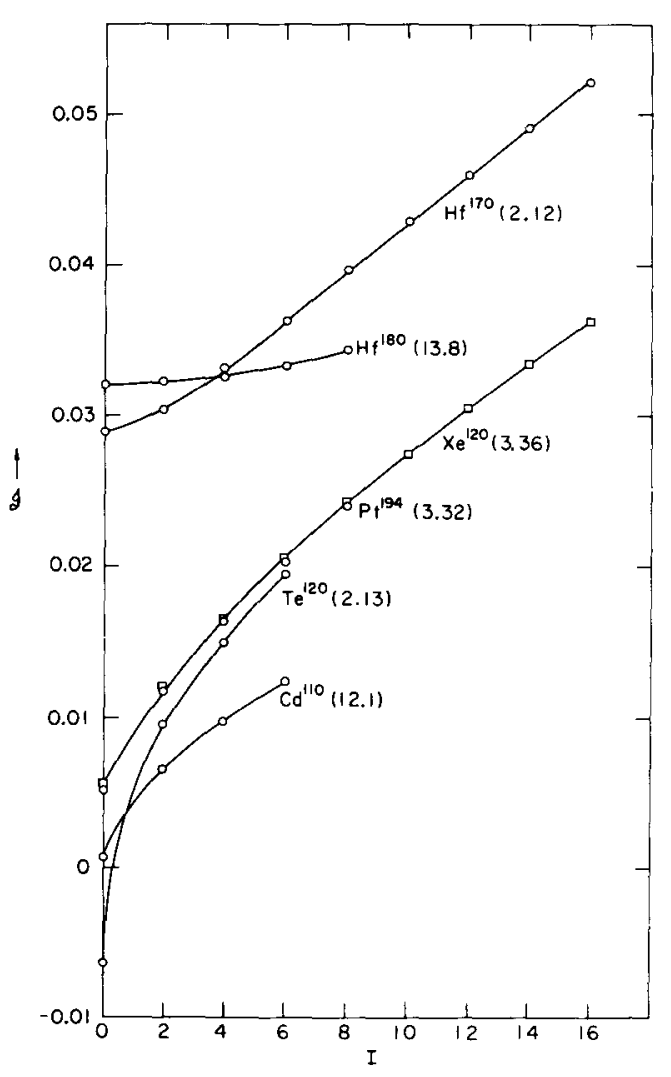

Figure 30. Dependence of the moment of inertia $\mathcal{J}$ on the angular momentum $I$ for several representative nuclei according to the VMI model. Values of $C$ are given in parentheses.

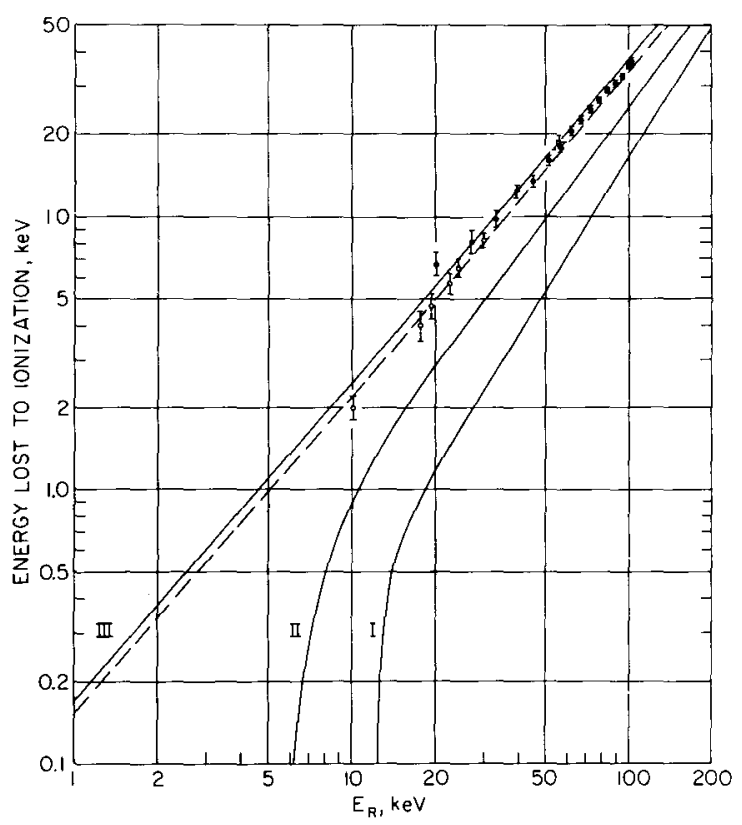

Figure 31. Comparison of experimental results and theory for the energy lost to ionization by a germanium atom stopping in germanium. The open points from 10 to 30 $\mathrm{keV}$ are taken from current work. All other points stem from earlier work at this Laboratory. The three solid curves are theoretical fits from the theory of Linhard calculated with the constant of proportionality $(k)$ between the electronic stopping power and the square root of the energy $=0.15$, and with (I), a sharp cutoff in the electronic stopping power; (II), an intermediate cutoff; and (III), no cutoff. The broken curve is calculated with $k=0.14$. 
however, $\mathcal{J}$ may increase from a negligibly small $J_{0}$ to a value several orders of magnitude higher for the $2+$ state. The increase of the moment of inertia with angular momentum for several typical nuclei is shown in Figure 30.

The variable moment of inertia (VMI) model makes plausible a fact observed by L. Grodzins which contradicts the previous models. He showed that for all even-even nuclei with mass numbers $A$ ranging from 12 to 240 the probabilities for $\gamma$ transitions from the $2+$ state to the ground state, divided by the appropriate single-particle transition probabilities, are inversely proportional to the energies of the first $2+$ states, whereas the proportionality constant for deformed nuclei was expected to be $\approx 2.4$ times larger than that for spherical nuclei. The hitherto puzzling static quadrupole moments of $2+$ states of spherical nuclei, measured by the reorientation effect, are shown to be correlated with the moments of inertia of these $2+$ states.

The effect of the energy gap on the ionization produced in a semiconductor by slow heavy particles has been studied. When a heavy particle moves through matter it loses energy in collisions with atoms and with electrons. Particles that are heavier or moving more slowly lose more energy in the atomic collision process than lighter or more rapidly moving particles. In the case of $\approx 23-\mathrm{keV}$ germanium atoms stopping in a germanium detector, it might be imagined that energy could no longer be transferred to electrons because the band-gap energy, $E_{g}$, in germanium exceeds the amount of energy such an atom can transfer to an electron in a direct collision. This implies that a $\mathrm{Ge}(\mathrm{Li})$ detector would be unable to detect low energy germanium ions or, more generally, ions of any mass with energy significantly below the threshold for transferring the band-gap energy to an electron in a direct collision. Alternatively, the point of view might be taken that at energies below this critical energy, electrons can still be elevated to the conduction band by a mechanism in which the electron clouds of the moving and stationary atoms interpenetrate during the collision, and the outer electrons are set free.

Measurements were made of the ionization produced by a slowly moving germanium atom stopping in a germanium detector as a function of the initial energy of the moving germanium atom. On the basis of a hard-sphere collision model a cutoff in the amount of electronic excitation would be anticipated at about 23-keV germanium ion energy due to the $0.67-\mathrm{eV}$ band-gap energy. However, no evidence was found for an abrupt cutoff at energies down to at least 0.1 to 0.2 of that expected from the band-gap energy. The conclusions are consistent with the conjecture that the electronic stopping power of heavy particles is proportional to the particle velocity, even at energies far lower than this cutoff energy. In Figure 31 the most recent data together with early results obtained at this Laboratory are compared with the theoretical calculations of J. Linhard (University of Aarhus, Denmark).

Recent neutron capture experiments have focused interest on theoretical internal-conversion coefficients for energies well above the limits of existing tabulations. For example, data are available for the $\operatorname{Cd}^{113}(n, \gamma) \operatorname{Cd}^{114}$ reaction up to $\approx 9$ $\mathrm{MeV}$, while existing tables extend to only $\approx 2.5$ $\mathrm{MeV}$. It is well known that in the high energy limit all coefficients for a given $Z$ and atomic shell become equal and proportional to $1 / \hbar \omega$. To permit preliminary analysis of experiment and to guide future studies, $K$-shell coefficients have been calculated at intermediate energies by first evaluating analytic values for a point-unscreened nucleus, and then applying approximate corrections for the static and dynamic effects of the finite nuclear size and for atomic screening. The first is estimated from the leading terms in the long-wavelength limit ( $p R \approx 0.3$ for $\mathrm{Cd}$ at $10 \mathrm{MeV}$ ); the second, by renormalizing the ground-state wave functions near the nucleus. At low energies these results agree with existing tabulations, while at $10 \mathrm{MeV}$ they lie above the high energy limit and still show dependence on the transition multipolarity. Although results for $\mathrm{Cd}^{\mathbf{1 1 4}}$ disagree with earlier measurements in the literature by factors of $\approx 2$, they are in agreement with re-evaluations based on remeasured $\gamma$-ray intensities. With increasing

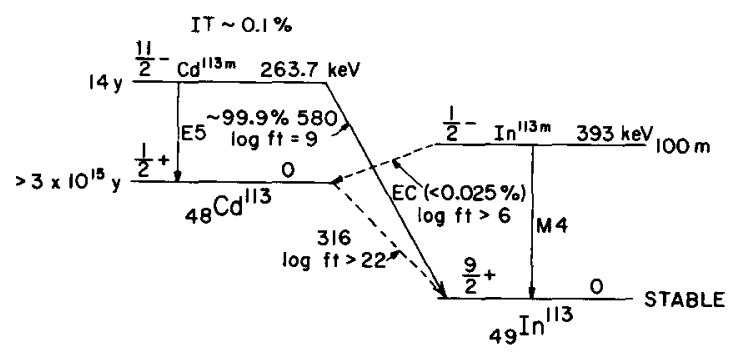

Figure 32. Decay scheme of $\mathrm{Cd}^{113 m_{-}} \operatorname{In}^{113 m}$. 
atomic number the magnitudes of the internalconversion coefficients, their dependence on the transition multipolarity, and their sensitivity to dynamic finite-size effects all tend to increase, which suggests the particular usefulness of conversion coefficient measurements as a tool for the study of highly excited states in heavy nuclei.

Although one of the isobaric pair $\mathrm{Cd}^{113}-\mathrm{In}^{113}$ is expected to be unstable, the radioactive decay

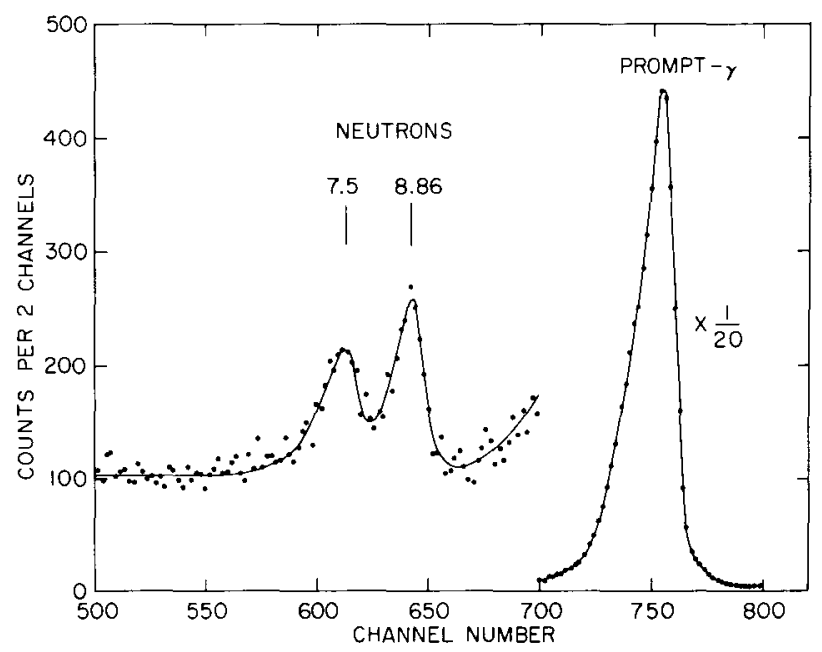

Figure 33. $\beta$-neutron time-of-flight spectrum in the decay of $\mathbf{B}^{13}$ for a flight path of $35 \mathrm{~cm}$. Time increases from right to left. The two small peaks correspond to the neutron decay of the 8.80 and $7.5-\mathrm{MeV}$ levels of $\mathrm{C}^{13}$ which have been populated by $\mathrm{B}^{13} \beta$ decay. These peaks are separated from the $\beta$ - $\gamma$ prompt peak by 13 and $16 \mathrm{nsec}$, respectively.

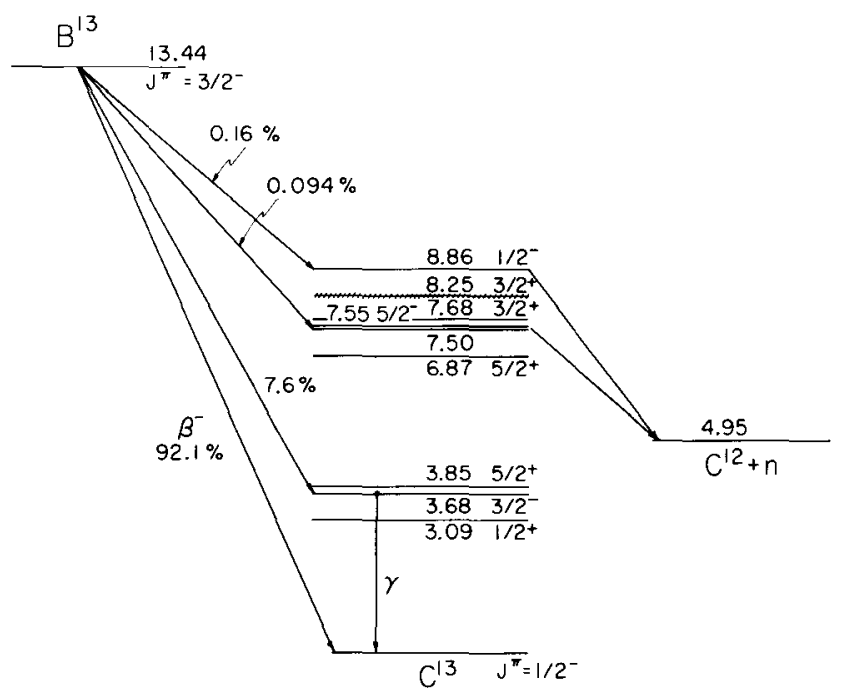

Figure 34. Proposed decay scheme of $\mathrm{B}^{\mathbf{1 3}}$. of neither of these nuclides normally found in nature has been detected. A study of the isomeric states of these isobars has revealed that $\mathrm{Cd}^{113}$ must be the unstable, or "labile," nuclide, with an available $\beta$-decay energy of $316 \mathrm{keV}$. A summary of the information gathered concerning the decays of $\mathrm{Cd}^{113 m}$ and $\operatorname{In}^{113 m}$ is given in the decay scheme shown in Figure 32. The partial half-life determined for the isomeric transition of $\mathrm{Cd}^{113 m}$ is $6 \times 10^{4} \mathrm{yr}$, which is 20 times longer than the singleparticle prediction for an $E 5$ transition. The lower limit set for the $\log f_{0} t$ value $(>22)$ for the $\beta$ decay of the $\mathrm{Cd}^{113}$ ground state is compatible with a fourth-forbidden transition and agrees with an estimate of the $\log f_{0} t$ expected for this decay obtained by considering a well-established fourthforbidden $\beta$ transition in $\mathrm{In}^{\mathbf{1 1 5}}$.

In the 3.5-MeV Van de Graaff research program, a tritium facility was installed in the accelerator and numerous experiments were carried out with use of the triton beam. Radioisotopes formed by triton bombardment include $\mathrm{Be}^{\mathbf{1 1}}, \mathrm{B}^{\mathbf{1 3}}$, $\mathrm{O}^{20}, \mathrm{~F}^{21}, \mathrm{Mg}^{28}$, and $\mathrm{Al}^{29}$. One of the most interesting of these was $\mathrm{B}^{\mathbf{1 3}}$, which decays by $\beta$-ray emission with a half-life of $18.6 \mathrm{msec}$. Theoretical shellmodel calculations have predicted the energies, spins, and parities of excited states in $\mathbf{C}^{\mathbf{1 3}}$, as well as the $\log f t$ values of the $\mathbf{B}^{\mathbf{1 3}} \beta$-decay branches to these levels. Previous work elsewhere on $B^{13}$ has shown that the $\beta$ decay goes mainly to the ground state of $\mathrm{C}^{13}$, with a branch to the $3.68-\mathrm{MeV} \gamma$-emitting level. The higher states of $\mathrm{C}^{13}$ fed by the predicted $\mathrm{B}^{13} \beta$ branches would have to decay by neutron emission; hence an experiment was designed to search for neutron groups from these levels. $\mathrm{B}^{13}$ was produced in a target by the $\mathrm{B}^{11}(t, p) \mathrm{B}^{13}$ reaction. A $\beta$-ray detector was placed close to the target, and a neutron detector was positioned at a distance of $35 \mathrm{~cm}$ on the opposite side of the target. Although the emission of a $\beta$ ray and of the corresponding neutron occur simultaneously, the neutron moves relatively slowly and therefore arrives at the neutron detector after a time delay depending on its velocity. Figure 33 shows the $\beta$-neutron time spectrum for $\mathbf{B}^{13}$. The strong prompt peak results from $\beta$ - $\gamma$ coincidences, since the neutron detector is also sensitive to $\gamma$ rays. Two small peaks are observed which have energies of 2.4 and 3.6 $\mathrm{MeV}$ and correspond to neutron-emitting states of $\mathrm{C}^{13}$ that are populated in the $\mathrm{B}^{13} \beta$ decay. The decay scheme deduced for $\mathrm{B}^{13}$ is shown in Figure 34. All the theoretical predictions are confirmed, 


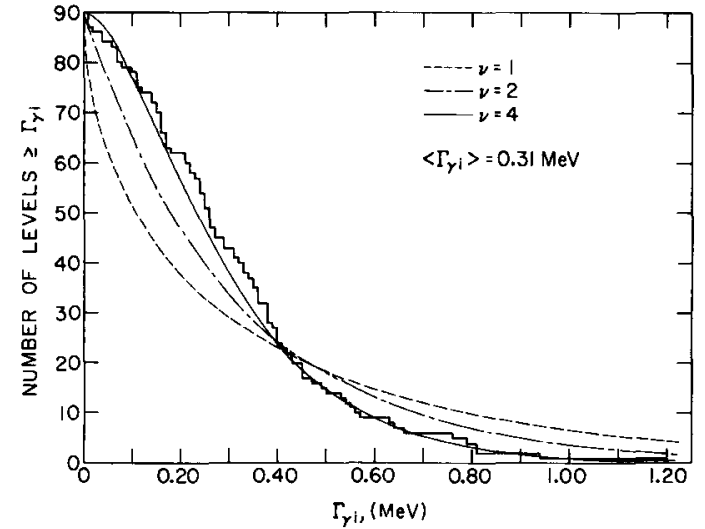

Figure 35. The distribution of radiative widths in $\mathrm{U}^{238}(n, \gamma) \mathrm{U}^{239}$.

including the properties of the $\mathrm{C}^{13}$ levels and the $\mathrm{B}^{13} \beta$-decay rates to these levels.

Measurement of the lifetimes of energy levels in light nuclei has represented much of the effort of the 3.5-MeV Van de Graaff Group during the past few years. A new technique for lifetime measurements, first developed at Chalk River in Canada, has now been applied in a number of cases at Brookhaven. This is the so-called plunger method, which has been made feasible by the high resolution of the recently developed lithium-drifted germanium detector for $\gamma$ rays. The Van de Graaff beam produces a reaction in a target such that the excited product nucleus recoils in vacuo. A plunger is located a short distance behind the target. If the excited recoil nucleus decays between the target and the plunger, the emitted $\gamma$ ray will be Doppler-shifted if observed in either the forward or backward direction. If the nucleus is stopped in the plunger before it de-excites, the $\gamma$ ray will then show no Doppler shift. Two peaks are therefore observed, their relative intensities depending on how far the plunger is from the target. As the targetplunger distance $d$ is increased, the ratio of the stopped peak intensity to the sum of the intensities of the two peaks decreases exponentially with $d$. From the resulting curve the mean lifetime of a state can be calculated. The lifetimes of a number of energy levels in $\mathrm{Na}^{22}, \mathrm{Ne}^{22}$, and $\mathrm{F}^{19}$ have been measured in this way.

\section{NEUTRON PHYSICS}

Neutron physics research at Brookhaven is concerned with the properties of the neutron and its interaction with nuclear matter. The neutron experiments, carried out at the High Flux Beam Research Reactor (HFBR), employ both time-offlight and crystal-spectrometer techniques. The excitation of nuclei by neutrons and study of the subsequent decay of the excited levels yield fundamental information on the neutron capture reaction mechanism and on the spin, parity, and other parameters of the excited states. Precise measurements of neutron cross sections give data that are significant for basic nuclear physics, as well as for technological applications involving neutrons. Additional data are obtained from studies of interactions of polarized neutrons with nuclei oriented in magnetic fields at ultralow temperatures.

With the HFBR fast neutron chopper, the radiative capture of neutrons by nuclei can be studied as a function of neutron energy over a range of several thousand electron volts. The complex $\gamma$-ray spectrum accompanying this process is a rich source of information on not only the reaction mechanism itself but also the structure of the residual nucleus. Accordingly, experiments with this facility are directed toward one or more of the following goals: (1) to obtain information about the highly excited quasi-stable nuclear states formed by neutron capture (the neutron resonances); (2) to obtain nuclear structure information on the low-lying states populated by the $\gamma$-ray de-excitation; and (3) to obtain information about the nature of the reaction process through the comparison of spectra measured on and between neutron resonances.

The nature of the resonant states formed by neutron capture can be studied by examining

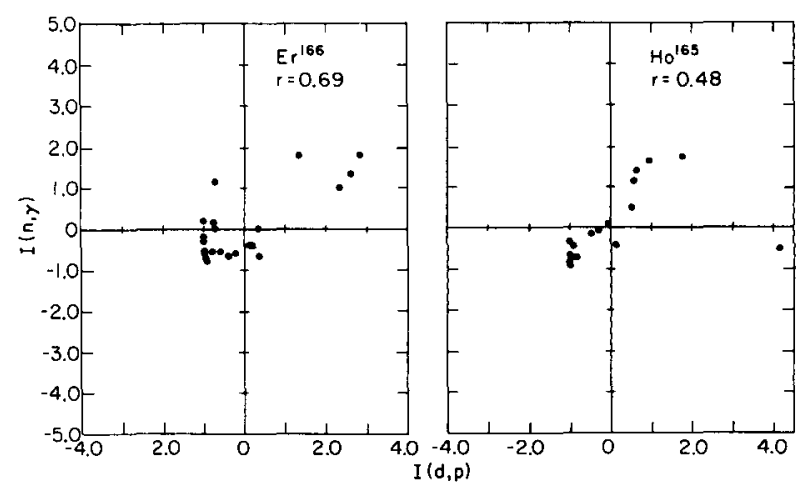

Figure 36. Radiative intensities compared with $(d, p)$ stripping intensities for like final states. The intensities are normalized to unit mean values placed at the origin. 


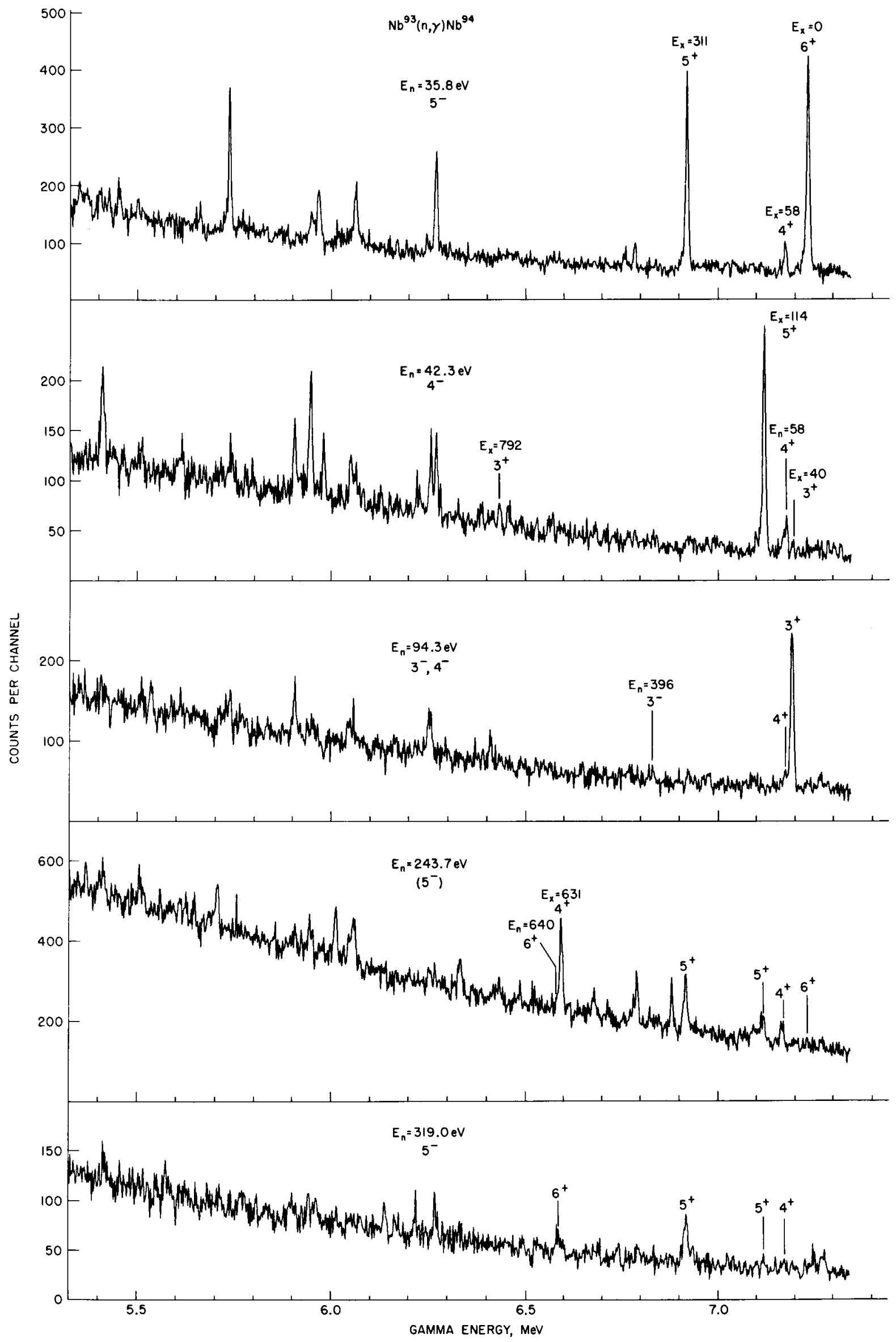

Figure 37. Several resonance $\gamma$-ray spectra from $\mathrm{Nb}^{93}(n, \gamma) \mathrm{Nb}^{94}$. 
Table 2

\begin{tabular}{rrr}
\hline$E_{0}$ & $J \pi$ \\
\hline $\mathrm{Nb}^{93}$ & 35.8 & $5^{-}$ \\
42.3 & $4^{-}$ \\
94.3 & $3-, 4^{-}$ \\
105.8 & $4^{+}$ \\
119.2 & $4^{+}$ \\
193.8 & $4^{+}$ \\
243.7 & $5^{-}$ \\
319.0 & $5^{-}$ \\
335.5 & $4^{+}$ \\
$\mathrm{Zr}^{91}$ & $3^{-}$ \\
182 & $1^{-}, 2^{-}, 3^{-}$ \\
240 & $2^{+}$ \\
293 & $3-, 4^{-}$ \\
885 &
\end{tabular}

the statistical properties of the radiative widths of these states. The random matrix hypothesis of compound nucleus theory predicts that these widths obey a distribution function known as the $\chi^{2}$ distribution with one degree of freedom (PorterThomas). The theory further predicts that these reaction widths will be independent and therefore uncorrelated. Recent experiments have indicated that both predictions are violated in some cases. Figure 35 gives the distribution of 90 radiative widths from resonances in $\mathrm{U}^{238}(n, \gamma) \mathrm{U}^{239}$. These show a departure from the Porter-Thomas distribution and are better fitted by a $\chi^{2}$ distribution with four degrees of freedom. Other experiments have indicated a correlation between reaction widths. Two examples of such a correlation are shown in Figure 36, in which the radiative width is plotted as a function of the final states' reduced neutron width in $\mathrm{Ho}^{165}$ and $\mathrm{Er}^{166}$, as measured

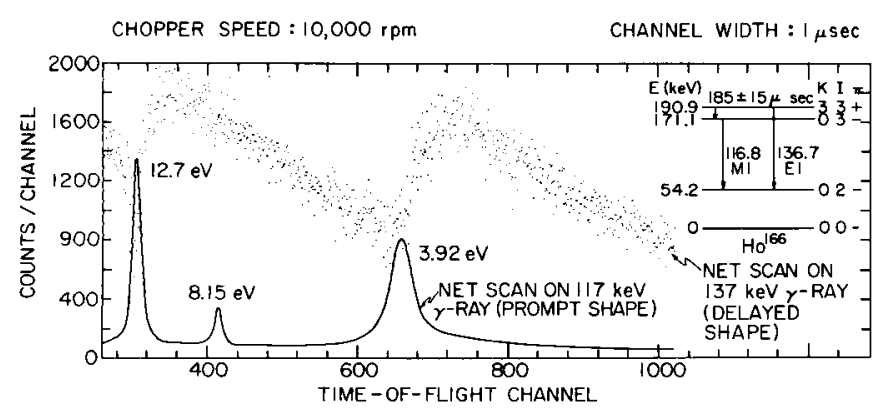

Figure 38. A comparison of the time-of-flight dependence of the 117 and $137-\mathrm{keV}$ transitions in $\mathrm{Ho}^{166}$. The $191-\mathrm{keV}$ state has a half-life of $185 \mu \mathrm{sec}$. by the $(d, p)$ cross section for these states. The figure displays the tendency for the preferred population of final states with strong single-particle characteristics by direct $\gamma$-ray transitions from the neutron resonances. Correlations have been observed between initial-state neutron widths and radiative widths and among radiative widths themselves. The evidence indicates that the highly excited resonant states possess some simple structure superposed on the complicated configurations usually associated with a compound nucleus state. A mathematical model, involving the decomposition of the compound nucleus wave functions in Hilbert space into two groups, has been formulated. One group has a large dimensionality and the other a small dimensionality. This two-group theory can accommodate the statistical anomalies found in the experiments.

One of the most difficult problems in neutron resonance spectroscopy is the assignment of spin and parity to the resonances. Such assignments can be inferred from cross-section measurements, but only with considerable uncertainty, by measuring the statistical weight factor of the resonance. The examination of the capture $\gamma$-ray spectrum offers a much more reliable way of making spin assignments, that of making use of the simple angular momentum selection rules which apply for dipole transitions. Such a technique is particularly valuable for the small resonances induced by capture of $l=1$ ( $p$-wave) neutrons. Measurements of several resonance spins and parities have been made in the isotopes $\mathrm{Zr}^{91}$ and $\mathrm{Nb}^{93}$, where $p$-wave levels are especially important. Figure 37 illustrates the assignment of the resonance at $35.8 \mathrm{eV}$ in $\mathrm{Nb}^{93}$ as $5^{-}$. The assignment is based on the strong groundstate transition $5^{-} \rightarrow 6^{+}$which appears in the spectrum. Table 2 indicates the spin assignments that have been made for $\mathrm{Nb}^{93}$ and $\mathrm{Zr}^{91}$.

A novel application of the HFBR chopper has been the measurement of the half-lives of isomeric states populated in the $(n, \gamma)$ reaction. The method is illustrated in Figure 38, which shows the timeof-flight spectrum of $\gamma$ rays from the 117 and 137$\mathrm{keV}$ transitions in $\mathrm{Ho}^{166}$, which has been formed by neutron capture in resonances of $\mathrm{Ho}^{\mathbf{1 6 5}}$. The striking difference in shape between these transitions is due to the long life of the $190.9-\mathrm{keV} \mathrm{3+}$ level compared with the short lifetime of the 171.1$\mathrm{keV} 3-$ level. The shape of the $137-\mathrm{keV}$ time-offlight spectrum may be analyzed relative to the "prompt" $117-\mathrm{keV}$ spectrum to obtain the half- 


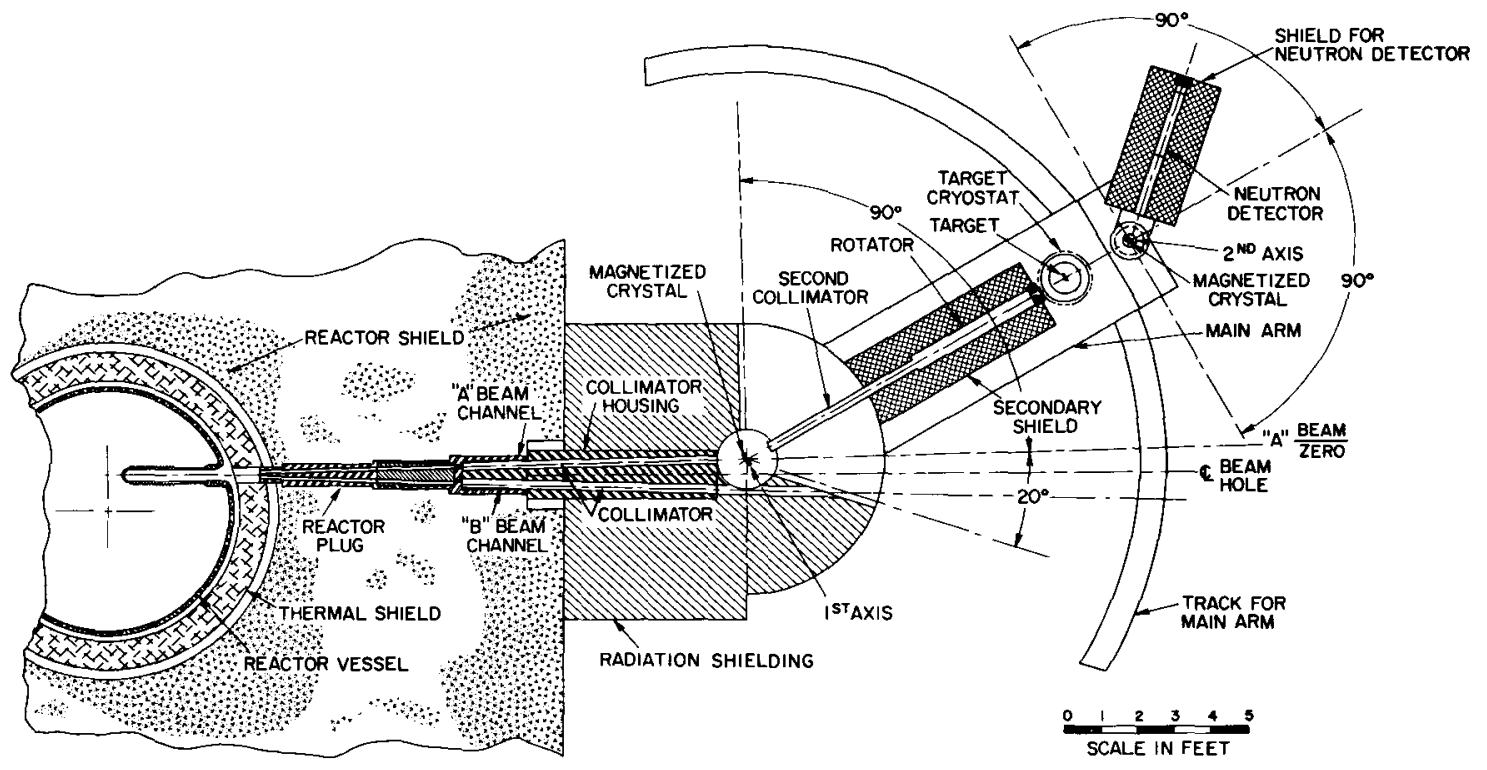

Figure 39. The polarization spectrometer at the HFBR. The spectrometer is a large, double-axis instrument capable of carrying several tons of research apparatus and shielding. The first axis, housed in a massive radiation shield, selects a monoenergetic polarized neutron beam. The second axis analyzes the degree of polarization of the beam. The vertical frame is the support for the cryogenic target. Despite its large size and massive construction, the instrument has unusually high precision, being capable of setting angles to an accuracy of within \pm 3 seconds of arc.

life of the $190.9-\mathrm{keV}$ transition, namely $185 \mu \mathrm{sec}$. The method is generally applicable in the lifetime range 1 to $1000 \mu \mathrm{sec}$.

This was the first year of effective operation at the HFBR of a new experimental facility designed primarily for studies of interactions between polarized neutrons and polarized nuclei. This highly versatile facility has provision for two independent neutron beams so that the unique epicadmium spectrum available at the $\mathrm{H}-1$ beam port can be exploited by several simultaneous experiments.

The major instrument, shown schematically in Figure 39, is a high-precision two-axis polarized neutron spectrometer mounted for use at the " $A$ " beam of the facility. It is equipped with a refrigerated target holder and a superconducting magnet which permit studies of target specimens at temperatures in the vicinity of $0.01{ }^{\circ} \mathrm{K}$ and in magnetic fields of 50,000 oersteds. The specially designed monochromator shielding permits wide variation of incident beam energies without interference with experiments carried out with the " $B$ " beam. Although setups for the latter are expected to change from time to time, experiments will generally make use of certain pieces of basic equipment now on hand. Three separate experiments were set up at the " $B$ " beam during the past year, and the present configuration now provides (1) a neutron monochromator for a capture $\gamma$-ray program in collaboration with Queens College of the City University of New York, (2) a double-crystal spectrometer for precise cross-section measurements, and (3) a triple-axis spectrometer for inelastic scattering experiments in which relatively large momentum transfer is desired.

The facilities have been used for a variety of cross-section measurements, particularly those for which unusual accuracy is needed or those that can serve as standards. Among the completed data are total cross sections for $\mathrm{He}^{4}, \mathrm{C}^{13}, \mathrm{Ne}, \mathrm{Ar}$, and $\mathrm{Kr}$. Measurements are in progress on the coherent scattering cross section of $\mathrm{He}^{3}$, capture cross sections of several elements, and a new determination of the parahydrogen total cross section.

In addition to the more conventional crosssection and polarization measurements, a new type of experiment has been tried which yields the value of the nuclear magnetic dipole moment for the compound state formed immediately upon neutron capture. Such states decay very rapidly (lifetimes in the range of $10^{-14} \mathrm{sec}$ ), and the dipole moments associated with these states have never been observed. In this experiment the neutron beam is polarized but, although the target is in a strong magnetic field, the target nuclei are maintained at a temperature high enough to keep them 
unpolarized. A neutron energy corresponding to a resonance in the cross section of the target isotope is selected. The total energy of the compound nucleus formed by capture of the neutron depends not only on the neutron binding energy (several $\mathrm{MeV}$ ) but also on the orientation of its newly created magnetic moment relative to the magnetic field. Since the nuclear moment is extremely small, the energy difference correspoding to the various allowed orientations is only a few $\mu \mathrm{eV}$. Reversal of the neutron polarization reverses the orientation of the moment, which produces a small shift in the energy of the cross-section resonance. Despite the small magnitude of the energy shift, it has been observed for two resonances in the compound nucleus $\mathrm{Er}^{168}$. The magnitude of the shift is proportional to the strength of the moment, and the direction of the shift determines the sign.

\section{SOLID STATE PHYSICS}

The research program in solid state physics is concerned with the understanding of the physical nature of condensed systems, with emphasis on crystal imperfections and on the structure and dynamics of solids. Some representative accomplishments are described below.

\section{Theory}

Among magnetic metals, the rare earths are unusual in that they have magnetic moments, the open $4 f$ electron shells, which are localized in the interiors of the ion cores. These cannot interact directly with one another (as happens in iron or nickel) and hence a sample would not become magnetized. However, the $4 f$ moments induce a magnetization density in the conduction electrons in their vicinity, and this polarization interacts, in turn, with neighboring $4 f$ magnetic moments. A theoretical estimate of such a polarization, obtained recently, appears in Figure 40; it is the magnetization density induced on the "free" conduction electrons by a gadolinium moment centered at the origin. The oscillations in the induced magnetization's direction, parallel and antiparallel to the polarizing $4 f$ moment at the origin, are typical. The size and sign of these oscillations determine the coupling to neighboring rare-earth moments (one such moment is indicated schematically at a typical neighbor distance). This coupling is at the heart of magnetic, transport, and other properties of the rare-earth metals and alloys and causes, among other things, the spiral magnetic

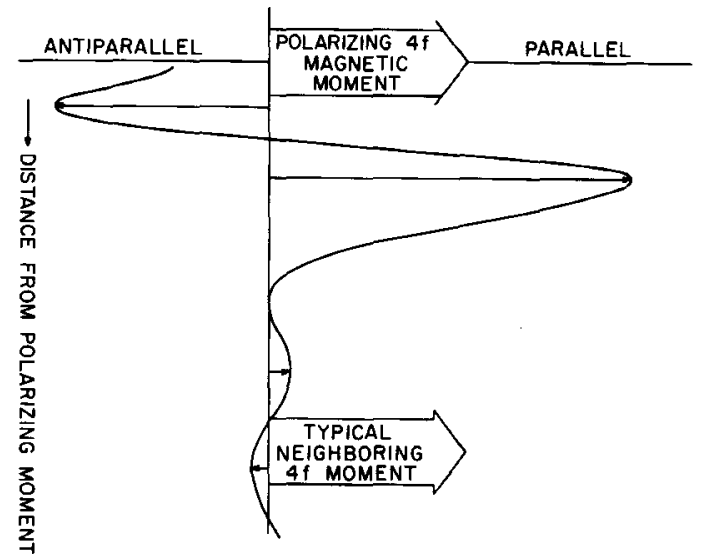

Figure 40. Theoretical estimate of induced magnetic density as a function of distance from polarizing moment.

structures often observed. Theoretical work has centered around the role played by the rare-earth moment-conduction electron interaction, which drives the polarization, and by the conduction electron properties as they affect the polarization response. The polarization effects are very sensitive to the details of these interactions, and a quantitative understanding of rare-earth magnetism requires an increasingly realistic assessment of them.

The phenomenon of Faraday rotation, in which the plane of polarization of light is rotated by passage through a medium in an external magnetic field, is familiar from classical optics. Similar effects should also be observable with $\gamma$ radiation, and they have indeed been detected recently in experiments using the Mössbauer effect. These observations are complicated by the fact that the $\gamma$ rays are absorbed in a manner depending on their polarization. A general theory of magneto- and electrooptical effects has been developed to treat this complication for substances in which absorption and rotation occur together. The theory is applicable to $\gamma$ rays and optical wavelengths alike. Several unusual phenomena occur when both absorption and rotation are present which affect the intensity and polarization of transmitted radiation. In some circumstances, for example, absorption followed by rotation will give a different transmitted intensity than rotation followed by absorption. This produces an apparent asymmetry in the selection rules for absorption. Such an effect was found in an experiment using the Mössbauer effect in an attempt to detect time-reversal non- 
invariance in a nuclear decay. The magnetooptic effect in fact accounted for all the observations in the experiment, and quantitative agreement with observation was found. The theory will be of importance in the interpretation of Mössbauer-effect experiments on magnetic materials and on single crystals of nonmagnetic substances.

In the tunneling model of ferroelectrics it is assumed that the ions can be found in one of two equivalent positions and that tunneling between these positions is possible. The model has been extended to cubic crystals of the perovskite structure in which there are at least six equivalent positions. The theory was then applied to calculations of the neutron scattering cross section. A "soft" mode was predicted for which the frequency vanishes as the critical temperature for ferroelectric ordering is approached. For particular values of the parameters the transition may become first order, with a discontinuous change in the polarization and accompanying latent heat. This is the case for the transitions in barium titanate and lead niobate, and the theory should be applicable to these substances.

As part of the cooperative project between BNL and Picatinny Arsenal, the quantum mechanical calculations on the $\mathrm{N}_{3}$ - ion, reported last year, were extended to the hydrozoic acid $\left(\mathrm{HN}_{3}\right)$ molecule. Wave functions were computed for different conformations (geometrical arrangements of the nuclei) of the molecule by using the Gaussian functions obtained during the $\mathrm{N}_{3}$ - calculation and published hydrogen atom functions. The minimum energy was obtained for a conformation in which the three nitrogen atoms are coplanar. The $\mathrm{H}-\mathrm{NNN}$ bond angle is $111^{\circ} 21^{\prime}$, the $\mathrm{H}-\mathrm{N}$ bond length is 2.776 atomic units, and the $\mathrm{N}-\mathrm{N}$ bond lengths are 2.348 and 2.148 atomic units, with the largest separation between the nitrogens nearest to the proton. The structure is illustrated by constant electron density contours in Figure 41 .

The rate equations governing the clustering and annealing of vacancies in metals were studied with use of computer techniques. Single vacancies were considered to be immobile at temperatures at which small clusters migrate, clusters with up to seven vacancies were explicitly treated, and approximations were made to account for the formation and breakup of large clusters. Various cases involving different cluster migration and binding energies were studied. As the limits of small or large binding energies were approached, the results were reasonably regular. For small binding energies, cluster concentrations were small, and the annealing involved divacancy migration to sinks and some increase in the single-vacancy concentration. For large binding energies very few defects reached sinks as clustering dominated. The results were very complex for a broad range of intermediate cluster binding energies. In general, isothermal and isochronal annealing curves gave little indication of the actual processes occurring, so that the input data could not be inferred from standard analyses of the data. The total annealing could be quite large with an appreciable decrease in concentration of single vacancies, and anomalously low activation energies and several distinct annealing stages were often found.

The general theoretical technique for treating defects in ionic crystals was improved by replacing a much-used approximation for the polarization contributions by an exact procedure. This step is particularly important when rather subtle interactions are investigated. During the last year the detailed characteristics of several defects were determined. A study of $\mathrm{Cu}^{+}$and $\mathrm{Ag}^{+}$centers in alkali halides gave the following displacements for those ions along the $\langle 111\rangle$ direction away from the normal lattice site: $0.21 \AA, 1.36 \AA, 1.65 \AA$, and $1.84 \AA$ for $\mathrm{Cu}^{+}$in $\mathrm{NaCl}, \mathrm{KGl}, \mathrm{RbCl}$, and $\mathrm{RbBr}$, and 0.54 $\AA$ for $\mathrm{Ag}^{+}$in $\mathrm{RbCl}$. $\mathrm{Ag}^{+}$is on site in $\mathrm{NaCl}, \mathrm{KCl}$, and $\mathrm{RbBr}$. These results are in good agreement with experiment. The formation and stability of two adjacent $H$-centers (an $H$-center is a $\mathrm{Cl}_{2}-$ molecular ion squeezed into a normal $\mathrm{Cl}^{-}$site) were investigated. This is primarily a strain interaction, with the strains reaching quite far into the lattice;

Figure 41. The electronic structure of the hydrozoic acid $\left(\mathrm{HN}_{3}\right)$ molecule, as shown by one of the higher occupied molecular orbitals. Each line represents a contour of constant electron density.

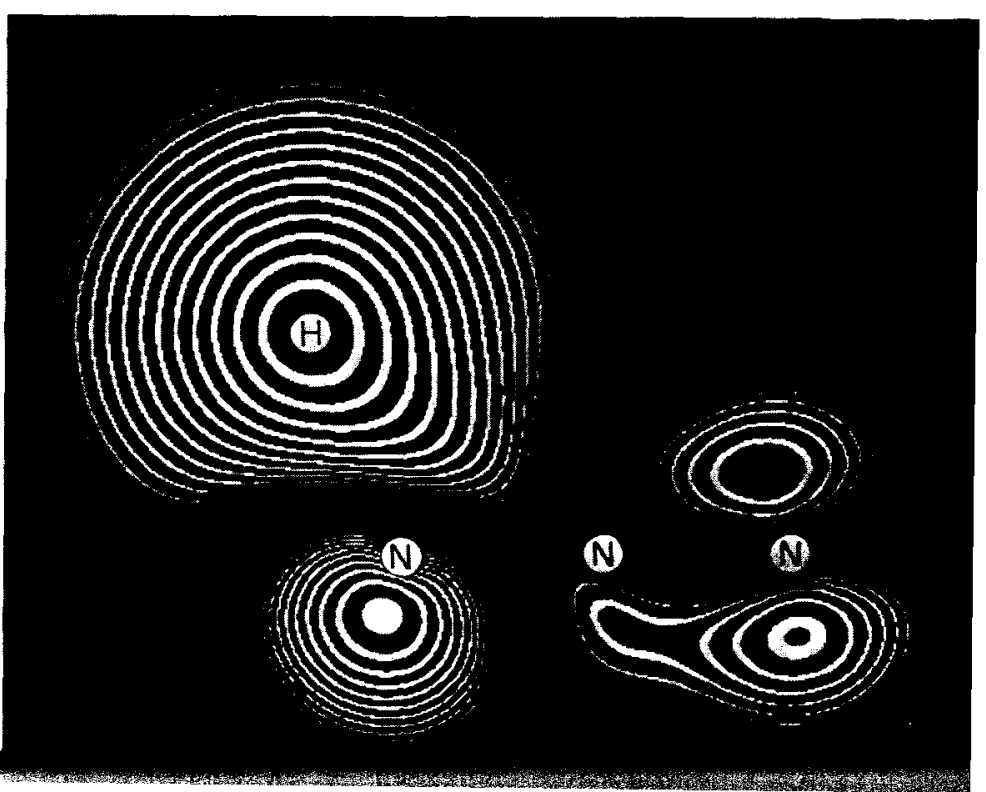


for instance, at the fifth neighbor in the outward direction the strain is $1 \%$ of the interionic distance, while it is about $0.3 \%$ in the inward direction. The effective interaction volume between two $H$-centers in $\mathrm{KCl}$ turns out to be about $2 \times 10^{3}$ compared with the experimental value of $(1.6$ to 3.2$) \times 10^{3}$ unit volumes.

\section{Crystal Imperfections}

The experimental study of defects in crystals continued as a major research activity in solid state physics. Basic investigations of charged-particle interactions with crystals and of the formation and subsequent behavior of defects in solids are in progress. The program concerned with electron transport properties of organic crystals has become an important part of the over-all research effort.

The thermally activated electrical current in anthracene, an organic crystal with widely spaced and weakly bound molecules, is qualitatively similar to that in normal semiconductors but is many orders of magnitude smaller. Two alternative mechanisms are possible for current conduction in this material: band-type conduction or a thermally activated hopping process. These two processes can be distinguished by measurement and analysis of the Hall mobility. Measurements were completed of the Hall mobility of holes in anthracene at room temperature. Carriers were photoinjected so that their sign could be determined, and Hall voltages were measured when the magnetic field was applied. When the magnetic field was perpendicular to the $a b$ plane, the Hall mobility was anomalous, as predicted by band theory, and had a value of $1.3 \mathrm{~cm}^{2} / \mathrm{Vsec}$ with the primary current in the $a$ direction, and $2.5 \mathrm{~cm}^{2} / \mathrm{Vsec}$ with the primary current in the $b$ direction. The Hall mobility is said to be anomalous when the holes respond as if they were electrons. When the magnetic field was perpendicular to the $a c^{\prime}$ plane, the Hall voltage was not anomalous, again in accord with the predictions of band theory, and the values of the Hall mobility were $7.8 \mathrm{~cm}^{2} /$ Vsec with the primary current in the $a$ direction, and $6.2 \mathrm{~cm}^{2} / \mathrm{Vsec}$ with the primary current in the $c^{\prime}$ direction. These high values for the Hall mobilities are attributed to the narrow width of the hole bands. None of these results would be in agreement with the predictions of a "hopping model" for current conduction. Thus, the evidence is quite strong that band theory gives the appropriate theoretical description of the electronic properties of anthracene.
The results of preliminary studies, in cooperation with New York University, point the way to a new and potentially powerful technique for investigating the concentration of positive-ion vacancies in crystals. Positive-ion vacancies (missing positive ions) constitute an important class of defects in crystal lattices which are difficult to investigate by conventional methods. The new technique is based on the decay of positrons localized at defects. In alkali halides typical lifetime data of positrons exhibit a short-lived component $(\approx 0.2$ nsec) as well as a long-lived component $(\approx 0.5$ nsec). The short-lived component is generally attributed to annihilation of the positrons by interactions with electrons in the bulk of the crystal, while the long-lived component is attributed to annihilation at defects and other perturbations. A positive-ion vacancy is negatively charged and should attract positrons while repulsing electrons. Because of the reduced likelihood of interacting with an electron at such a site, the lifetime of any positron that reaches a positive-ion vacancy should be extended. For these reasons, it has been postulated that the intensity of the decay of the longlived component of positron annihilation should correlate with the concentration of positive-ion vacancies. The decay of positrons was measured in $\mathrm{NaCl}$ crystals both before and after straining the crystals by plastic compression. The intensity of the long-lived component was found to increase after straining. This observation is consistent with the postulated action of positive-ion vacancies and with the generally accepted concept that plastic compression introduces vacancies. Experiments using $x$-ray irradiation showed that the intensity of the long-lived component was decreased following irradiation and was restored after thermal treatment at $400^{\circ} \mathrm{C}$. The positive-ion vacancies trap holes (positive charges) as a result of $\mathrm{x}$-ray irradiation, and consequently can no longer attract positrons, a result again consistent with the concept that positive-ion vacancies are indeed centers for delayed positron annihilation. Consequently, positron annihilation is expected to provide a new, powerful tool for studying a variety of defect problems.

One of the important defects induced by radiation in calcite $\left(\mathrm{CaCO}_{3}\right)$ is the $\mathrm{CO}_{2}-$ ion. Electron spin resonance (ESR) techniques were used to study the width of the ESR absorption lines of the $\mathrm{CO}_{2}-$ defect as a function of temperature. These lines are extremely narrow at $120^{\circ} \mathrm{K}$, become un- 
observably broad as the temperature is increased to $260^{\circ} \mathrm{K}$, and, as the temperature is further increased, merge into a single broad band whose width decreases with increasing temperature. This unusual behavior can be explained by an extension of the Bloch-Gutowsky-Hecht treatment. Each $\mathrm{CO}_{2}$ - center can occupy one of three possible orientations. The transitions between orientations were assumed to be describable by a single thermally controlled relaxation time, $\tau=\tau_{0} e^{E / k T}$. When this parameter is inserted into the three coupled Bloch equations necessitated by the three possible orientations, extremely complex algebraic equations are obtained. The three equations were solved simultaneously on a computer for each set of values of frequency, magnetic field, and relaxation rate. These results were then matched to the experimentally determined widths at one point in the low-temperature region where the line was very narrow. The resulting equations fit the data very closely over five orders of magnitude. The most significant result of this work is the demonstration that this extremely large variation in ESR line width can be explained on the basis of a single thermally activated process.

Recent work in cooperation with the University of Pennsylvania has shown that cyclic straining of a metal at low amplitude (i.e., fatiguing within essentially the elastic range) produces defects that can accelerate diffusion. The enhanced diffusion effect has been detected by measuring the decrease in electrical resistivity, and hence the increase in short-range order, in $\alpha$ brass as a function of the number of fatigue cycles applied to the specimen. These results have important implications with respect to metallurgical processes occurring during fatigue as well as with respect to the mechanism of defect production by small-amplitude dislocation motion. It has been known for a decade that irradiation with energetic particles accelerates diffusion in alloys by means of the production of an excess concentration of lattice defects. By analogy, straining of the specimen should also accelerate diffusion, but it has been very difficult to demonstrate this effect experimentally. The reason is that conventional tracer experiments, which see a few tracer atoms making many jumps, are seriously influenced by surface, grain boundary, and dislocation effects. The change in the ordering rate of an order-disorder alloy, which measures the motion of many atoms, each making a few jumps, is free of these difficulties. The experiments showed that a sample of $\alpha$ brass, prepared originally in a state of low short-range order by quenching, increased in short-range order during cyclic deformation. Since the increase in short-range order can occur only by diffusion, this experiment demonstrated clearly that cyclic straining accelerates diffusion. Equilibrium short-range order was not achieved at the temperatures so far investigated. Therefore a model was proposed in which the number of potential defects depends on the initial preparation of the material; when these defects are exhausted by defect recombination, the diffusion enhancement stops.

The solid state electron accelerator operated routinely during the year. Irradiations were performed in connection with experiments on electron damage in $\beta$ brass, vacancy doping of platinum for positron annihilation studies, and optical emission from metal films. Structural defects created by energetic-electron irradiation can markedly affect the kinetics and equilibrium state of a system. Liquid-nitrogen-temperature electron irradiations of $\beta$-brass wires were performed at an energy of $1.5 \mathrm{MeV}$ to doses of about $2 \times 10^{18}$ electrons $/ \mathrm{cm}^{2}$. Isochronal recovery of electrical resistivity following these irradiations has revealed equilibrium states unobtainable through normal thermal-diffusion processes. Enhanced diffusion has been observed after liquid-nitrogen-temperature irradiations.

In the last few years, a few measurements have been reported of angular correlation spectra of positron-electron annihilation in plastically deformed metals and in metals near their melting points. The changes in spectra may well be due to the presence of lattice vacancies. A series of experiments has been started, in cooperation with Western Michigan University, to determine whether vacancies introduced by electron irradiation can be measured by this technique. A definite effect was observed in a platinum specimen irradiated at $90^{\circ} \mathrm{K}$ with $2.5-\mathrm{MeV}$ electrons to a dose of $1.6 \times 10^{19}$ electrons $/ \mathrm{cm}^{2}$. This new technique looks very promising for defect studies, particularly if combined with positron lifetime measurements.

The apparatus for observing optical emission for thin metal film during electron irradiation has been completed. This appears to be the first time that transition radiation and optical bremsstrahlung spectra produced by relativistic electrons have been observed. Previous experiments elsewhere have measured only the total integrated intensity, and thus any structure arising from the 
electronic properties of the metals has been obscured. The light emitted by a metal film during exposure to a well-collimated electron beam should consist of parallel-polarized transition radiation plus unpolarized (or partially polarized) bremsstrahlung. Data obtained on a $1000-\AA$ silver film showed that the parallel-polarized component is primarily transition radiation. This component exhibits a marked dip at about $3250 \AA$ corresponding to a minimum in the extinction coefficient of silver. The perpendicular component shows no structure.

During the year a new program was undertaken to study the effects of stress upon surface layers of metals. These effects are of particular current interest because of their relation to the force felt by electrons placed in a gravitational field. In a difficult series of experiments Witteborn and Fairbank at Stanford investigated the gravitational force acting upon electrons located inside a metal cylinder. The result of the experiments was that the electrons appeared to feel no force whatever. This remarkable result was in agreement with a calculation by Schiff in which it was argued that the downward gravitational force on the electrons in the metal container produced a downward shift in the conduction electrons, which yielded a vertical field of precisely the magnitude and sign to cancel the gravitational force on free electrons in the tube. This apparent agreement was not generally accepted, and there shortly appeared other papers arguing that the effect of lattice compression should be to produce large electric fields of such sign as to give the free electrons a downward acceleration $>10,000$ times gravity. The Brookhaven experiments were designed to search for the effect of lattice compression by observation of shifts in contact potential upon stressing in compression. The experimental procedure was to use the Kelvin method of measuring contact potential shifts. In this system a vibrating capacitor is so constructed that one electrode is a free surface that can be stressed. In experiments on brass, the contact potential outside of stressed brass was found to become more positive as the metal was compressed. This result is in agreement in both sign and magnitude with the predictions of a large effect arising from lattice compression. Similar results have been obtained with other metals. How to reconcile these results with the small electric fields observed in the Stanford experiments remains a subject for future speculation.

\section{Structure of Solids}

Another major experimental activity is centered around the use of neutrons in studies of the dynamical and structural properties of solids. Several other techniques, such as x-ray diffraction, Mössbauer spectroscopy, and low-temperature experiments, contribute significantly to the over-all effort. Critical scattering has emerged as an essentially new research topic, with advantage taken of the unique role of neutron scattering in the study of second-order phase transition dynamics.

A new and simple mechanism of second-order phase transitions in solids was elucidated by neutron scattering measurements, namely the condensation of a soft phonon mode at an appropriate zone boundary. This transition may be called antiferroelectric because of its similarity to the antiferromagnetic transition. The first example of this new type of phase change was demonstrated at the $110^{\circ} \mathrm{K}$ transition in $\mathrm{SrTiO}_{3}$, which had long been an unsolved puzzle because of its well-defined, yet unexplained, characteristics. Neutron inelastic scattering measurements have revealed that this transition is caused by a $\Gamma_{25}$ soft mode at the [111]

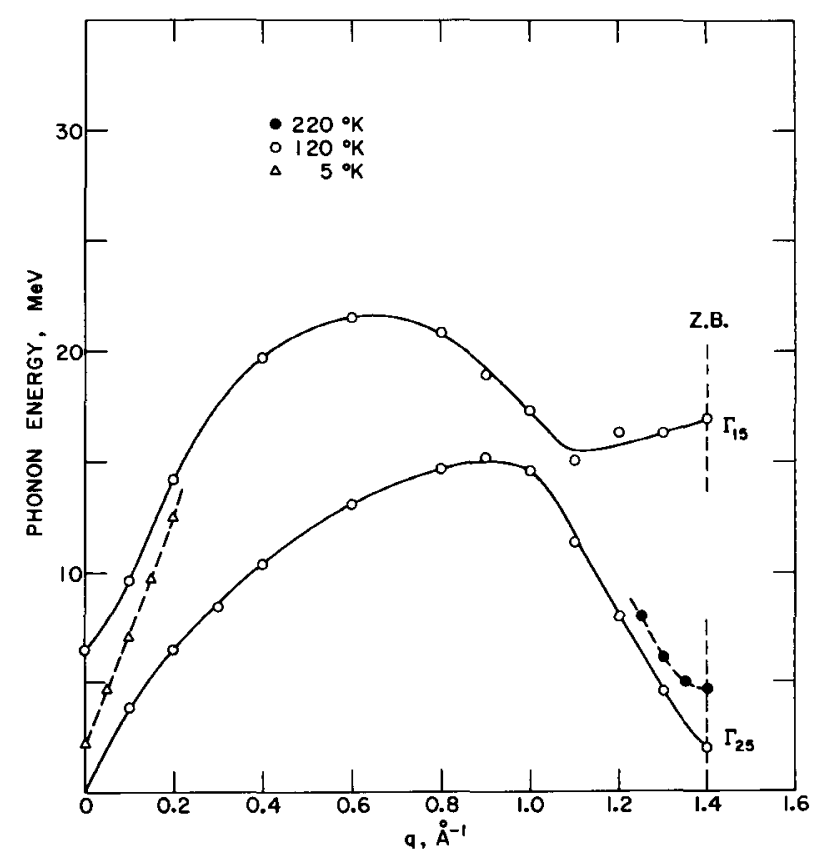

Figure 42. Phonon dispersion relation for $\mathrm{SrTiO}_{3}$ at several temperatures along a [111] direction. The condensation of the $\Gamma_{25}$ mode at the zone boundary is clearly indicated by the $120^{\circ} \mathrm{K}$ data. The low-temperature data (at $5^{\circ} \mathrm{K}$ ) show the condensation of a $q=0$ mode. 
zone boundary. The square of the frequency of this soft mode is proportional to $T-T_{0}$ above the transition temperature, $T_{0}$. At the transition, this zone boundary phonon condenses into a superlattice Bragg point; thus the low-temperature phase has a structure logically derived from atomic displacements of the soft phonon. Figure 42 illustrates the phonon dispersion relation in $\mathrm{SrTiO}_{3}$ which, in addition to the $\Gamma_{25}$ soft mode at $120^{\circ} \mathrm{K}$, also shows the ferroelectric $(q=0)$ soft mode at low temperatures.

Study of the $\Gamma_{25}$ transition was extended to two other perovskite compounds, $\mathrm{KMnF}_{3}$ and $\mathrm{LaAlO}_{3}$, with results that shed further light on the lattice dynamics of the transition. This characteristic $\Gamma_{25}$ mode is a simple rotation of an $\mathbf{M X}_{6}$ octahedron. The rotation axis may take one of the three cubic axes creating the triply degenerate mode: $A, B$, and $C$. The transition in $\mathrm{SrTiO}_{3}$ corresponds to the condensation of the $A$ mode, which results in a tetragonal structure, and the phase transition in $\mathrm{KMnF}_{3}$ at $184^{\circ} \mathrm{K}$ corresponds to the $A+B$ mode, which results in an orthorhombic structure. The final case, condensation of $A+B+C$ to the rhombohedral phase, was observed in $\mathrm{LaAlO}_{3}$ at $535^{\circ} \mathrm{C}$.

In a similar investigation, inelastic neutron scattering measurements have been performed on crystal quartz in the vicinity of the $\alpha-\beta$ phase transformation at $573^{\circ} \mathrm{C}$. In agreement with previous optical work, it was found that the lowest frequency $A_{1}$ zone-center phonon mode in $\alpha$ quartz (207 $\mathrm{cm}^{-1}$ at room temperature) shifts to lower frequency and broadens remarkably as the temperature is increased. In the high-temperature $\beta$-phase quasi-elastic scattering was observed, which can be ascribed to an overdamped zone-center optical phonon. The intensity exhibits a Curie-law divergence characteristic of critical fluctuations. A study of the relative intensity of this critical scattering about several Bragg reflections in the $(h 0 l)$ zone showed that the "soft" phonon eigenvector closely resembles the atomic displacements associated with the phase transformation.

Extensive investigation has been carried out on magnetic scattering from the $3 d$ metals iron and nickel near the Curie temperature. These metals are the oldest known ferromagnets, yet their magnetic properties are the least understood because of difficulties in treating long-range magnetic interactions involving itinerant electrons. Satisfactory knowledge of the dynamics of magnetic interactions through the Curie temperature is es- sential for better understanding. The long-wavelength inelastic scattering by iron and nickel was studied over a broad temperature range, from temperatures well below the critical temperature, $T_{c}$, at which spin-wave modes are well defined, through the critical region to temperatures above $T_{c}$, at which diffusive types of spin fluctuation are dominant. Below $T_{c}$, in the temperature range $0.004<\left(1-T / T_{c}\right)<0.2$, the spin-wave energies vary as $\left(1-T / T_{c}\right)$ to the powers 0.37 and 0.39 for iron and nickel, respectively. Slightly below $T_{c}$ the spin waves become overcritically damped. There is no indication of a peak in the scattering below $T_{c}$ corresponding to a diffusive mode. At the critical temperatures, the line-shapes scale as the wave vector to the powers 2.46 and 2.7 for iron and nickel, respectively. Above $T_{c}$ there exists a region in which the scattering is accurately described by diffusion theory. The diffusion constant varies as $\left(1-T_{c} / T\right)$ to the powers 0.14 and 0.51 for iron and nickel, respectively. For a Heisenberg ferromagnet the predicted exponent is 0.33 . At present, the different behavior of the diffusion constant for the two metals is not understood.

Basic problems of the dynamical scattering of neutrons were investigated with use of perfect silicon crystals. Observations were made on the appearance of Pendellosung interference fringes within neutron Bragg reflections. These were used to determine the atomic scattering a mplitude of silicon for neutrons as $(0.41644 \pm 0.00022) \times 10^{-12}$ $\mathrm{cm}$. Conclusions were drawn concerning the spatial extent of the neutron wave packet from the fringe characteristics. Another experimental observation was concerned with single-slit diffraction of slow neutrons on passing through fine slits of widths between 21 and 4.1 microns. The observed broadening agrees well with that calculated, and this supplied evidence on the width of the coherent wave front of a neutron wave packet. The experiments imply that the individual neutron wave packets possess a length $>\approx 3000 \AA$ as they emanate from the crystal. Further, it can be concluded from the slit experiments that the neutron wave front, as it approaches the slit, must be coherent over a transverse width at least that of the largest slit studied, namely 21 microns. Thus, the coherence volume of a neutron wave packet must be considered to be very extended relative to the deBroglie wavelength scale.

The satellite reflections appearing about normal Laue-Bragg reflections in holmium were investi- 
gated. These reflections arise from the aspheroidicity of the $4 f$ charge density as distinct from the core charge density. In holmium metal the $5 d^{1}$ and $6 s^{2}$ valence electrons are believed to enter the conduction bands and to leave a tri-positive ion core approximated by the configuration ${ }^{5} I_{8}$. The $4 f$ shell lacking four electrons is screened from the crystalline environment by the filled $5 s^{2}$ and $5 p^{6}$ shells. Because of the unpaired electrons in the $4 f$ shell the ions have a magnetic moment which between $20^{\circ}$ and $132^{\circ} \mathrm{K}$ tends to aline them in a flat spiral with a propagation vector lying along the hexagonal axis of the crystal. This tendency of the ions to order into a spiral, coupled with the aspherical charge density, induces periodicities in the scattering which produce $\mathrm{x}$-ray satellite reflections. At a finite temperature the ions are disordered to the extent that they are distributed among states with magnetic quantum number $M$ and inner quantum number $J=8$. By using the molecular field approximation suggested by Nagamiya to describe this disorder, it was found that past observations of neutron satellite intensities of Koehler et al. were explained. The theory was then used to calculate the average $x$-ray scattering factor from the scattering factors for the various ionic states with use of parameters derived by Blume et al. from recently determined HartreeFock wave functions. The agreement with experimentally observed values of the scattering of the first satellite pair about the $(22 \overline{4} .0)$ reflection is excellent.

The properties of lattice vibrations at low temperatures were investigated with Mössbauer spectroscopy. It was shown recently that this technique can be used to determine directly a certain average of the lattice vibrational frequencies which, in a pure lattice, is the important quantity in the theory of strong-coupled superconductors. According to McMillan's theory this vibrational quantity, rather than the usual electronic ones, governs the transition temperature of a class of superconductors. The local effect of an impurity atom on this lattice quantity was investigated theoretically. Such a calculation can be used to reduce the Mössbauer measurements to the pertinent vibrational average of the pure lattice. Further, the effect of impurities on the superconducting transition temperature can be estimated. It is found that, for a number of lattice models, heavy mass impurities should increase the superconducting transition temperature.

During the past year funds were made available to implement the cold neutron moderator project for the HFBR. The moderator is to be installed in the $\mathrm{H}-9$ beam port and is expected to considerably enhance the beam intensity available to the slow neutron chopper. The design calls for a hemispherical shell $3 \mathrm{~cm}$ thick and $\approx 20 \mathrm{~cm}$ in diameter, in which liquid hydrogen will be circulated. Cooling of the hydrogen will be by heat exchange, with $14^{\circ} \mathrm{K}$ helium gas supplied by a $3-\mathrm{kW}$ helium refrigerator. Preliminary design studies have been made for both the beam plug and moderator and for the refrigeration and gas-handling systems. In addition, a mock-up of the proposed moderator and beam plug has been built for study and tests at the Brookhaven Medical Research Reactor. Work on the safety analysis, which is necessarily quite elaborate, is in progress.

The Solid State Group takes an active part in a joint program on superconductivity involving members of the Metallurgy and Materials Science Division of the Department of Applied Science. Research in this area is reviewed in the section on Applied Science.

\section{THEORETICAL PHYSICS}

Work in the Theoretical Group ranges over nuclear structure theory, nuclear models, nuclear reactions, many-body systems, and atomic physics. Close contacts are maintained with experimental nuclear physics groups, as well as with theoretical activities in solid state and high energy physics. Some typical studies are described briefly below.

Based on deduction from the Coulomb energy separation between the analogue states of lead, the suggestion was made recently that the neutron radius is considerably larger than the proton radius as known from electron scattering. Since this was in apparent contradiction with the results of the $\pi^{ \pm}$-lead scattering experiment of Abashian, Cool, and Cronin, it seemed necessary to reanalyze the experimental data with very general neutron distributions. Agreement was obtainable only by admitting neutron distributions with sharp edges and with peaks near the surface. Since these are implausible, the problem of understanding a large neutron radius was therefore emphasized. On modification of the original analysis of the Coulomb energy separation data, acceptably smaller neutron radii resulted.

How to derive the well-known nuclear rotational motion from the nuclear Hamiltonian has been an interesting problem. Some time ago, 
Thouless and Valatin showed that it could be extracted from the Hamiltonian within the formalism of the random-phase approximation. A number of problems remained that had to do with the detailed interpretation of the results of this formalism: the rotational energies form a continuum and the corresponding eigenfunctions cannot be normalized. On examination the difficulty was shown to originate in the fact that the randomphase approximation has the properties of a smalloscillation approximation, and such difficulties are known and understood. Once this is recognized, a straightforward prescription follows that allows the difficulties to be bypassed and the results to be used for practical calculations. Thus static and transition matrix elements can be calculated. Further, the connection was made with the selfconsistent cranking model method of calculating the parameter defining the rotational energy, the moment of inertia. A critical criterion, that of Kelson for the moment of inertia, originally set up for the self-consistent cranking model, can be discussed again directly, and it can be shown that within the approximations it is met.

A reaction matrix calculation was completed which deduces a realistic residual interaction for the shell model from a solution of the Brueckner equation. The residual interaction, obtained from a separable potential fitted to the two-body scattering data, was found to be more sensitive to the single-particle shell model spectrum than to possible uncertainties assumed in the composition of the force, such as the amount of tensor force or degree of short-range repulsion. The eventual result is an easily calculated residual interaction which can be used in further shell model studies.

The reaction matrix calculation still lacks a treatment of the so-called core-polarization contribution to the residual interaction. It is hoped that the matrix elements obtained in this calculation can be used with already existing codes to deduce the part of the force arising from a breakup of closed nucleon shells. When the core polarization terms have been included, it should be possible to do a few many-nucleon calculations to test the general validity of the derived residual interaction.

An attempt was made to calculate the effect of using more realistic wave functions on shell model diagonalizations. The results for low-lying spectra were dramatic in the $A=18$ nuclei, but were of less significance for heavier nuclei. Previously obtained fits to the spectra of $\mathrm{O}^{18}$ and $\mathrm{F}^{18}$, which used the realistic Kuo-Brown force and harmonic oscillator wave functions (as a definite example), would be considerably distorted by the introduction of Wood-Saxon (WS) wave functions. In the lead region of the periodic table residual interactions are generally weak, and the effect of using WS functions is of less significance.

A model for nuclei with masses 15,16 , and 17 that consists of an exact shell model calculation for all configurations of four particles moving in the lowest three orbits outside an inert core of $\mathrm{C}^{12}$ had been presented earlier. It correlated the energies of some 21 levels in these nuclei with essentially no arbitrary parameters. Also included was a successful calculation of the magnitude of the $E 0$ (pair production) matrix element for the decay of the first excited state of $\mathrm{O}^{16}$, and it was demonstrated that $\mathrm{O}^{16}$ behaves in many respects as a closed shell nucleus, even though the ground-state wave function is highly correlated. Later work showed that about 60 energy levels can be accounted for with the simple model in its original form. Calculations of electromagnetic transition rates and spectroscopic factors for transfer reactions support the validity of the treatment.

The original model was extended to mass 18 nuclei with very encouraging results. Levels that could not be adequately explained in earlier treatments are accounted for easily in the present work. Furthermore, the usual treatments of $\mathrm{O}^{18}$ based on calculations of two-particle states outside a closed-shell $\mathrm{O}^{16}$ are inconsistent with the present work, since the new model brings in many coreexcited components. The model was enlarged to take account of configurations that are important for $\mathrm{N}^{15}$ but were neglected in the first applications. This process requires careful estimation of the importance of various configurations in order to keep the calculations manageable without omitting correlations. The scheme finally decided on leads to an accurate description of the spectrum of $\mathrm{N}^{15}$ which appears to be supported by the latest experimental evidence. In addition, the properties of several levels are predicted.

A new method of solving the Boltzmann equation for degenerate Fermi systems was devised. The resulting expressions for transport coefficients differed from previously accepted results by factors that involved the quasi particle lifetime. The correction was most significant for the thermal conductivity, which was changed by a factor of two, and it removed a discrepancy between theory and 
experiment for dilute mixtures of $\mathrm{He}^{3}$ in liquid $\mathrm{He}^{4}$. The method was also used to calculate the next order term in the expansion of transport coefficients in powers of the temperature. This term is particularly large for systems that are near a phase transition, and it seems to account for the deviations from Fermi liquid theory that have been observed in liquid $\mathrm{He}^{3}$.

An eikonal approximation for nonlinear equations, proposed by Whitham, was derived from an expansion in powers of space and time derivatives. The approximation is of interest because it arises frequently when there is a change of level of approximation or of physical understanding, but in the past it has been applied to linear equations only. A general solution for each order of the expansion was obtained, and the discontinuity for a fluid with a free surface was derived in a form that was suitable for application of the method.

The electronic dipole polarizabilities $\alpha_{d}$ of the alkali atoms were recalculated with the inclusion of the shielding effect of the ion core in the perturbation equations. The resulting calculated values of $\alpha_{d}$ are $24.72 \AA^{3}$ for $\mathrm{Li}, 22.3 \AA^{3}$ for $\mathrm{Na}, 43.0 \AA^{3}$ for $\mathrm{K}, 45.5 \AA^{3}$ for $\mathrm{Rb}$, and $61.2 \AA^{3}$ for Cs. These results are in very good agreement with the recent experimental values of Hall and Zorn. The dipole polarizabilities $\alpha_{d}$ of the $\mathrm{Rb}^{+}$and $\mathrm{Cs}^{+}$ions were recalculated with use of the appropriate HartreeFock wave functions (including exchange), which have recently become available. The resulting values, namely $\alpha_{d}\left(\mathrm{Rb}^{+}\right)=2.02 \AA^{3}$ and $\alpha_{d}\left(\mathrm{Cs}^{+}\right)$ $=3.76 \AA^{3}$, are in much better agreement with experiment than those obtained in a previous calculation by using Hartree wave functions (without exchange).

\section{NUCLEAR MOMENTS}

The Nuclear Moments Group has been engaged in a continuing program of studying hyperfine structure, primarily for radioactive nuclei, with use of atomic beam magnetic resonance. The principal objectives have been to determine nuclear spins, nuclear magnetic hyperfine energies, nuclear electric quadrupole energies, and nuclear magnetic moments. With the Brookhaven equipment, transitions may be studied with relative precision of the order of $10^{-8}$ to $10^{-9}$, which permits separating out of the interaction of the nuclear magnetic moment with an external magnetic field in the presence of the normal hyperfine interaction. Measurement of magnetic moments in this manner is not subject to perturbations due to molecular or lattice environments, nor need it be analyzed by utilizing atomic wave function calculations.

The determination of nuclear moments is of particular interest if made to a precision of $10^{-3}$ or better, since this permits evaluation of the nuclear hyperfine anomaly. The latter is an effect resulting from penetration of valence electrons into the nuclear volume and interaction with the magnetic field due to the particular configuration of intrinsic particle and orbital magnetization.

In a typical problem studied during the past year, the method was applied to $\mathrm{K}^{42}(12 \mathrm{hr}$ ) to determine the parameters

$$
\begin{aligned}
a & =-503,550,779(5), \\
\mu_{i} & =1.1424(2), \text { and } \\
{ }_{39} \Delta_{42} & =+0.000344(35) .
\end{aligned}
$$

A similar study confirmed the spin of $\mathrm{Pt}^{197}(20 \mathrm{hr})$ and yielded a rough preliminary value of $a$ of about $1600 \mathrm{MHz}$. The final value of the latter may approach a precision of about $\pm 10 \mathrm{kHz}$. Because of the complex electronic state, it is not feasible to measure $\mu$ directly.

The experiment undertaken at the HFBR to determine the electric dipole moment of the neutron was reported in part last year. This experiment utilized the basic technique of magnetic resonance, as used by Smith, Purcell, and Ramsey in 1950, and as a short-term goal sought to reduce the previous upper limit by a factor of 50 or more. Final results were limited by systematic errors to an upper limit of $\approx 10^{-21} e \mathrm{~cm}$. No further work on this problem is anticipated until the cold neutron facility is installed at the HFBR. 


\section{High Energy Accelerators}

The Accelerator Department, in addition to operating, maintaining, and improving the Alternating Gradient Synchrotron (AGS), is also responsible for planning, procuring, and erecting apparatus for the experimental program. The AGS conversion program to improve the intensity and performance of the AGS and the research and development of new ideas in accelerator design and exploitation are also being carried out by the Department. The present status and programs are described in the following sections.

\section{ALTERNATING GRADIENT SYNCHROTRON}

The performance of the AGS again improved significantly during the past year. As a result of major improvements in the injector and the linear accelerator (linac) rf structure, it was possible to inject higher currents into the AGS. In addition, by energizing a series of backlog windings mounted on the AGS magnets, it became possible to increase the aperture of the synchrotron and thereby capture more of the injected beam. A peak intensity of $3.1 \times 10^{12}$ protons/pulse already has been achieved. The successful reliable operation of the new high-gradient dc accelerator column of the preinjector system, coupled with its beam intensity performance, has justified the long developmental time invested in this device. The slow external beam facility has been successfully used for three experiments in high energy physics, an indication of its present reliable operational status. Performance has been good, and during the past year increased knowledge has been gained of the various possible modes of carefully time-controlled beam extraction.

In order to make efficient use of the increased AGS beam intensities, the external beam system is being expanded into a beam "switchyard" to provide better distribution of the available number of protons.

The activities of the AGS Division related to the operation of the facility, accelerator studies, and AGS development are summarized below.

\section{Operation}

The AGS continued this year to run for considerable periods with reduced intensity to specifi- cally suit the requirements of experimenters. The average intensity performance of the AGS is illustrated in Figures 1 and 2, which also indicate the steady rise in yearly average intensity for the past four calendar years. The reliability of the machine continues to improve. This was coupled this year with a rise in scheduled time which resulted in an increase of almost $10 \%$ in the number of hours available to experimenters. Biweekly efficiency and utilization of the AGS are indicated in Figures 3 and 4, respectively. The rise in efficiency during the last four years is shown in Figure 5.

The average number of experiments being run at any one time during the year declined slightly (3.6 for calendar year 1968, as opposed to 4.0 for 1967). This resulted in a decline in the number of hours experimenters were utilizing the AGS, as shown in Figure 6.

Shutdowns during the fiscal year were as follows: a scheduled shutdown from Sept. 24 to Oct. 28 , a scheduled shutdown from Dec. 26 to Jan. 2, and an unscheduled shutdown from Jan. 18 to Feb. 2, 1969. The first shutdown for conversion work started on May 26, 1969, and is scheduled to last for five months.

During the first shutdown the high-gradient column and the rf multiport system were installed in the linac. In the slow extracted beam the "optics" were modified and additional instrumentation was installed. Installation of the fast extracted beam for the Southwest Experimental Area was started in the B-10 area of the ring. Vacuum-chamber shorts were checked and eliminated. The backleg lowfield correction windings and circuits were installed. Metal seals were installed on vacuum chambers in selected high radiation areas. An earth radiation shield was constructed over the ring from A-10 to B-10.

During the second shutdown, the preinjector beam transport was modified, the B-10 ejector magnet installation was completed, and the monitoring circuits for the low-level backleg windings were completed.

On Jan. 15 electrical sparking was observed in the rotor air gap of the AGS magnet power supply alternator. Operation was halted and the rotor removed. The sparking, which was due to loose 
damper bars on the rotor body, was eliminated by swaging the bars tight in their slots. The alternator was brought into service again in $2 \frac{1}{2}$ weeks, considerably ahead of original estimates.

Major failures of shorter duration are listed in Table 1.

Improvements and additions made at the AGS during other brief shutdowns included installation of bellows-type extraction magnets at I-10

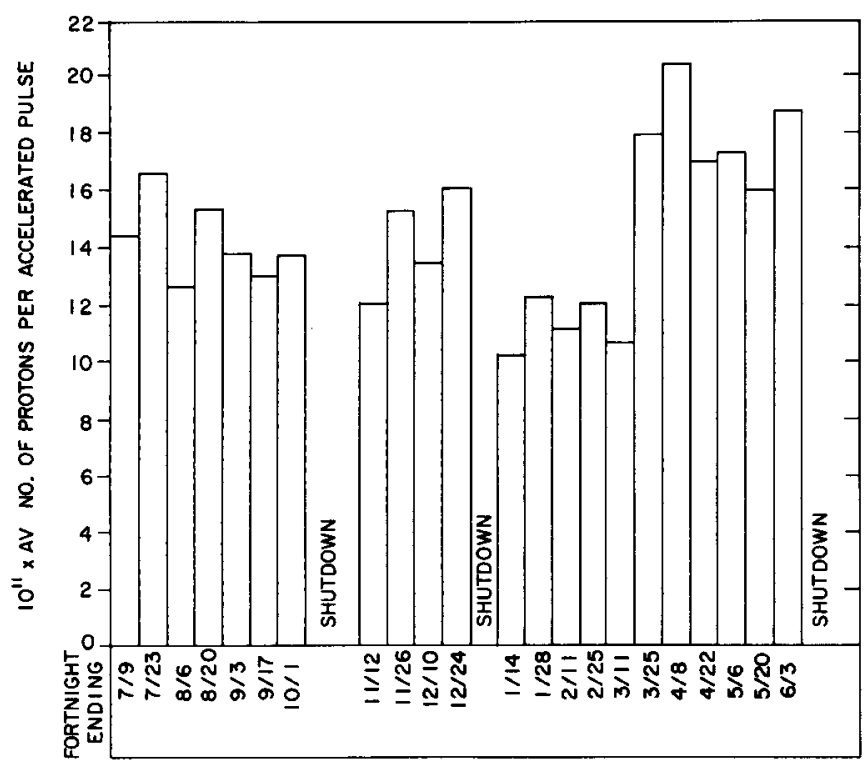

Figure 1. Biweekly average beam intensity of the AGS, July 1968 - June 1969.

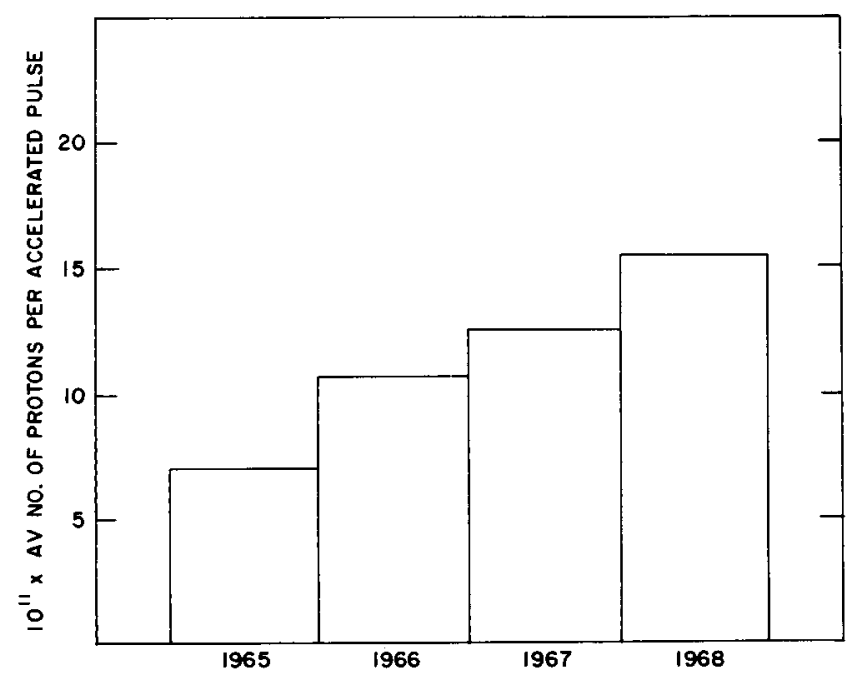

Figure 2. Yearly average beam intensity of the AGS, calendar years 1965-68.

Table 1

Cause of failure

Duration, hr

Ignitron vacuum failure in main magnet power supply

Irradiated O-ring failure at K2

Pneumatic leak into vacuum at circulating beam stopper

Linac timing and FTH-tube problems

Vacuum leak at rf cavity $K$

Water leak in F-10 ejector magnet

Water leak in G-20 rapid beam deflector coil

Vacuum leak at rf cavity I J

Arcing in inflector

Hard-tube modulator problems, including circuit breaker

54

9.5

24.6

9.4

14.6

13.8

8.6

11.9

21.1

12.9

and B-10, an airlock and other instrumentation in the slow extracted beam, an airlock for a beam clean-up target, and five "conversion" vacuum chambers and sputter-ion pumps; and recommissioning of the fast extracted beam for the Southwest Experimental Area.

All AGS cooling systems ran with normal maintenance and only minor interruptions of machine operation during the year. During the Sept. 1968 shutdown period the three AGS wells were shocktreated with phosphate to restore their yields. Two portable coolers and their associated piping were reactivated in the Southwest Experimental Area to provide cooling for the 7 - $\mathrm{ft}$ bubble chamber. Further, a new chilled-water cooling system was installed for the B-10 magnet power supply. A third $850-\mathrm{gal} / \mathrm{min}$ pump was installed in the North Experimental Area. This addition made possible the removal of two portable coolers that had been providing supplementary cooling.

Humidity control in the linac-Cockcroft-Walton pit was improved by the addition of a steam humidifier coil and associated controls and the relocation of the dehumidifier cooling coil. The air and water lines to the linac FTH triodes were modified during the Sept. 1968 shutdown, in line with the general rebuilding and upgrading of the units. The linac air-conditioning system was modified to provide additional cooling to the relay room. Equipment is being purchased that will make it possible to use demineralized water in the existing closed cooling system in the linac and thus eliminate "plugging" of magnets and other equipment.

As part of the continuous radiation safety program for personnel, the AGS Health Physics Group 
improved the dosimetry records system. About 400 Accelerator Department personnel were regularly monitored with the aid of film badges, pocket ion chambers, and thermoluminescent dosimeters. The information thus obtained was processed by the CDC 6600 computer, and an up-to-date record is continuously available.

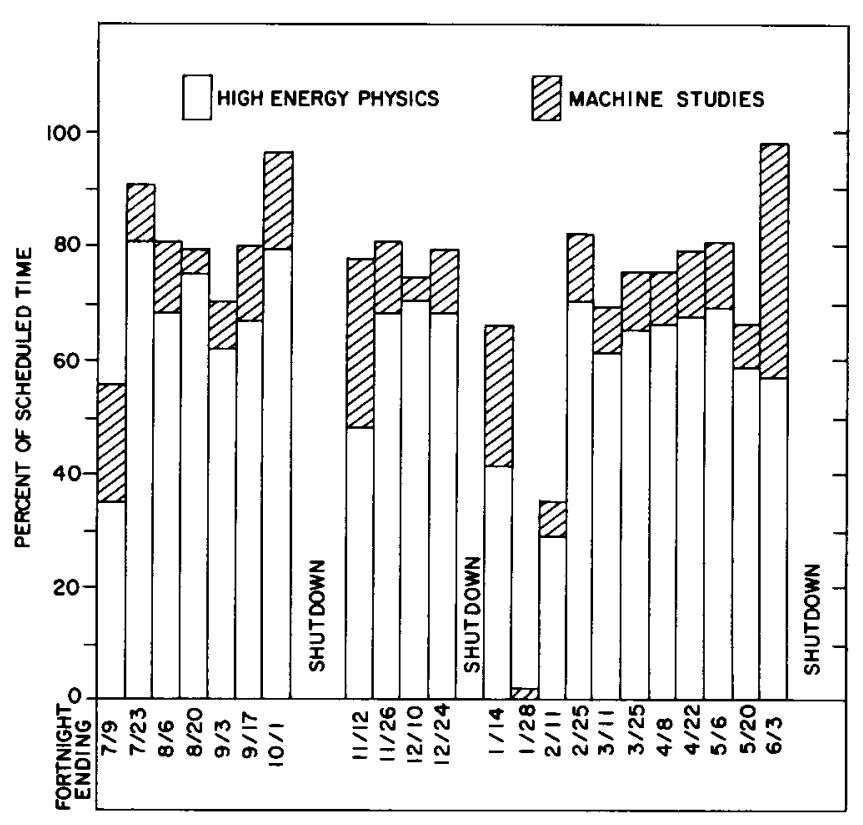

Figure 3. Biweekly efficiency of the AGS, July 1968 - June 1969.

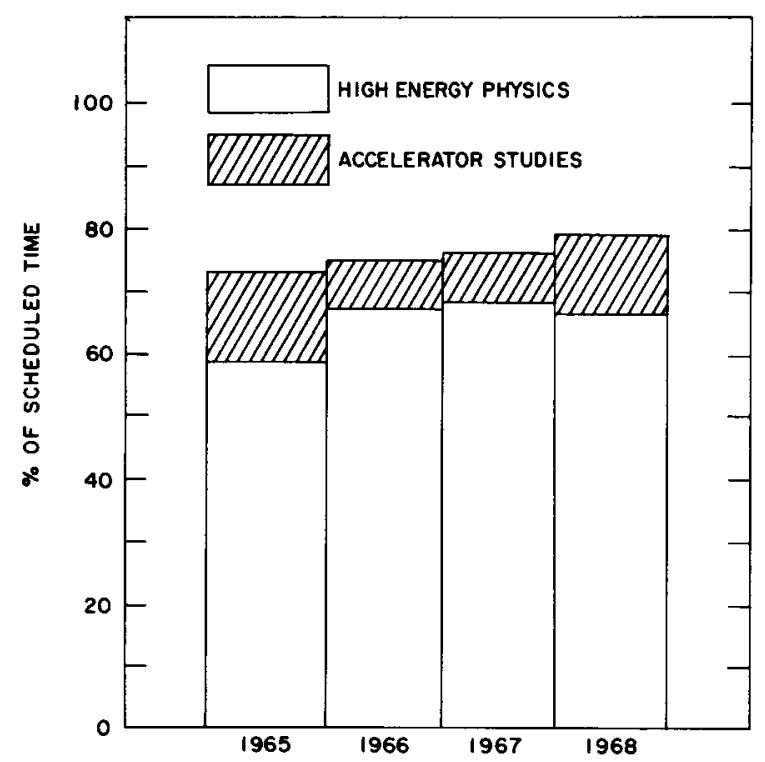

Figure 5. AGS efficiency, calendar years 1965-68.
In addition, a color-coding system for film badges was initiated which allows the yearly exposure record of any person working in the AGS to be determined immediately. The total 1968 radiation exposure of Accelerator Department personnel was about $504 \mathrm{Rem}$. This figure is $\approx 5 \%$ lower than that for 1967.

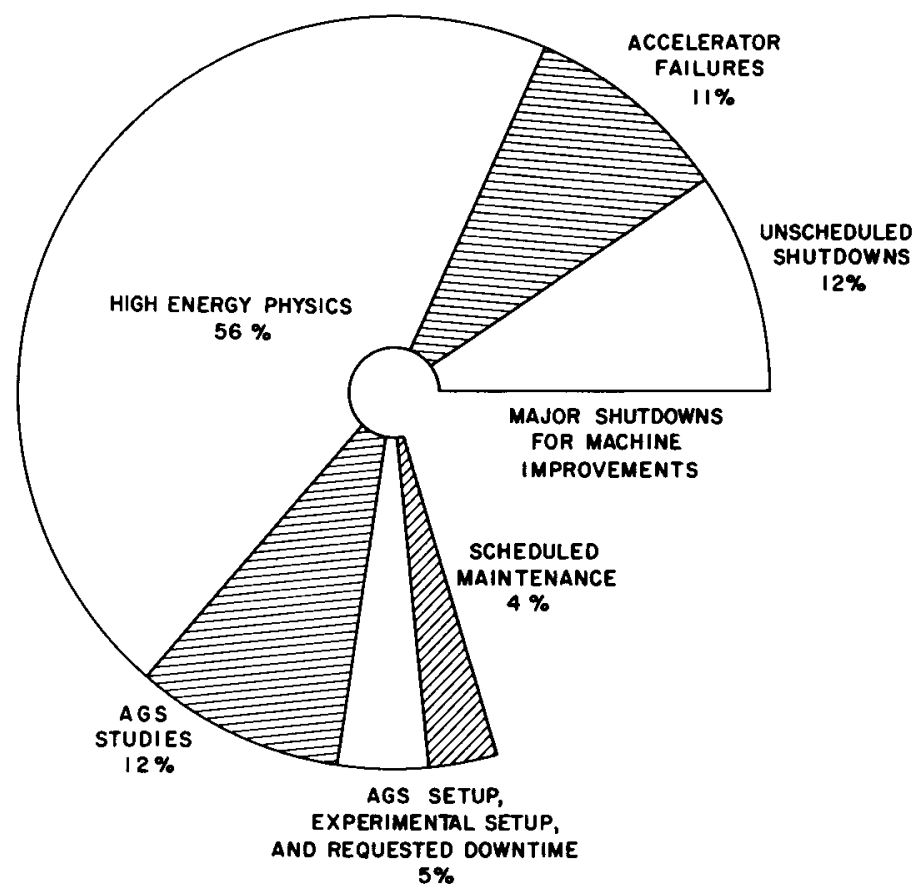

Figure 4. AGS utilization during fiscal 1969. Sum of enclosed areas represents scheduled operation time. Percentages refer to scheduled time, which is $80 \%$ of maximum possible running time.

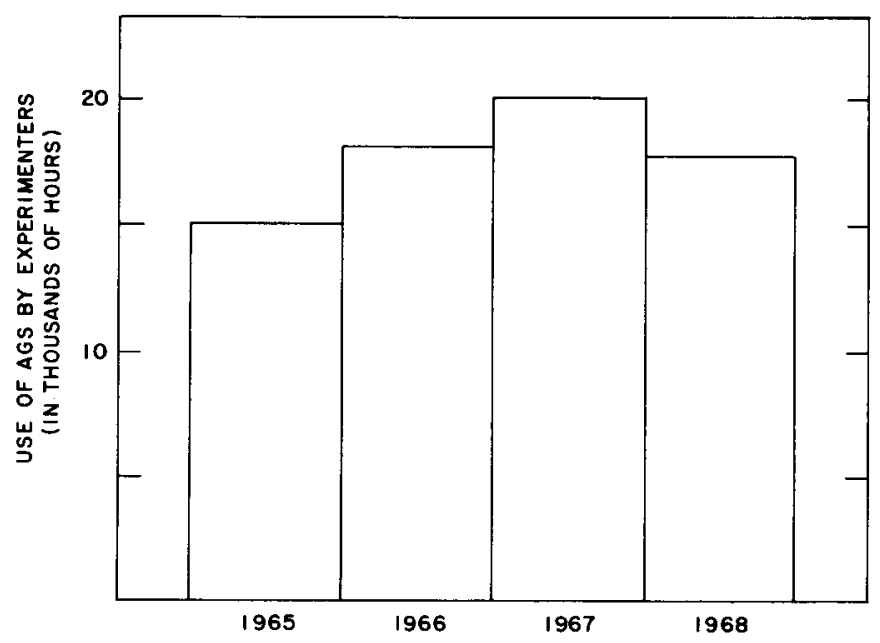

Figure 6. Total experimental time, calendar years 1965-68. 


\section{Accelerator Studies}

During the year considerable effort was expended in studying the effects of dipole and quadrupole errors in the AGS. In particular, quadrupole effects were investigated by observing the beam loss as the horizontal half-integral resonance was crossed. Shorting a single vacuum chamber $(\approx 43 \mathrm{~A}$ peak circulating current) was found to produce a barely discernible loss. Detailed studies of correcting the half-integral stopband loss produced with a single dipole perturbation were also made. The results are in good agreement with the linear theory for stopbands. When the machine was tuned with maximum intensity from the linac, introduction of the divide-by-two intensity attenuator in the linac AGS beam line did not change the time at which the horizontal half-integral resonance occurred. This indicates that the incoherent space-charge depression for the horizontal betatron oscillation frequency $\left(\nu_{H}\right)$ is as yet no problem. This observation was made during AGS operation with an accelerated beam of $3 \times 10^{12}$ protons/pulse.

In addition, the loss produced by insertion of the slow external beam septum magnet at F-10 was found to be a gradient perturbation produced by a local distortion of the earth's field. This could be eliminated with the standard quadrupole corrections, but local correcting fields were installed to keep these quadrupoles free for correcting other errors.

The effect of dipole errors produced by backleg or vacuum-chamber short circuits, as distinct from those resulting from magnet displacement or $\mathrm{dc}$ fields, was investigated by using the 36 -point orbit measuring system. Because of the changing $d B / d t$ $(\dot{B})$ present in the AGS $(\approx 4.4: 1$ from injection to $200 \mathrm{msec}$ ), subtraction of orbits taken at different values of $\dot{B}$ under the same conditions can be used to find large dipole errors $\approx \dot{B}$. Subtraction of orbits taken when $\dot{B}$ is near maximum and $B \gg B_{\text {inj }}$ can also be used to find changes in $\dot{B}$ errors. The first method turned up a series of vacuum-chamber ground loops in the F-10 area, while the latter pinpointed backleg winding shorts in the G-15 magnet.

The quadrupole distortions present in the AGS at injection were greatly reduced by connecting some of the backleg windings of every AGS magnet in such a manner as to completely correct for the remanent gradient differences that existed between open and closed magnets and produced a
$25 \%$ distortion of the horizontal and vertical $\beta$ functions. Other backleg windings were connected to provide tuning of the machine (i.e., shifting the $\nu$ values in opposite directions) without distortion of the $\beta$-function. The previous $\sin , \cos 8 \theta$ and $\sin$, $\cos 9 \theta$ backleg corrections were retained ( 16 and 18 points respectively), and $\sin 12 \theta, \cos 12 \theta(24$ points) were added.

When the $\beta$-function correction and tuning windings were energized for the first time, a 25 to $30 \%$ increase in captured beam was observed. Subsequent tuning of other corrections resulted in an accelerated beam of $3 \times 10^{12}$ protons/pulse. This intensity was obtained with a linac beam output of 50 to $55 \mathrm{~mA}, 80-\mu \mathrm{sec}$ duration, which is now regularly available as a result of recent linac improvements, mentioned below.

Intensive programs investigating slow and socalled slow-fast beam ejection from the AGS are being carried out. These programs relate to future multiple cycling of the bubble chambers per single AGS cycle, the potential of using the G-10 internal target simultaneously with the slow external beam system, and the study of alternative beam ejection modes of specified short duration. The slow external beam has been successfully time-shared with internal G-10 flat-top targeting on an alternate machine-pulse basis. Studies are in progress to determine the feasibility of either simultaneous or sequential internal and external targeting during the same magnet cycle flat-top. Methods of obtaining a fast-slow spill, of interest in bubble chamber operations, have been studied in which a fraction of the internal beam is excited by the slow external beam nonlinear resonance, as follows: $\tau_{\text {spill }} \approx 0.2 \mathrm{msec}$, induced by fast kicker; $\tau_{\text {spill }} \approx 0.5$ msec, induced by rapid beam deflector; and $\tau_{\text {spill }} \approx 5$ msec, by spill at "invert" time in the AGS cycle. Extraction efficiencies of $<0.5$ of the normal slow external beam efficiencies are obtained when the above methods are used, and it is therefore planned to study alternative means of stimulating the resonance.

\section{Technical Development}

Linear Accelerator. The new high-gradient accelerating column has been installed on the AGS Cockcroft-Walton set and is operating satisfactorily. The maximum operating voltage to date is 770 $\mathrm{kV}$, with a maximum output current of $150 \mathrm{~mA}$. Many of the mechanical aspects of the column were explained in last year's report. One of the 

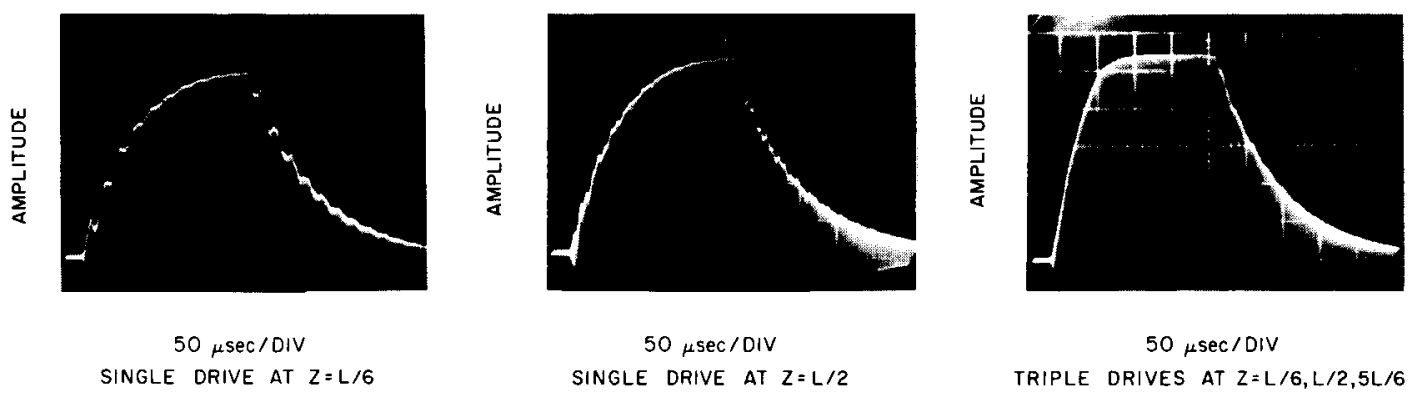

Figure 7. Tank patterns observed at the low energy end $(Z=0)$ of the linac for three different drive configurations.

features of this device is its ability to condition rapidly and remain conditioned for a long period of time, with little deterioration.

The arrangement of the transport system between the Cockcroft-Walton set and the linac was changed. The primary purpose of the new line-up was to improve trapping of the preinjected beam in the linac by moving the buncher farther downstream. A pair of 2000-liters/sec mercury diffusion pumps has been installed on the accelerating column and low energy beam transport line. These pumps are primarily used as backup for the evaporion pumps.

To handle adequately the higher beam intensity from the ion source, a fast beam compensation system was installed with the Cockcroft-Walton highvoltage power supply. This fast compensator, or "bouncer," produces high-voltage compensation signals, coincident with the ion source discharge, which are coupled to the high-voltage terminal through a $400-\mathrm{k} \Omega$ surge-limiting resistor and the 4500-pF filter capacitor of the Cockcroft-Walton high-voltage set. Feedback information, extracted from the high-voltage terminal by a capacitor plate mounted on the Cockcroft-Walton enclosure wall, is combined with preprogrammed signals for driving the output stage of the fast regulator. The hard-tube "bouncer" system has been operating satisfactorily and is able to compensate for up to $150 \mathrm{~mA}$ of beam current. Development is continuing on the low-level stages to raise this capability to $250 \mathrm{~mA}$.

A new high-power $r$ system has been installed to accelerate the higher beam intensity now available. The total power requirement for the linac cavity loaded with a 100 -mA beam is $\approx 8$ MW. This could be supplied by two TH-515 amplifiers operating at their recommended datasheet maximum. The use of three tank-drive ports, each powered by a TH-515 triode, provides a conservative solution. Multiple feed ports (properly located) reduce unwanted transients that are excited by the compensation pulse and reduce the operating gradients on the tank feed lines. Correct positioning of the ports cancels the $T M_{01 n}$ modes up to and including $n=5$. To facilitate tuning of the multiport system, each TH-515 amplifier is mounted on a carriage, which enables it to be moved through a distance of $\lambda / 2(\approx 20$ in.). As part of this feature, both the 8-in. and the 3-in. transmission lines are provided with telescoping sections. Figure 7 shows the variation of the tank pattern at $\mathrm{Z}=0$ under three different drive configurations. The faster rise time for the threeport drive system is due to overcoupling, since the loops are set for a beam-loaded cavity. At present the normal linac output current is $50 \mathrm{~mA}, 80-\mu \mathrm{sec}$ pulse (10-turn injection). The observable halfmaximum-width energy spread is $250 \mathrm{keV}$, comparable to that obtained previously with a $25-\mathrm{mA}$ beam. As ion source development continues, higher currents are expected to be accelerated in the linac.

With the new preinjector and multiport rf system, the linac has increased its beam intensity from about 25 to $50 \mathrm{~mA}$, with an increase of emittance of only $30 \%$. This improved the two-dimensional phase-space density by about $40 \%$. The beam loading in the preaccelerator as well as in the linac tank can be compensated completely.

Synchrotron Components. Improved versions of the fast external beam septum and ejector magnet carriage and ram mechanisms have been installed in the AGS. Organic materials have been eliminated, where possible, to make the units less susceptible to radiation damage. The components have been arranged to allow for their rapid interchange in case of failure. One of the major improvements is the introduction of dynamic bellows vac- 
uum seals with which a better vacuum can be maintained in the AGS than was possible with the conventional V-type Viton seals.

The slow external beam facility installed last year has been extended and is developing into a major AGS facility. At present, seven television stations are distributed along the slow beam array. The stations monitor fluorescent screens used to set up the beam. To keep the beam centered on the external target, two vertical insulated aluminum plates with a 0.005 -in. gap between them are used. When struck by a proton beam, these plates emit a signal that is proportional to the number of protons transversing them. This in turn is used to generate a steering signal to keep the beam centered on the target.

Long, thin, insulated aluminum plates are mounted edgewise to the beam on linear actuators and are used to measure the beam's horizontal and vertical profiles. To obtain a beam profile, an actuator-driven emittance device, consisting of 12 insulated plates stacked alternately between 13 bias shield plates, is placed in the beam's path. The resulting signal can be fed to the PDP-8 computer to give an almost instantaneous beam profile.

The five conversion vacuum chambers installed in the AGS this year are constructed of Inconel-X and have a flame-sprayed aluminum oxide coating on the external surface and a ceramic coating on the flange face. Both coatings provide electrical insulation. Metal C-ring seals were used in the vacuum joints. By wrapping 0.005-in. indium foil over previously used C-rings, a recent innovation, the C-rings can be reused and substantial savings effected. More than $250 \mathrm{C}$-rings are now in use on the AGS and the slow external beam lines.

A new, nonrammed, fast-extraction scheme calls for the development of a new full-aperture fast kicker and a 1/16-in. thin septum ejector magnet to extract the high energy beam from the AGS. Currently under design is a one-quarter-length model of the new fast kicker. This model will represent all the equipment required for full-scale operation. A $63 / 8 \times 10 \times 15$-in. ferrite magnet has been machined and assembled with use of a nonorganic cement. The magnet was fired at $300^{\circ} \mathrm{F}$ to assist curing, and mechanical tests have shown that the cement will withstand normal use. Highvoltage cable connections and terminations have been developed. These assemblies are currently in use in the power supply tank operating at $100 \mathrm{kV}$.
Design of the high current density, short-pulse, thin septum-ejector magnet for this fast extraction scheme has been completed. It is the first magnet assembly designed to be free of all organic materials. In addition, the entire assembly, including the vacuum enclosure and position mechanism, has been modularized so that it can be replaced in minutes.

All the regulated pulsed power supplies used in the slow beam extraction process have performed satisfactorily. However, to permit operation at higher energies, high-field excitation of the horizontal and vertical quadrupoles in the AGS ring was required to shift the $\nu$ value and avoid deleterious coupling resonances. This called for two new controlled rectifier power supplies capable of being pulsed to several hundred amperes. At the same time, injection-field correction in these magnets could be maintained with proper isolation between low and high-field power supplies.

The emphasis of the computer effort at the AGS has shifted from the development and installation of computer hardware and accelerator interface connections to the writing and operation of accelerator-oriented data-acquisition and analysis programs. Changes in the computer complex itself have been limited to items such as cathode-ray tube displays which are directly related to the problem of operator-computer-accelerator interface and to relatively minor changes in configuration which permit improved processing of multiple, complex, real-time accelerator service programs. The accelerator-computer interface channels are steadily being increased in number and range. Operational applications now include computerassisted operation of the slow external beam; statistical analysis of machine performance, including tuning indicator displays; data logging; and automated measurement systems for basic machine parameters.

Extracted Beams. During the year the slow external beam facility became operational and was successfully used in three experiments in the high energy physics program. A concurrent program of beam development has extended the useful energy range from 20 to $29 \mathrm{BeV}$ and the spill duration to $\leqslant 400 \mathrm{msec}(500 \mathrm{msec}, 28-\mathrm{BeV}$ flattop) and has yielded improvements in spill modulation and beam position stability. The low-frequency modulation has been reduced to $\approx \mathbf{4 0 \%}$ (peak-topeak/average) by a combination of control devices: (1) a closed-loop spill servo mechanism in 
which a beam-derived signal is fed back to the voltage regulator of the main magnet power supply to decrease the $\leqslant 60-\mathrm{Hz}$ current ripple components in the AGS magnets; (2) a quadrupole spill servo capable of correcting higher frequency modulation ( $\leqslant 2 \mathrm{kHz}$ ) that uses a ring quadrupole in straight section C-5 which is programmed with a signal from an AGS magnet backleg winding to modulate the horizontal $\nu$ value in synchronism with the main magnet current ripple; (3) improved gain of the electronic ripple filter to reduce the $720-\mathrm{Hz}$ fundamental ripple frequency from the 12-phase rectifier of the main magnet power supply; (4) a resistor matrix that applies orthogonal harmonic corrections to the firing angles of the ignitrons in the main magnet rectifier to reduce subharmonics of the $720-\mathrm{Hz}$ ripple; and (5) a computer-generated correction program, updated from spill data of the previous pulse to maintain uniform spill in a preselected time interval. $\mathrm{Rf}$ modulation at the fundamental $4.45-\mathrm{MHz}$ bunching frequency of the AGS has been reduced to $\approx 1 \%$ by shifting the phase of the accelerating voltage by $\approx 170^{\circ}$ at $\approx 2.5 \mathrm{msec}$ before $\mathrm{rf}$ turn-off. This phase shift introduces a momentum spread of about $1 \%$ on the circulating beam to enhance debunching and minimize rebunching, which occurs on flat-top because of beam cavity interactions and negative-mass instability.

Maximum slow-beam extraction efficiency is about $80 \%$. To attain this efficiency at energies $\geqslant 27 \mathrm{BeV}$ it has been necessary to shift the machine tune with AGS superperiod quadrupoles to avoid beam blow-up at the $\nu_{h}=\nu_{v}$ coupling and the $2 \nu_{v}+\nu_{h}=26$ coupling resonance. A shadow target in straight section F-4 has increased the extraction efficiency by $3 \%$ and reduced beam loss on the 0.030 -in. F-5 septum magnet by $50 \%$. A thin $(\approx 0.002$-in.) electrostatic septum will be installed upstream of the present F-5 septum to further reduce these losses. Preliminary measurements of horizontal and vertical emittance of the slow external beam give $E_{h} \approx 0.035 \pi$ and $E_{v} \approx 0.09 \pi$ in.mrad. These values are about 2.5 times larger than expected. Modifications were made in the external optics to reduce beam scraping on external apertures resulting from the larger beam size. New instruments, such as secondary emission chambers, insulated plates, and counter telescopes, together with their amplifiers, integrators, and other associated electronics, were developed for beam analysis and beam steering control. Several of these instruments provide outputs that can be readily displayed on oscilloscopes or digital voltmeters, while others are tied in with the on-line PDP-8 computer so that data may be handled by the computer at high rates and then subsequently displayed or printed out.

Developmental studies are in progress on extending the extracted beam system into a beam "switchyard." Initially the primary proton beam will be channeled to a target location in the new target building, which is essentially completed.

The principal fast external beam development in the past year has been reactivation of the Southwest Experimental Area facility; this required reinstallation of the B-10 fast external beam ejector magnet. Operational capability has been demonstrated for single-bunch extraction through both the B-10 and I-10 ejector magnets during the same AGS cycle. The extraction efficiency of the fast external beam has been improved during the past year as a result of several changes in circuits and targeting techniques.

\section{EXPERIMENTAL PLANNING AND SUPPORT}

The AGS is steadily used by research groups from universities throughout the United States as well as resident Brookhaven physicists. These groups develop research proposals for high energy physics experiments using the research facilities at the AGS. After approval of a proposal, the staff of the Experimental Planning and Support Division assists the experimenter in planning the experimental setup. This often calls for the design of a new particle beam or an unusual magnet. When the equipment is ready, Experimental Area personnel make the necessary beam changes and operate the magnets, separators, and targets for the experimenter. Fifteen counter experiments were in operation at the AGS during the past year. In most cases data were taken and the experiment was completed. In others, testing or preparing for an experiment was involved. On the average, two bubble chambers operated during the year, and a total of 3.7 million pictures were taken. Further details of the experimental program and the equipment developed are presented in the following sections.

\section{Experimental Facilities and Program}

During the year the experimental program for counter experiments was successfully inaugurated 
at the F-10 straight section of the AGS with experiments matched to the increasing sophistication of the slow external beam system. Two experiments in which the protons were used directly were completed, and data-taking was begun in a third experiment for which the first secondary beam from a target in the proton beam was used. A BNL-Yale University group using the full 28.5$\mathrm{BeV} / c$ proton beam on a massive uranium target set an upper limit on the cross section for the intermediate boson $W^{ \pm}$, up to a mass of $6 \cdot \mathrm{BeV} / c^{2}$. A Columbia University group, again utilizing a twoton uranium target and protons at $28.5 \mathrm{BeV} / c$, collected data in a search for massive bosons in the 2 to $6-\mathrm{BeV}$ mass range decaying into two $\mu$ mesons (muons). With the same apparatus they were able to study the annihilation of antiprotons and protons into muon pairs and to extend the cross-section and mass-range limits of the search for the $W^{+}$. In March the first secondary beam for counter experiments from the slow external beam was delivered to a BNL group utilizing a massive wire-chamber array on-line to the PDP-6 computer to study a wide range of interactions of $\pi$ and $K$ mesons in hydrogen. This single secondary beam facility is capable of delivering particles of either sign of charge over a range of $\approx 10$ to 25 $\mathrm{BeV} / c$.

A full program of counter experiments was also carried out from the internal target station at G-10, and an efficient mode of sharing a beam on a pulse-to-pulse basis between G-10 and the slow external beam has been evolved. On beam No. 6 (G-10 $+4.7^{\circ}$ on the outside of the AGS ring), a group from the State University of New York at Stony Brook completed a study of the associated production of $K$ mesons and $\Sigma$ hyperons involving $500 \mathrm{hr}$ of running time. In the same beam a University of California group from La Jolla completed a run of some $300 \mathrm{hr}$ in a study of charge conjugation, parity $(C P)$ violation in $K$-meson decay. A third group from Cornell used optical spark chambers to study backward peaks in neutral meson production for $350 \mathrm{hr}$. Finally, a group from MIT, Brown University, and the University of Padua began running an experiment to study various aspects of $\pi^{-}-p$ interactions with the use of optical spark chambers. This run will be concluded on termination of the shutdown that started at the end of May. During the winter shutdown a neutral beam port was installed by utilizing the front end of the G-10+4.7 beam, and a Princeton
University group has begun running a very sensitive test of $C P$ violation in $K^{0}$-meson decay.

At beam No. 5 (G-10+10 $10^{\circ}$, the enriched counter beam utilizing electrostatic separators) a series of $K$-meson experiments was carried out during the year. In the first of these an MIT group used a $\mathrm{BeO}$ target, which proved to be a significant advance in target technology, and collected some $560 \mathrm{hr}$ of data on various aspects of $K^{0}$ decay. A group from California Institute of Technology, BNL, and the University of Rochester completed a $640-\mathrm{hr}$ run on the scattering of $K^{+}$mesons at large angles from hydrogen. A Princeton group, using wire chambers coupled to computers, has studied $C P$ violation in the $\tau$ decays of $K^{ \pm}$mesons for $400 \mathrm{hr}$. At present, a Yale group is using a polarized proton target set up on one of the two branches of this beam to study the scattering of $K$ mesons, and a BNL group is using the other branch to study a particular mode of $K$-meson decay.

On the neutral beam on the inside of the AGS ring (beam No. 7, at G-10-20 ), a ColumbiaHarvard University group completed a run of some $1000 \mathrm{hr}$ to study charge asymmetry in $K_{L}{ }^{0}$ leptonic decay. A Princeton group completed a run of $\approx \mathbf{4 0 0} \mathrm{hr}$ in an investigation of other aspects of $K_{L}{ }^{0}$ decay. A Cornell-Princeton University experiment is being installed to utilize this beam after termination of the current shutdown.

A significant development during the period was the increasing use of wire-chamber arrays and large spectrometers on-line to computer facilities, either the experimenter's own computer or the Laboratory's PDP-6, or both computers linked. The state-of-readiness of computer facilities will have increasing impact on the efficiency of utilization of the AGS by counter groups.

During fiscal 1969 a change was made in the scheduled mode of operating the two smaller bubble chambers at the AGS. The 30 and 31 -in. bubble chambers now alternate their running periods, with reduced man power and electric power needs as emphasis shifts to other areas of the accelerator. These two chambers are each provided with separated particle beams. $K$ mesons, from zero up to about $900 \mathrm{MeV} / c$ in momentum, may be obtained in the 30 -in. chamber, while the beam to the 31 -in. chamber provides $K$ mesons from about $1.5 \mathrm{BeV} / c$ to $3.0 \mathrm{BeV} / c$, with $\pi$ mesons and antiprotons available to $4.0 \mathrm{BeV} / c$. In the North Experimental Area, the 80-in. bubble 
chamber is still serviced by two separated beams: No. 3, which is effective to $5.0 \mathrm{BeV} / c$ for $K$ mesons or $8.0 \mathrm{BeV} / c$ for antiprotons; and No. 4 , an rf separated beam that can give purified $K$ mesons to $12.8 \mathrm{BeV} / c$, antiprotons to $18 \mathrm{BeV} / c$, and pions or protons to $28 \mathrm{BeV} / c$.

In the first of two running periods, the 30 -in. bubble chamber completed 146,000 additional pictures of $K^{+}$and $\pi^{+}$events in liquid deuterium for Princeton University. In the winter-spring period, 297,000 additional pictures of $K$ - and antiprotons in $\mathrm{H}_{2}$ and $\mathrm{D}_{2}$ and 441,000 additional $K^{+}$ pictures were taken, or a total for the year of 884,000. Experimental groups have come from the Universities of Melbourne, Wisconsin, and Maryland, from Tufts and Brandeis Universities, and from the Illinois Institute of Technology.

The program for the 31-in. chamber has also been divided into two parts. In the summer and fall, eight research groups came from Syracuse, Brandeis, Vanderbilt, Columbia, and CarnegieMellon Universities, and from the Universities of Kansas, Maryland, and Michigan. Beams of $\pi^{-}$, $K^{-}$, and antiprotons were photographed in both liquid $\mathrm{H}_{2}$ and $\mathrm{D}_{2}$ chamber fillings. A total of 1.072 million pictures was accumulated during this period. In the late spring, physicists from four of these universities obtained some 136,000 more exposures, to bring the year's total for this beam and chamber to 1.2 million pictures before the shutdown late in May.

Operation of the 80-in. bubble chamber in the North Experimental Area was confined this year to the use of the $\mathrm{rf}$ separated beam. During the summer and fall, exposures in liquid $\mathrm{H}_{2}$ totaled 940,000. These were for experiments being carried out by the State University of New York at Stony Brook, Yale and Harvard Universities, by the Universities of Wisconsin, Notre Dame, and California at Davis, and by Brookhaven National Laboratory. With a liquid $\mathrm{D}_{2}$ filling in the 80 -in. bubble chamber, about 459,000 pictures were taken for some of the schools mentioned above, as well as for groups from the Universities of Colorado, Hawaii, and California at Los Angeles and from Purdue and Vanderbilt Universities. Just prior to the summer shutdown, about 72,000 more pictures in liquid $\mathrm{H}_{2}$ were taken for the Rutgers University group.

In the Southwest Experimental Area a new facility was constructed to provide a neutrino beam and a charged-particle test beam to the $7-\mathrm{ft}$ cryogenic bubble chamber. This work consisted of refurbishing and supplementing the existing fast ejected beam from B-10, lengthening the concrete shielding cave by $85 \mathrm{ft}$, and relocating 10,000 tons of steel to form the new filter for the neutrino beam. This chamber will be ready for experiments next year.

\section{Experimental Equipment}

During the year there were, on the average, 12 beams in operation or under construction in the East, West, North, and Southwest Experimental Areas. Figure 8 shows the configuration of beams in the Target and East Experimental Buildings in the spring of 1969. The scheduling and use of these areas was such that construction of new beams could proceed without interrupting operations on other beams. Members of the Experimental Division and the Laboratory's Plant Engineering and Planning Division expended 38 man-years of effort on new construction.

A summary of the inventory and usage of major experimental service items is given below.

\begin{tabular}{lccc}
\multicolumn{1}{c}{ Item } & Inventory & & $\begin{array}{c}\text { Usage, } \\
\%\end{array}$ \\
\cline { 1 - 1 } Magnets & & & 85 \\
Power supplies & 216 & & 88 \\
Beam separators & 12 & & 83 \\
Shielding & 90,000 tons & & 85 \\
Experimental power & $45 \mathrm{MVA}$ & 87 \\
Experimental cooling water & $4,050 \mathrm{gal} / \mathrm{min}$ & 94
\end{tabular}

The Power Supply Group has purchased ten $450-\mathrm{kW}$ and five $120-\mathrm{kW}$ dc power supplies. Incorporated in these units are three design changes that will improve reliability and usefulness: a standard, low-level firing circuit that is interchangeable with all power supply sizes, a remotely operated reversing or transfer switch, and a low-voltage air circuit breaker. Work continues on other power supply improvements, the major improvements this year being in current regulation and remote controls that will afford a greater degree of regulation.

About 8 MVA of new experimental power was made available along the east wall of the East Experimental Area via 16 distribution boxes. This power was provided by purchasing a $3750-\mathrm{kVA}$ transformer and by relocating a similar unit from the Cosmotron. The power to these transformers was provided by splicing into an existing feeder cable. An additional 9 MVA of new experimental 
power was made available in the addition to the East Experimental Building via 36 distribution boxes. This power is supplied from the new $M$ substation as part of the AGS conversion project.

The Mechanical Service Group continues its efforts to reduce machine downtime due to experimental magnet failure and to increase the radiation resistance of all magnets in high radiation areas. This program consists of moving the magnet coil jumper hoses from the aperture area to an area under the magnet away from the beam, fitting these jumper hoses with quick disconnects, replacing the cooling-water hoses with flexible copper tubing, providing an external electrical connection for reading coil voltages quickly when coil shorts are suspected, and providing water-sensing mats under the magnets that detect leaks immediately and alarm remotely. In addition, ceramic insulators have been installed on two magnets and are currently being life-tested to evaluate their usefulness in radiation areas.

Four 18C72 magnets have been received and are being assembled in the East Experimental Area. These magnets, after initial testing, will be installed in the slow external beam. Purchase orders have been issued for seven additional 18D72 bending magnets and five N3Q36 quadrupole magnets for use as general beam transport magnets. All existing 18D72 Mark I magnet cores are being modified to Mark II cores to provide interchangeability of coils and thereby reduce the number of spare coils required. Vacuum boxes designed to utilize the full aperture of the 18D72 and 30D72 magnets in the slow external beam have been ordered.

A large magnet-transporting device, capable of lifting, moving, and positioning a 100 -ton spectrometer magnet within the necessary alignment tolerances, has been fabricated and tested. This device is to be used with a $48 \mathrm{D} 48$ spectrometer magnet in the BNL double vee experiment.

The efforts of the Magnet Study Group have been devoted principally to the design and study of cryogenically cooled window-frame dipole magnets for use in general beam transport and in synchrotrons. The group has also routinely maintained and provided precision-calibrated Hall probes for use by various experimental groups. Precision calibration curves of all beam transport magnets are available, the newest type of magnet being the 18Q36 quadrupole which has replaced the rectangular quadrupoles in separated beam No. 3 at the
AGS. Magnetic measurements on the septum and ejector magnets of the slow external beam were made, and corrective measures were devised for reducing the fringing field of the ejector magnet.

An effort of appreciable significance has been that involved in backleg-winding improvements for the accelerator magnets. Because of the difference in magnetic reluctance between the open and closed magnets in the AGS, a difference in magnetic gradient at low fields has always existed between the two types of magnet and has caused a shrinking of the AGS aperture at injection. A series of backleg windings was devised to correct the gradient differences, and the result has been a considerable gain in the intensity of the AGS. These windings also provide $\nu$-tuning at injection and correction of the $8 \theta$ and $9 \theta$ stopbands, as well as any other periodic correction that may be necessary, e.g., $4 \theta$ and $12 \theta$.

A laboratory dipole magnet using cryogenically cooled coils was designed and constructed for use in studies of the highly saturated magnetic circuit. Results of these tests, which are in essential agreement with computer studies, indicate that a field of $41 \mathrm{kG}$ can be achieved with modest sextupole aberrations of $2.5 \%$. Further testing, both electric and magnetic, in liquid $\mathrm{H}_{2}$ of a dipole model with a coil constructed of $99.9999 \%$ pure aluminum indicates that this type of magnet at fields $>40 \mathrm{kG}$ is practical for a synchrotron. Tests in which highpurity aluminum is used as a stabilizer for superconducting materials are continuing.

A 2.5-in.-diam quadrupole magnet using high current density, cryogenically cooled coils was designed, constructed, and tested. The maximum gradient was $20 \mathrm{kG} /$ in., and the harmonic aberrations were as small as those of a standard AGS beam transport quadrupole. The use of high-purity aluminum for beam splitter magnets is also being studied and a model is being constructed. In conjunction with the magnet testing program, properties of conductors at cryogenic temperatures as well as insulation systems for cryogenic coils and cores are being studied.

The Beam Separator Group operated 10 electrostatic separators in four beams and the rf separated beam. The two $\mathrm{rf}$ deflectors were opened during the fall because of organic contamination, and sections of the internal structures were cleaned. In a program initiated last year, all organic seals in the deflector vacuum system were replaced with metallic seals, except those in the pneumatic valves. 
As soon as suitable bellows-sealed valves can be obtained, the changeover to metallic seals will be completed.

In October, as a result of a series of tests begun last year on the test separator, the two separators in the 30-in. bubble chamber beam were removed from the beam and modified with grounded shields. After being returned to operation the separators showed the same improvements demonstrated by the test separator. A new separator control area for the $10^{\circ}$ beam at $\mathrm{G}-10$ was also put into operation during October.

The testing program on the test separator was continued during the year. A series of tests in which all-metal and glass-metal electrode systems were used was conducted on gaps of 5 and $10 \mathrm{~cm}$. The voltage gradients obtained are shown in Table 2. The test separator is limited to $600-\mathrm{kV}$ total gap voltage by the present means of electrode support and the feedthrough insulators. Of the various gases used during the tests, helium appears to give the best over-all operation. Further testing is planned in which improved corona treatment of the glass electrodes and the feedthrough surfaces will be used.

New rf deflectors have been ordered for eventual use as a third deflector and a spare. A test facility is being constructed in order to operate these deflectors at high power levels in the acceptance tests. During the year many modifications were made in the modulator units to improve the accessibility and reliability of the electronic components.

The Cryogenic Group's major efforts have been directed toward the design, fabrication, and operation of liquid hydrogen or liquid deuterium targets. Five targets, each consisting of a Mylar flask surrounded by superinsulation and a vacuu $m$ container, were designed and operated at the AGS. Supporting equipment includes a liquid reservoir, two liquid-level detectors, vapor vent lines, vacuum electronics, and an electrical power control chassis. Cryogenic liquid transfer lines were constructed for a number of experimental setups.

A system was developed to fill liquid $\mathrm{H}_{2}$ targets automatically. The system consists of a pressurizing $\mathrm{H}_{2}$ gas supply, a 175-liter liquid hydrogen Dewar, a vacuum-jacketed transfer line and cold valve, a liquid $\mathrm{H}_{2}$ reservoir and target system, and a main electrical control chassis. This chassis includes all the control relays, air control valves, timer, and other controllers required to sequence the valves and controls that start and stop liquid
Table 2

Gap Voltages per Centimeter With Crossed Magnetic Field

\begin{tabular}{lcccccc}
\hline & \multicolumn{2}{c}{$\begin{array}{c}\text { All-metal } \\
\text { electrode }\end{array}$} & & \multicolumn{2}{c}{$\begin{array}{c}\text { Glass-metal } \\
\text { electrode }\end{array}$} \\
\cline { 2 - 3 } & $5 \mathrm{~cm}$ & $10 \mathrm{~cm}$ & & $5 \mathrm{~cm}$ & $10 \mathrm{~cm}$ \\
\hline Laboratory test results & 80 & 60 & & 120 & 60 \\
Operational results & 70 & 50 & & 100 & 50 \\
\hline
\end{tabular}

$\mathrm{H}_{2}$ transfer to the liquid $\mathrm{H}_{2}$ target system. The front face of the main control panel includes an annunciator panel and a complete piping schematic.

A cryogenic purification system for use with wire chambers was designed. This system will purify $200 \mathrm{ft}^{3} / \mathrm{hr}$ of a $90 \%$ neon- $10 \%$ helium gas mixture. Impurities (water vapor, sulfur dioxide, alcohol, argon, oxygen, and nitrogen) are removed by absorption at ambient temperature, condensing at $\mathrm{CO}_{2}$ temperature, and condensing and absorption at liquid nitrogen temperature. The purifier system also includes a pressure-controlled make-up system for argon, $\mathrm{SO}_{2}$, and alcohol to mix with the 90\% neon-10\% helium gas mixture.

The Beam Instrumentation Group's major efforts have been to improve the instrumentation and operation of beams No. $1 \mathrm{~A}$ and No. 2. The shutter magnet in beam No. 1A was improved and modified for multipulse operation. In beam No. 2, time-of-flight instrumentation for monitoring beam purity was provided. A small, special-purpose digital computer is being used in preliminary design and preparation work to further improve the quality of instrumentation and efficiency of operation in these beams.

The High Energy Electronic Equipment Pool has an inventory of some 2500 components, which they lend, maintain, and repair for use at the Laboratory. The counter experiments at the AGS employed a major portion of this equipment, each piece being used in 2 or 3 experiments during the year. Equipment utilization was about $85 \%$.

A new electronic chassis-washing facility has improved the cleaning techniques and allows a faster turn-around for the equipment. In addition, the original Brookhaven logic (Nanocards) has been retired and is being replaced with $200-\mathrm{MHz}$ logic modules in an effort to provide the best and most reliable equipment for general use. 


\section{AGS CONVERSION}

During fiscal 1969, good progress was made on the AGS conversion project. Almost all building and facility construction work is under contract, and several of the buildings are essentially complete. Preliminary design of the accelerator components has been finished, and detailed design is about $75 \%$ complete. All major machine components have been ordered, and many have been delivered. Some installation of machine components began in April, and major installation will begin in July. By the end of the fiscal year, almost $70 \%$ of the project funds had been committed. Some aspects of the project have not progressed as rapidly as had been hoped; nevertheless, injection of 200 $\mathrm{MeV}$ protons into the AGS is expected to begin in the last quarter of 1971.

Four major new buildings became available for occupancy during the year. The $50,000-\mathrm{ft}^{2}$ addition to the East Experimental Building was completed and turned over to the Department. The new Magnet Power Supply Building and the Rf System Building, both located inside the AGS ring, were essentially completed, and installation of equipment in the buildings was started. The complex of buildings for the $200-\mathrm{MeV}$ linac was also completed, except for the final commissioning of the electrical and mechanical services. Concrete arches were placed in the $\mathrm{AB}$ sector of the ring and an additional $10 \mathrm{ft}$ of sand shielding was successfully added. The 50-MW substation to supply additional power to the north part of the AGS complex was completed. A contract was signed to do the construction work around the AGS ring during the five-month shutdown that started at the end of May. This work, which involves removing shielding from the magnet enclosure tunnel, must be done while the AGS is shut down. It includes construction of the conjunction section to connect the $200-\mathrm{MeV}$ linac to the AB sector of the AGS tunnel. It also includes the placement of arches for shielding over the magnet enclosure for several hundred feet north and south of the Target Building, construction of the conjunction section at $\mathrm{H}-10$ for the new fast external beam to the North Experimental Area, extensive modifications at both ends of the Target Building for additional shielding, and numerous smaller jobs in connection with the magnet enclosure.

Theoretical studies of proton linacs have continued throughout the year, even though the de- tailed design of the linac is nearly complete. The detailed understanding of beam dynamics with space-charge effects has continued to improve, although the mathematical descriptions are still far from perfect. Studies of the electromagnetic field distributions in the linac cavities are being applied to the problem of servo-control of the cavity field amplitude and phase. These studies continue to confirm the belief that proton beams of 100 to $200 \mathrm{~mA}$ can be accelerated with good emittance.

The 750-keV Cockcroft-Walton generator has been received from Switzerland and installation started in the 200-MeV Linac Building. The highgradient column for this generator has been fabricated, following the successful design of the highbrightness source and high-gradient column for the $50-\mathrm{MeV}$ linac. Auxiliary components for the preinjector were designed and fabricated. Design of the beam transport section between the preinjector and the linac was completed, and many of the components are being fabricated. The doubledrift buncher method (two buncher cavities, operating on the fundamental frequency and separated from each other and from the linac by drift spaces) was adopted. This technique gives a beam superior in quality to that obtainable with the conventional system.

The first four linac cavities have been delivered. The drift tubes (single stem) for the first cavity were completed and installed. Preliminary alignment of drift tubes and cavity field adjustment were completed preparatory to installing the cavity in the Linac Building. Work on the multistem drift tubes for the other cavities proceeded and installation was commenced.

Detailed design and procurement of components for the linac vacuum system and cooling system continued. Some difficulty was encountered in the design of the 1500-liter/sec sputter-ion pumps for the cavities. The first model of the pump supplied by the vendor was a complete failure and major redesign was required.

The prototype 5-MW, 200-MHz rf system operated satisfactorily throughout the year and was used extensively to test transmission-line components and other devices. Based on the successful prototype, detailed design and specifications were prepared and contracts placed for all units of the rf systems. The first units of each type were delivered by the end of the year. All components of the 12-in. coaxial transmission-line system were de- 
signed and tested during the year. Some difficulty was experienced with the vacuum window and coupling-loop assembly, but the problem has been solved.

The quadrupole power supplies and pulsing system were redesigned during the year to improve reliability and reduce cost. Procurement of these devices has been initiated. The general control system philosophy for the linac has been decided upon and is being implemented. Studies continued on the use of a small computer for controlling certain functions in the machine. Detailed design and prototypes of many of the printed circuit cards for the control system were completed. The details of the beam transport and analysis system between the linac and the AGS were largely determined, and detailed design has been commenced. Studies of several types of nondestructive beam monitors were initiated during the year.

The modifications to the AGS itself will be carried out in two steps. The first, to be accomplished during the latter half of calendar 1969, will consist of installing the new main magnet power supply and the new $\mathrm{rf}$ power system. Some modification of the existing accelerating cavities is planned, to match them to the new rf system. These modifications will permit operation of the AGS with a duty cycle of up to $50 \%$ with slow beams and/or a higher repetition rate. In 1971, the new accelerating cavities will be installed and the modularization and radiation hardening of the main ring completed.

The complete new main magnet power supply was delivered from West Germany and installation commenced. The designs of the interconnecting bus bars and the control system were completed and procurement is under way. A considerable amount of the copper bus bar is to come from the main magnet coils of the Cosmotron, and that copper has already been removed from the Cosmotron. The $14 \mathrm{rf}$ power amplifiers and their power supplies and drivers were completed and readied for installation in the new building.

A complete set of seven prototype vacuum chambers for the magnet ring was received and installed with excellent results. Fabrication of the main order for 240 chambers has commenced. Design of the vacuum pumping system was completed and delivery of components is under way. Design of the modifications to the main ring magnets was reviewed and adopted. Procurement of components is proceeding.
The design of the prototype for the new accelerating cavities was completed and procurement initiated. These new cavities will have about three times as much accelerating voltage as the old cavities but a smaller frequency swing in order to match the 200-MeV injection. Theoretical and experimental studies of the $\mathrm{rf}$ properties of ferrites have improved the understanding of certain instabilities and have increased confidence in the new cavity design. The ferrite for these cavities has been ordered because of the long delivery time.

Design of the new radiation monitoring system has been completed and components have been procured. The modifications to the main control room have been generally defined and detailed planning is proceeding. Design of the fast beam extraction equipment for the $\mathrm{H}-10$ beam is progressing.

\section{ADVANCED ACCELERATOR DEVELOPMENT}

\section{Superconducting Synchrotron Magnets}

A year ago, the losses in superconducting magnets under pulsed conditions appeared to be so high as to make impractical their use as synchrotron magnets. In addition, discontinuous jumps in magnetic field were observed as the flux penetrated the superconductors. The "flux jumps" were accompanied by generation of heat, which frequently resulted in loss of superconductivity and limited peak magnetic fields to values materially below those expected.

Many measurements of these properties were made at Brookhaven and elsewhere, and much better understanding of their mechanisms was finally gained. They were the subject of much discussion during the 1968 Brookhaven Summer Study on Superconducting Devices and Accelerators. A high point of the Summer Study was the presentation of several proposals by P.F. Smith, of the Rutherford Laboratory, for amelioration of the undesirable effects. Smith predicted that a conductor including a number of fine strands of superconductor could be made essentially stable against flux jumps. He then proposed that twisting, or transposing, of the strands in the composite could result in materially reduced losses under pulsed conditions.

These ideas have been given a number of tests at Brookhaven during the past year, with very encouraging results. Smith's predictions have been borne out qualitatively and quantitatively. Conductors with a hundred or more strands of super- 
conductor have been manufactured by several vendors and, when wound into magnet coils, display unprecedented stability. The strands are of the order of $0.001 \mathrm{in}$. in diameter and are embedded in copper or some other matrix to form a conductor with an over-all diameter of the order of $0.020 \mathrm{in}$. When this conductor is twisted around its own axis and then wound into a magnet coil, the losses under pulsed conditions are indeed materially reduced. Moreover, the loss rate, which formerly rose in proportion to the cube of the magnetic field, now rises only linearly with field.

Tests have also been made on braided conductors, formed out of fine strands of superconductor. The braided conductors also display reduced losses rising linearly with field.

These encouraging results have led to an expanded measurement program of superconductor properties as a function of strand diameter and configuration and matrix material. It is now thought that adequate performance for use in conventional synchrotron magnets will be achieved during the coming year. As a result of this conclusion, work on accelerators of the FFAG type, using $\mathrm{dc}$ superconducting magnets, has been abandoned.

\section{Cryogenic Synchrotron Magnets Using Coils of Pure Aluminum}

It has recently been demonstrated that aluminum of high purity (less than a few parts per hundred thousand of impurity) shows very low resistance at temperatures of $20^{\circ} \mathrm{K}$ or less. At this temperature (approximately the boiling point of liquid hydrogen), the resistance may be lower by a factor of 2000 or more than the resistance at room temperature. Although the losses will be materially higher for an aluminum coil at liquid hydrogen temperatures than for a superconducting coil at liquid helium temperatures, refrigeration to liquid hydrogen temperatures is materially cheaper than refrigeration to the temperatures at which superconductors operate.

These observations have led to the conclusion that pure aluminum coils may be competitive for use in synchrotron magnets, and a program of study of such cryogenic coils has been initiated at Brookhaven. Measurements on magnets with aluminum coils of various purities give results in general agreement with predictions under dc conditions. Measurements on pulsed magnets are in progress to establish optimum coil and magnet configurations.

\section{Use of Iron in High-Field Magnets}

It is hoped that aluminum-cored magnets can be run at fields up to $50,000 \mathrm{G}$ and that superconducting magnets will reach fields $>60,000 \mathrm{G}$. Since iron saturates at fields of about $20,000 \mathrm{G}$, its use in such high-field magnets has been questioned. Theoretical and experimental studies have indicated that it is advantageous to use iron as a shield, even when it is completely saturated. First, it adds 10,000 to $20,000 \mathrm{G}$ to the useful field for the same current in the winding. Second, it serves as a shield to contain the magnetic flux, which otherwise could seriously disturb nearby apparatus. It has been concluded that the iron should surround the coil as closely as possible; hence it appears that the iron shield must also be cooled to the low temperature of the coil. Although this will require the removal of more heat during cool-down, it will make no serious contribution to losses during operation of the magnet.

\section{Superconducting Covities}

Superconducting cavities seem to have major advantages for use in $\mathrm{rf}$ particle separators, since the losses would be reduced to levels at which the separator could be operated continuously instead of in the short pulses to which room-temperature rf separators are limited. This would add greatly to their usefulness in high energy physics.

A program of study of superconducting cavities has been in progress at Brookhaven for about three years. Very low losses are observed in cavities plated with superconducting lead, but the coating is easily damaged by exposure to the atmosphere. Niobium appears to be an even more promising superconductor, and measurements are in progress on cavities of various geometries machined from niobium. Many troublesome effects have been observed, resulting in anomalous high losses and in deterioration with time. It is hoped that these difficulties will be less serious in niobium cavities made by electroforming and then given a treatment under vacuum at high temperature. Such cavities are now in preparation.

\section{RESEARCH}

Analysis was completed of a Cosmotron experiment that measured the Wolfenstein triple scattering parameters $R, D$, and $A^{\prime}$ at $1.9 \mathrm{BeV}$ for $p+p$ scattering at $90^{\circ}$ in the center-of-mass system. It 


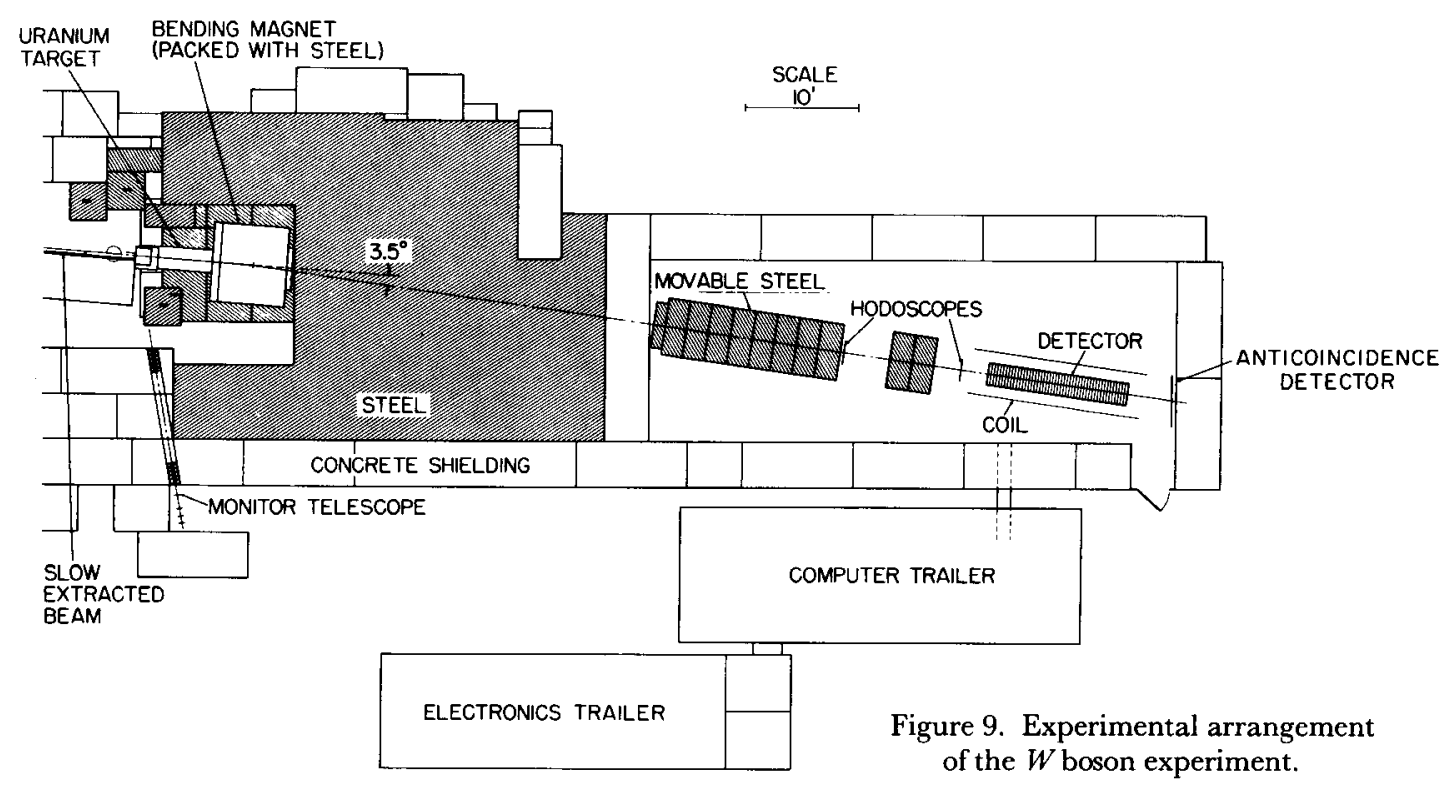

was found that $R=0.11 \pm 0.16, A^{\prime}=-0.54 \pm 0.16$, and $D=0.91 \pm 0.21$ when these parameters were defined in the center-of-mass system. It was concluded that neither a single vector meson exchange nor a single pseudoscaler meson exchange can account for the data. Spin effects are found to remain an important part of the nucleon-nucleon interaction at four-momentum transfer $-t=1.8(\mathrm{BeV} / c)^{2}$.

A search for the $W$ boson (see Figure 9), presumed to mediate weak interactions, was conducted by measuring the polarization and flux of high energy muons produced through the interaction of $28-\mathrm{BeV}$ protons with uranium. Protons from the slow external beam of the AGS were focused on a target constructed of sections that could be separated so as to vary the effective target density. Forward muons passed through a magnet which directed the positive muons through a thick steel shield into a range-polarization detector consisting of twenty-three 4 -in. aluminum slabs separated by scintillation counters. The stopped muons precessed in a magnetic field imposed on the detector. The muon range, the decay time, and the direction of the decay electron were recorded. The variation of the front-back ratio with time determined the muon polarization independent of many biases. The muon flux from the decay of mesons varies inversely with the target density, and the polarization direction is opposite to the beam direction: A flux from $W$-boson decays should be independent of the density, and the polarization would be aligned with the beam. A preliminary analysis, based on an extrapolation to infinite target density, suggests that the cross section for the production of $W$ bosons by protonnucleon interactions is $<5 \times 10^{-36} \mathrm{~cm}^{2}$ if the $W$ boson decays to a muon about $25 \%$ of the time.

A spark-chamber spectrograph in an all-zenith mount is being constructed. The instrument is intended for use in the study of the zenith angle distribution of cosmic-ray muons in the range 0.5 to $1.5 \mathrm{TeV} / c$. The spectrograph has an aperture of $\approx 200 \mathrm{~cm}^{2}$ sr and a field integral $\geqslant 40 \mathrm{kG}-\mathrm{m}$ and is about $8 \mathrm{~m}$ long. The spark chambers, three on each side of the magnet, are photographed by two cameras in $90^{\circ}$ stereo. A counter hodoscope is used to reject low energy events with cutoff at $15 \mathrm{BeV} / c$ and about $50 \%$ efficiency at $45 \mathrm{BeV} / c$, which keeps the rate of vertical events below $2000 /$ day. This rate of pictures can be easily handled by the optical scanner coupled to a GDC 924 computer that was used to measure a previous muon experiment.

A test of $C P$ violation was made for the neutral $K$-meson (kaon) decay into three pions. If the longlived and short-lived neutral kaons, $K_{L}$ and $K_{S}$, have a common decay mode (in the same quantum state), then the decay into that mode violates the principle of $C P$ invariance. The long-lived neutral kaon is known to decay into three pions. Therefore the detection of the decay of the shortlived kaon,

$$
\kappa_{S} \rightarrow \pi^{+} \pi^{-} \pi^{0},
$$

and its interference with the decay products from $K_{L}$ decay would demonstrate $C P$ violation. In col- 
laboration with groups at Yale and the University of Massachusetts, the initial phase of such an experiment has been completed. A coherent sample of $K_{L}$ and $K_{S}$ was produced in the charge exchange reaction $K^{-}+p \rightarrow \mathcal{N}+K^{0}$ in the 30 -in. bubble chamber at the AGS. From 80,000 measurements (40,000 at BNL), a sample of 30 three-pion decays was obtained within the first six $K_{S}$ lifetimes. The data reveal no $K_{S}-K_{L}$ interference effects and show no evidence for the three-pion decay of $K_{\boldsymbol{S}}$. No large $C P$ violation is found.

In the same bubble-chamber exposure, the ratio of vector to axial vector coupling constants, $G_{v} / G_{a}$, in the decay

$$
\Sigma-\rightarrow e^{-\nu \mathcal{N}}
$$

was measured by correlating the direction of electron emission with the spin of the $\Sigma$ hyperon. The ratio $G_{v} / G_{a}$ is important to test theories of weak interactions, especially those that relate the strangeness changing and strangeness nonchanging decays. With use of the Yale PEPR film reader and BNL digitizers, 90,000 events were measured (30,000 at BNL). This large number of events will be used to obtain precise measurement of the $\Sigma-$ lifetime and the asymmetry parameters in the decay mode

$$
\Sigma-\rightarrow \pi^{-} \mathcal{N}(\gamma)
$$

The exposure is also being used for strong interaction experiments. The reactions $K^{-}+p \rightarrow \Sigma-+\pi^{+}$, $K^{-}+p \rightarrow \Lambda^{0}+\pi^{0}$, and $K^{-}+p \rightarrow \Sigma^{0}+\pi^{0}$ are being studied. This year 80,000 events with topologies corresponding to these reactions have been measured. 


\section{Instrumentation}

The Instrumentation Division provides a number of specialized services to other Laboratory departments and, in addition, engages in a modest research program. Services include the design and construction of research and test instruments and data-gathering devices, consultation on instrumentation problems, and the design and construction of computer-experiment interface systems, including development of the associated software. The Division also provides calibration and repair services and operates a vacuum technology shop, a glassblowing shop, and an electron microscopy facility.

The research effort is devoted primarily to the development of radiation detectors for both high and low energy applications and the design of special circuitry for fuller exploitation of the detectors' resolution and speed capabilities.

Some of the results of the research effort are discussed below, and some of the devices and systems built during the past year are described.

\section{SEMICONDUCTOR DETECTORS AND ASSOCIATED CIRCUITS}

Work on semiconductor detector systems has been directed toward improving the resolution of large-volume lithium-drifted germanium detectors and identifying and reducing the importance of noise sources in the measurement of $x$ rays with small silicon detectors.

Several 30-cc coaxial $\mathrm{Ge}(\mathrm{Li})$ detectors have been fabricated which have yielded a measured line width for the $\gamma$ rays from $\mathrm{Co}^{60}$ of $<2 \mathrm{keV}$ full width at half maximum. This has been accomplished by extending the lithium drift compensation to reduce the diameter of the uncompensated core, which reduces the device capacitance, and by using great care with the electronics.

Several small commercial silicon $\mathbf{x}$-ray detectors have been mounted in the Division's cryostats with selected preamplifier components (field-effect transistors and high-value feedback resistors), and line widths of about $250 \mathrm{eV}$ have been achieved. On the other hand, efforts to make $\mathrm{x}$-ray detectors from thin slices of planar germanium diodes, ir- radiated from the side, have so far been largely unsuccessful because of difficulties with an excessive dead layer which is opaque to $\mathrm{x}$ rays below 14 $\mathrm{keV}$.

The best feedback resistors selected to date are still responsible for a significant part of the electronic noise in the $\mathrm{x}$-ray measuring systems, and two parallel efforts are proceeding to eliminate this noise source. An attempt is being made to make high-value resistors $(\approx 5000 \mathrm{M} \Omega$ ) from single crystals of high-resistivity GaAs. Work on surface treatments is proceeding in order to eliminate a frequency-dependent change in resistance which leads to increased noise in the system pass band. The second approach, which is potentially promising, is to eliminate the feedback resistor entirely and to remove the detector charge by periodically pulsing the field-effect transistor (FET) gate into forward conduction with very short pulses whose amplitudes are controlled by negative feedback. This has the advantage over alternative photoelectric techniques of eliminating the need to connect additional components to the amplifier input and of avoiding additional shot effect noise.

Two rather different high counting rate problems have been worked on during the past year. In order to perform a Mössbauer resonant absorption experiment in which a weak, interesting $\gamma$ ray at $122 \mathrm{keV}$ is accompanied by a much more intense $\gamma$ ray at $136 \mathrm{keV}$, it was necessary to maintain high resolution at counting rates up to $10^{5} / \mathrm{sec}$. A system featuring Gaussian pulse shaping and gated integration was used.

The other high counting rate problem involved a pulsed accelerator that produces a very intense $\gamma$-ray flash followed by $\gamma$ rays at a moderate rate which must be measured during the ensuing few milliseconds. Conventional preamplifiers were driven into heavy saturation from which they did not recover for as much as $100 \mathrm{msec}$. A fast recovery modification was added to one of the Division's standard preamplifiers. It employs a second, normally cut-off FET which is pulsed on by an overload-detecting discriminator. This FET quickly removes the excess charge (in about $10 \mu \mathrm{sec}$ ) while increasing the noise of the preamplifier by only the 
small amount associated with the added $2 \mathrm{pF}$ of capacitance.

\section{SPARK AND PROPORTIONAL CHAMBERS}

Work on multiwire digital gas-discharge devices has proceeded in a continuing effort to improve time resolution, spatial resolution, and multiple particle efficiency and to simplify readout systems.

A proportional mode device was assembled which was capable of 10 -nsec time resolution. However, its practical implementation on a large scale will depend upon the availability of low-cost, high-speed, sensitive amplifiers.

Another device, also operating in the proportional mode, makes it possible to digitize the position of the intersection of a particle track with a plane and to characterize it with two coordinates (or more, if multiparticle redundancy is required), with all the coordinates referred to a single plane, regardless of the inclination of the particle track. An initial proportional discharge is produced in a first wire plane. Then some of the electrons from the first discharge are accelerated normally to subsequent planes at progressively higher voltages. Each subsequent plane determines a position coordinate. If desired, an adjustable time delay can be introduced by varying the potential across a delay gap to synchronize the measurement with other event-selecting devices.

A promising variation on this multiplane transfer chamber, capable of combining the fast timing of proportional chambers with the substantially simpler readout systems of spark chambers, has been shown to be feasible. A proportional gap is followed by a delay gap (to compensate for triggering delays without extending the memory time) and then by one or more pulsed spark gaps which can be read out either magnetostrictively or with magnetic cores.

The momentum resolution of high energy magnetic spectrometers is determined by the size of the magnet and the spatial resolution of the detectors marking the particle trajectories. Since the magnets used are already large and costly, economic considerations suggest that it is worth while to improve the spatial resolution of the detectors as much as possible. Already $0.07-\mathrm{mm}$ spatial resolution has been achieved with a magnetostrictivereadout digitized spark chamber in the detection of highly ionizing low energy protons ( $15 \mathrm{MeV})$, and an attempt is being made to determine whether similar resolution can be obtained with minimum ionizing particles.

In conventional spark chambers, the sparking efficiency decreases markedly as the number of simultaneous sparks is increased. By building the chamber like a low-impedance terminated transmission line and by selecting a gas mixture to yield relatively high impedance sparks, it is possible to maintain efficiency even with many sparks. A shower-detecting chamber of this type was built and found to have a sparking efficiency $>90 \%$ with over 100 simultaneous sparks.

\section{COMPUTER SYSTEMS}

A multiple-detector x-ray diffraction spectrometer operating in association with a Varian $620 \mathrm{I}$ computer is nearing completion. The system employs 128 silicon-junction $\mathrm{x}$-ray detectors mounted on a computer-controlled movable semicircular arc. Each detector has its own preamplifier, amplifier, and differential discriminator. The discriminator outputs go to a diode encoding matrix which generates a 7-bit word characterizing the detector number. These words are entered into a fast (100-nsec) 6-word derandomizing buffer from which they are read periodically and used to address the computer memory via a dual access controller. The contents of the addressed locations are incremented in an external fast adder to avoid overburdening the central processor of the computer at the expected high data rates. The system can record a maximum of $385,000 \mathrm{x}$ rays $/ \mathrm{sec}$ from the detector array. At $100,000 \times$ rays $/ \mathrm{sec}$, the counting loss is only $1 \%$. The operating system permits manual control of the detector array, or control via a stored program. It is anticipated that, in some applications, program modifications will be made automatically, based on data already accumulated, in order to optimize data collection.

The multiple spectrometer control system at the High Flux Beam Research Reactor makes use of an SDS 920 computer to control the positioning and data collection from nine neutron diffraction spectrometers. In addition, it performs some of the data reduction, and in some instances alters the control program in response to the recorded data. Experience has shown the two input-output and control teletypewriters to be a serious bottleneck in the system because of the large number of timesharing users. The system has therefore been expanded with the addition of a telecommunication 
system under the control of a Varian 620 I computer. This permits input-output and control to be exercised by any of 16 remotely located teletypewriters without interference from the others.

A photographic film densitometer under the control of a CDC 924 computer has been constructed for the automatic scanning and measuring of $x$-ray diffraction photographs. It uses a highquality cathode ray tube to achieve a spatial resolution of $50 \mu \mathrm{m}$ in a $125-\mathrm{mm}$ square, corresponding to $6.25 \times 10^{6}$ resolved spots. Optical density is measured at computer-selected locations by using a photomultiplier and logarithmic amplifier in each of two channels, one observing the light spot through the film and the other observing the unattenuated light from the cathod ray tube. The difference of the two signals is a linear function of the density of the film and is digitized with a resolution of 0.01 optical density in the range from 0 to 2 , and the result is communicated to the computer. In addition, certain point, line, and scan-generating functions are available to the operator to aid in positioning the film and displaying graphic, as well as density, information on a "slave" oscilloscope for purposes of communication with and direction of the computer program.

Some of the small computers associated with control of experiments are equipped with only rudimentary input-output hardware, which often makes the assembly of new programs a tedious and time-consuming process. To alleviate this problem, a generalized assembler program has been written which, with some modifications, can be adapted to a number of small computers. This permits programs for small computers to be assembled rapidly on the CDC 6600 computer at the Central Scientific Computing Facility.

\section{OTHER INSTRUMENTS}

To indicate the variety of activity in the Instrumentation Division, some of the other instruments and devices worked on during the past year are briefly described below.

A fast function generator capable of generating the value of a fourth-order polynomial as a function of a rapidly varying independent voltage variable has been built. It uses two multiplier circuits based on junction field-effect transistors and several fast operational amplifiers to yield an output accurate to within $0.5 \%$ in as few as 200 nsec.
A multichannel pulse-height analyzer which records individual digitized pulse amplitudes on punched paper tape is now operating at the bottom of a gold mine in South Dakota in a neutrino detection experiment. In addition to the pulse amplitude and time of day, the record of each signal indicates whether or not the signal occurred in coincidence with one from an anticoincidence guard counter, whether the signal had an undershoot (valid detector signals are unipolar, whereas the majority of spurious signals appear as damped oscillations), and which of four detectors produced the signal. Each detected signal is followed by a pair of calibration pulses bracketing the energy range of interest. The calibration pulses are digitized and recorded on the tape, with suitable identifying bits, so that a continuous record of the system performance is maintained. This is important in very low counting rate experiments which must run for many months to yield adequate statistical accuracy. The tape perforator is solenoid-operated because motor-driven punches tend to wear out in the course of one or two measurements.

A plutonium assay device, based on the detection of spontaneous fission, has been built. It uses a set of four $\mathrm{BF}_{3}$ proportional counters connected in parallel in a moderator block and characterizes an event as fission if two neutrons are detected within the moderator lifetime of about $100 \mu \mathrm{sec}$. A precise mechanism is included for evaluating the accidental coincidence rate because, with so long a resolving time, this rate is comparable with the detected fission rate with even moderate source strengths.

Circuitry for a parity nonconservation experiment involving the measurement of the right-left asymmetry in the circular polarization of $\gamma$ rays is nearing completion. Because the effect may be as small as 1 part in $10^{7}$, very intense sources must be used to complete the experiment in reasonable time. Direct counting is out of the question because of the high rates and unreasonable timing requirements. Instead, a synchronous detection scheme is employed which searches for a correlation between the fluctuations in the current from a scintillation counter and the reversals of a magnetic field. Because the expected signal may be very much smaller than the noise due to statistical fluctuations in the detector current, dynamic range considerations have led to the use of a double synchronous detection scheme. The first detection employs conventional analogue tech- 
niques to narrow the noise bandwidth. The second detection is digital in order to avoid the use of unreasonably long filter time constants.

A sensitive bridge circuit has been built for the measurement of fine structure in the volt-ampere characteristic of superconducting tunneling junctions. Conductivity and its derivative are measured over a range of bias voltages with excitation voltages $<100 \mu \mathrm{V}$. The derivative of conductivity is determined by measuring the amplitude of the sum frequency beat note produced when the bridge is excited by two signals with a digitally fixed frequency ratio of 3 to 2 .

A circuit has been added to a constant velocity Mössbauer drive system which provides for a graceful flyback between well-determined position limits, with accelerations programmed to minimize shock-excited ringing.

Electronics for a new system for scanning bubble-chamber photographs called SICKLE is being tested. The system searches for the best fit between a set of arcs (of continuously varying curvature) and film tracks from a selected vertex, and communicates digitized angles and a measure of the fit to a digital computer.

An automatic control and recording system has been added to a dual-beam vacuum ultraviolet spectrophotometer. The system adjusts the gain of a photomultiplier to accommodate to the wide range of variation of the source illumination spectrum, alternately digitizes the reference and sample beams, and, when enough signal information has been integrated, records the results on punched tape and signals the monochromator to advance. In addition, analogue circuitry computes the logarithm of the ratio of the sample and reference beams and plots optical density on a chart recorder.

An optically coupled beam pulser has been added to a Van de Graaff generator. Separate start and stop optical channels are employed to permit an unlimited range of duty cycles with the ambient light independence and stability of an accoupled system.

\section{METEOROLOGY}

\section{Wind and Turbulence}

Measurements of the wind and temperature structure in the same forested area that is being used for particulate studies have been expanded. The data obtained from the series of earlier, short-

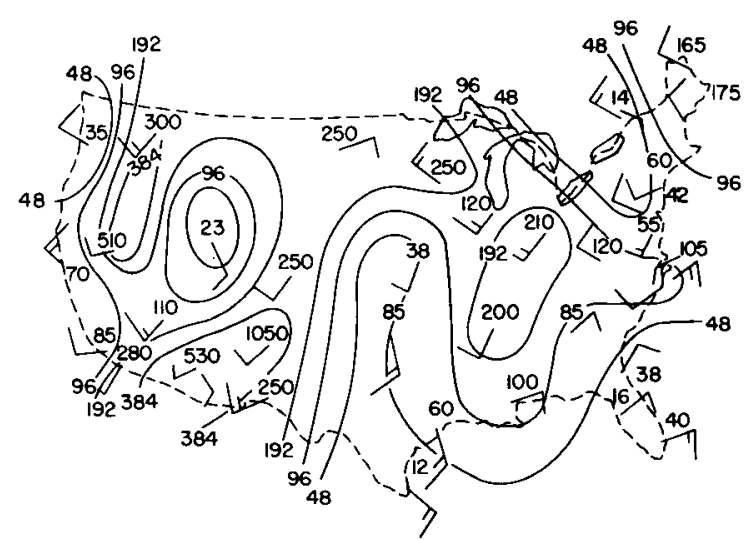

Figure 1. Map showing the recurrence intervals (in months) that may be expected between periods when the wind direction remains almost invariant (steadiness $=1.0$ ) for four consecutive days. The shortest interval $(12 \mathrm{mo})$ is observed in southern Texas, and the longest in Albuquerque (1050 mo). A 12-mo interval may seem quite long to the casual observer, but a steadiness value of $1.0 \mathrm{implies}$ almost no change at all in wind direction.

term experiments have been classified according to larger-scale meteorological parameters, and more recently the 25 anemometers and 10 temperature sensors have been connected to the central data acquisition system for routine recording. This new series of continuous data, correlated with the records from the $420-\mathrm{ft}$ meteorological tower, is processed to give a complete forest climatology.

On a much broader scale, recurrence interval maps of wind steadiness during periods of 2,4 , and 8 days have been completed. These maps show consistent and logical patterns of wind steadiness over the United States and should be valuable in evaluating reactor siting problems. Figure 1, for example, shows clearly that the coastal areas of the country and the lower Mississippi Valley have a high probability of winds remaining relatively fixed in direction for extended periods. Conversely, the interior areas show wide variability of wind direction. These results agree with what would be expected from the study of the major synoptic meteorological features, such as the position of the polar front and the stationary high-pressure systems, but they have the advantage of providing quantitative estimates of the frequency of the very steady wind conditions and the meteorological conditions accompanying them.

\section{Dispersion and Deposition}

Experimental studies of particulate dispersion and deposition from low-level, continuous, cross- 
wind line sources were continued. Seventeen tests were made using pollens emitted naturally from 80-meter-long lines of potted plants as tracers. Five other tests were conducted in which particles were sprayed from a vehicle moving slowly back and forth along a path upwind of the sampling grid. Preliminary results from 1967 experiments indicate that the dispersion of these particles is close to that predicted by theory.

Studies of particulate dispersion into, through, and out of a forested region were also continued. Fifteen point source releases were made, most of them in and above the forest to study transfer through the forest crown. Five additional runs were made from an area source of pollen upwind of the forest. The effect of particle characteristics on forest filtration efficiency was studied by releasing particles of several sizes and shapes simultaneously. Physical improvements were made in the samplers at additional levels above the forest to allow more detailed measurements of tracer concentration at these levels.

An important part of the study of dispersion and deposition has been the perfection of equipment for collecting airborne particles, and an extensive study of the relation between wind speed and the effiiciency of filter particle collectors has been conducted. The atmosphere is commonly sampled for gases and particulates by using filter samplers and other devices that draw air into an entrance passage before removal or measurement of selected constituents. Such samplers can seldom be operated isokinetically in the free atmosphere, and the larger particles often are sampled inaccurately because they fail to follow faithfully the air flow into the entrance. Collection efficiencies of typical filter holders for particles $0.7,6.0$, and 20.0 microns in diameter were tested at various wind speeds, angles of attack, and flow rates in the wind tunnel. The efficiency varied from $<1 \%$ at the highest wind speed and lowest flow rate to $>100 \%$ at forward angles where impaction aided suction. Efficiency was generally lowest when the filter holder entrance was at right angles to the air stream, increased as the entrance was tilted forward toward the wind, and also increased, but more slowly, as the entrance was tilted backward and away from the wind. As would be anticipated, the larger particles proved to be difficult to collect efficiently.

\section{Plume Rise Study}

A thorough study of the rise of buoyant plumes in the atmosphere has been completed, resulting in a set of simple equations for the general approximations of plume behavior. Perhaps the most significant result of this study is an increased awareness of the importance of certain parameters, such as the variation of turbulence intensity with height, about which far too little is known, and of the paucity of plume data acceptable for determining the accuracy.

\section{Lidar System}

The Lidar (light detection and ranging) system developed for the remote quantitative measurement of oil-fog plumes in the atmosphere has been calibrated in the Meteorology Group's calibration room and is now ready for field use.

\section{Atmospheric Diagnostics Program}

The meteorological portion of this program has been concentrated in two areas, the development and perfection of sampling equipment and the modeling of multiple source emissions. An important result of the work is the development of a simple, efficient, airborne sampling system which permits the simultaneous collection of $\mathrm{SO}_{2}$ and airborne sulfates with samples of the tracer gas, $\mathrm{SF}_{6}$. The simultaneous use of these two collection techniques should be valuable in comparing the behavior of the sulfur compounds that undergo physical and chemical changes in the atmosphere with a conservative tracer that does not.

A unique environmental test chamber was installed in the Meteorology Building. This chamber contains $3400 \mathrm{ft}^{3}$ of air, and in it a wide range of meteorological conditions can be simulated. Temperature, relative humidity, and ultraviolet radiation can be controlled over the ranges normally found in the atmosphere, and fog and rain can be simulated. Conditions can be changed drastically within an hour's time.

Although designed primarily for the study of air pollution and the performance of sampling equipment, the chamber can be used for many other experiments. Entire instrument assemblies, up to and including complete monitoring stations, could be subjected to the full range of weather changes within a day or two, and it is possible for several people to work normally or exercise in the chamber under controlled meteorological conditions. 


\section{Chemistry}

Studies carried on in the Chemistry Department, although they may be described generally as centering on the structural and dynamic properties of matter, encompass a range in this area from the behavior of nucleons and particles in nuclei to that of atoms and molecules in complex and condensed molecular systems. Thus, in the sections below, results are summarized from a number of different and related studies, many of which could not have been done without the special large facilities available at BNL. This work has been carried out by staff members of the Department, including a number of American and foreign postdoctoral Research Associates, and by visitors and colleagues from other laboratories and universities.

\section{HIGH ENERGY NUCLEAR REACTIONS}

Experimental investigations of the reactions of high energy protons with complex nuclei have continued at the Alternating Gradient Synchrotron (AGS). While for some time the emphasis in this work has been on the measurement and interpretation of cross sections, new experiments to measure energy spectra of products (particularly those having low masses) are under way. Detailed analysis of the backlog of data acquired at the Cosmotron is progressing in parallel. Theoretical studies are carried on to supply a framework for the interpretation of the experimental results.

\section{Product Yield Distributions}

The combination of chemical separation and mass separation was used to measure cross sections for forming rare-earth nuclides from the interaction of $28-\mathrm{BeV}$ protons with heavy-element targets. The study of uranium $\left(\mathrm{U}^{238}\right)$ as a target has been essentially completed, and some data have been acquired from thorium and tantalum targets. Charge dispersion curves for products having mass $\approx 146$ are shown in Figure 1 . A striking feature of the results is the general similarity of the yield patterns of neutron-deficient products $(\mathcal{N} / Z$ $<1.4)$ from all three targets, concomitant with the progressive decrease of neutron-excessive products with decreasing target atomic number. From tanta- lum practically no neutron-excessive nuclides are produced. These results confirm the conclusion of earlier studies that at least two processes are important. The neutron-excessive products appear to be formed by fission, when it can occur at moderately low excitation energies; neutron-deficient products have characteristics of processes with high excitation energy.

Production cross sections of rare-gas $(\mathrm{He}, \mathrm{Ne}$, $\mathrm{Ar}, \mathrm{Kr}, \mathrm{Xe}$ ) isotopes from a variety of targets with 3 and 28-BeV protons have been measured in collaboration with scientists at the State University of New York at Stony Brook. Both mass spectrometric and radiochemical techniques were used in these measurements. The results are being analyzed to give information on the charge distributions and yield-vs-mass curves for the various processes (e.g., spallation, fission, or fragmentation) involved. By combining the results of the rare-gas and rare-earth measurements with results obtained previously at the AGS, a clearer picture of yield-vs-mass curves (shown in Figure 2) for the $28-\mathrm{BeV}$ proton irradiation of heavy-element tar-

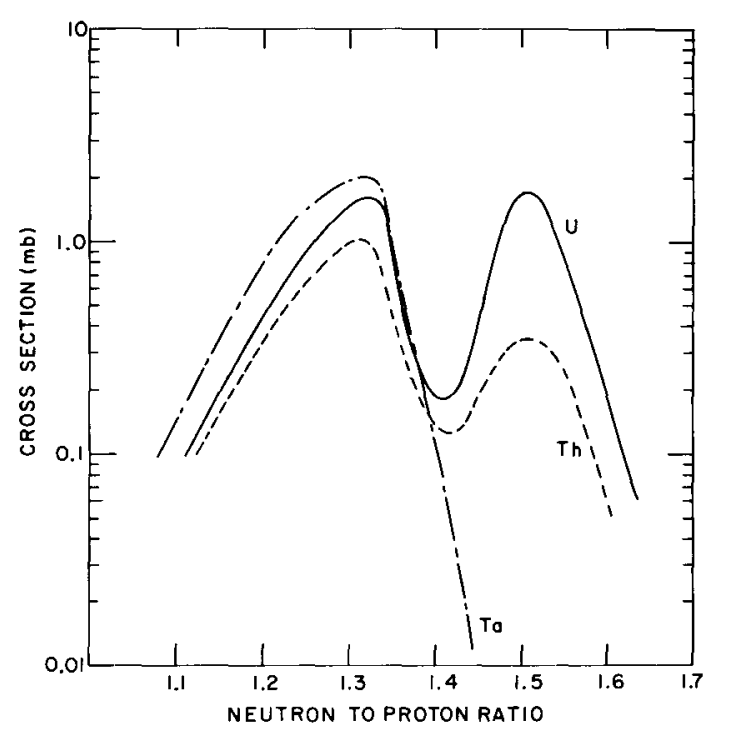

Figure 1. Charge dispersion curves for products having mass $\approx 146$ from the irradiation of uranium, thorium, and tantalum with $28-\mathrm{BeV}$ protons. 


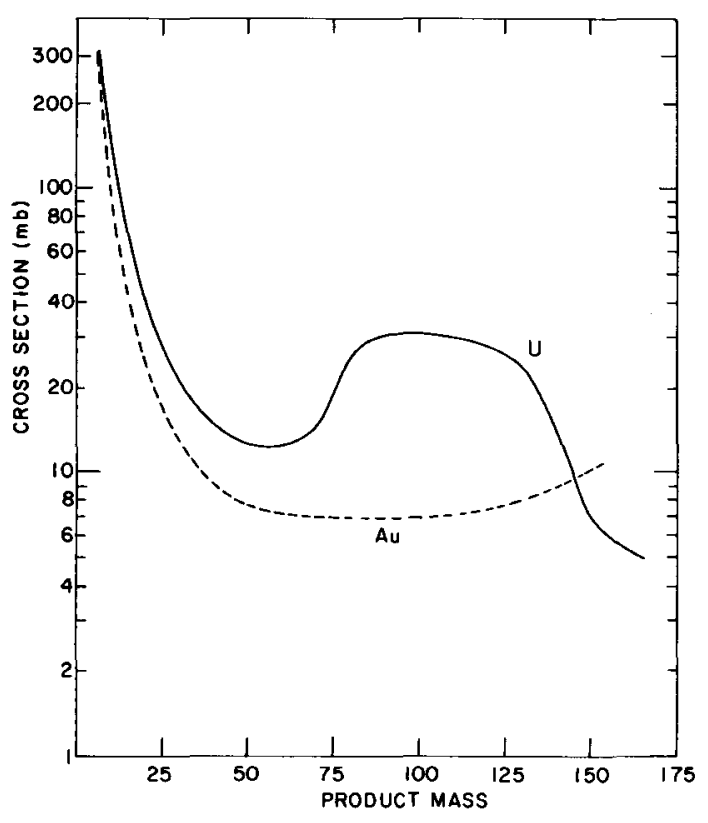

Figure 2. Yield-vs-mass curves for uranium and gold irradiated with $28-\mathrm{BeV}$ protons at the AGS. The curve labeled $\mathrm{Au}$ is based on data for both gold and lead targets, the differences between the two being small in the mass region shown.

gets emerges. The prominent fission-product peak in the uranium curve is not seen in the curve for gold. Yields from uranium fall below those from gold in the rare-earth region at the right-hand side of the figure.

Work is proceeding on the determination of reaction cross sections with high-resolution $\mathrm{Ge}(\mathrm{Li})$ detectors. Because chemical separations are not necessary, data are accumulated rapidly. Preliminary experiments with copper targets at the CERN proton synchrotron have shown that at least 16 individual products may be identified from their $\gamma$-ray energies and half-lives in the $\gamma$ spectra of untreated irradiated targets.

\section{Momentum Spectra and Angular Distributions}

Analysis has been completed of measurements of the angular distribution and energy spectra of $\mathrm{Tb}^{149}$ produced from gold by $2.2-\mathrm{BeV}$ protons. The data are consistent with a two-step mechanism, a fast intranuclear cascade-excitation followed by a de-excitation sequence. The momentum calculated to be imparted to the $\mathrm{Tb}^{149}$ by the second step would indicate that particles even heavier than $\mathrm{Li}^{7}$ are emitted in the evaporative de-excitation. With the assumption that this re- action is typical of "complex spallation" and with data from other similar reactions, a scheme has been developed which allows prediction of momenta imparted to products of such reactions from many different targets. From comparison with a similar study of fission described last year, it is evident that the mean momentum of a product formed by fission should be significantly greater than that resulting from a spallation mechanism. An application of these spallation-fission systematics is shown in Figure 3. Mean momenta measured for iodine isotopes produced from uranium in irradiations with $18-\mathrm{BeV}$ protons at the CERN laboratory decrease from values corresponding to a fission mechanism at the higher masses to values approximating those of spallation for isotopes of lower mass.

An improved targeting facility has been installed at the AGS to allow collection in a stack of thin plastic foils of the nuclear fragments ejected from thin uranium targets irradiated with $28-\mathrm{BeV}$ protons. Subsequent radiochemical analysis of the separate collectors has yielded energy spectra for a number of isotopes between $\mathrm{Na}^{22}$ and $\mathrm{Sr}^{91}$. These data will serve to complement results obtained with semiconductor detectors and with plastic-foil track detectors. The latter are being developed as particle identifiers, since it has been shown recently that energetic particles leave damage tracks which can be etched chemically at rates dependent on the particle energy and atomic number. Calibrations are being carried out with various heavy ions, from carbon to chlorine, obtained at the Yale Heavy Ion Linear Accelerator and the Rochester Tandem Van de Graaff facility.

\section{Simple Reactions}

Two studies of simple nuclear reactions in light nuclei have been completed. Cross sections for the $\mathrm{Mg}^{25}(p, 2 p) \mathrm{Na}^{24}$ reaction and effective ranges of the $\mathrm{Na}^{24}$ product nuclei were measured at eight energies from 0.43 to $2.7 \mathrm{BeV}$. All the results were found to be consistent with the "clean knockout" mechanism - knockout of a proton in the target nucleus by the incident proton.

The cross section for the $\mathrm{He}^{4}(p, 2 p) \mathrm{H}^{3}$ reaction was measured to be $30.2 \pm 3.6 \mathrm{mb}$ at $2.2 \mathrm{BeV}$. This result is of astrophysical interest in that, together with the $\mathrm{He}^{4}(p, p n) \mathrm{He}^{3}$ cross sections, it may allow determination of the amount of matter traversed by cosmic-ray $\mathrm{He}^{4}$ from a measurement of the $\mathrm{He}^{3} / \mathrm{He}^{4}$ ratio in the high energy cosmic rays. 


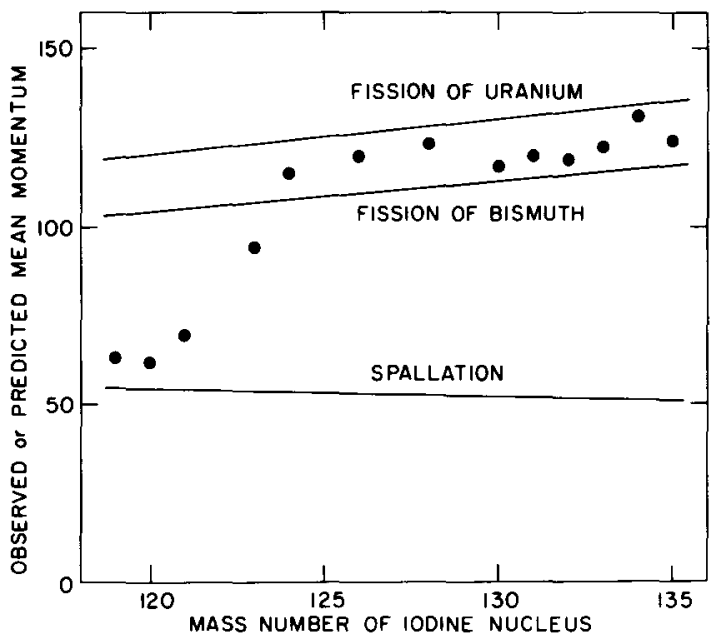

Figure 3. Dependence of experimental and theoretical mean momenta of iodine isotopes on product mass. The points were obtained from the irradiation of uranium with $18-\mathrm{BeV}$ protons. The upper two curves are the theoretical results from a liquid drop model for the fission of uranium and bismuth nuclei. The lower curve is based on spallation systematics (see text).

\section{Theoretical Studies}

The Monte Carlo calculations of intranuclear cascades induced in complex nuclei by nucleons of energy up to $\approx 380 \mathrm{MeV}$ were extended to include a velocity-dependent potential. This was done to explore the possibility of improvement of the nuclear model in which classical refraction and reflection are used. Only slightly better agreement was obtained between calculated and experimental results than was found previously with a velocity-independent potential. Isobar model calculations have been performed for reactions with low energy (up to $180 \mathrm{MeV}$ ) incident pions.

To complete the calculation of the course of high energy nuclear reactions, the de-excitation of highly excited nuclei, which often have large angular momenta, must be considered. This has now been done with combinatorial calculations of nuclear level densities, based on a realistic shell model that includes pairing effects. Extensive comparisons with experiment show this calculation to be remarkably accurate for masses $>50$. In contrast, the algebraic expression for level densities most commonly used in previous calculations often gives highly erroneous predictions. Calculations of the de-excitation of $\mathrm{Br}^{75}$ are now being made for comparison with data recently obtained at the
Yale Heavy Ion Linear Accelerator by Columbia University scientists. These experiments definitely show effects of high angular momenta and probably offer the best opportunity available to test calculations of densities of levels having high angular momenta.

\section{NUCLEAR SPECTROSCOPY}

\section{Direct Measurement of the Decay Energies of the Electron-Capture Nuclides $\left.\right|^{125}$ and Cd 109}

These energies, previously known only indirectly from measured $L / K$ capture ratios and the theoretical variation of the $L / K$ capture ratio with decay energy, were measured directly by way of the end-point energies of the emitted bremsstrahlung spectra. The energy differences between ground states of the parent and daughter atoms were found to be

$$
\mathrm{I}^{125} \rightarrow \mathrm{Te}^{125}: \quad 177 \pm 2 \mathrm{keV}
$$

(compared with the indirect value of $148.9 \mathrm{keV}$ )

$$
\mathrm{Cd}^{109} \rightarrow \mathrm{Ag}^{109}: \quad 182 \pm 3 \mathrm{keV}
$$

(compared with indirect values of 159 to $184 \mathrm{keV}$ ).

\section{The Energy Level Diagram of Os 186}

Ir ${ }^{186}$, which decays to levels in Os ${ }^{186}$ with a 15.8-hr half-life, is one of a class of especially interesting nuclides lying in a region of the nuclide chart in which the nuclear shapes are changing from spherical to spheroidal. An extensive, but incomplete, analysis of the complicated level scheme of $\mathrm{Os}^{186}$ was made some years ago with data from high-resolution conversion electron spectrometry obtained both at BNL and at Oak Ridge National Laboratory (ORNL). This work has now been supplemented by analysis of high-resolution measurements of the $\gamma$-ray energies and intensities performed at Clark and Texas A\&M Universities. In all, $127 \gamma$ transitions were observed. In combination, the electron and $\gamma$-ray results give the conversion coefficients and multipole orders of most of the $127 \gamma$ transitions. This new information serves to fix the level scheme more rigorously. In particular, it resolves many discrepancies between the level schemes previously proposed by the BNL and ORNL groups, it provides confirmation of the previously established ground-state and $K=2(\gamma-$ vibrational) bands, and it supports a previous tentative suggestion that there is a $K=\mathbf{4}$ band. A number of new levels were proposed to accommo- 

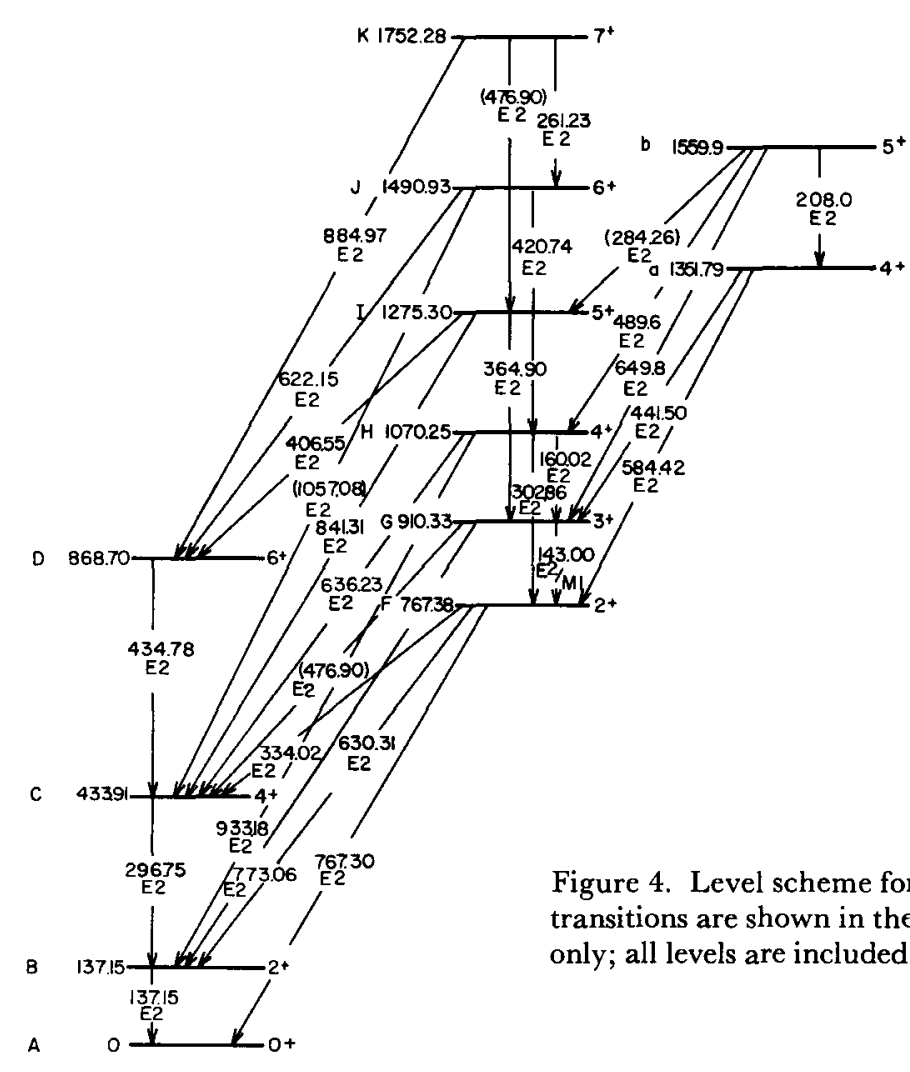

Figure 4. Level scheme for $\mathrm{Os}^{186}$. Inter- and intraband transitions are shown in the upper section of the diagram only; all levels are included in the lower section.

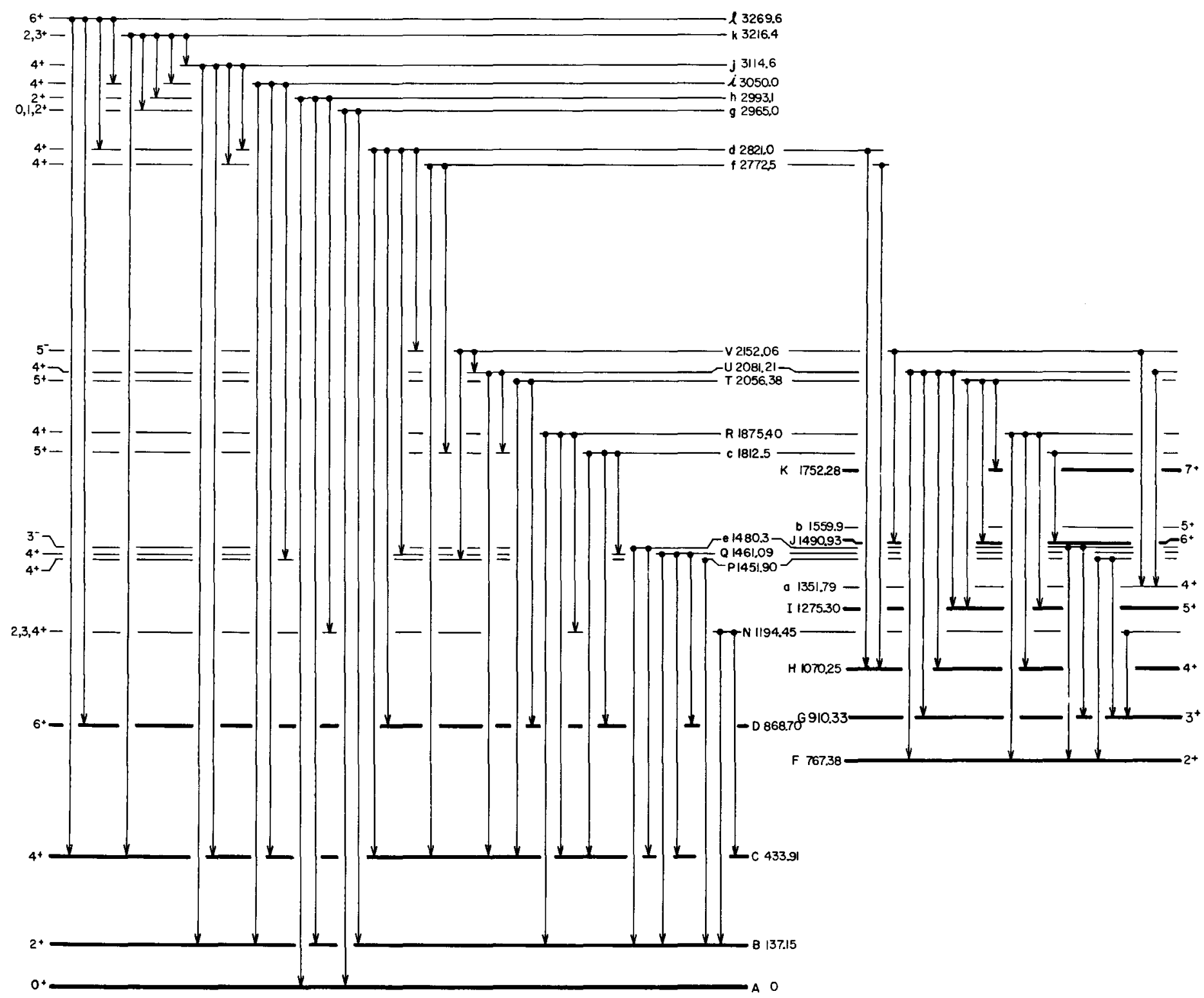


Table 1

\begin{tabular}{llcr}
\hline Nuclide & Half-life & $\begin{array}{c}\gamma \\
\text { Energy, } \\
\text { keV }\end{array}$ & $\begin{array}{c}\text { Branching } \\
\text { ratio, } \\
\%\end{array}$ \\
\hline Gd $^{146}$ & 48 days & 115 & 78.7 \\
& & 155 & 39.5 \\
Gd ${ }^{147}$ & $38 \mathrm{hr}$ & 229 & 59.4 \\
& & 370 & 13.8 \\
& & 397 & 29.7 \\
& & 625 & 7.9 \\
Eu149 & & 765 & 11.1 \\
& & 928 & 17.3 \\
Dy153 & & 277 & 2.9 \\
& & 328 & 3.3 \\
& & 81.5 & 9.0 \\
& & 100 & 10.3 \\
& & 149 & 3.5 \\
& & 246 & 3.0 \\
& & 256 & 6.6 \\
& & 275 & 5.7 \\
\hline
\end{tabular}

date the observed high energy transitions, some of which have $E 0$ components. The improved version of the Os ${ }^{186}$ energy level diagram, which consists of 29 levels, accounts for 91 of the $127 \gamma$ rays assigned to $\operatorname{Ir}^{186}$ decay (Figure 4).

\section{Chemical Bonding Effects on the Internal Conversion Spectra of $\mathrm{Te}^{125}$}

This work, like the work on $\mathrm{Sn}^{119 m}$ described in the 1967 Annual Report, is a study of how nuclear transition probabilities are affected by the chemical bonding of the atom containing the nucleus. Such measurements allow inferences to be drawn concerning the way in which chemical bonding affects the density of the bonding electrons at the nucleus. The $O$-shell conversion coefficients of $\mathrm{Te}^{125 m}$ transitions were measured for tellurium as metal and as $\mathrm{Ag}_{2} \mathrm{Te}^{125 m}$. These forms were chosen because in work done at Harvard University they had been shown to have different half-lives. On the basis of the results obtained so far, the two forms appear to differ by $<5 \%$ in $5 s$ electron density at the nucleus. This small difference cannot account for the difference in half-lives. Whether the latter is due to differences in $5 p$ electron density near the nucleus is now being investigated.

\section{Decay Characteristics of Various Nuclides}

Chemically and isotopically pure samples of several neutron-deficient rare-earth nuclides pro-
Table 2

Calculated Solar Neutrino Capture Rates

\begin{tabular}{|c|c|c|c|}
\hline $\begin{array}{l}\text { Neutrino } \\
\text { source }\end{array}$ & $\begin{array}{c}\text { Energy, } \\
\mathrm{MeV}\end{array}$ & $\begin{array}{c}\text { Flux } \\
\text { at earth, } \\
\mathrm{cm}^{-2} \sec ^{-1}\end{array}$ & $\begin{array}{l}\text { Capture } \\
\text { rate } \\
\text { per day }\end{array}$ \\
\hline \multicolumn{4}{|c|}{$\mathrm{H}+\mathrm{H}+e^{-} \rightarrow$} \\
\hline $\mathrm{D}+\nu$ & 1.44 line & $1.7 \times 10^{8}$ & 0.05 \\
\hline $\mathrm{Be}^{7}$ decay & $0.861 "$ & $2.9 \times 10^{9}$ & 0.16 \\
\hline $\mathrm{N}^{13}$ decay & $0-1.19$ spectrum & $2.2 \times 10^{8}$ & 0.01 \\
\hline $\mathrm{O}^{15}$ decay & $0-1.74 \quad " \quad$ & $2.2 \times 10^{8}$ & 0.03 \\
\hline \multirow[t]{2}{*}{$\mathrm{B}^{8}$ decay } & $0-14$ & $3.6 \times 10^{6}$ & 0.93 \\
\hline & & Total & 1.18 \\
\hline
\end{tabular}

duced in AGS irradiations were prepared by ion exchange and mass separation methods and their $\gamma$ rays studied by high-resolution $\gamma$-ray spectroscopy. The results obtained are shown in Table 1 . Most of this information is new; the $\mathrm{Gd}^{\mathbf{1 4 7}}$ results confirm values that others have reported in the literature.

In a study of $\mathrm{Tb}^{147}$, which according to the literature has a 24-min half-life, no evidence of such a half-life was found either in AGS-produced samples or in $\mathrm{C}^{12}$ irradiations of $\operatorname{Pr}^{141}$ at the Yale Heavy Ion Linear Accelerator. Instead, $\mathrm{Tb}^{147}$ was found to have two isomers, with halflives of $1.65 \mathrm{hr}$ and $2.5 \mathrm{~min}$. For the 1.65-hr isomer the $\gamma$ energies and their abundances per daughter $\mathrm{Gd}^{147}$ are $118.5 \mathrm{keV}(0.051), 139.4 \mathrm{keV}(0.236)$, and $694.4 \mathrm{keV}(0.315)$. The assignment of the 2.5min isomer of $\mathrm{Tb}^{147}$ is inferred from the rate of growth of the $\mathrm{Gd}^{147}$ daughter, and its $\gamma$ energies (relative intensities in parentheses) are $395.9 \mathrm{keV}$ (1.00), $787.3 \mathrm{keV}$ (2.03), and $886.2 \mathrm{keV}$ (2.14).

\section{THE SEARCH FOR NEUTRINOS FROM THE SUN}

A large-scale apparatus, whose construction was completed in 1967, has been used to search for and measure neutrino radiation from the sun. Because the thermal fusion reactions that are the source of the sun's energy produce neutrinos, these very penetrating particles provide a means for study of the nuclear processes occurring in the solar interior. Initial measurements showed the neutrino flux to be much lower than that expected on the basis of theoretical model calculations, and measurements made over the past year confirm the early observations. 


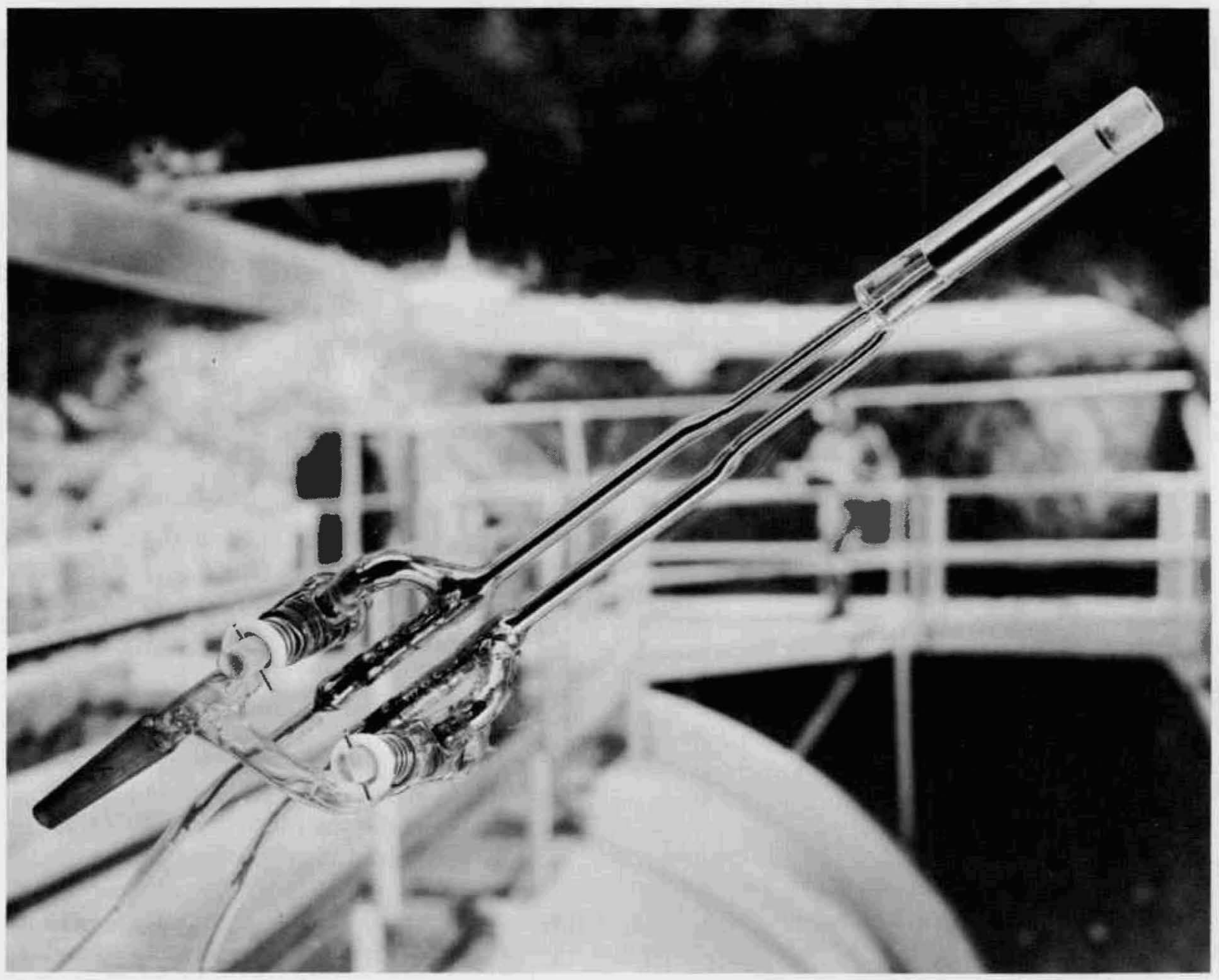

Figure 5. Miniature, low-background proportional counter for measurement of radioactivity of $0.2 \mathrm{cc}$ of argon extracted from 100,000 gallons of cleaning fluid. The top of the fluid-containing tank is in the lower part of the photograph. The counter cathode is a 20 - $\mathrm{mm}$-long, 3-mm-i.d. cylinder of zone-refined iron; the envelope is fused silica. At one end of the counter the silica is thin, to allow calibration with an external source of $\mathrm{Fe}^{55}$,

Neutrino detection depends upon the capture of neutrinos via the reaction

$$
\nu+\mathrm{Cl}^{37} \underset{\text { decay }}{\stackrel{\text { capture }}{\rightleftarrows}} \mathrm{Ar}^{37}+e^{-}
$$

that leads to the formation of the 35-day radioactive isotope $\mathrm{Ar}^{37}$. Because the neutrino capture cross section is extremely small, it is necessary to use a large amount of $\mathrm{Cl}^{37}$. In the Brookhaven detector, the $\mathrm{Cl}^{37}$ is in the form of 100,000 gallons of liquid perchloroethylene, $\mathrm{C}_{2} \mathrm{Cl}_{4}$. Argon, removed from the liquid by purging with helium gas, is purified and placed in a very small $\left(0.3-\mathrm{cm}^{3}\right)$ lowlevel proportional counter to observe the characteristic radiations from $\mathrm{Ar}^{37}$ decay (see Figure 5). To eliminate background effects from cosmic radiation the apparatus was built a mile underground in the Homestake Gold Mine at Lead, South Dakota.

No $\mathrm{Ar}^{37}$ activity has been found in experiments performed to date. These observations have already set an upper limit on the solar neutrino flux that is significantly lower than the flux calculated from theoretical solar models. Specifically, the neutrino capture rate in the detector was $<0.5$ per day, a value to be compared with a theoretical rate of 1.2 per day. Table 2 lists the specific neutrinoemitting processes expected to be occurring in the sun along with their calculated neutrino fluxes and corresponding neutrino capture rates in the detector. The energetic neutrinos from the decay of $\mathrm{B}^{8}$ 
should produce the dominant effect in the detector. If $\mathrm{B}^{8}$, contrary to model prediction, is not produced, the lower neutrino fluxes from the $\mathrm{H}\left(\mathrm{H}, e^{-}, \nu\right) \mathrm{D}$ reaction and from $\mathrm{Be}^{7}$ decay would not be detected, because their intensities are too low for the present sensitivity level. It is possible to improve the neutrino detection sensitivity, now limited by background rate in the $\mathrm{Ar}^{37}$ counter, by a factor of five. A new recording system and associated heavy shielding have been built and installed underground in the Homestake Mine. It is anticipated that a lower background will be achieved, which will make it possible to search for lower neutrino fluxes.

The flux limit set by this experiment has evoked a number of possible explanations. Perhaps the most reasonable is that there are errors in some of the experimental parameters used in the solar model calculations. For example, the accepted hydrogen, helium, and heavy-element composition of the sun may not be correct, or certain nuclear cross sections may be in error. Another possibility is that there are some processes occurring in the interior of the sun that are not included in the calculations. One of these could be mixing within the body of the sun arising from differential rotation and from effects of magneticfields in the interior. These experimental questions and new theoretical ideas are being pursued at various laboratories.

\section{RADIOCHEMICAL STUDIES OF EXTRATERRESTRIAL MATERIALS}

\section{The Lunar Surface}

One of the objectives of the Apollo flight series is to make available for study at the NASA Manned Spacecraft Center in Houston samples of the lunar crust and of the attenuated lunar atmosphere. Under a contract with NASA, members of the Chemistry Department are planning to measure the radioactivity in several separated rare-gas fractions to be obtained from these samples, and systems have been constructed and tested for carrying out the necessary gas separations and purifications and for determining the radioactivities at the low levels expected. Specifically, there may be in the atmosphere and in gas samples extracted from crust by vacuum melting $\mathrm{Rn}^{222}$ (3.8 days), $\mathrm{Xe}^{133}$ (5.3 days), and $\mathrm{Kr}^{85}$ (10.6 yr) - species produced by $\alpha$ decay or spontaneous fission of crustal uranium. Cosmic-ray bombardment of lighter crustal elements should be producing the isotopes $\mathrm{Ar}^{37}$ (35-day), $\mathrm{Ar}^{39}$ (269-yr), and stable $\mathrm{Ar}^{36}$, $\mathrm{Ar}^{38}$, and $\mathrm{Ar}^{40}$. Analysis of the data from these gases should make it possible to deduce the cosmicray exposure age of the lunar surface and should help to answer questions about surface turnover effects of meteoritic bombardment and possible volcanic activity.

\section{The Alandroal Meteorite}

An iron meteorite that fell in Portugal on Nov. 14,1968 , provided one of the rarely available opportunities for acquiring information about the spatial intensity distribution of galactic cosmic radiation. Measurements of the $\mathrm{Ar}^{37}$ (35-day)/ $\mathrm{Ar}^{39}$ (269-yr) activity ratio were made with the lowlevel counting system designed for the lunar gas sample analysis. The $\mathrm{Ar}^{37}$ activity is proportional to the cosmic-ray intensity to which the meteorite was exposed during about the last 60 days before striking the earth; that for $\mathrm{Ar}^{39}$ is proportional to the average cosmic-ray intensity during about the last 500 years. Since it has a highly eccentric orbit, a meteorite spends most of its time at a distance of several astronomical units from the sun, and it can therefore be used to test for cosmic-ray intensity variations within the solar system.

A small, low-level proportional counter was used for the measurements so that the characteristic 2.8-keV Auger electron spectrum from $\mathbf{A r}^{37}$ decay could be distinguished from the continuous $\beta$ spectrum from $\mathrm{Ar}^{39}$. A plot of the pulse-height spectrum observed with a counter filling of argon extracted from the Alandroal meteorite is shown in Figure 6. From this spectrum the $\mathrm{Ar}^{37} / \mathrm{Ar}^{39}$ activity ratio at the time of fall was calculated to be $0.55 \pm 0.05$; this value is to be directly compared with the production ratio by cosmic-ray particles in meteoritic iron, $1.01 \pm 0.05$. The relatively low activity ratio in Alandroal clearly shows that the cosmic-ray intensity near the earth at the time of the meteorite's fall was about one-half that of the average cosmic-ray intensity to which the meteorite had been exposed in its orbit. Such a local intensity decrease is attributable to modulation of the galactic cosmic rays by the solar wind, a flux of low energy charged particles. Solar activity is now near peak in the 11-yr cycle, and the magnetic field accompanying the relatively intense wind would seem to be modulating the high energy ( 0.5 to $5-\mathrm{BeV})$ cosmic-ray flux that produces the activities measured. 


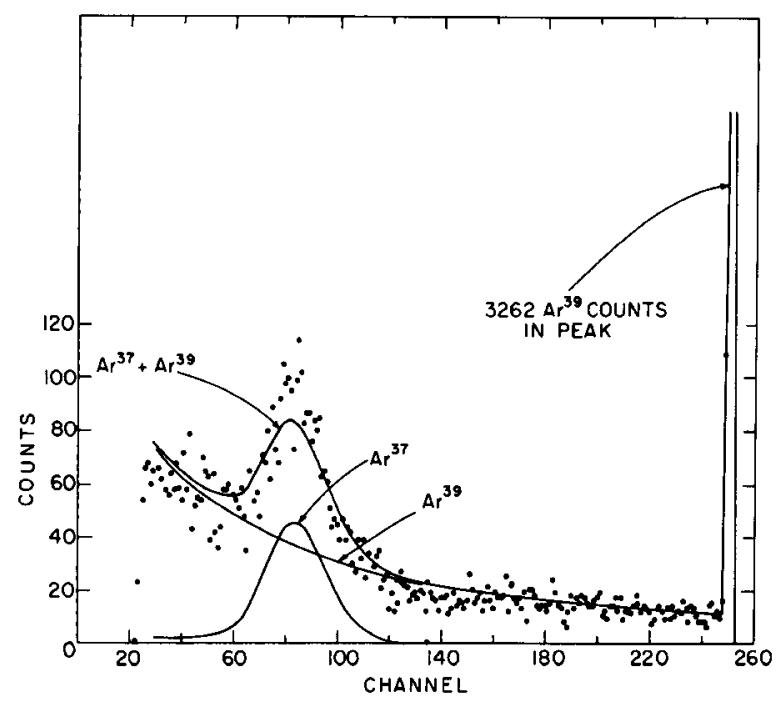

Figure 6. Pulse-height spectrum of radioactive argon extracted from Alandroal, an iron meteorite that fell in Portugal on Nov. 14, 1968. Argon from a 23.6-g sample dissolved in $2 \mathrm{~F}_{2} \mathrm{SO}_{4}$ was counted from Dec. 31, 1968, to Jan. 21, 1969. $\mathrm{Ar}^{37}$ activity $=12 \pm 1$ dis $\mathrm{min}^{-1} \mathrm{~kg}^{-1}$; $\mathrm{Ar}^{39}$ activity $=22 \pm 1$ dis $\mathrm{min}^{-1} \mathrm{~kg}^{-1} ; \mathrm{Ar}^{37} / \mathrm{Ar}^{39}=0.55 \pm$ 0.07. Total counts: $\mathrm{Ar}^{37}, 1440 ; \mathrm{Ar}^{39}, 9038$.

Radioactivity of $\mathrm{Cl}^{36}$ (308,000 yr), also measured in Alandroal, was found to be only $75 \%$ of the amount expected on the basis of $\mathrm{Cl}^{36} / \mathrm{Ar}^{39} \mathrm{re}$ sults from other iron meteorites. A collision in space that occurred perhaps a few hundred thousand years ago could account for a low $\mathrm{Cl}^{36}$ value. Alandroal does have voids and an unusual metallographic pattern, features that could also be produced by collision processes.

\section{NEUTRON SCATTERING BY MAGNETIC MATERIALS}

Neutron scattering methods are being used to study in detail the nature of the transition from the magnetically ordered state to the disordered, or paramagnetic, state. The behavior of magnetic crystals in the vicinity of their Curie or Néel points is closely analogous to that of fluids, binary solutions, ordered alloys, and a number of other physical systems near their critical points. In all these systems, cooperative interactions of large numbers of atoms result in transitions characterized by the loss of long-range order as temperature is raised to a critical point and the persistence of short-range correlations beyond this temperature. These correlations become long-ranged and long-lived in the vicinity of the critical point, which gives rise to large fluctuations in space and time of the degree of order. The energy and momentum changes in neutron scattering produced by these fluctuations provide a unique way of studying the full spacetime correlation function in the vicinity of the critical point.

Critical magnetic scattering of neutrons and the sublattice magnetization have been measured for the antiferromagnet $\mathrm{RbMnF}_{3}$ in the vicinity of the Néel point $\left(83.03^{\circ} \mathrm{K}\right)$. The critical scattering was measured with use of a two-crystal spectrometer, i.e., without energy analysis; and critical indices for the temperature variation of parameters describing the transition were obtained by using the quasi-elastic approximation. These indices, together with the defining relations, are given below.

$$
\begin{aligned}
\beta=0.316 \pm 0.008 ; & M \approx(\Delta T)^{\beta} \\
\nu=0.724 \pm 0.008 ; & \kappa_{1} \approx(\Delta T)^{\nu} \\
\gamma=1.397 \pm 0.034 ; & \chi T \approx(\Delta T / T)^{-\gamma}
\end{aligned}
$$

$M$ is the sublattice magnetization, $\kappa_{1}$ is the inverse correlation range parameter, and $\chi$ is the staggered susceptibility. For $\Delta T / T_{N}<0.1 \%$ the observed critical scattering showed a marked deviation from the Lorentzian shape that would be expected if the time-independent pair-correlation function had the classical Ornstein-Zernike form. Excellent agreement was obtained, however, by using a cross section of the form $\left[A /\left(\kappa_{1}{ }^{2}+q^{2}\right)\right]^{1-\eta / 2}$, where $\mathrm{q}$ is the momentum transfer relative to the (111) magnetic reciprocal lattice point and $\eta$ is a small quantity which modifies the Ornstein-Zernike pair-correlation function. This form was originally proposed by Fisher, and the values of the critical indices given above are based on this modification of the cross section. The best value of $\eta$, obtained by least-squares analysis, is $0.067 \pm 0.01$, in excellent agreement with Fisher's estimate of $\approx 0.08$. It should be noted, moreover, that the best values of $\nu$ and $\gamma$ obey the predicted relation $\gamma=(2-\eta) \nu$, which indicates that there is good internal consistency.

In addition, the inelasticity of the scattering in the critical region was examined in great detail with use of a three-crystal spectrometer. The present theoretical understanding of the more general space-time pair-correlation function is restricted to the so-called hydrodynamic region, i.e., a region in which the fluctuations obey a diffusion equation. By making assumptions about the behavior of the time-dependent correlation function 
near the critical point, Halperin and Hohenberg have developed a theory of dynamic scaling which predicts that the energy half-width, $\Gamma(\omega)$, in the hydrodynamic region should follow the relation $\Gamma(\omega) \approx \kappa_{1}{ }^{3 / 2}$. The measurements made here are in reasonable agreement with this prediction and give added support to the theory of dynamic scaling.

\section{MAGNETIC MOMENTS OF TRANSITION ELEMENTS}

The magnetic properties of transition element salts, such as ferrocyanides, ferricyanides, and cobalticyanides, are of interest because in these crystals the ions are sufficiently far apart to constitute magnetically dilute systems. In such systems measured magnetic moments may be compared with theoretical values in order to investigate quantitatively spin-orbit coupling effects which are still incompletely understood.

A number of well-defined compounds have been prepared and their magnetic susceptibilities measured as a function of temperature:

$$
\begin{gathered}
\mathrm{NH}_{4} \mathrm{Fe}^{3}+\mathrm{Fe}^{\mathrm{II}}(\mathrm{CN})_{6}, \quad \mathrm{Zn}_{3}\left[\mathrm{Fe}^{\mathrm{III}}(\mathrm{CN})_{6}\right]_{2} \cdot 10 \mathrm{H}_{2} \mathrm{O}, \\
\mathrm{Mn}_{3}\left[\mathrm{Co}(\mathrm{CN})_{6}\right]_{2} \cdot 12 \mathrm{H}_{2} \mathrm{O},
\end{gathered}
$$

and

$$
\mathrm{Ni}_{3}\left[\mathrm{Co}(\mathrm{CN})_{6}\right]_{2} \cdot 12 \mathrm{H}_{2} \mathrm{O}
$$

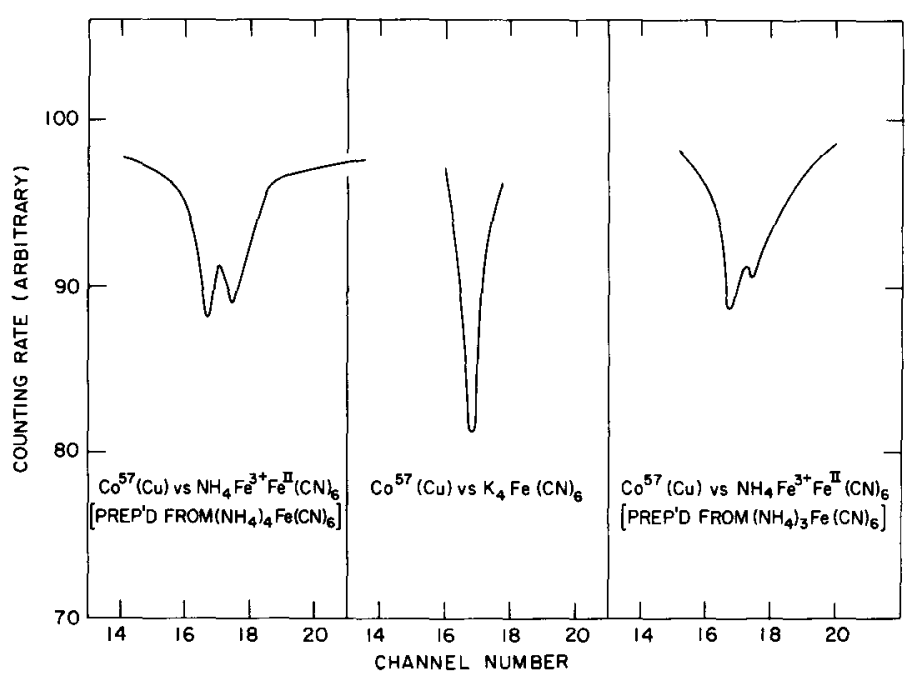

Figure 7. Mössbauer absorption spectra of two preparations of $\mathrm{NH}_{4} \mathrm{FeFe}(\mathrm{CN})_{6}$, one prepared from $\mathrm{Fe}^{\mathrm{II}}(\mathrm{CN})_{6}{ }^{4-}$ and the other from $\mathrm{Fe}^{\mathrm{III}}(\mathrm{CN})_{6}{ }^{3-}$. These spectra show that the Prussian Blue complex structure is independent of cyanide structure used in preparation.
In $\mathrm{NH}_{4} \mathrm{Fe}^{3+} \mathrm{Fe}^{\mathrm{II}}(\mathrm{CN})_{6}, \mathrm{Fe}^{3+}$ is in a ${ }^{6} S_{5 / 2}$ state with a "spin-only" moment. The susceptibility obeys a Curie law,

$$
\chi_{\mathrm{mol} \mathrm{Fe}}=4.365 / T,
$$

as expected for a magnetically dilute system. The $\mathrm{Fe}^{3+}$ moment is

$$
\mu=5.92 \beta,
$$

to be compared with the theoretical spin-only value of $5.92 \beta$ (Bohr magnetons). $\mathrm{NH}_{4} \mathrm{FeFe}(\mathrm{CN})_{6}$ has been prepared by the two reactions

$\mathrm{Fe}^{3+}+\mathrm{Fe}^{\mathrm{II}}(\mathrm{CN})_{6}{ }^{4-}$ and $\mathrm{Fe}^{2+}+\mathrm{Fe}^{\mathrm{III}}\left(\mathrm{CN}_{6}{ }^{3-}\right.$.

The Mössbauer spectra of crystals from the two preparations were found to be nearly identical and to consist of two peaks, one corresponding to the low-spin $\mathrm{Fe}^{\mathrm{II}}$ and the other to $\mathrm{Fe}^{3+}$ in the highspin state (see Figure 7). This confirms evidence from other sources that the Prussian Blue complex is always $\mathrm{Fe}^{3+}-\mathrm{N} \equiv \mathrm{C}-\mathrm{Fe}^{\mathrm{II}}$.

For $\mathrm{Zn}_{3}{ }^{2}+\left[\mathrm{Fe}^{\mathrm{III}}(\mathrm{CN})_{6}\right]_{2} \cdot 10 \mathrm{H}_{2} \mathrm{O}, \mathrm{Fe}^{\mathrm{III}}$ is in a ${ }^{2} T_{2 g}$ state with a moment dependent on temperature and spin-orbit coupling. The moment at $296^{\circ} \mathrm{K}$ is $2.38 \beta$, very close to that found in $\mathrm{K}_{3} \mathrm{Fe}^{\mathrm{III}}(\mathrm{CN})_{6}$.

In the case of $\mathrm{Mn}_{3}{ }^{2+}\left[\mathrm{Co}^{\mathrm{III}}(\mathrm{CN})_{6}\right]_{2} \cdot 12 \mathrm{H}_{2} \mathrm{O}$, $\mathrm{Mn}^{2+}$ occupies both cube corners and centers and is in a ${ }^{6} S_{5 / 2}$ state. Although spin-spin exchange would appear to be possible in this compound because of the small distances between manganese ions, none was found; Curie's law is followed with

$$
\chi_{\mathrm{mol} \mathrm{Mn}}=4.5 / T \text {, }
$$

and the moment is

$$
\mu=6.08 \beta \quad \text { (theor. } 5.92 \beta \text {, spin only). }
$$

In the compound $\mathrm{Ni}_{3}{ }^{2+}\left[\mathrm{Co}^{\mathrm{III}}(\mathrm{CN})_{6}\right]_{2} \cdot 12 \mathrm{H}_{2} \mathrm{O}$, the state of the $\mathrm{Ni}^{2+}$ ion at both the cube corners and centers is ${ }^{3} A_{2 g}$. Experimentally a Weiss-Curie law is observed for the susceptibility as a function of temperature,

$$
\chi=1.33 /\left(T+9^{\circ}\right) .
$$

Thus, the moment is calculated to be

$$
\mu=3.26 \beta \text {. }
$$

This value is a little larger than that observed for the hydrated ion, $\mathrm{Ni}^{2+} \cdot 6 \mathrm{H}_{2} \mathrm{O}$, and it indicates a slightly larger ratio of spin-orbit coupling to crystal-field splitting than is the case for $\mathrm{Ni}^{2+} \cdot 6 \mathrm{H}_{2} \mathrm{O}$. 


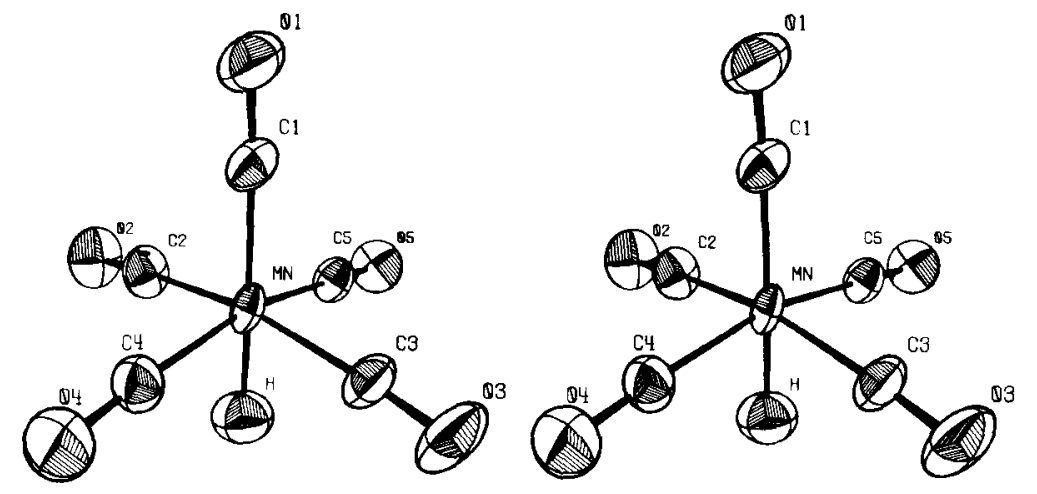

Figure 8. Molecular structure of $\mathrm{HMn}(\mathrm{CO})_{5}$. Stereo pair.
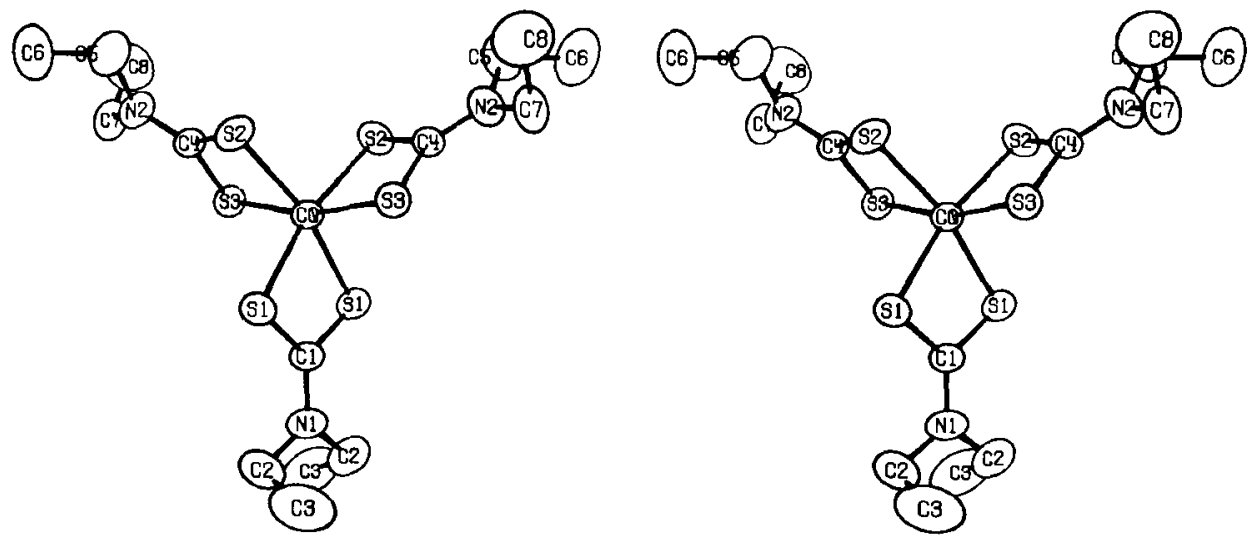

Figure 9. Structure of tris(N,N-diethyldithiocarbamato)Co(III). Stereo pair.

\section{CHEMICAL CRYSTALLOGRAPHY}

\section{Structures of Transition Metal Complexes}

A long-standing controversy in the stereochemistry of transition metal hydride complexes was resolved by the neutron diffraction study of single crystals of manganese pentacarbonyl hydride, $\mathrm{HMn}(\mathrm{CO})_{5}$. This study showed clearly that, contrary to earlier conjectures from other laboratories, the hydrogen atom occupies a definite stereochemical position and the $\mathrm{Mn}-\mathrm{H}$ bond length is that predicted from consideration of normal covalent radii, $1.60 \pm 0.01 \AA$. A stereoscopic drawing of the octahedral molecule is shown in Figure 8. This result provides strong confirmation of the suggestion from a number of $x$-ray studies that there is nothing unusual about the stereochemical behavior of hydrogen as a ligand in inorganic complexes.
It has been postulated that the anomalous magnetic behavior of iron in hemoglobin can be explained by the assumption that the spin ground state of $\mathrm{Fe}$ (III) in that compound is a quartet $(S=3 / 2)$. Because the spin ground state of $\mathrm{Fe}(\mathrm{III})$ in a variety of sulfur-ligated compounds has also been claimed to be a quartet, an investigation of the crystal structures of compounds containing sulfur-ligated $\mathrm{Fe}$ (III) was begun. The catalytic properties of some important enzymes have also been traced to sulfur-ligated iron. Previous measurements at Brookhaven on the crystallography and chemistry of some of these compounds had raised questions about earlier reports of anomalous magnetic properties.

To obtain information about the bonding characteristics of dithiocarbamates of iron, bis(cyclopentamethylenedithiocarbamato $) \mathrm{Fe}(\mathrm{CO})_{2}$ was 
synthesized and the structure of its stable crystals determined. The dithiocarbamate ligand behaves in a normal stereochemical way; the bond lengths are normal and the piperidine ring is in the chair configuration. Tris( $\mathrm{N}, \mathrm{N}$-diethyldithiocarbamato)$\mathrm{Fe}$ (III) is isomorphous with the stable $\mathrm{Co}(\mathrm{III})$ derivative, and the crystal structure of the latter compound was studied. The magnetic and structural characteristics (see Figure 9) of this compound also are normal. Both of these compounds have the $3 d^{6}$ configuration; hence, reported chemical instabilities and anomalous magnetic properties would seem to be confined to the $3 d^{5}$ configuration.

Anomalous magnetic properties have been reported also for bis[cis-1,2-bis(trifluoromethyl)ethylene-1,2-dithiolato]Fe triphenylarsine. This neutral molecule is stated to be diamagnetic, with its anion in a spin-quartet $(S=3 / 2)$ ground state, which implies that the introduction of a single electron into the parent compound decouples two electrons when the anion is formed. A BNL study of the crystal shows that the compound is monomeric, although the dimeric structure found here earlier for other bis-dithiolates of iron would be more consistent with the magnetic properties of the anion. Preliminary studies of the tetra- $n$-butylammonium bis[cis-1,2-bis(trifluoromethyl)ethylene-1,2-dithiolato]Fe triphenylarsenate salt indicate that this anion also may be monomeric. If this is verified, the anion will be the first well-documented example of $\mathrm{Fe}(\mathrm{III})$ with a spin- $3 / 2$ ground state.

Several new compounds were synthesized and characterized both spectrally and structurally. $\mathrm{Co}\left(\mathrm{NH}_{3}\right)_{6} \mathrm{CdCl}_{5}$ is cubic and contains the trigonal bipyramidal anion $\mathrm{CdCl}_{5}{ }^{3-}$; this is the first actual isolation and structural proof of such an anionic pentacoordinated species. The compound $\mathrm{Co}\left(\mathrm{NH}_{3}\right)_{6} \mathrm{ZnCl}_{5}$, however, contains $\mathrm{ZnCl}_{4}{ }^{2-}$ and separate $\mathrm{Cl}-$ anions.

\section{Hydrogen Bonding}

Two compounds containing short, probably symmetric, hydrogen bonds were studied, one of them by neutron diffraction. In this compound, bis(2-amino-2-methyl-3-butanone oximato) Ni(II)Cl • $\mathrm{H}_{2} \mathrm{O}$, the hydrogen atoms were found to lie almost, but not exactly, at the center of the short hydrogen bond. A study of the dynamical variables suggests that the hydrogen atom lies in the lowest vibrational energy level of a broad, single-minimum potential function.

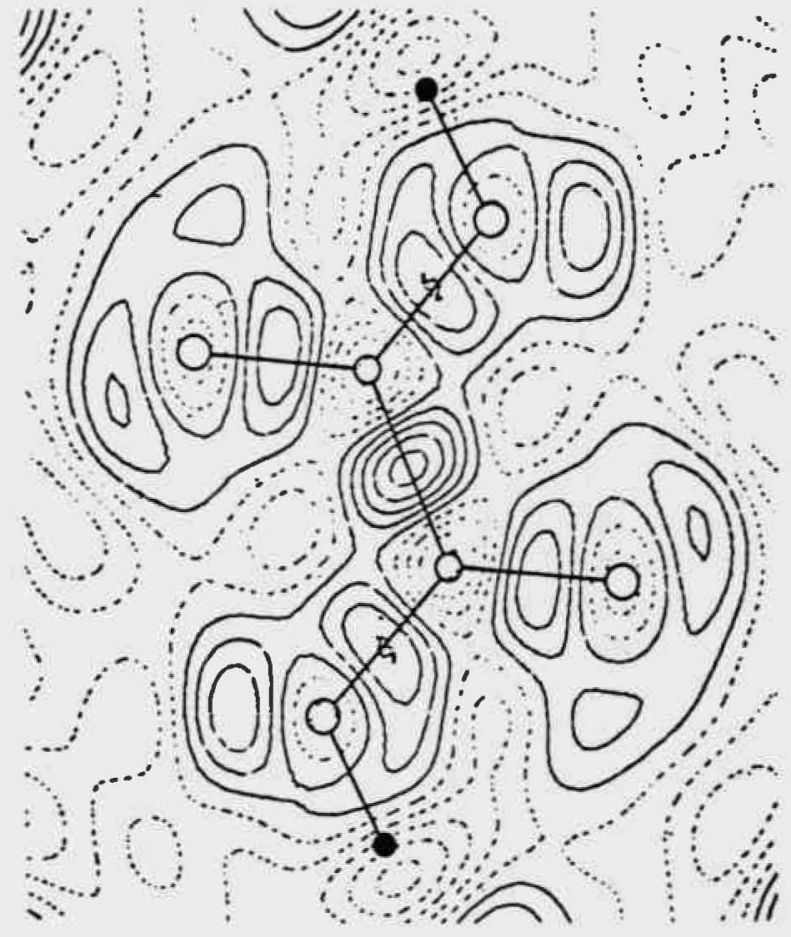

Figure 10. Electron density map of oxalic acid showing differences in density in the molecule with respect to isolated atoms. Densities in bonds as well as in unbonded electron pairs may be seen. $O$, oxygen; $O$, carbon; $\bullet$, hydrogen.

An x-ray diffraction study of the related compound of cobalt reveals almost identical bond lengths, although the hydrogen atom in this case is forced to lie in a symmetry position.

Neutron diffraction data on the polymorphs of solid water, ice V and ice IX, were analyzed. Ice IX (the low-temperature phase of ice III) has a structure in which the protons are well ordered, as they are in ice II. Order-disorder phenomena in ice structures are particularly complex. The structure of ice $\mathrm{V}$ as studied in a quenched form exhibits only partial order in the proton positions. Some sites are occupied almost $100 \%$, others in the neighborhood of $50 \%$, although even here there is some variability. The number of hydrogens bonded to each oxygen is one in every case.

\section{Electron Density Determination}

Electron density maps and accurate thermal parameters were obtained for cyanuric acid, and the work on several phases of oxalic acid hydrate was completed. By comparison of the $\mathrm{x}$-ray and neutron diffraction data it is possible to see clearly 
the bonding and lone pair electrons in organic molecules. An example is shown in Figure 10, in which the bonding electron density, that is, the difference between the electron density in the molecule and that of isolated atoms, is clearly shown.

\section{Molecular Biology, Protein Structure}

In a collaborative study with the Biology Department, it has been demonstrated that, with equipment on hand, significant neutron diffraction data can be collected from proteins. Such data on single crystals of myoglobin have been used to calculate scattering density maps of good quality. The applicability of neutron anomalous scattering to the solution of the phase problem would appear to be assured; and, most important, it has been demonstrated that protein crystals suffer no significant radiation damage when exposed to the thermal neutron beams in the diffraction apparatus.

\section{Instrumentation and Techniques}

A variable-temperature liquid-helium cryostat for the collection of three-dimensional neutron dif- fraction data was built. A similar cryostat has been designed for $\mathrm{x}$-ray diffraction work; it will be the first of its kind anywhere. With these cryostats, accurate electron-density and potential-function information will be more readily obtainable than at present. Figure 11 shows the neutron cryostat mounted on the full-circle goniometer at the High Flux Beam Research Reactor.

Development work was continued on other advanced instrumentation, chief among which is the 128-detector $x$-ray diffractometer AESOP (automatic equi-inclination spectrometer operating in parallel). The design of this advanced instrument has been completed, along with the computercontrolled system and associated software. The use of this diffractometer should speed up the collection of data from protein crystals by a factor of ten. Work has also continued on techniques for the display of three-dimensional crystal structures on the color television screen of a computer monitor. The computer generates the drawings and allows direct manipulation by means of a teletype terminal and a coordinate digitizer. Molecular drawings may be rotated, expanded, and amended at will.

\section{CHEMICAL APPLICATIONS OF THE MÖSSBAUER EFFECT}

neutron diffraction measurements on samples maintained at controlled temperatures as low as that of liquid helium.

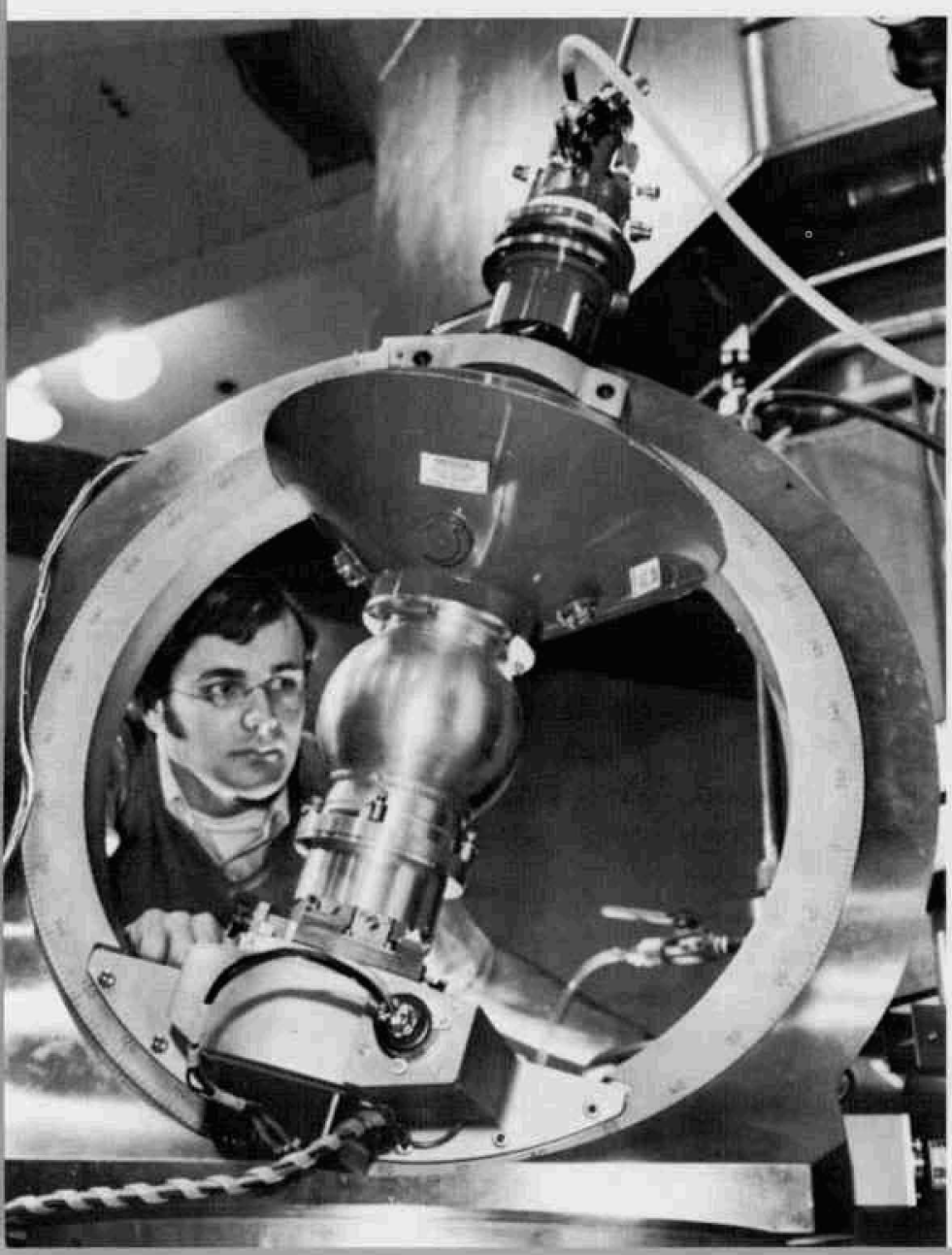

\section{Sign of the Electric Quadrupole Field Gradient in Organo-Tin Compounds}

The sign of the field gradient at the position of the Mössbauer nucleus is obtained by comparing spectra observed in strong magnetic fields (about $50 \mathrm{kOe}$ ) with spectra calculated for model structures. For these computations a computer program developed at Argonne National Laboratory is used.

Signs of the field gradient were measured in compounds of the type $\mathrm{R}_{2} \mathrm{Sn}$ (diethyldithiocarbamate) $)_{2}$, with $\mathrm{R}$ either an alkyl group or a phenyl group. The signs of the gradients in the alkyl compounds were found to be opposite to those in the phenyl compounds. Both types of compound have asymmetry parameters close to zero. It can now be inferred that the structure of the phenyl compounds is cis-octahedral, and that of the alkyl compounds is trans-octahedral, rather than tetrahedral as reported in the literature. To demonstrate that the literature interpretation cannot be correct, a compound known to be tetrahedral, dibutyl tin bis(isooctylthioglycolate), was studied. 
It proved to have a large asymmetry parameter. It is therefore inferred that the alkyl compounds, which, like the octahedral phenyl compounds, have a small asymmetry parameter, cannot be tetrahedral.

The signs of the electric field gradients at the tin nucleus were shown to be the same in the compounds $\left(\mathrm{CH}_{3}\right)_{3} \mathrm{SnF}$ and $\left(\mathrm{CH}_{3}\right)_{3} \mathrm{SnOH}$, which are both five-coordinated with trigonal bipyramidal structures. If these signs were opposite, as had been reported in the literature, it would mean that the sign depends in a sensitive way on bonds other than the carbon-tin bonds. That no such sensitive dependence obtains was shown by measurements of the field gradient made on a dozen organo-tin compounds of known structure.

It was earlier found at BNL that in a series of compounds like $\mathrm{R}_{3} \mathrm{SnCl}, \mathrm{R}_{2} \mathrm{SnCl}_{2}$, and $\mathrm{RSnCl}_{3}$, which all presumably have tetrahedral structures, the relative magnitudes of the field gradients differ from theoretical expectations. To determine whether this could be due to intermolecular effects in the crystal, measurements were made on frozen solutions of these compounds in a noncoordinating solvent. The spectrum of $\mathrm{RSnCl}_{3}$ in dilute solution is much the same as that in the crystal, but there are large changes in the cases of $\mathrm{R}_{3} \mathrm{SnCl}$ and $\mathrm{R}_{2} \mathrm{SnCl}_{2}$. This indicates that in crystals of the two last-named the intermolecular forces are important, an interpretation supported by recently reported crystallographic results.

\section{Standard Reference Sources for Mössbauer Spectroscopy of Tin Compounds}

The sign of the electric quadrupole field gradient at the tin nucleus in $\left(\mathrm{CH}_{3}\right)_{2} \mathrm{SnF}_{2}$ was established unambiguously about one year ago at BNL. As a consequence of the fact that this now wellunderstood Mössbauer absorption spectrum consists of two intense, well-separated, narrow, individual lines, $\left(\mathrm{CH}_{3}\right)_{2} \mathrm{SnF}_{2}$ is being proposed to the National Bureau of Standards as a reference material for Mössbauer measurements of tin compounds. The Bureau has judged it to be promising and is providing samples to various laboratories for further investigation.

\section{Structure of $\operatorname{Sn}($ DTC)}

To check a claim in the literature that tin in $\operatorname{Sn}(\mathrm{DTC})_{4}(\mathrm{DTC}=$ diethyldithiocarbamate $)$ is eight-coordinated, with each DTC anion acting as a bidentate ligand,the compound $\left[\operatorname{Sn}(\mathrm{DTC})_{3}\right]^{+-}$ $\left[\mathrm{B}\left(\mathrm{C}_{6} \mathrm{H}_{5}\right)_{4}\right]^{-}$, which cannot have an eight-coordinated structure, was prepared for a Mössbauer study. The two compounds proved to have identical Mössbauer shifts, an indication that tin has the same coordination number in both. From known regularities in shifts and from the infrared spectra of both compounds it was inferred that this coordination number was six. This has recently been confirmed by crystal structure analyses at the University of Missouri, which show that $\operatorname{Sn}(\text { DTC })_{4}$ contains two monodentate and two bidentate DTC ligands.

\section{Mössbauer Studies on $\mathrm{Te}^{125}$ Compounds}

A study has been made of the compound Te(II)$(\mathrm{TmTr})_{4} \mathrm{Cl}_{2} \cdot 2 \mathrm{H}_{2} \mathrm{O}(\mathrm{Tm} \mathrm{Tr}=$ tetramethylthiourea). This is the first true $\mathrm{Te}^{2+}$ compound ever to be subjected to Mössbauer study. The observed quadrupole splitting, $\approx 17.4 \mathrm{~mm} / \mathrm{sec}$, is more than twice as large as the largest splitting previously reported for tellurium compounds. Large splittings are expected for other $\mathrm{Te}^{2+}$ compounds, and possibly the magnitude of splittings may serve to distinguish $\mathrm{Te}^{2+}$ from $\mathrm{Te}^{4+}$.

\section{INORGANIC REACTIONS IN SOLUTIONS}

Previous studies at BNL and elsewhere have shown that many oxidation-reduction reactions involving metal ions and their complexes proceed via intermediates in which the metal ions are connected by a common bridging group. This group of reactions, which are called inner-sphere reactions, proceeds in a number of steps. The first step is the formation of a precursor complex from the separated reactants; the second step, which is usually the slowest, involves the reorganization of the precursor complex. The subsequent steps are comparatively rapid. An important advance recently made at BNL was the recognition of a new class of reactions in which the formation of the precursor complex is the slowest step. This reaction mechanism was substantiated by comparisons, made recently at BNL and elsewhere, of the activation parameters for the reduction of vanadium(IV), chromium(III), and cobalt(III) complexes by vanadium(II) with the activation parameters for substitution of water on vanadium(II). These comparisons show that the rate-determining step in the reductions by vanadium(II) is the replace- 
ment of a water molecule coordinated to the metal center. On the basis of this finding it is possible to rationalize the effects of a variety of anions on the rate of oxidation-reduction reactions involving vanadium(II) and to explain why the reactivity patterns observed in these systems differ from those observed in other inner-sphere reactions.

The oxidation of chromium(II) by hydrogen peroxide in the presence of cyanide ions proceeds by a variety of paths. The relative contributions of these paths depend upon the $p \mathrm{H}$ and cyanide ion concentrations of the solution. At $p \mathrm{H} 11$ to 12 and high cyanide ion concentrations, the reaction proceeds by a two-step outer-sphere mechanism:

$$
\begin{aligned}
& \mathrm{Cr}(\mathrm{CN})_{6}{ }^{4-}+\mathrm{H}_{2} \mathrm{O}_{2} \rightarrow \mathrm{Cr}(\mathrm{CN})_{6}{ }^{3-}+\mathrm{OH}^{-}+\mathrm{OH}, \\
& \mathrm{Cr}(\mathrm{CN})_{6}{ }^{4-}+\mathrm{OH} \rightarrow \mathrm{Cr}(\mathrm{CN})_{6}{ }^{3-}+\mathrm{OH}^{-},
\end{aligned}
$$

with the first step being the slower. At higher $p H$ values chromium(II) and hydrogen peroxide form a relatively stable complex, which only slowly decomposes into products.

$$
\begin{gathered}
\mathrm{Cr}(\mathrm{CN})_{6}{ }^{4-}+\mathrm{H}_{2} \mathrm{O}_{2} \rightleftarrows \\
\mathrm{Cr}(\mathrm{CN})_{4} \mathrm{HO}_{2}{ }^{3-}+2 \mathrm{CN}-+\mathrm{H}^{+} \\
\mathrm{Cr}(\mathrm{CN})_{4} \mathrm{HO}_{2}{ }^{3-} \rightarrow \text { products }
\end{gathered}
$$

The above mechanisms may operate quite generally in reactions involving hydrogen peroxide and metal centers. Further studies of reactions of this type are in progress.

Thallium(II) has frequently been proposed as an intermediate in reactions of thallium(III) with reducing agents, but convincing experimental evidence is limited to only a few instances, of which the thallium(III)-iron(II) reaction is the best documented. Earlier work on this reaction was extended, and the mechanistic conclusions were verified. It was also found that the rate of the thallium(I)-thallium(III) exchange reaction is increased in the presence of iron(II), evidently through the intervention of thallium(II). In conjunction with photochemical and radiation-chemical studies performed elsewhere, the results of these exchange studies should provide significant new information on the reactions of thallium(II).

In a publication from another laboratory it was recently proposed that the mechanism of the thallium(III) oxidation of ferrous tris(1,10-phenanthroline) is

$$
\begin{aligned}
& \mathrm{Fe}(\text { phen })_{3}^{2+}+\mathrm{Tl}^{3}+\underset{k_{-1}}{\stackrel{k_{1}}{\longrightarrow}} \mathrm{Fe}(\text { phen })_{3}{ }^{3+}+\mathrm{Tl}_{2}+ \\
& \mathrm{Fe}(\text { phen })_{3}^{2+}+\mathrm{Tl}^{2+} \stackrel{k_{2}}{\longrightarrow} \mathrm{Fe}(\text { phen })_{3}{ }^{3+}+\mathrm{Tl}+
\end{aligned}
$$

However, the rapid reaction (studied earlier at BNL) between $\mathrm{Fe}(\text { phen })_{3}{ }^{3+}$ and $\mathrm{Fe}^{2+}$, which is formed by aquation of $\mathrm{Fe}$ (phen $)_{3}{ }^{2+}$, was neglected. The above system has therefore been re-examined. New data on the oxidation-reduction reaction suggest that an iron(IV) species, rather than thallium(II), is involved in the reaction between $\mathrm{Fe}(\mathrm{phen})_{3}{ }^{2+}$ and thallium(III).

In solvent extraction studies it was established that thallic bromide is extracted from aqueous solution into tributylphosphate-octane mixtures as the solvated molecular species $\mathrm{TlBr}_{3} \cdot 2 \mathrm{TBP}$ and the solvated ion pair $\mathrm{HTlBr}^{4} \cdot 4 \mathrm{TBP}$, and evidence was obtained that the $\mathrm{TlBr}_{4}-$ anion in the latter species is solvated. Values were obtained for the stability constants of the complexes $\mathrm{TlBr}_{2}{ }^{+}, \mathrm{TlBr}_{3}$, and $\mathrm{TlBr}_{4}$ - in the aqueous phase. From conductivity measurements on methyl-isobutyl ketone extracts of $\mathrm{HCl}, \mathrm{HClO}_{4}$, and $\mathrm{HFeCl}_{4}$, it was concluded that all three acids form ion pairs and triple ions $\left(\mathrm{HX}_{2}{ }^{-}\right)$in the organic phase.

\section{REACTION MECHANISMS IN ORGANIC SYSTEMS}

\section{Recombination of Caged Radicals}

Some azo compounds undergo thermal decomposition by stepwise rupture of two carbon-nitrogen bonds:

$$
\begin{aligned}
& \mathbf{R}-\mathbf{N}=\mathbf{N}-\mathbf{R}^{\prime} \rightarrow \mathbf{R}-\mathbf{N}=\mathbf{N} \cdot+\mathbf{R}^{\prime} \cdot, \\
& \mathbf{R}-\mathbf{N}=\mathbf{N} \cdot \quad \rightarrow \mathrm{R} \cdot+\mathrm{N}_{2} .
\end{aligned}
$$

The fact that nitrogen is evolved relatively slowly when decomposition is carried out in highly viscous solvents has been regarded as an identifying characteristic of such a mechanism and has been considered as supporting the notion that the caged pair of radicals produced in (1) undergoes reversible recombination.

$\alpha$-Phenylethylazomethane, which has been shown by secondary $\alpha$-deuterium, $\mathrm{C}^{\mathbf{1 3}}$, and $\mathrm{N}^{15}$ kinetic isotope effects to undergo decomposition by steps (1) and (2), has been prepared optically active. Reversible rupture and re-formation of one carbon-nitrogen bond would lead to loss of optical activity without nitrogen formation. Experimental 
results, however, appear to indicate that the rates of nitrogen production and loss of optical activity are very nearly the same.

\section{Enzyme-Related Model System}

A study has been in progress of a model isomerization reaction related to the enzymatic cis-trans isomerization of maleylacetoacetic acid. Recently, the effect of Schiff base formation on the rate of isomerization, which may have a role for the enzyme, has been investigated. Such a base, the semicarbazone of $c i s-\beta$-acetylacrylic acid, has been prepared, and the kinetics of its hydrolysis and isomerization to the trans compound have been studied. The rate of isomerization catalyzed by thiocyanate ion turns out to be about the same for cis- $\beta$-acetylacrylic acid as for its semicarbazone. This type of Schiff base thus does not accelerate the isomerization; however, the possibility that others may do so is not excluded.

\section{Hot Atom Chemistry}

Studies of hot atom chemistry in organic systems with accelerator-produced isotopes were resumed with the rebuilt 60 -in. cyclotron, after a three-year hiatus due to lack of irradiation facilities. Three fundamental mechanism studies were carried forward. In pure fluorocarbons, product distributions of both $\mathrm{C}^{11}$ - and $\mathrm{F}^{18}$-containing compounds are determined. A second program is concerned with the relative reactivity toward hot carbon atoms of the components in fluorocarbon-hydrocarbon mixtures. Perfluoromethane-ethane-oxygen and perfluoroethane-ethane-oxygen mixtures were studied. The results support previous postulates that carbon atom-substrate complexes of long lifetime are formed. The perfluorohydrocarbon turns out to be an effective moderator of the carbon atom and provides a new system for the study of thermalized carbon atoms. A third study is directed toward details of carbon atom reactivity as influenced by bond type and structure. Specifically, deuteriumlabeled ethyl and $n$-propyl aromatics, e.g., toluene$\alpha-{ }^{2} \mathrm{H}_{3}$ and the various side-chain-labeled ethyl and $n$-propylbenzenes, were prepared for irradiation. The double-label technique previously developed at BNL is being applied to determine the point of attack on the molecule by the carbon atom and the selectivity it shows in discriminating between aromatic and aliphatic moieties.
A study was completed of isotope distributions in the reaction of carbon atoms with hydrocarbons and alkylfluorides; a large number of specifically deuterated and fluorinated hydrocarbons were investigated. Deuterium distributions observed in product $\mathrm{C}^{11}$-acetylene as compared with the distributions in the reactant molecules reflect the intramolecular nature of the reaction and the lifetime and excitation of the intermediate complex. The results strongly support mechanistic hypotheses previously put forth at BNL.

A study of $n$-butylcarbene production in the photolysis of diazo- $n$-butane showed very little correspondence with the results from a study of $n$-butylcarbene production in the reaction of propane with a hot carbon atom. Bond scission occurred, but products reflecting the lower energy state of the photo-produced carbene were found. Products from a typical low-pressure experiment are shown in the following equation.

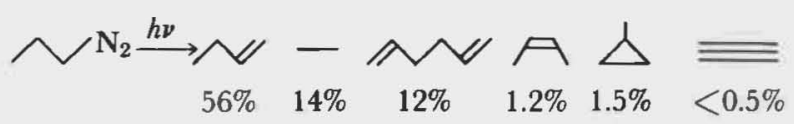

This work, along with results obtained at BNL in previous years, supports the hypothesis that the thermal, ground-state carbon atom reacts slowly, if at all, in insertion reactions. It is thus different in reactivity from carbon atoms in the first two excited states and from the kinetically hot atom in any electronic state.

\section{Application of Hot Atom Reaction Studies in Medicine}

Carbon-11 was used to prepare compounds of interest in medical research. This interdisciplinary program was begun last year. Success was attained in the preparation of labeled dopamine (used in research and treatment of Parkinsonism) by a combination of recoil method and organic synthesis. The technique developed involves hot atom preparation of cyanide followed by incorporation of the cyanide into an organic precursor of dopamine. Chemical methods then lead to dopamineC11. The entire procedure can be completed in one hour. Direct recoil labeling of L-dopa, tryptophan, and other amino acids at usable levels was also accomplished. In these recoil syntheses, chemically pure materials were obtained in $30 \mathrm{~min}$ or less after the end of proton irradiation (see Figure 12). This work represents an important practical 


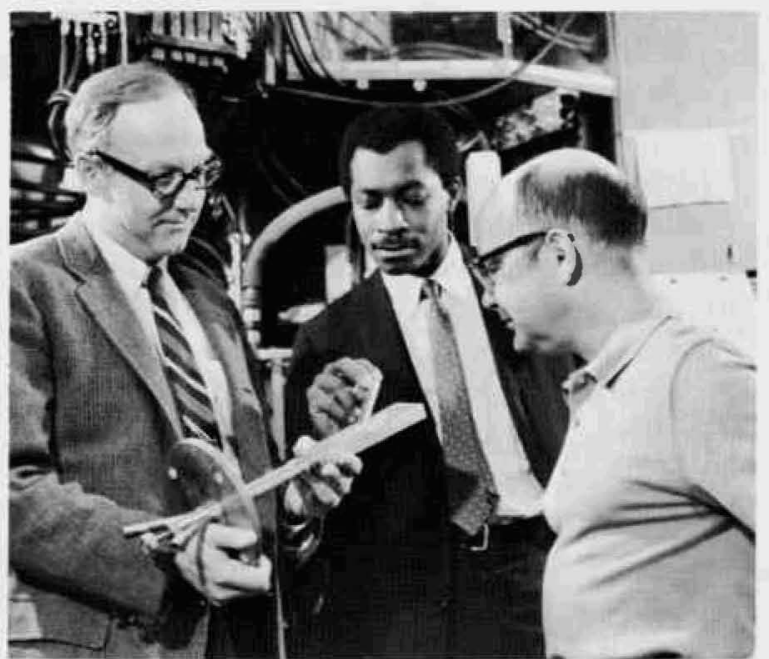

Figure 12. Preparation of target for nuclear recoil labeling of a drug by irradiation of the material with protons at the BNL 60 -in. cyclotron.

application of the research in hot atom chemistry and labeling techniques carried out at BNL over a period of years.

\section{RADIATION CHEMISTRY}

\section{Properties of $\mathrm{H}_{2} \mathrm{O}_{3}$ and $\mathrm{HO}_{2}$}

$\mathrm{H}_{2} \mathrm{O}_{3}$, a "super peroxide" of hydrogen, is an interesting but unstable compound, which has to date been made only by irradiation of water containing dissolved oxygen gas. The deuterium compound $\mathrm{D}_{2} \mathrm{O}_{3}$ has now been made by irradiation of heavy water containing oxygen, and its decomposition rate has been studied as a function of $p \mathrm{H}$. At room temperature, in any acid concentration, the lifetime of $\mathrm{D}_{2} \mathrm{O}_{3}$ in $\mathrm{D}_{2} \mathrm{O}$ is just six times that of $\mathrm{H}_{2} \mathrm{O}_{3}$ in $\mathrm{H}_{2} \mathrm{O}$ (Figure 13). At lower temperatures the ratio is somewhat greater than six. It was calculated that if $\mathrm{D}_{2} \mathrm{O}_{3}$ could be obtained at $-80^{\circ} \mathrm{C}$ it would be essentially stable.

The deuterium analogue of the $\mathrm{HO}_{2}$ radical, $\mathrm{DO}_{2}$, has been studied in flow systems with use of both chemical detection and electron spin resonance measurement. The bimolecular decay constant for $\mathrm{DO}_{2}$ is six times smaller than that for $\mathrm{HO}_{2}$ under the same conditions $\left(0.4 \mathrm{M} \mathrm{H}^{+}\right)$.

\section{Accurate Yield Values for Water Radiolysis}

Very careful determinations were made, with use of a weak source of $\gamma$ rays, of the true initial yields in aerated aqueous solution for three reactions: (1) the oxidation of ferrous sulfate over the $p \mathrm{H}$ range from 0.3 to $6,(2)$ the formation of hydrogen peroxide in aerated solutions of ethanol and of formate ion, and (3) the reduction of tetranitromethane. These observations are interpreted in terms of the number of free radicals formed in the irradiation of water. Certain previously accepted values have been revised as a consequence of this work. In all these reactions the products tend to react with the radicals formed, so that the yields at higher conversions are smaller than the true initial values. The new values, when carefully extrapolated back to zero dose, agree with one another and with results for certain other systems reported in the literature; they do not agree with other results from experiments in which larger doses were used. It appears that this problem has at last been satisfactorily settled and that no further work need be done.

\section{Radiolysis of Aqueous Sodium Linoleate}

The study of radiation oxidation of fatty acids in solution was undertaken because the results may throw some light on radiation effects on permeability of biological membranes. Like other soaps,

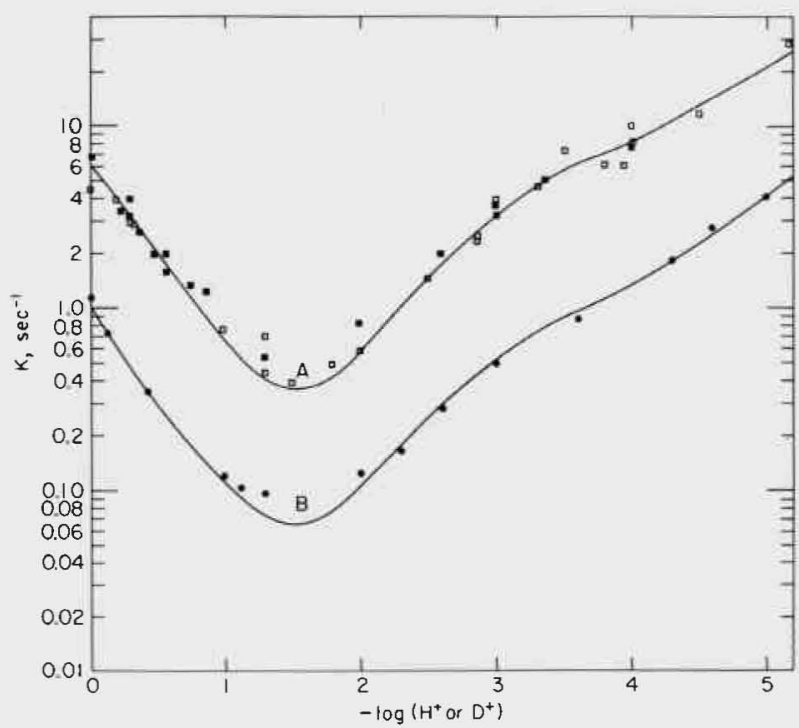

Figure 13. Rate constants for first-order decay of $\mathrm{H}_{2} \mathrm{O}_{3}$ and $\mathrm{D}_{2} \mathrm{O}_{3}$ as a function of acidity. $\bullet, \mathrm{D}_{2} \mathrm{O}_{3}$ in $\mathrm{D}_{2} \mathrm{O}$. and $\square, \mathrm{H}_{2} \mathrm{O}_{3}$ in $\mathrm{H}_{2} \mathrm{O}$ : $\square$, old data obtained by flow method; $\square$, new data obtained by pulse radiolysis. The curves are theoretical, derived from an assumed acid-base catalysis mechanism. 
sodium linoleate at sufficiently low concentrations in alkaline solutions is present as molecules; but at a certain critical concentration, depending upon the alkalinity and the nature of the anions present, it begins to aggregate into colloidal micelles. Under irradiation this soap may be oxidized by a chain reaction. The chains are not of appreciable length in the molecularly dispersed solution, but solutions just above the critical concentration for micelle formation show a very large rise in the oxidation yield; $G$-values are as high as 170 . Thus the oxidation proceeds very readily when the fatty acid molecules are agglomerated into lipidlike phases, but not when they are dispersed.

\section{lonization of Liquids Measured by Electrical Methods}

Of the physical processes involved in the interaction of radiation with the various forms of matter, the least well understood is the production of ionization in liquids and the fate of the free electrons thus formed. Recently, considerable progress in understanding this subject has been made at BNL as the result of a new electrical method developed here for measuring yields of free ions formed in irradiation of liquids with high energy $x$ rays. The yield is determined by the probability that an electron, given a certain kinetic energy in the ionization process, can escape immediate recombination with the positive ion from which it came. The escape probability depends in an obvious way on the density and dielectric constant of the medium, but beyond this it is found to depend strongly on the molecular structure of the substance. Generally, in a set of isomers, free ion yields increase with increasing branching in the structure, and quaternary atoms have an especially large effect. The temperature coefficient of the yield tends to be smaller for liquids that exhibit higher yields. The results have been interpreted in terms of the cross sections for energy loss by slow electrons to the vibrational and rotational modes of the liquid molecules.

During exposure to radiation, neopentane and tetramethyl silicon show an anomalously high conductivity which disappears extremely rapidly on termination of the exposure. The effect is due to the presence of a population of electrons of very high mobility, comparable to that of electrons in liquid argon. This phenomenon does not occur in the presence of a small concentration of any electron-trapping substance, such as oxygen.
The study of the basic processes of ionization in liquids, which had languished for many decades, was revived in recent years in large part through the efforts of the Radiation Chemistry Group in this Department. It has now become an exciting and rapidly developing field which has been taken up by an increasing number of laboratories.

\section{Ionization and Charge Transfer Studied by Optical Methods}

Ionization in a liquid may be studied by adding small quantities of a substance that readily accepts the positive or negative charge from the ion present to produce a compound with a distinctive color ("optical absorption spectrum"), which can be observed, even though its lifetime be very short, by the methods of pulse radiolysis. Several such substances in a variety of solvents have been studied at BNL with a view to using them to determine the free ion yields in liquids of relatively high inherent conductivity, to which electrical methods of yield determination cannot be applied. It is found that a generally applicable charge acceptor is the stable radical galvinoxyl, which easily accepts electrons and forms a negative ion having a distinctive absorption spectrum. Yields of the galvinoxyl ion are equal to the total free-ion yield as determined by the electrical method in several hydrocarbon and other low-dielectric-constant solvents. Yield of the ion in ethanol was found to be equal to the yield of solvated electrons, as determined by pulse radiolytic studies described in the literature. Ion yields in several other alcohols have been determined. The method of pulse radiolysis opens up possibilities of studying ionization in a wide range of solvents for which other methods are not readily applicable.

\section{Radiation Chemistry of Heterogeneous Systems}

The way in which energy introduced into one phase of a heterogeneous system can produce chemical effects in another phase is of obvious importance for the study of radiation effects in biological and in practical industrial systems. A study has been completed of the effects of $\gamma$ radiation on ethanol adsorbed on silica. Radiation energy deposited in the silica induces decomposition of the ethanol, with many of the same products formed as when ethanol is irradiated directly. However, additional ethane and ethylene are formed in amounts that vary in a peculiar manner with the 


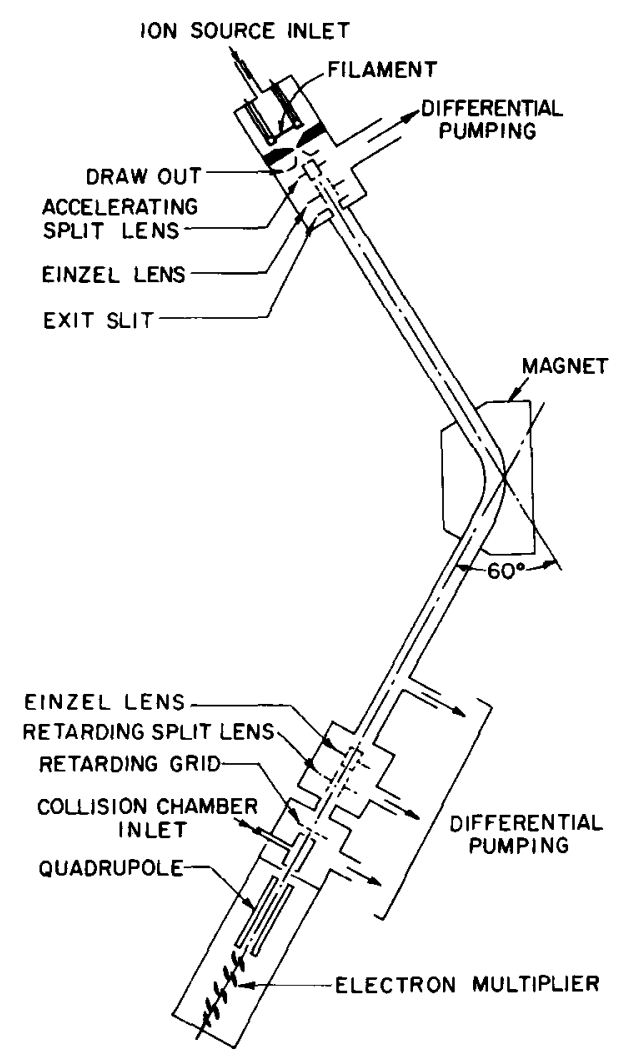

Figure 14. Tandem mass-spectrometer system with highpressure ion source and collision chamber for study of ionmolecule reactions.

quantity of ethanol present; the results suggest that one effect of the radiation is to produce on the surface of the silica acidic sites similar to those present in commercial silica-alumina catalysts.

\section{GASEOUS ION CHEMISTRY}

A high-pressure ion source has been developed and incorporated into a tandem mass-spectrometer system for the study of ion-molecule reactions. This source was designed for the production of ions that require more than one or two ion-molecule collisions for their synthesis. Furthermore, ions initially produced in excited states are deexcited by collisions in the source prior to their use in the study of collision-induced dissociation thresholds. Figure 14 is a sketch of the apparatus for these studies. The rather long path between the primary ion source, which operates at pressures of up to a few torr, and the collision chamber insures the nearly complete separation of source neutral gases from the scattering gas in the collision chamber.

The first material investigated in this apparatus was hydrogen. The binding of the proton to the hydrogen molecule in the $\mathrm{H}_{3}+$ ion presents a problem of considerable interest to the theoretical chemist. Experimental estimates of this binding energy based on kinetic studies are significantly lower than values obtained from supposedly accurate calculations. The $\mathrm{H}_{3}+$ ion represents the simplest polyatomic system available for theoretical study and is therefore especially important as an object of quantum mechanical calculations.

The experiment on the hydrogen system consisted of generating $\mathrm{D}_{3}+$ in the high-pressure ion source, separating it by magnetic mass analysis from all other ions prepared in the source, and introducing it with controlled kinetic energy into the collision chamber, which contained a neutral inert gas (helium, neon, or argon). The collision of $\mathrm{D}_{3}+$ with the inert gas produced $\mathrm{D}^{+}$ions and $\mathrm{D}_{2}$ molecules. The yields of $\mathrm{D}^{+}$ions were determined by mass analysis, by means of the quadrupole mass filter, of the ions emerging from the collision chamber. From the variation of the amount of $\mathrm{D}^{+}$ produced with the initial kinetic energy of the $D_{3}+$ ion beam, the threshold energy for the dissociation reaction, in other words, the binding energy of $\mathrm{D}^{+}$ to the $\mathrm{D}_{2}$ molecule, was determined.

The measurements demonstrated that there is significant shift in threshold energy for $\mathrm{D}_{3}+$ dissociation with increasing pressure in the primary ion source. The high-pressure threshold provided a basis for estimating the proton affinity of $\mathrm{H}_{2}$ to be $\approx 5 \mathrm{eV}$, in excellent agreement with recent theoretical values.

In the investigation of the kinetics of the deexcitation of $\mathrm{H}_{3}+$, a very efficient process requiring only one collision, on the average, was discovered. A resonant proton transfer mechanism is suggested which produces ground-state $\mathrm{H}_{3}{ }^{+}$and highly excited $\mathrm{H}_{2}$ neutral molecules. This mechanism requires the transfer of a proton from $\mathrm{H}_{3}+$ to neutral $\mathrm{H}_{2}$ to take place rapidly, with very little interaction between donor and acceptor hydrogen molecules.

\section{THEORETICAL CHEMISTRY}

\section{Semiempirical Molecular Orbital Theory}

Several semiempirical quantum-mechanical methods developed recently show promise as tools 
for understanding certain empirical correlations observed in reactions that appear to have regularities in electron density and potential energy changes. A major problem, however, is to define the areas of trustworthiness of the approximate theory, i.e., to determine for which of the molecular properties predictions are correct, and of these which will be fundamentally plausible and which accidental or predetermined trivially. Some of the most important questions to be asked are: How sensitive are the wave functions and hence the expectation values to the representation of molecular geometries? Are formally charged species as well represented as neutral molecules? Are nonequilibrium (including transition-state) structures acceptably represented by these theories? Work was recently completed in one area of this problem, derivation of the energetics and details of charge flow accompanying distortion of simple molecules from their equilibrium configurations. Specifically, both angular and stretching deformation effects in the first-row dihydrides were analyzed in closed form by two different approximate methods. Comparisons with experimental results, with empirical rules, and with the results of Hartree-Fock calculations confirmed that serious deficiencies exist in the extended Hückel theories. However, the true SCF(CNDO2)* method of Pople appears to be more soundly grounded in its approximations, especially in its explicit recognition of important repulsion effects, as judged by the geometric distortion analysis. This method seems at present to be the best choice for the reactivity analysis.

\section{Reactivity Correlations by Linear Free Energy Relations}

The complementary study involving statistical analysis and improvement in linear free energy (LFE) equations has progressed past the stage at which solvent effects upon the reactivity constants ( $\rho$ 's) in the Taft dual substituent equation, log $k / k_{0}=\rho_{\mathrm{I}} \sigma_{\mathrm{I}}+\sigma_{\mathrm{R}} \sigma_{\mathrm{R}}$, have been recognized and correlated with the dielectric properties of the solvent. The present stage involves the refining of the substituent constants ( $\sigma$ 's) to eliminate the small yet potentially important effects that solvent may have on these constants, especially as regards their correlation with the results of theory on intramolecular electronic effects accompanying reaction.

*Self-consistent field (complete neglect of differential overlap).
Development of somewhat more complicated models, including, for example, specific site hydrations, is being initiated to test the differences noted between the refined and unrefined LFE parameters.

\section{Ab Initio Caussian Molecular Orbital Calculations}

Gaussian orbital SCF calculations on the reaction system $\mathrm{C}_{2} \mathrm{H}_{6} \rightarrow \mathrm{C}_{2} \mathrm{H}_{5}++\mathrm{H}^{-}$were essentially completed. The energetics of the reaction calculated from theory appear reasonable when compared with experimental appearance potential data. Analysis of the electronic distributions in the $\mathrm{C}_{2} \mathrm{H}_{5}+$ ion showed the simple concept of hyperconjugation to be valid insofar as donation of labile $\pi$ and quasi- $\pi$ electrons to the empty methylene orbital is concerned. Population and true density analysis also revealed that with ion formation there occur significant $\sigma$ electron shifts which may actually exceed the hyperconjugative effects in importance. The relative importance of these effects may be better understood from consideration of the torsional conformers of the ion; detailed comparison of the wave functions for the staggered and eclipsed conformations of the ions continues. The interest in the hyperconjugation phenomenon has arisen because kinetic $\beta$-deuterium isotope effects in $S_{N} 1$ solvolysis reactions are taken to be related to hyperconjugation.

\section{Isotopic Equilibria}

Previous work at BNL on the exact formulation of the effect of anharmonicity on isotope effects was extended. The formula for the zero-point energy of a polyatomic molecule is

$$
E_{0} / h c=G_{0}+\frac{1}{2} \sum \omega_{i} g_{i}+\frac{1}{4} \sum_{i<j} X_{i j} g_{i} g_{0},
$$

where the $g$ 's are degeneracy factors, $\omega_{i}$ 's are harmonic frequencies, and $X_{i j}$ 's are usual anharmonicity corrections. $G_{0}$ has usually been ignored in calculating isotope effects on the zero-point energy. It is actually a non-negligible factor. $G_{0}$ consists of one part that arises from anharmonicity of vibration and another that arises from rotationalvibrational interaction. The latter part has now been reduced to very simple form, and the magnitude of $G_{0}$ has been calculated for a number of diatomic and triatomic molecules. For diatomic "self-exchange equilibria" such as $\mathrm{H}_{2}+\mathrm{D}_{2}=2 \mathrm{HD}$, it is found that there is neither a $G_{0}$ nor an $X_{i j}$ contribution to the equilibrium constant. For the similar triatomic equilibrium $\mathrm{H}_{2} \mathrm{O}+\mathrm{D}_{2} \mathrm{O}=2 \mathrm{HDO}$, 


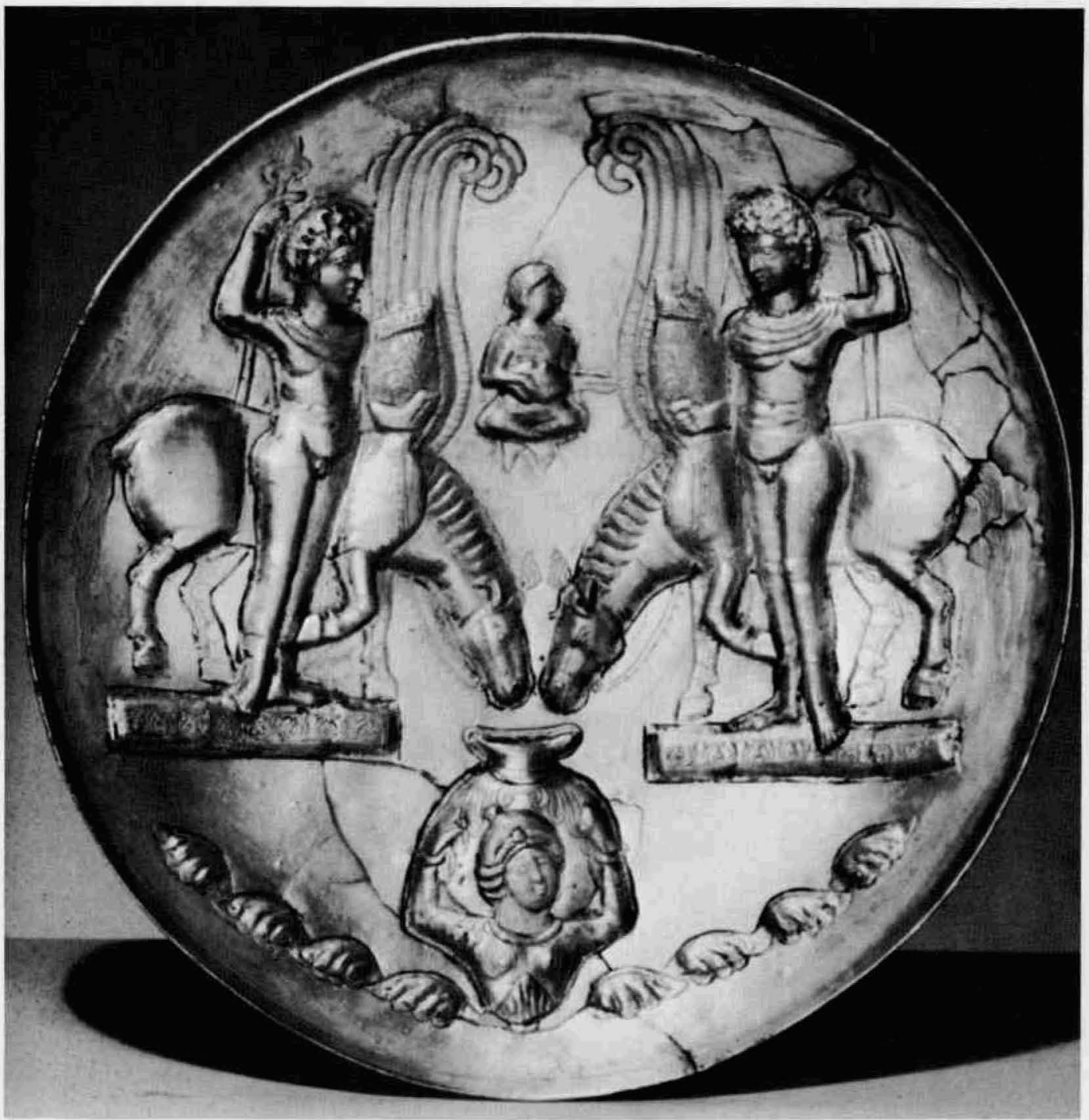

Figure 15. Sasanian silver bowl with inlaid figures (Castor and Pollux) and gilt surface decoration.

there is a large $X_{i j}$ contribution to the equilibrium constant, as has long been known, but now it is found that this contribution is almost perfectly canceled by the $G_{0}$ factor. Reasonable agreement between theory and experiment is now obtained for the above equilibrium constant.

\section{INVESTIGATIONS OF ARCHAEOLOGICAL AND HISTORICAL MATERIALS}

The tracing of geographic origins and the establishment of criteria for authentication of arti- facts of archaeological, anthropological, and artistic interest are being done at BNL by neutron activation analysis of small amounts of impurities in these objects, which are of many kinds.

During the past year many Mayan pottery objects found in Central America have been examined. Although the utilitarian pottery is variable in impurity concentration, the fine orange ware in a large central area several hundred miles across is remarkably uniform. Fine orange ware from each of two peripheral areas appears to be of common composition within each area, but the area 
compositions differ. Efforts are under way to locate pottery sources by examination of clay samples. Sasanian silver objects, made in Persia from $\approx 200$ to 600 A.D., have been investigated to explore origins and fabrication techniques. These objects have been found to have one of two basic characteristic compositions. One of the inlaid bowls studied is shown in Figure 15. Results of neutron activation analysis show that the inlays were made of the same basic typical silver alloy as the bowl itself and that a gold-silver alloy was used for the gilding. The gilt layers retain traces of the mercury used in what was evidently an amalgamation gilding technique. 


\section{Applied Science}

In January 1969 the Trustees of Associated Universities, Inc., approved changing the name of the Nuclear Engineering Department to the Department of Applied Science. The new name recognizes that much of the present work of the Department is applied scientific research rather than engineering, and also that some of its work is not of a nuclear character.

The Department continued to engage in scientific and developmental activities, principally of an applied nature, which fulfill recognized objectives of a National Laboratory and are consistent with the character of Brookhaven. These activities are of a pioneering nature with a high degree of technical sophistication; they either are required for the general research program of the Laboratory or fulfill a national need for which the Laboratory has unique capabilities.

Within this general framework, the activities of the Department can be grouped into three categories, as follows.

1. Research and development, not necessarily of a nuclear nature, directed toward applications of atomic energy.

2. Research and development, and engineering when required, in support of other scientific programs of the Laboratory.

3. Research and development in the service of other national needs, with the agreement of the AEC, in cases in which Laboratory facilities and staff capabilities are superior to those of other agencies and in which the foregoing guidelines are clearly satisfied.

BNL personnel were involved in the study of the U.S. nuclear power industry for the period 1970 to 2020 for the US AEC's Division of Reactor Development and Technology. Other work for this Division concerned the development of codes and standards applicable to the reactor field.

In the Reactor Physics Division, theoretical work and reactor analysis emphasized analytical and computer studies to promote understanding of the Liquid-Metal-Cooled Fast Breeder Reactor (LMFBR) and of the cross sections important in LMFBR calculations. This included reactor dynamics and stability, transport and slowing-down of fast spectrum neutrons, and fluid dynamics problems, as well as the analysis of fast and epithermal critical experiments. Physics work for the AEC's Division of Reactor Licensing was concerned with feedback mechanisms in power reactors and analysis of control rod worth.

The Nuclear Materials Division continued to provide technical advice and guidance to the US AEC's Office of Safeguards and Materials Management.

A meeting of the Cross Section Evaluation Working Group was hosted by BNL's National Neutron Cross Section Center in September 1968.

The program of the Liquid Metal Center of the Metallurgy and Materials Science Division is also directed toward the AEC's fast breeder reactor program.

The program on superconductivity in cooperation with the Cryogenics Group of the Physics Department was continued.

A meeting was held at BNL in November 1968 under the Japanese-American intergovernmental information exchange agreement on the radiation-produced polyethylene program and the chemonuclear reactor program. The Japanese delegation was headed by Dr. I. Munekata, Chairman of the Japan Atomic Energy Research Institute, and Dr. S. Sawayanagi, Director of the Institute's Takasaki Laboratory.

A seminar on radiation chemical processing was organized for the AEC's Division of Research and held at BNL in February 1969. It was attended by 40 faculty members from departments of chemical or nuclear engineering.

Information meetings were held at BNL for the AEC's Division of Isotopes Development on concrete-polymer materials in October 1968 and on the atmospheric sulfur pollutants program in November 1968 . These were attended by representatives of industrial and governmental organizations.

The close collaboration that has existed for several years between the Hot Laboratory Division and the Medical Department continues to increase, and the development of radioisotopes for medical applications constitutes a significant part of the Division's program. The Hot Laboratory 
Division has also collaborated with the Chemistry Department in writing a computer program to calculate nuclear level densities.

\section{EVALUATION AND TECHNICAL ASSISTANCE}

The Evaluation and Technical Assistance Group continued to assist the AEC in program reviews and the evaluation of specific areas of interest. BNL personnel participated in the study of the U.S. nuclear power industry for the Reactor Development and Technology Division of the AEC. They served as members of the Research and Development Subcommittee and as a special working group to help in writing the final systems analysis report. Assistance was rendered in writing several sections of the final report, in evaluating the linear program output, and in devising ways of presenting the data, which included the preparation of over 100 graphs encompassing each reactor case investigated.

Final draft reports of ihe heavy-water-moderated, organic-cooled and the high-temperature, gas-cooled converter systems, and advanced converter task force and thorium systems task force evaluations were reviewed, and comments were submitted to the AEC.

BNL is participating in the development of codes and standards applicable to the reactor field, as part of an AEC-sponsored program at Oak Ridge National Laboratory and Atomics International for the water-reactor and LMFBR programs, respectively. Detailed comments on some 60 standards have been submitted thus far. BNL is also represented on subcommittees of the USASI-NSB* Standards Committee N-101.

A study was initiated to investigate the effect of pumped storage on the plant factor of power plants.

\section{REACTOR PHYSICS}

\section{Theoretical Reactor Physics}

Theoretical reactor physics studies have treated problems in reactor dynamics, neutron slowingdown and transport, and fluid dynamics.

Perturbation techniques were used for analyzing space and space-time problems with nonlinear feedback. The method has been applied to problems in which the reactor starts from zero power as well as from equilibrium. The technique is well

*USA Standards Institute, Nuclear Standards Board. suited to the investigation of the effect of the sodium void coefficient in fast reactors. At the same time it casts additional light on the method of solution by modal analysis. A perturbation method based on the dominant roots of the linearized equations has been examined and applied to excursions and cases with xenon poisoning. Higher order corrections to the prompt jump approximation have given a firmer basis to the conventional prompt jump treatment. This extension has shown the way to greatly increase the size of time steps in the numerical integration of the reactor kinetics equation. The effect of delayed neutrons on power oscillations has been studied, and conditions have been established under which delayed neutrons allow unstable limit cycles to exist in reactors in which feedback is linear with power.

Study of unstable pulsed neutron decay has elucidated the behavior of the decay constant for large bucklings in cases in which the decay constant is no longer discrete and real. Conditions under which the behavior at large times continues to resemble an exponential decay were examined. The experimental techniques of measuring time constant vs buckling and relaxation length vs poisoning and vs frequency of oscillation of driving source are all fundamentally related. These techniques were analyzed, and the changes in the nature of the measured quantity when a parameter (buckling, poisoning, frequency) exceeds a critical value were studied. Time-dependent slowing-down has been investigated for fast subcritical assemblies with use of the exact elastic scattering kernel and diffusion or asymptotic transport theory.

Two-phase flow studies have shown the importance of a crucial condensing length as well as the crucial boiling length. The analogy between boiling and condensing flows was treated. The method of iterative solution has been applied to several problems, including resonance absorption. A more rigorous analysis of the method has shown how to establish error bounds on the solutions.

Reactor Physics Analysis. The work of the Reactor Analysis Group included studies of experiments, development of analytical methods, and code development.

The ZPR 3 assemblies 11 and 48 were studied as part of the BNL contribution to the Cross Section Evaluation Working Group intercomparison in the Evaluated Nuclear Data File for Reactor 
Applications (ENDF/B) program. Analysis of Los Alamos experiments with critical reflected plutonium spheres yielded information on energy and angular distribution of spectra to be used for setting up tests of ENDF/B data. Some analysis of heterogeneity effects in fast lattices was carried out.

The poisoned $\mathrm{Pu}-\mathrm{D}_{2} \mathrm{O}$ lattice experiments performed at BNL have been analyzed with use of ENDF/B data and as nearly rigorous methods as possible. Monte Carlo results were coupled with those from the HAMMER code. Up-scattering through the low energy $\mathrm{Pu}^{240}$ resonance was investigated. Some thermal, slightly enriched uranium-water critical experiments were recalculated for testing ENDF/B data.

Programming was completed on NøAH, a onedimension one-group dynamics code with a number of feedback and control options. This code produces an equilibrium power distribution and then calculates the evolution of a transient. In another code, two-phase flow was incorporated in a steady-state calculation of power distributions.

A new Monte Carlo resonance capture code, REPCDC, has been produced. Using ENDF/B data and an improved statistical treatment of unresolved resonances, the code is useful for fast and thermal reactor calculations. The DOT two-dimension $S_{N}$ code was modified to allow rigorous inclusion of a transverse buckling.

The 2DB two-dimension multigroup diffusion code was brough into operation on the BNL computer system together with an associated perturbation code. ENDF/B service routines obtained from other laboratories required a number of modifications and corrections.

A synthesis approach has been used for solving the transport equation for the space, energy, and angle flux in multiregion critical experiments. Discontinuous trial functions and improved weighting techniques were employed. Numerical tests show the method to be effective.

Division of Reactor licensing. Technical assistance to the AEC's Division of Reactor Licensing included work on reactor temperature and power coefficients and on reactor stability, and study of control-rod calculational methods, as well as other day-to-day problems.

An extensive series of calculations was carried out for comparison with zero-power experimental data on reactivity variation with water temperature and boron content in a pressurized water reactor (PWR). Satisfactory agreement was obtained over the range studied. Calculations for the power coefficient of a PWR were begun. Consistent power and cross-section distributions are being obtained at several power levels. The uncertainty in the Doppler coefficient of a PWR due to uncertainties in the resonance parameters was evaluated.

Calculation of control-rod worths in PWR's has been studied and comparisons have been made with experimental data on worth of control clusters. A survey of methods of calculating rod worth in boiling water reactors was begun.

The stability of nonboiling and boiling reactors has been studied for a range of reactor sizes with various feedback parameters.

The flux of high energy neutrons on the pressure vessel of a PWR was calculated in one- and twodimension transport and removal studies.

\section{Experimental Reactor Physics}

The experimental reactor physics program includes measurements of neutronic parameters in clean and well-defined assemblies of fissionable and moderating materials. These experiments are coordinated with parallel programs in theoretical reactor physics. The intent is to provide data over a wide range of variables which can be used as bench marks in evaluating the accuracy of theoretical methods and neutron cross sections used in designing nuclear reactors. In addition to the experimental program, a compilation of data from other fast reactor critical experiments is being prepared.

The FS-1 (the first fast critical assembly at BNL) contains $\approx 150 \mathrm{~kg} \mathrm{U}^{235}$. It is being used to study heterogeneity and spectral effects in fast reactors. For this purpose a central cell was constructed of especially well machined pieces of $\mathrm{U}^{235}$, graphite, and steel having the same densities as the rest of the critical assembly. Measurements of neutron capture and fission distributions at various points within the cell are indicative of neutron spectra and self-shielding and will be compared with theoretical predictions. In this manner the theoretical methods may be improved to properly account for the heterogeneity associated with fuel rods and coolant channels in fast reactors. Concurrent measurements of neutron spectra in FS-1, using proton recoil chambers, are being used to study the neutronics of fast reactors.

Measurements are being made in a subcritical assembly of plutonium and heavy water to assist in the evaluation of plutonium neutron cross- 
section data in the epithermal energy range. The lattices of $\mathrm{Pu}-\mathrm{Al}$ rods in heavy water are undermoderated and poisoned with boron to emphasize the importance of epithermal neutrons. Measurements of material buckling, neutron flux distributions, and neutron activation rates were made in 11 lattices having various fuel to heavy water ratios. A preliminary examination of the experimental data has suggested that the ratio of neutron capture to fission in $\mathrm{Pu}^{239}$ is somewhat greater than that published in the ENDF/B library. A detailed analysis of the data is yet to be made.

A compilation of data from fast reactor critical experiments throughout the United States is being prepared. This past year all the data were collected and a draft of the compilation was prepared. The completed compilation is expected to be especially useful in the design of fast reactors. A second volume, containing data from foreign countries, has been begun and will follow soon after the first.

\section{NUCLEAR MATERIALS}

The Nuclear Materials Division, which acts as a technical support organization for the US AEC's Office of Safeguards and Materials Management (OSMM), continued to provide technical advice and guidance in a number of areas related to research and development in the safeguarding of nuclear materials.

A simple version of the "strategic point" concept, an approach for safeguarding nuclear facilities, has been studied to provide a basis for comparison with the material balance concept currently employed in the United States. Similarities and differences are being clarified to compare such factors as cost effectiveness, credibility, and political acceptability.

On the basis of visits and discussions with OSMM contractors and other institutions with similar interests, and after evaluation of the status of instrumentation and data-handling in this country, recommendations have been prepared for conducting future research and development. The Division has also reviewed a number of proposals for research and development received by the OSMM.

In conjunction with the need to develop new techniques for inventory measurement, a survey was made to categorize by type and form the special nuclear materials that are being processed in industry. The survey included visits to typical nuclear facilities, and an informal report was submitted to OSMM.

Scintillation detectors and analyzers now used by inspectors for the United States and the International Atomic Energy Agency (IAEA) were evaluated. In cooperation with the Arms Control and Disarmament Agency, several portable passive analysis instruments are being procured for field tests. Techniques in high-resolution $\gamma$-ray spectroscopy and passive neutron measurements that might be applied to safeguards have been reviewed.

The program of evaluating seals as safeguard tools is being conducted in two parts. First, IAEA and Internal Revenue Service seals are being tested to determine their effectiveness under present IAEA procedures. Second, a system-oriented analysis of sealing operations has been started to determine the applicability and limitations of seals as compared with alarm devices and attackresistant volumes.

System studies at the National Bureau of Standards and at Pacific Northwest Laboratory are being continued. The Division closely follows these studies, since they will be used to identify the sensitive points in the nuclear fuel cycle and to determine where special emphasis should be given to research and development.

A report giving an estimate of IAEA costs and manpower requirements under the nonproliferation treaty was prepared for the OSMM, for presentation to the U.S. Senate's Foreign Relations Committee.

A glossary of special terms used in connection with safeguards is being assembled. The information is being stored on IBM cards to permit sorting and classification.

\section{NATIONAL NEUTRON CROSS SECTION CENTER}

The National Neutron Cross Section Center (NNCSC) maintains and develops a computerized system for the storage and retrieval of neutron cross-section information. The information handled is divided into two classifications, experimental and evaluated. The evaluated data files consist of data choices from among the experimental data files and nuclear model calculations. The NNCSC also advances new methods of cross-section analysis.

The program is divided into three major areas: (1) support of the experimental data file, scisRs; (2) support of the evaluated nuclear data file, 
ENDF; and (3) generalized computer development to achieve improved data-handling procedures and automated publication of data and bibliographies.

\section{Experimental Data}

All the energy-dependent data and resonance parameters from the old Sigma Center files have been prepared for insertion into the scisRs library. Thermal cross-section values from the Second Edition of Neutron Cross Sections and its supplements (BNL 325, 1958-1966) have been prepared for merging into scisrs. Completion of this task will conclude the effort to bring all old usable Sigma Center file data into the data libarary.

Efforts were begun to detail the "content" of scisRs-II, a new data storage and retrieval system. Assistance was obtained from experimental and evaluation groups in ascertaining what was necessary and what was useful to make the new library an appropriate tool for all users. Input format problems were investigated in collaboration with the NNCSC programmers and personnel from the data centers of the IAEA and the European Nuclear Energy Agency (ENEA).

A system for sending out author proofs in the form of listings and curves has been completed. It is planned to begin sending these out for all new data.

\section{Evaluated Data}

The first version of the ENDF/B library was distributed to cross-section users in the United States and Canada and to member countries of the ENEA. The ENDF/B library was assembled in a cooperative program among US AEC-supported laboratories to increase the number of completed data sets available to reactor designers. These tasks are performed by the Cross Section Evaluation Working Group. A meeting of this group took place at BNL September 16-18, 1968. In addition to crosssection evaluation the meeting was devoted to defining ground rules for testing data, defining the criteria for re-evaluation of data, and conducting seminars and workshops on special topics.

A study of the resolved resonance region of $\mathrm{Co}^{59}$ was initiated. To date the work has shown that while one bound level is needed to match the thermal capture data, two bound levels (one for each $s$-wave spin state) are needed to satisfy polarization data.

Previously reported calculations for holmium [Stephenson and Ferrer, (A) Bull. Amer. Phys.
Soc. 12, 650 (1967)] were extended. Resonance parameters for 76 resonances of holmium below $500 \mathrm{eV}$, selected from the literature and from values compiled and recommended in BNL 325, were used as the starting point in fitting the total neutron cross-section data to a Breit-Wigner multilevel scattering and single-level absorption formula. The addition of two bound levels, one for each $s$-wave spin state, yielded a calculated ratio of thermal neutron capture cross sections for the two spin states which agreed with experiment $(\approx 60 \%$ for $J=3$ ), as did the calculated value of the thermal capture cross section, $67 \mathrm{~b}$. In addition, the two bound levels enabled the fit of the total crosssection data to be extended to very low energies $(0.2 \mathrm{mV})$. The energy-dependent paramagnetic scattering cross section $(23.5 \mathrm{~b}$ at $0.0253 \mathrm{eV})$ and the capture and scattering resonance integrals $(\approx 672$ and $125 \mathrm{~b}$, respectively) were also calculated.

Neutron cross sections for several isotopes have been calculated with use of both spherical and deformed nuclear potentials.

Calculations for the isotopes $\mathrm{U}^{238}$ and $\mathrm{Cf}^{252}$ which exhibit rotational spectra have been carried out by using the coupled-channel code JUPITER for both the $2^{+}$and $4^{+}$states. When the $\mathrm{U}^{238}$ results were compared with experiment, the agreement was found to be superior to that obtained when only the $2^{+}$state was coupled or when an adiabatic approximation was assumed. Since no experimental data existed for $\mathrm{Cf}^{252}$, the calculations must be considered tentative.

Further analysis on $\nu_{p}$, the average number of prompt neutrons per fission, and $E_{k}$, the average kinetic energy of fission fragments, has shown improvement when a $Z^{2} A^{1 / 2}$ correlation is assumed rather than the conventional $Z^{2} / A^{1 / 3}$.

The influence of direct reactions on cross-section distributions in the continuum region is being investigated numerically with the help of random matrix techniques. The results will be compared with those of the older Ericson method.

An effort is being made to derive an analytic expression for the two-angle distribution law corresponding to the $F_{2}$ ensemble of unitary matrices. The single-angle distribution law was derived previously.

The polarization studies in the continuum region originally limited to incident spin-1/2 polarized nuclei were extended to include incident polarized nuclei of any spin, provided that spin tensor moments of higher order vanish. The 
methods were also applied to the calculation of the average polarization induced by the scattering of unpolarized nuclei.

\section{Computer Applications}

A new data storage and retrieval system was developed to replace scisRs; its tentative name is scisRs-II. The main characteristics of the new system are that bibliographic and numeric information will be stored separately and that the system format will be expandable to include a wide variety of data. An elemental version of the system now exists. This program will retrieve data based on criteria such as $Z, A$, reaction type, data type, and energy range. The first-round scisRs-II retrieval package has been extended to include CALCOMP-835 output. The scisRs-I to scisrs-II translator is in an advanced state of development. Tentative agreement has been reached on the specifications for a center-to-center transmission format.

A computer routine is being prepared to plot an evaluated curve or calculated curve over scisRs data, including its error bands. This routine will be incorporated as an option into multilevel codes to provide a graphic check of the fit of calculation to data within the error bands.

Progress was made in finding a way to treat angular distribution data taken directly from the scisRs library in such a manner that usable computer-based plots could be obtained, including curves through the data points. Studies are continuing on ways to reduce the amount of human labor involved in the process, which is still large.

A study of the computation needs of the NNCSC clearly indicates the need for a computer that can be devoted largely to data file manipulation. Bids from several manufacturers have been received in response to specifications issued by BNL. A computer configuration has been selected and submitted to the AEC for approval.

\section{METALLURGY AND MATERIALS SCIENCE}

\section{Sodium Technology Program}

The corrosion behavior of fuel-cladding materials in sodium and the chemistry of solutions of impurities in liquid sodium are being studied in support of the LMFBR program.

Corrosion tests are conducted in several natural and two forced circulation loops. Alloys under test include austenitic stainless steels, cobalt-base al-

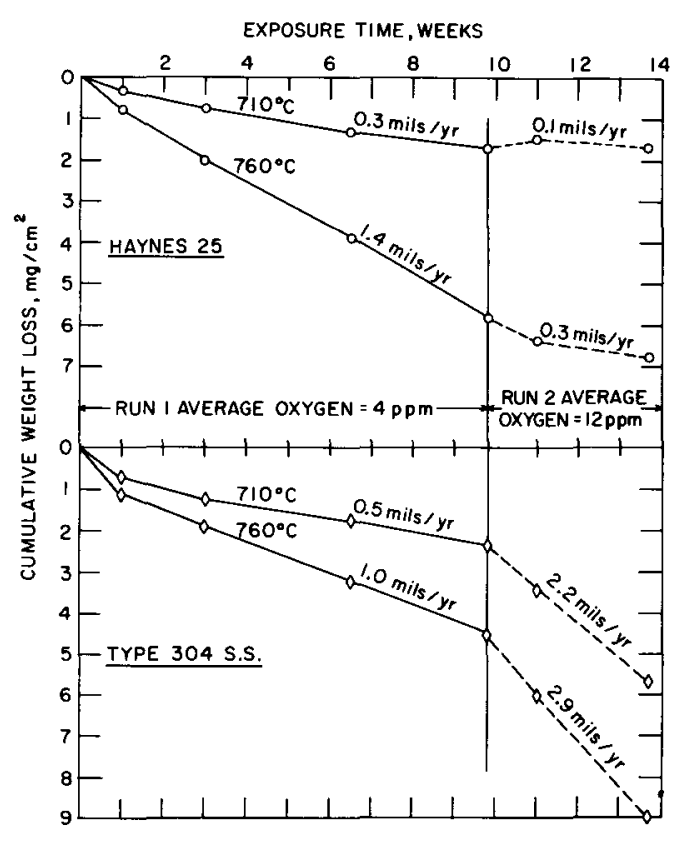

Figure 1. Effect of oxygen on corrosion rate of Haynes 25 and type 304 stainless steel. Velocity $=22 \mathrm{ft} / \mathrm{sec}$. Haynes 25: $20 \% \mathrm{Cr}, 15 \% \mathrm{~W}, 10 \% \mathrm{Ni}, 2 \% \mathrm{Fe}$, balance Co. Type 304 stainless steel: $19 \% \mathrm{Cr}, 10 \% \mathrm{Ni}$, balance $\mathrm{Fe}$.

loys, and refractory vanadium-, niobium-, and molybdenum-base alloys. Corrosion rates calculated from weight-change data from the first forced circulation loop test are shown in Figure 1. At the low oxygen concentration the weight losses of both alloys are approximately the same; however, at the higher oxygen concentration (12 ppm) the corrosion rate of the type 304 stainless steel increased by a factor of 3 to 4 , while the corrosion rate of Haynes 25 decreased by a factor of 3 to 5 . The effect of these test variables on surface composition of the two alloys, as determined by $\mathrm{x}$-ray fluorescence, is shown in Figure 2. The lower weight losses of Haynes 25 in the sodium containing 12 ppm oxygen may be due to the observed increase in iron on its surface.

Refractory metal alloys were evaluated in a second forced circulation loop in the temperature range $710^{\circ}$ to $760^{\circ} \mathrm{C}$. All the vanadium-base alloys gained weight in sodium containing $1 \mathrm{ppm}$ oxygen, probably because of their reaction with nitrogen and carbon in the sodium. Hardness measurements showed that the specimen had "disturbed layers," which indicated that significant changes in mechanical properties had occurred. The vanadium alloys all scaled badly in the 


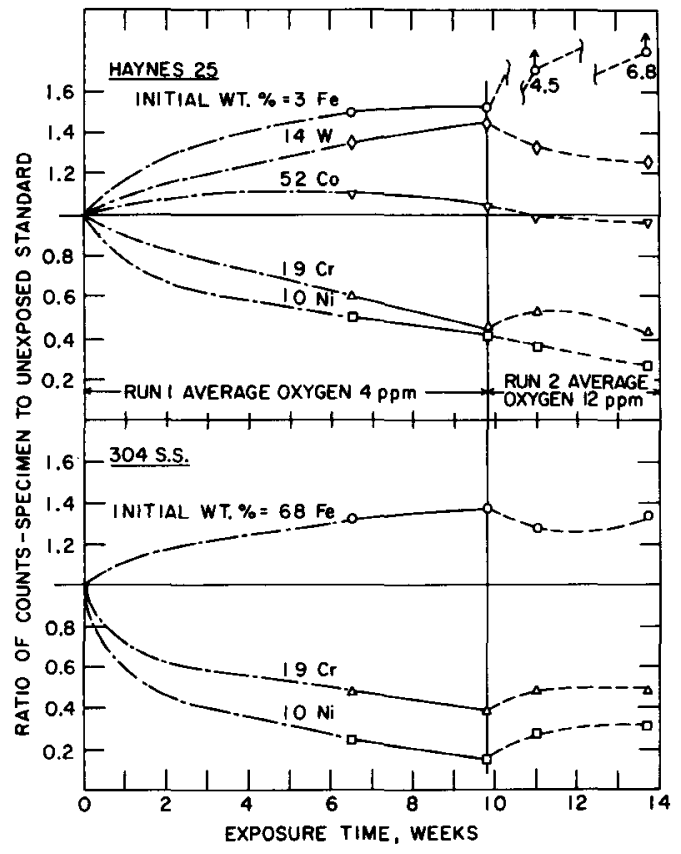

Figure 2. X-ray fluorescence results (Forced Circulation Loop 1).

sodium containing $3 \mathrm{ppm}$ oxygen and lost considerable weight. The $\mathrm{Nb}-1 \% \mathrm{Zr}$ alloy behaved similarly, but the molybdenum alloy was virtually unaffected by exposure to sodium of either purity. Except for molybdenum, these materials would not be acceptable LMFBR cladding materials if used in sodium containing $\approx 1$ to $3 \mathrm{ppm}$ oxygen at $710^{\circ}$ to $760^{\circ} \mathrm{C}$.

The effects of velocity, oxygen concentration, and temperature on the corrosion rate of pure metals in sodium are being studied. At $600^{\circ} \mathrm{C}$ the rate of corrosion of pure iron increases almost linearly with velocity in the range 1 to $2.5 \mathrm{ft} / \mathrm{sec}$ in sodium containing $60 \mathrm{ppm}$ oxygen.

The solubilities of nickel and iron in sodium have been determined from $450^{\circ}$ to $600^{\circ} \mathrm{C}$ and found to be $\log _{10}(\mathrm{ppm} \mathrm{Ni})=1.545-1230 / T\left({ }^{\circ} \mathrm{K}\right)$ and $\log _{10}(\mathrm{ppm} \mathrm{Fe})=5.16-4300 / T\left({ }^{\circ} \mathrm{K}\right)$, respectively. The solubility of iron in sodium is independent of the chemical activity of $\mathrm{Na}_{2} \mathrm{O}$ dissolved in sodium up to a value of 0.2 ; at higher $\mathrm{Na}_{2} \mathrm{O}$ activities, the apparent solubility of iron increases. The solubilities of $\mathrm{Ni}, \mathrm{Fe}$, and $\mathrm{Cr}$ (in $\mathrm{Na}$ containing $<1$ ppm oxygen) at $650^{\circ} \mathrm{C}$ (the design LMFBR reactor outlet temperature) are estimated to be 1.6, 3.2 , and $0.3 \mathrm{ppm}$, respectively.

Uranium has been found to remove oxygen from sodium at $500^{\circ} \mathrm{C}$ at a rate controlled only by the concentration and diffusion rate of oxygen in - the sodium. The rate of weight gain of the uranium thus offers an extremely accurate and specific method for determining oxygen concentrations in sodium to levels from $<1$ to $>30 \mathrm{ppm}$ oxygen. This reaction is also applicable to the determination of precise mass transfer coefficients in sodium.

The electrochemistry of solid electrolyte galvanic cells is being studied to improve commercial cells and to determine the interactions between oxygen and other impurities in liquid sodium. The conduction mechanism in the $\mathrm{ThO}_{2}-\mathrm{Y}_{2} \mathrm{O}_{3}$ solid electrolyte is largely ionic in the oxygen pressure range from air to $10^{-30}$ atm at temperatures between $300^{\circ}$ and $850^{\circ} \mathrm{C}$. The $\mathrm{Cu}-\mathrm{Cu}_{2} \mathrm{O}$ reference electrode in the commercial meters was found to be sluggish below $350^{\circ} \mathrm{C}$. Alternative reference electrodes of $\mathrm{Na}-\mathrm{Na}_{2} \mathrm{O}$ (sat.) and gaseous $\mathrm{O}_{2}$ improve the cell performance substantially.

Studies with these cells have shown that the oxygen activity in sodium contained in stainless steel vessels is buffered by the chromium component of the steel. With use of an $\mathrm{Na}-\mathrm{Na}_{2} \mathrm{O}$ (sat.) reference electrode, an expression for the solubility of $\mathrm{Na}_{2} \mathrm{O}$ in sodium from $350^{\circ}$ to $600^{\circ} \mathrm{C}\left[\log _{10}\right.$ (ppm oxygen $\left.)=6.55-2600 / T\left({ }^{\circ} \mathrm{K}\right)\right]$ has been obtained which agrees well with experimental solubility measurements.

Development of an electrochemical meter for measuring carbon activities in sodium is in progress, based on the use of $\mathrm{CaC}_{2}$ as electrolyte and iron as a diffusion barrier for carbon between the electrolyte and the liquid sodium. Preliminary experiments using a cell made of graphite and $\mathrm{ZrC}$ electrodes have been successful, and the first results with sodium are encouraging.

Reactions between excess liquid sodium and various gases have been studied. The reaction of $\mathrm{HCl}$ with both sodium and sodium-barium solutions takes place in two consecutive stages, the first producing $\mathrm{H}_{2}$ and $\mathrm{NaCl}$ (or $\mathrm{BaCl}_{2}$ ) and controlled by processes of a physical nature, the second consisting of the chemical reaction between $\mathrm{H}_{2}$ and sodium. The reaction of $\mathrm{H}_{2}$ with sodium containing $18 \mathrm{ppm}$ oxygen at $400^{\circ} \mathrm{C}$ shows first-order behavior with respect to $\mathrm{H}_{2}$, with a simultaneous decrease in oxygen activity. The reaction with carbon monoxide proceeds in three stages at $200^{\circ} \mathrm{C}$ : an induction period accompanied by film formation, a rapid absorption stage in which the metal surface appears clear, and a second, slow stage with no further film formation. 
Stainless steel container surfaces have been found to contribute 1 to $2 \mu \mathrm{g}$ per linear inch of $1 / 2$-in.-o.d. tubing to the oxygen content of sodium samples analyzed by the amalgamation method without prior extrusion of the sodium. Determinations of trace-metal impurities in sodium, such as $\mathrm{Cr}, \mathrm{Ni}$, $\mathrm{Cu}, \mathrm{Ca}$, and $\mathrm{Mg}$ down to the $1 \mathrm{ppm}$ level, are now performed routinely by atomic absorption spectroscopy. The samples are now dissolved in $n$-butyl chloride to avoid the strong $\mathrm{NaOH}$ produced by dissolving the sodium in alcohol, which attacked the glass vessels and contaminated the samples.

\section{Irradiation and Point Defect Studies}

In the irradiation program on fuel-cladding materials for fast breeder reactors, cobalt-base alloys are being developed as an advanced cladding material, and methods of improving the postirradiation high-temperature ductility of austenitic stainless steels are being studied. The results show that cobalt-base alloys are promising cladding materials for use at a temperature of $650^{\circ} \mathrm{C}$ or higher and that refining the grain structure by thermal-mechanical processes improves significantly the high-temperature ductility of irradiated cobalt-base alloys and austenitic stainless steels.

Studies of irradiated iron and niobium have shown that neutron irradiation can change the rate-determining process of plastic deformation. This result, which is new, presents a way of studying the influence of various defects upon such ratedetermining processes in crystals. Deformation studies of tungsten crystals have shown, for the first time, quantum-mechanical tunneling of dislocations. Experiments measuring the influence of impurity and simple point defects in such processes indicate clearly that the barrier is purity dependent.

Radiation damage studies have been made in platinum. The defect density and the distribution of vacancy clusters were measured by field ion microscopy; the temperature dependence of the yield stress of irradiated specimens was also measured. The irradiated specimens were annealed at a variety of temperatures, and the change in the distribution of vacancy clusters was measured, as well as the temperature dependence of the yield stress. Under these conditions a constant activation energy for the hardening process is observed. This indicates that a constant barrier to dislocation motion exists in the irradiated and in the irradiated and annealed specimens.

\section{Liquid Metals}

A model for the composition dependence of the resistivity of binary mercury alloys involving a simple volume-dependent pseudopotential has been developed. Anomalous electrical resistivities of alloys exhibiting miscibility gaps have been observed and may be interpreted as arising from critical concentration fluctuations near the consolute temperatures.

Molecular dynamic techniques have been used to investigate the mass effect in diffusion in liquid mixtures. Whereas a heavy impurity appears to obey the Enskog rule, the motion of a light impurity is governed by the relaxation of the surrounding liquid.

In current investigations the electromigration properties of combinations of polyvalent and monovalent metals in binary alloys have been found to deviate from those obtained with the previous model for electromigration. These deviations probably arise from the widely differing electron densities of the components.

\section{Superconductivity}

Equilibrium Irreversibility. From a generalized treatment, hysteresis can be subdivided into two types that differ by continuity and discontinuity of the Gibbs free energy within the hysteretic domain. The existence of isothermal reversible paths peculiar to the hysteretic domain is associated with continuity in the Gibbs free energy. The condition that this energy be continuous in a hysteretic domain also requires that different states in the domain have the same free energy. Irreversibility, which arise as a result of equilibrium, occurs when intrinsic restraints exist for the number of and interactions between the generalized forces and displacements that define the Gibbs function. These general concepts have been applied to properties of hysteretic superconductors to account for and determine the unique restrictions for new reversible processes that are created by the hysteretic states. The data show that in fact, as well as in principle, the properties of hysteretic superconductors lie between two limiting types, one of which can be explained from equilibrium concepts.

Superconducting Films. Experimental and theoretical work on films is concerned with two problems: (a) factors influencing the transition temperatures and critical field of ultrathin films, and (b) the dynamics of superconductors, including their flux flow and fluctuation resistivity. 
Experiments on thin layers of soft metals such as $\mathrm{Al}, \mathrm{Sn}, \mathrm{Pb}$, and In have finally led to a unified explanation of the increases in the critical temperature $\left(T_{c}\right)$ of these films. The recent theoretical ideas of McMillan and the suggestion that the phonon frequencies are drastically lowered in disordered and very thin films have been used to explain the results in "soft" metal films. This work has been extended to transition metals such as niobium, in which the $T_{c}$ is drastically lowered in disordered films. In further studies, low energy diffraction work and Auger spectroscopy will be used to help characterize metals in the ultrathin film state. Measurements made of the critical field in ultrathin tin and gallium films allow estimates of the spin-orbit scattering time and the upper critical field obtainable in a given metal.

Initial measurements on the fluctuation rounding of the resistive transition are being continued as a function of temperature and applied magnetic field, with specific emphasis on theoretical work which indicates an anomalous behavior near the transition due to the nonanalytic character of expansions in powers of the order parameter near the transition.

Nuclear Magnetic Ordering and Very Low Temperatures. Measurements of the nuclear magnetism of solid $\mathrm{He}^{3}$ by the method of nuclear resonance absorption have been made down to a temperature of $0.006^{\circ} \mathrm{K}$ and reveal the onset of an ordered state. An attempt is being made to reach the region of $0.001^{\circ} \mathrm{K}$ by nuclear adiabatic demagnetization. It should be possible to observe the complete transition to the ordered nuclear state in this temperature region.

The apparatus has a downtime for the range five to ten millidegrees of about two days. Temperature is measured by the nuclear magnetic resonance absorption of platinum powder. With this method of thermometry, heat contact problems at these very low temperatures can be studied. The techniques developed should prove valuable for research in general in the millidegree region.

\section{CHEMISTRY}

\section{Fused-Salt Chemistry}

Cold-neutron scattering definitely proved its usefulness for study of the fused-salt state in an extended series of experiments. Neutron velocity spectra were obtained at four different scattering angles for zinc chloride at $400^{\circ} \mathrm{C}$, for potassium
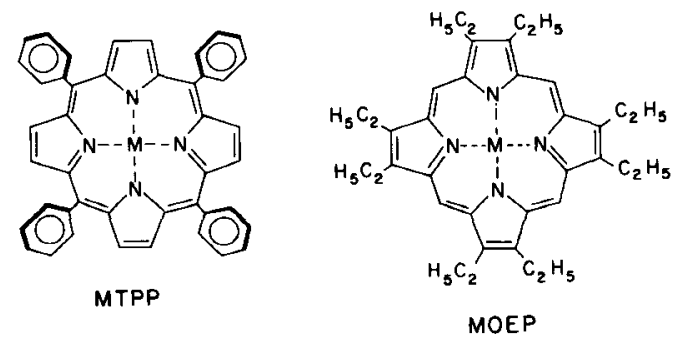

Figure 3. Structures of two typical metalloporphyrins. $M$ is a divalent such as $\mathrm{Mg}, \mathrm{Zn}$, or $\mathrm{Co} ; T P P$ is tetraphenyl porphyrin; $O E P$ is octaethyl porphyrin.

chloride at $850^{\circ}$, and for the equimolar mixture at $500^{\circ}$; and at three different angles for $2 \mathrm{ZnCl}_{2}: \mathrm{KCl}$ at $400^{\circ}$. The results for zinc chloride may be interpreted in terms of a quasi-lattice liquid structure having a triatomic "primitive cell" giving rise to 6 distinct phonon modes, 3 of which are doubly degenerate. The dispersion of the longitudinal acoustic mode provides a tentative estimate of the sound velocity in zinc chloride at $400^{\circ} \mathrm{C}$ at 535 meters sec-1 and also provides an estimate of $3.8 \AA$ for the chlorine-chlorine distance between neighboring "primitive cells." The results for potassium chloride are consistent with a quasi lattice having a diatomic "primitive cell" giving rise to just 4 distinct phonon modes, 2 of which are doubly degenerate.

\section{Porphyrins}

Porphin is the parent compound of chlorophyll, which converts visible light into chemical energy in plants, and of heme, the oxygen-carrying blood pigment. The oxidation-reduction properties (loss or gain of electrons) of porphin derivatives, or porphyrins (see Figure 3), play a key role in their biological function in photosynthesis and respiration. In recent work information about the oxidized and reduced states has been obtained by electrochemical and spectroscopic techniques.

Both cation radicals (in which the compound has lost one electron and is thus positively charged) and dications (two electrons lost) of porphin derivatives were prepared by electrolytic and chemical oxidation $\left(\mathrm{XeF}_{2}, \mathrm{Cl}_{2}, \mathrm{Br}_{2}\right.$, and $\mathrm{I}_{2}$ were used as oxidants). The resulting data provided information about ease of oxidation, stability, and magnetic and optical properties. The latter were quite consistent with molecular orbital calculations and thus supported a theory developed to predict porphin reactivity and characteristics. Of 
particular interest were green radical ions obtained from doubly oxidized cobalt porphyrins, where one electron was abstracted from the metal and the second from the porphin ring. It has long been known that the green "Compound I" forms of the heme enzymes, catalase and peroxidase, have also lost two electrons, one from the metal, the other from sources unknown. The results with the cobalt compound suggest a similar behavior for the heme.

The cation radicals of ethylchlorophyllide $a$ and chlorophyll $a$ were also investigated. The magnetic (electron paramagnetic resonance) spectra of these radicals closely resemble the electron spin resonance signal found in the initial stages of photosynthesis. These results lend further support to the current theory that cation radicals of bacteriochlorophyll are the first steps in photosynthesis.

\section{Graphite Vaporization}

The study of the graphite vaporization process was extended to a still higher temperature region, up to nearly $4000^{\circ} \mathrm{K}$. In the process, an important source of error was found in temperature determined by an optical pyrometer. In extreme cases the observed temperature was several hundred degrees too low. The effect was noticed while graphite specimens were being resistively heated in 0.5 atm He. The photoelectric pyrometer output dropped rapidly when the temperature reached $3200^{\circ} \mathrm{K}$, even though the power dissipated remained constant. The drop was apparently caused by microscopic carbon particles which formed in the vapor from the recombination of evaporated species. The absorbing species or particles were completely dispersed or condensed on the cell walls in $\approx 0.1 \mathrm{sec}$. When the graphite was heated in $0.5 \mathrm{~atm} \mathrm{H}_{2}$ the effect was not observed, since the vaporized carbon reacts to form hydrocarbons.

An interesting new phenomenon was observed when graphite was vaporized in an evacuated vessel whose walls were cooled with liquid nitrogen. The material that condensed (in trace amounts) on the walls was in an unstable state and gave off flashes of light as soon as it was allowed to warm up slightly.

\section{ENGINEERING}

\section{Fission-Product Release (Nuclear Safety) Studies}

Research in this area is primarily concerned with the development of a fundamental under- standing of the chemical reactions, mechanisms of release, and transport behavior of the fission products and radioactive aerosols emanating from nuclear materials under conditions likely to be encountered in reactor accidents. All the work carried out during the past year was done in connection with the safety of sodium-cooled fast breeder reactors.

Because of the health hazard associated with plutonium, considerable effort was expended in elucidating the behavior of $\mathrm{PuO}_{2}$ aerosols. Experiments were carried out in both inert and oxidizing environments, with pure $\mathrm{PuO}_{2}$ and mixtures of $\mathrm{PuO}_{2}$ and $\mathrm{UO}_{2}, \mathrm{PuO}_{2}$ and sodium, and $\mathrm{UO}_{2}$ and sodium vaporized into settling chambers 30 $\mathrm{cm}$ and $1 \mathrm{~m}$ in height. The initial aerosol mass concentration covered a range extending from $\approx 10^{-2}$ to $30 \mathrm{~g} / \mathrm{m}^{3}$. The experimental results show that, for all aerosols, the airborne mass concentration drops very rapidly per meter of vessel height during the first hour of settling. Subsequently, the rate of aerosol settling decreases continually until the airborne $\mathrm{PuO}_{2}$ concentration

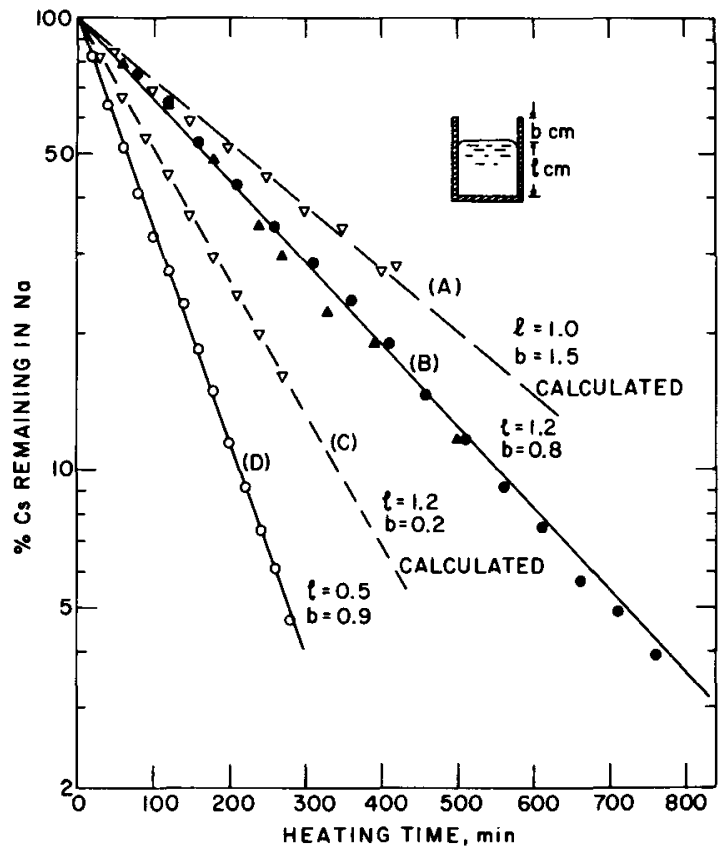

Figure 4. Vaporization of cesium from liquid sodium at $730^{\circ} \mathrm{K}$ into a helium stream. Indicated on the lines are the geometric parameters: $l$, the liquid depth, and $b$, the thickness of a gas layer through which cesium diffuses. The points represent experimental data, while the broken lines $(A)$ and $(C)$ are the theoretical release calculated from the transport model. 
reaches $\approx 10^{-6} \mathrm{~g} / \mathrm{m}^{3}$, a level that often persists for several days.

Theoretical studies are also in progress to establish the important parameters affecting the time dependence of the aerosol mass concentration and number of particles per unit volume. Recently, analytical studies were initiated to develop equations for calculating particle size distributions, surface area for absorption, and mass concentration of well-stirred heterogeneous aerosols. This was accomplished by solving the general basic equations expressing the rate of change of aerosol concentration due to agglomeration and settling, with use of a CDC 6600 computer. A comparison of the preliminary results shows good agreement between the experimental and computer-predicted mass concentration time dependence for pure $\mathrm{PuO}_{2}$ aerosols.

Experimental studies of the parameters controlling the rate and extent of fission-product vaporization from liquid sodium were completed during the year, and mathematical equations were developed for calculating the release rates as a function of time. The excellent agreement between predicted and experimental release rates is shown in Figure 4 for some typical cesium vaporization experiments made at $730^{\circ} \mathrm{K}$. The final results conclusively show that the vaporization of cesium from sodium solutions into an inert gas stream is controlled by both gas and liquid phase resistances. Relationships suitable for predicting the maximum extent of fission-product vaporization were also developed. In addition, a theoretical equation for accurately calculating the diffusivities of solutes in liquid metals was developed to provide the data needed in predicting the release rates of other fission products.

In another phase of the program, the chemical states of fission products released from uranium and plutonium fuels under accident conditions are under study. A thermochromatographic apparatus is being constructed inside an $\alpha-\gamma$ hot cell to separate the fission products released from the heated fuels. A Knudsen effusion apparatus, designed to determine the molecular weights and thermodynamic properties of fission products and their compounds, has been constructed and tested with $\mathrm{Kr}^{85}$ and atomic $\mathrm{I}^{131}$.

\section{Heat Transfer Research}

The work in this area continues to be devoted primarily to the study of forced-convection heat

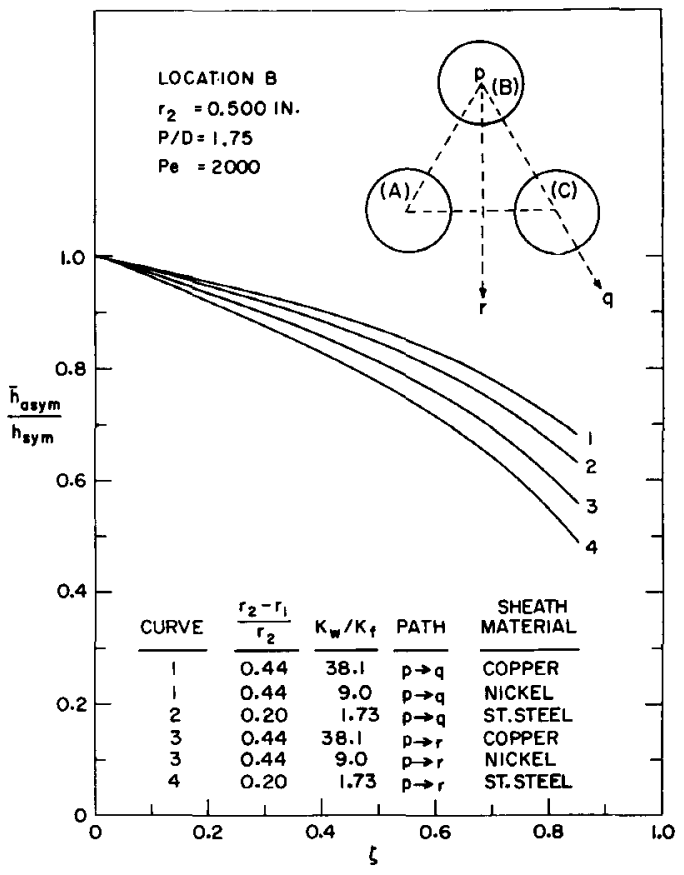

Figure 5. Effect of displacement of a rod on its average heat-transfer coefficient, illustrating the relative influences of cladding thickness, cladding conductivity, and displacement direction.

transfer in liquid metals. It currently includes both analytical and experimental studies of liquidphase and boiling heat transfer, and most of it is applicable to the design of liquid-metal-cooled reactor plants. Two of the studies are reviewed here.

In a continuing study, the effect of rod displacement on heat-transfer behavior was determined for in-line turbulent flow of mercury through an unbaffled rod bundle. The rods were special electrical heaters consisting of a spiral resistor surrounded by $\mathrm{MgO}$ insulation and swaged within a metal tube. The tube is therefore analogous to the cladding on a reactor fuel rod. Three claddings of different thicknesses and conductivities were tested. Some typical results are shown in Figure 5, in which $P / D$ represents the pitch: diameter ratio; $\mathrm{Pe}$ is the Peclet number; $r_{1}$ and $r_{2}$ are the inside and outside diameters of the cladding, respectively; and $k_{w}$ and $k_{f}$ are the thermal conductivities of the cladding and liquid metal, respectively. The central $\operatorname{rod}(\operatorname{rod} B$ in the figure) was displaced from its symmetrical position along two paths, $p \rightarrow q$ and $p \rightarrow r$, as indicated. The curves show the effect of displacement on the average heat-transfer coefficient, where $\zeta$ is the ratio of 


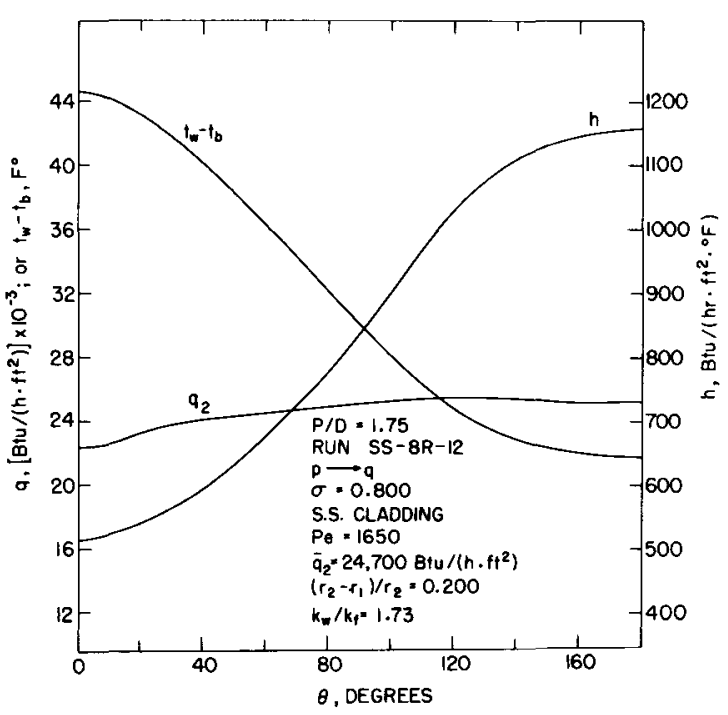

Figure 6. Heat-transfer behavior for in-line flow of mercury through an unbaffled rod bundle, showing the circumferential variations of wall heat flux, temperature driving force, and local heat-transfer coefficient for a rod that is moved $80 \%$ of the distance directly toward a neighboring rod.

actual to maximum displacement and $h_{\mathrm{asym}} / h_{\mathrm{sym}}$ is the ratio of the average coefficient for a displaced rod to that for a centered rod. It can be seen that large reductions in heat-transfer capability can be caused by rod displacement and that the reduction is affected little by variations in cladding thickness and conductivity but is affected appreciably by flow rate.

Rod displacement can result in large circumferential variations of the surface temperature of the rod, for rods with relatively low cladding thicknesses and thermal conductivities. This is illustrated in Figure 6 for the situation in which a rod with stainless steel cladding is moved 0.8 of the distance directly toward a neighboring rod. In this case, there is $180^{\circ}$ symmetry. The symbols $t_{w o}$ and $t_{b}$ represent the local surface temperature and the bulk-stream temperature, respectively.

In both Figures 5 and 6 , the adverse effects of displacement would be more severe for closer rod spacings.

A second continuing project involves studies of boiling and two-phase flow problems with alkali liquid metals. Recent experiments in a forcedconvection loop, using potassium as the test fluid, measured (a) the effect of flow rate on the superheat required to cause incipient vaporization and (b) the pressure drop of two-phase flow, with and

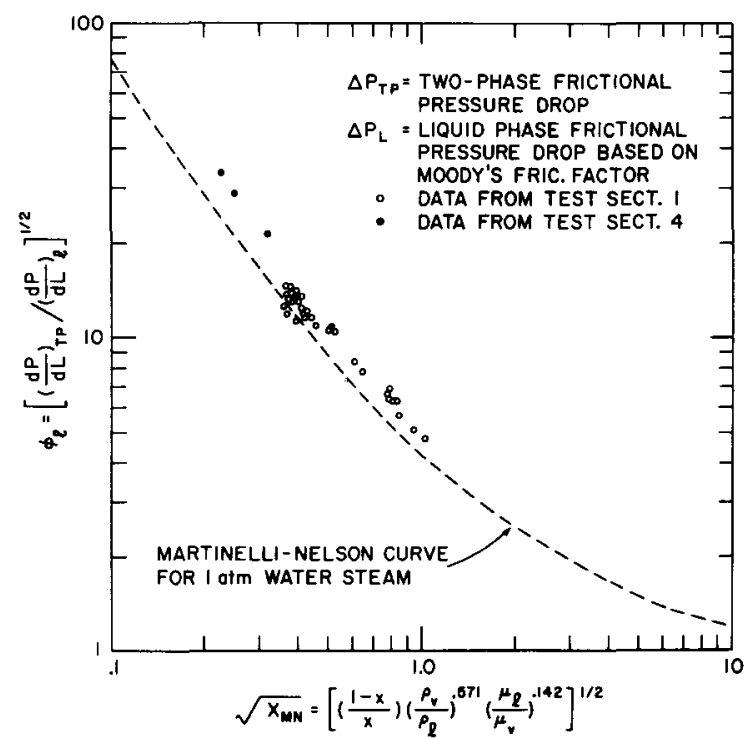

Figure 7. Two-phase adiabatic pressure drop for potassium.

without net vaporization. The superheat data indicated a strong dependence on flow rate. With all other parameters held constant, the incipientboiling superheat decreased almost linearly with increasing velocity. A theoretical model is being developed to correlate this effect with flow turbulence. Some of the two-phase pressure drop results, for adiabatic conditions, are shown in Figure 7. The results are seen to be well correlated by the Martinelli-Nelson parameter, $X_{\mathrm{MN}}$, although the values of the pressure drop parameter, $\phi_{l}$, for the potassium data are $\approx 15 \%$ higher than those predicted by the Martinelli-Nelson correlation for water at $1 \mathrm{~atm}$ pressure.

Other current projects in heat-transfer research include (a) development of a probe for void detection in liquid metals, (b) study of void propagation in liquid metals flowing through rod bundles, (c) measurement of local heat-transfer coefficients for turbulent flow of liquid metals through closely packed rod bundles, and (d) investigation of the effects of solid-liquid interface characteristics on heat transfer with sodium.

\section{Pulsed Research Reactor Project}

The repetitively pulsed fast reactor is under study as a high-intensity source for neutron beam experiments. Two alternative reactor concepts are being investigated: a pulsed reactor in which the reactivity is modulated by mechanical means, and an accelerator-injected pulsed reactor in which 


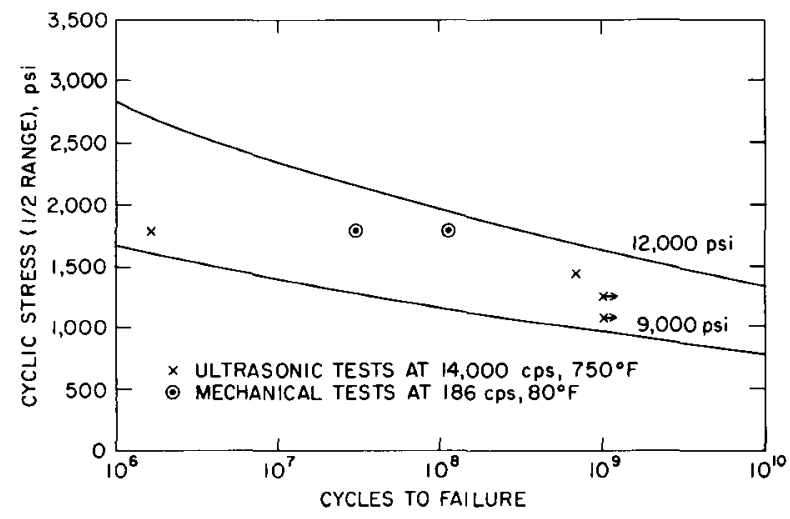

Figure 8. High-cycle fatigue life of $60 \mathrm{vol} \% \mathrm{UO}_{2}-\mathrm{Mo}$ cermet. Curves are predictions for material with an ultimate tensile strength of 9000 or 12,000 psi. All specimens were tested with a mean applied stress of 4500 psi.

neutrons are injected into the system from the target of an electron accelerator coincidentally with mechanical modulation of the reactivity. The effort during the year has concentrated on the reference design of a pulsed reactor and on supporting work in fuel development and experimental physics.

The fuel in a pulsed reactor is subjected to between $10^{8}$ and $10^{10}$ stress cycles during the life of a core loading. These cyclic stresses are induced in the fuel by the extremely rapid heating during the repetitive power pulses. A form of the $\mathrm{PuO}_{2}$ Mo cermet reference design fuel has been subjected to fatigue tests to determine its high-cycle fatigue behavior. In actual testing, uranium was substituted for plutonium and tested in the unirradiated condition. The fatigue tests were performed by a mechanical technique at a frequency of $186 \mathrm{cps}$ and by an ultrasonic technique at 14,000 cps. The fatigue test results are plotted in Figure 8.

Development work was also performed on alternative fuel materials. Battelle Memorial Institute has been successful, in subcontract work, in manufacturing a ternary alloy $(\mathrm{U}, 27 \% \mathrm{Zr}, 20 \%$ $\mathrm{Nb}$ ) that exhibits a stable $\gamma$ phase. The material was ductile, with an ultimate tensile strength of 91,200 psi and a $32.5 \%$ area reduction in the unirradiated condition. Battelle has also fabricated specimens of a dispersion fuel with $40 \%$ uranium in a thorium matrix.

A critical assembly was designed and operated to assist in the design of the pulsed fast reactor. In a series of experiments, measurements were made of the neutron lifetime, the critical mass, and the reactivity effect of movable reflector pulse blocks. The experimentally determined values of critical mass and pulse-block reactivity effect are in excellent agreement with calculated values of these parameters. The critical assembly was fully reflected except on the face adjacent to the pulse block, and in some experiments a poison liner was installed between the reflector and core to reduce the neutron lifetime. Although calculated and measured values of the lifetime in unpoisoned cores disagreed, agreement was quite good when the poison liner was added. In one experimental series, moderator blocks were located in the reflector with a layer of poison material and a layer of steel between the core and moderator. The thermal-neutron yield from various moderator blocks was measured.

\section{Ultimate Waste Disposal}

Investigation was continued of the phosphate glass process as a means of fixing fission products in stable solids. The BNL pilot plant was operated in support of Pacific Northwest Laboratory's demonstration prototype program, and a number of new waste feed compositions were investigated to establish flowsheets for use in the prototype plant.

Development was emphasized of flowsheets for a new type of Purex waste, PW-4m, which is low in iron and sodium and related to the reprocessing of 45,000 MWD/tonne of uranium burnup fuel. A particular requirement was that the glass product have a very high simulated fission-product loading, representing sufficient internal heat generation to maintain the center-line temperature of an 8-in.-diam glass billet at $900^{\circ} \mathrm{C}$. A number of formulations with varying iron-sodium ratios were optimized in laboratory studies and evaluated in pilot-plant operation. Very satisfactory results were obtained with respect to smoothness of plant operation, reduced tendency for settling of solids, and improved transfer of heat to the central region of the glass pool. The formulations were submitted for use in the scheduled runs of the prototype plant at Pacific Northwest Laboratory.

Experiments were initiated to evaluate performance characteristics of a new type of melter. The objective is to increase heat input capacity through the use of induction heating and minimized path length for heat transmission during glass formation.

Investigation of the treatment of sulfate-rich melter off-gas condensates by distillation was con- 
tinued and the basic outlines for a promising practical process were established. The process involves volatilization of the nitric acid under reduced pressure and temperature, to avoid formation of $\mathrm{RuO}_{4}$, and subsequent distillation of the sulfuric acid with very small carry-over of ruthenium.

Investigation of the removal of krypton and xenon from bulk gas streams was continued, with emphasis on stagewise adsorption on charcoal. A schedule of adsorption tests with dilute mixtures of krypton in air or nitrogen was completed, and information was obtained to permit calculation of the first stage of an adsorber system.

\section{Engineering Assistance}

The Engineering Assistance Group contributed more than 45,000 man-hours to the support of departmental and Laboratory programs during the year. Most of this effort was concerned with equipment for radiation processing, liquid-metal heat transfer, experimental reactor physics, and advanced reactor concepts. These activities are discussed in more detail in the section on Technical Operations and Services under the heading "Mechanical Engineering."

\section{RADIATION RESEARCH AND DEVELOPMENT}

\section{Basic Radiation Research}

Solid-State Reactions. Gaseous molecules of varying sizes were diffused into preirradiated crystalline amides. The ensuing reactions indicated that the reactive free radicals formed in the solid crystals tend to concentrate near lattice defects, although originally formed in a more uniform distribution. A mechanism of radical migration by hydrogen atom abstraction is postulated.

Polymerization Studies. Radiation-produced polystyrene has a molecular weight distribution that changes with the temperature of polymerization, depending on the type of initiation. For ionically polymerized samples, the average molecular weight and the spread of the distribution increase with decreasing temperature. The opposite behavior is observed in the free-radical initiated polymerization.

Radiolytic Decomposition of Nitrous Oxide. Investigation of the radiolytic decomposition of nitrous oxide established that the yield of nitrogen gas, $G_{\mathrm{N}_{2}}$, is independent of the physical state of the nitrous oxide, independent of its density in the gaseous state, and independent of the quality of the incident radiation.

Radiolysis of Adsorbed Gas. The radiolysis of nitrous oxide adsorbed on molecular sieves was studied over a wide range of surface coverage. The constancy of the radiolytic yield established that the exciton or electron responsible for energy transfer must have a range of at least $1000 \AA$ in the molecular sieve.

Radiolytic Formation of Ozone. An analysis of combined radiation and electric discharge fields in oxygen led to the prediction of conditions under which a several-fold increase in the efficiency of ozone formation may be expected.

\section{Radiation Chemical Processing}

Concrete-Polymer Materials. In cooperation with the Bureau of Reclamation and the Office of Saline Water (OSW) of the Department of the Interior, a program on the development of concrete-polymer materials is under way. The program has evaluated impregnation methods and measured the strength and durability of several test series. Fundamental studies of the nature of concrete-polymer composites are being undertaken. Four monomers have been evaluated for AEC-type applications and an equal number for OSW high-temperature applications. All the composites tested have considerably improved strength and durability (see Figure 9).

The Naval Civil Engineering Laboratory is considering concrete-polymer materials, because of their high compressive strength and low water permeability, for a variety of underwater applications, including instrument packages, mines, buoys, and habitats.

A number of highway applications, including bridge decks, sign posts, and light poles, are being considered by the Bureau of Public Roads of the Federal Highway Administration.

The U.S. Department of Agriculture is interested in using concrete-polymer for precast panels, both steel-fiber reinforced and nonreinforced, in lowcost rural housing. An increase by about a factor of 5 in flexural strength is obtained with fiber reinforcing and polymer impregnation.

With use of the same techniques as for the preparation of concrete-polymer materials, stabilized soil mixtures impregnated with methyl methacrylate are being developed by the U.S. Army Corps of Engineers for use as a building material for low-cost housing in underdeveloped 
countries. The results with soil samples from abroad indicate strength increases of up to $1000 \%$.

Heterogeneous Radiation Chemistry. The reactions under current study are the fixation of nitrogen and the formation of ozone. The purposes of the work are the elucidation of the mechanisms for homogeneous formation via irradiation and the investigation of the use of solid additives for possible energy transfer effects. The effects of temperature, pressure, flow rate, radiation intensity, and nitrogen-oxygen ratio have been explored for the fixation of nitrogen. Studies down to $-100^{\circ} \mathrm{C}$ have given $G$-values as high as 14 for ozone production, and a strong effect of a small amount of nitrogen in the feed oxygen on the rate of formation was observed.

Polymerization of Ethylene and Copolymers. Because several industrial firms are continuing their development activities in this field, no further work has been done at BNL. An information exchange meeting was held with scientists from the Japan Atomic Energy Research Institute Laboratories at Tokai-mura and Takasaki.

Polymer Composites. An area that shows promise for the application of radiation processing is that of aggregate-polymer composites that do

Figure 9. Comparison of freeze-thaw durability of impregnated and unimpregnated concrete. Left, unimpregnated concrete; after 590 cycles in test, cylinder shows $26.5 \%$ weight loss. Right, concrete impregnated with methyl methacrylate, polymerized by radiation; after 4000 cycles in test, cylinder shows $0.5 \%$ weight loss.

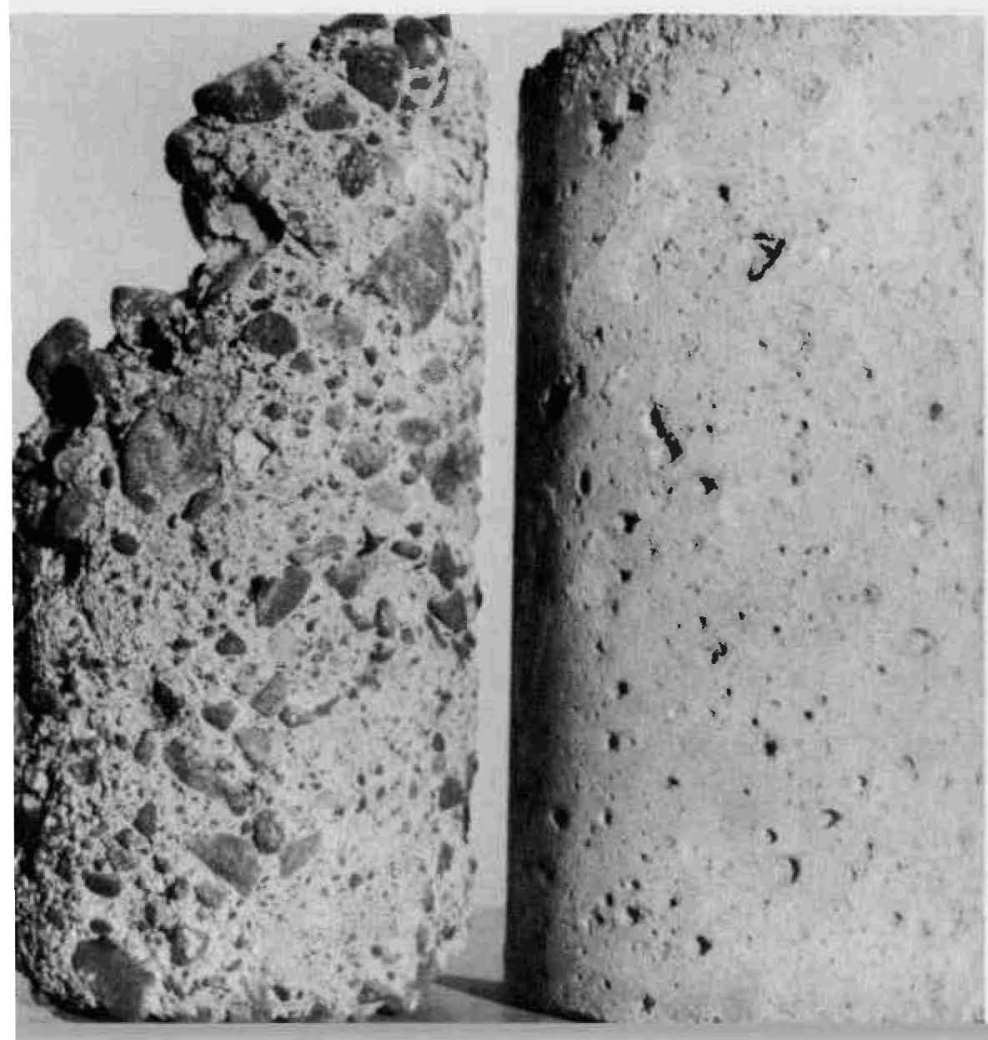

not contain cement. A wide range of aggregate, filler, and binder materials is being considered.

Cryogenic Radiation Chemistry. Radiation reaction studies at low temperatures have been started with the irradiation of liquid nitrogenhydrogen mixtures at $-160^{\circ} \mathrm{C}$ and $40 \mathrm{~atm}$.

Electrode Reactions. The study of electrode reactions under the influence of $\gamma$ radiation was continued with a study of the oxygen electrode, and $\gamma$ effects were noted.

Chemonuclear In-Pile Research Loop. The Brookhaven Chemonuclear In-Pile Research Loop was tested over its full range of operating conditions and found to meet specifications. It is stable in operation and very little effort is required to maintain desired conditions. (See Figure 10.)

Four runs have been made with the Brookhaven Graphite Research Reactor operating, but without chemonuclear fuel installed. One run was made to establish the flux pattern and the dose distribution. The other three runs, of about $100 \mathrm{hrs}$ each, were made with oxygen as the process gas, for the production of ozone. Operating temperatures were varied between $+70^{\circ}$ and $-30^{\circ} \mathrm{F}$. Additions of nitrogen up to about $30 \%$ were found to enhance both the rate of ozone production and its steady-state concentration. Further runs are planned to establish more fully the effect of these parameters. The maximum effective $G$-value for ozone production found was nearly 10 , with a maximum steady-state concentration of about $500 \mathrm{ppm}$, at a dose rate of $2.5 \times 10^{6} \mathrm{rads} / \mathrm{hr}$. Preparations are being made for fueled runs, pending authorization by the AEC.

Emphasis on chemonuclear ozone production results from the growing interest here and abroad in the use of ozone in tonnage quantities for water treatment and pollution control. Process studies made recently both at BNL and by others indicate that the use of a chemonuclear reactor may provide the most economical way of producing ozone in quantities of 400 tons/day and up. Economic studies of production and utilization systems are continuing.

\section{Radiation Chemical Engineering}

Theoretical and experimental investigations were conducted on radiation chemical reactor performance with the objectives of providing a framework for the design and scale-up of radiation chemical reactors and for the interpretation of experiments on the kinetics of radiation chemical reactions. 
Previous theoretical work on the existence and direction of effects of mixing on fraction conversion in reactors with space-dependent residence time and reaction-rate distributions has been extended to include the entire range of conversion in chain reactions.

Flow-dosimetry problems were investigated in two tubular reactors employing $\mathrm{Sr}^{90} \mathrm{Y}^{90} \beta$-particle sources and in a continuously stirred tank reactor with a $\mathrm{Co}^{60} \gamma$-ray source.

A theoretical study of the performance characteristics of continuously stirred tank reactors when used to conduct radiation-induced polymerizations has been carried out.
Experiments have been conducted on a new type of quartz dosimeter suitable for use in the 5 to 50-krad range.

\section{Radiation Engineering}

High Intensify Radiation Development Laboratory.

1. Operations and services. Source inventories maintained for research and development activities included $>2.5 \times 10^{6} \mathrm{Ci} \mathrm{Co}^{60}, 400,000 \mathrm{Ci} \mathrm{Cs}^{137}$, and $5000 \mathrm{Ci} \mathrm{Sr}^{90}$. Several hundred thousand curies of corroded, damaged, or initially rejected $\mathrm{Co}^{60}$ sources were recovered.

The preparation and radiation cells at the HIRDL were operated at near capacity. Typical

Figure 10. Control panel of the Brookhaven Chemonuclear In-Pile Research Loop, now in operation in the Brookhaven Graphite Research Reactor.

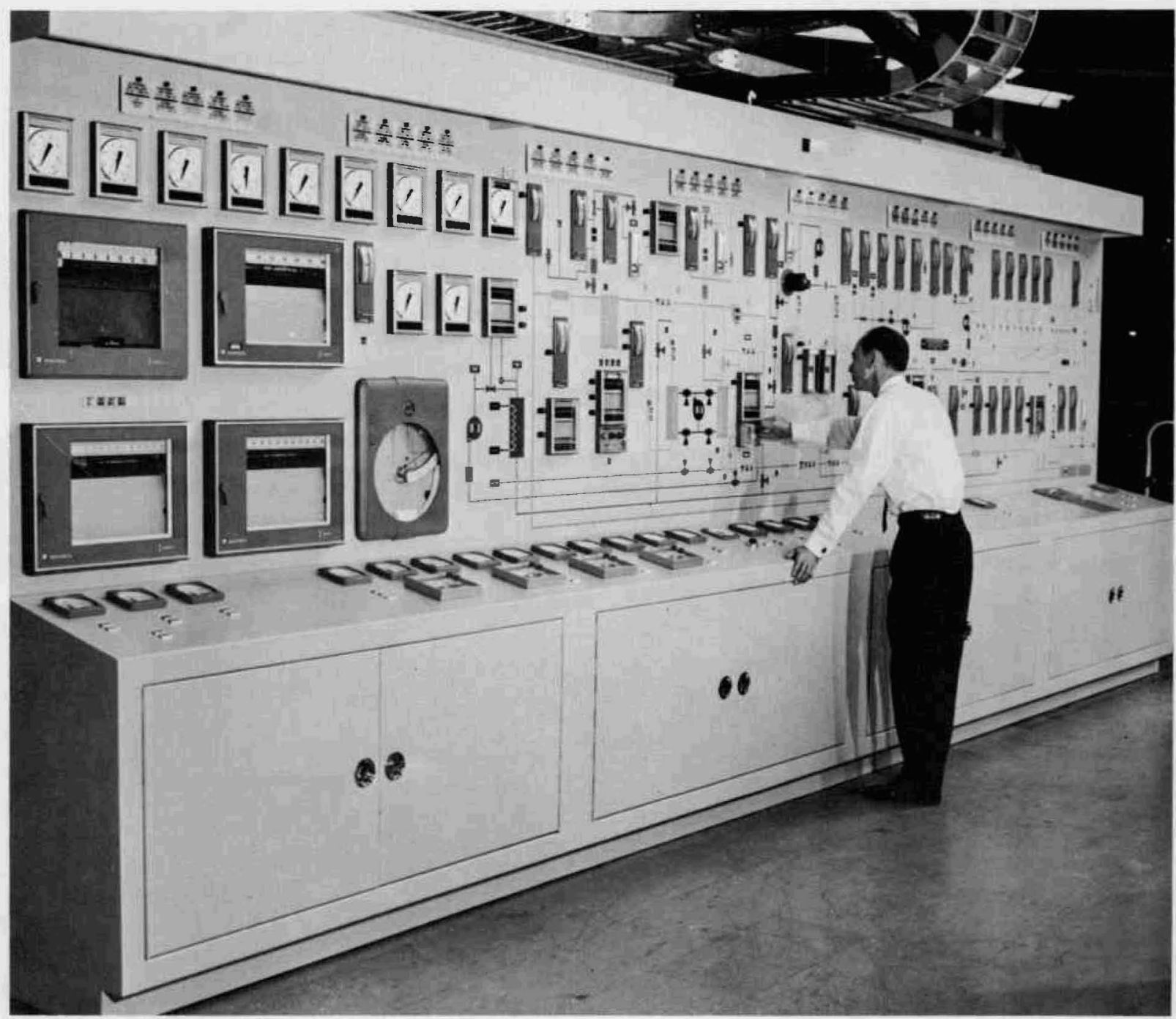


operations included the development and use of remote-welding equipment for the secondary encapsulation of cylindrical sources; the remote loading of numerous units, including portable cesium irradiators, the shark brain irradiator, and a blood irradiator; the irradiation of monomer-loaded concrete test specimens; verification of meat-irradiator pilot plant design data on a mock-up of the proposed conveyor system; and the fabrication and operation of a continuous-flow, $\gamma$-irradiated liquid system in support of reactor vessel design research.

An underwater $\gamma$ facility was constructed which can deliver calibrated $\gamma$ doses from $5 \times 10^{3}$ to $10^{7}$ rads/hr. About 5000 sample-hours of irradiation were performed each month.

2. Source development and testing. The first batch (about $5000 \mathrm{Ci}$ ) of the newly developed, stainless-steel encapsulated, aluminum-strontium silicate matrix $\mathrm{Sr}^{90}$ sources is under test. A larger batch (about $50,000 \mathrm{Ci}$ ), modified to increase $\beta$ efficiency by about $65 \%$, is in preparation.

An automated cycling apparatus for the accelerated study of source corrosion rates was designed and put into service.

Cooperative efforts are under way with investigators at Battelle Memorial Institute and in pri- vate industry on the development of nondestructive testing techniques (primarily ultrasonic) for the examination of bonding and end-weld integrity in source strips.

3. Dose distribution measurement and prediction. The major effort this year was the refining and publishing of predictive codes, including GIRD, FUDGE 4A, and RAD. An improved fit for buildup factors was developed for the FUDGE 4A code. Extensive "look-up" tables were published to permit rapid determination of dose rate and dose uniformity for most practical irradiators.

4. Dosimetry. The study of "effects" dosimetry in wheat was continued. Water extracts of methyl bromide-fumigated wheat were shown to have optical-absorption spectra peaks inversely proportional to the radiation dose administered, in the 10 to $50-\mathrm{krad}$ range. Further development of this technique will be carried out by the USDA in Savannah, Ga.

A report on $\mathrm{Co}^{60}$ dosimetry in radiation research and processing was prepared and published.

$\gamma$ Dosimetry standardization, in support of AEC food irradiator interests, was stressed this year. Units calibrated or checked included two shipboard irradiators, three portable cesium irradia-

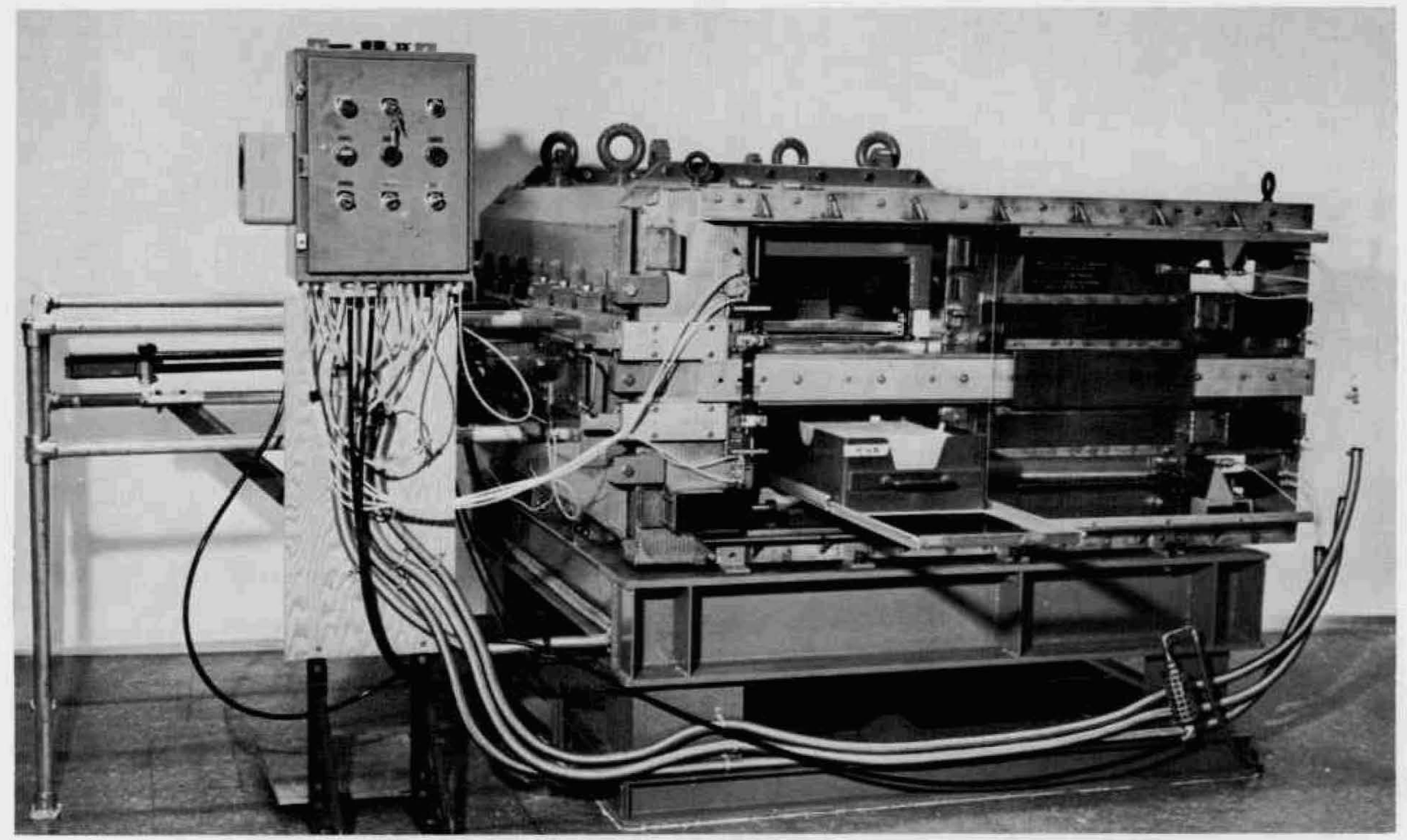

Figure 11. Brookhaven portable cesium development irradiator prior to shipment to Argentina. 
tors, and facilities in Hawaii, California, Washington, and Massachusetts. Because of continuing problems with the gathering and reporting of data in support of FDA petitions, an extensive irradiator calibration manual was prepared. It is currently undergoing review and trial use by selected investigators.

5. Irradiator design, fabrication, and support. The.portable cesium irradiator was completely rebuilt and installed in a new trailer, in conformance with Department of Transportation requirements. A shipboard irradiator was removed from a Bureau of Fisheries vessel in Seattle and installed at the University of Nebraska.

Three BNL-designed portable cesium development irradiators (see Figure 11) were received from the vendors. After modification, one unit was installed in Argentina. The second and third units will be installed in India and Chile early next year.

Atmospheric Diagnostic Program. The isotope ratio tracer (IRT) method has been developed as a usable technique for tracing sulfur in the atmosphere. The method makes use of the natural variation in the ratios of the stable isotopes of sulfur in fuels from different deposits. There is enough variation in the ratio of $\mathrm{S}^{32}$ to $\mathrm{S}^{34}$ in coals and oils to make it possible to select a fuel having an isotope ratio differing sufficiently from that of the sulfur prevailing in the atmosphere at the point of use to permit the identification of sulfur oxides as having been emitted by a specific plant. The sensitivity of the method is such that it has been possible to trace a stack plume for as far as 12 miles from its point of origin.

The basic elements of the IRT method essential for its application to meteorological problems are in routine use. These include a filter pack for the quantitative sampling of $\mathrm{SO}_{2}$ and $\mathrm{SO}_{3}$ particles in the atmosphere, an airborne sampling system that collects samples of adequate size in a reasonable length of time (see Figures 12 and 13), the methods and equipment for processing the small samples collected and preparing them for mass spectrometric measurement without fractionation of the sulfur isotopes, and an isotope ratio mass spectrometer that permits measurements to be made with a precision of within $\pm 0.02 \%$. Crude oils have been located that contain adequate a mounts of sulfur with $\delta$ values varying by 10 to 15 parts per thousand from those of the background sulfur.

The program has been expanded to include responsibility for field work with sulfur hexafluoride

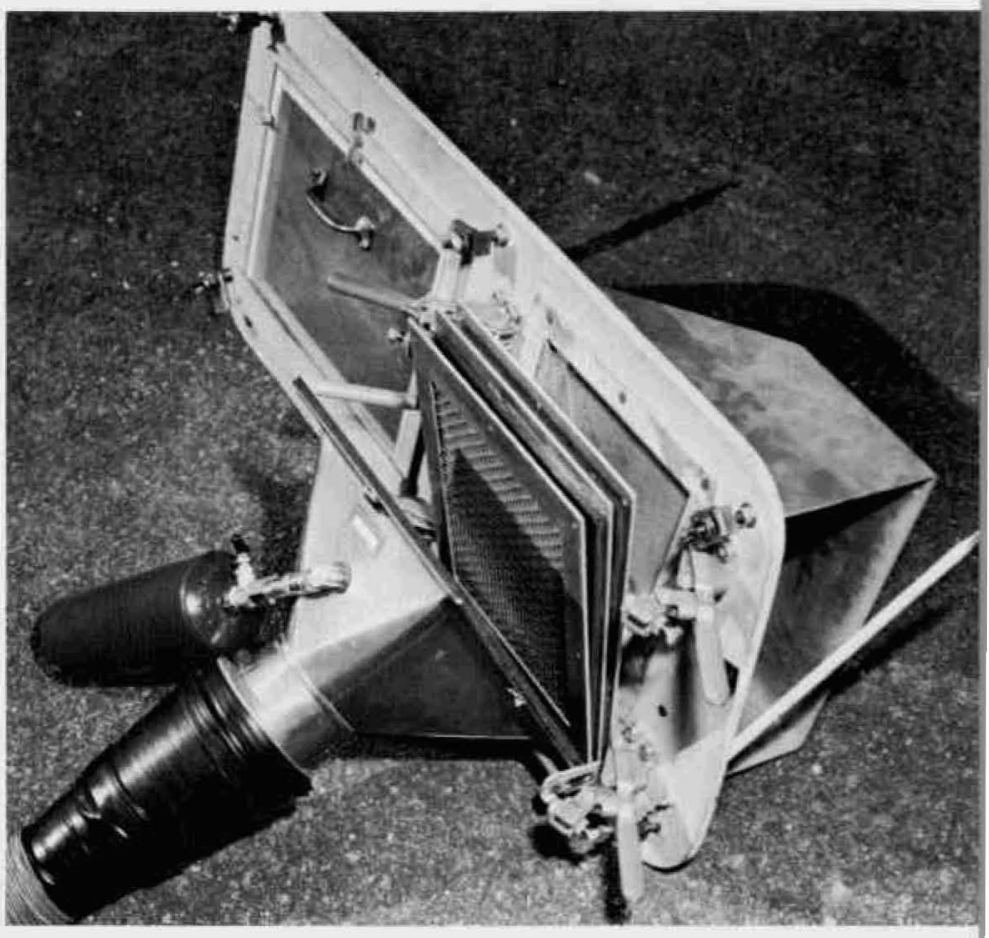

Figure 12. Closeup of sampling assembly. The air scoop with its shutter is on the right. In the center is the gasketed holder for the $8 \times 10$-in. filter sheets. Attached to the air duct by a quick-disconnect fitting is an evacuated cylinder for the collection of a sample for $\mathrm{SF}_{6}$ analysis.

Figure 13. Sampling assembly mounted in window of the plane used for aerial survey. The duct is connected to a high-volume fan. Also shown is the analyzer for measuring ambient $\mathrm{SO}_{2}$ concentrations and the strip chart that records concentration, temperature, and altitude-pressure.

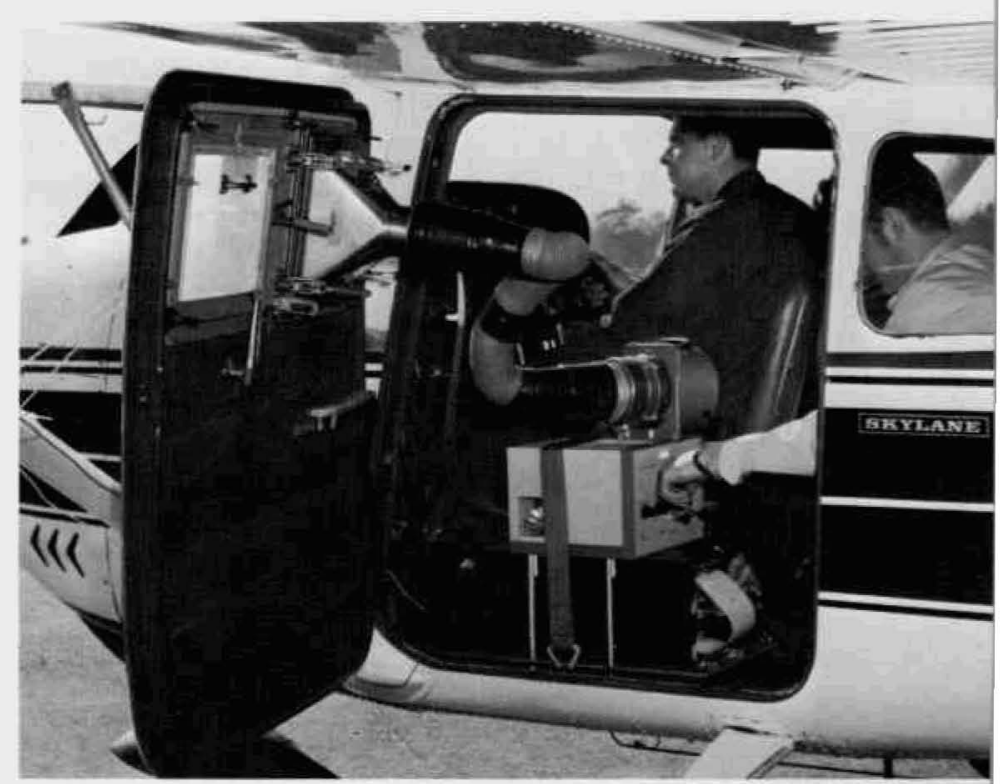


$\left(\mathrm{SF}_{6}\right)$ as a tracer. The simultaneous use of the IRT and $\mathrm{SF}_{6}$ methods provides a powerful tracer system for studying both the chemical and physical behavior of sulfur oxides in the atmosphere.

Three experiments are under way to demonstrate the utility of the IRT method. One is the development of a mathematical model for $\mathrm{SO}_{2}$ pollution patterns in New Haven, Conn., and its verification by use of the IRT method. A second is the study of the rate of conversion of $\mathrm{SO}_{2}$ to $\mathrm{SO}_{3}$ in stack plumes, using the coal-fired Keystone plant in Pennsylvania and the oil-fired plant of the Long Island Lighting Company at Northport. The third is a study of the efficacy of tall stacks in dispersing pollutants under inversion conditions in metropolitan areas. The Arthur Kill Plant of the Consolidated Edison Corporation on Staten Island, N.Y., will be used for the experiment, which will be run on a selected off-ratio fuel. In preparation for these experiments, samples have been collected and analyzed of fuels (both oil and coal), stack gas and plumes, and atmospheric backgrounds both at ground level and aloft.

The program is a joint operation with the Analytical Group of the Hot Laboratory Division and the Engineering Division, both of this Department, and the Meteorology Group of the Instrumentation and Health Physics Department. It is being undertaken with the cooperation of and support from the Public Health Service of the Department of Health, Education, and Welfare.

\section{HOT LABORATORY}

\section{Analytical and Physical Chemistry Research}

The behavior of metals with organic acids continues to be studied in order to determine the requirements for forming mixed-metal complexes, and their stability. For example, many hydroxy acids with a malic acid structure were found to form complexes containing two metals such as indium and chromium, while aspartic acid, which has a $-\mathrm{NH}_{2}$ group in place of the $-\mathrm{OH}$ group, did not. Such complexes contain one atom of each metal and two molecules of acid. The uraniumindium citrate complex is 30 times stronger than the uranyl citrate dimer, which indicates (in view of the ubiquity of citric acid as an analytical reagent) the importance of considering the influence of mixed-metal complexation on analytical procedures.
The electrochemical reduction of pertechnetate ion $\left(\mathrm{TcO}_{4}{ }^{-}\right)$was shown to proceed by rapid (diffusion-controlled) disproportionation of the technetate ion $\left(\mathrm{TcO}_{4}{ }^{2-}\right)$. The small value observed for the rate constant is explained by Coulombic repulsion; and gelatin, generally used as a polarographic maximum suppressor, plays a unique role by selectively blocking the thermodynamically favored reaction $e^{-}+\mathrm{Tc}_{4}{ }^{2-} \rightleftarrows \mathrm{TcO}_{4}{ }^{3-}$. Other chemical systems involving electron transfer coupled with chemical kinetics were studied in collaboration with groups outside the Laboratory. The principal Brookhaven contribution was the development and application of the theory required for kinetic or mechanistic interpretation. Such studies included determining the rates of dimerization of triphenylamine cation radicals, of oxidation of cyanide ion by trianisilamine cation radical, and of hydrolysis of 1,4 di-iminobenzene.

A recently developed theory quantitatively explains electrode double-layer relaxation, in excellent agreement with experimental data.

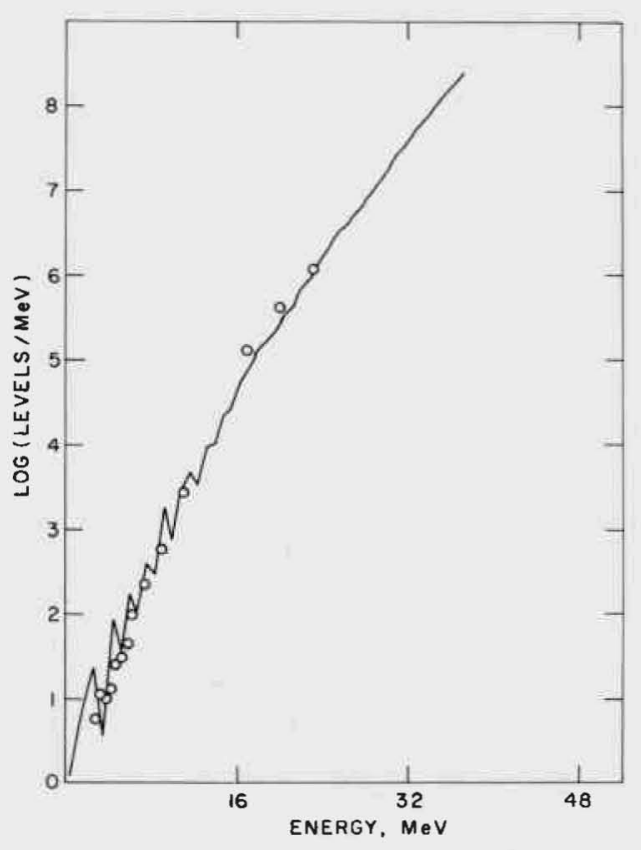

Figure 14. Nuclear level densities of $\mathrm{Fe}^{56}$. The open symbols represent the experimental points, and the solid, jagged line represents the calculated level densities. This is one of two nuclides for which there are experimental data up to $23 \mathrm{MeV}$. The high energy experimental points were determined with use of a correction for spin distribution now considered invalid. However, agreement with the experimental data is not expected to change significantly after use of a more appropriate correction. 


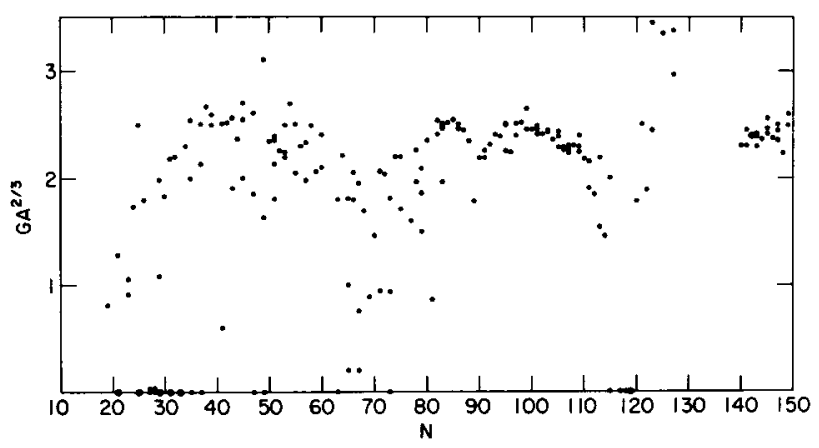

Figure 15. Plot of the values of $G$ (the pairing parameter) necessary to cause the calculated level densities of $192 \mathrm{nu}$ clides to pass through the experimental points at the neutron binding energies. The plot is in the form of $G A^{2 / 3} \mathrm{vs}$ $\mathcal{N}$ (the neutron number). Its significance lies in the fact that for most nuclides the value of $G A^{2 / 3}$ is constant. The large scattering observed in some of the points is attributed to difficulties near closed shells and in light nuclides.

\section{Nuclear and Radiochemistry Research}

Identical chemical effects found for the isomeric decay of $\mathrm{Te}^{127 m}$ and $\mathrm{Te}^{129 m}$ indicate that a proposed $5 \%$ branch in the decay scheme of the latter is not required to explain chemical results. The chemical effects of the reactions of neutrons with biscyclopentadienyl dichlorides of hafnium and zirconium were found to support previous conclusions concerning similar reactions with phthalocyanines of these elements. The much lower retention of activity of the metallocenes results from the greater difficulty of recombining the three bodies formed relative to the two bodies formed by the phthalocyanines. A continuing program to measure cross sections in the transplutonium region yielded $14 \pm 8 \mathrm{~b}$ for the epithermal neutron capture resonance integral of $\mathbf{C f}^{253}$. Recoil range measurements indicate that a fraction of the $\mathrm{Ca}^{48}(\alpha, \alpha n) \mathrm{Ca}^{47}$ reaction involves a direct interaction mechanism.

The measured mass distribution from ternary fission of $\mathrm{Pu}^{240^{*}}$ and $\mathrm{Pu}^{241^{*}}$ confirms earlier results from other nuclides and eliminates the possibility that the observed events are due to scattering. Further, the yield of $\mathrm{Si}^{32}$ (if any) from $\mathrm{U}^{236^{*}}$ is $<10^{-10}$ per fission. An experiment in progress to determine the number of prompt neutrons coincident with ternary fission of $\mathrm{Cf}^{252}$ permits observation of up to two ternary events per week; thus far five events have been observed, each associated with relatively few neutrons. Preliminary calculations indicate the possibility of increasing the flux of a neutron beam by focusing with tapered tubes. Five of the six possible products of the $\mathrm{Mg}(t, x)$ reaction were observed with a single irradiation.

Results of a computer program to calculate nuclear level densities by using a combinatorial method, the spherical single-particle levels of the shell model, and Bardeen-Cooper-Schrieffer pairing forces are in very good agreement with known experimental data (Figure 14). Analysis shows that the previously held convictions concerning the Gaussian distribution of spins and the association of the dispersion of this distribution with the rigid body moment of inertia of the nucleus were incorrect and that the pairing parameter $G$ is probably proportional to $A^{-2 / 3}$ instead of $A^{-1}$, as previously thought (see Figure 15).

\section{Development of lsotopes \\ for Medical Applications}

As part of this interdisciplinary effort, the Medical Research Center was supplied with $>200$ radiopharmaceutical preparations for evaluation. A technique developed for preparing high specific activity $\mathrm{Tc}^{99 m}$-labeled human serum albumin extended the usefulness of this isotope, as did development of a simple, inexpensive, solvent extraction process for the separation of high purity $\mathrm{Tc}^{99 m}$ from the low specific activity $\mathbf{M o}^{99}$ parent. The latter process is of special utility wherever only low flux reactors (which are unsuitable for producing high quality $\mathrm{Mo}^{99}$ ) are available. Studies of the $\mathrm{Tc}^{99 m}$-DTPA chelate indicate considerable potential for brain-tumor localization and kidney diagnosis. Biological distribution of tagged colloids is being correlated with particle size as a means of increasing the utility of radioactive colloidal preparations. Reactor production of $\mathrm{Mg}^{28}$ was discontinued on shutdown of the Brookhaven Graphite Research Reactor, and a superior quality of this important medical tracer is now being produced by bombarding enriched $\mathrm{Mg}^{26}$ metal with 3.5$\mathrm{MeV}$ tritons in a Van de Graaff generator. About $50 \mu \mathrm{Ci}$ per microampere-hour and specific activities of $>400 \mathrm{mCi} \mathrm{Mg}{ }^{28}$ per $\mathrm{g} \mathrm{Mg}$ are obtained. 


\section{Applied Mathematics}

The Applied Mathematics Department conducts research in mathematics and computer science and performs service functions for the over-all research program at Brookhaven. The research program consists of three activities designated as mathematics research, programming research, and computer research and development. The work done is not only of general value in these fields, but also of direct significance to many of the other research activities at the Laboratory.

The Department's service functions include operation of the Laboratory's Central Scientific Computing Facility (CSCF), maintenance of a library of service programs for general use, provision of consulting services in certain areas of mathematics, Laboratory-wide training in computer programming, and programming services. In fulfilling these functions the Department engages in a number of research efforts related directly to the use of the CSCF. Consequently the Department's research program is also concerned with increasing the effective utilization of the CSCF and extending the application of CSCF computers to new areas of research in the physical and life sciences.

\section{COMPUTER OPERATIONS}

The CSCF provides large-scale general computational support to the various research activities at Brookhaven.

\section{Central Scientific Computing Facility Equipment}

The CSCF currently utilizes two CDC 6600 computers and a CDC 924 computer. The CDC 6600 system (see Figure 1) performs the bulk of the Laboratory's general-purpose processing, whereas the CDC 924 computer is used principally as peripheral support for the large-scale machines. The two GDC 6600's are coupled directly to a onemillion-word extended core storage (ECS). The first half of the ECS was installed in the fall of 1968 , and the second half during the following spring. An IBM 7094 computer, which is devoted almost entirely to bubble-chamber data proc- essing, was transferred to the Physics Department in July 1968.

Several significant additions were made to the library of diagnostic programs. The new programs provided more exhaustive diagnostic testing of the central memory and central processor of the GDC 6600 's. Several of the existing programs were revised to enable them, like the others, to be run under the CDC 6600 operating system in the same manner as any other job, which eliminated the need for taking the computer system out of production during diagnostic testing. The computer time required for the detection of malfunctions was reduced by the use of these diagnostic programs.

\section{Computer Scheduling and Usage}

The two CDC 6600 computers were in operation 7 days a week for 3 shifts a day throughout fiscal 1969. Figure 2 shows the usage by quarters for the combined CDC 6600 computers. Only productive time was included in the usage figures; hardware maintenance and engineering changes were excluded. The variation in usage during the first three quarters of fiscal 1969 resulted from the installation and testing of the ECS and engineering changes.

\section{COMPUTER PROGRAMMING}

Maintaining the software systems for the CSCF, updating and expanding the general-purpose program library, supplying programming services to the various research activities of the Laboratory, and conducting Laboratory-wide training in computer programming are all functions of the Department's programming staff.

\section{Software System Maintenance and Improvement}

The SCOPE 2 operating system for the CDC 6600 computers was replaced by the SCOPE 3 system early in the year. Facilities and features that had been added to SCOPE 2 at BNL to improve it and adapt it to the Laboratory's needs were carried over. ECS software was incorporated subsequently into the SCOPE 3 system. Other improvements to 


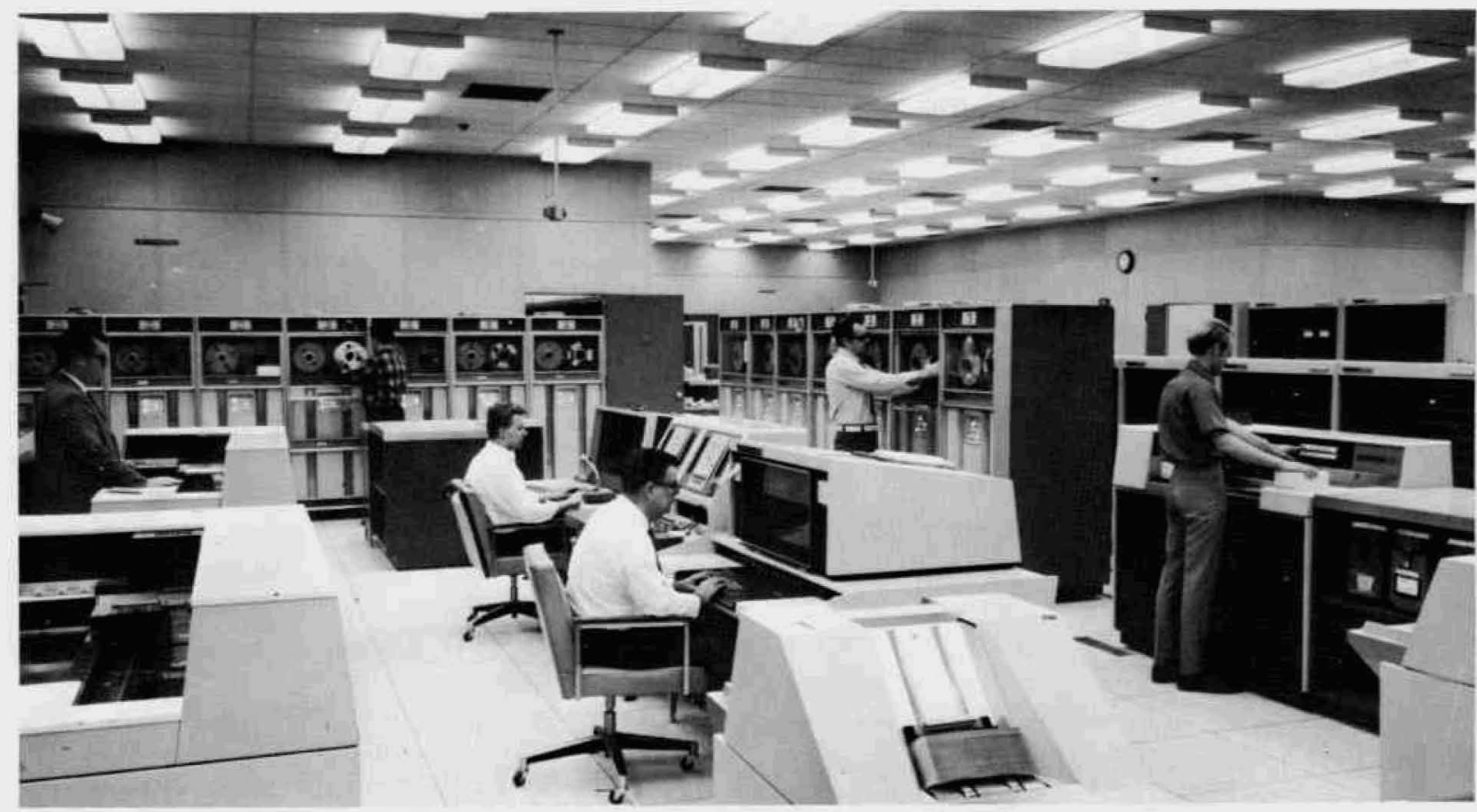

Figure 1. Area of the C.SCF computer room in which CDC 6600 operating activities are conducted. The two computer operators in the center are seated at the consoles for the two CDC 6600 computers. On the left an operator is loading the card reader for the first CDC 6600 . The operator in the left background is loading a reel of tape on one of the magnetic tape units, while the operator in the right background is removing one. Both function under instructions from the console operators. In the right foreground another operator is preparing to read cards into the second CDC 6600 computer.

SCOPE 3 developed by the manufacturer and by Department personnel were incorporated at various times during the year. As with any complex new operating system, debugging continued throughout the year as subtle difficulties were detected.

\section{Programming Services}

Programming consultation, for users who do their own programming, continued to be provided by a programmer-of-the-day in the CSCF users' room. Information on computer center procedures, software systems, hardware acquisition, and planning was disseminated to computer users through the Computer Newsletter. A limited amount of programming service was provided for the preparation of users' problems for computer processing. Some typical examples are included below.

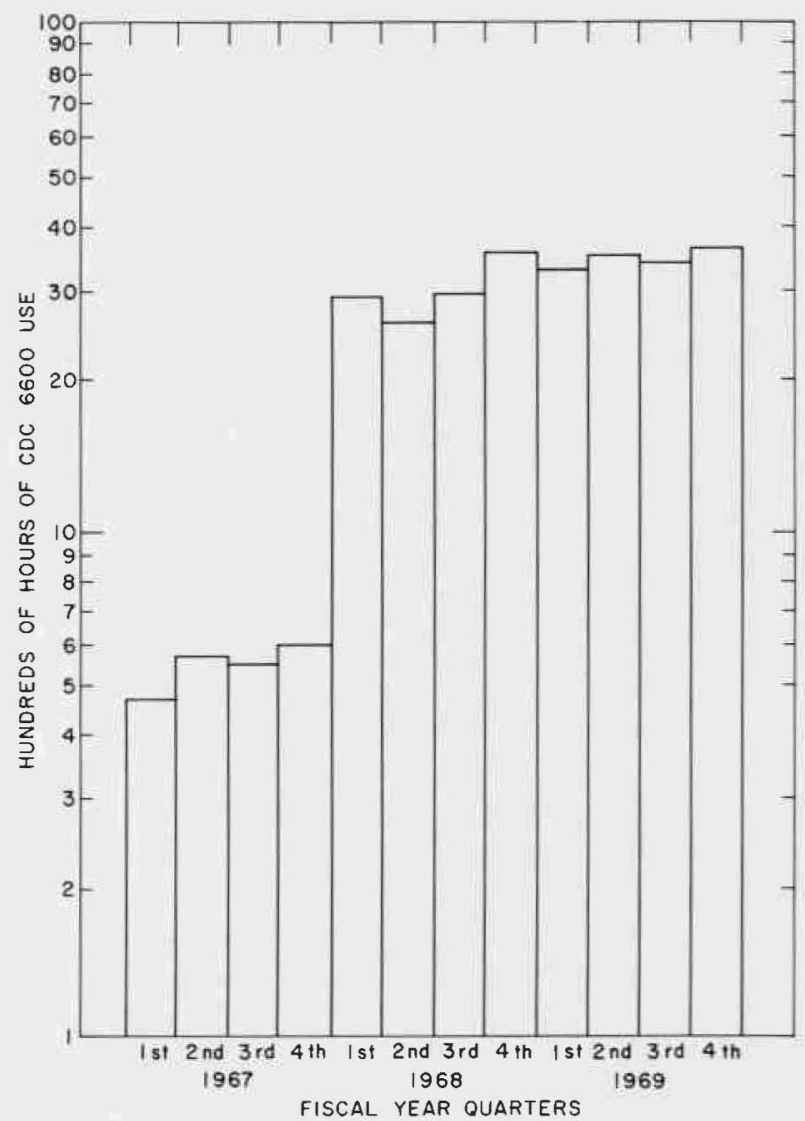

Figure 2. Histogram of CDC 6600 computer usage at Brookhaven National Laboratory. 
A program was written for solving the basic integrodifferential equations that describe the behavior of an aerosol distribution with respect to time. Various forms of this equation have been investigated elsewhere. In the present program considerable flexibility is permitted in the specification of such factors as particle sources, conglomeration, and settling. The program replaces the basic equation by a system of approximating ordinary differential equations. This approach allows the solution curve for each successive time step to be fitted by an approximating curve defined over a relatively short interval, without undue loss of accuracy. As these distribution curves shift with time, the program automatically shifts and contracts the interval of integration. These techniques allow a solution to be obtained with a minimal amount of computer time.

Construction of the linear accelerator involves the installation of an elaborate network of cables and wires. The technicians installing the components need detailed information on the cables of wires involved, the physical routes followed, the physical (geographical) location of each terminal or connection point, and the exact nature of the components that terminate or connect the wires. A program was written to create and store on magnetic tape tables of information concerning every wire in the network. Specifically, the stored information consists of wire terminals (geographical location and electrical type), wire numbers (for identification), groupings of wires (into cables), and cable routes. Means are provided for altering the nature of the network and for retrieving information about the entire network or specified sections. Paths can be traced from any designated point in the network. The program also makes a number of internal consistency checks on the structure of the network and any changes made in it.

A densitometer was constructed which measures the intensity of any selected point on a $5 \times 5$-in. photographic negative in steps of 256 intensity levels. In order to use the apparatus for densitometer measurements of diffraction patterns, a computer program was written for the CDC 924, which is connected on-line to the densitometer. Photographs are analyzed in packs of related exposures. As each negative is scanned (over a userspecified region), the following information is written on magnetic tape for subsequent analysis by a CDC 6600 program: coordinates of lattice points (in terms of the true crystallographic axes of the frame), the integrated difference intensity of the lattice-point spot, the weighted background intensity, and a descriptive code dependent on various characteristics of the lattice point. Lattice points with intensities falling below a user-supplied cutoff value are ignored. Subsequent negatives in a given pack retain the general lattice pattern but with decreasing intensity for corresponding points. The program makes use of this trend by remembering the positions of the lattice points used in the immediately preceding negative in the pack. Thus, when the intensity of a lattice point falls below the cutoff value, the corresponding points are ignored without examination on all subsequent negatives in the same pack.

\section{PROGRAMMING RESEARCH}

The Programming Research Division, which is closely allied with the Computer Research and Development Division, also is concerned with the development of new means for increasing the effective utilization of very large-scale central computing facilities and extending the boundaries of computer application in research in the life and physical sciences. Some of the effort is devoted to the development of advanced computer software. Both Divisions have been engaged in the development of commercially unavailable software and hardware for BROOKNET, the Brookhaven Digital Data Communications Network, which will provide on-line intercomputer communication at internal memory speeds between the CDC 6600 computers of the CSCF and small to mediumsized computers. The BRoOKNET hardware and software system will make the computational capability of the CDC 6600's available to remotely located on-site computers engaged in local processing functions such as monitoring remote experiments, acting as input-output stations, handling time-shared terminals, and performing reduction and analysis of experimental data. Other principal efforts have been concerned with the development of advanced software for effective utilization of the ECS and the development of a highly advanced operating system.

\section{BrOOKNet Software}

Software development for BROOKNET consists of three phases. The first phase was the development of an initial version of a general-purpose system to provide some of the basic BROOKNET functions re- 
quired for remote station operation and on-line processing through the GDC 6600 operating system. The design of the initial system was completed, programming was finished, and operation was checked out in the CSCF, with a PDP-8 computer used as the remote computer. The system was subsequently revised to incorporate additional functions which emerged from the overlapping effort on the second phase of development. By the end of the fiscal year, remote operation utilizing the initial system and the prototype remote input/ output station installed in the Chemistry Building had been achieved.

Phase 2 is the development of a full-scale general-purpose system which incorporates, in addition to the functions of the initial system, means for file management, scheduling, detection and diagnosis of hardware and software failures, recovery of BROOKNET files, and manual control of BROOKNET. This system will make the full logical and computational capability of the CDC 6600 computers available to remote users. Most of the design work was completed, portions of the system were coded, and debugging was started.

Phase 3 calls for expanding the capability of the general-purpose system to include the means for transmitting data directly between the remote computer and the ECS by utilizing the commercially unavailable ECS direct-access hardware developed at BNL. Provisions were made in the phase 2 software for the subsequent addition of this capability.

\section{Advanced Operating System}

The first stage was completed of the research and development effort on the design of an advanced operating system for a central computing facility configuration based on two CDC 6600 computers sharing the use of a one-million-word ECS unit. In this phase, an initial version was implemented, as planned, by the time of delivery of the ECS hardware. The approach taken was to partition ECS into separate areas for each attached computer. These areas were then subdivided into two parts. One was used to allow executing programs direct access to a portion of ECS for use in any manner that the programmer specified. This part of ECS was allocated to running programs in a manner exactly analogous to the way in which central memory is allocated. The other part of ECS was used for file storage and was integrated into the standard operating system input/ output procedures as if it were a small fast zerolatency disk. As a result, the file storage area can be used to contain not only users' files but the file of system programs, which reduces system overhead by allowing faster access to these programs. A procedure was implemented to move files created by a given job from ECS to the disk at job termination if the file storage area is not to be immediately released. The area is then available to currently active files. Studies of the effectiveness of the initial version of the operating system indicate that an increase of between 10 and $20 \%$ in the productivity of the computers is obtained.

The second stage of work on an advanced operating system is concerned with the design and development of a more sophisticated system which will provide a larger increase in the productivity of the central computers. Previous work in this area took into consideration the expressed intent of the Control Data Corporation to make available in the near future a highly advanced operating system, which, with appropriate modification, would be suitable for Brookhaven's use. The manufacturer's intent, as well as schedule, has changed, and the development effort at BNL has had to be expanded to satisfy the Laboratory's requirements and schedule. The present approach is to move toward such a system in steps by successively modifying the design of the initial system currently in use. Redesign for the first step was frozen and implementation was nearly completed. Debugging of the completed portions was begun. This first step consists of moving the portion of the system that controls and coordinates all other system activities from a peripheral processor to the central processor. This was done for two reasons: (1) peripheral processors cannot have direct access to ECS, and (2) the extreme speed of ECS would make the overhead associated with communicating with a peripheral processor (and even within the peripheral processor) unacceptably high.

A statistical sampling and analysis was made of the degree of saturation of various components under the current CDC 6600 operating system to obtain statistics with a direct bearing on the development of the advanced operating system and future acquisitions of computing equipment. The analysis was designed to reveal bottlenecks, weak points, and deficiencies and to aid in deciding which hardware and software additions or modifications would be most effective in raising the throughput capability of the hardware and software system. 


\section{Interactive Computing}

The work on graphics and interactive programming was continued with a study of the methods of interaction that BROOKNET operation will allow and appropriate graphic display techniques for each of these methods. Studies were also made of the corresponding modes of operation of the algebraic manipulation programs that will interact with the remote display terminal.

\section{COMPUTER RESEARCH AND DEVELOPMENT}

In addition to advancing the state of the art in data communications and advanced computer hardware systems, the Computer Research and Development Division is also concerned with developing new means for increasing the effective utilization of very large-scale computing facilities. During the year primary emphasis continued to be placed on the development of commercially unavailable devices for improving the operational efficiency of the CSCF and extending further its application in research at Brookhaven. This includes hardware implementation of BROOKNET. Work on the flying image digitizer (FID), interactive computing, and graphics was continued and a study of associative memory begun.

\section{BrOOKNET}

Work on BROOKNET continued throughout the year. Previously completed hardware for the medium-distance branch of BROOKNET to a Sigma7 computer in a user group was fully checked out from the CDC 6600 end to the remote computer adapter at the Sigma-7 end. Final checkout will take place after the completion of Sigma-7 software for conducting the BROOKNET dialogue.

An ECS coupler was designed to allow the BROOKNET branch path to be diverted from the CDC 6600 input/output channel and directed to an unused port of the ECS controller. The coupler appears to ECS as a third CDC 6600 computer. The new data path will be established selectively under program control by a series of devices called multiswitches, which interface with the coupler. Data transfers can then bypass the input/output channel, peripheral processor, and central memory buffer, and yet the first path can be re-established for executive dialogues between the CDC 6600 and the remote computer. In addition, switching devices were designed, constructed, and installed to allow the BROOKNET synchronizer chain to be referenced from either CDC 6600 computer.

\section{Flying Image Digitizer}

The feasibility of the design developed in fiscal 1968 for the FID was studied and some design changes were made. The FID still promises to be simple in structure but fast enough to meet the requirements of automatic data processing for superconductive bubble chambers. Digitizer-to-computer coupling, digitizer-to-operator communication, and the characteristics of the output signal from the detectors were studied. A highly simplified test model was designed and construction begun. A computer-controlled high-speed optical shutter was designed and the digital circuitry for finding a tract segment was devised.

\section{Quest}

A language called QUEST was developed to provide the means for using and troubleshooting any on-line peripheral hardware of the CDC 6600 computers during system operation. Previously, the checkout of peripheral hardware on the CDC 6600 necessitated dedication of the total computer system to the peripheral hardware during the checkout. The new language allows the hardware checkout programs to be written and run on the CDC 6600 system without system dedication to the peripheral hardware. Checkout programs written in QUEST are run under the CDC 6600 operating system and are handled like any other job.

\section{Interactive Computing/Graphics}

Operation and evaluation of a preliminary graphic station, consisting of a small computer, a cathode ray tube display, an alphameric keyboard, and an electrostatic printer, pointed the way to a more advanced system for multiple graphic terminals working interactively with the large computer and, ultimately, the CDC 6600 computer through brooknet. Preliminary examination was made of engineering support software programs produced elsewhere that may be used immediately in the batch system environment and also are capable of extended use on an interactive graphic system. 


\section{Associative Memory}

A study of associative memory techniques was initiated in an attempt to utilize advances in the semiconductor field to improve the efficiency of content addressable memory designs. The question of applications of an associative system centered around such a memory also was considered.

\section{MATHEMATICS RESEARCH}

The objectives of the mathematics research activity are to make broad general contributions in the field of mathematics, support and advance the scientific program at Brookhaven, and expand the utility and applicability of computers in research. Some of the efforts involved are conducted in collaboration with other researchers at Brookhaven and elsewhere who are engaged in physical, life science, and mathematics research. Other efforts are totally independent. In addition, the work done in this area complements some of the service functions of the Department.

\section{Combinatorial Analysis}

Combinatorial analysis encompasses problems from all branches of science. Many of these problems require determination of the number of elements in a collection that possess a specified set of attributes. Hence, some type of counting is necessary. Research at Brookhaven is concerned with the particular type of counting that involves the systematic use of the structure of a set to solve counting problems on that set. Consequently the research is concerned with the relationship of combinatorial theory to algebraic structures, particularly semigroups, lattices, and groups. At present, work is directed toward the generalization of some analytic methods for counting on semilattices to semigroups and sets. During the past year, many of the algebraic concepts leading to analytic formulas for solving some combinatorial problems on lattices and partially ordered sets were generalized and shown to apply to sets with only a multiplication operation defined. A well-known and useful formula for solving combinatorial problems on partially ordered sets is the Möbius inversion formula. Furthermore, the Möbius function is known to be an invariant on lattices. This is to say that certain types of lattices each have the same functional formula for the Möbius function. All these results are completely analogous to theories of transforms and inversions in calculus. During the past year a Möbius inversion formula was developed for partial groupoids (i.e., sets with only a multiplication operation defined) and semigroups. In addition it was shown that the Möbius function is also a natural invariant on certain classes of semigroups. Specific examples are being developed to show the applicability of these results to the solution of combinatorial problems on these structures.

When a set is specified in an enumerative manner, no difficulties are experienced in counting the number of elements in the set. Difficulties arise when the set is specified by attributes not directly relatable to the enumeration of its elements. The Burnside lemma is a powerful mathematical tool for determining the number of elements in sets such as these. Counting is accomplished by overcounting in a manner governed by the specifications of the set and then reducing the count by making use of symmetries that are necessarily introduced by the overcounting. This lemma is the basis for the Polya counting theorem so widely used today in counting problems. During the past year refinements and variations of the Burnside lemma were developed and applied to some specific problems. One of the problems is concerned with the number of pairings of classes of like objects. A general expression was found for the number of distinguishable pairings of $k n$ elements belonging to $n$ distinct "genotypes" each consisting of $k$ indistinguishable members. These expressions are applicable in many areas, such as the study of genealogical relationships in plant and animal life, the collision of like particles in nuclear and molecular physics, and the proof of theorems on pfaffians in mathematics. Another problem dealt with was the classification of the number of distinct groupoids containing $n$ elements. An expression was derived which gives the number of distinct functions of two variables that can be formed from $n$ elements. This is the number of groupoids.

\section{Padé Approximants}

Perturbation theory is concerned with the problem-solving approach in which successive corrections to the known solution of a simple problem are calculated in order to reduce the difference between the answer to the simple problem and that to a far less tractable one which cannot be calculated directly. However, perturbation theory suf- 
fers from the deficiency that the accuracy obtained after a number of corrections have been calculated is not readily ascertainable. In some cases the higher corrections may be farther from the correct answer than the earlier one. Research is being conducted at Brookhaven on a Padé approximant technique for the solution of difficult physical problems and on the development of a general theory of the Padé approximant. For a special, although commonly occurring, class of function, convergence has been proved and best-error bounds have been established for all values of the argument for which the function is nonsingular.

The Padé approach in many cases allows upper and lower limits to be placed on the correct solution, based on perturbation theory corrections and a careful analysis of the problem under consideration. This technique has been applied successfully to several problems and its applicability to many scientific problems has been demonstrated. Questions of convergence and the provision of error bounds for values of analytical functions that arise in physical research are being studied through the use of Padé approximants. An investigation using Padé techniques is being conducted into the behavior in the neighborhood of the critical point of various thermodynamically significant properties of the Ising and Heisenberg models of ferromagnetism, order-disorder phenomena, and lattice gases through the use of exact series expansions. Here rigorous bounds on the errors of the solution of the model equations have been established and detailed predictions made of certain experimental properties whose measurement should serve as a precise check on certain aspects of the models. A new method is being used, with appreciable success, to determine the behavior below the critical temperature of the Heisenberg model of ferromagnetism. Previously, reliable results were available only for temperatures above the critical temperatures. The same method is being applied to the better-understood Ising model of magnetism in order to obtain sharper results and to verify the method. Reasonable success has been achieved in all cases.

Valid procedures were sought for calculating the ground-state energy of a many-fermion system by making use of the idea of an appropriately formulated expansion in the interaction strength at fixed density. The fourth order in the perturbation expansion parameter of the many-fermion energy for a potential closely simulating that for nuclear matter is being calculated in an effort to gain a qualitative result for the binding energy in that system. These expansions will be summed by means of Padé approximants. The results should be much more reliable than those given by previous methods because of the greater attention given to the singular points in the energy function.

\section{Neutron Transport Theory}

Two classes of problems in neutron transport theory (thermal and fast systems) were investigated. By means of a mathematical technique called the method of analytic continuation some basic new facts regarding the Boltzmann equation have been discovered. These facts have been applied to explain discrepancies between theory and experiment in the neutron poisoning problem, in which the spatial decay of a neutron population in a moderator is studied as a function of the amount of poisoning for neutron absorbing material placed in the moderator. It was found that previous theoretical analyses were inadequate and that the experimental results are perfectly consistent with the mathematical properties of the Boltzmann equation. The mathematical experience gained with the thermal systems was applied to fast systems for which the analysis was similar, but more complex. The results of the analysis were found to be in qualitative agreement with some experiments and numerical studies recently completed in France. A mathematical analysis of the Boltzmann equation was also applied to give further insight into the problem of high-frequency ground propagation in a rarefied gas. A firm analytical foundation was provided which explains the rationale of some earlier numerical work on this problem. In a standard reference on higher transcendental functions (the Bateman manuscript project), an important function that occurs in neutron transport theory (for fast systems) and in the theory of radiative transfer had been incorrectly described. A correct analysis of this special function and a related class of special functions was made and applied to the study of fast pulsed neutron systems.

\section{Three-Body Rearrangement Process}

The rearrangement process, $A+B C \rightarrow A B+C$, is of fundamental importance in atomic and molecular scattering problems. Many models and approximation methods have been devised because 
the general dynamics of three-body rearrangement collisions are not amenable to exact mathematical treatment. Classical mechanics plays an important role in the theories used to gain insight into this phenomenon. Therefore the conditions under which the classical approach might be valid have been extensively considered. The classical equations describing this rearrangement process are analogous to the equations describing the motion of a particle moving on a potential surface. Classical mechanics should be valid if the de Broglie wavelength (which is inversely proportional to the momentum) is sufficiently small in the reactive region. Hence, a better classical result is expected if the interaction potential is represented by a depression in the potential surface that the particle slides down, rather than a barrier that it must climb. A model of a three-body rearrangement process was defined which is solvable in both the classical and quantum mechanical formulations. A study of several cases, using the model to compare classical and quantum results, indicated that if classical mechanics is valid at all, it gives better results in the case of a potential barrier than a potential well. This contradicts the earlier argument. The quantum mechanical results also yielded information about the existence of an "activated complex" during the rearrangement process. An intrinsic difficulty with classical mechanics is its inability to take proper account of vibrationally excited states without an artificial assumption regarding the distribution of energy into discrete states. The quantum mechanical study made it possible to consider, without further hypothesis, vibrational excitation.

In another effort, a time-dependent scattering theory for both the wave and Schrödinger equations was developed. Formulas were obtained for the transmission and reflection coefficients associated with the problems concerning transmission lines and wave guides. In addition, a study of nonselfadjoint differential operations was conducted. Results were obtained concerning general second-order perturbations of certain differential operators.

\section{Linear Operators and Elementary Symmetric Functions}

The ultimate goal of the work in this area is a deeper analysis of the structure of linear operators. Such an analysis will clarify the mathematical model for quantum mechanics and mathematical models in other fields. Toward this end a better understanding of the relationship between linear operators and eigenvalues is being sought. Three approaches for studying this relationship were explored: determinantal inequalities, linear transformations on $\mathrm{C}^{*}$-algebras, and numerical range of bounded operators. As a result of the work of the past year, it is now possible to extend Hadamard's classical determinantal inequality for positive semidefinite matrices to the broader class of complex elementary symmetric functions. Moreover, for the first time a definition was obtained for elementary symmetric functions on quaternionentried matrices. The generalized Hadamard inequality was shown to hold in the quaternion case as well. In a related exploration it was proved that any bounded linear transformation sending one $\mathrm{C}^{*}$-algebra to another is uniquely decomposable as a linear combination of four completely positive transformations. Finally, the classical HausdorffToeplitz theorem on numerical range was extended to all the elementary symmetric functions. Further research is expected to reveal information pertaining to quantum mechanical situations described by a $\mathrm{C}^{*}$-algebra context.

\section{Monomer-Dimer Problem}

An important problem in physical research is to understand what occurs during a change of state, or so-called phase transition. In the field of lattice statistics, phase transitions are studied by applying the procedures of statistical mechanics to models of physical systems. Work was directed toward the calculation of the properties of the monomerdimer model, one of the simplest, yet unsolved, problems in lattice statistics. The model consists of a regular lattice in which some sites are vacant while others are linked to one of their neighboring sites. The vacant sites are regarded as occupied by "monomers," and two linked sites are occupied by either end of a "dimer." This model has been used, for example, in connection with the theory of absorption of diatomic molecules and in the cell cluster theory of liquids. The central problem is to calculate, with use of statistical mechanics, the thermodynamic properties of the model in the thermodynamic limit, with particular emphasis on the possible existence of a phase transition. The model was studied for several two- and threedimensional lattices by deriving the leading coeffi- 
cients of exact series expansions. Numerical analysis of the series enabled the thermodynamic properties to be calculated and showed that, while there is no phase transition, close-packing is a singular point. A novel feature of this model is that for lattices of fixed dimension the singularity is found to depend on the lattice structure. The reason for this behavior is being studied further. 


\section{LIFE \\ SCIENCES}




\section{Biology}

The present report provides brief summaries of projects conducted by members of the scientific staff of the Biology Department. Research is in progress at all levels of biological study, from ecology to biophysics, and is related to the development of basic information that will lead to improved understanding of the manner in which ionizing radiations affect living things and in which these effects may be used constructively for specific biological purposes. Insofar as the problems examined permit, emphasis is given to the molecular approach and mechanistic rather than descriptive studies are stressed. Thus, a common theme throughout is the correlation of macromolecular structure with biological organization and function.

A number of the projects derive immdiate benefit from the availability of research reactors and particle accelerators at Brookhaven, while more specialized irradiation sources are utilized directly within the Biology complex. A noteworthy feature of the program is the number of projects that relate to studies with plants. In addition to extensive field and greenhouse facilities, specialized growthchamber space is available for precise manipulation of the environments in which plants are maintained.

The Biology Department maintains a number of collaborative programs with visiting scientists from other institutions, both in this country and abroad, and sponsors the Brookhaven Symposia in Biology, a series of annual conferences at the Laboratory on topics of current interest in biological research. The topic of this year's symposium is Diversity and Stability in Ecological Systems. The proceedings will appear as the 22 nd volume in the series.

\section{PLANT SCIENCES}

\section{Increased Mutagen Efficiency in Plants}

Ethyl methanesulfonate (EMS) induces mutations in plants with high frequency and presumably by specific reaction with genetic material. Since EMS treatments may induce physiological damage, ways were sought to reduce such damage without affecting mutagenic activity. Damage by EMS is thought to be caused mainly by watersoluble decomposition products, and therefore water-extraction of seeds treated with the mutagen was tried. This is feasible in maize, since the seed tolerates soaking in water and drying. When seeds were soaked in water for 4 to 6 days after treatment with EMS, dried, and then planted, damage to the plant was eliminated. The soaking treatment with water also reduced the mutation frequency. However, the efficiency of the mutagen (mutation frequency compared with plant damage) was maximized, i.e., the damage was reduced more than the mutation frequency.

\section{Photoperiodism and Photomorphogenesis}

Many aspects of plant development depend on red and far-red light, probably acting through the chromoprotein phytochrome. Phytochrome can take two relatively stable forms, reversibly interconvertible by light: $\mathrm{Pr}$, absorbing predominantly in the red, and Pfr, in the far-red. Pfr is probably the active form. The relationship between physiological response and tissue content of measureable phytochrome is complex. For example, there appear to be no differences in response attributable to differing phytochrome contents among pea seedlings de-etiolated with various red and farred treatments. However, de-etiolation itself brings about differences in the apparent rate of response to Pfr, estimated physiologically, and in the spectrophotometrically detectable Pfr transformations that take place in darkness. De-etiolated seedlings, whether high or low in total phytochrome, appear to be less sensitive to Pfr than etiolated (totally dark-grown) seedlings, and the ratio of Pfr destruction to Pfr reversion is higher than in etiolated tissue. Much further work is needed to establish whether the suggested relationship between the rate of Pfr action and Pfr transformations is physiologically significant or merely fortuitous.

The differences between the chemical properties of $\mathrm{Pr}$ and Pfr presumably reflect photochemically induced changes in structure. Study of the differences should eventually bear on the mechanism 
of phytochrome action. Peas contain a smallmolecular-weight substance that attacks Pfr with substantial specificity relative to $\mathrm{Pr}$, which causes it to lose photoreversibility. Methods for concentrating, purifying, and identifying this "Pfr killer" are being developed. Although Pfr was found to be more labile than $\operatorname{Pr}$ in all previous work with nonenzymic reagents, it is now clear that several aldehydes, including glutaraldehyde and acetaldehyde, affect Pr more than they do Pfr. This suggests that the $\varepsilon$-amino groups of lysine, with which aldehydes are believed țo react with some specificity, are more accessible in Pr than in Pfr.

\section{Plant Bile Pigments}

Bile pigments are found in all plants. They can function as photoreceptors in photosynthesis and in the regulation of growth and development. The pigments, generally, are linked covalently at unknown sites to specific proteins. In continuing work concerned with the sites of attachment between pigments and proteins, enzymatic methods are devised for preparation of bile pigments linked to a small portion of the amino acid backbone of their proteins. By ion-exchange separation some bilipeptides were purified to homogeneity.

Large areas of the sea are occasionally colored red by the marine ciliate Cyclotrichium meunieri. The red pigment in the ciliate was found to be a cryptomonad-type phycoerythrin, a bile pigment. The ciliate also contained chlorophylls $a$ and $c$. Thus, the numerous photosynthetic symbionts in each ciliate belong to the Cryptophycae.

\section{Protochlorophyll Holochrome}

Following photoreduction of protochlorophyll to chlorophyll in dark-grown leaves, there occurs a series of dark reactions characterized by spectral shifts from long to shorter wavelengths. Analogous shifts were observed in preparations of the protochlorophyll holochrome isolated from dark-grown bean leaves after exposure to red light. They apparently reflect changes in protein conformation following photoreduction of protochlorophyll, since the spectral shifts are sensitive to $p \mathrm{H}$, temperature, and mild protein perturbants.

\section{The Mechanism of Electron Transport} and Energy Conversion in Photosynthesis

Illuminated chloroplasts oxidize water by way of a series of electron transfers involving one photo- chemical and several thermal steps, known collectively as photosystem II. Chloride ion ( $\left.\mathrm{Cl}^{-}\right)$is a necessary component of photosystem II. The Clrequirement provides means for selective inhibition of electron flow and identification of electroncarrying components of the system. According to the results of present work, the lack of $\mathrm{Cl}$ - depresses the fluorescence yield in pea chloroplast preparations because of a drop in maximum yield at saturating light intensity and in quantum efficiency. The fluorescence emission spectra, at $77^{\circ} \mathrm{K}$, in the presence and absence of $\mathrm{Cl}-$ differ around 693 $\mathrm{nm}$. This is thought to indicate an effect of $\mathrm{Cl}^{-}$on the redox state of the photosystem II light trap in unfrozen material. The fluorescence data support location of the site of $\mathrm{Cl}^{-}$action between the water-splitting enzyme and the photoact. $\mathrm{Hy}$ droxylamine, hydrazine, and related compounds, in high concentration, donate electrons to photosystem II and thus bypass the site at which $\mathrm{Cl}^{-}$is required. These same donors also reverse the inhibitory effect of high concentrations of amines or ammonia on electron flow. Chloroplasts from manganese-deficient Euglena cultures have weak photosystem II activity but the electron flow is likewise restorable by the donor systems.

\section{Control of Cell Division}

In excised pea root tips, carbohydrate deprivation results in the accumulation of meristematic cells in either the $G_{1}$ or $G_{2}$ stages of the mitotic cycle. Following carbohydrate restoration, $G_{1}$ cells initiate deoxyribonucleic acid (DNA) synthesis and $G_{2}$ cells enter mitosis. Thus, initiation of DNA synthesis and mitosis both require a relatively large supply of energy. According to further analysis of the energy requirement for cell replication, initiation of the processes indicated depends upon aerobic metabolism. Continuation and completion of DNA synthesis also require oxygen. Experimentally, the synthesis can be arrested by anaerobiosis for up to $24 \mathrm{hr}$ and restored to the control rate by resupply of oxygen. The accumulation of cells in $G_{1}$ and $G_{2}$ is associated with loss of ribonucleic acid (RNA) and protein required for DNA synthesis and mitosis proportional to the duration of carbohydrate deprivation. Provision of an energy source enables the cells to synthesize the requisite RNA and protein. Apparently the $G_{1}$ and $G_{2}$ periods of the mitotic cycle represent distinct physiological states during which proliferative cells prepare for DNA synthesis and mitosis. 
Further, the main expenditure of energy in cell division occurs in preparation for these processes.

\section{Plastid Development in Plants}

The plastids in the leaves of higher plants begin as relatively simple, polymorphic structures in germinating seedlings. In the light, these structures develop membranes finally arrayed in rather specific three-dimensional patterns. In the dark, seedling plastids first develop a complex of tubular membranes known as the prolamellar body. According to the reports of others, light is required for the next two steps in development: a small amount of light is needed for the first expansion of membranes into lamellae that grow out from the prolamellar body into the stroma of the plastid; more light is required for the second step - pairing of these lamellae into the close association found in functional chloroplasts. To test this proposed sequence in development, kidney beans were germinated in complete darkness and fixed in the dark after suitable periods of growth. Thin sections of portions of the leaves were examined in the electron microscope. In plastids examined after 5 days of seedling growth in the dark, lamellae were observed extending out from the prolamellar body. The lamellae are actually flattened sacs limited by a membrane. The outer surfaces of these lamellae

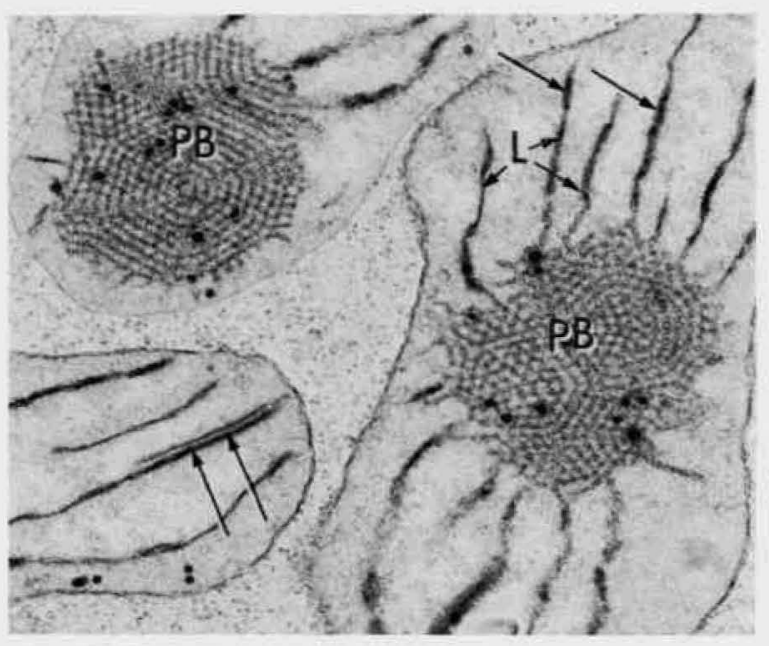

Plastid development. Plastids in the palisade cells of a kidney-bean seedling grown in the dark for five days are shown. $P R=$ prolamellar body; $L=$ lamellae; the arrows indicate paired membranes. Neither expansion of the membranes arising from the prolamellar body into membranes nor membrane pairing requires light. are appressed to one another. These results are contrary to reports of others and indicate that expansion of the membranes to form lamellae and membrane pairing are not light-dependent processes.

\section{Relative Biological Effectiveness of Heavy lons in Producing Mutations, Tumors, and Growth Inhibition}

Dry seeds of Arabidopsis thaliana, a short-life-cycle Crucifer, were irradiated in the heavy ion linear accelerator (Hilac) at the University of California with the accelerated heavy ions $\mathrm{He}^{4}, \mathrm{Li}^{7}, \mathrm{C}^{12}, \mathrm{O}^{16}$, $\mathrm{Ne}^{20}$, and $\mathrm{Ar}^{40}$. The small size and simple geometry of Arabidopsis seed embryos made it possible to locate the Bragg peak of maximum ionization, for ions of widely different linear energy transfer (LET), within the meristem region from which the plant develops. For reference in computing relative biological effectiveness (RBE), exposures were also made to $250-\mathrm{kVp}$ x rays and to fullenergy helium ions, for which the respective LET's were 2 and $18 \mathrm{keV} / \mu$, as compared with Braggpeak-adjusted heavy ions for which the LET ranged from $74 \mathrm{keV} / \mu(\mathrm{He})$ to $1890 \mathrm{keV} / \mu(\mathrm{Ar})$. Response to the irradiations was measured on the basis of three criteria: frequency of induced tumors, percent decrease in plant dry weight, and frequency of an induced locus-specific somatic mutation. The relative response was the same for each criterion: $x$ rays $(L E T=2)$ were least effective, full energy helium ions $(\mathrm{LET}=18$ ) were about twice as effective as $\mathrm{x}$ rays, Bragg-peak $\mathrm{He}^{4}$

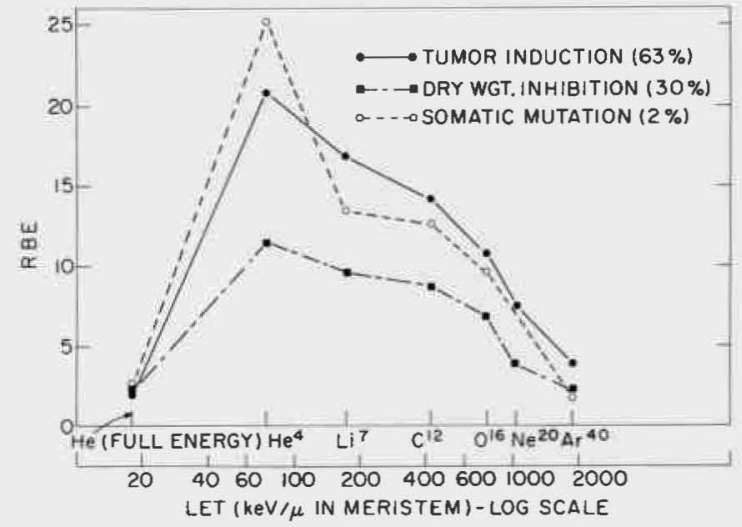

The relative biological effectiveness (RBE) compared with $\mathbf{x}$ rays of heavy accelerated ions of widely different linear energy transfer (LET) based on three different criteria of response. 
ions (LET $=74$ ) were the most effective, and there was then a declining effectiveness from $\mathrm{Li}^{7}$ ions $(\mathrm{LET}=172)$ to $\mathrm{Ar}^{40}$ ions $(\mathrm{LET}=1890)$. The RBE's for Bragg-peak helium ions were high compared with $\mathrm{x}$ rays $-25,21$, and 12 for mutation, tumorization, and growth inhibition, respectively. Since all three criteria gave the same relative response over a wide range of LET, the results suggest that the targets sensitive to radiation damage are the same for each. Since for induction of mutation these targets are the chromosomes, the results support the conclusion that radiation damage to normal processes of growth and of differentiation (as shown by tumorization) is due to chromosome damage.

\section{Long-Term Survival in Chronically Irradiated Trees}

Young trees of 13 species were planted in the field in the spring and exposed to $\gamma$ radiation during 7 years. For each species, the daily and accumulative radiation doses $(\mathrm{R})$ required to kill $50 \%$ of the trees $\left(\mathrm{LD}_{50}\right.$ 's) were determined annually. The

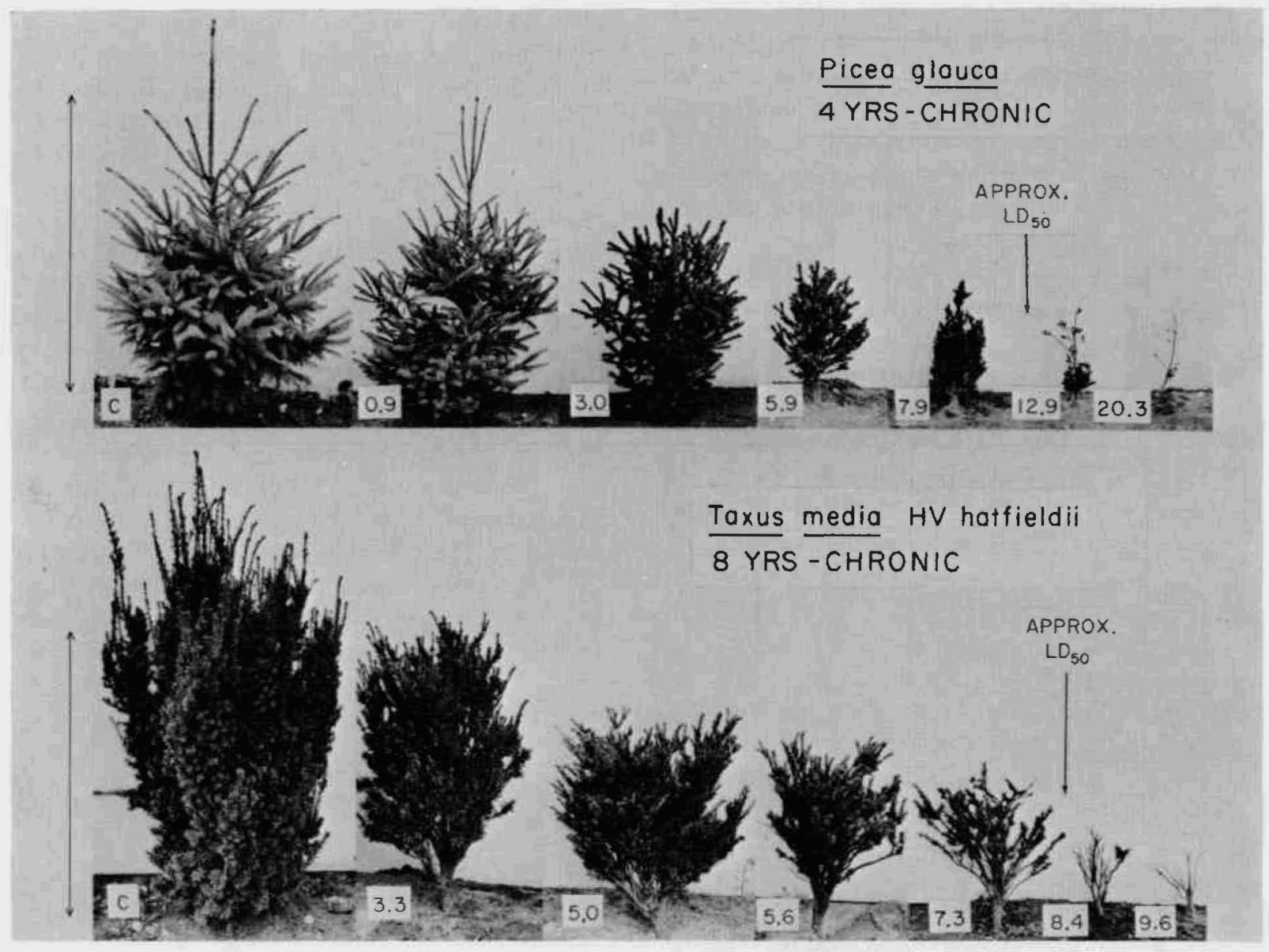

Effects of chronic $\gamma$-irradiation in white spruce and yew. Representatives of white spruce (Picea glauca) and Hatfield yew (Taxus media $\mathrm{HV}$ hatfieldii) subjected to various levels of chronic $\mathrm{Co}^{60}$ $\gamma$-irradiation for 4 and 8 years, respectively, are shown. $C$ indicates controls; the numbers, the daily dose of radiation in roentgens. The approximate daily exposures at which half of the two species of trees survived are denoted by the arrows. Growth was reduced to about half that of the controls by exposures much smaller than those required to produce $50 \%$ killing. Note the tendency in Picea glauca for a change in growth habit with increasing exposure: the control and $0.9-\mathrm{R} /$ day groups are excurrent in habit, while with increase in dose rate there is an increase in deliquescence (one terminal leader no longer dominant). This change in habit obtains in other irradiated species that are normally excurrent. (Arrows at left of each set of trees indicate one meter.) 
daily LD's decreased, whereas the accumulative LD's increased during the irradiation period. The rates of change in the LD's became stabilized by the third year. The daily 7-year $\mathrm{LD}_{50}$ 's were about half, the accumulative $L_{5} D_{50}$ 's about four times, the corresponding 1-year values. Apparently, a highly effective repair system developed in the plants during the third year of exposure to the radiation. The daily 3-year $L_{50}$ 's ranged from 8 to $31 \mathrm{R}$ in 5 species of gymnosperms and from 85 to $138 \mathrm{R}$ in 7 species of angiosperms.

\section{Radiobiological Studies of Tradescantia Plants Carried in Earth Orbit by Biosatellite II}

In the first concerted American attempt to study basic biological and radiobiological processes in space, a satellite was launched from Cape Kennedy and recovered near Hawaii after making 30 orbits of the earth during about $45 \mathrm{hr}$. The spacecraft carried 13 experiments involving 10 species of organisms and a $\gamma$ radiation source.

The Tradescantia experiment probed for effects of space environment on spontaneous and radiationinduced mutation rates and cytological changes in clone 02 . Thirty-two young flowering plants were arranged to allow the flower buds to be exposed to $223 \mathrm{R}$ of $\gamma$ rays and the roots, immersed in nutrient, to 116 to $282 \mathrm{R}$ during the flight. Control plants were carried behind a tungsten radiation shield. Additional irradiated and unirradiated controls were maintained at the launching site. After retrieval of the spacecraft, intact plants and samples of root tip, ovary, and stamen tissues were taken to Brookhaven for observations on the following: somatic mutations (blue to pink or colorless cells), cell size (giant and dwarf), loss of reproductive integrity (stunted stamen hairs), pollen grain mortality (early and late stages), disturbed mitotic spindle function, and chromosome aberrations. The data indicated no significant modifications of somatic mutations or cell size and no chromosome aberrations attributable to weightlessness or dynamic flight factors. However, pollen abortion and loss of reproductive integrity in irradiated material increased as a function of weightlessness. Disturbed mitotic spindle function in root tip and microspore cells was associated with orbital flight in irradiated and control plants. Clearly, differences obtained between flight and nonflight material. Explanation of the differences and assessment of their significance are being sought in continuing nonflight tests.

\section{Radiation Mułation Program}

Facilities at the Laboratory are used to irradiate biological material for workers in this and other countries. Some of the irradiations are made for plant breeders who use the irradiated material in the search for improved varieties; others are for individuals concerned with various aspects of radiation biology. Illustrative of the work involving use of irradiated material is that of D.I.T. Walker and colleagues at the West Indies Sugar Cane Breeding Station, Barbados. They are concerned with the development of nonflowering varieties of sugar cane. Such varieties are desired because in the absence of flowering the cane continues vegetative growth and sugar production. Nonflowering types have been found in material irradiated with $\gamma$ rays. These are undergoing yield trials and perhaps may soon be in commercial production.

\section{Chlorophyll-Protein Complexes} and Photochemical Reaction Centers

Through use of the detergent sodium dodecyl sulfate, the membranous structures of photosynthetic bacteria, blue-green algae, and higher plants were dissolved to yield well-defined chlorophyll-

Spindle malfunction in the root tip of Tradescantia clone 02. The cells shown are from unirradiated material carried by Biosatellite II in earth orbit. The multinucleate condition indicated by the arrow suggests that during mitosis the chromosomes scattered throughout the cell, presumably because the spindle did not form or did not function. $\times 1000$.

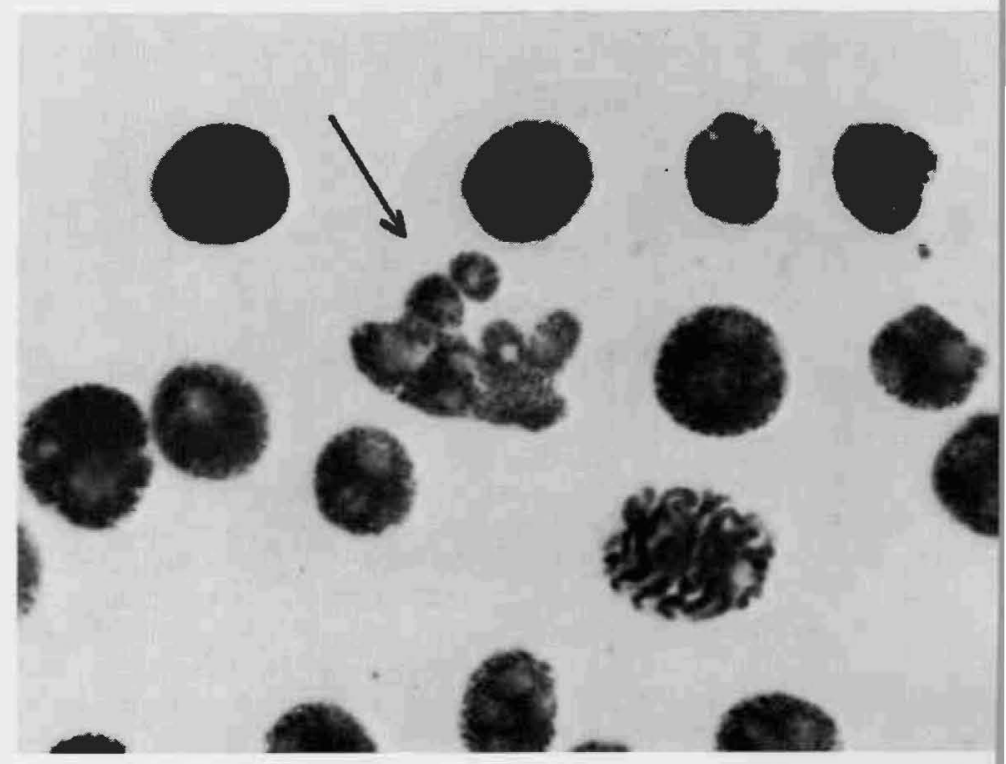


protein complexes which in most cases contain photochemical reaction centers. In the case of Rhodopseudomonas viridis, the reaction center preparation contains chlorophyll, cytochrome, and carotenoid in the molar ratio $3: 7: 1$ and can carry out a light-driven cyclic electron flow. From the purple sulfur bacterium Chromatium the detergent releases two chlorophyll-protein complexes, each associated with a different reaction center. One drives cyclic electron flow; the other appears to be associated with a noncyclic electron transport chain from exogenous donors to an unknown acceptor, possibly ferredoxin. The chlorophyll $a$ protein from blue-green algae contains $P 700$, the reaction center chlorophyll of system I in oxygen-

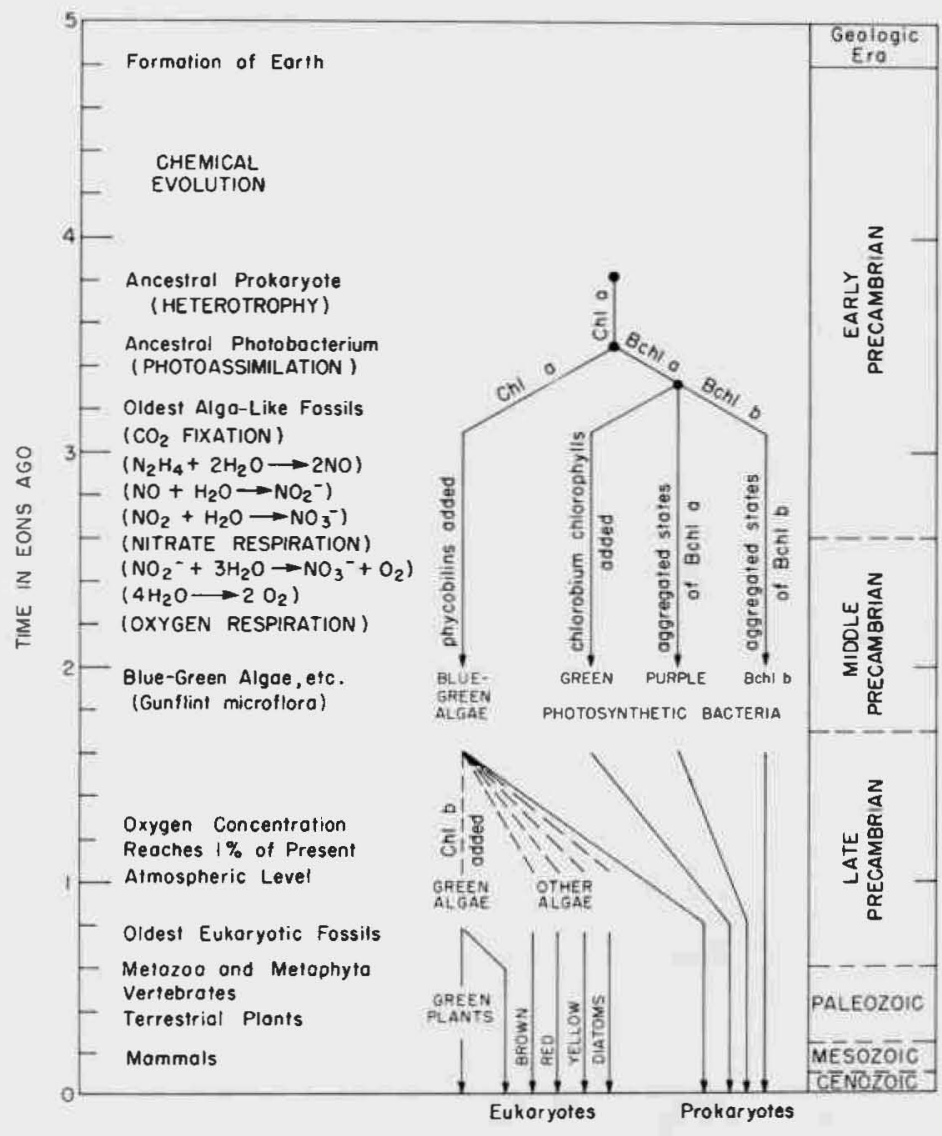

Evolution of photosynthesis. The hypothesis presented relates the photosynthesis of bacteria and blue-green algae to that of a common ancestor. According to the hypothesis, the algae developed the capacity to evolve oxygen after a long period of evolution in which nitrogen in various oxidation states served as electron donor for $\mathrm{CO}_{2}$ fixation. The scheme is consistent with thermodynamics and current views about the nature of the earth's atmosphere $3 \times 10^{9}$ years ago. evolving organisms. Similar chlorophyll $a$ proteins exist in green algae and higher plants. However, these organisms also contain a system II chlorophyll protein complex having chlorophylls $a$ and $b$ in a $1: 1$ ratio.

\section{GENERAL PHYSIOLOGY}

\section{The Role of Cyclic 3',5'-Adenosine Monophosphate in the Epinephrine Insensitivity of the Hypophysectomized Dog}

Cyclic 3',5'-adenosine monophosphate (cyclic AMP) is known to initiate the activation of phosphorylase induced by epinephrine. The AMP is formed inside the cell in response to external application of the hormone. The activation of phosphorylase causes increased breakdown of glycogen. The final product of the breakdown in liver is glucose, which passes into the blood and raises the blood sugar level.

In the hypophysectomized dog, epinephrine administration increases the blood sugar relatively little. Thus, some link in the chain of events between application of the hormone to liver cells and eventual increase in blood sugar differs in the hypophysectomized animal. To partially identify this link, comparison was made of the effects of the dibutyryl derivative of cyclic AMP on blood sugar in the normal and the hypophysectomized animal. The derivative when given by intravenous infusion penetrates liver cells to some extent and thus increases the blood sugar. Surprisingly, the output of glucose in response to the dibutyryl derivative is greater than normal in the epinephrine-insensitive hypophysectomized animal. The simplest conclusion is that some link in the chain of events between application of epinephrine to the liver cells and increase in cyclic AMP is aberrant in the hypophysectomized animal. Another, but less likely, possibility is that an intracellular enzyme (phosphodiesterase) which splits cyclic AMP is more active in the liver of the hypophysectomized than of the normal animal and that this enzyme does not hydrolyze the dibutyryl AMP. Testing of both hypotheses is under way.

\section{Effect of X-Irradiation on Hemoglobin Synthesis in Duck Erythrocytes in vitro}

Decreased rates of heme and globin synthesis, turnover of free protoporphyrin, transfer of plasma iron to erythrocytes, and incorporation of plasma 
iron into heme in duck blood in vitro and decreased levels of blood porphyrin and reticulocytes were observed 1 to 8 days after $x$-irradiation of the animal. The decreased rates and porphyrin level presumably reflected the decreased levels of reticulocytes, i.e., they were a consequence of erythropoietic arrest. The various parameters were unchanged at 5 and $30 \mathrm{~min}$ after irradiation of the animal and after direct irradiation of the blood. The latter findings are in contrast to reports that stimulation of the in vitro synthesis of heme and globin in spleen and of heme in bone marrow follows irradiation of the rabbit and that stimulation of heme and globin synthesis in dog erythrocytes in vitro follows irradiation of both the animal and blood alone. The contrast may reflect differences in the several systems of hemoglobin synthesis.

\section{Radiation-Induced Aging}

The late effects of irradiation in animals closely resemble those of natural aging. Based on the close parallel observed between chromosome stability and both natural and radiation-induced aging, a somatic mutation theory of aging was proposed. Although findings in support of the theory accumulated steadily, some were inconsistent. Thus, a composite theory was developed which proposes that aging is due not to a single chance event such as a mutation, but to a series of such events. This theory correlates much of the information on aging and predicts an average of about five events as necessary for induction of a degenerative disease. By using a method of estimating the mutation rate in somatic cells based on the incidence of liver tumors developing in a special strain of mice, two of the steps postulated in the composite theory were identified. The steps consist of a mutation followed by a stimulus for cell division. Attempts to identify a third step indicate only that it is not another mutation.

It is generally thought that nondividing mammalian cells, e.g., those in liver, accumulate faults with increase in age, whereas dividing cells, e.g., those in bone marrow, eliminate faults through cell selection. This supposition was tested in the mouse with use of a method developed for scoring chromosome aberrations in bone marrow. The aberration frequency tends to increase late in life. It increases immediately after irradiation but returns to normal within $48 \mathrm{hr}$, only to increase late in life sooner than in control animals. Work is being continued, but it is now apparent that the dividing cell systems of the mammal cannot be considered immortal in the same sense as are bacterial cultures. Loss of chromosome stability in bone marrow cells late in life perhaps reflects depletion of chromosomal repair enzymes. To test this possibility the bone marrow cells of mice of different ages were scored for chromosome aberrations as a function of time after irradiation. The aberration frequency was found to increase and then return to the control level at the same rate in mice of all ages. Thus, chromosomal repair mechanisms function well into extreme old age.

\section{Material Transfer Across Biological Barriers}

The effect of solutes added to the homogeneous phases $\alpha$ and $\beta$ of the material transfer system, phase $\alpha /$ membrane/phase $\beta$, upon the exchange resistance of the membrane to water was determined. For both Millipore filters and cellophane membranes, the exchange resistance was found to be linearly related to the square of the concentration of the added substance. Each solute had a specific effect on the magnitude of the resistance. Sucrose, for example, increased the magnitude sixfold when the concentration in the terminal phases was $2 M$.

\section{MOLECULAR BIOLOGY}

\section{Glycoprotein Structure}

The ribonuclease present in the pancreatic secretion of the pig is a highly heterogeneous glycoprotein which contains some $35 \%$ polysaccharide. The availability of this enzyme provides for structure-function studies of a well-defined glycoprotein and permits assessment of the at present obscure biological significance of the attachment of poly-

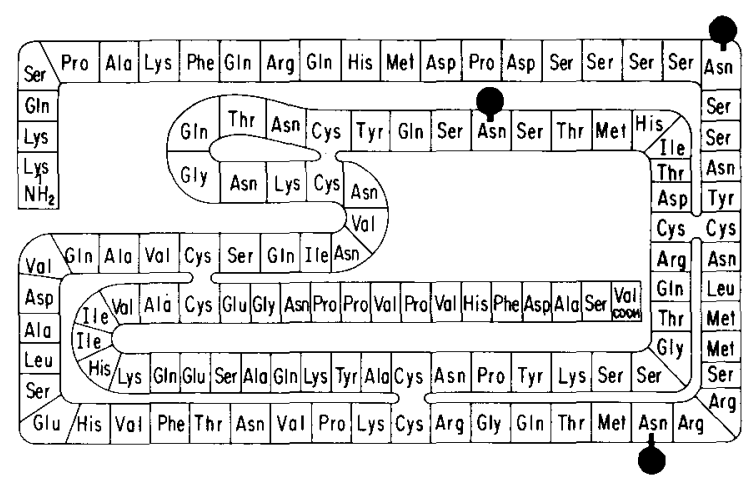

The primary structure of porcine ribonuclease. 
saccharide side-chains to enzymes. As a preliminary to such studies, the structure of porcine ribonuclease is under investigation. Like the wellknown bovine enzyme, porcine ribonuclease is a single-chain protein of 124 amino acid residues cross-linked by 4 disulfide bonds. The primary structure is now known, as are the points of polysaccharide attachment. The disulfide bonds occupy the same positions as in bovine ribonuclease and, although there are 28 a mino acid replacements in the two structures, there is conservation of those residues known to be important in the bovine enzyme for the formation of a hydrophobic "core" around which the tertiary structure of the protein appears to develop. Also conserved are all residues implicated in bovine ribonuclease as essential for binding substrate and for catalytic activity. The polysaccharide side-chains are attached in regions of the molecule shown by $x$ ray crystallography to be remote from the active center. Of the three side-chains, that at asparagine-34 occurs at the same sequence position as in bovine ribonuclease $\mathrm{B}$. The heterogeneity of porcine ribonuclease originates in the attached polysaccharides, particularly the side-chains at asparagine-19 and asparagine- 76 , which are larger than that at asparagine-34. The present results constitute the most complete description yet achieved of the structure of a complex glycoprotein.

\section{Deoxyribonuclease-Deficient Mutants of Pneumococcus}

The two enzymes that account for most of the deoxyribonuclease (DNase) activity in cell extracts of Diplococcus pneumoniae are an endonuclease and a phosphatase-exonuclease. Since the latter enzyme converts double-stranded DNA to single strands and mononucleotides, and since precisely these products are formed when transforming DNA enters a pneumococcal cell, it was supposed that this enzyme plays a role in the entry of DNA, by degrading one strand and thereby drawing the complementary strand into the cell. To test this hypothesis and to see whether this enzyme or the endonuclease is essential for either transformation or cell viability, mutant strains lacking the enzyme were sought as follows. After treatment of the bacteria with a chemical mutagen, DNase-deficient mutants were identified on agar plates containing DNA and methyl green. Methyl green binds to intact DNA to give a persistent blue color, but around a colony that contains DNase and there- fore degrades the DNA in its vicinity, a colorless zone develops. The size and time of development of the zone depend on whether one or both of the DNases are present.

Cells of mutant strains lacking both DNases grew normally, so neither enzyme appears to be essential for viability. Similarly, neither enzyme was required for the uptake of DNA or any other phase of transformation. Newly introduced DNA was still converted to single strands and mononucleotides in the double-mutant recipient. Residual exonuclease activity in the double-mutant strain was found associated with the DNA polymerase. This enzyme, therefore, could conceivably play the role in DNA entry previously assigned to the phosphatase-exonuclease.

\section{Structure and Function of Enzymes}

Enzymes have two important characteristics, specificity and the ability to promote chemical change. Currently a search is being made for the structural features of trypsin responsible for these properties, i.e., for the identity and interrelationship of the amino acid residues at the active center. Residues related to catalysis, namely histidine- 46 and serine-183, were identified earlier. The specificity of trypsin is thought to depend on ionic interaction between a carboxylic acid side-chain of the enzyme and its positively charged substrates. Identification of this group has become the goal of chemical modification studies. Isoxazolium salts, which activate carboxyl groups in chemical synthesis, were selected for study in the hope that the positive charge of the reagent might lead to a substratelike attachment and subsequent activation of the specificity-site carboxylic acid. Conditions were found under which limited reaction was achieved, with considerable alteration of enzymic activity. The observed and hoped-for loss of specificity indicated chemical modification of the carboxyl group responsible for this property. In addition to identification of the modified residue, it is planned to explore the possibility of producing new enzymes by altering the existing binding site.

A number of enzymes are related to trypsin in specificity although their physiological roles are different. Among these are thrombin and plasmin, essential in blood clotting and fibrinolysis, respectively. Attempts are being made to define the catalytic differences of these enzymes in order to arrive at inhibitors that may be useful in controlling their activities selectively. 
The Replication of Bacteriophage $\phi R$

Intracellular development of the small bacteriophage, $\phi R$, is restricted in one of its bacterial hosts, Salmonella typhimurium. As indicated earlier by comparison of this restricted development with normal replication in another host, Escherichia coli, the restriction is due to host-induced changes in the structure of the replicating DNA. These changes affect only the newly synthesized (progeny) DNA, leaving intact and functional the infecting (parental) DNA, which suggests localization of these two types of molecules within the host cell. Elsewhere, association of parental DNA with a cellular structure, probably the cell membrane, has been demonstrated. In present extension of these observations to $\phi \mathrm{R}$ infection, progeny DNA was also found to be associated with cellular structures, distinct from those that bind parental DNA. Test for the association was as follows. Bacterial cells were subjected to gentle lysis $10 \mathrm{~min}$ after infection with $\phi R$ and fractionated into three layers of host components. Selective labeling experiments and electron-microscopic examination demonstrated that the first layer contained host DNA which was not being actively replicated at the time of lysis, the second layer contained the parental viral DNA and actively replicating host DNA, and the third layer contained most of the infectivity of the lysate, but virtually none of the parental or host DNA. Freely sedimenting replicative DNA was not found, although the cytoplasmic contents of the cell had been released.

\section{Amino Acid Changes in Mutant Proteins and the Genetic Code}

Frame-shift mutant hisD3018 of bacterium Salmonella typhimurium was induced by the chemical mutagen ICR-191, while hisD2550 arose spontaneously. Spontaneously occurring double mutants were isolated from his D2550, whereas chemically induced [by ICR-191 and a nitrosoguanidine compound (NG)] as well as spontaneous double mutants were isolated from his D3018. The protein product of the his $D$ gene, histidinol dehydrogenase, from the normal or wild-type and several double mutants, was purified and examined for amino acid differences. Histidinol dehydrogenase from one double mutant of hisD2550 was found to contain an extra leucine residue and to lack a proline residue, while the protein from another double mutant lacked both. The findings suggest that the his D2550 frame-shift may have arisen through a deletion of one or a few nucleotides. One doublemutant protein of hisD 3018 was found to contain an extra amino acid, proline, while another contained the extra proline and at least three additional changes. The multiple amino acid substitutions found in the latter strain are in agreement with the in vitro triplet code word assignments, an additional confirmation of their validity. The results strongly indicate that his D3018 carries an extra nucleotide in the his $D$ gene as the basis of the frame-shift. An NG-induced double-mutant protein hisD3018 was unexpectedly found to be identical to the wild type, the first substantial evidence that this mutagen is capable of deleting nucleotides from DNA.

\section{Renaturation and Aggregation of Single-Stranded DNA}

The native DNA molecule consists of a pair of specifically intertwined single strands. In solution under certain conditions, the strands separate and, once apart, may reunite specifically (renaturation) or imperfectly (aggregation). The bearing of renaturation and aggregation in solution on events in vivo is not known, but since renaturation is a very specific reaction, it is presumably involved in genetic recombination and incorporation of viral DNA into that of a corresponding host, a process perhaps involved in tumor formation. Renaturation is also used as a technique for study of hydrolysis between different populations of DNA molecules or between DNA and RNA. In current work, the molecular details of renaturation and aggregation were determined under a wide range of conditions. Particular conditions were found under which single strands of DNA can exist as long strings or fold back upon themselves many times to form a compact structure. These changes in shape are deducible from the rates at which the molecules sediment or from the viscosities. The shape of the single strands in solution determines whether or not renaturation occurs. Unfolded strands intertwine freely to form the native, doublestranded structure; the folded strands combine to only a limited extent, with formation of nonspecific aggregates. When the native DNA structure is stable, perfectly specific renaturation of unfolded single strands is attained. Perfect renaturation occurs under conditions similar to those obtaining in vivo. In tests for homology, conditions can be chosen such that renaturation is complete, inter- 
ference from nonspecific aggregation is eliminated, and degraration of molecules is minimum.

\section{Exocrine Pancreatic Secretory Proteins}

Pancreatic acinar cells elaborate polypeptides that inhibit trypsin and certain "trypsinlike" proteolytic enzymes that participate in blood clotting, clot dissolution, and release of hypotensive peptides. To obtain information on their biological role, the inhibitors of cow, pig, and human pancreas are being compared with respect to amino acid sequence, mechanism of interaction with proteolytic enzymes, and intracellular distribution.

The amino acid sequence and location of the disulfide bridges of the bovine pancreatic secretory trypsin inhibitor (Kazal's inhibitor) have been determined. There are no major similarities in amino acid sequence between Kazal's inhibitor and the intracellular inhibitor of Kunitz. Both inhibitors are synthesized by the same cell and form $1: 1$ molar complexes with trypsin, yet they differ in structure, inhibitor properties, and intracellular localization.

Lyophilized bovine and porcine pancreatic juice have been used for replacement therapy in canine pancreatic insufficiency. The results obtained suggest that these preparations would be clinically useful.

\section{Determination of Biological Structure by $X-R a y$ Diffraction}

The three-dimensional protein structures so far established by x-ray diffraction support the belief that many more must be determined before rules sufficient to predict conformations of proteins from their amino acid sequences can be formulated. The purpose of present work is therefore twofold: to determine protein structures and, in the course of such determination, to materially simplify the necessary procedures, both by development of instrumentation and by employment of advances in crystallographic theory. Determination of the structure of crystalline subtilisin continues by the conventional isomorphous replacement method; a set of heavy-atom isomorphs prepared by a previously described technique is used. A full set of data for the neurohypophyseal hormone oxytocin has been collected, and solution of the structure by a "direct phasing" method, symbolic addition, is being attempted in the belief that the method may become sufficiently powerful to largely supplant isomorphous replacement. In this eventuality, determination of large structures would be radically simplified. The first general computer program for employment of this method has been developed and is in use. It has already made possible the direct determination of several small structures for which previous methods were unavailing.

\section{The Cell Wall of Pneumococcus and Meningococcus}

The bacterial cell wall is especially suitable for study of the relation between macromolecular structure and biological function. In the species examined, the wall consists of heteropolymeric substances, two of which are relatively distinct. One of these is the ubiquitous bacterial mucopeptide. The nature of the other polymer varies with the species, but is frequently the group-specific polysaccharide. Present work is concerned with the structure of group-specific polysaccharides and involves development of methods for quantitative analysis of polysaccharide components, estimation of molecular size of the polysaccharides, elucidation of the linkages between the polymers, and definition of enzymes involved in the biosynthesis and depolymerization of the carbohydrates. The $C$-polysaccharide from the cell wall of pneumococcus, a gram-positive bacteria, was found separable into two components, one a typical mucopeptide, the other a polymer containing $\mathrm{N}$-acetyl-galactosamine phosphate and an unknown diamino sugar. The two polymers differ in immunological properties and are cross-linked covalently through the phosphodiester bond of muramic acid. The cell wall of meningococcus, a gram-negative bacteria, also yields a mucopeptide and a carbohydrate polymer of either $\mathrm{N}$-acetyl-mannosamine phosphate or sialic acid. However, these polymers are held together by noncovalent bonds. Based on this, a new procedure was developed for isolation of high-molecular-weight $\left(>10^{5}\right)$ polysaccharides from meningococcus.

\section{Conformational Studies of Macromolecules}

Preliminary studies were completed of the applicability of neutron diffraction to protein structure determination. Data were collected for a large myoglobin crystal $\left(30 \mathrm{~mm}^{3}\right)$ by using a monochromatic (1.5- $\AA$ ) flux of $10^{6}$ neutrons $/ \mathrm{cm}^{2}$-sec and a counting time of 3 to $10 \mathrm{~min}$ per reflection. From the data and with use of the $\mathrm{x}$-ray phases, projection maps as well as a three-dimensional density map were calculated. The maps compare well 
with those calculated from x-ray data alone. Thus, neutron diffraction data suitable for structural determination can be obtained. Work is under way to determine whether the anomalous dispersion effect of isotopes such as those of cadmium, samarium, or xenon can be used to determine the phases directly.

To gain information about the distribution of rotational and vibrational modes in proteins, a study of the inelastic neutron scattering behavior of macromolecules was initiated. Early experiments using the slow neutron chopper and polycrystalline myoglobin show that a large number of unresolvable energy transfers do take place, which indicates a multitude of low-lying energy levels.

An attempt to determine the structure of thermolysin by $x$-ray diffraction is under way. Of particular interest are the structural features that enable thermolysin and certain other enzymes to function at elevated temperatures (up to $95^{\circ} \mathrm{C}$ ). Thermolysin, a protease, consists of two identical subunits of molecular weight 38,000 . The protein contains zinc and does not have any disulfide bridges. Although water insoluble, the enzyme was found to be readily crystallizable from $40 \%$ dimethyl-sulfoxide solution that contained calcium to protect the protein from autodigestion. The crystals are hexagonal and belong to the space group $P 6_{5} 22$ with 12 molecules per unit cell. An automatic computer-controlled densitometer was developed to allow efficient collection of $\mathrm{x}$-ray diffraction data with use of a film method.

\section{ECOLOGY}

\section{Mineral Nutrient Recycling}

The mineral nutrient budget of any unit of landscape such as a pond or forest appears to be a major determinant of the quantity and diversity of life within the unit. Yet such budgets are poorly known and certainly abused. One flagrant abuse that is becoming a world-wide problem arises from dumping nutrients discarded by cities into nearby watercourses to flow ultimately to the sea. The nutrients dumped by cities are those that are accumulated in crops from agricultural land. For a city they may represent the annual harvest from many thousands of acres. These nutrients are discharged through sewage treatment plants into watercourses of limited area. The result is that these water bodies are being spectacularly overloaded with nutrients and "degraded" in the sense that the natural, mostly aerobic, communities are replaced by simple, frequently anaerobic, communities. The degraded communities produce few harvestable products and may also produce such noxious substances as hydrogen sulfide and various amines. The classical solution has been to hasten passage of the nutrients to the sea by increasing flushing rates. The more desirable objective of recycling the nutrients into agriculture is overlooked.

The possibility of developing natural and manmade ecosystems to trap the nutrients in the effluent of sewage treatment plants is currently being explored in detail. The most promising ecosystems are combinations of ponds and marshes. Interest has focused on two combinations providing for ready harvest: natural and man-made marshes associated with ponds containing species of carp and mullet, and especially designed rice paddies. With refinements in detail it appears reasonable to expect recycling of $50 \%$ or more of the nutrients in sewage-plant effluents with use of land areas of reasonable size. The current exploration is bringing new insights into the general problem of how nutrient elements control life in both natural and man-made communities. 


\section{Medical Research}

In developing its research program, the Medical Department has several responsibilities.It has the broad responsibility to advance medical knowledge and the more specific responsibility to advance understanding of the effects of ionizing radiations in man. Some of the investigations are directed toward beneficial applications of these radiations and toward improvement of measures to prevent or counteract their detrimental effects. Other investigations are centered on elucidation of disease states and development of improved methods of diagnosis and therapy. Investigations in these two areas complement each other, since the study of radiation effects contributes to the understanding of diseases, and vice versa, yet the pace of progress in both is controlled by the advances in knowledge of the normal biological processes of man.

A great influence on these advances is the rapid development of new techniques for the examination of structures and mechanisms at the molecular as well as the subcellular and biochemical levels of organization. Information revealed through these techniques and with the aid of such instruments as the electron microscope dictates re-examination of such medical and biological phenomena at even finer levels of organization. Findings from such investigations present the Department with the additional responsibility to exploit them for clinical applications.

The diversified talents and extensive facilities uniquely concentrated at Brookhaven provide the Department with unusual advantages for pursuing its objectives. Its broadly based program incorporates the experience and skill not only of those devoted to research in the field of medicine but of many from the various disciplines in other departments and from collaborating institutions. The interchange of ideas, information, facilities, and services is essential for the staff to keep abreast of the new developments that have medical implications, and within the framework of the program opportunities are limited only by the scope of the vision and interest of the individual investigator.

Thus, in its role of service to Associated Universities, Inc., to the Atomic Energy Commission, and to the medical community, the Department's primary responsibility is the assemblage of talented scientists capable of elucidating biological phenomena at the finest level of organization and experienced physicians capable of relating these researches to clinical studies.

Specific examples of research under way are given in the following pages. The report is not comprehensive, and the reader may obtain additional information from publications of the Department.

\section{EFFECTS OF RADIATION}

\section{Mathematical Modeling of the Relationships of Cellular, Tissue, and Animal Radiation Effects}

Certain effects of radiation on the physiology of the intact animal can be reasonably assumed to depend directly on the radiation response of only a single critical cell type. For example, the acute hemopoietic syndrome may depend on the response of the hemopoietic repopulating or stem cell, and radiation-induced leukemia may depend on the delayed response of a cell type as yet unidentified. In such instances it appears feasible to relate, by means of a mathematical model, the animal doseeffect curve to the cellular dose-effect curve. In general, account must be taken not only of the primary radiation response of cells of the critical type but also of the proliferation that they initiate and the animal's requirement for, or sensitivity to, their progeny. Of use in the analysis are the theory of random variables and stochastic processes, Fourier transforms, computer simulation, and the theory of competing risks. Current work is concerned with models to account for radiationinduced leukemia in mice and the effect of dose protraction on the width of the mouse dosesurvival curve. A newly completed model of the acute, hemopoietic radiation syndrome is being extended and compared with suitable new data as they become available, and such data are being augmented by experimentation, especially with mice of strains showing large differences in radioresistance.

\section{Influence of Radiation Quality and Dose Upon Lens Opacification in the Mouse}

A comprehensive analysis and compilation has been carried out on data from a series of investiga- 


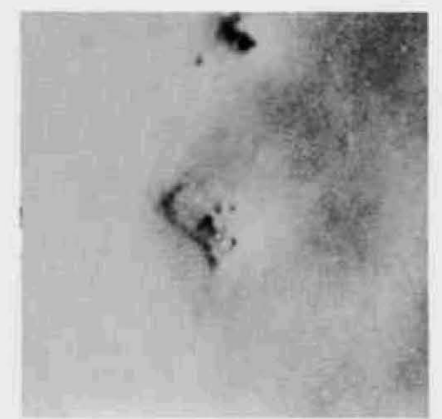

52 WK

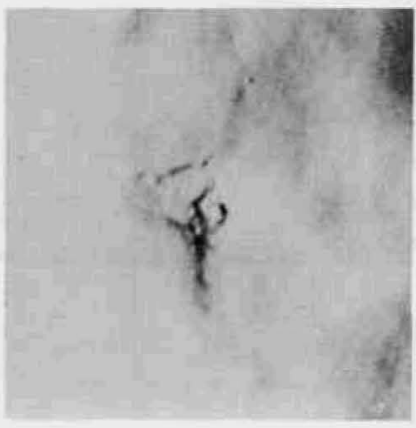

56 WK

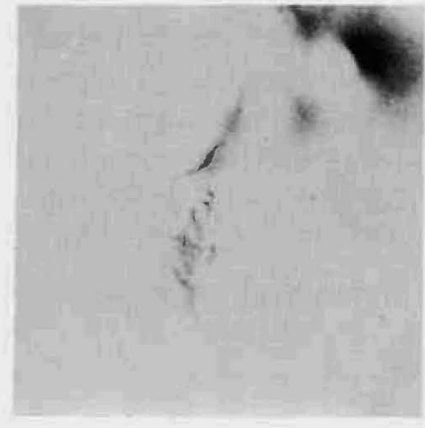

58 WK
Figure 1. Opacification in the anterior polar region of the lens in mouse exposed to $50 \mathrm{rads}$ of $250-\mathrm{kVp}$ x rays (at noted intervals post irradiation).

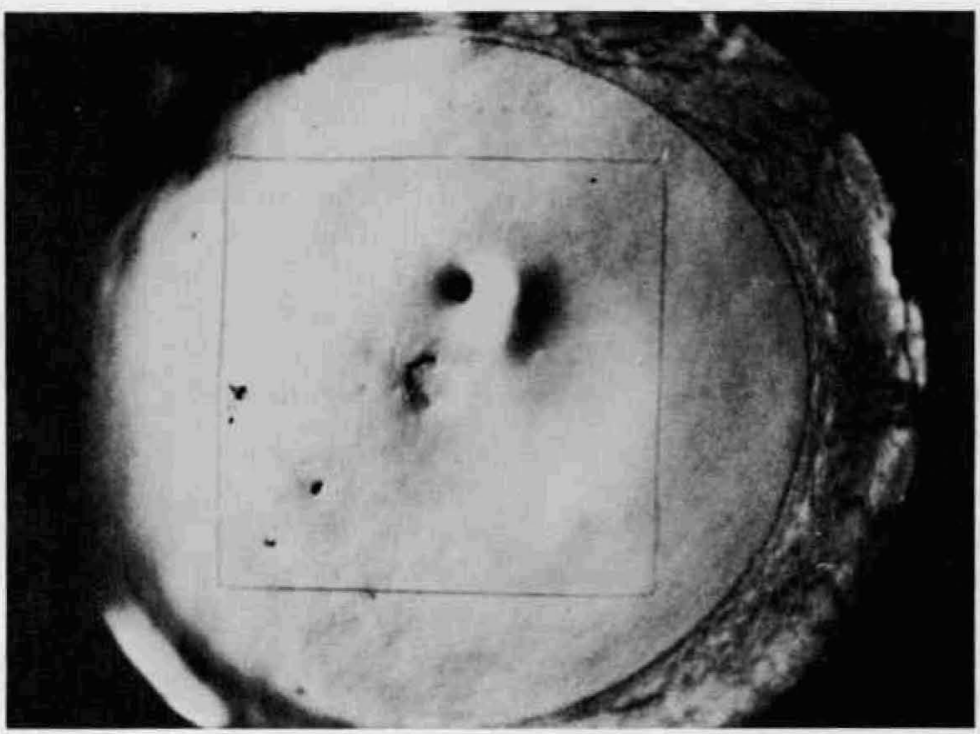

$48 \mathrm{WK}$
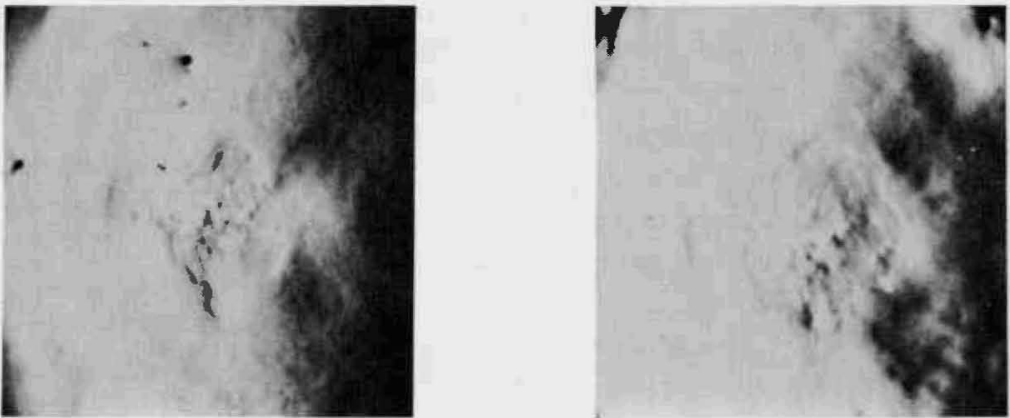

60 WK

64 WK tions of optic lens opacification resulting from exposures of mice to $250-\mathrm{kV}$ x rays or "fast" neutrons of $0.43,1.80$, or $14-\mathrm{MeV}$ energy. The resultant changes in both the anterior polar (see Figure 1) and posterior subcapsular regions of the lens have been found to be indistinguishable from those present in the aged, unirradiated animal. The less frequently described anterior opacities begin as unifocal hyperplastic areas and appear later and in lesser degree than the multifocal degenera- tive posterior changes described so adequately by other workers. Despite the differences in morphology and development, these two biological parameters yielded similar relative biological effectiveness (RBE) values under all conditions tested. Neutrons of each energy were found to have an RBE compared with $250-\mathrm{kVp}$ x rays that rose with declining dose. Thus the RBE of 0.43 $\mathrm{MeV}$ neutrons was about 9 when compared with 500 rads of $x$ rays, but rose to 30 against 100 rads 
of $x$ rays and reached 60 against 50 rads of $x$ rays. A similar, but lower, relation of RBE to dose was obtained for $1.80-\mathrm{MeV}$ neutrons, and an even lower relation of RBE to dose was found for 14$\mathrm{MeV}$ neutrons. Simple empirical expressions in energy-mean linear energy transfer (LET) are being derived which it is hoped will adequately describe the relative effectiveness between the various radiations employed in these lens opacification studies. Human lens examination techniques are being developed with the split-lamp biomicroscope in preparation for evaluation of opacification in a series of people exposed to fallout radiation doses ranging from $\approx 15$ to 175 rads.

\section{Medical Survey of the People of the Marshall Islands Accidentally Exposed to Fallout}

Medical surveys of the Marshall Islanders who were exposed accidentally to radioactive fallout in 1954 are conducted by Brookhaven National Laboratory on an annual basis in conjunction with the Trust Territory of the Pacific Islands. The early, acute effects have been reported previously. What appears to be the most important of the later effects has been the development of abnormalities of the thyroid gland and its function. Of the 82 people exposed, 66 are now living, and no deaths have been attributed to radiation exposure. Twenty-one have developed abnormalities of the thyroid gland, including 2 cases of marked hypothyroidism. Of the 19 Marshallese who were $<10$ years of age at the time of exposure, 17 have developed thyroid abnormalities. In all, 14 Marshallese have had thyroid surgery, 13 exhibited adenomatous goiter, and there was one case of thoyroid cancer in an adult. Some of these individuals were brought to Brookhaven for additional study and treatment and then returned to their homes. Some of the exposed children exhibited an impairment in growth, but they have responded well to thyroid hormone therapy.

\section{Californium-252 as an Interstitial Neutron Source}

Californium-252 is a man-made transuranium element that undergoes spontaneous fission with a half-life of 2.6 years. It can be fabricated into small sources to be used for interstitial (and intracavity) irradiation of tumors. About $1 \mu \mathrm{g} \mathrm{Cf}{ }^{252}$ is the equivalent, in a radiological sense, of $1 \mathrm{mg}$ radium. Dosimetric studies of $\mathrm{Cf}^{252}$ have indicated a distribution of doses in tissue that is similar to that of radium. A computer program has been set

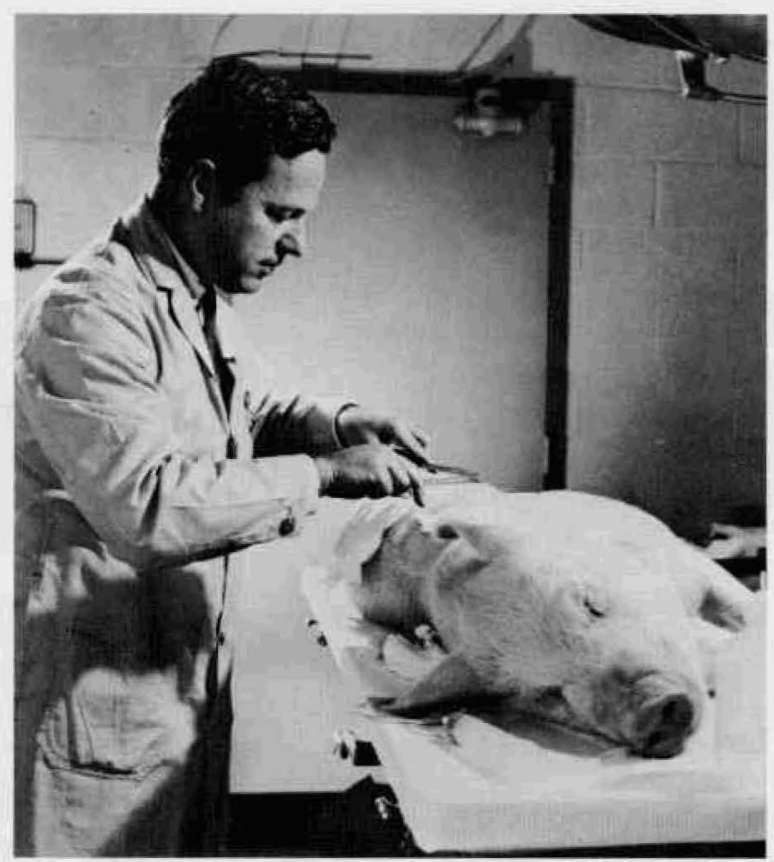

Figure 2. Application of californium plaque to the skin of a pig for testing of skin tolerance.

up to calculate dose distributions from arrays of sources. Studies with HeLa cell cultures have given an $\mathrm{RBE}$ of 2.34 for $\mathrm{Cf}^{252}$ with respect to a single dose of $x$ rays and 2.96 with respect to radium and an oxygen enhancement ratio of 1.2. Plaques of $\mathrm{Cf}^{252}$ or radium have been placed on the skin of swine and are now being observed to determine the relative skin tolerance (see Figure 2).

\section{CLINICAL AND METABOLIC STUDIES}

\section{L-Dopa and Parkinsonism}

The therapeutic effectiveness of $\mathrm{L}$-dopa in the treatment of Parkinsonism has now been firmly established, both at Brookhaven and in other medical centers. Often the therapeutic effects of L-dopa on Parkinsonism have been dramatic. A marked diminution of the therapeutic dose of $\mathrm{L}$ dopa has been achieved by blocking one of its catabolic pathways in the periphery, that is, not in the brain, with $\alpha$-methyl dopa hydrazine. This indicates that chemoreceptors are located outside the blood-brain barrier. The induction of involuntary movements with L-dopa in some patients with Parkinsonism led to the control of choreiform movements with $\alpha$-methyl dopa in a case of 


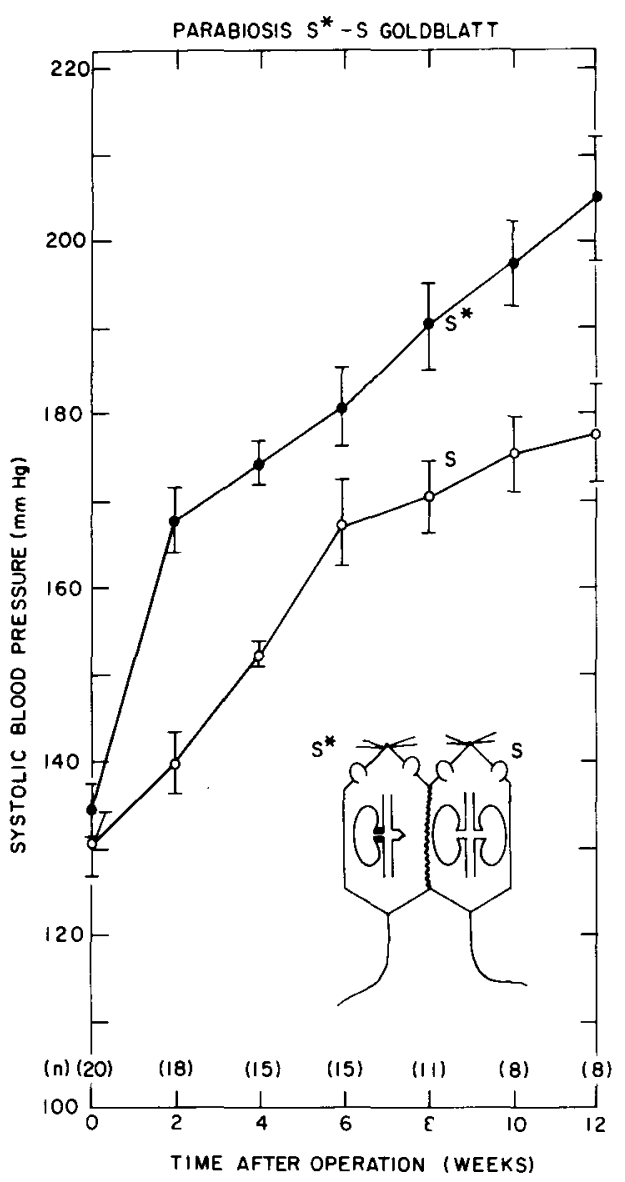

Figure 3. Blood pressure response of $S^{*}-S$ parabionts after Goldblatt procedure in only one rat $\left(\mathrm{S}^{*}\right)$.

Huntington's chorea. A continuing study has been designed to detect whether or not the progression of Parkinsonism can be diminished by L-dopa. The results are encouraging thus far. A collaborative study conducted in Santiago, Chile, has yielded an impressive dividend: dystonia muscularum due to chronic manganese poisoning in miners was ameliorated greatly by treatment with $\mathrm{L}$-dopa. This indicates that dopa treatment is applicable to selected diseases other than Parkinsonism. Along with the clinical studies mentioned above, laboratory studies concerned with the following have been accomplished. Experiments permitting the issuance of IND* numbers from the Food and Drug Administration for use in humans of 5-OH tryptophan, $\alpha$-methyl dopa, $\alpha$-methyl dopa hydrazine, phenylalanine, tyrosine, and phenylalanine-deficient diets have been finished. Other

${ }^{*}$ IND = investigatal new drug.

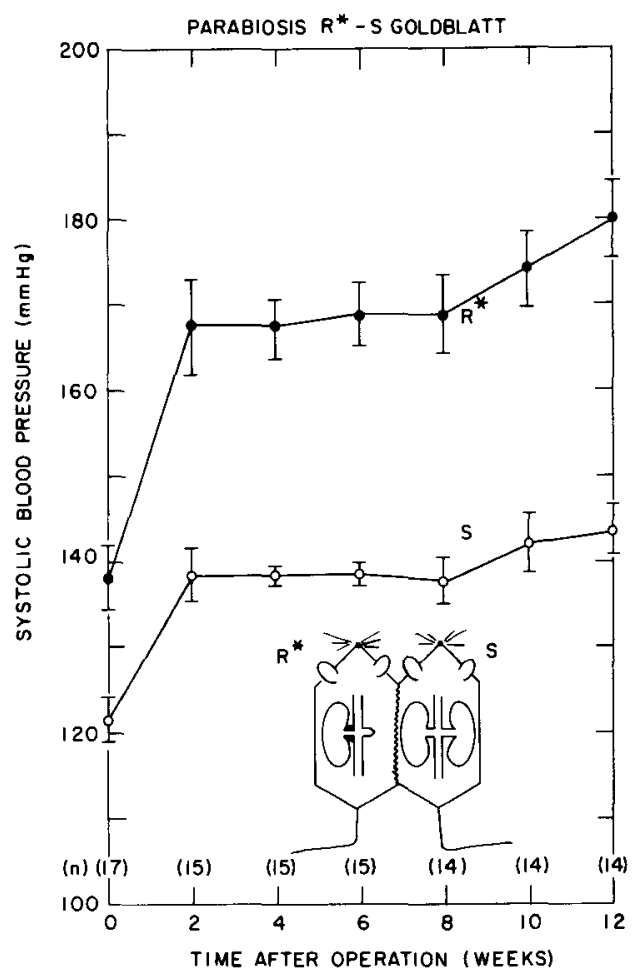

Figure 4. Blood pressure response of $R^{*}-S$ parabionts after Goldblatt procedure in only one rat $\left(R^{*}\right)$.

experiments have shown a biochemical link between the metabolism of amines and of metals like manganese by way of $3^{\prime}, 5^{\prime}$-adenosine monophosphate. Techniques for the nondestructive analysis of metals like manganese have been worked out, as well as techniques to quantitate amines and their metabolites in human materials. These two techniques are to be combined in the hope that the same small sample of brain tissue can be analyzed for both metals and amines.

\section{Studies on Hypertension}

In man, there is good evidence relating genetic factors to the development of hypertension, and there is also evidence that chronic excess salt $(\mathrm{NaCl})$ ingestion is involved. In two strains of rats developed by selective inbreeding it has been possible to demonstrate unequivocally that genetic factors and $\mathrm{NaCl}$ can interact to produce experimental hypertension. One strain will develop rapidly fulminating hypertension from the same $\mathrm{NaCl}$ intake from which the other strain develops little or no hypertension. Subsequently, it was found that these two strains of rats also showed opposite innate predispositions for developing ex- 
perimental hypertension from the steroid desoxycorticosterone acetate plus salt, unilateral renal artery compression without salt, cortisone, adrenal regeneration, or from uninephrectomy. On the basis of these data, it has been proposed that the effectiveness of all nongenetic (environmental) factors considered to play a primary role in the etiology of experimental hypertension would be profoundly modified by the genetic substrate of the individual concerned. It was considered likely that genetic factors play a decisive role in determining whether or not an individual will develop hypertension after exposure to one or more of the "causal" factors. There is a considerable body of clinical evidence in support of this hypothesis, and therefore the working assumption that a similar relationship between genetic and environmental factors operates also in man has been adopted.

The effect of various renal manipulations on the two strains of rats with opposite, genetically determined tendencies to hypertension were studied. On single rats, the following results were obtained. (1) Uninephrectomy produced mild hypertension in rats on low $\mathrm{NaCl}$ diets from the sensitive strain but not in those from the resistant strain; (2) unilateral renal artery constriction produced severe hypertension in members of the sensitive strain on a low $\mathrm{NaCl}$ diet but no, or only mild, hypertension in rats from the resistant strain; (3) unilateral renal artery constriction and contralateral nephrectomy (Goldblatt procedure) resulted in fulminating hypertension of approximately equal severity in both strains. Taken together, the uninephrectomy and unilateral renal artery constriction studies suggest that at least two factors are at work, one that "protects" against hypertension, and another that "promotes" hypertension. These findings on single rats were further explored in pairs of rats united in parabiosis (see Figures 3 and 4). It was concluded that the pressor signal was different in the two strains of rats, although it is not yet clear whether the difference is quantitative or qualitative.

\section{Studies of Carbohydrates and Lipid Metabolism in Diabetes and Obesity}

Studies with $\mathrm{C}^{14}$-labeled pyruvate and lactate have indicated that excessive production of glucose from these intermediate compounds characterizes the metabolism of obese patients and may help account for the common coexistence of diabetes.
Conversion of $\mathbf{C}^{\mathbf{1 4}}$-labeled glycerol to glucose in obese patients does not show as much abnormality as does the conversion of $\mathrm{C}^{14}$-labeled pyruvate and lactate, although formation of $\mathrm{C}^{14} \mathrm{O}_{2}$ from all three compounds is typically decreased in obese as well as diabetic patients. The studies of gluconeogenesis from $\mathrm{C}^{\mathbf{1 4}}$-labeled precursors have been extended to a species of desert rodent, Psammomus obesus, which becomes both diabetic and obese on a laboratory diet. In these "sand rats" the mechanism of the effect of hypothalamic lesions and certain drugs that act on the central nervous system to improve carbohydrate tolerance is under investigation.

Studies of the metabolism of labeled glucose during oral glucose tolerance tests in diabetic and obese patients have shown additionally that the apperance of $\mathrm{C}^{14} \mathrm{O}_{2}$ from oxidation of glucose$1-\mathrm{C}^{14}$ is decreased in obese subjects to a greater extent than is the simultaneous formation of tritiated water $\left(\mathrm{H}^{3} \mathrm{OH}\right)$ from glucose- $1-\mathrm{H}^{3}$. The extent of reduction of $\mathrm{C}^{14} \mathrm{O}_{2}$ formation during a cortisone-glucose tolerance test is greater than the extent of elevation of the blood glucose and is similar to the degree of reduction of intravenous glucose tolerance. The differences in the rates of formation of $\mathrm{C}^{14} \mathrm{O}_{2}$ and $\mathrm{H}^{3} \mathrm{OH}$ suggest a retention of carbon in some intermediates or excessive recycling of carbon to glucose in obese subjects. Comparisons of oxidation of $\mathrm{C}^{14}$ - and $\mathrm{H}^{3}$-labeled glycerol in obese and nonobese subjects are confirmatory of the findings with labeled glucose.

Studies with $\mathbf{C}^{\mathbf{1 4}}$ - and $\mathbf{H}^{3}$-labeled carbohydrates have repeatedly demonstrated excessive hepatic lipogenesis in genetically obese-diabetic mice. Pancreatic release of insulin is also high, with elevated levels in the blood. Chronic administration of mannoheptulose, which inhibits release of insulin from the pancreas in some species, was associated with even higher levels of insulin and perhaps acceleration of obesity. Lipogenesis from labeled glucose was not changed. Evidently synthesis of insulin is not impaired by mannoheptulose, and effects on release are variable.

Other recent studies of lipogenesis from labeled carbohydrates in rats have revealed interesting differences among glucose, fructose, and sucrose. Fructose and sucrose (containing fructose) are much more extensively converted to triglycerideglycerol than glucose, which may help explain the higher plasma triglyceride levels produced by sucrose vs glucose or starch feeding. Sucrose feed- 
ing predisposes to greater incorporation of $\mathrm{C}^{14}$ from various precursor carbohydrates into glycerol and fatty acids of plasma triglycerides than do glucose or fructose feeding. However, glucose feeding has predisposed to greater incorporation of $\mathrm{H}^{3}$ from the malate into fatty acids. A synergistic effect of the glucose and fructose moieties of sucrose may account for the superior lipogenic effect of sucrose.

Glucose may exert its lipogenic action via stimulation of the pancreas to produce and release insulin. Currently being studied are the characteristics of insulin output by isolated rat pancreatic islets as affected by varying modes of stimulus by glucose, tolbutamide, and other influences and by inhibitory agents such as mannoheptulose.

\section{Clinical Research in Leukemia With \\ Extracorporeal Irradiation of Blood and Cell-Labeling Techniques}

Treatment of adult patients with leukemia by extracorporeal irradiation of blood (ECIB) was started in November 1964 and 35 patients have been treated. The technique involves the diversion of blood from the radial artery past a source of radiation $\left(\mathrm{Co}^{60}\right)$, via a chronic arteriovenous shunt. ECIB is carried out from 2 to $4 \mathrm{hr}$ each day per patient or less frequently. The median survival time in 16 patients with acute myelocytic leukemia was 8.0 months, equivalent to that obtained after intensive chemotherapy. There was an apparent positive correlation of survival time with transit doses $>340$ rads. Results obtained in six patients with chronic myelocytic leukemia have been too variable for any definitive conclusions. Thirteen patients with chronic lymphocytic leukemia (CLL) have had a marked decrease in the concentration of lymphocytes in the peripheral blood, and many have had a decrease in splenomegaly and lymphadenopathy. The results indicate that the early treatment of CLL with ECIB may be particularly beneficial. As a result of the information obtained from these 35 patients, the indications for treatment of leukemia in adults by ECIB have become more sharply defined, and the number of patients considered suitable for this mode of therapy has been sharply curtailed. In the future, the major effort will be devoted to the early treatment of CLL.

Studies of DNA synthesis time, with $\mathrm{H}^{3}$-thymidine used as a cell label, were continued in an effort to understand the basic aberrations in leu-

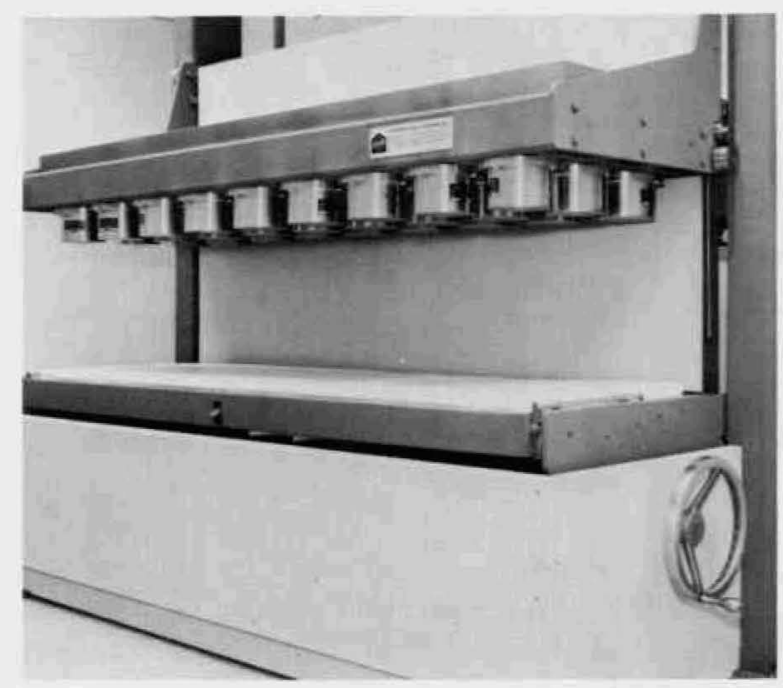

Figure 5. View of 54-detector whole-body counter.

kemia. No difference was found in the DNA synthesis time ( $14.2 \pm 0.8 \mathrm{hr}$ ) of blood and bone marrow myelocytes in patients with chronic myelocytic leukemia. RNA turnover in vivo, as estimated by the change in the mean grain count of in vitro labeled ( $\mathrm{H}^{3}$-cytidine) autotransfused lymphocytes in CLL patients, was found to be inversely related to the clinical course of disease and radiosensitivity of lymphocytes as indicated by response to ECIB.

\section{Methods of Extending and Improving Radiographic and Isotopic Diagnostic Techniques}

The recently developed chelated form of $\mathrm{Tc}^{99 \mathrm{~m}}$ has been studied in humans. This compound is excreted by glomerular filtration and no specific localization has been noted. Renal visualization is readily accomplished following intravenous administration of the compound, and it is useful as a scanning agent for brain, since no undesirable localization occurs in the choroid plexus or salivary glands. Tc ${ }^{99 m}$ sulfur colloid has been used successfully in lymph-node scanning. Good scans of high resolution have been obtained of both pelvic and abdominal nodes as well as axillary and supraclavicular nodes.

The new 54-detector whole-body counter (Figure 5) has been calibrated for the in vivo measurement of low levels of radionuclides in man. The new counter is unique in that it incorporates the following properties: high sensitivity and spectral resolution, an invariant response to both body size 
and spatial distribution of radionuclides, and the ability to provide data on the spatial distribution of rapidly translocating radionuclides in the body. The operational characteristics of the counter and its associated computer facility were studied with use of point sources and distributed sources in phantoms. An empirical technique was developed to correct for the variable response to both geometry and attenuation of photons by the human body. The new counter was used in a series of clinical studies to provide data for the evaluation of the response of the system to variation in counting geometry and body size. These studies indicate a considerably greater accuracy in the absolute measurement of the levels of internally deposited radionuclides compared with that obtainable with other whole-body counting systems.

The whole-body counter was used in kinetic studies employing the tracers $\mathrm{Ca}^{47}$ and $\mathrm{Sr}^{85}$ to determine the factors involved in the control of skeletal metabolism in various states of health and disease. The therapeutic effects of administering Thyrocalcitonin to patients with osteoporosis was evaluated in terms of the $\mathrm{Ca}^{47}$ tracer technique. On the basis of concurrent animal studies, it appears that Thyrocalcitonin, although it does not affect the bone accretion rate, does produce a diminished bone resorption rate which results in an increased mass and/or density of bone.

A stochastic model (within a compartmental analysis) using the likelihood function was formulated to fit long-term strontium retention data. This model is an analytical representation of a system in which there are unique outcomes for a given set of values which can be predicted within a probabilistic range.

The program for development of an in vivo neutron activation analysis of whole-body calcium content has continued. It appears possible with a whole-body dose to a phantom man of $<0.24$ rad from 14- MeV neutrons to measure the in vivo whole-body concentration of calcium. Because of its sensitivity and accuracy, activation analysis may prove to be an important tool in medical research, particularly in studying trace metal metabolism.

\section{Metabolic Interrelationships of $\mathrm{D}$ - and L-Kynurenine in Rats}

The metabolism of DL-tryptophan has been studied extensively in animals and humans. Despite the availability of the pure $\mathrm{L}$ isomers, the racemic

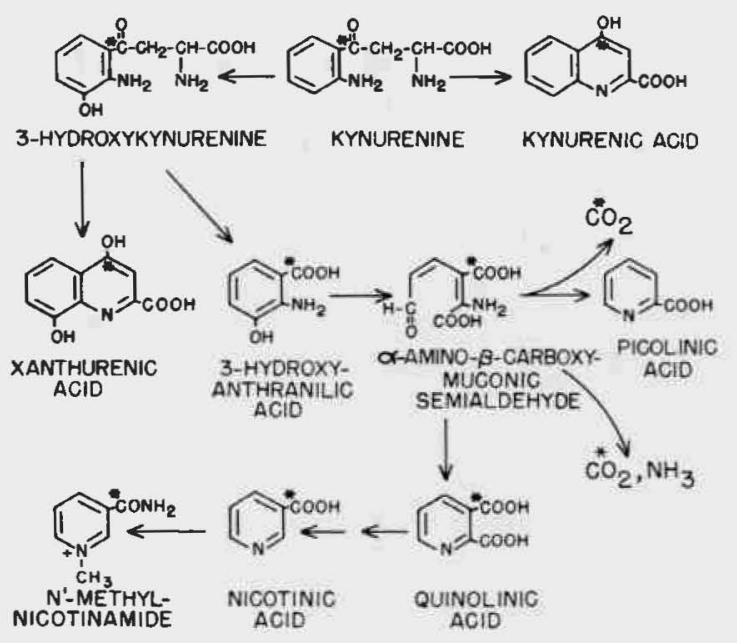

Figure 6. Metabolism of kynurenine-keto-C $\mathbf{C}^{\mathbf{1 4}}$.

mixtures of amino acids are still used in laboratory and clinical studies. Reports that humans and rabbits fed D-tryptophan excreted D-kynurenine in their urine emphasize the importance of studies dealing with the metabolism of the enantiomers of tryptophan along the kynureninehydroxyanthranilic acid pathway (Figure 6). A study was conducted concerning the metabolism of the $\mathrm{D}$ and $\mathrm{L}$ isomers of kynurenine-keto- $\mathrm{C}^{14}$ and the effect of the opposite unlabeled isomer on these metabolic pathways in vivo in rats.

The $\mathrm{D}$ and $\mathrm{L}$ isomers of both the unlabeled DLkynurenine-sulfate and DL-kynurenine-keto- $\mathrm{C}^{\mathbf{1 4}}$ sulfate were isolated by separation of their respective DL mixtures on a paper pulp column. Rats were injected intraperitoneally with aqueous solutions of a $\mathrm{C}^{14}$ isomer either alone or together with an equimolar a mount of unlabeled enantiomer or $30 \mathrm{~min}$ after a loading dose of the unlabeled enantiomer, and the respiratory and urinary radioactivities were measured. L-Kynurenine was more rapidly metabolized to $\mathrm{C}^{\mathbf{1 4}} \mathrm{O}_{2}$ than was the $\mathrm{D}$ isomer and was also a better precursor of quinolinic acid. A load of $D$ isomer inhibited the metabolism of the L-C ${ }^{14}$ isomer to $\mathrm{C}^{14} \mathrm{O}_{2}$, and, on the basis of changes in urinary metabolites, it is suggested that $\mathrm{D}$-kynurenine inhibits L-kynurenine hydroxylation. A load of the $\mathrm{L}$ isomer increased the metabolism of the $\mathrm{D}$ isomer very slightly. Kynurenic acid was the main product of D-kynurenine- $\mathrm{C}^{14}$ metabolism identified in the urine. It probably arose by deamination and cyclization as a result of the action of a transaminase 
or D-amino acid oxidase. The appearance of label from $\mathrm{D}$-kynurenine- $\mathrm{C}^{14}$ in niacin metabolites indicates the presence of metabolic pathways in the rat capable of converting D-kynurenine to niacin without conversion to the $\mathrm{L}$ form.

\section{Computers}

The Medical Department's Sigma-2 computer was programmed for on-line data acquisition for the Department's 54-crystal whole-body counter and for the electron paramagnetic resonance (EPR) machine. The EPR programs provide an initial analysis of the spectrum during a run and make it possible to adjust the equipment to optimize the operating parameters.

A large part of the computer programming effort has gone into computation of the kerma (first collision radiation dose) rate for objects of various sizes (e.g., drosophila, mice) that are irradiated by neutrons generated when particles accelerated at the new Van de Graaff facility react with a given target. The accelerated particles used for bombarding the target may be protons, deuterons, or other light atomic nuclei. The target may include tritium, deuterium, lithium-7, beryllium-9, or some other nuclide, supported by a base of a metal such as titanium. The neutron energy is a function of the energy of the original bombarding particle (which may range from zero to $6 \mathrm{MeV}$ ), the composition of the target, and the angle at which the neutron is emitted. The kerma rate depends on the neutron energy, the beam current (which in turn determines the neutron flux), the distance from the target to the object, and the composition of the object. Values for the parameters required in these calculations are available in the literature, and particular use has been made of those for $p-t, d-t, d-d$, and $p$-Li reaction cross sections and of tables of kerma as a function of neutron energy for hydrogen, carbon, oxygen, and nitrogen. These four elements account for almost all of the energy deposition in mammalian soft tissues. The main program is coded under the acronym ANY, for accelerator neutron yield. One variant of ANY has been used to tabulate relative kerma rates for energies of the bombarding particle from the threshold for neutron production to $6 \mathrm{MeV}$ at $50-\mathrm{keV}$ intervals for the $p-t$, $d-t$, and $d-d$ reactions and for angles from 0 to $180^{\circ}$ at $5^{\circ}$ intervals for an idealized target. Another version gives more detailed information, including the range of kerma rate values for irradiations of specific objects at a given position relative to a real target. The more detailed calculations include corrections for energy loss by the bombarding particle during its passage through the target and for the finite geometry involving the sizes of the target and object. Program any and its subroutines are coded in FORTRAN IV and adapted to the CDC 6600 computer. Although designed to provide dose parameters for completed experiments, the principal use of these calculations to date has been in planning experiments in connection with the converted Van de Graaff accelerator formerly used as the injector for the Cosmotron.

\section{NUCLEIC ACID STRUCTURE AND FUNCTION}

\section{Interaction of Daunomycin and Deoxyribonucleic Acid}

The interaction of proflavin, daunomycin, and ethidium bromide with natural and synthetic polynucleotides is of interest because (1) these tgents have significant clinical and laboratory use; (2) they have been used to study replication and transcription mechanisms in vivo and in vitro; and (3) structural studies of drug-nucleic acid complexes have contributed to understanding of forces important in stabilizing secondary and tertiary polynucleotide structure. Daunomycin is typical of this group of agents and binds strongly to deoxyribonucleic acid (DNA). Recent studies have shown that the interaction is an intercalation of the chromophore of the drug between successive DNA base pairs. However, intercalation is only a general description of the mode of binding of a drug, and additional studies have been done on the stereochemistry of the binding process itself. These three compounds all have a strong positive charge. In addition to studying the interaction of the planar hydrophobic faces of their chromophores with the DNA base pairs, molecular model-building techniques were used which disclosed possible interactions between this charge and the negatively charged DNA phosphate. Intercalation results in an increase in the average pitch of the DNA helix because (1) the insertion of each chromophore increases the effective translation per nucleotide pair from 3.4 to $5.8 \AA$, and (2) since the sugar phosphate chain in the native DNA helix is already almost completely extended, this increase in translation per nucleotide pair. can be achieved only by a concurrent untwisting of the DNA helix. Molecular model-building 
studies show that the amount of untwisting required depends on the pucker of the nucleic acid sugar. In nucleic acids and model compounds two main types of pucker have been observed. The range of untwisting allowed per insertion for each of the sugar conformations has been defined. Xray diffraction studies of oriented fibers of DNAdaunomycin complexes have provided data on the increase in helix pitch as a function of the amount of antibiotic bound to the DNA. These results have been correlated with molecular modelbuilding studies and can be accounted for by an untwisting of $\approx 12^{\circ}$ for each drug molecule intercalated. In the studies on the stereochemistry of the daunomycin intercalation into DNA it has been assumed that the nature of the bases at or near an intercalation site has only a minor effect, since there is no evidence that the binding of daunomycin is base specific. It was found that the eight principal models for daunomycin could be intercalated with the daunosamine group in either the large or small groove of DNA. A position and orientation of the drug could be found in which most, if not all, of the triple aromatic ring system was overlapped by the adjacent base pairs and the sugar amino group was brought close to a phosphate group. In this way two bonds would be formed: a hydrophobic interaction between the bases and the chromophore, and an electrostatic interaction at the phosphate. Acetylation of the sugar nitrogen could be expected to reduce or prevent the ionic interaction. These models show that the intercalation is possible and indicate what other interactions might occur, but no particular model is favored at this time. Further modelbuilding will be necessary before the various models can be described in more detail. It would seem unlikely from these model-building studies that a different model would be required for the interaction of an antibiotic to be complexed to the outside of the DNA molecule.

\section{Interaction of Actinomycin and DNA}

Only a few positive conclusions can be made on the mode of binding of actinomycin to DNA. $\mathrm{X}$-ray diffraction patterns from fibers containing this drug showed a gradual change of the pattern from that of $B$ DNA as the proportion of drug increased, but at no time showed a change in the layer line spacing or in the $3.4-\AA$ reflection in $B$ DNA. These results, consistent with earlier observations, suggest that in the fiber the actinomycin is bound to the outside of the DNA; since no increase in helix pitch was observed, intercalation can be excluded. It seem that if the published model for the external binding is to be refined, involvement of the cyclic pentapeptides must be considered. Previously only the hydrogen-binding scheme between the phenoxazone chromophore and a guanine residue had been considered in detail, with reference being made to the possibility that the four peptide NH groups might be hydrogen bonded to the DNA phosphate oxygens. Conformational analysis of the cyclic pentapeptides of actinomycin is being attempted in the hope that it will provide the basis for a study of the interaction of the whole molecule with DNA.

\section{Messenger Ribonucleic Acid Synthesis}

Transfer of the genetic message involves two steps: (1) transcription from DNA to messenger ribonucleic acid (RNA) and (2) translation of this information from messenger RNA to protein. Step 1 is studied by determining in some detail the three-dimensional conformation of DNA and RNA, synthetic polynucleotides, and the interaction of synthetic polyribonucleotides and polydeoxynucleotides, and by examining deoxyribonucleohistones from cells isolated to preserve as much as possible of the original chromosome structure, especially the messenger RNA-DNARNA polymerase complex.

Deoxyribonucleohistone extracted at neutral $p \mathrm{H}$ and in low salt concentrations from mouse liver always contains small amounts of RNA. This RNA is of low molecular weight $(<20,000)$ and comprises $\approx 4 \%$ of the total nucleotides, and most of it is bound to DNA at low but not at high ionic strength. About $25 \%$ is associated with nucleohistone in such a way that it is resistant to pancreatic RNase. The structure is destroyed by heat, freezing, and high ionic strength, which suggests that protein is involved, and by urea, which suggests that hydrogen bonding is important, but not by exposure to citrate or ethylenediaminetetraäcetic acid, which indicates that the structure is probably not stabilized by metal ions.

\section{Inferaction of Synthetic Polynucleotides}

To complement studies of natural DNA-RNA hybrids, synthetic complexes from polyribonucleotides and polydeoxyribonucleotides have been prepared. X-ray diffraction patterns of poly- 
deoxyadenylic acid (poly $\mathrm{dA}$ ) and polyribo-uridylic acid (poly rU) have suggested that this complex has a structure very similar to the threestranded complex of poly A:2U. The threestranded structure is helical with a pitch of 26 $\AA$, and at $97 \%$ relative humidity the intermolecular separation in the fibers is $27 \AA$. It is interesting that the complex with one deoxyribo strand is so similar in structure to one in which all the polynucleotide chains have ribose sugars. The fact that such a complex is three stranded may be related to the process of transcription in the natural hybrid of DNA-RNA. Other forms have been seen in which the poly $\mathrm{dA}$ and the poly $\mathrm{rU}$ are two-stranded.

\section{Structure and Function of Ribosomal Ribonucleic Acid}

The ribosome plays a central role in protein synthesis. Its structure is exceedingly complex, and its function is at present poorly understood. Studies to provide information on the primary and secondary structure of ribosomal RNA and on the interaction of the various segments of the RNA with ribosomal proteins have been designed to further understanding of the molecular interactions and functions of the ribosome and its RNA. Ribosomal RNA's from mammalian and avian liver have been shown to have ribonucleaseresistant regions containing primarily guanine and cytosine residues. Conformational studies on the fraction of 28S RNA most resistant to ribonuclease show that it has an ordered and rigid structure. This fraction is primarily double stranded, but has single-stranded regions in it. Thermal denaturation studies reveal that most of the hydrogen bonding is between guanine and cytosine, although $15 \%$ of the hydrogen bonds are between adenine and uracil. A model has been proposed for the secondary structure of the ribonuclease-resistant fraction. This fraction, which has strong guanine and cytosine interactions, may stabilize the three-dimensional structure of the large ribosome subunit. Other studies on bacterial and plant cells do not reveal high guanine and cytosine regions. Digestion of bacterial strains Escherichia coli and Clostridium pasteurianum and of wheat germ ribosomal RNA's with ribonuclease under the conditions used to obtain the guanineand cytosine-rich fraction from animal tissues, does not produce the stable high guanine and cytosine fraction found in animal RNA. Thus the fraction rich in guanine and cytosine may be confined to animal ribosomal nucleic acids to stabilize the larger ribosome subunit.

\section{Control Mechanisms in Chloroplast Replication and Synthesis}

An action spectrum was measured for the red light effect on photoreactivation of chloroplast replication. Sublethal doses of uv light specifically inhibit the replication of the DNA of Euglena chloroplasts; the uv effect is highly photoreactivable. A pulse of red light causes loss of photoreactivability. The red light effect is overcome by cyclohexamide, but not by chloramphenicol, which indicates that the effect is mediated by cytoplasmic (nonchloroplast) ribosomes. Growth of Euglena in the light at $32^{\circ}$ or $34^{\circ} \mathrm{C}$ results in permanent loss of chloroplasts. Growth in the dark at these temperatures has no effect on chloroplasts. This effect is mediated by red and blue light and may involve a heat-sensitive RNA (or DNA) polymerase unique to chloroplasts. The role of light is as yet unclear but may relate to the induction of nucleic acid synthesis in Chlorella by blue light. It has been shown that red light is required for the synthesis of the lamellar proteins and photosynthetic enzymes of chloroplasts of Euglena. Further, there are probably at least three light reactions involved in chloroplast synthesis: one for the protochlorophyll step, one for the synthesis of structural elements, and one for the synthesis of the enzymes of photosynthetic $\mathrm{CO}_{2}$ fixation. Analysis of the fluorescence kinetics of mutants of Euglena unable to carry out photosynthetic electron transport indicates that these mutants may lack the first electron acceptor from system II of photosynthesis. Heretofore, the existence of this acceptor, " $Q$ ", was hypothetical, but such mutants may allow for its isolation and characterization.

\section{ANTIGENS AND ANTIBODIES}

\section{Studies of Lymphocyte Kinetics and Immunological Mechanisms in Experimental Animals}

Continuous extracorporeal irradiation of thoracic duct lymph (ECIL) in calves has proved to be of value in the understanding of the mechanism of skin allograft rejection. It was shown that skin allografts placed in the drainage bed of thoracic duct could be protected from the rejection mech- 
anism by continuous postgrafting ECIL. Further confirmation of this finding was obtained with triplet calves, one member of the triplet set functioning as sensitized donor of thoracic duct lymph to the other two members. These experiments provided direct evidence that immunologically activated lymphocytes rather than the humoral antibodies are essential for the rejection of firstset skin allografts. Humoral isoantibodies have been detected in the sera of animals and humans rejecting allografts. Recent evidence indicates that these antibodies are directed against transplantation antigens and may play a major role in the rejection of vascularized transplants such as the kidney. An attempt was made to measure the antibody (lymphocytotoxin) response to kidney transplants in goats, in order to determine whether the onset and degree of the response can be used in predicting the course of clinical rejection. Results from the preliminary studies indicate that the cytotoxin response may be independent of graft rejection and that the antigens responsible for cytotoxin production may be independent of the antigens responsible for accelerated graft rejection.
Studies of lymphocyte kinetics utilizing extracorporeal irradiation of blood (ECIB) and ECIL, in vitro and in vivo labeling of cells, isotopic labeling of individual lymphoid organs in situ, size distribution analysis of lymphocytes, and thymectomy have been continued. It was shown that the lymphoid cells proliferating in the thoracic duct of the calf represent at least two populations, if classified according to size distribution and the degree of cytoplasmic basophilia. The cells with more basophilic cytoplasm have shorter DNA synthesis and generation times. The mitotic time of these cells was not altered by prior ECIB. DNA synthesis time of calf thoracic duct lymphocytes, as measured by analysis of labeled mitosis and the double-labeling technique, was 4 to 6 $\mathrm{hr}$ and $4.6 \mathrm{hr}$, respectively. The newly synthesized thymic lymphocytes enter the circulation via the thymic vein and thymic efferent lymphatics, and the newly synthesized lymph node cells enter the circulation via the efferent lymphatics. Labeled thymic lymphocytes were observed in the thoracic duct lymph. Size distribution analysis of blood and thoracic duct lymphocytes of the calf did not reveal any significant differences.
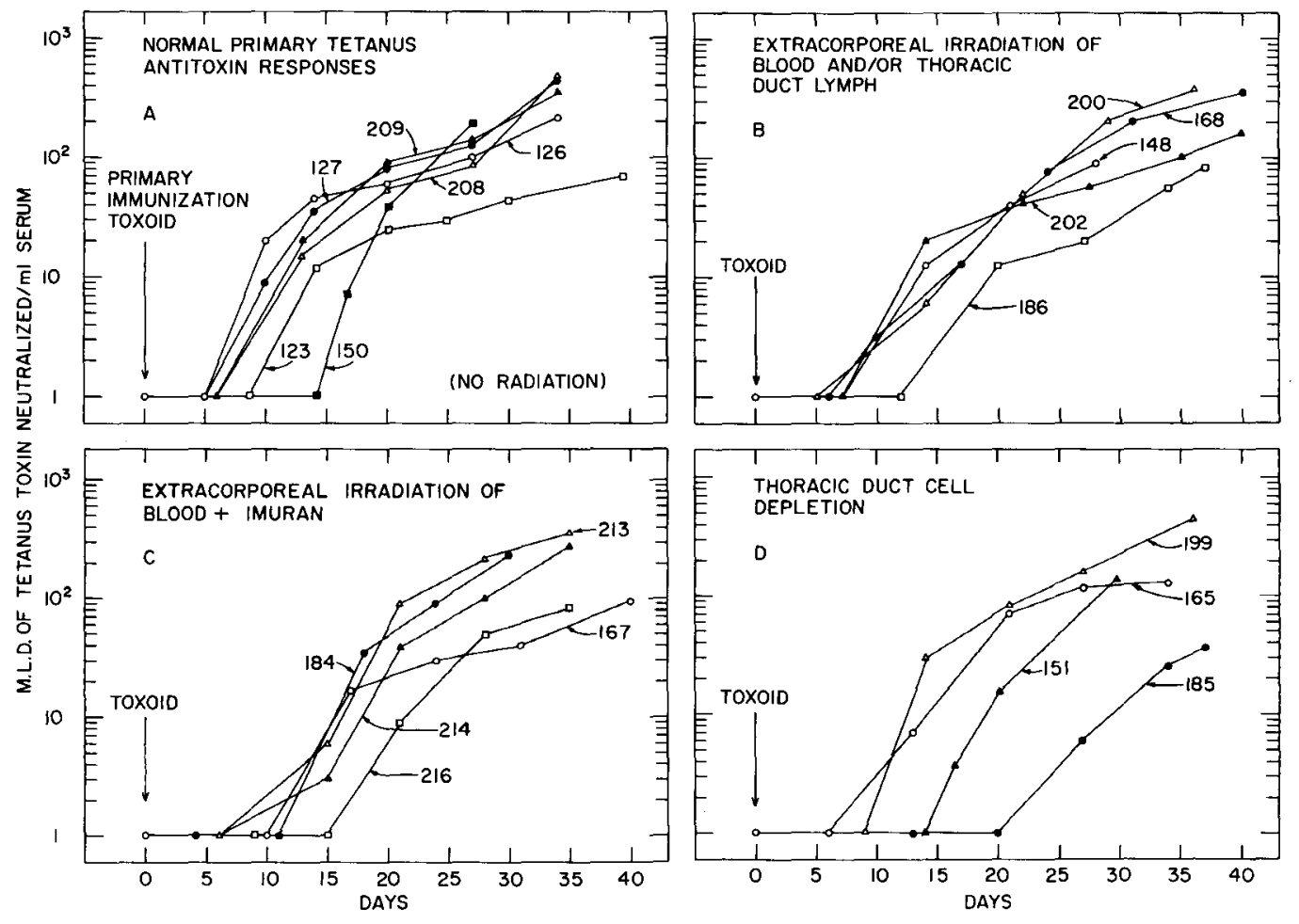

Figure 7. Primary antibody responses in calves elicited with aluminum phosphate adsorbed tetanus toxoid. 


\section{Pertussin-Induced Lymphocytosis}

The application of ECIB in the treatment of chronic lymphocytic leukemia and for immunosuppression is based upon its ability to deplete the blood and lymphoid organs of lymphocytes. In order to improve the therapeutic effectiveness of ECIB, greater than normal numbers of tissue lymphocytes must be induced to enter the blood. Pertussin produces lymphocytosis, but the mechanism of its action is not fully known. The toxic anaphylaxis-producing properties of pertussin preclude its use in patients. An attempt has been made to study the mechanism of the action of pertussin in goats, sheep, and calves. $\mathrm{H}^{3}$-thymidine has been used as a cell label in these studies. An attempt has also been made to separate the toxic and lymphocytosis-producing factors by sephadex column chromatography and analysis of the various fractions thus obtained. In a series of pilot studies performed on mice, sheep, goats, and calves, it has been observed that pertussin-induced lymphocytosis is reproducible and dose dependent. At higher dose levels severe anaphylactic reactions and deaths have occurred. Lymphocytosis induced by a single intravenous injection of pertussin is sustained for 10 to 14 days. Preliminary results obtained by the use of fractions produced by sephadex column chromatography indicate the possibility of separating the toxic and lymphocytosis-producing factors.

\section{The Effects of Extracorporeal Irradiation of Blood and Lymph Upon Antibody Production}

A series of experimental situations has been explored in 22 calves to determine the effects of ECIB, ECIL, and thoracic duct lymphocyte depletion by centrifugation (TDC) upon antibody responses. Repetitive daily ECIB, continuous ECIL, and extended TDC produce a severe depletion of lymphocytes in the circulating blood and lymph. The outer cortical zones of lymph nodes and the germinal centers remain intact, although the cuffs surrounding the germinal centers in the spleen are depleted of small lymphocytes. The combination of a radiation-induced blood lymphocytopenia with intact cortical zones of lymph nodes and actively proliferating germinal centers in lymphoreticular organs provides a unique situation for studying the relative significance of these elements versus circulating lymphocytes in primary antibody responses to adsorbed tetanus toxoid and secondary responses to fluid tetanus toxoid.
Repetitive ECIB and/or ECIL was delivered prior to immunization; in some instances, radiation was also continued after immunization. The primary antibody responses for 6 control calves are shown in Figure $7 a$. Detectable neutralizing antibody for tetanus toxin appeared in the sera 5 to 15 days after immunization. Antibody responses for 5 calves given different doses of radiation by ECIB or ECIL are shown in Figure $7 b$. Calves 148, 168, and 200 received repetitive ECIB for 10 to 31 days with accumulated doses of 65,000 to 92,000 rads. Although total circulating lymphocytes were reduced by 73 to $88 \%$ at the time of immunization, these animals responded, after a delay of about 5 days, with nearly normal primary antitoxin responses. Calves 186 and 202 received continuous ECIL for 23 and 68 days, respectively. Although total thoracic duct lymphocyte counts were reduced by $90 \%$ of normal, these calves eventually responded with typical primary antibody response; calf 186 showed an initial delay of about 10 days. It should be noted (Figure $7 a$ ) that several nonirradiated control animals also responded slowly to primary immunization.

The data in Figure $7 c$ are from animals given treaments of ECIB along with daily injections of Imuran (azothioprine) prior to and after primary immunization with adsorbed tetanus toxid. Although primary antibody responses were delayed 5 to 10 days, it is evident that the combined treatments failed to alter significantly the capacity of these animals to respond to primary antigenic stimulation.

Thoracic duct-venous shunts were established in the calves for which data are shown in Figure $7 d$. The lymph was centrifuged continuously and lymphocytes were discarded. Cell-free lymph was returned to the calves via the jugular vein. Centrifugation of lymph and discard of lymphocytes was continued in calves 151 and 185 for 10 and 30 days, respectively. The appearance of serum antibody was delayed about 5 days in calf 151 and about 15 days in calf 185 . In spite of the severe stress of these experiments, these animals eventually responded to primary immunization.

Repetitive ECIB of 250 to 860 blood volumes during a period of 10 to 30 days with accumulated doses of 93,000 to 250,000 rads failed to repress significantly secondary antitoxin responses to fluid toxoid. These findings are in sharp contrast to the well-known radiosensitivity of antibody responses when total does of 50 to 600 rads of ionizing radia- 

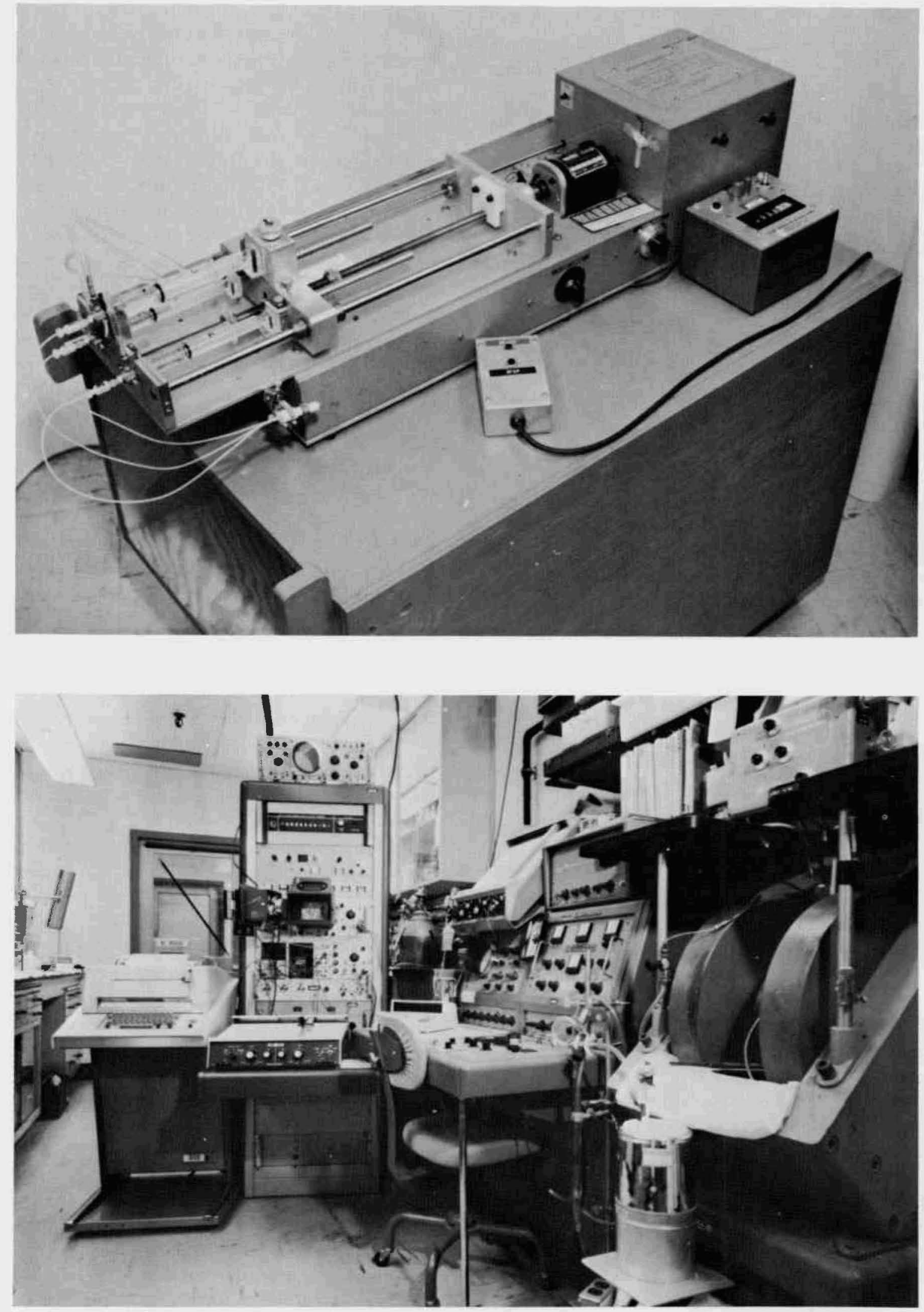
tion are delivered by whole-body exposure prior to immunization.

The present findings indicate that, under the conditions of the experiments, ECIB, ECIL, and TDC did not adequately reduce the numbers of either immunocompetent or previously sensitized cells with the capacity to respond to antigenic stimulation. It should be noted that in the present experiments the return of cell-free lymph or irradiated cells to the animals provides an opportunity for circulating antigen to reach and be accepted by nonirradiated immunocompetent lymphoid cells in lymphoreticular organs.

\section{BIOCHEMICAL STRUCTURE AND FUNCTION}

\section{Collagen}

In animals the amino acids hydroxylysine and hydroxyproline occur only in collagen, an important structural protein. They are formed by oxidation of lysine and proline during collagen formation and after the amino acids are incorporated into a polypeptide chain. During the past year additional understanding has been gained of the biochemical mechanism of hydroxylysine and hydroxyproline formation and of the importance of hydroxylysine to collagen structure. Several refinements have been developed for purification of the hydroxylating enzyme in terms of both the yield of enzyme and the reproducibility of the procedure. A tentative molecular weight of 380,000 has been established for the

$\leftarrow$ Figure 8. Top: Reaction-mixer for $Q$-band (35 GHz) EPR of rapidly flowing aqueous solutions. The three-inlet system permits the control of reaction conditions over a wide range of solvent mixtures and of $p \mathrm{H}$. Siphoning between multiple reservoirs of reactants is avoided, and precise control of flow velocity is achieved with a pumping system employing several syringes. These are driven by a push-block connected via a magnetic clutch to a variable-speed motor equipped with a multiratio gearbox and electronic speed control. In use, the stainless-steel micromixer assembly fits just below the floor of the $Q$-band microwave cavity, which is held between the poles of the EPR spectrometer's electromagnet. Bottom: General view of the spectrometer and console area. A cryogenic temperature controller is in place in front of the electromagnet at the right, where the high-velocity apparatus may also be set. An $X$-band microwave bridge is on the high table over the magnet. To the left of the control console stand the interface and teletypewriter for on-line operation of the Department's Sigma-2 computer in association with the EPR spectrometer. enzyme and it has been found to be isoelectric at $p \mathrm{H} \mathrm{5.0.} \mathrm{Reagents} \mathrm{that} \mathrm{block} \mathrm{sulfhydryl} \mathrm{groups} \mathrm{in}$ proteins inhibit the enzyme, but the sulfhydryl compound dithiothreitol can either enhance or suppress activity, depending on the concentration of iron in the solution. At low iron concentrations, increasing amounts of dithiothreitol first stimulate and then depress activity. At higher iron concentrations, increasing amounts of dithiothreitol only depress activity. New and unusual types of adsorbants have shown considerable promise for the chromatographic purification of the hydroxylase, and their use is now being actively pursued. The hydroxylase probably hydroxylates lysine residues only in that part of the collagen peptide chain in which the amino acid glycine occupies every third position. Synthetic polypeptides and tripeptides containing lysine and glycine are being made as possible substrates. A variable amount of the hydroxylysine of collagens is unavailable for oxidation by sodium periodate. This unreactive hydroxylysine probably is bound glycosidically through the hydroxyl group to galactose. In collagen from most sources glucose is linked to some of the galactose units. A micromethod has been developed which permits quantitative cleavage of the sugar units from the hydroxylysine so that they may be determined by gas chromatography. Hydroxylysine is determined on the amino acid analyzer. With use of this procedure, it has been found that the amount of hydroxylysine unreactive to periodate in a derivative of bovine bone collagen is exactly equal to the amount of galactose present and is twice the amount of glucose present. This method will also permit accurate analyses of small quantities of collagen subunits and degradation products and of collagenlike proteins from various tissues.

\section{Bioenergetic Mechanisms Involving Free Radicals}

Studies have been continued on the properties of free radicals that may be involved in many of the pathways of oxidative metabolism and in the chemical reactions that give rise to some of the effects of ionizing radiations on living systems. The stable forms of most organic molecules and biological compounds contain even numbers of electrons. Free radicals are half-oxidized or halfreduced forms that possess odd numbers of electrons. The magnetic moments associated with the spins of their unpaired electrons make free radicals paramagnetic. Hence they may be detected and 
analyzed by the complex but sensitive and nondestructive physical method known as electron paramagnetic resonance (EPR) spectrometry (also referred to as electron spin resonance, ESR).

During the year there was further development of continuous-flow apparatus for spectrometry of short-lived free radicals in liquids, using EPR at $35 \mathrm{GHz}$ (Q-band microwaves). This project at BNL has provided spectra with the consumption of $<5 \%$ of the amounts of scarce biochemical reactants required by EPR flow apparatuses used elsewhere. Fabrication of a three-inlet stainless steel mixer for $Q$-band EPR, used in conjunction with a precisely controllable pumping system em-

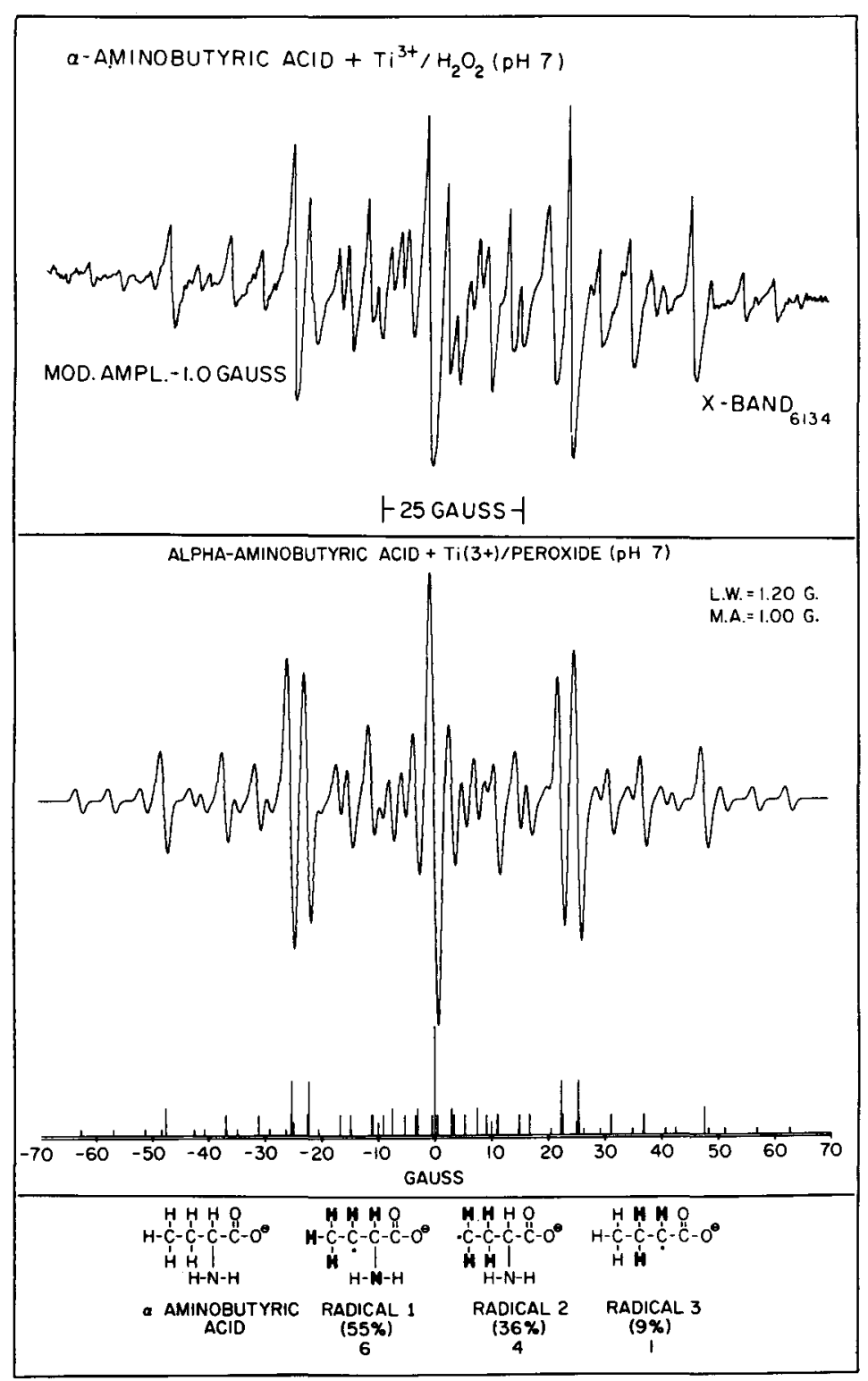

ploying multiple syringes (see Figure 8), has permitted reactions to be run at $p \mathrm{H}$ ranges at which the primary reactants were unstable. Other progress in instrumentation is aimed at providing on-line computerization of the EPR spectrometer to facilitate markedly the analysis of spectra and to improve the resolution of weak signals, such as those from biological samples. Already real-time EPR input to the Department's Sigma-2 generalpurpose computer system has been achieved, following completion of the interface components (Figure 8), and the first stages of programming for data manipulation have been completed.

Further experiments using EPR flow systems were carried out to evaluate chemical mixtures that rapidly form hydroxyl radicals $(\mathrm{OH} \cdot)$ as models of some of the reaction mechanisms of ionizing radiations. The action of this radiomimetic system on amino acids more complex than glycine gave rise to complicated EPR spectra. From analysis of these results, multiple free radical fragments existing simultaneously in solution were

Figure 9. EPR spectrum and analysis of a reaction mimicking a free radical chain produced by ionizing irradiation of an amino acid in aerated aqueous solution. There is no way, at present, for EPR spectra to be obtained directly from aqueous samples while they are undergoing irradiation, but hydroxyl radicals $(\mathrm{OH} \cdot)$ produced from water are thought to give rise to most of the so-called indirect effects which cause the biological and chemical changes that follow $\beta-, \gamma-$, and $x$-irradiation of wet, oxygenated materials. Therefore, the combination of rapid chemical formation of $\mathrm{OH}$ - in association with equipment that permits detection of transient free radical inermediates may serve to model the mechanisms that are also involved in the radiation case. In this example hydroxyl radicals produced by the action of $\mathrm{Ti}(\mathrm{III})$ upon $\mathrm{H}_{2} \mathrm{O}_{2}$ are reacted with $\alpha$-aminobutyric acid, and the short-lived reaction products are studied by EPR spectrometry with the BNL rapid-flow apparatus. Top: Actual EPR spectrum a few milliseconds after reaction (first derivative of microwave power absorbed by the sample plotted against magnetic field strength). Center: Simulation by means of a CDC-6600 computer program, ignoring certain minor effects that cause the experimental spectrum to be noisy and slightly asymmetrical. The spectral envelope, made up of Gaussian line widths (LW) compensated for modulation amplitude (MA), is found to represent the superimposition of three separate free radical spectra, admixed in different proportions $(6: 4: 1)$. Bottom: Molecular diagrams denoting the possible distribution of unpaired electron (spin) density on the three free radical fragments. In boldface are the symbols of atoms with detectable spin density. It should be noted that EPR spectra do not directly reflect spin localization on carbon or oxygen. 
identified (e.g., Figure 9). Transient intermediates detected following the chemical reaction included products that had undergone hydrogen abstraction (radicals 1 and 2 of Figure 9, for example) and deamination (radical 3 of Figure 9), results that are comparable to the effects of ionizing irradiation on amino acids.

Development was undertaken of apparatus and techniques for studying human and animal tissues directly with EPR. EPR signals from tissue have been recognized for 15 years, but their low intensity and lack of spectral character have precluded identification and have limited their exploitation as possible biological correlates. To increase the sensitivity of measurements, one BNL program has sought to provide EPR spectrometry at $X$-band (3-cm microwaves at $9.5 \mathrm{GHz}$ ) of large samples (several grams) of frozen tissue. Another program seeks to enhance the analysis of spectral shape by studying very small samples (micrograms) of tissue with EPR at $Q$-band (8-mm microwaves). Both programs depend heavily upon computer recording and treatment of data, as reported briefly above. Preliminary findings at BNL indicate that tissue EPR signals may possess more spectral character than was previously recognized.

Investigations involving free radicals related to nicotinamide and to pyridine nucleotides were made, and earlier findings regarding light-induced free radicals of visual pigments were extended. An active new program of research was initiated on cationic free radicals of porphyrins involved in photosynthesis or related to the heme groups of many enzymes. This work entails extensive collaboration with persons in BNL's Department of Applied Science (J. Fajer) and at Harvard (D. Dolphin) and Georgia Institute of Technology (R.H. Felton). 
SUPPORTING

ACTIVITIES 


\section{Technical Operations and Services}

The technical services and facilities essential to the Laboratory's research programs are provided by a number of organizational units whose operations are described below under appropriate headings.

\section{REACTOR OPERATIONS}

\section{High Flux Beam Research Reactor}

The High Flux Beam Research Reactor (HFBR) successfully completed a full year of operation at rated power level without any major operating difficulties. All experimental facilities were used during the year, and two new sample irradiation systems were placed in service.

Operation. The reactor operated as scheduled, with an accumulation of 10,147 MWd for the year. The total power accumulated by this reactor to date is $30,050 \mathrm{MWd}$. In the course of the year, 13 full operating cycles were completed. Routine operation was interrupted by two unscheduled shutdowns (compared with eight in fiscal 1968). The first, on Feb. 8, 1969, was the result of an electrical power failure that occurred at the end of a normal operating cycle, and operation was resumed after the reactor had been refueled. The second, caused by malfunction of the nuclear safety system, occurred on May 16 and lasted for 23 minutes.

During the shutdown periods, 182 used fuel elements (13 half-cores) were discharged from the reactor and replaced with new elements. A total of 24 shipments (336 elements) of spent HFBR fuel were made to the Atomic Energy Commission's reprocessing plant at Savannah River.

The change in isotopic purity of the reactor system $\mathrm{D}_{2} \mathrm{O}$ is shown in Figure 1 for the period from reactor start-up to date. All the degradation occurred during periods when the reactor primary system was open for maintenance.

Material Surveillance Program. The material surveillance program monitors the effects of radiation, as it exists in the reactor environment, on the materials used in construction of the reactor vessel and control-rod blades. Specimens of the aluminum used for the reactor vessel have been placed in the reactor in a region of high neutron flux. Irradiations of 20, 80, and 200 days' duration have been completed, and the 800 and 4000-day samples are currently in the reactor.

In June, auxiliary control-rod blade A-7 was removed from the reactor for metallurgical examination. It had been in the reactor for 29,194 MWd.

Exfended Fuel Element Irradiation Program. A fuel element irradiation program was initiated to determine whether the current HFBR fuel element design is capable of sustaining higher burnup levels. Four standard elements were irradiated in the HFBR for periods of up to 34 days beyond the normal 40-day exposure. Visual and metallographic examination of the fuel elements following irradiation has shown no evidence of hot spots, pitting, blistering, or distortion of the elements or of individual plates. A small amount of swelling was observed over the fueled portion of the plates, but this was no greater than the swelling seen after normal exposures. Radiochemical analyses of punchings taken from selected fuel-plate locations have shown that burnup levels of up to $65.7 \%$ $\left(16.7 \times 10^{20}\right.$ fissions $\left./ \mathrm{cm}^{3}\right)$ were achieved. The results of the program indicate that an increase of $50 \%$ in the fuel element design lifetime is feasible.

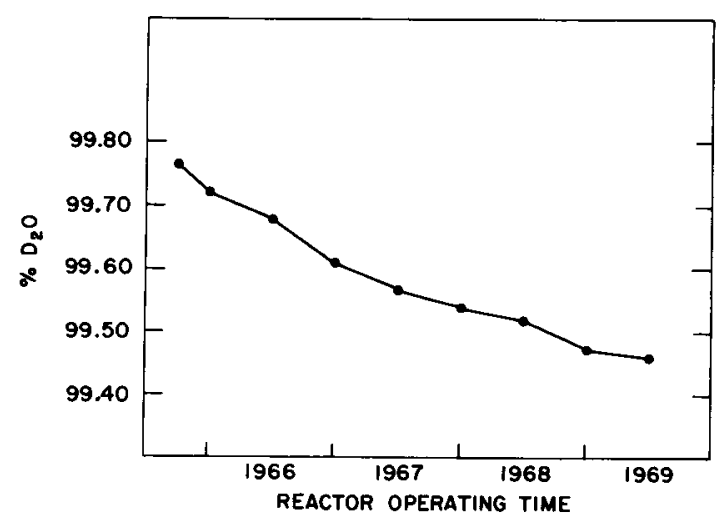

Figure 1. Changes in the isotopic purity of the $\mathrm{D}_{2} \mathrm{O}$ in the HFBR from reactor start-up (Oct. 25, 1965) to date. 


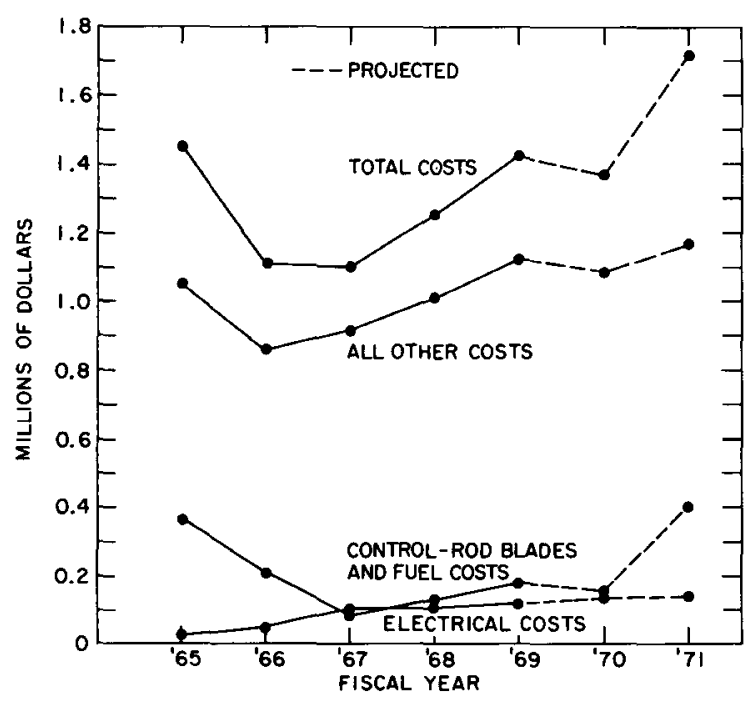

Figure 2. Costs associated with operating the HFBR

Improved Fuel Element. It may be noted from Figure 2 that one of the major elements driving the cost of reactor operation upward is the cost of fuel. Shown here is anticipated fabrication cost only. The increase can be offset to a great extent by extending the life of each element. Studies indicate that by increasing the $\mathrm{U}^{235}$ content by $15 \%$, the life of the element can be extended by $50 \%$. If this is done, fewer refueling shutdowns of the reactor will be required and the utility of the reactor on a time basis will be increased from $\approx 70 \%$ to $>80 \%$.

A review of the safety aspects of an improved element is in progress.

Reacfor System Improvements. The secondary water holdup system is now in service and provides for the rapid and complete isolation of the secondary water system should a heat exchanger failure occur.

Experimental Facilities. All the horizontal beam holes are now occupied by experiments that are operating routinely, with holes $\mathrm{H}-1, \mathrm{H}$ 4, and H-6 utilizing dual-beam spectrometers.

During the year two irradiation facilities were installed in the vertical irradiation thimbles: (1) the hydraulic irradiation facility in reflector thimble V-11, in which "rabbits" (sample containers) are charged and discharged by flowing $\mathrm{D}_{2} \mathrm{O}$; and (2) a cable-operated irradiation facility in reflector thimble $\mathrm{V}-12$, in which rabbits are attached to the end of a flexible titanium Teleflex cable and are charged and discharged mechanically.
A long-term experiment was installed in onehalf of the V-16 in-core vertical thimble for the Westinghouse Electron Tube Division. The irradiation is a life-test on miniaturized nuclear detectors.

The usage of the vertical irradiation thimbles is summarized in Table 1.

Operating Costs. Since no support for the operation of BNL reactors is budgeted directly by the AEC, each scientific program using a reactor supports it in proportion to the program's portion of the total use of the reactor. Table 2 shows the distribution of this support over a three-year period for the HFBR. In Figure 2 the cost of operating this reactor in fiscal 1970 and 1971 is projected. For fiscal 1970, no increase in operating costs, compared with 1969, is projected because of staff reductions and the ability to operate the HFBR for the entire year with fuel purchased in previous years. A set of control-rod blades ordered in 1969 will be paid for over a three-year period. The projection for fiscal 1971 includes the cost of fuel of the improved design, previously discussed, and a portion of the control-rod order.

\section{Brookhaven Graphite Research Reactor}

Routine scheduled operation of the Brookhaven Graphite Research Reactor (BGRR) was curtailed at the end of fiscal 1968. This year the BGRR was operated on 27 days; the remainder of the time it was maintained on standby. In this condition operation is possible, with appropriate advance notice, for periods of up to five days by using the HFBR operating crews during scheduled HFBR shutdowns.

The Chemonuclear Group of the Department of Applied Science was the only group to take advantage of this situation. This group had completed the rather large loop installation at about the same time that routine operation of the reactor was curtailed, and there was great interest in running the reactor at least long enough to determine the capability of the loop. The initial runs were for dosimetry and calibration. Later runs consisted of operation under various conditions of temperature, oxygen partial pressures, and total pressure to study the production of ozone in a radiation field. The loop was designed to study chemical reactions in the presence of energetic fission fragments. As yet, however, no fuel has been used in the loop.

The total energy produced by this reactor to date is $99,857.8 \mathrm{MWd}$. 
Table 1

Usage of HFBR Vertical Irradiation Thimbles, Fiscal 1969

\begin{tabular}{|c|c|c|c|c|c|c|c|c|}
\hline \multirow[b]{2}{*}{ User } & \multicolumn{3}{|c|}{ Reflector } & \multicolumn{2}{|c|}{ Core edge } & \multicolumn{2}{|c|}{ In-core } & \multirow[b]{2}{*}{ Total } \\
\hline & $\mathrm{V}-10$ & $\mathrm{~V}-11$ & $\mathrm{~V}-12$ & $V-13$ & $V-14$ & V-15 & $V-16$ & \\
\hline Applied Science & 1 & & 1 & & 18 & 16 & 24 & 60 \\
\hline Chemistry & 25 & 1 & & & 4 & & & 30 \\
\hline Instrumentation and Health Physics & & & & & 1 & & & 1 \\
\hline Medical & 1 & 1 & & & & & & 2 \\
\hline Physics & 12 & 2 & 4 & & 9 & & & 27 \\
\hline HFBR materials surveillance experiment & & & & 26 & & & & 26 \\
\hline Outside organizations & 1 & 3 & & & & & 6 & 10 \\
\hline Total & 40 & 7 & 5 & 26 & 32 & 16 & 30 & 156 \\
\hline
\end{tabular}

Table 2

Distribution of HFBR Support From Scientific Programs

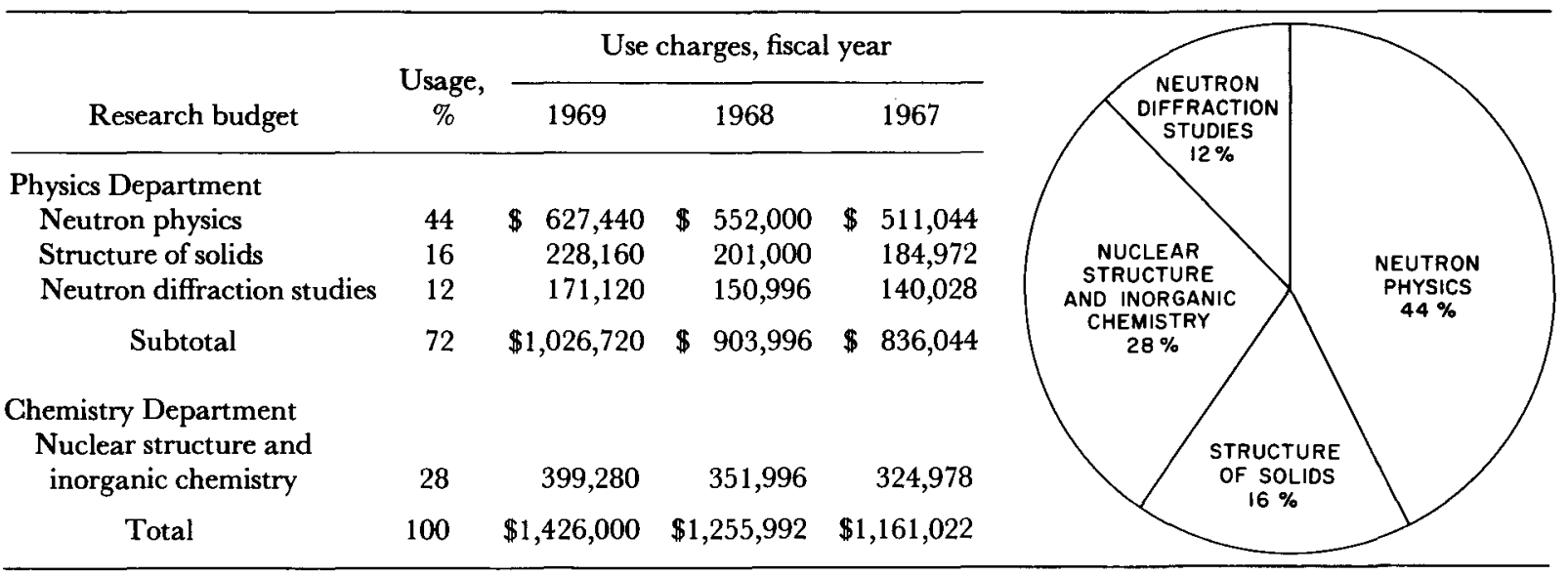

\section{Brookhaven Medical Research Reactor}

Operations. The Medical Research Reactor (MRR) was operated on 203 days during the year, and 1,764.997 MWh of operation were accumulated. Total integrated energy to date is $5,994.059$ MWh.

There were four unscheduled shutdowns of the reactor during the year for the following causes: (1) a control-rod magnet failure, (2) actuation of a manual scram by a student visitor who thought it was an airlock door release button, (3) high primary $\mathrm{H}_{2} \mathrm{O}$ temperature due to insufficient secondary $\mathrm{H}_{2} \mathrm{O}$ flow through the heat exchangers, and (4) a spurious "blip" on one of the period amplifiers of the nuclear safety system during replacement of a faulty unit, which caused two units in a two-out-of-three scram system to be tripped. All shutdowns were of short duration and caused little delay in programs.

Improvements. A fission neutron irradiation facility was placed in operation in the animal exposure room during Dec. 1968. It consists of three fission plates containing $1529.00 \mathrm{~g}$ uranium 93.16\% enriched in $U^{235}$, arranged in a holder in such a way that the effective fission plate area is $12 \times 12$ in. Ninety-two percent of the incident thermal neutrons are absorbed, to give a fast neutron flux of $3 \times 10^{10}$ neutrons $/ \mathrm{cm}^{2}$-sec at $3.0-\mathrm{MW}$ reactor power.

An off-gas system was installed during Sept. 1968. The system incorporates eight available tiein locations placed so as to permit off-gassing of the pneumatic-tube dry box and other experimental facilities as required. The air flow is provided by the main reactor exhaust air fan, with the air 
Table 3

Irradiations at the MRR, Fiscal 1969

\begin{tabular}{|c|c|c|c|c|c|c|c|c|}
\hline \multirow[b]{2}{*}{ User } & \multirow[b]{2}{*}{ In-core } & \multirow[b]{2}{*}{$\begin{array}{l}\text { Core } \\
\text { edge }\end{array}$} & \multirow[b]{2}{*}{$\begin{array}{l}\text { Pneumatic } \\
\text { tube }\end{array}$} & \multirow[b]{2}{*}{$\begin{array}{l}\text { Radial } \\
\text { hole }\end{array}$} & \multicolumn{3}{|c|}{ Beam hole } & \multirow[b]{2}{*}{ Tota } \\
\hline & & & & & $\begin{array}{l}\text { Broad } \\
\text { beam }\end{array}$ & $\begin{array}{l}\text { Patient } \\
\text { facility }\end{array}$ & $\begin{array}{l}\text { Animal } \\
\text { facility }\end{array}$ & \\
\hline Accelerators & & 5 & & & & & & 5 \\
\hline Applied Science & 2 & & 8 & 15 & 10 & 5 & & 40 \\
\hline Biology & & & 11 & & 2 & & 404 & 417 \\
\hline Chemistry & 4 & 38 & 131 & 1 & & 13 & & 187 \\
\hline Instrumentation and Health Physics & & & 5 & & & 1 & & 6 \\
\hline Medical & & 7 & 55 & & & 48 & 55 & 165 \\
\hline Physics & & 13 & 86 & & 1 & & & 100 \\
\hline Outside organizations & & 9 & 11 & 23 & 2 & & & 45 \\
\hline Total & 6 & 72 & 307 & 39 & 15 & 67 & 459 & 965 \\
\hline
\end{tabular}

Table 4

Summary of Services to Outside Users

(Handling and other charges included)

\begin{tabular}{|c|c|c|c|c|c|c|}
\hline & \multicolumn{2}{|c|}{ Fiscal 1969} & \multicolumn{2}{|c|}{ Fiscal 1968} & \multicolumn{2}{|c|}{ Fiscal 1967} \\
\hline & Number & Volume, $\$$ & Number & Volume, \$ & Number & Volume, $\$$ \\
\hline Reactor irradiations & 43 & 19,564 & 166 & 18,341 & 237 & 19,484 \\
\hline Processed radioisotopes & 43 & 8,052 & 67 & 12,401 & 269 & 51,080 \\
\hline $\mathrm{Co}^{60}$ and $\mathrm{Cs}^{137}$ sources & 9 & * & 4 & $*$ & 3 & * \\
\hline Cyclotron irradiations & 1 & 230 & 0 & 0 & 0 & 0 \\
\hline Total & 96 & 27,846 & 237 & 30,742 & 509 & 70,564 \\
\hline
\end{tabular}

*AEC transfers - $\mathrm{Co}^{60}$ and $\mathrm{Cs}^{137}$ not produced by Brookhaven.

passing through the main exhaust filters before being exhausted to the stack.

Use. During the year, 965 irradiations were made, 45 of them for outside organizations. A breakdown by facilities and departments is given in Table 3.

The annual use of the reactor is plotted (Figure 3) as number of runs and as energy produced. The increase in use during 1969 was caused by the curtailment of BGRR operations and the shifting of irradiation programs to the MRR.

\section{Procurement of Special Materials and Services}

The procurement for the scientific departments of all radioactive and stable isotopes, as well as special materials controlled by the AEC, is a responsibility of the Isotopes and Special Materials Group. In this connection, 266 purchase orders were placed for radioisotopes, 25 for stable iso- topes, and 35 for special materials. Against these orders, $\approx 395$ shipments were received and processed. The totals for orders placed and shipments received were essentially the same as those for the previous fiscal year. Of the orders placed, $34 \%$ were for the Medical Department, $20 \%$ for $\mathrm{Bi}$ ology, $14 \%$ for the Department of Applied Science, $11 \%$ for Chemistry, $15 \%$ for Physics, and $6 \%$ for Instrumentation and Health Physics. These percentages are similar to those for the past two years.

The Isotopes and Special Materials Group is also responsible for annual inventories of radium sources and purchased stable isotopes and for negotiations for the loan of valuable isotopes. The number of radium sources on hand remained at 34 , with a total activity of 5.86 curies and a total value of $\approx \$ 39,500$. The inventory of purchased stable isotopes valued at $\$ 29,350$ shows a decrease from last year's value of $\$ 42,204$, since consump- 


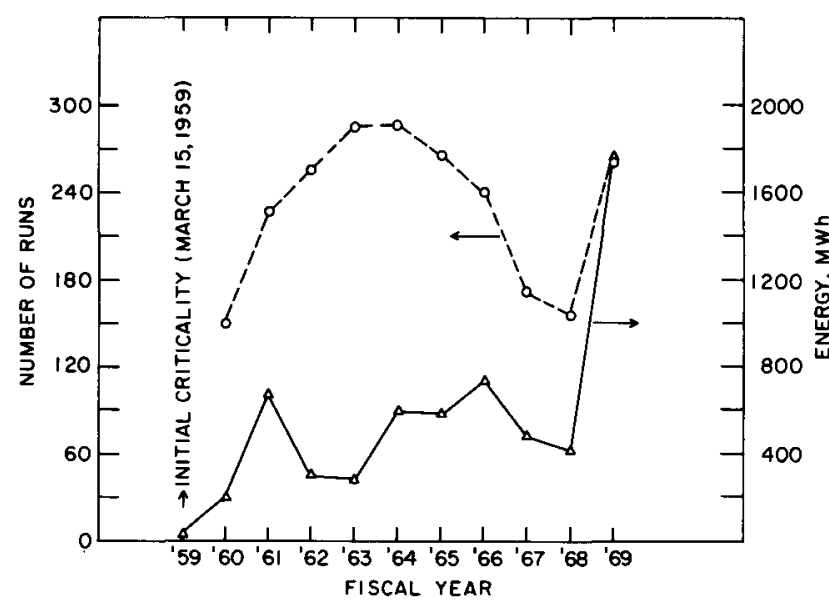

Figure 3. Use of the MRR.

tion $(\$ 17,026)$ during the year exceeded the cost of purchases (\$4165). The number of stable isotopes on loan increased from 22 to 29 , but the value decreased from $\$ 1,785,438$ to $\$ 1,549,310$.

\section{Irradiation Services and Isotope Production}

Table 4 is a summary of irradiation services to outside organizations during the past three years. These services have been continuously decreasing in number because of the AEC's policy of noncompetition and the increasing availability of similar services commercially. The dollar volume for the past year did not drop correspondingly because of the higher rates for services performed in the MRR and HFBR since the BGRR was placed on standby. The volume of these services is expected to remain at a minimal level in the near future.

\section{Source and Special Nuclear Material Accountability}

In Table 5, a three-year summary is presented of the amounts of source and special nuclear (SS) materials on hand at Brookhaven at the end of each fiscal year. Based on dollar values published by the AEC, the current inventory of SS materials represents a value in the range of $\$ 15.8$ million. The decrease of about $\$ 1.7$ million from the previous year is associated with the decrease in the inventory of $U^{235}$ enriched to $>75 \%$ resulting from shipments of spent fuel from the HFBR and the BGRR and with the large decrease in the $U^{235}$ $<75 \%$ resulting from the return of this material to AEC production channels.

The annual audit of SS materials at Brookhaven and the associated accounting records and
Table 5

Source and Special Nuclear Materials on Hand at End of Year (in kilograms)

\begin{tabular}{|c|c|c|c|}
\hline & \multicolumn{3}{|c|}{ Fiscal year } \\
\hline & 1969 & 1968 & 1967 \\
\hline \multicolumn{4}{|c|}{ Facility BZA } \\
\hline Natural uranium & 358 & 361 & 505 \\
\hline Depleted uranium & 28,114 & 23,766 & 23,144 \\
\hline $\mathrm{U}^{235}>75 \%$ & 811 & 860 & 633 \\
\hline $\mathrm{U}^{235}<75 \%$ & 612 & 6,244 & 3,898 \\
\hline $\mathrm{U}^{233}$ & 0 & 23 & 23 \\
\hline $\mathrm{Pu}^{239}$ & 58 & 58 & 59 \\
\hline Thorium & 85 & 817 & 820 \\
\hline Heavy water & 52,351 & 49,378 & 53,959 \\
\hline \multicolumn{4}{|c|}{ Facility BZB (Graphite Reactor Fuel) } \\
\hline $\mathrm{U}^{235}>75 \%$ & 67 & 79 & 82 \\
\hline \multicolumn{4}{|c|}{ Facility BZC (Medical Reactor Fuel) } \\
\hline $\mathrm{U}^{235}>75 \%$ & 5 & 5 & 3 \\
\hline
\end{tabular}

procedures was performed by the Brookhaven AEC Operations Office from Jan. 20 to Feb. 17, 1969. In line with the recommendations from this and prior audits, a procedure is in effect for sealing unused materials to reduce periodic auditing efforts, and a major effort was completed to remove surplus material from the inventory. Transfer was made of $23 \mathrm{~kg}$ of $\mathrm{U}^{233}$ and $731 \mathrm{~kg}$ of thorium to the AEC's Bettis Atomic Power Laboratory as surplus, and $5874 \mathrm{~kg}$ of slightly enriched uranium containing $89.3 \mathrm{~kg}$ of $\mathrm{U}^{235}$ were returned to the AEC at Fernald, Ohio, for recovery. In addition, 26 shipments of spent reactor fuel containing 90 $\mathrm{kg}$ of highly enriched uranium, of which $78 \mathrm{~kg}$ were $U^{235}$, were made to the Savannah River Plant of the AEC.

The contract for HFBR fuel elements with the United Nuclear Corp. was completed this year with the delivery of 112 elements. It is expected that a new contract will be initiated early next year in which some design changes that have been under study will be incorporated. Thirty unused surplus fuel elements were acquired at no cost from the Pennsylvania State University for use in future refueling of the MRR.

The 14th and 15th IAEA inspections of Facility BZB (Graphite Reactor fuel) were performed by Mr. T. Mori of the IAEA on Aug. 18 and 19, 1968, 
and on Dec. 11 and 12, 1968. Because of the standby condition of the BGRR, the inspections were mostly concerned with how to seal and monitor the shut-down reactor. During the second inspection, neutron flux dosimeters provided by the IAEA were inserted in hole E-W-45 and the hole covers were sealed by the inspector. On May 28, 1969, another inspector, Mr. R. Skjoeldebrand, broke the seals and removed the dosimeters for analysis to check the amount of reactor operation that had occurred.

\section{HEALTH PHYSICS}

The major exposure control problem continues to be at the AGS, where the effect of improvements in equipment and technique has been offset by the achievement of higher beam intensities. Control of the contamination generated by bombardment of certain targets has become more of a problem than in the past. A reduction in health physics work at the BGRR has been counterbalanced by an increase in activities at the MRR.

Radiation surveys included coverage of the initial use of accelerated tritons for bombardments at the Physics Department's Van de Graaff accelerator, initial testing of a $13,000-\mathrm{Ci} \mathrm{Co}{ }^{60}$ source, and initial operation of the new radiological research accelerator facility at the Cosmotron Building. A new radiation monitoring system has been installed at the Physics Department's Van de Graaff, and improvements have been made in the nuclearincident alarm system at the critical assemblies complex.

A 10-wk on-the-job training course in applied health physics was provided for a group of 12 AEC and US PHS Fellows during the summer. Members of the Division participated in activities sponsored by the International Commission on Radiation Protection, the National Council on Radiation Protection, the International Commission on Radiation Units and Measurements, the AEC Advisory Panel on Radiation Safety, the FAA Standing Committee for Radiation Biology Aspects of the Supersonic Transport, and the Intersociety Committee on the Manual of Methods for Ambient Air Sampling and Analysis.

\section{Synchrotron Monitoring and Dosimetry}

Work has continued on the development of a practical dose-equivalent survey meter based on the Rossi-type linear energy transfer (LET) spec- trometer chamber. An analysis was made of several possible methods, the most promising being an amplifying arrangement with a suitable nonlinear relation between current output and the size of the input pulses. The gain requirements have been established, and a circuit providing a good approximation to that gain has been designed, built, and tested. Three parallel amplifying circuits are used, with the input bias and gain of each adjusted to yield the desired output. The readout can be changed from dose equivalent to absorbed dose by means of switch-controlled changes in the circuit parameters. Satisfactory performance of the chamber for several months after sealing-off has been achieved. Limited tests indicate that the instrument is indeed measuring dose equivalent for a variety of radiation qualities. A battery-operated model suitable for more extensive testing is under development.

\section{Fundamental Dosimetry}

Data on the radial distribution of dose for 1 to 3- $\mathrm{MeV}$ protons and helium nuclei in hydrogen and tissue-equivalent gas have been obtained with a 3-ft-long cylindrical ionization chamber operated at reduced pressure. LET $_{r}$ can be determined by varying the pressure. In addition, measurements of ionization as a function of radial distance from the charged particle beam can be made with use of a small probe-type chamber inside the larger chamber.

A study is being made of cell survival data for particles with various charges and velocities in an attempt to correlate biological effects with LET and the microdistribution of ionization. The analysis suggests that two classes of sensitive site are involved, with effective thicknesses of $<33 \AA$ each. One class of site relates to inactivation of the single-hit type, whereas the second class relates to a loss of repair process or a repairable type of injury. Two models have been developed that fit a family of curves obtained at different values of LET. Various "track core" refinements in the models as well as the effects of $\mathrm{O}_{2}$ and protective agents are being studied to elucidate the relationship between LET, the microdistribution of ionization, and fundamental mechanisms of biological damage.

\section{Waste Disposal and Reclamation}

Present plans call for discharge of cooling water from a beam stop at the new $200-\mathrm{MeV}$ linac di- 
rectly into the Laboratory sewage system. Analysis of the expected activities indicates that this is an acceptable procedure, but an experimental program to verify this conclusion is being carried out. Tests have been made with use of purchased quantities of $\mathrm{H}^{3}$ and $\mathrm{Be}^{7}$ tracers to establish decay times and the removal of activity prior to reaching the site boundary. More tests are planned in which water activated by the beam of the present $50-\mathrm{MeV}$ injector will be used.

Data relating to the liquid waste system are presented in Table 6 . The amount of gross $\beta$ activity, other than tritium, leaving the site in the stream to which the effluent is discharged totaled $13.4 \mathrm{mCi}$ during the fiscal year. The average concentration was $1.4 \times 10^{-8} \mu \mathrm{Ci} / \mathrm{cc}$, which is $1.3 \%$ of the applicable AEC standard. Tritium amounting to 10.7 $\mathrm{Ci}$ was discharged at an average concentration of $1.1 \times 10^{-5} \mu \mathrm{Ci} / \mathrm{ml}$, which is $0.4 \%$ of the applicable standard. With the BGRR operating only a small fraction of the time, activity discharged per month from the BGRR-HFBR stack averaged only $16,000 \mathrm{Ci}$ of $\mathrm{Ar}^{41}, 0.61 \mathrm{mCi}$ of $\mathrm{I}^{131}$, and $36 \mathrm{Ci}$ of $\mathrm{H}^{3}$. Four vaults of solid waste were shipped to the disposal site at West Valley, N.Y.

\section{Environmental Monitoring}

A stream monitoring and sampling station has been installed at the new east perimeter of the site, $4300 \mathrm{ft}$ downstream from the old perimeter. Activity and flow data from the new location have been used since Jan. 1, 1969. The amount of activity carried across the new site boundary is much less than that at the old boundary because of losses to ground water.

During fiscal 1969 the highest external radiation level at the site boundary occurred at a point north of the Gamma Forest source and averaged $3.24 \mathrm{mR} / \mathrm{wk}$. This may be separated into three components of $1.61,0.02$, and $1.61 \mathrm{mR} / \mathrm{wk}$, due to background, $\mathrm{Ar}^{41}$ from the BGRR, and the forest source, respectively. The permissible level above background is $500 \mathrm{mR} / \mathrm{yr}$, or an average of 10 $\mathrm{mR} / \mathrm{wk}$.

Samples of air particulates, including both longer-lived natural and fallout isotopes, averaged $0.19 \mathrm{pCi} / \mathrm{m}^{3}$ of gross $\beta$ activity, while the average activity in precipitation was $4.4 \mathrm{nCi} / \mathrm{m}^{2} / \mathrm{mo}$.

\section{Personnel Monitoring}

A new hand-and-foot monitoring service using thermoluminescent dosimeters has been very useful in recording and controlling hand exposures in the handling of radioactive materials. Results are far superior to those obtained previously with film dosimeters.

In a cooperative program with the Central Automatic Data Processing Group, conversion of the processing of personnel monitoring records from Remington Rand to IBM equipment was completed.

Personnel monitoring service was provided for 2800 persons during calendar year 1968, a 4⿺辶⿸\zh14𠄌

Table 6

Summary of Liquid Waste Data

\begin{tabular}{|c|c|c|c|c|}
\hline & \multicolumn{2}{|c|}{ Fiscal 1969} & \multicolumn{2}{|c|}{ Fiscal 1968} \\
\hline Input to filter beds, gal/day & \multicolumn{2}{|c|}{995,000} & \multicolumn{2}{|c|}{960,000} \\
\hline Output from filter beds, gal/day & \multicolumn{2}{|c|}{759,000} & \multicolumn{2}{|c|}{810,000} \\
\hline Net loss in filter beds, $\%$ & \multicolumn{2}{|c|}{24} & \multicolumn{2}{|c|}{16} \\
\hline Stream above discharge point, gal/day & \multicolumn{2}{|c|}{15,000} & \multicolumn{2}{|c|}{21,200} \\
\hline at site boundary, gal/day & & & & \\
\hline \multirow[t]{2}{*}{ Rainfall, in./mo } & \multicolumn{2}{|c|}{2.9} & \multicolumn{2}{|c|}{3.7} \\
\hline & Gross $\beta$ & Tritium & Gross $\beta$ & Tritium \\
\hline Activity concentration at input to filter beds, $\mathrm{Ci} / \mathrm{cc}$ & $1.6 \times 10^{-8}$ & $1.1 \times 10^{-5}$ & $2.9 \times 10^{-8}$ & $1.4 \times 10^{-5}$ \\
\hline at output from filter beds, $\mathrm{Ci} / \mathrm{cc}$ & $1.4 \times 10^{-8}$ & $1.0 \times 10^{-5}$ & $2.1 \times 10^{-8}$ & $1.2 \times 10^{-5}$ \\
\hline at site boundary, $\mathrm{Ci} / \mathrm{cc}$ & $1.4 \times 10^{-8}$ & $1.1 \times 10^{-5}$ & $2.1 \times 10^{-8}$ & $1.3 \times 10^{-5}$ \\
\hline Activity at input to filter beds, $\mathrm{mCi} / \mathrm{mo}$ & 1.4 & 1280 & 3.2 & 1620 \\
\hline at output from filter beds, $\mathrm{mCi} / \mathrm{mo}$ & 1.2 & 900 & 2.0 & 1180 \\
\hline at site boundary, $\mathrm{mCi} / \mathrm{mo}$ & 1.1 & 890 & 2.0 & 1300 \\
\hline
\end{tabular}

Figures are averages for each fiscal year. 
Table 7

Distribution of Exposures of Individuals Receiving Regular Personnel Monitoring Service

\begin{tabular}{crr}
\hline Exposure range, Rem & Calendar 1968 & Calendar 1967 \\
\hline $0.00-0.49$ & 2445 & 2525 \\
$0.50-0.99$ & 163 & 190 \\
$1.00-1.49$ & 82 & 99 \\
$1.50-1.99$ & 32 & 52 \\
$2.00-2.49$ & 22 & 29 \\
$2.50-2.99$ & 8 & 15 \\
$3.00-3.49$ & 20 & 14 \\
$3.50-3.99$ & 10 & 6 \\
$4.00-4.49$ & 13 & 0 \\
$4.50-4.99$ & 5 & 0 \\
5.00 and over & 0 & 0 \\
Total & 2800 & 2930 \\
\hline
\end{tabular}

decrease from the 1967 figure. Film dosimeters were provided for $\approx 5000$ visitors. The distribution of whole-body exposures to penetrating radiation for the 2800 persons regularly monitored is shown in Table 7. All exposures were $<3$ Rem per 13-wk period. The total exposure for all individuals monitored was $658 \mathrm{Rem}$, nearly the same as the 1967 value of $687 \mathrm{Rem}$. Seventy-seven percent of the total exposure was received at the AGS by $32 \%$ of all personnel monitored at the Laboratory.

\section{Laboratory Safety}

The 1968 BNL injury frequency rate of 2.9 represents the fifth consecutive calendar year with a rate below 3.0 injuries per million man-hours worked. Since this rate roughly represents accident costs, including the workmen's compensation benefits paid by the Laboratory, a considerable saving was achieved.

Fire and other property damage losses amounted to $\$ 21,000$ during 1968 . This is slightly lower than the losses during each of the last several years. The major part (estimated at $\$ 12,000$ ) of the 1968 loss resulted from a fire in a $600-\mathrm{kW}$ power supply unit in the AGS West Experimental Area.

During 1968 the BNL Safety Policy Manual and the Emergency Plan Manual were revised and distributed.

Implementation was begun of the results of the 1967 Facility Fire Protection Survey. Smoke detection systems were provided for the dormitories, and fire detection systems were added to the West
Experimental Area at the AGS, to various Grounds Maintenance buildings, and to the Heavy Machine Shop. Eleven new fire alarm code areas were added to the BNL Fire Protective Signaling System. Engineering work was commenced on $\mathrm{a} \mathrm{CO}_{2}$ standpipe hose system for the HFBR and on added fire protection features for the two major computer installations.

\section{MECHANICAL ENGINEERING}

During fiscal 1969 the number of personnel in the Mechanical Engineering Division remained fairly constant, with a year-end total of 187 . Their assignments to the various departments of BNL are shown in Table 8.

An important part of the operation of this past year involved movement of personnel from one department to another as some programs contracted and others expanded. In addition, there has been increased technical consultation between sections of the Division to utilize special knowledge or expertise in various engineering fields.

A closer working relationship with the Central Shops has developed with the new organizational relationship. This has resulted in better work scheduling and technical collaboration.

Funds were made available for the purchase of a complete aperture card drawing record system. This should eventually make it possible to discard all tracings more than five years old and should also effect considerable savings in the production of prints for use in fabrication.

Members of the Division at the Accelerator Department are assigned to the Conversion Division, the AGS Division, and the Experimental Planning and Support Division.

For the latter the Design Group has assembled a magnet transporter and is conducting tests involving electrical and hydraulic circuit checks as well as measurement of pressure, flow rate, and cycle control. The tests indicate that the unit is operating as designed, although a full-load test is yet to be accomplished.

The transporter is in the form of a pedestal base, electrohydraulically powered to cycle automatically while moving the load in a steplike manner. It is designed to transport and position large magnets or other equipment weighing up to $300,000 \mathrm{lb}$ in the $x, y$, and $z$ axes. It also provides $360^{\circ}$ rotation of the load in the $z$ axis, which also is the method by which change in direction of travel is 
Table 8

Assignment of Mechanical Engineering Division Personnel as of June 30, 1969

\begin{tabular}{|c|c|c|c|c|}
\hline & Engineers & $\begin{array}{c}\text { Design engineers, } \\
\text { designers, and } \\
\text { draftsmen }\end{array}$ & Clerical & Total \\
\hline \multicolumn{5}{|l|}{ Accelerators } \\
\hline Advanced accelerator development & 3 & 1 & & 4 \\
\hline Alternating Gradient Synchrotron (AGS) & 9 & 19 & 2 & 30 \\
\hline \multicolumn{5}{|l|}{ AGS conversion } \\
\hline Magnet ring & 9 & 7 & & 16 \\
\hline Linear accelerator & 9 & 14 & & 23 \\
\hline \multicolumn{5}{|l|}{ Experimental planning and support } \\
\hline Floor operations & 7 & 2 & & 9 \\
\hline Cryogenics and design & 8 & 10 & & 18 \\
\hline Administration & 1 & & & 1 \\
\hline Applied Science & 5 & 14 & & 19 \\
\hline Central Design & 1 & 6 & 1 & 8 \\
\hline Chemistry & 1 & & & 1 \\
\hline \multicolumn{5}{|l|}{ Physics } \\
\hline Bubble chambers & 14 & 16 & & 30 \\
\hline General & 9 & 18 & & 27 \\
\hline Safety & 1 & & & 1 \\
\hline Total & 77 & 107 & 3 & 187 \\
\hline
\end{tabular}

obtained. Horizontal motion is limited to $12 \mathrm{in./}$ cycle in the $x$ direction and $13 / 4$ in./cycle in the $y$ direction. Rotation is $20^{\circ}$ /cycle. Movement may be manually controlled or fully automatic at either low or high speed. Slow speed is about $2.2 \mathrm{in} . / \mathrm{min}$ and allows for positioning the load with a precision of $0.010 \mathrm{in}$. High speed will provide a horizontal motion averaging about $1 \mathrm{ft} / \mathrm{min}$.

The employment of a 35 -in.-diam hydrostatic journal bearing is unique. The piston that provides vertical motion and is the shaft for rotation also must counteract by hydraulic pressure the eccentric loading imposed on the bearing by the horizontal movement of the load off the axis. Two groups of four oil passages within the piston, at two locations along the piston, serve to center the piston within the cylinder.

In a new technique for fabricating a multipleport hydraulic manifold, precut laminations were used to provide the hydraulic circuits in compact block form.

All load-carrying bearings are of the hydrostatic type and are fully enclosed to contain the oil flow and exclude environmental dirt. These bearings are expected to have coefficients of friction of the order of $7 \times 10^{-7}$ at load.

The transporter, which weighs $20,000 \mathrm{lb}$, is equipped with an air-cushion bearing and can be positioned under a load by hand. The air cushion utilizes building air reduced to $20 \mathrm{psi}$ pressure, and electric power is delivered to the transporter by a single $440-\mathrm{V}$ cable.

A new spectrometer magnet has been designed in cooperation with the Columbia University High Energy Physics Group. The poles of this magnet will be 96 in. wide and 40 in. deep with a gap of 22 in., and it will weigh $200,000 \mathrm{lb}$. It will have a field strength of $9 \mathrm{kG}$ when coupled to one $450-\mathrm{kW}$ power supply and of $\approx 13 \mathrm{kG}$ when two power supplies are used.

The design of the 48D48 dipole magnet has been improved and acquisition is under way. The improvements make changing the magnet gap or moving the magnet easier by having the coil and water-cooling manifolds mounted integrally with the upper and lower core halves, which then move as a complete subassembly. Gap changes are easily accomplished by the addition or removal of yoke shim pieces and electric bus extensions.

Two magnet aperture vacuum boxes of a new design are being constructed for two dipole magnets that are $72 \mathrm{in.} \mathrm{long}$ and 18 and $30 \mathrm{in}$. wide, respectively. This design provides for minimum pole gap height and therefore maximum magnetic field strength consistent with particle beam optics. The top and bottom of each vacuum box, which are the 
pole tips, are constructed of high-permeability metal, but the sides and ends are of nonmagnetic metal.

A number of magnet shield assemblies for the large-aperture magnets have been fabricated to reduce the fringe field along the beam axis. This is a common problem with these magnets, since adjacent experimental equipment must be shielded from the magnetic field so as to function properly. Such assemblies are large, costly, and complicated to assemble. In future magnet designs provision will be made for their installation, and where possible they will be costed and acquired with the magnet. A typical shield installation recently constructed has a sandwich of 1,2, and 6-in. plates spaced $1 / 2$ in. apart. These are of full magnet width and height, with an aperture cut in each the approximate size of the magnet gap. Two such shields were made, one for each end of the magnet, together with a support structure for attachment to the magnet. With the shields in place the stray field $2 \mathrm{ft}$ from the magnet was lowered to $\approx 10 \mathrm{G}$ to avoid influencing magnetostrictive readout cable in adjacent wire chambers.

The use of large wire spark chambers in high energy physics experiments has necessitated the construction of purification systems for sparkchamber gas mixtures. Circulation of gas (mainly neon) through the chamber is necessary, since nitrogen from air diffusion or leakage inhibits sparking thresholds and oxygen acts to damage the grid wires, and because certain additives must be maintained at specific levels. Reprocessing of this gas is mandatory for large arrays of wire spark chambers because of its high cost.

A large purification system has been constructed and is in operation. This system recirculates 200 scf $/ \mathrm{hr}$ of a $90 \%$ neon-10\% helium gas mixture through the chambers. Provision is included for the controlled addition of isopropyl alcohol, sulfur dioxide, and argon to the mixture being delivered to improve the chamber performance.

The return gas from the chamber is stripped of impurities and additives by (1) adsorption of water vapor on silica gel, (2) condensation of alcohol and sulfur dioxide with dry ice and acetone refrigeration, and (3) adsorption of argon, oxygen, and nitrogen on a molecular sieve at liquid nitrogen temperature. Dual adsorbers are provided with integral electric heating for continuous operation.

Gas circulation is effected by an unlubricated diaphragm compressor. Control of gas pressure and overpressure protection to the wire chambers are of high precision, since these large panels are of light construction.

In the Cryogenics Group the low-temperature end of a 30 to $60-\mathrm{W}$ helium refrigerator at $4.7^{\circ} \mathrm{K}$ using a helium-gas bearing turbine expander has been delivered and is being installed in the hydrogen liquefier facility. The compression and purification equipment still remaining at the facility has been modified for helium service and is being coupled to the refrigerator, which is designed to supply liquid helium to a series of superconducting magnets in a particle beam transport system. The unit, which was in design and development for more than a year, was acquired to evaluate the performance of turbine expanders in small helium refrigerators. Of particular interest are the longterm performance and reliability requirements for unattended continuous operation. The turbine rotor is $\approx 1 \mathrm{in}$. in diameter and $3 \mathrm{in}$. long. Energy extracted from the process stream is taken up in an ambient-temperature helium-gas brake and ultimately removed to building cooling water. The rotor speed is 3000 to $4000 \mathrm{rev} / \mathrm{sec}$.

Another part of the project was an attempt to fabricate extremely compact heat exchangers, with $600 \mathrm{sq} \mathrm{ft}$ of heat transfer surface per cu ft of exchanger. This was not successful and the more conventional plate fin exchangers were used. In spite of this the entire package of heat exchanger and turbine expander is contained within a cylinder $31 \mathrm{in.}$ in diameter and $57 \mathrm{in.}$ long.

Considerable effort has gone into the design of the cryogenics system for the cold neutron moderator for the HFBR, in cooperation with the Physics Department section. This comprises a 1.2in.-thick hemispherical container of liquid hydrogen at the innermost end of the $\mathrm{H}-9$ hole thimble.

A $3.5-\mathrm{kW}$ refrigerator located on the equipment floor is to provide $14.1^{\circ} \mathrm{K}$ helium gas to a heat exchanger adjacent to the $\mathrm{H}-9$ hole at the reactor's face. Return gas from the heat exchanger or subcooler will be at $17.1^{\circ} \mathrm{K}$. The subcooler, consisting of two helium-cooled submerged pumps in parallel with automatic switching, will circulate 50 liters/ min of liquid hydrogen at $14.1^{\circ} \mathrm{K}$ between the moderator and heat exchanger. The liquid hydrogen return temperature will be $17.1^{\circ} \mathrm{K}$, and the loop circulating pressures will be 22 psia discharge and 16 psia suction. The entire helium refrigerator including the subcooler is insulated by vacuum and multilayer reflecting shields. The liquid hy- 
drogen loop is surrounded by both an insulating vacuum space and an ambient-temperature helium gas blanket at 5 psig to provide two levels of protection against air condensation on surfaces within a high radiation flux area.

Design of a new dc short separator has been completed and the components are being delivered. This design incorporates ground shields to reduce the charged volume and coupling between the magnetic and electric fields. New corona hardware for the electrical feedthrough was designed. The cable end was designed to eliminate oil migration along the cable, which had previously required bimonthly downtime for inspection purposes. In addition, the magnetic field coil was redesigned with double the number of turns to provide a better match with the power supplies used and was simplified into a two-part design. The new hardware is being designed for ease of service and replacement and for improved reliability. As a result of the test program, new hardware installed in two existing separators has improved performance so that the former 3-day conditioning period has been extended to every 2 to 3 weeks.

Assistance is being given in studies of superconducting rf cavities for beam separators. At present electroformed pure niobium experimental cavities, as well as identically sized, lead-plated, machined copper cavities for comparison purposes, are being obtained.

Service has continued to be given to the Supply and Materiel Division on various aspects of the acquisition and delivery of liquid helium and liquid hydrogen.

In the AGS Division, the new high-gradient accelerating column has been installed on the AGS Crockcroft-Walton set and is operating satisfactorily. The maximum operating voltage to date has been $770 \mathrm{kV}$, with a maximum output current of $150 \mathrm{~mA}$. Many of the mechanical aspects of this device were explained in last year's report. One of the features of the column is its ability to condition rapidly and remain conditioned for a long period of time, with no deterioration. This is due to the use of inorganic materials in the construction and the sealing of the epoxy between the ceramic and metal joints with an indium seal, which prevents outgassing of the epoxy and contamination of the inside of the column when under vacuum. The column is supported at one end by a frame adjustable in all planes for purposes of alignment. To reduce the stresses in the ceramic-to-metal joints, a 3/4-in. Dacron rope is used to support the high voltage end. The rope is enclosed in a 4-in. Pyrex glass tube to prevent voltage breakdown.

The beam transport system between the Cockcroft-Walton set and the linear accelerator (linac) was rearranged. The primary purpose of the new line-up was to improve trapping of the preinjected beam in the linac by moving the buncher farther downstream. A pair of $2000-$ liter/sec mercury diffusion pumps has been installed on the accelerating column and low energy beam transport line. These pumps are primarily used as backup for the evapor-ion pumps.

More rf power has been installed at the linac to accelerate the higher intensity proton beam from the preinjector. Revision of the rf system involved the coupling of power to the linac cavity at three points instead of one. In formulating the design of the multiport system it was planned that the linac coupler loops and the FTH-515 power amplifier couplers be made variable. Coupling to the linac cavity may be varied by manually rotating the loop about its axis, and the power output of the FTH-515 amplifiers can be varied by regulating the depth of penetration of a capacitor plate. To further facilitate tuning of the septum, each FTH515 amplifier powering a loop is mounted on a carriage to allow it to be moved. In order to do this the 3-in. and 8-in. transmission lines connecting these tubes to the linac tanks were fabricated with telescoping sections.

These linac improvements have led in part to the new record beam intensity of $>3 \times 10^{12}$ protons/pulse attained early this year.

Improved versions of the fast external beam septum and ejector magnet carriage and ram mechanisms have been installed in the AGS. Organic materials have been eliminated, where possible, to make the units less susceptible to radiation damage. The components have been arranged to allow for their rapid interchange in case of failure. One of the major improvements leading to a better vacuum in the AGS is the introduction of dynamic bellows vacuum seals to replace the conventional V-type Viton seals.

The slow external beam facility installed last year has been extended and is developing into a major AGS facility. At present, seven television stations distributed along the slow beam array monitor fluorescent screens used to set up the beam. To keep the beam centered on the external target, two vertical insulated aluminum plates 
with a 0.005-in. gap between them are used. When struck by a proton beam, these plates emit a signal that is proportional to the number of protons traversing the plates. This in turn is used to generate a steering signal to keep the beam centered on the target.

Long, thin, insulated aluminum plates perpendicular to the beam are mounted on linear actuators and are used to measure the beam's horizontal and vertical profiles. To obtain a beam profile, an actuator-driven emittance device, consisting of 12 insulated plates stacked alternately between 13 bias shield plates, is placed in the beam's path. The resulting signal can be fed to the PDP-8 computer to give an almost instantaneous beam profile.

A higher gap and longer duty cycle thin septum magnet for postconversion slow extraction is being built. This magnet incorporates a new design to edge-cool the thin septum, which will produce a magnet with less leakage field than the present design. A model under test to check its reliability has attained $3 \times 10^{6}$ cycles.

At the major internal targel location in the AGS, small-cross-section beryllium oxide targets have been successfully used. This target material has a life $\approx 4$ times as long as that of the beryllium wire targets formerly used.

The five conversion vacuum chambers installed in the AGS this year are constructed of Inconel-X and have a flame-sprayed aluminum oxide coating on the external surface and a ceramic coating on the flange face. Both coatings provide electrical insulation. Metal C-ring seals were used in the vacuum joints. By wrapping 0.005 -in. indium foil over previously used C-rings, a recent innovation, the C-rings can be reused and a substantial saving effected. More than $250 \mathrm{C}$-rings are now in use on the AGS and the slow external beam lines.

A prototype elliptical quadrupole magnet for low-field correction that clamps to the main-ring vacuum chambers and is self-centered has been designed and fabricated. This magnet fits over the upstream bellows between the main magnet end windings. The coil, which consists of No. 14 triple Formvar-coated copper wire laced through three epoxy Fiberglas boards that properly space the wires and hold them parallel, is potted in epoxy resin. Since the coil is vertically split to facilitate installation in the ring, the current is conducted through the clamping rods, which eliminates external wiring. This magnet is now undergoing electrical tests.
A new, nonrammed, fast extraction scheme calls for the development of a new full-aperture fast kicker and a $1 / 16$-in.-thick septum ejector magnet to extract the high energy beam from the AGS. Currently under design is a one-quarter-length model of the new fast kicker. This model will represent all the equipment required for full-scale operation. A $6 \% 3 \times 10 \times 15$-in. ferrite magnet has been machined and assembled with use of a nonorganic cement. The magnet was fired at $300^{\circ} \mathrm{F}$ to assist curing, and mechanical tests have shown that this cement will withstand normal abuse. High-voltage cable connections and terminations were also developed. These assemblies are currently in use in the power supply tank operating at $100 \mathrm{kV}$.

The design of the high current density, shortpulse, thin septum-ejector magnet for this fast extraction scheme has been completed. It is the first magnet assembly designed to be free of all organic materials. In addition, the entire assembly, including the vacuum enclosure and position mechanism, has been modularized so that it can be replaced within minutes.

In the AGS Conversion Division, work has continued on the design, development, and fabrication of the new $200-\mathrm{MeV}$ injector for the AGS. The design is $90 \%$ completed, and the fabrication and procurement of machine components is well advanced. Assembly and installation of these components will begin in Aug. 1969.

The design of the accelerator tanks was completed in mid-1968, and an order for their fabrication was placed with the Youngstown Welding and Engineering Corp. The tanks consist of 26 tank sections, $3 \mathrm{ft}$ in diameter and $20 \mathrm{ft}$ long, made of copper-clad steel, rolled, welded, and machined accurately. Fabrication is progressing well, and Division personnel are engaged in the follow-up work of inspection and vacuum leak checking.

The drift-tube program, one of the largest commitments of this project, is well under way. Design and development of various components for this program were carried out in 1968. An electron beam welder was installed and successfully operated for the final closure of end covers on the drift tubes. The hydrogen brazing techniques for the difficult joints involved were well established. The production of complete drift tubes is now in progress. Each of the 280 drift tubes required for the machine is dimensionally different and consists of a water-cooled, vacuum-tight, copper body con- 
taining a quadrupole magnet supported by stems providing passages for water and power. These drift tubes will be installed in the accelerator tank and positioned to an accuracy of within $0.003 \mathrm{in}$.

Design and procurement of auxiliary components for the system are also nearly complete. Among the major items involved are the focusing quadrupole magnets contained in the drift tubes, which were developed at the Laboratory and contracted to industrial firms. Procurement is progressing satisfactorily, and a program is under way to test all the magnets as they are delivered.

The accelerator is powered by $5-\mathrm{MW}$ pulses fed through 12-in. coaxial transmission lines. Since these are not available commercially, a design program was carried out in 1968 to develop properly matched $50-\Omega$ coaxial components. Particular attention was paid to fabrication techniques to minimize the manufacturing costs. More than $3000 \mathrm{ft}$ of line are needed, and a special order of 6 and 12-in.-diam aluminum tubing was obtained. The packaging of heavy electronics making up the rf systems was also completed. This involves the design and procurement of cabinets and watercooling and air-handling equipment.

In the AGS Ring Conversion Group, prototype vacuum chambers were received late in the year. Two of these have been installed in the ring, with the balance to follow soon. The order will be completed this summer.

The Siemens power supply was completed in Dec. 1968, factory tests were started in Jan. 1969, and delivery was made late in the spring. Bids for erection and alignment of the motor-generator set were solicited, and an award was let to R. Doughty and Sons of Hoboken, N. J.

A decision was made to use 50 -cm-o.d. ferrite rings and 10 cavities for beam acceleration in order to avoid the unstable region observed in all sample ferrite rings furnished. Bids for the total ferrite order were requested during the last quarter of the year. With the decision to use 50-cm-o.d. rings the new cavity design was completed, and the order for the ferrite cooling plates was placed.

Prototype distributed-current-sheet, low-fieldcorrection coils were built and are undergoing magnetic measurements. The new bus bar design was completed late in the year, and bids are being solicited.

Members of the Division assigned to the Physics Department support all areas of that Department's effort.
The horizontally shafted rotor of the slow neutron chopper (HFBR beam hole H-9) has been operated with one set of bearings for $>2000 \mathrm{hr}$ at speeds between 12,000 and 12,500 rpm. Samples of oil were checked periodically for contamination and deterioration. Based on bearing performance during this period of high-speed operation, the replacement interval is expected to be $10,000 \mathrm{hr}$, or $\approx 1 \mathrm{yr}$ of operation at the HFBR.

A multiple detector system being designed for the slow neutron chopper will provide for setting up arrays of stationary detectors at different radial and angular locations. The present safety and radiation shield is being modified to accommodate the new detector system and provide more convenient access to the sample axis.

A bearing and motor development program for the fast neutron chopper (HFBR beam hole $\mathrm{H}-2$ ) is in the testing phase and is scheduled to be installed early in fiscal 1970. A unique scheme is being used to circulate the oil for the main rotor support bearings. The lubricating oil is contained in a sump below the vertically oriented bearings, and the level is adjusted to submerge the lower bearing of a tandem pair. The pumping action of the bearings forces the oil upward through the bearings to a slinger and then down through drain tubes to the sump, which also contains a heat exchanger. In this arrangement reliability is greatly enhanced by elimination of external pressure and scavenger pumps. This scheme has been tested at speeds of up to $15,000 \mathrm{rpm}$, with excellent results.

The capture gamma spectrometer (HFBR beam hole $\mathrm{H}-3$ ) continues to perform well, with some design activity centering about external collimation for the " $B$ " beam.

Installation was completed of some major accessories for the polarization spectrometer (HFBR beam hole $\mathrm{H}-1$ ). A beam rotator was designed which has its outer end mounted on the main arm and its inner end mounted on a portion of the crystal cavity shielding. This device is a tube surrounding the beam with provisions for evacuation, for maintaining the magnetic field originating in the crystal cavity, and for future coupling to downbeam collimators. Its principal function is to permit a choice of either a maintained or a reversed orientation of the neutron spin axes. This is accomplished by providing two magnet windings, one with the wires parallel to the beam path and the other with wires laid in helices, the axis being coincident with the beam center line. 
The problem of moving the rotator through the spectrometer working arc without loss of shielding effectiveness was solved by providing a stainless steel and borated paraffin structure comprising two eccentric rolling cones, disposed to move a bore in the smaller of them along an approximation to a straight-line path in all cross sections perpendicular to the beam. The working clearance and departure from true straight-line travel are accommodated by stepping the bore and the outside of the rotator.

This cone device was installed at the same time as the rotator and was provided with a temporary drive system requiring manual operation from the main instrument console. A permanent system with automatic servo-control has now been installed.

Other work in connection with the $\mathrm{H}-1$ port included design, construction, and installation of a drive control for automatic positioning of the moving balcony, fabrication and installation of permanent control devices to replace the "breadboard" drum and filter positioners initially used, and continuing design of apparatus needed for achieving the ultimate capability of the $\mathrm{H}-1$ polarization spectrometer.

A large number of experimental accessories in support of the solid-state spectrometer research program (beam holes $\mathrm{H}-4$ through $\mathrm{H}-8$ ) were designed, fabricated, and put into operation. Among them were a Dewar stand, variable collimators, beam shutters, general-purpose collimators, an encoder test fixture, goniometers, and three new cantilever arm assemblies. Other accessories are in various stages of design.

Installation and checkout of a cable-operated irradiation device was completed at HFBR reflector thimble $\mathrm{V}-12$. With this device a researcher can automatically load up to eight sample capsules at a time, unload them automatically after irradiation into a transport cask, and, using a guillotine device, remove the irradiated material from each capsule.

Design work has been started on a tandem goniometer to be used for precision $\gamma$-ray angular distribution measurements at the new Tandem Van de Graaff Facility. The device will provide variable angular and radial positions on two 6-ft arms pivoting about a central target and will have facilities for remote control drives and angle readouts.
At the 3-MeV Dynamitron, design and fabrication have been concentrated on beam transport components, including remotely controlled beam gates, electromechanical beam profile scanners, and evacuated beam pipes.

The members of the Mechanical Engineering Division assigned to the Tandem Van de Graaff project maintained liaison with the architectengineers during the final phases of the building construction and conducted operational tests of the insulating gas system. The major components of beam transport hardware have been designed and either have been fabricated or are in the shop. One neutron beam plug has been installed at the 60 -in. cyclotron and is now in operation.

A liquid nitrogen distribution line to fill the cold traps of the mercury diffusion pumps on the accelerators was designed and ordered and is being installed.

Design work is continuing on the terminal ion source system. The ion source, inflector, and vacuum system have been tested on the wooden model of the terminal structure. The control-rod drive assemblies have been designed and partially fabricated. Enclosures for the electronics in the terminal have been designed to minimize the damage due to sparking. A high-pressure housing for a terminal ion pump is being designed.

Work has been continued on the design of a cold neutron moderator for HFBR beam hole H-9. A preliminary safety analysis report has been prepared and submitted to both the Reactor Safety and the Cryogenic Safety Committees. The report examines the various safeguards introduced in the design and assesses the potential hazards to the reactor and personnel of introducing liquid hydrogen in the HFBR.

Work is being done for the Nuclear Interactions Group on a prototype measuring machine intended to handle bubble-chamber film data. Unique features of the machine are the "sickle" method of particle track recognition, the combination air-vacuum pad arrangement used for support, and $x-y$ motion guidance of the stage.

In the sickle method a trace of a nuclear event is scanned in one step, which avoids the digitizing procedure of many points required on present equipment. The projected trace line on the film is rotated at high speed around its apex by use of a rotating dovetail prism, behind which an opaque disk with transparent lines on it is rotated at lower speed. At a certain instant the trace line on the 
film, the dovetail prism location, and a particular transparent line on the opaque rotating disk will line up and allow light to hit a photomultiplier tube. This instant is recorded for relative location of all elements and fed into a computer programmed to recognize the trace.

The use of this optical system reduces the amount of manipulation by the operator required to locate the apex of the trace line on the film. This is done by moving the film so as to bring the apex of the trace into the center line of the optics. The film is also projected onto the working table and enlarged.

The stage must be very light, to reduce inertia, and frictionless. A totally air-suspended stage was designed and a prototype mock-up tested. A thinfilm pressure air pad will lift a certain weight and hold the air gap constant as long as the volume of air supplied is constant. If vacuum pads are added adjacent to or concentric with the pressure pad, their faces being on the same plane, an adherent air film is created. This film can be applied in any direction, i.e., horizontally, vertically, upside down, or at any angle, as long as the vacuum forces are greater than the weight of the components.

The magnetic-finger device that focuses both $\pi$ and $K$ mesons to provide a high-intensity $\nu$ beam was subjected to life tests. A number of mechanical failures occurred, and design modifications to the fingers were made. The critical section of the first inner finger was strengthened, and the inner first and second fingers were redesigned to eliminate longitudinal welds. A new quick-removable front and a ferrule type of joint were designed and fabricated. The electrical contacts on the third fingers were improved. Water leaks were repaired, and controls for cooling water and water distribution piping were installed. Gas pressurization and leak detection controls for the first and second fingers were installed, including safety interlocks for minute leaks which might indicate fracture in the fingers.

Promising results were obtained, and the magnetic fingers are expected to withstand mechanical stresses during the neutrino experiment and possibly be of use in other experiments as an efficient particle concentrating device.

Much of the General Physics Group's effort was directed toward design and fabrication of equipment for an experiment to test for $C P$ invariance in $K$-meson decays. This experiment, the largest worked on by the group, involved a Cerenkov counter, 13 optical spark chambers, and 7 doublegap wire spark chambers ranging up to $56 \times 74$ in. in sensitive area. In addition, there were numerous hodoscope arrays using hundreds of scintillator light-pipe assemblies. Of particular interest were the optical mirrors and lenses, wire chambers, and special magnetic measurement instruments used in this experiment. The optical mirrors ranged in size from $20 \times 30$ in. to $74 \times 32$ in. and were used in a $90^{\circ}$ stereo optical system.

Because of severe space limitations, it was necessary to fold the optic path up toward the ceiling of the AGS Building and to position the four largest mirrors 15 to $20 \mathrm{ft}$ above the floor. To maintain the optical flatness of reflecting surfaces and keep the weight of the mirror assemblies to a minimum, a $1 / 4$-in.-thick front surface mirror was bonded to one side of a 4-in.-thick slab of aluminum honeycomb, and a $1 / 4$-in.-thick piece of plate glass was bonded to the other. This laminated assembly was then mounted on resilient pads in an aluminum frame. The largest of these assemblies weighs 210 lb, compared with $600 \mathrm{lb}$ for a conventional mirror, and can be mounted with no loss of rigidity or quality. The field lenses used in conjunction with the mirrors were acrylic, plano-convex lenses $6 \mathrm{ft}$ in diameter, with a 40 -ft focal length. They were fabricated by the Central Shops on numerically controlled machines using computer generated tape.

A magnetic measurement device originally fabricated for the University of Rochester was modified for use in an expanded-gap 48D48 magnet, with a resultant field plot that is accurate to within $0.2 \%$ in the main field.

A good deal of engineering effort was applied in the structural analysis of elements of this experiment to ensure the safety of personnel on the AGS floor.

In the Bubble Chamber Group of the Physics Department, two research and development programs have been carried on in the 30 and 31-in. bubble chambers. The 30-in. chamber was used, in cooperation with the Accelerator Department, as a cryostat. Tests involved putting a magnet constructed of aluminum conductor in the chamber and surrounding it with liquid hydrogen as a coolant and then powering the coils. These tests are intended to lead to the development of cryogenic magnets for very high energy accelerators.

Both bubble chambers have been used in the development of an "internal, track-sensitive target." This device will allow a target containing a 
fluid different from that in the main body of the chamber to be placed in the chamber. The target fluid will be expanded, through a flexible diaphragm, by the chamber fluid and thus become sensitive to radiation. The objective is to reduce the amount of material, such as deuterium, needed for very large chambers. The target will be a pure substance, to allow simpler analysis of the primary interaction, while the main body of the fluid surrounding the target can have a shorter radiation length for analysis of the products of the event. The aim of this research is to develop a body for the target that can withstand the slight differential pressure and still be optically acceptable for measurements of the primary event, and a flexible membrane that will withstand many flexures, without breaking or leaking, at liquid hydrogen temperature.

The major portion of the Group's effort has been directed toward the final design and assembly of the 7-ft test facility. Full-scale tests of both the chamber and magnet are under way.

Studies of an even larger chamber have been under way throughout the year. Conceptual design leading to a proposal submitted jointly by BNL and the National Accelerator Laboratory (NAL) for a 25- $\mathrm{ft}$ chamber to be erected at NAL has resulted. The information gained in the development of the 7-ft bubble chamber was used to produce a design incorporating a large visible volume and vessels of spherical shape, with a consequent reduction in cost. The most unusual feature is the conductor and cooling for the superconducting magnet. This conductor is to be copper-stabilized superconductor with a square cross section and a cooling hole in the middle. Instead of submerging the conductor in a bath of liquid helium, subcooled helium gas will be circulated, at a pressure of 10 to $30 \mathrm{~atm}$, through the cooling hole. This system eliminates the necessity for a coil containment vessel and may provide more positive cooling. A program has been instituted to obtain and test samples of this type of conductor and develop the necessary pumping equipment for supercritical helium. Work on the Title I design for this chamber is expected to begin soon.

Division personnel assigned to the Department of Applied Science have been involved in many diverse areas.

Members of the Engineering Group have continued cooperative efforts with the Laboratory's Plant Engineering and Planning Division in the fields of water conservation and reactor improvement.

In the water conservation area, the HFBR chilled water system for air conditioning was converted from a once-through to a closed-loop system. When completed next fiscal year, this change will result in a net saving of 600 to $700 \mathrm{gal} / \mathrm{min}$ of well water.

The water system in the Medical Department was modified for more efficient water usage. Since the secondary cooling system of the Medical Research Reactor (MRR) now re-uses water rejected from the Department's air conditioning system, both units can now be operated simultaneously at full capacity.

The Engineering Group has become increasingly active in a BNL research group working on air pollution. This group is developing equipment for injecting $\mathrm{SF}_{6}$ tracer gas into power-plant flue gases and, in conjunction with the Meteorology Group, is devising airborne sampling equipment for the tracer. Equipment for quantitatively sampling $\mathrm{SO}_{2}$ and $\mathrm{SO}_{3}$ in power-plant flue gases is being developed in cooperation with the Analytical Chemistry Group.

A portable ozone generator has been designed for use with the "Camp System" of air monitoring pollution control devices employed by the U.S. Department of Health, Education and Welfare. A $500-\mathrm{mCi} \mathrm{Sr}{ }^{90}$ source is used to convert oxygen fed from a standard cylinder, and the flow rate may be varied from 600 to $15,000 \mathrm{cc} / \mathrm{min}$ by means of critical orifices.

A test cell for insertion into the core of the fast source assembly was completed. This cell consisted of a number of 1 -in. ${ }^{2}$ stainless steel, graphite, and $U^{235}$ plates. The plates are stacked in a cell 3 in. $^{2}$ by 10 in. long. Considerable radioactive machining work was necessary to modify the adjacent graphite and fuel to accept the test cell.

An experiment involving continuous production of delayed neutrons is currently in the design stage. It consists of a continuous uranium-bearing steel tape, $0.75 \mathrm{in}$. wide, $0.030 \mathrm{in}$. thick, and $100 \mathrm{ft}$ long, moving past the reactor core at speeds of 0.003 to $200 \mathrm{ft} / \mathrm{sec}$. The speed must be controlled to within $\pm 1 \%$. Both tape steels and welding techniques borrowed from bandsaw technology proved to be most satisfactory.

An experiment was designed to measure the neutron capture to fission ratio in $\mathrm{Pu}^{239}$. A capsule with a boron carbide-aluminum shield will be in- 
serted into the HFBR core through thimble V-15. A test capsule for evaluation of the shield material is also being built.

In support of the Pulsed Fast Reactor Fuel Development Program, a commercial fatigue-testing machine was modified to expand its environmental range. Candidate fuels may now be tested in sodium at $1000^{\circ} \mathrm{F}$ or steam at 600 psig and $500^{\circ} \mathrm{F}$.

The enhanced strength and corrosion properties of plastic-impregnated concrete prompted an investigation of the possible uses of this new material. Detailed evaluations were made of several applications, including a prestressed concrete reactor vessel, a prestressed concrete undersea habitat, and prestressed or reinforced concrete beams, columns, and pipes. Computer techniques were utilized in the design of these complex structures. A prestressed concrete desalinization vessel is now under evaluation.

A BNL radioactive waste disposal cask was redesigned to meet the new Federal requirements. A $30-\mathrm{ft}$ free fall to a hard surface and a drop onto a 6-in.-diam steel rod were among the tests for the container. The new unit has successfully qualified and is now in routine use.

The decision of the U.S. Department of Transportation to tighten specifications for the transport of radioactive materials has made obsolete practically every shipping cask and portable irradiator in the country. BNL is modifying units designed and built here and several others now being used in AEC research.

The shipboard irradiators were the first to be modified. There are three units in existence today. One was sent to Iceland on loan. The same crash frame was used to ship another to the University of Nebraska. A third is now on loan to Israel, and the same crash frame will be used to return the unit to the United States.

A portable cesium irradiator, designed by the Vitro Engineering Co. and built by the American Nuclear Corp., is a trailer-mounted unit that has traveled the country as a demonstration irradiator. It is now at BNL being outfitted with a new trailer, crash frame, and other modifications.

The Brookhaven portable cesium development irradiator has also been modified. One of these units has been shipped to Argentina, and a second is awaiting shipment to India. A third will be modified and shipped to Chile early next year. The cask containing the cesium source is shipped in its own crash frame.
Design work has been started on the modification of another trailer-mounted unit, the mobile gamma irradiator, currently located at the University of California at Davis. Another project under way is a crash frame for the cask of the bulk grain irradiator located in Savannah, Georgia. Both units were designed by Vitro and built by the Lockheed-Georgia Co. These casks have no lifting lugs on them and must be modified in the field, since they cannot be moved from their present locations.

An irradiator with a $4000-\mathrm{Ci} \mathrm{Cs}^{137}$ source for extracorporeal treatment of animal fluids has been built. An important feature of its design is that fresh sections of plastic tubing are continuously exposed to the radiation, which eliminates shutdowns for retubing, with the attendant danger of infection of the animal.

A remotely controlled electrically driven robot was designed and built to aid in the recovery of a $\mathrm{Co}^{60}$ irradiation source. The source could not be returned to its safe position remotely, and direct action within the cell was required. The robot, with attached TV camera, was maneuvered through the entrance labyrinth and up to the source, and the necessary remedial action was taken. Total lost time on the use of the facility 10 days.

Mechanical Engineering activities at the Department of Chemistry were focused on the detail design of AESOP (automatic equi-inclination spectrometer operating in parallel). A monochromatized x-ray beam impinges on a crystal mounted in a Euler cradle, and the reflections from each reciprocal lattice layer are collected rapidly by 127 discrete $\mathrm{SiO}_{2}$ diffused junction detectors arrayed in a $180^{\circ}$ arc of $14-\mathrm{cm}$ radius. The detectors are mounted in a vacuum housing and maintained at liquid nitrogen temperatures.

The extensive and precise motions of the detector housing the cradle have challenged AESOP's designers. The housing rotates $90^{\circ}$ on one axis, $45^{\circ}$ on another (to accuracies of within $0.01^{\circ}$ ), and translates $5.5 \mathrm{in}$. (reproducible to $\approx 0.001 \mathrm{in}$.). The cradle rotates $360^{\circ}$ and $45^{\circ}$ on separate axes with the resulting crystal runout of $\approx 0.001$ in. Some motions are computer controlled; others are controlled by hand.

Engineering assistance was given for a preliminary design of an automatic sample changer and counter for neutron-activated samples and for a compact cryostat to be mounted upon a Picker goniostat for use in $\mathrm{x}$-ray analysis of crys- 
tals at helium temperatures. Continuing assistance was given in the improvement of existing equipment, such as redesign of the vacuum system and power transistor heat sink for the isotope separator and a sample holder for use in the cyclotron. Miscellaneous devices were designed for the molecular beam apparatus and for use in experiments on returned lunar samples.

\section{MACHINE SHOPS}

The Central Shops Division operates 8 shops and 3 inspection and quality control stations located in 6 Laboratory buildings to provide on-site fabrication of machined, welded, and formed metal parts for the construction, modification, repair, and servicing of scientific and Laboratory equipment. The Division also arranges for off-site services for which no facilities are available and for vendor fabrication as required. The acquisition of Laboratory machine tools and the selection, training, and placing of all machinists, welders, and sheet metal mechanics are also functions of the Central Shops.

To administer, supervise, and provide these diversified services, a staff of technical personnel with varied backgrounds in metal working and related areas and well acquainted with BNL's scientific programs is at the disposal of all Laboratory departments. During fiscal 1969 the Division's personnel complement reached 133 employees, with an additional 41 assigned to staff area shops.

Employee training has become a continuing program at both the supervisory and operating levels. To insure familiarity with new technologies and their effective implementation in metals fabrication and inspection and quality control, seminars and work shops were attended this past year covering such topics as the metallurgical properties of titanium, IBM auto-spot adapt numerical control, ultrasonic testing, and radiographic and nondestructive testing. Shops supervisors also attended seminars on management theories, training, labor relations, and communications. On-the-job training produced additional operators for the electrical discharge, numerically controlled, and plasma-arc cutting machines.

Shops services provided to the various Laboratory departments during fiscal 1969 varied as in past years; many jobs were routine and repetitious and many others were complex and exacting, requiring unique fabrication techniques. During the
Table 9

Contributions to Central Shops Workload (\% man-hours)

\begin{tabular}{lrrr}
\hline & \multicolumn{3}{c}{ Fiscal year } \\
\cline { 2 - 4 } & 1969 & 1968 & 1967 \\
\hline Accelerators & 38.8 & 26.3 & 25.3 \\
Applied Science & 12.6 & 18.1 & 21.8 \\
Biology & 2.2 & 1.7 & 1.6 \\
Chemistry & 3.4 & 3.5 & 3.9 \\
Instrumentation and Health Physics & 0.7 & 0.8 & 0.5 \\
Medical & 2.9 & 2.2 & 2.5 \\
Physics & 33.0 & 39.8 & 36.4 \\
Reactors & 3.3 & 5.4 & 5.3 \\
Miscellaneous & 2.8 & 1.5 & 2.2 \\
Outside contracts & 0.3 & 0.7 & 0.5 \\
\hline
\end{tabular}

year $157,272^{1 / 2}$ productive man-hours were supplied. Table 9 shows the distribution of this time to the various departments and a comparison with fiscal 1968 and 1967.

While most of the Division's efforts were chargeable to regular shop work orders, distributed shop time also included (1) short-order work - shortrun jobs of $15 \mathrm{hr}$ or less, generally of an emergency nature; (2) machine repair and maintenance work - repair of Laboratory machine tools, generators, pumps, and many other large pieces of machinery, and routine lubrication and preventive maintenance of this equipment by machine maintenance mechanics; and (3) the services of on-loan employees - skilled mechanics working under the supervision of requesting department personnel on particular jobs that could not be handled feasibly within the shops.

The contracting of fabrication work to vendors is of major importance to the Division. Central Shops personnel are actively involved in the surveying and evaluation of vendor facilities. To control Central and Staff Shops backlog and meet delivery dates, the Vendor Fabrication Section arranges for some jobs to be either wholly or partially fabricated off-site and administers the Laboratory's open contracts for short-order machining, plating, anodizing, and heat-treating. This accounted for the purchase of $\$ 180,360$ worth of parts and services involving 58 different vendors during the fiscal year.

Complete mechanical inspection of all parts procured by or fabricated within the Central Shops, nondestructive testing, and periodic calibration of 
Laboratory precision measuring tools is routine for the Division's eight-man Inspection and Quality Control Section. During this past year a third inspection station was completed within the machine shops complex to provide mass spectrometer leak detection and hydrostatic pressure testing, as well as increased in-process inspection of fabricated parts. Assistance to the scientific departments in providing in-process or acceptance inspection of more than 2000 pieces of purchased research equipment during fiscal 1969 required that Division inspectors visit out-of-state as well as local vendor plants.

The nature of research at BNL requires unique scientific pieces of instrumentation and associated hardware not commercially available. To aid in the design and modification of this equipment a Central Shops capabilities list was compiled and distributed in the Mechanical Engineering Division during the year.

Building modifications continued during 1969. The elimination of material handling equipment on the main shop floor of the Heavy Machine Shop was accomplished with the installation of a traveling overhead crane (see Figure 4). Through the efforts of the Plant Engineering and Planning Divison, all overhead services were removed and placed under the shop floor or rerouted to permit crane coverage of all the large machine tools, as shown in the photograph.

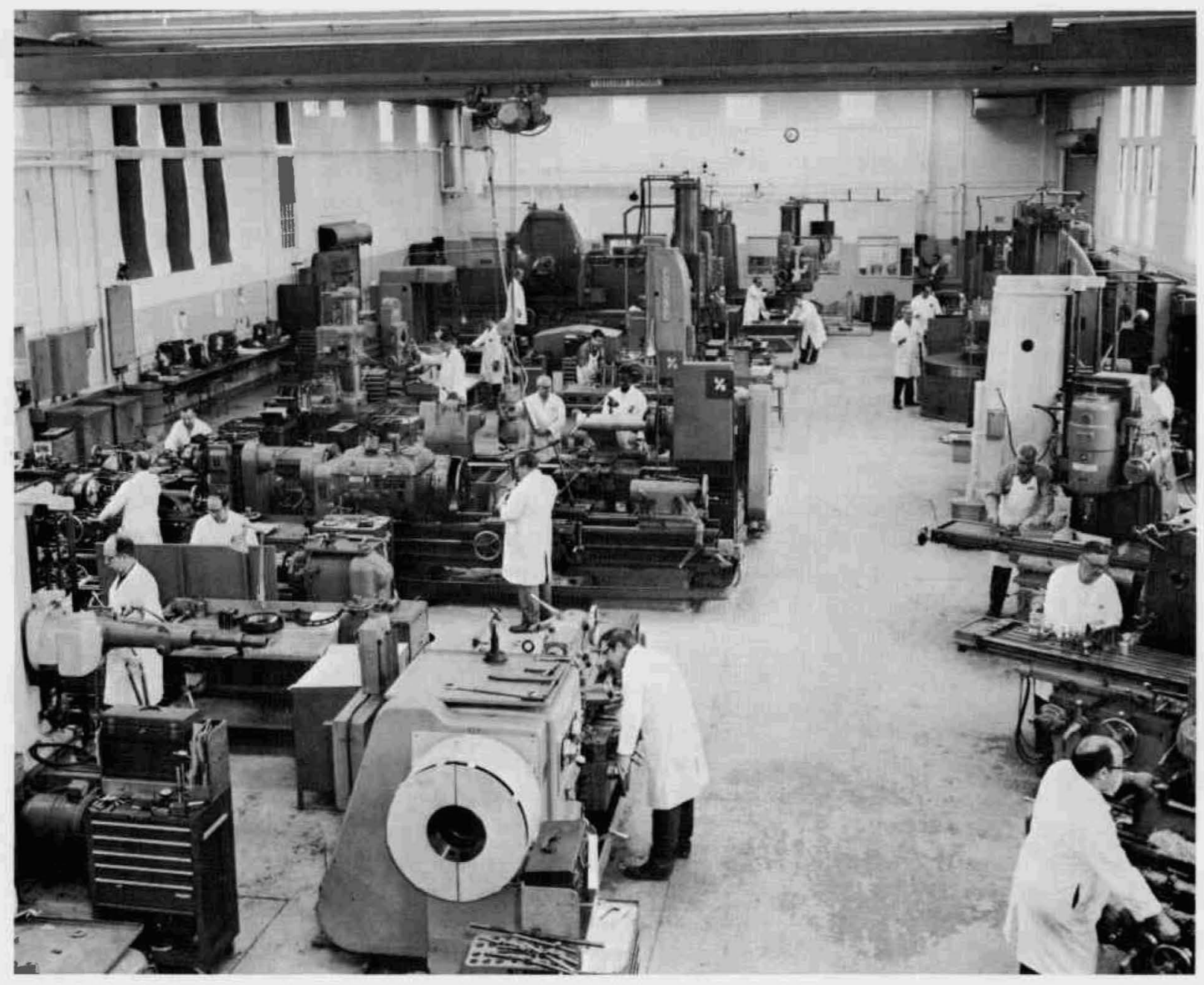

Figure 4. A section of the shop floor of the Central Shops Division's Heavy Machine Shop. Horizontal and vertical boring mills, radial drill presses, and several of the large swing lathes can be seen. All machine tools are now serviced by the recently installed overhead traveling crane. 


\section{TECHNICAL INFORMATION}

The responsibilities of the Information Division include the operation of the Research and Classified Libraries, the editing, processing, and distribution of Laboratory reports, patent review, and some of the aspects of public information and education. These activities are discussed below.

The construction and renovation program at the Research Library is now nearly completed. Relocation of members of the staff and the installation of sound-absorbent materials on the ceiling and floor have produced a high degree of noise reduction and improved working conditions.

Particular attention has been given to the Serials and Circulation Section. Here an attractive unit in walnut and black has been built and installed by the Laboratory's carpenter shop. Shelving for the display of current periodicals is being constructed; when this has been installed, together with steel shelving for the reference and abstract journal collection, the entire collection of bound journals will be moved for the final time.

Despite the overcrowded stacks, cramped working areas, and loss of reading space during the construction program, circulation records have been about normal. Records from the Reference Section show a decided increase in service via telephone, whereas usage of reference material in the Library dropped considerably.

In a review of the Library's lists of journal subscriptions and annual monographs, infrequently used back journal sets were examined and several titles were removed from the shelves.

There has been a $10 \%$ increase in the Laboratory's requirements for translation services. In addition to this work, the Library's translator has served at international conferences and as a member of the escorting party for foreign scientists visiting the Laboratory.

During the last few months the Long Island Library Council Information Network was inaugurated. This program, administered by the Council, provides a fast loan or permanent copy service to six Nassau and Suffolk County academic and special libraries, namely, those at Hofstra University, Adelphi University, the State University of New York at Stony Brook, C.W. Post College, Grumman Aircraft Engineering Corp., and Brookhaven. Sources upon which each of these libraries can draw, in addition to those within the above group, include the New York Public Library, the
Engineering Societies Library, the New York Academy of Medicine Library, and the Medical Research Library of Brooklyn. Over half of Brookhaven's interlibrary loan requirements are filled by the Council, with material being received within three days of filing a request.

As an index of the Laboratory's steadily decreasing involvement in classified information, it has been possible to reduce the staff of the Classified Library from three to two. Nevertheless, some unclassified research still requires reference to information contained in classified documents, and a library of material of possible interest must continue to be maintained. During the year, 356 classified reports were received. More than 40 reports have been declassified and about 4800 copies have been destroyed. As of December 31, the total holdings consisted of $\approx 11,600$ reports, of which 3234 are secret $\mathrm{AEC}$ research and development reports and 286 are secret Department of Defense reports. The Classified Library is no longer receiving reports in micronegative form on standard distribution. During the year, 91 pieces of correspondence were received, and 538 copies were destroyed. Thus, the correspondence holdings have been reduced to $\approx 3200$.

This year a total of 1177 scientific and technical manuscripts resulting from BNL-supported research were processed by the Information Division for publication in the open literature. This compares with a total of 1109 in the previous year. The activities of the Editorial Section in processing formal Laboratory reports remained at about the same level as in previous years.

The film library, a comprehensive collection of $16-\mathrm{mm}$ motion pictures covering many aspects of nuclear energy at both popular and professional levels, was very active, particularly during the winter months. Films are lent, free of charge, to schools, civic groups, and research institutions on Long Island. During the year, 367 such loans were arranged.

The BNL Patent Office is responsible for the review and clearance for patent matters of all scientific and technical manuscripts covering the Laboratory's research. The number of inventions reported by this office during fiscal 1969 dropped for the second year, probably because of the decrease in the number of scientific manuscripts processed and in research of an applied nature. The number of patent applications was $\approx 22 \%$ greater than in 1968 , but still within the range of 
Table 10

\begin{tabular}{lrrr}
\hline & \multicolumn{3}{c}{ Fiscal year } \\
\cline { 2 - 4 } & 1969 & 1968 & 1967 \\
\hline Patent clearances & 1208 & 1218 & 1367 \\
Records of invention & 16 & 38 & 54 \\
Patent applications & 22 & 18 & 22 \\
\hline
\end{tabular}

the long-term average. A summary of the activities of the Patent Office during fiscal 1969 and the two previous years is given in Table 10.

\section{INTERNATIONAL COOPERATION}

The spirit of international cooperation in science is greatly fostered by the many arrangements whereby foreign scientists visit and come to work at Brookhaven and Laboratory scientists attend overseas meetings and conferences. In addition, the Laboratory has been involved in several formal programs of scientific cooperation. Those currently active include arrangements with the Italian Laboratorio di Fisica Nucleare Applicata del Centro di Studi Nucleari della Casaccia, the Greek Nuclear Research Center, and the Puerto Rico Nuclear Center.

The first of these programs, arranged as an appropriate activity under the U.S.-Italian Agreement of June 19, 1967, for scientific cooperation, became formally effective only in March 1969, and activities thus far have been concerned with the development of plans for specific research projects. The general nature of proposed work will be the study of the structure and dynamics of magnetic spin systems, with neutron scattering employed as the principal experimental tool and nuclear magnetic resonance used in complementary studies. It is expected that preparation of specimens and their study by nuclear magnetic resonance and neutron diffraction will be carried out at Casaccia. Neutron inelastic scattering studies on the same specimens will be done at the HFBR at Brookhaven. An exchange of selected scientists from the two laboratories is anticipated during the three-year term of the cooperative program.

Under the auspices of the Atomic Energy Commission's Division of International Affairs, a cooperative program between BNL and the Greek Nu- clear Research Center (Demokritos) has been maintained since 1965. As part of this program, visits are arranged between staff scientists of both institutions for discussion of problems of mutual interest and exchange of information. BNL has supplied several items of equipment, on a loan basis, to Demokritos to expedite their research activities. The most recent loan is that of a special low energy radiation collimator to be used in radioscanning of body organs. Although foreign travel limitations have seriously curtailed the scope of the program in the past year, three visits by BNL scientists took place between July 1968 and June 1969 as part of the cooperative effort. Two of these visits involved the particle physics program at Demokritos, while one was devoted to a survey of the reactor-based research program.

For the past two years, a limited number of Greek graduate students who have been working at Demokritos have been given the opportunity of working at BNL during the summer. These students have participated in such diverse research projects as the structure of RNA, emission spectroscopy, neutron diffraction, and peptide synthesis. This experience has given the students a unique opportunity to broaden their research experience and has helped acquaint BNL staff members with the excellent levels of scholarship maintained by the Greek universities and at Demokritos.

Contact between Brookhaven and the Puerto Rico Nuclear Center is now maintained principally through joint or complementary research projects. The principal areas of common interest have continued to be neutron diffraction and organic semiconductors. Although now at a much reduced level from the early days of the cooperative program, an exchange of visiting scientists for short periods has continued. This exchange is still very useful in overcoming the problem of Puerto Rico's relative isolation and in contributing to the effectiveness of joint research efforts.

During February and March the Head of BNL's Information Division served as Director of the Technical Information Center at the Atoms in Action Exhibit at Manila, Republic of the Philippines. This exhibit, one of the US AEC's Demonstration Centers in foreign countries, is designed to stimulate public interest in the peaceful uses of atomic energy, as well as to assist scientists in the host country in the development of their own research programs. 


\section{Administration and Operations}

The management operations reviewed in this section have been designed to facilitate and encourage the research activities of the Laboratory's scientific staff.

\section{PERSONNEL}

\section{Scientific Staff and Students}

For the first time in a decade, the number of regular staff members at Brookhaven decreased during the 12 months ending May 31. Turnover remained at a significantly high level; 36 scientists were appointed to the staff, and 48 terminated their appointments. Comparative year-end statistics for 1969 and 1968 are shown in Table 1.

The number of salaried visitors at the Laboratory decreased to the lowest total since 1960 . This was due to a reduction in the number of postdoctoral Research Associates, which also reached a nine-year low. During the year 36 recent graduates began postdoctoral research investigations and 41 completed their terms. Of the latter, 10 were promoted to the regular staff.

In contrast to the decrease in the number of salaried appointments, the number of scientists and students holding nonsalaried appointments continued to increase. More than 750 individuals in this category participated intermittently for periods of up to several weeks at a time.

May 31, instead of June 30, was used in Table 1 to exclude from the statistics those who work at Brookhaven only during the summer months. The same date was used in Table 2 to include participants in only one summer program.

Table 1

Scientific Staff and Students on May 31, 1969 and 1968

\begin{tabular}{|c|c|c|c|c|c|c|}
\hline & & & \multicolumn{4}{|c|}{ Visitors } \\
\hline & \multicolumn{2}{|c|}{ Regular staff } & \multicolumn{2}{|c|}{ Salaried } & \multicolumn{2}{|c|}{ Nonsalaried } \\
\hline & 1969 & 1968 & 1969 & 1968 & 1969 & 1968 \\
\hline \multicolumn{7}{|l|}{ By appointment category } \\
\hline \multicolumn{7}{|l|}{ Staff } \\
\hline Senior Scientist & 88 & 85 & 1 & 2 & 76 & 65 \\
\hline Scientist & 189 & 182 & 7 & 2 & 160 & 143 \\
\hline Associate Scientist & 139 & 149 & 5 & 8 & 198 & 198 \\
\hline Assistant Scientist & 48 & 60 & 8 & 10 & 90 & 106 \\
\hline Senior Research Associate & - & - & 4 & - & - & - \\
\hline Research Associate & - & - & 67 & 72 & 93 & 76 \\
\hline \multicolumn{7}{|l|}{ Students } \\
\hline Junior Research Associate & - & 一 & 4 & 6 & 174 & 144 \\
\hline Research Assistant & - & - & 0 & 0 & 131 & 98 \\
\hline Total & 464 & 476 & 96 & 100 & $922^{a}$ & $830^{b}$ \\
\hline \multicolumn{7}{|l|}{ By academic degree } \\
\hline Ph.D. or M.D. & 333 & 347 & 89 & 90 & 584 & 554 \\
\hline Master & 57 & 59 & 3 & 6 & 122 & 104 \\
\hline Bachelor & 68 & 64 & 4 & 4 & 202 & 154 \\
\hline No degree & 6 & 6 & 0 & 0 & 14 & 18 \\
\hline
\end{tabular}

a72 of these appointees were at BNL on a full-time basis as of May 31, 1969.

b66 of these appointees were at BNL on a full-time basis as of May 31, 1968 . 
Table 2

Classification of Visiting Scientists and Students Participating in BNL Program for One Month or More, June 1, 1968 - May 31, 1969

\begin{tabular}{|c|c|c|c|c|c|c|c|c|}
\hline & \multicolumn{4}{|c|}{ Guests and salaried visitors } & & & & \\
\hline & \multicolumn{2}{|c|}{$\begin{array}{l}\text { More than } \\
3 \text { months }\end{array}$} & \multicolumn{2}{|c|}{$\begin{array}{l}\text { Less than } \\
3 \text { months }\end{array}$} & \multicolumn{2}{|c|}{$\begin{array}{c}1968 \\
\text { Summer program }\end{array}$} & \multicolumn{2}{|c|}{ Total } \\
\hline & Salaried & Guest & Salaried & Guest & Salaried & Guest & Individuals & Institutions \\
\hline University staff & 14 & 54 & 5 & 65 & 72 & 43 & 253 & 102 \\
\hline Thesis students & 8 & 44 & 0 & 29 & 0 & 8 & 89 & 38 \\
\hline Student research assistants & 1 & 29 & 0 & 18 & 121 & 32 & 201 & 84 \\
\hline Subtotal & 23 & 127 & 5 & 112 & 193 & 83 & 543 & 160 different \\
\hline Industry & 0 & 4 & 0 & 2 & 0 & 0 & 6 & 6 \\
\hline Other institutions & 12 & 38 & 3 & 19 & 2 & 10 & 84 & 52 \\
\hline Total & 35 & 169 & 8 & 133 & 195 & 93 & 633 & 218 different \\
\hline
\end{tabular}

Table 2 lists the numbers of visiting scientists (salaried Research Associates excepted) and students who worked at the Laboratory for cumulative periods of one month or more. The total of 633 individuals compares with the all-time high of 644 reported last year.

Table 3 shows the extent to which consultants' services were employed during each of the past three years.

\section{Summer Program for 1969}

Arrangements have been made for 300 visiting scientists and students to work at the Laboratory during the summer of 1969 . Of this number, 133 are staff members from colleges, universities, and other institutions, and 167 are students.

Special groups include 100 undergraduate participants in the Laboratory's 18th annual summer student program, 17 Health Physics Fellows, 22 graduate students in the first Brookhaven summer school in theoretical elementary particle physics, and the first two summer semester students in the Boston University-BNL Honors Program.

\section{Employment, Training, and Education}

Because the Laboratory's operating budget has remained fairly constant over the last several years, plans for increasing the size of the staff have, for the most part, been precluded. This condition has, in fact, led to reductions in some employee groups. Total Laboratory employment numbered 3333 in 1967,3337 in 1968, and 3261 in 1969. Because of

\section{Table 3}

Consultants' Services

\begin{tabular}{lrrr}
\hline & \multicolumn{3}{c}{ Fiscal year } \\
\cline { 2 - 4 } & 1969 & 1968 & 1967 \\
\hline Total contracts in effect June 30 & 101 & 104 & 109 \\
Number of consultants involved & 61 & 74 & 58 \\
Number of man-days of service & 475 & 617 & 478 \\
\hline
\end{tabular}

budgetary restrictions, many vacated positions have remained unfilled, and departments have been highly selective in filling the openings that are approved. Employment statistics for fiscal 1968 and 1969 are given in Table 4.

Despite a general curtailment in hiring, progress has been made in the hiring of minority employees, consistent with BNL's affirmative action objectives. Three new programs to provide assistance to disadvantaged young people were initiated during the year.

In September the Laboratory and the Regional Cooperative Association for Science and Mathematics, under a grant from the National Science Foundation, started a program entitled "Brookhaven Semester." This educational program gives students from predominantly Negro colleges the opportunity to spend an academic semester in residence at Brookhaven. The six students selected each semester pursue courses for full academic credit under the instruction of Brookhaven scien- 
Table 4

Employment Statistics*

\begin{tabular}{|c|c|c|c|c|}
\hline & \multicolumn{2}{|c|}{ June 30, 1969} & \multicolumn{2}{|c|}{ June 30, 1968} \\
\hline Scientific staff** & \multicolumn{2}{|c|}{545} & \multicolumn{2}{|c|}{555} \\
\hline $\begin{array}{l}\text { Scientific } \\
\text { professional staff }\end{array}$ & \multicolumn{2}{|c|}{376} & \multicolumn{2}{|c|}{393} \\
\hline Nonscientific staff & \multicolumn{2}{|c|}{2340} & \multicolumn{2}{|c|}{2389} \\
\hline Total & \multicolumn{2}{|c|}{3261} & 33 & 37 \\
\hline Turnover data & Number & $\begin{array}{c}1969 \\
\text { Annual } \\
\text { rate }(\%)\end{array}$ & Number & $\begin{array}{c}1968 \\
\text { Annual } \\
\text { rate }(\%)\end{array}$ \\
\hline
\end{tabular}

\begin{tabular}{|c|c|c|c|c|}
\hline \multicolumn{5}{|l|}{ Accessions } \\
\hline $\begin{array}{l}\text { Scientific staff** } \\
\text { Scientific }\end{array}$ & 77 & 14 & 91 & 16 \\
\hline professional staff & 30 & 8 & 52 & 13 \\
\hline Nonscientific staff & 201 & 9 & 247 & 10 \\
\hline Total & 308 & 9 & 390 & 12 \\
\hline \multicolumn{5}{|l|}{ Separations } \\
\hline $\begin{array}{l}\text { Scientific staff** } \\
\text { Scientific }\end{array}$ & 87 & 16 & 99 & 18 \\
\hline professional staff & 47 & 13 & 48 & 12 \\
\hline Nonscientific staff & 250 & 11 & 239 & 10 \\
\hline Total & 384 & 12 & 386 & 12 \\
\hline \multicolumn{5}{|l|}{ Net Accessions } \\
\hline $\begin{array}{l}\text { Scientific staff** } \\
\text { Scientific }\end{array}$ & -10 & -2 & -8 & -1 \\
\hline professional staff & -17 & -5 & 4 & 1 \\
\hline Nonscientific staff & -49 & -2 & 8 & 0.3 \\
\hline Total & -76 & -2 & 4 & 0.1 \\
\hline
\end{tabular}

*Figures do not include 78 temporary summer nonstudent employees. Guests and temporary student employees are included in Table 2.

**Includes Research Associates and visitors.

tists. In addition, the program provides an opportunity for two faculty members from the participating colleges to spend a full academic year at the Laboratory. The ten colleges participating in this joint venture are Miles College, Tuskegee Institute, and Talladega College in Alabama; Grambling College in Louisiana; Tougaloo College in Mississippi; Langston University in Oklahoma; Prairie View A \& M College, Texas College, and Jarvis Christian College in Texas; and Knoxville College in Tennessee.

Also in September the Laboratory began to participate in federally supported programs with Dowling College and the Neighborhood Youth
Corps. These, coupled with the annual Youth Opportunity Campaign and the High School Cooperative Education programs, gave 75 disadvantaged youngsters the opportunity to receive on-the-job training in technical and clerical positions throughout the Laboratory.

The effect of these various programs has been not only to provide valuable training for the disadvantaged but also to give them employment credentials that are considered marketable. In this way affirmative action continues to be a meaningful commitment, benefitting the Laboratory, the community, and industry.

\section{Labor Relations}

Negotiations with the International Brotherhood of Electrical Workers (IBEW), which represents $\approx 630 \mathrm{craft}$ and maintenance employees, led to settlement of a three-year contract effective until July 1,1971 . The settlement provided annual increases of 5, 6, and 7\%, all based on June 1968 rates. In addition, the Laboratory reached agreement with the IBEW on the adoption of a three-platoon schedule for the Fire Fighter Group. This schedule provides $40 \%$ more surveillance, with no increase in manpower.

A new three-year contract, terminating Feb. 29, 1972, was negotiated with the Long Island Guards Union. This settlement, which covers 28 patrolmen, resulted in wage increases comparable with those of the IBEW.

The Laboratory was involved in two arbitration proceedings during the year. The first concerned a charge by the Oil, Chemical, and Atomic Workers (OCAW) that the Laboratory granted pay increases that were discriminatory against certain employees because of their Union activities. The Laboratory's contention that the increases were awarded in accordance with published merit increase policy was sustained by the arbitrator.

The second arbitration involved a charge by the IBEW that the Laboratory had arbitrarily reassigned work from the IBEW membership to employees outside their Union. The arbitrator ruled in favor of the Laboratory, holding that such reassignment was within the Laboratory's prerogatives and not in violation of the collective bargaining agreement.

\section{Employee Services}

Recreation. Laboratory-sponsored social, cultural, and sports events continued to be diversified. 
Activities included seasonal dances, plays, bowling and golf entries in the National Industrial Recreation Tournament, and, as a special musical presentation, a concert by the Baja Marimba Band and the Brothers Four. These were but highlights of programs that brought enjoyment to some 11,000 employees and guests.

Benefits Administration. Efforts to improve employee benefits led the Laboratory to increase its retirement-plan contribution rate from $7 \frac{1}{2}$ to $10 \%$ of the employee's base salary and to reduce the employee's contribution on the first $\$ 7800$ of base salary to $2 \frac{1}{2} \%$. This action not only resulted in increased take-home pay for each participating employee but also increased the probability of greater retirement income.

\section{PLANT ENGINEerING AND PLANNING}

Plant Engineering and Planning has completed its second year as a department in its own right. As now organized, the Department discharges its functional and professional responsibilities through the Planning Group, the Design and Construction Division, the Electrical Division, and the Operation and Maintenance Division, whose reports follow.

\section{Plonning}

A simulated model of the site's domestic water system has been programmed for the CDC 6600 computer system. Computer runs of this program were checked against measured pressures and flow, and the model proved to be adequate for evaluating changes in the water system. Several practical applications of these runs have resulted in hardware changes, and more are in the engineering design stage for future implementation. Now that cross-connections have been made, the present model will be expanded to include the AGS and Medical Department systems in order to study over-all site water usage, quality, re-use, and costs as related to safety, conservation, and pollution. Studies have been initiated for a simulated model of the electrical distribution system.

Consideration of other site environmental questions this year led to a plan to automate collection, transport, and separation of trash, garbage, and sewage with use of the site sewerage system. An end use of separated solids as a new material of construction was proposed jointly with the Department of Applied Science, and an advanced water-treatment process for removal of dissolved nutrients and other materials from liquid wastes was proposed jointly with staff ecologists in the Biology Department. The integrated plan was offered to many interested federal, state, and city agencies in April 1969.

Members of the Planning Group's staff presented invited papers on the above subjects at the winter annual meeting of the American Society of $\mathrm{Me}$ chanical Engineers and at seminars during the year. Extensions of these initial definitions of the Laboratory physical environmental systems are a continuing effort of the Department's Planning Group.

\section{Operation and Maintenance}

At midyear, the Head of the Operation and Maintenance Division retired and was not replaced. The Division is administered by four group superintendents who report directly to the Department Manager. Through these superintendents the Division maintains all buildings and services and plant equipment in all buildings and outside, keeps the grounds, and operates, maintains, and repairs all plant utilities. In addition, the Division provides personnel on a chargeable basis to all departments to supply skilled labor for the scientific and service effort in the form of contributed technical services. Tables 5 and 6 present, respectively, the breakdown of manpower utilization and the distribution of costs among these efforts for fiscal 1968 and 1969.

\section{Electrical}

The function of the Electrical Division is the integration of the design, construction, maintenance, and operation of the Laboratory's electrical distribution system and plant electrical equipment.

Electric power capacity was increased by $80 \%$ with the energizing of the $40,000-k V A$ Fifth Avenue Substation in Feb. 1969. This substation was built in connection with the AGS conversion project and necessitated construction of $1 / 2$ mile of $69,000-\mathrm{V}$ transmission line by the Long Island Lighting Co. The two existing $69,000-\mathrm{V}$ incoming lines were utilized to provide an alternate source of power for both the existing and the new primary substations. Loss of power in the normal source results in automatic switching to the alternate line.

A reliable emergency power source was provided for the Life Sciences buildings by the installation of two diesel generators totaling $650-\mathrm{kW}$ capacity. 
Table 5

Manpower Utilization

\begin{tabular}{|c|c|c|c|c|}
\hline \multirow[b]{2}{*}{ Type of work } & \multicolumn{2}{|c|}{ Fiscal 1969} & \multicolumn{2}{|c|}{ Fiscal 1968} \\
\hline & $\begin{array}{l}\text { Productive } \\
\text { man-years }\end{array}$ & $\begin{array}{l}\text { Percent of } \\
\text { total work }\end{array}$ & $\begin{array}{l}\text { Productive } \\
\text { man-years }\end{array}$ & $\begin{array}{l}\text { Percent of } \\
\text { total work }\end{array}$ \\
\hline Maintenance, repairs, and utilities operations & 153 & 43.2 & 154 & 42.2 \\
\hline Major maintenance programs & - & - & - & - \\
\hline Facility improvements & 6 & 1.7 & 3 & 0.8 \\
\hline Research program support & 105 & 29.7 & 107 & 29.3 \\
\hline Building janitor services & 83 & 23.4 & 93 & 25.5 \\
\hline Decontamination & 1 & 0.3 & 2 & 0.6 \\
\hline Hot Laundry operation & 6 & 1.7 & 6 & 1.6 \\
\hline Total & 354 & 100.0 & 365 & 100.0 \\
\hline
\end{tabular}

Table 6

Costs of Supplies, Materials, and Contracts

\begin{tabular}{|c|c|c|}
\hline & Fiscal 1969 & Fiscal 1968 \\
\hline Maintenance and utilities materials & $\$ 334,653$ & $\$ 355,026$ \\
\hline Major maintenance materials and contracts & 144,960 & 421,012 \\
\hline Facility improvement materials & 288,102 & 450,029 \\
\hline Building janitor supplies & 63,895 & 69,508 \\
\hline Decontamination & 518 & 499 \\
\hline Hot Laundry & 10,903 & 9,283 \\
\hline Fuel oil & 421,644 & 433,810 \\
\hline Electricity & $1,713,145$ & $1,828,576$ \\
\hline Total & $\$ 2,977,820$ & $\$ 3,567,743$ \\
\hline
\end{tabular}

The generators are located in a remote semiunderground building to obviate the problems of noise, fumes, and vibration. This installation replaces a 250-kW generator that provided emergency power to the Medical Research Center Hospital only.

During fiscal 1968 further progress was made in the elimination of overhead electric and telephone lines. Along Cornell Ave. west of Railroad St., 1000 $\mathrm{ft}$ of pole lines were removed. Design was completed for underground power and communication duct banks along Rochester St. and South Harvard St. between Brookhaven Ave. and Machine Shop B, but installation was delayed by the freeze on new construction. During the last 5 years $\approx 7$ miles of overhead power and communication lines have been removed from service; about $2^{1 / 2}$ miles remain.

Further information is being obtained on the use of electric energy for building heating. The new Supply and Materiel offices are heated with electric resistance heaters. Two similar warehouses are heated with electric resistance heat and infrared heat, respectively. Installation and operating costs are being compiled and analyzed in terms of broader use of electric heating in future buildings.

Progress was made in the establishment and updating of Laboratory standards for electrical design and construction. Training of electricians and linemen was continued, with further instruction in the National Electric Code and in resuscitation.

No major electrical power outages occurred during fiscal 1969. A 1000-kVA transformer in the Medical Building failed in service, but the contingency provisions in the substation design allowed full service to be maintained through a second transformer until Electrical Division personnel completed repairs of the high-voltage insulation.

\section{Design and Construction}

Three major facilities with a total value of slightly more than $\$ 62,000,000$ were under construction or completed during fiscal 1969. 
Tandem Van de Graaff Accelerator Facility Lecture Hall-Cafeteria

AGS conversion

The contracts for architect-engineer and construction work are held by the AEC and administered by its Brookhaven Office, except those for the AGS conversion project, which are held by Associated Universities, Inc., and administered at BNL by the Accelerator Department. Certain portions of the Lecture Hall-Cafeteria were administered by the Design and Construction Division, which also acted on behalf of the Laboratory in all other work to provide the liaison required from early planning through construction.

Small projects initiated or undertaken during fiscal 1969 amounted to $\$ 1,295,000$ under the General Plant Projects program. There were about 100 of these additions to or modifications of facilities, as well as miscellaneous site improvements. Many of these presented special engineering problems and required considerable design effort. As in the past, the Design and Construction and Operation and Maintenance Divisions worked closely to coordinate many of these projects; in several cases Operation and Maintenance undertook management of the construction. The projects exceeding $\$ 50,000$ included refrigeration system improvements, Physics Building; compressor room addition, 80-in. Bubble Chamber Building; and air conditioning system improvements, Biology Building.

The Design and Construction Division coordinates the issuance and reporting of all proposals for accelerator and reactor additions and modifications (ARAM projects) and participates to a considerable degree in the design of a number of these projects.

Early in 1969 the Division began preparing the data sheets for construction projects in the budget request for fiscal 1971. Work included site selection, preliminary building plans, cost estimates, and description for the projects listed below (total estimated cost, $\$ 10,000,000$ ).

\section{Electron accelerator Bio-medical conjunct facility General plant projects, fiscal 1971}

During fiscal 1969, the Division continued to assist the Physics Department in delineating the scope of future large-scale bubble-chamber facilities and building construction. Assistance was given to the Accelerator Department in connection with evaluation of Title I, II, and III design for the
AGS conversion, and two staff members were assigned on a full-time basis to aid AGS staff members in execution of the work.

In concert with corporate staff members, the Division prepared a proposal for the phased design, construction, and operation of a tropical marine science center in Puerto Rico. The proposal for this $\$ 28$-million complex was submitted to the $\mathrm{Na}$ tional Science Foundation on Oct. 15, 1968.

\section{PLANT PROTECTION AND SECURITY}

The Plant Protection and Security Division continued to perform its assigned functions during fiscal 1969.

Although the scope of the Security function at the Laboratory has contracted substantially since Brookhaven's early days, the Laboratory continues to maintain an active security clearance program. At the end of fiscal 1969, about 350 employees and affiliates required active " $Q$ " clearance, and about 250 had active " $L$ " clearance. These clearances are required in connection with classified documents at the Laboratory; they also give Brookhaven scientists and technical personnel full access, when necessary, to classified data at BNL and at other laboratories and institutions in the Atomic Energy Commission's complex.

The physical growth of the Laboratory and the expansion of its research programs have necessitated ever-increasing emphasis upon the Laboratory's plant protection program. Adaptation of industrial plant protection techniques and practices to the specialized requirements of Brookhaven has had gratifying results in effectiveness of operation and economy of manpower. The number of Laboratory employees, coupled with a substantial increase in the number of persons visiting the Laboratory, has given rise to automobile traffic and parking problems which have been effectively resolved.

The Laboratory's professional Fire Group has maintained its excellent record in meeting potentially dangerous fire situations at the Laboratory. An active fire-inspection and fire-prevention program is a continuing and important aspect of the Fire Group's activities. Close liaison with scientific and technical personnel engaged in research experiments and other activities presenting potential fire hazards is regularly maintained, with excellent results. A major change in work schedules in fiscal 1969 allowed a greater number of fire fighters to be on duty at any one time. Fire-fighting equip- 
ment was increased and modernized, mutual aid fire-fighting agreements with nearby communities were continued, and training of fire fighters was accelerated.

Responses to fire alarms are tabulated below.

\begin{tabular}{|c|c|c|c|}
\hline & 1969 & 1968 & 1967 \\
\hline Responses on site & 224 & 195 & 213 \\
\hline Responses off site & 2 & 6 & 6 \\
\hline Investigations & 43 & 60 & 67 \\
\hline Total & 269 & 261 & 286 \\
\hline
\end{tabular}

\section{BUSINESS MANAGEMENT AND ADMINISTRATION}

As a result of personnel and organizational changes in the Technical Photography and Graphic Arts Division, the Graphic Arts Supervisor was promoted to the new post of Deputy Manager for the Division, and new supervisors were appointed for the Technical Photography and Graphic Arts Groups.

Among the projects completed by the Management Services Office since it was established last

Table 7

Distribution of Personnel

\begin{tabular}{|c|c|c|c|c|}
\hline Division & Staff & $\begin{array}{l}\text { Technical } \\
\text { and } \\
\text { clerical }\end{array}$ & $\begin{array}{c}\text { Fiscal } \\
1969 \\
\text { total }\end{array}$ & $\begin{array}{c}\text { Fiscal } \\
1968 \\
\text { total }\end{array}$ \\
\hline Purchasing & 21 & 15 & 36 & 41 \\
\hline $\begin{array}{l}\text { Supply and Materiel } \\
\text { Systems and Data }\end{array}$ & 12 & $79^{\mathrm{a}}$ & 91 & 91 \\
\hline Processing & 19 & 13 & 32 & 33 \\
\hline $\begin{array}{c}\text { Technical Photograph } \\
\text { and Graphic Arts }\end{array}$ & 10 & 40 & 50 & 56 \\
\hline Total & 62 & 147 & 209 & 221 \\
\hline
\end{tabular}

aTwo more employees in this category are assigned to Supply and Materiel but are on the AGS payroll.

\begin{tabular}{lrr}
\hline \multicolumn{3}{c}{ Table 8} \\
\multicolumn{3}{c}{ Purchasing and Supply Operations } \\
\hline & Fiscal 1969 & Fiscal 1968 \\
\hline Number of procurements & 31,187 & 34,853 \\
Value of procurements & $\$ 28,147,202$ & $\$ 32,505,002$ \\
Number of receiving actions & 38,200 & 42,687 \\
Number of stores issues & 289,078 & 330,619 \\
Value of inventory turnover & $\$ 3,524,466$ & $\$ 3,615,219$ \\
\hline
\end{tabular}

year are an analysis of leave-of-absence policies, a survey of all reports required by the AEC on the Laboratory's administrative data processing equipment and activities, the design of a computerized system for reporting purchasing statistics, the design of an edge-punched card system for mechanically preparing bidder lists, and a number of administrative and functional analyses for various Laboratory organizational units. Current activities include the development of an integrated, computerized system to meet the combined management information needs of the Laboratory's purchasing, receiving, and accounts payable functions.

The Management Services Office has also completed a computer model for simulating the overall operation of Brookhaven's relatively large water distribution system. This model is being used by the Plant Engineering and Planning Department to study in detail the capabilities of the existing water system, investigate the relative merits of possible water conservation measures, and explore alternative design possibilities for upgrading and extending the system to meet increasing demands for water. Preliminary work has been started on a

Table 9

Technical Photography Operations

\begin{tabular}{lrrr}
\hline & \multicolumn{3}{c}{ Fiscal year } \\
\cline { 2 - 4 } & \multicolumn{1}{c}{1969} & 1968 & 1967 \\
\hline Photographs & 17,797 & 18,058 & 14,565 \\
Photomicrographs & 12,637 & 8,728 & 9,682 \\
Lantern slides & 23,957 & 20,437 & 14,952 \\
Prints & 58,336 & 53,526 & 60,231 \\
Film processed, ft & $3,729,366$ & $5,228,415$ & $5,810,049$ \\
Charts and graphs drawn & 4,704 & 4,904 & 5,433 \\
Motion picture footage & 9,664 & 25,372 & 20,706 \\
\hline \hline
\end{tabular}

Table 10

Graphic Arts Operations

\begin{tabular}{|c|c|c|c|}
\hline & \multicolumn{3}{|c|}{ Fiscal year } \\
\hline & 1969 & 1968 & 1967 \\
\hline $\begin{array}{l}\text { Photo-offset } \\
\text { impressions }\end{array}$ & $12,419,030$ & $11,410,856$ & $14,850,193$ \\
\hline $\begin{array}{l}\text { Quick Copy } \\
\text { impressions* }\end{array}$ & $6,613,125$ & $6,413,952$ & $6,586,014$ \\
\hline
\end{tabular}

*Includes Xerox copies. 
model for the Laboratory's electrical power distribution system.

Comparative statistics for business management and administrative functions are presented in Tables 7 through 10.

\section{Purchasing}

Because of recent general progress in the area of administrative data processing, the Purchasing Division has reassessed and substantially revised its over-all plans for computerizing its operations. These revisions have been incorporated in a new five-year master plan for the Division. They take into account the progress made in converting data processing operations to the new IBM $360 \mathrm{com}$ puter, the new computer's advanced capabilities, which include on-line teleprocessing, and the development of an integrated management information system for the Laboratory. They also provide for coordination with other plans and efforts in this area in order to integrate various but interrelated data input, control, and reporting requirements, particularly those for the accounts payable and supply and materiel functions.

Preparations were made for converting the expediting-reports system to the IBM 360 . They include the introduction of new codes that permit open orders to be reported by appropriate categories and provisions for the input and reporting of rescheduled delivery dates. An edge-punched card system for updating and mechanically preparing lists of approved bidders was also established.

As previously reported, an explanation of the Brookhaven Offer of Sale concept was given by W. J. Catacosinos at a hearing of the House Technical Program Subcommittee of the United States Congress. As a result, the Subcommittee recommended that several Government agencies, including the Department of Defense and the General Services Administration, investigate the use of this concept in their procurement activities. In turn, several installations were directed by their agencies to test the use of the Offer of Sale technique and evaluate the results of its application. One of the installations, the United States Army's Harry Diamond Laboratory, has reported that their trial application of the Offer of Sale concept resulted in considerable savings not previously obtained through other means and that they plan to expand their application of the Offer of Sale concept.

Through the continued efforts of all Purchasing Division personnel, documented cost savings for the
Laboratory of more than $\$ 600,000$ were achieved during the year. Representative of these efforts is an unusual procurement technique incorporated in long-term contracts for liquified gases in which the unit price paid for all gas is based on the total amounts purchased during the life of a contract. This new idea was accepted by the industry after a lengthy period of negotiation. Progress is also being made in extending the practice of combining Brookhaven requirements with those of other institutions, New York State, or Government agencies, such as the Federal Aviation Administration and the Department of Defense, in order to obtain better prices than the Laboratory could by itself.

The Division recently introduced a program in which each member of the purchasing staff investigates an assigned topic on purchasing techniques or contract terms and conditions and then presents the information to the rest of the staff. Other training activities included the attendance by several staff members at a series of lectures on Government contracting given at a nearby community college and the attendance of two staff members at a training seminar sponsored by the Sandia Corp. in Albuquerque, N.M.

\section{Supply and Materiel}

To improve the effectiveness and efficiency of its working operations, the Supply and Materiel Division has completed the consolidation and relocation of the majority of its functions within a single conveniently located area. By centralizing its administrative and clerical staff together with the bulk of its supply operations, the Division has realized maximum utilization of the available manpower.

A physical inventory sample technique was implemented by the Division at the beginning of fiscal 1969. The use of this technique has significantly reduced the time and manpower expended by the Supply and Materiel, Fiscal, and Administrative Systems and Data Processing Divisions in activities related to the physical inventory function. The results of using the sample technique during the past year will be analyzed to determine its full effectiveness.

A continuing effort to establish nomenclature for all direct purchase items used by AGS development groups is nearing completion. A computerized system now being implemented will utilize 
the established nomenclature to consolidate purchases for the various AGS groups and promote cross-utilization of materials. The elimination of duplicate procurement activities is expected to result in substantial savings for the Laboratory.

The organization of a photographic equipment pool was completed during the year. Descriptions of all photographic equipment at Brookhaven are recorded on edge-punched cards which are used periodically in publishing updated lists of available equipment. By regularly informing potential users about equipment available on a loan basis, these listings promote fuller utilization of existing photographic equipment and thus aid in curtailing unnecessary purchases of new equipment.

Because of increased activities associated with the AGS conversion project, a receiving dock was established in the project area for direct receipt of large volumes of materials. Two imprest stockrooms, one at the Graphite Research Reactor and another at the Hot Laboratory, have been shown to be insufficiently active to warrant continued operation and will be closed. The personnel involved will be reassigned to the high energy physics area.

\section{Systems and Data Processing}

Throughout the fiscal year the Systems and Data Processing Division continued to focus its efforts on the design and implementation of a new integrated management information system for the Laboratory. The system is centered on the use of the data processing capabilities of the Division's third-generation IBM 360 Model 30 computer installation. Its design takes into account the longrange as well as immediate management-information requirements of both the Laboratory as a whole and the AEC.

Conversion and upgrading of all existing applications from the old Univac SS/90 computer for use with the new IBM 360 computer is expected to be completed by Aug. 1, 1969. At that time the Univac computer is scheduled for release to excess.

While continuing to maintain and operate its two separate and incompatible computer systems in parallel, the Division has successfully converted and upgraded 11 of the former Univac operations, including those for payroll, personnel, job labor and material cost, capital equipment, stores inventory, and accounts payable. Remaining conver- sion activities involve final work on an accounting and budget control system and a computeroriented budget forecasting system. During the entire conversion process, great care has been taken to generate a sound, integrated data base for the various applications and thus provide a realistic foundation for the development of an integrated, total management information system.

In addition, the Division satisfied more than 150 requests during the year for improvements in systems, programs, and operations involving both the Univac and IBM computer installations. Work on these improvements was absorbed into the Division's already tight conversion schedule.

Existing Univac applications formerly required $2 \frac{1}{2}$ shifts on the Univac computer. When conversion is completed, only $1 \frac{1 / 2}{2}$ shifts will be required on the IBM 360, even with upgrading. Most of this saving in shift time has already been achieved. Other cost savings have been realized by standardizing all input and output documentation for the new system and thereby reducing the use of custom-printed forms.

A standard input system has been implemented for the IBM 360 system. By evaluating all input and controlling its acceptance into the total information system, the input system has established firm control over the quality of output for all areas of application, and its use has resulted in a general improvement in output accuracy and timeliness.

The continued upgrading of services provided to management has led to the need for more on-line data storage capacity. Consequently two more IBM 2311 disk drives, with a combined capacity of 15 million characters, have been added to the basic IBM 360 installation. A 7-track tape drive was also installed to accommodate the tape translation needs of the Department of Applied Science.

An on-line inquiry system for the IBM 360 installation, in the pilot stage a year ago, has proved to be an important tool for Laboratory management and is now in regular operation. Present plans include its further development to serve an increased number of on-line terminals at a more sophisticated level. Three additional terminal units are on order, and the addition of a large directaccess storage device is being considered.

The Division has continued its program to provide systems and programming staff members with an intimate knowledge of the capabilities of thirdgeneration computer hardware and software. This program has been an important factor in the suc- 
cessful development of a standard input system and a standard report generator, which are used by all the Laboratory's IBM 360 application systems. The Division extended its training effort during the year to include a number of information sessions for users of its new computer installation. The purpose of these sessions is to provide users with a thorough understanding of the capabilities of the new installation and encourage their involvement in the design and subsequent implementation of new application systems.

\section{Technical Photography and Graphic Arts}

Services provided to the Laboratory by the Technical Photography Group during the fiscal year ranged from assisting members of the Medical Department in developing standard procedures for preparing serially ordered photomicrographic prints to preparing both still and motion picture material for public information purposes. In addition, the Photography Group continues to meet the weekly needs of the Brookhaven Bulletin staff for photographic services.

In the area of photomicrography, a system was developed for the orderly preparation and display of serial mouse-spleen sections. This system, now a routine production procedure, involves taking consecutive photomicrographs of serial sections on microfilm and then preparing enlarged prints in order on a continuous roll of print paper. The rolls are delivered uncut so that the set of prints for each series of sections may be viewed in the proper serial order.

Although no major projects were undertaken by the Motion Picture Section, it has demonstrated its usefulness in providing needed documentation and training material for a number of Laboratory activities. A detailed training film on the operation of remote target-handling equipment at the AGS has been completed and used successfully both for introducing experimenters to the equipment and as an instructional aid for training operators in methods of using the equipment for making target changes and adjustments. Computer-produced displays provided by the Applied Mathematics Department were assembled into an animated film that demonstrates, in slow motion, the generation of events in a bubble chamber. A program for documenting on film the progress of patients afflicted by Parkinson's disease was improved by the addition of synchronized sound.
In connection with the Laboratory's public information activities, 150 sets of color slides were prepared for distribution to high schools. Each set contains 40 slides that depict major equipment installations at Brookhaven and illustrate a number of the Laboratory's research programs. Soundsynchronized motion picture footage on a concretepolymer materials project at Brookhaven was supplied by the Motion Picture Section for a special news report on a national television network.

A new and highly efficient camera for making $2 \times 2$-in. slides from negatives of various sizes was purchased in line with a continuing trend toward using $2 \times 2$ rather than $3^{1 / 4} \times 4$-in. slides. At present more than $90 \%$ of all slides prepared are of the $2 \times 2$-in. size. To provide a quick, low-cost means of producing multiple copies of computer output recorded on microfilm, a one-step procedure has been developed for directly enlarging microfilm output frames onto offset printing plates.

During the year the Graphic Arts Group further improved its efficiency by automating several operations previously done by hand. The output from the Xerox 2400 copier is now jogged, stitched, and stacked automatically by a single machine, and a Cummins Planax binder has been installed to bind fanfold computer printouts.

In an attempt to improve both the efficiency and the quality of its operations, the Composition Section is implementing a scheme for color-contrasting all its type fonts. The use of color is expected to decrease eye strain among compositors and to lessen the possibility of wrong type-font selection. One Fotosetter underwent extensive repairs during the year. In particular its camera section, which must accurately expose type-character images and record them on film, was carefully rebuilt.

An internal inventory control system instituted during the year has reduced the amount of time required for inventory maintenance activities within the Graphic Arts Group. With the pertinent and timely data provided by this system, the Group can now exert closer control over its supply levels and take full advantage of quantity-purchase discounts.

To obtain a more thorough understanding of their problems and responsibilities, six Graphic Arts supervisors attended a one-week supervisory development seminar offered by Rutgers University.

Some of the larger reports produced within the BNL printing plant during the year are listed below. 


\begin{tabular}{|c|c|c|}
\hline Title & Pages & $\begin{array}{c}\text { Total } \\
\text { impressio }\end{array}$ \\
\hline \multicolumn{3}{|l|}{$\begin{array}{l}\text { Annual Report, July 1, 1968, BNL } 50106 \\
\text { (AS-22) }\end{array}$} \\
\hline \multicolumn{3}{|l|}{$\begin{array}{l}\text { Brookhaven Symposia in Biology No. } 21 \text {, } \\
\text { Structure, Function, and Evolution in } \\
\text { Proteins, BNL 50116 (C-53): }\end{array}$} \\
\hline Volume I & 270 & $1,215,000$ \\
\hline Volume II & 180 & 810,000 \\
\hline \multicolumn{3}{|l|}{$\begin{array}{l}\text { Proceedings of the Brookhaven Conference } \\
\text { on Industrial Needs and Academic Re- } \\
\text { search in Reactor Kinetics, BNL } 50117\end{array}$} \\
\hline \multicolumn{3}{|l|}{$\begin{array}{l}\text { Proceedings of the } 1968 \text { Proton Linear } \\
\text { Accelerator Conference, BNL } 50120\end{array}$} \\
\hline (C-54): Part 1 & 458 & 377,850 \\
\hline Part 2 & 374 & 308,550 \\
\hline \multicolumn{3}{|l|}{$\begin{array}{l}\text { Concrete-Polymer Materials, First Topi- } \\
\text { cal Report, BNL 50134(T-509) and } \\
\text { USBR Gen. Rep. } 41\end{array}$} \\
\hline \multicolumn{3}{|l|}{$\begin{array}{l}\text { Annual Report, Nuclear Engineering De- } \\
\text { partment, December } 31,1968, \text { BNL } \\
50149 \text { (S-71) }\end{array}$} \\
\hline \multicolumn{3}{|l|}{$\begin{array}{l}\text { Proceedings of the } 1968 \text { Summer Study on } \\
\text { Superconducting Devices and Accelera- } \\
\text { tors, BNL } 50155 \text { (C-55): Part I }\end{array}$} \\
\hline & 350 & 525,000 \\
\hline Part III & 442 & 663,000 \\
\hline $\begin{array}{l}\text { Bulletin of the Medical Department, } \mathcal{N}_{0-} \\
\text { vember } 1,1968\end{array}$ & & 273,000 \\
\hline
\end{tabular}

\section{STAFF SERVICES}

The general level of activity in the Staff Services Division continued high during fiscal 1969. The Division provides the following services and assigned functions for the Laboratory staff and visitors.

\section{Housing}

On-site housing includes 1 to 4 -bedroom family units, men's and women's residences, and a guest house. Total capacity (nearly 880 persons) is fully utilized each summer. Assignment, billing, and janitorial services are the responsibilities of the Housing Office. A rental rate survey pursuant to Bureau of the Budget Circular No. A-45 is now in process and should be completed early in fiscal 1970.

\section{Travel}

A complete Travel Office is maintained. Tickets, reconfirmations, travel reservations, and accommodations are obtained for all personnel traveling in an official capacity. Tickets and associated reservations for space and cars for more than 7940 trips were processed this year. The Laboratory uses agreements that result in reduced rates for hotel and motel reservations and car rentals under certain circumstances for Laboratory employees on official travel status.

\section{Telecommunications}

Telephone, telegram, and teletype services for the Laboratory are operated by the Division. At present 1900 lines and 3200 telephones are installed. The increase in lines and telephones from the previous year is due mainly to the partial completion and occupancy of the Tandem Van de Graaff Accelerator Facility and to alterations and enlargements in other buildings.

\section{Mail and Wire Service}

Mail distribution service is maintained to effect frequent pickup and delivery of United States and interoffice mail at each of 94 Laboratory mail drops. About 3,200,000 pieces of mail were handled during fiscal 1969. Standard distribution lists are maintained to facilitate efficient distribution of material to appropriate personnel with use of an Elliott "Dymatic 7000" addressing machine, on which more than 430,000 addresses were run off this year. Three types of wire service are in daily use at the Laboratory: telegraph, teletype, and the Secure Automatic Data Information System (SADIE).

\section{Transportation}

A pool of $\approx 24$ vehicles is available on a trip basis for official business. Limited driver service is also available when use of a passenger-operated vehicle is not practical.

\section{Motor Vehicles}

The entire fleet of 259 vehicles is acquired, maintained, assigned for use, rotated in assignment, and readied for disposal by the Division. Most vehicles are assigned to user departments or divisions and operated on a subpool basis to eliminate the need for drivers, dispatchers, and radios. Total mileage for the year was 1.5 million.

\section{Food Service}

Food service is provided for Laboratory staff and guests by a concessionaire in the new cafeteria, the Brookhaven Center, and vending machines. These operations are responsibilities of the Division. 


\section{Special Staff Services}

The Laboratory is host annually to more than 130 scientific seminars, conferences, meetings, and special events, and a similar number of intraLaboratory functions are conducted. Each requires all or some of the services described above and, in some instances, additional services unique to the situation. The necessary planning, acquisition, and coordination of these services are additional functions of the Division.

\section{Personnel}

The Division is composed of 61 persons, consisting of housing, travel, and mail clerks, telephone and teletype operators, automobile and truck mechanics, janitors and matrons, and appropriate supervisory personnel.

\section{CONFERENCES, PUBLIC INFORMATION, PUBLIC RELATIONS, AND EDUCATION}

The tenth annual series of George B. Pegram Lectures was presented on April 17, 22, and 24 by Professor Roger Revelle, who is internationally recognized for his investigations on the physical nature of oceans and who has devoted much of his time in recent years to the problems of the developing countries. The title of the series was "The Population Problem and What Can Be Done About It." The three lectures were subtitled "Mankind Divided - The Rich and the Poor," "Can the Poor Countries Feed Themselves?" and "What Must Be Done To Solve the Population Problem?"

The seventh AUI Distinguished Lecture was delivered on Nov. 7 by Professor Adam Yarmolinsky, internationally known lawyer and government advisor and Professor of Law at Harvard University. The title of his lecture was "The Limits of Violence." The eighth AUI Distinguished Lecture was presented on March 20 by the Honorable Emilio Q. Daddario, member of the Congress of the United States and of the Joint Committee on Atomic Energy. His lecture was entitled "Shaping the Environment of Federal Laboratories."

Ten major conferences were held at BNL during fiscal 1969: the International Conference on Lymphocytes, Sept. 12-13; Seminar on Medical Planning and Care in Radiation Accidents (sponsored by the US AEC), Oct. 21-23; IAEA Panel on Neutron Data Compilation, Feb. 10-14; Seminar on Radiation Chemical Processing, March 3-4; Subcommittee on Radiobiology of the Committee on Nuclear Science of the National Academy of Sciences, March 15-17; Lidar Uses in Atmospheric Research, April 15-16; High-Temperature Superconductors, May 1-2; Biology Symposium No. 22, entitled "Diversity and Stability in Ecological Systems," May 26-28; the IAEA International Nuclear Data Committee, June 2-6; and Planning and Care for Radiation Accidents (sponsored by the US AEC), June 15-18.

In addition, a number of smaller conferences were held: Cross Section Evaluation Working Group Meeting, Sept. 16-18; University-AECLaboratory Relations Meeting, Oct. 24-25; Program presentation to AEC Materials Branch, Nov. 21-22; Atmospheric Diagnostic Meeting, Nov. 2526; Joint US AEC and Canadian AEC Meeting, Dec. 17-18; Information Meeting on ConcretePolymer Materials for Department of the Army (sponsored by the US AEC), March 21; Approach to Pollution Control Systems Meeting, April 25; Heat Transfer Meeting (sponsored by the US AEC), May 22-23; and Atomedic '69, June 8-10.

The following meetings took place during the fiscal year: AEG High Energy Staff Program Review, July 1-2; High Energy Physics Advisory Panel to the AEC, July 19-20; High Energy Discussion Group, July 22; Polymer-Concrete Meeting, Oct. 31-Nov. 1; Information Exchange Meeting on Polyethylene and Chemonuclear Programs, Nov. 6-8; National Neutron Cross Section Advisory Committee, Nov. 19-20; Task Force on Cross Sections for Fissile and Fertile Materials, June 9-10; AEC Budget Review Committee, June 10; AEC Physics and Mathematics Low Energy Research Program Review, June 11-12; Advisory Committee to the AEC on Reactor Physics, June 11-12; and Canada-USA Safeguard Development Group, June 26.

The 15th annual High School Visitors' Day was held on Oct. 19 and was attended by 3800 students representing 153 high schools and 38 junior high schools. The 19th Visitors' Day, held on Oct. 26, was attended by 6091 persons.

On February 18, BNL observed the 13th Science Youth Day in commemoration of Thomas Alva Edison's birthday. The program was an in-depth study of the various projects of the Department of Applied Science. The event was attended by 84 students and 17 teachers from 25 Suffolk County high schools. 
Ten lectures in the BNL Lecture Series were delivered by staff members, who discussed their work.

Three National Science Foundation teachers-intraining seminars, comprising 76 teachers, were given talks on the Laboratory's research program and tours through the major facilities. In addition, 11 professional groups visited Brookhaven for a first-hand view of the research in progress, and 51 teachers and 478 college students were also given tours.

Foreign groups visiting the Laboratory during the year included Fulbright scholars, Japanese science writers, a Rumanian scientific delegation, USSR high energy physicists, Japanese science educators, and many others, among them N. Nabeshima, Minister of the Japan AEG, accompanied by four of his staff, July 19; Congolese students, Aug. 7; Venezuelan students with the Director of the University of Venezuela, Aug. 9; R.L. Kopel, Israeli Ambassador to Central America, Aug. 28; Dr. Jean-Baptiste Romain, President of the University of Haiti, Oct. 31; Brazilian labor coordinators, Nov. 7; foreign nurses in the Ex-
change-Visitor Program, Dec. 4; physics students from McMaster University, Hamilton, Ont., Jan. 2; students from the Republic of El Salvador, Feb. 6; Brazilian science students, Feb. 20; USSR high energy physicists, March 10-11; and the Chairman of the Greek AEC, accompanied by four members of his staff, June 9 .

Members of the Brookhaven Town Industrial Commission and the Suffolk County Planning Commission visited the Laboratory on Dec. 5 and July 17 respectively.

Six speeches were delivered by staff members to civic, church, school, and educational groups.

A total of 463 foreign scientists visited BNL during the report period.

The Public Information Office experienced a steady increase in requests for information and advice from students and teachers. Most of these requests are for assistance in the preparation of science projects and term papers. An extensive stock of booklets and information sheets suffices in the majority of cases, but some require specialized attention. 


\section{Appendix A}

\section{PUBLICATIONS, JULY 1, 1968 - JUNE 30, 1969}

This list includes official Laboratory publications, abstracts of papers which were or will be presented at scientific meetings, and publications by staff members, consultants, and guests. All these listings result from work done at the Laboratory; they were submitted during the review period.* Abstracts are indicated by (A); letters to the editor, $(\mathrm{L})$; and notes, $(\mathrm{N})$. Acceptance for future publications is designated by (in press).

\section{GENERAL PUBLICATIONS}

Annual Report, July 1, 1968. BNL 50106 (AS-22).

Annual Report, Nuclear Engineering Department, Dec. 31, 1968. BNL 50149 (S-71).

Conference Reports:

Brookhaven Symposia in Biology No. 21. Structure, Function and Evolution in Proteins. BNL 50116 (C-53), Vol. I and II.

Proceedings of the 1968 Proton Linear Accelerator Conference, May 20-24, 1968. BNL 50120 (C-54), Pt. 1 and 2.

Proceedings of the 1968 Summer Study on Superconducting Devices and Accelerators, June 10- July 19, 1968. BNL 50155 (C-55), Pt. I, II, and III.

Brookhaven Lecture Series:

68. Information Theory and Biology: A Critique and Resynthesis, H.A. Johnson. BNL 50158 (T-524).

69. The A GS Conversion Project, G.W. WheEler. BNL 50131 (T-506).

76. Interatomic Forces in Chemical Reactions, R.E. WESTON, JR. BNL 50133 (T-507).

High-Temperature Liquid-Metal Technology Review:

Vol. 6, No. 4, Aug. 1968. BNL 50137 (PR-34).

Vol. 6, No. 5, Oct. 1968. BNL 50153 (PR-35).

Vol. 6, No. 6, Dec. 1968. BNL 50163 (PR-36).

Vol. 7, No. 1, Feb. 1969. BNL 50170 (PR-37).

Vol. 7, No. 2, Apr. 1969. BNL 50179 (PR-38)

Vol. 7, No. 3, June 1969. BNL 50190 (PR-39).

Bibliographies:

Bibliography of Technical Publications and Reports of the Radiation Division, Brookhaven National Laboratory, July 1965 June 1968 Inclusive, D. J. METZ AND L.E. KOPP. BNL 50132 (L-407).

Informal Reports:

Brookhaven Portable Cesium Development Irradiator Manual, A. Oltmann. BNL 13506.

*Also included are those listings from the last annual report [BNL 50106 (AS-22)] for which complete reference information was not then available.
ENDF-110. Description of the Endf/B Processing Codes Chegrer, Crect, Dammet, Pløtfr, and Retrieval Subroutines, National Neutron Cross Section Center. BNL 13582.

Pulsed Fast Research Reactor Project, Management Plan, Revision I, K.C. Hoffman and J.M. Hendrie, Editors. BNL 13370.

Pulsed Fast Research Reactor Project, Progress Reports, K.C. Hoffman and J.M. Hendrie, Editors:

May 1, 1968-Oct. 31, 1968. BNL 12750.

Nov. 1, 1968-April 30, 1969. BNL 13605.

Miscellaneous:

Progress Report of the Work Sponsored by the Sodium Technology and Fuels and Materials Branches, Division of Reactor Development and Technology, United States Atomic Energy Commission (Official Use Only):

Quarterly, July 1-Sept. 30, 1968. BNL 50141 (T513).

Annual, Jan. 1-Dec. 31, 1968. BNL 50161 (T526).

Quarterly, Jan. 1-March 31, 1969. BNL 50176 (T-534).

Quarterly, April 1-June 30, 1969. BNL 50193 (T-546)

Weekly Bulletin 22, No. 4-52; 23, No. 1-4.

Weekly Selected Reading List 21, No. 8-52; 22, No. 1-7.

\section{STAFF PUBLICATIONS AND ABSTRACTS}

\section{Accelerator Department}

ADAIR, R.K. Diffraction-disassociation model of very-highenergy nucleon-nucleon interactions and the diffusion of cosmic rays through the atmosphere. Phys. Rev. 172, 1370-80 (1968).

Adair, R.K. - See also Carithers, W.C. Jr.; Wanderer, P. J. JR.

Agritellis, C. and Chasman, R. Design of a buncher and the transport system in its drift space for the $200 \mathrm{MeV}$ injector linac. In Proc. 1968 Proton Linear Acceleratcor Con- 
ference, Brookhaven National Laboratory, May 1968, Pt. 1, Pp. 328-37. BNL 50120 (C-54), Dec. 1968.

Agritellis, C., Chasman, R., and Sluyters, T.J.M. Design of the low-energy beam transport system of the Brookhaven 200-MeV injector linac. IEEE Trans. Nucl. Sci. NS-16, No. 3, Pt. 1, 221-6 (1969).

Allinger, J.E., Danby, G., Jackson, J., Polk, I., and Prodell, A. High field iron magnets for operation at cryogenic temperatures. Ibid. 728-33.

Allinger, J.E. - See also Danby, G.T.

Ankenbrandt, C.M. - See Wanderer, P. J. Jr.

BARTon, M.Q. Computers in accelerator control rooms. A personal appraisal. IEEE Trans. Nucl. Sci. NS-16, No. 3, Pt. 1, 810-2 (1969).

Barton, M.Q., Blumberg, L.N., Fox, J.D., GlenN, J.W., Nawrocky, R. J., and Soukas, A.V. Performance of the slow extracted beam at the Brookhaven AGS. (A) In Proc. 7th Int. Conf. High Energy Accelerators, Yerevan, Armenian SSR, Aug. 27-Sept. 2, 1969 (in press).

Barton, M.Q., Frankel, R., and Month, M. Determination of accelerator tune from perturbed closed orbits. Rev. Sci. Instr. (in press).

Barton, M.Q. - See also Blumberg, L.N.

Bennet, G.W. - See Blumberg, L.N.; Fox, J.D.; Friedes, J.L. (Physics).

BEnton, A. Multigap debunchers. IEEE Trans. Nucl. Sci. NS-16, No. 3, Pt. 1, 210-11 (1969).

Berley, D., Yamin, P., Chan, S., Button-Shafer, J., Hertzbach, S.S., Kofler, R.R., Meisner, G.W., Yamamoto, S.S., Schiff, M., Thompson, J., AND WILLIs, W.J. Study of $r_{1}^{*}(1385) \pi$ production near the $r_{0}{ }^{*}$ resonance. (A) Bull. Amer. Phys. Soc. 14, 560 (1969).

Berley, D., Yamin, P., Kofler, R., Mann, A., Meisner, G., Yamamoto, S., Tanenbaum, W., Thompson, J., and Willis, W. A search for the decay $K_{S^{0}} \rightarrow \pi^{+} \pi^{-} \pi^{0}$. In Proc. Int. Conf. Weak Interactions, CERN, Geneva, Jan. 1969 (in press).

Berley, D., Yamin, P., Kofler, R., ManN, A., Meisner, G., Yamamoto, S., Thompson, J., and Willis, W. An isospin conservation test from $K-p$ interactions at 400 $\mathrm{MeV} / c$. Submitted to Phys. Rev. Letters.

Berley, D. - See also Bertanza, L. (Physics); Yamin, P. Ветн, R.A. Analytical design of superconducting multipolar magnets. In Proc. 1968 Summer Study on Superconducting Devices and Accelerators, Brookhaven National Laboratory, June 10-July 19, 1968, Pt. 3, pp. 843-59. BNL 50155 (C-55), April 1969.

BETH, R.A. Currents and coil forces as contour integrals in two-dimensional magnetic fields. J. Appl. Phys. 40, 2445-9 (1969).

BETH, R.A. Evaluation of current produced two-dimensional magnetic fields. J. Appl. Phys. (in press).

BLEWETT, J.P. Acceleration of charged particles to high energies. In Handbook of Physics, 2nd Ed., Pt. 9, pp. 24054, E.U. Condon and H. Odishaw, Editors, McGrawHill, New York, 1967.

BLEWETT, J.P. The future of high energy accelerators. IEEE Trans. Nucl. Sci. NS-16, No. 1, 19 (1969).

BLEWETT, J.P. Iron shielding for air core magnets. In Proc. 1968 Summer Study on Superconducting Devices and Accelerators, Brookhaven National Laboratory, June 10-July 19, 1968 , Pt. 3, pp. 1042-51. BNL 50155 (C-55), April 1969.
BLewetT, J.P. Proton alternating-gradient synchrotrons. Article in Encyclopaedia Britannica (in press).

BLEWETT, J.P. Summary of sixth week of 1968 summer study. In Proc. 1968 Summer Study on Superconducting Devices and Accelerators, Brookhaven National Laboratory, June 10-July 19, 1968, Pt. 3, pp. $1127-32$. BNL 50155 (C-55), April 1969.

BLEWETT, J.P. 200-BeV proton synchrotron. In 1968 Yearbook of Science and Technology, pp. 291-3, D.I. Eggenberger, Editor, McGraw-Hill, New York, 1968.

BlewetT, J.P. Use of superconductors in high energy physics. In Proc. 1968 Summer Study on Superconducting Devices and Accelerators, Brookhaven National Laboratory, June 10-July 19, 1968, Pt. 2, pp. 544-9. BNL 50155 (C-55), April 1969.

Blewett, J.P. - See also Sampson, W.B.

Blumberg, L.N., Barton, M.Q., Bennett, G.W., Fox, J.D., GlenN, J.W., Hsieh, H.C.H., NaWrocky, R. J., AND Soukas, A.V. Initial performance of the AGS slow external beam. IEEE Trans. Nucl. Sci. NS-16, No. 3, Pt. 1, 234-41 (1969).

Blumberg, L.N. - See also Barton, M.Q.

Britton, R.B. Brookhaven superconducting dc beam magnets. In Proc. 1968 Summer Study on Superconducting Devices and Accelerators, Brookhaven National Laboratory, June 10-July 19, 1968, Pt. 3, pp. 893-907. BNL 50155 (C-55), April 1969.

BRITTON, R.B. Niobium tin and related superconductors. Ibid., Pt. 2, pp. 449-64.

Britton, R.B. 60-Hz flux pumps. Ibid., Pt. 2, pp. 679-80.

Britton, R.B. - See also Gillani, N.V.; Morgan, G.H.; Sampson, W.B.

Brown, H.N. An enriched-particle beam using superconducting rf deflectors. In Proc. 1968 Summer Study on Superconducting Devices and Accelerators, Brookhaven National Laboratory, June 10-July 19, 1968, Pt. 1, pp. 136-49. BNL 50155 (C-55), April 1969.

Buchanan, V. J. - See Jablonski, E.

Carithers, W.C. Jr., Adair, R.K., Hawkins, C. J.B., Kasha, H., Larsen, R.C., Leipuner, L.B., Smith, L.W., AND Wangler, T.P. Proton-proton triple scattering at $1.9 \mathrm{GeV}$. Phys. Rev. 179, 1304-14 (1969).

Chasman, R. Numerical calculations of the effects of space charge on six-dimensional beam dynamics in proton linear accelerators. In Proc. 1968 Proton Linear Accelerator Conference, Brookhaven National Laboratory, May 1968, Pt. 1, pp. 372-88. BNL 50120 (C-54), Dec. 1968.

Chasman, R. Numerical calculations on transverse emittance growth in bright linac beams. IEEE Trans. Nucl. Sci. NS-16, No. 3, Pt. 1, 202-6 (1969).

Ghasman, R. and Sluyters, T. J.M. Design of the low energy beam transport system of the BNL $200-\mathrm{MeV}$ injector linac. (A) Bull. Amer. Phys. Soc. 14, 210 (1969).

Chasman, R. - See also Agritelis, G.; Lee, M. J.

Glaus, J. On transverse space-charge effects in bunched beams. IEEE Trans. Nucl. Sci. NS-16, No. 3, Pt. 1, 21620 (1969).

Glaus, J. Transverse space-charge effects in bunched beams in a transport system. Informal Report BNL 13103.

Cornish, G.W. - See Watts, A.R. 
Cottingham, J.G. - See Post, J.A.

Courant, E.D. Accelerators for high intensities and high energies. Annu. Rev. Nucl. Sci. 18, 435-64 (1968).

Courant, E.D. Some high-current effects in accelerators. (A) In Proc. 7th Int. Conf. High Energy Accelerators, Yerevan, Armenian SSR, Aug. 27-Sept. 2, 1969 (in press).

Courant, E.D. - See also Lee, M.J.

Dahl, P.F., Morgan, G.H., and Sampson, W.B. Loss measurements on twisted multifilamentary superconducting wires. J. Appl. Phys. 40, 2083-5 (1969).

Dahl, P.F. and Sampson, W.B. Some recent ac loss measurements in normal and superconducting coils. Informal Report BNL 13502.

Dahl, P.F. - See also Morgan, G.H.; Sampson, W.B.

DANBY, G.T. Synchrotrons using high field iron magnets operating at cryogenic temperatures. (A) In Proc. 7 th Int. Conf. High Energy Accelerators, Yerevan, Armenian SSR, Aug. 27-Sept. 2, 1969 (in press).

Danby, G.T., Allinger, J.E., and Jackson, J.W. Synchrotron magnets with cryogenic exciting coils. In Proc. 1968 Summer Study on Superconducting Devices and Accelerators, Brookhaven National Laboratory, June 10-July 19, 1968, Pt. 3, pp. 1115-26. BNL 50155 (C-55), April 1969.

Danby, G.T. - See also Allinger, J.

DeVito, B. - See Keane, J.T.

FlataU, C.R. Compact servo master-slave manipulator with optimized communication links. (A) In Proc. 17th Conf. Remote Systems Technology, San Francisco, Nov. 1969 (in press).

FlataU, C.R. General purpose servo-manipulator for remote maintenance of accelerators. IEEE Trans. Nucl. Sci. NS-16, No. 3, Pt. 1, 594-8 (1969).

Forsyth, E.B. An accelerator design using constantgradient superconducting magnets. Informal Report BNL 12690.

Forsyth, E.B. Line harmonics at the AGS. Informal Report BNL 12691.

Forsyth, E.B. Modulator applications in high energy accelerators. In Proc. 10th Modulator Symposium, U.S. Department of Defense, New York, May 1968, pp. 1-9.

Foster, E.H. - See HAHN, H.

Fox, J.D., Bennett, G.W., Levine, G.S., Nawrocky, R. J., REPETA, L.E., AND SoukAs, A.V. Instrumentation and control of AGS slow external beam. IEEE Trans. Nucl. Sci. NS-16, No. 3, Pt. 1, 832-6 (1969).

Fox, J.D., Bennett, G.W., Levine, G.S., Repeta, L.E., AND Soukas, A.V. Instrumentation of slow external beam. (A) Bull. Amer. Phys. Soc. 14, 232 (1969).

Fox, J.D. - See also Barton, M.Q.; Blumberg, L.N.

Frankel, R. Automatic measurement of equilibrium orbits at the Brookhaven AGS. Rev. Sci. Instr. 40, 255-7 (1969).

Frankel, R. The use of computer developed and generated displays in the monitoring and control of accelerators. IEEE Trans. Nucl. Sci. NS-16, No. 3, Pt. 1, 837-9 (1969).

Frankel, R. - See also Barton, M.Q.

Gillani, N.V. and Britton, R.B. Critical currents of superconductors in low fields. (N) Rev. Sci. Instr. 40, 949-51 (1969).

Grordano, S. A new multiperiodic drift tube structure. Informal Report BNL 12910.
Giordano, S. and Hannwacker, J. Field measurements of variable $\beta$ multistem accelerating structure. In Proc. 1968 Proton Linear Accelerator Conference, Brookhaven National Laboratory, May 1968, Pt. 2, pp. 565-9. BNL 50120 (C-54), Dec. 1968.

Giordano, S. and Hannwacker, J. A preliminary study of the TS zero mode and its role in field flattening of multi and tunable stem accelerating structures. Ibid., Pt. 2, pp. 570-8.

Glenn, J.W. - See Barton, M.Q.; Blumberg, L.N.

Gluckstern, R.L. - See Lee, M.J.

GRAND, P. The Brookhaven 200-MeV linac injector construction progress report. In Proc. 1968 Proton Linear ACcelerator Conference, Brookhaven National Laboratory, May 1968, Pt. 1, pp. 17-29. BNL 50120 (C-54), Dec. 1968.

Green, G.K. Accelerator power systems. IEEE Trans. Nucl. Sci. NS-16, No. 3, Pt. 1, 1109-11 (1969).

$\mathrm{H}_{\text {AHN }}, \mathrm{H}$. Analysis of iris-loaded deflectors in the smallpitch approximation. Informal Report BNL 13261.

$\mathrm{H}_{\mathrm{AHN}}, \mathrm{H}$. Extension of the theory of uniform-periodic iris-loaded deflectors to $\pi / 2$-mode biperiodic structures. Informal Report BNL 13718.

$\mathrm{H}_{\mathrm{AHN}}, \mathrm{H}$. Optimization of alternating-periodic irisloaded deflectors for superconducting $\mathrm{rf}$ beam separators. In Proc. 1968 Proton Linear Accelerator Conference, Brookhaven National Laboratory, May 1968, Pt. 2, pp. 52332. BNL 50120 (C-54), Dec. 1968.

$\mathrm{H}_{\mathrm{AHN}}, \mathrm{H}$. And Halama, H.J. Investigation of superconducting niobium cavities at $S$-band. IEEE Trans. $\mathcal{N}$ ucl. Sci. NS-16, No. 3, Pt. 1, 1013-17 (1969).

$H_{\text {aHn, }}$ H. and Halama, H. J. $Q$ measurements of superconducting cavities at $2.868 \mathrm{GHz}$. (A) In Proc. 1968 Proton Linear Accelerator Conference, Brookhaven National Laboratory, May 1968, Pt. 2, p. 774. BNL 50120 (G-54), Dec. 1968.

Hahn, H., Halama, H. J., and Foster, E.H. Q measurements on superconducting cavities at $S$-band. In Proc. 1968 Summer Study on Superconducting Devices and Accelerators, Brookhaven National Laboratory, June 10-July 19, 1968, Pt. 1, pp. 13-17. BNL 50155 (C-55), April 1969.

Hahn, H. ANd Miller, J.M. Coupling losses in superconducting cavities. Ibid., Pt. 1, pp. 18-22.

Halama, H. J. Design problems in superconducting $\mathrm{rf}$ beam separators. Ibid., Pt. 1, pp. 150-64.

Halama, H. J. - See also Hahn, H.

Hannwacker, J. - See Giordano, S.

Hawkins, C. J.B. - See Carithers, W.C. JR.

Herrera, J.C. Experimental study of the horizontal halfintegral corrections in the AGS. Informal Report BNL 13711.

Herrera, J.C. Maximum transmittable phase-space in beam transport systems. Informal Report BNL 11768.

Herrera, J.C. AND Month, M. Investigation of gradient corrections for the AGS. IEEE Trans. Nucl. Sci. NS-16, No. 3, Pt. 1, 187-91 (1969).

Herrera, J.C. ANd Month, M. Theory of dipole perturbations in the AGS. Informal Report BNL 12756.

Herrera, J.C. and Month, M. Theory of gradient stopbands in the AGS. Informal Report BNL 11964.

Hsien, H.C.H. and Soukas, A.V. Slow external beam ejection magnets and power supplies. IEEE Trans. N Nucl. Sci. NS-16, No. 3, Pt. 1, 752-6 (1969). 
HSIEH, H.C.H. - See also Blumberg, L.N.

Jablonski, E. AND Buchanan, V.J. Engineering study of thermal problems in ferrites of AGS accelerating cavities. IEEE Trans. Nucl. Sci. NS-16, No. 3, Pt. 1, 535-9 (1969).

Jackson, J.W. - See Allinger, J.; Danby, G.

Jellett, K. and Parzen, G. Iron magnets without air gaps. Informal Report BNL 13347.

Kasha, H. and Stefanski, R. J. Search for heavy triplets in cosmic rays. Phys. Rev. 172, 1297-303 (1968).

Kasha, H. - See also Carithers, W.C. JR.; Wanderer, P. J. JR.

Keane, J.T., DeVito, B., and Menerney, A. J. The Brookhaven $50-\mathrm{MeV}$ linac rf multiport system. IEEE Trans. Nucl. Sci. NS-16, No. 3, Pt. 1, 362-6 (1969).

Keane, J.T., Lankshear, R.F., Sheehan, J.F., and WitKOVER, R.L. The prototype $\mathrm{rf}$ system for the $200-\mathrm{MeV}$ linac for the alternating gradient synchrotron. Ibid., 351-5.

Keane, J.T., Lankshear, R.F., Sheehan, J.F., and WitKover, R.L. Status of the rf system for the $200-\mathrm{MeV}$ linac injector for the AGS. (A) Bull. Amer. Phys. Soc. 14, 214 (1969).

Kovarik, V.J. and Sluyters, T.J.M. Discussion of H.V. power supply units connected with high gradient tubes in linac preinjectors. In Proc. 1968 Proton Linear Accelerator Conference, Brookhaven National Laboratory, May 1968, Pt. 1, pp. 251-68. BNL 50120 (C-54), Dec. 1968.

LANCASTER, J.H. Design and construction of physical facilities, AGS conversion project. IEEE Trans. $\mathcal{N} u c l$. Sci. NS-16, No. 3, Pt. 1, 174-5 (1969).

Lankshear, R. - See Keane, J.T.

Larsen, R.C. - See Carithers, W.C. JR.; Wanderer, P.J. JR.

LEe, M.J. Steady-state beam loading of a standing wave linac rf system. In Proc. 1968 Proton Linear Accelerator Conference, Brookhaven National Laboratory, May 1968, Pt. 1, pp. 114-22. BNL 50120 (C-54), Dec. 1968.

LEE, M.J. Transient beam loading in standing wave linacs. Ibid., Pt. 1, pp. 108-13.

Lee, M. J., Chasman, R., Peterson, H.K., and GluckSTERN, R.L. A method for calculating frequencies, mode structure, and field flatness in an Alvarez-type linac cavity. IEEE Trans. Nucl. Sci. NS-16, No. 3, Pt. 1, 340-4 (1969).

Lee, M. J., Courant, E.D., Pellegrini, C., And Sessler, A.M. Beam amplitude behavior upon crossing a linear coupling resonance with damping in one dimension. Ibid., 176-9; Informal Report BNL 13510.

Leipuner, L.B. - See Carithers, W.G. Jr.; Wanderer, P. J. JR.

LEvINE, G.S. The Brookhaven AGS ring radiation monitoring system. (A) In Proc. 2nd Int. Conf. Accelerator Dosimetry and Experience, Stanford Linear Accelerator Center, Nov. 1969 (in press).

Levine, G.S. AND Moore, W.H. Beam stop studies at the Brookhaven AGS. (A) Ibid.

LeVine, G.S. ANd Moore, W.H. Stopping a 200-MeV, 40-kW proton beam. (A) Bull. Amer. Phys. Soc. 14, 224 (1969).

LEVINE, G.S. - See also Fox, J.D.

MaInturf f, A.D. Composite materials. In Proc. 1968 Summer Study on Superconducting Devices and Accelerators,
Brookhaven National Laboratory, June 10-July 19, 1968, Pt. 2, pp. 465-77. BNL 50155 (C-55), April 1969.

McInturfF, A.D. Coupling of (Ti-Nb) filaments in a superconducting multifilament composite conductor. J. Appl. Phys. 40, 2080-2 (1969).

MaInturff, A.D. Observations of flux jump behavior related to various changes of geometry, and thermal and electrical environment. In Proc. 1968 Summer Study on Superconducting Devices and Accelerators, Brookhaven National Laboratory, June 10-July 19, 1968, Pt. 2, pp. 612-18. BNL 50155 (C-55), April 1969.

MaInTURFF, A.D. AND Paskin, A. Magnetization studies in $\mathrm{Nb}_{3} \mathrm{Sn}$ and Ti-Nb alloys. J. Appl. Phys. 40, 2431-40 (1969).

McInturff, A.D. - See also Schweitzer, D.G. (Applied Science).

MaNerney, A. J. - See Keane, J.T.

Miller, J.M. - See Hahn, H.

Month, M. Explanation of the $\nu$ shifts with momentum in the AGS. Informal Report BNL 13316.

Month, M. Momentum compaction change with quadrupoles. Informal Report BNL 13314.

Month, M. Shift of the $\nu$ value with sextupoles. Informal Report BNL 13315.

Month, M. - See also Barton, M.Q.; Herrera, J.C.; van SteEnbergen, A.

Moore, W.H. Jr. - See Levine, G.S.; Jesseph, J.E. (Medical); Tisljar-Lentulis, G.M. (Medical).

Morgan, G.H. The Bean model of AC losses in type II superconductors. Informal Report BNL 13200.

Morgan, G.H. A simple model of the Smith superconducting cable. Informal Report BNL 13716.

Morgan, G.H. Theory of the twisted multicore superconducting wire in a uniform magnetic field. Submitted to J. Appl. Phys.

Morgan, G.H. Two-dimensional, uniform current density, air-core coil configurations for the production of specified magnetic fields. IEEE Trans. Nucl. Sci. NS-16, No. 3, Pt. 1, 768-9 (1969).

Morgan, G.H. and Dahl, P.F. AC losses in magnets made of $\mathrm{Nb}_{3} \mathrm{Sn}$ ribbon. In Proc. 1968 Summer Study on Superconducting Devices and Accelerators, Brookhaven National Laboratory, June 10-July 19, 1968, Pt. 2, pp. 559-66. BNL 50155 (C-55), April 1969.

Morgan, G.H., Dahl, P.F., Sampson, W.B., and Britton, R.B. Measurements of energy losses in pulsed superconducting magnets. J. Appl. Phys. 40, 1821-9 (1969).

Morgan, G.H. - See also DaHL, P.F.; SAMPson, W.B.

Nawrocky, R.J. - See Barton, M.Q.; Blumberg, L.N.; Fox, J.D.

Nishikawa, T. Note on the dispersion relation of periodic cavity chains with two coupled passbands. Informal Report BNL 13348.

Oleksiuk, L. First-order beam dynamics programs using linearized space-charge forces. Informal Report BNL 13710.

Oostens, J. - See Prunster, S.

PARzEN, G. Iron magnets without air gaps. IEEE Trans. Nucl. Sci. NS-16, No. 3, Pt. 1, 770-1 (1969).

Parzen, G. A mesh-iteration program for field calculations in linac cavities. In Proc. 1968 Proton Linear Accelerator Conference, Brookhaven National Laboratory, May 1968, Pt. 2, pp. 537-42. BNL 50120 (C-54), Dec. 1968. 
Parzen, G. Superconducting FFAG accelerators. In Proc. 1968 Summer Study on Superconducting Devices and Accelerators, Brookhaven National Laboratory, June 10-July 19, 1968, Pt. 3, pp. 1052-8. BNL 50155 (C-55), April 1969.

Parzen, G. Superconducting magnetic dipoles. Ibid., Pt. 3, Pp. 860-5.

Parzen, G. - See also Jellett, K.

Pease, R.L. Longitudinal instabilities in synchrotrons at transition. Informal Report BNL 13266.

Pellegrini, C. - See Lee, M.J.

Peterson, H.K. - See Lee, M.J.

Polk, I. - See Allinger, J.

Post, J.A. and Cottingham, J.G. Component failure vs. voltage rating, Accelerator Department survey. Informal Report BNL 13525.

Prunster, S., Wang, C.L., Yuan, L.C.L., and Oostens, J. Transition radiation from relativistic charged particles passing aluminum and silver foils. Phys. Letters 28B, 47-50 (1968).

RAKA, E.C. Damping bunch shape oscillations in the Brookhaven AGS. IEEE Trans. Nucl. Sci.NS-16, No. 3, Pt. 1, 182-6 (1969).

Rakowsky, G. and Tranis, A. Nonlinear effects in "linear" ferrites at high rf fields. Ibid., 543-5.

Repeta, L.E. - See Fox, J.D.

Sampson, W.B. Pulsed superconducting magnets. In Proc. 1968 Summer Study on Superconducting Devices and Accelerators, Brookhaven National Laboratory, June 10-July 19, 1968, Pt. 3, pp. 908-12. BNL 50155 (C-55), April 1969.

SAMPSon, W.B. Summary of the fifth week of the 1968 summer study. Ibid., Pt. 3, pp. 962-6.

SAMPSON, W.B. A 2000-GeV superconducting synchrotron. Ibid., Pt. 3, pp. 998-1001.

Sampson, W.B., Britton, R.B., Morgan, G.H., Daht, P.F., AND BlewetT, J.P. Superconducting synchrotron magnets. IEEE Trans. Nucl. Sci. NS-16, No. 3, Pt. 1, 720-2 (1969).

Sampson, W.B. - See also Dahl, P.F.; Morgan, G.H.

Sessler, A.M. - See Lee, M. J.

Sheehan, J. - See Keane, J.T.

SLuYTERS, T. J.M. Characteristics of the AGS high brightness ion source. In Proc. Int. Conf. Ion Sources, Saclay, France, June 1969 (in press).

Sluyters, T.J.M. Recent progress in linac pre-accelerators. In Proc. 1968 Proton Linear Accelerator Conference, Brookhaven National Laboratory, May 1968, Pt. 1, pp. 22839. BNL 50120 (C-54), Dec. 1968.

SLuYTERs, T. J.M. Some considerations associated with high current accelerator ion sources. IEEE Trans. Nucl. Sci. NS-16, No. 3, Pt. 1, 29-34 (1969).

Sluyters, T. J.M. - See also Agritellis, C.; Chasman, R.; KovarIK, V. J.

Smith, C.A. - See Carroll, A.S. (Physics).

Smith, L.W. - See Carithers, W.C. Jr.; Wanderer, P. J. JR.

Soukas, A.V. - See Barton, M.Q.; Blumberg, L.N.; Fox, J.D.; Hsieh, H.C.H.

Stefanski, R. J. - See Kasha, H.; Wanderer, P. J. JR.

STEWART, C. Real-time executive for a small computer system. IEEE Trans. Nucl. Sci. NS-16, No. 3, Pt. 1, 8802 (1969).

Tranis, A. - See Rakowsky, G. van Steenbergen, A. Two-turn extraction from an injector synchrotron. IEEE Trans. Nucl. Sci. NS-16, No. 3, Pt. 1, 246-54 (1969).

van Steenbergen, A. and Month, M. Three-turn resonant fast extraction. (A) In Proc. 7th Int. Conf. High Energy Accelerators, Yerevan, Armenian SSR, Aug. 27-Sept. 2, 1969 (in press).

VoGEL, U. The measurement of AGS spatial beam density distributions by means of flipping targets. IEEE Trans. Nucl. Sci. NS-16, No. 3, Pt. 1, 905-8 (1969).

Vogel, U. A system for high field pulsing and demagnetizing of multipole magnets in the AGS. Ibid., 750-6.

Wanderer, P.J. Jr., Adair, R.K., Stefanski, R. J., Ankenbrandt, C.M., Kasha, H., Larsen, R.C., LeIPUNeR, L.B., AND SMITH, L.W. Search for an intermediate vector boson. (A) Bull. Amer. Phys. Soc. 14, 50 (1969).

WANG, C.L. Alpha production by $28.5-\mathrm{BeV}$ proton incident on beryllium oxide. Phys. Rev. Letters 22, 1011 -3 (1969); Errata: Ibid., 1233.

W ang, C.L. - See also Carroll, A.S. (Physics); PrunSTER, $\mathrm{S}$.

Wangler, T.P. - See Carithers, W.C. JR.

W ATts, A.R. AND CoRnish, G.W. Improving information exchange between the AGS and its experimenters. IEEE Trans. Nucl. Sci. NS-16, No. 3, Pt. 1, 840-3 (1969).

Wheeler, G.W. The AGS Conversion Project, Brookhaven Lecture Series No. 69, Oct. 18, 1967. BNL 50131 (T-506).

Wheeler, G.W. Progress in proton linear accelerators. IEEE Trans. Nucl. Sci. NS-16, No. 3, Pt. 1, 345-50 (1969).

Witkover, R.L. High voltage capacitor bank charging through a series vacuum tube. Rev. Sci. Instr. 40, 566-73 (1969).

Wrtkover, R.L. Pulse width modulated optical data link. Ibid., 469-72.

Witkover, R.L. - See also KEANE, J.T

Yamin, P., Berley, D., Kofler, R., Mann, A., Meisner, G., Yamamoto, S., Tanenbaum, W., Thompson, J., and

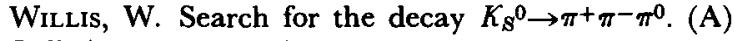
Bull. Amer. Phys. Soc. 14, 519 (1969).

Yamin, P. - See also Bìrley, D.

\section{Applied Mathematics Department}

Baker, G.A. JR. A certain unfolding problem. J. Comput. Phys. 3, 486-93 (1969).

Baker, G.A. JR. Error bounds for Padé approximants. J. Math. Phys. 10, 814-20 (1969).

BAKER, G.A. JR. Linked-cluster expansion for the graphvertex coloration problem. Submitted to J. Combinatorial Theory.

Baker, G.A. JR. The Padé approximant method, and some related generalizations. In Padé Approximants, Theory and Applications, G.A. Baker, Jr. and J.L. Gammel, Editors, Academic, New York (in press).

Baker, G.A. JR. ANd Gaunt, D.S. Low temperature exponents for the Ising model from high temperature series. (A) Presented at Amer. Phys. Soc. Meet., Washington, D.C., April 1969. 
BaKer, G.A. JR. AND Kahane, J. Critical point singularities of the perturbation series for the ground state of a many fermion system. J. Math. Phys. (in press).

BAKER, G.A. JR. - See also GAunt, D.S.

Baldwin, R. Chemical Titles. A Computer Information Retrieval Data System. BNL 50119 (T-499), May 1968.

Bowers, R.G. - See Domb, C.

Brenner, J. - See de Pillis, J.

Campbell, G., Fuchel, K., and Heller, S. Extended core storage as an adjunct to multiprogramming operating systems. Informal Report BNL 12273.

DE PILlis, J. Convexity properties of a generalized numerical range. Submitted to Trans. Amer. Math. Soc.

de Pillis, J. and Brenner, J. Generalized elementary symmetric functions and quaternion matrices. Submitted to J. Linear Algebra and Its Applications.

DOMB, G. AND Bowers, R.G. Specific heat of europium sulphide, a comparison between theory and experiment. Submitted to Phys. Rev. Letters.

Dürre, K. Properties of sets of colorations. Informal Report BNL 13768.

Fuchel, K. - See Campbelt, G.

Gaunt, D.S. Exact series-expansion study of the monomer-dimer problem. Phys. Rev. 179, 174-86 (1969).

GAUNT, D.S. AND BAKER, G.A. JR. Low temperature critical exponents from high temperature series: The Ising model. Submitted to Phys. Rev.

Gaunt, D.S. - See also Baker, G.A. Jr.

Goldstein, C.I. Analytic perturbations of the operator - $\Delta$. J. Math. Anal. Appl. 25, 128-48 (1969).

Goldstein, C.I. Eigenfunction expansions and scattering theory for certain infinite domains. (A) Amer. Math. Soc. Notices (in press).

GoldsteIN, C.I. Eigenfunction expansions and similarity for certain nonselfadjoint operators. Bull. Amer. Math. Soc. 75, 550-3 (1969).

Goldstein, C.I. Eigenfunction expansions associated with the Laplacian for certain domains with infinite boundaries. Pt. I, Trans. Amer. Math. Soc. 135, 1-31 (1969); Pt. II, Ibid., 33-50; Pt. III, Ibid. (in press).

Heller, S. - See Campbell, G.

KAHANE, J. Algorithm for reducing contracted products of $\gamma$ matrices. J. Math. Phys. 9, 1732-8 (1968).

KAHANE, J. Grassman algebras for proving theorems on pfaffians. Submitted to J. Combinatorial Theory.

KaHANE, J. On the number of pairings among classes of like objects. Submitted to J. Combinatorial Theory.

Kahane, J. - See also Baker, G.A. JR.

Karplus, M. - See TANG, K.T.

Kawagutr, M. Flying image digitizer. A high-speed digitizer for track chamber photographs. In Proc. Int. Conf. Instrumentation for High-Energy Physics, Versailles, France, Sept. 1968 (in press).

KawaGuTI, M. A proposal for a new digitizer of bubble chamber photographs. Nucl. Instr. Methods 63, 57-60 (1968).

KLEINMAN, B.S. Gaussian transform evaluation of multicenter integrals with a shielded-coulomb potential: $e^{-\gamma r} / r$. J. Chem. Phys. 49, 3323-4 (1968).

KLEINMAN, B.S. The transformation of four-center, twoelectron integrals into one-electron integrals. Submitted to J. Chem. Phys.
Kleinman, B.S. and TAng, K.T. A comparison of quantum and classical theories of an idealized three-body rearrangement collision. J. Chem. Phys. (in press).

K LeINMAN, B.S. AND TANG, K.T. Solution of the reduced wave equation in an infinite domain. (A) Presented at Amer. Math. Soc. Meet., New Orleans, Jan. 1969.

Kleinman, B.S. - See also TANg, K.T.

LICK, D.W. A divergence theorem for a non-linear Dirichlet problem. (N) J. Comput. Phys. 4, 142-3 (1969).

Lick, D.W. The remarkable Bernoulli family. Math. Teacher 62, 401-9 (1969).

LICK, D.W. A singular Cauchy problem. J. Math. Anal. Appl. (in press).

Lick, D.W. Why not mathematics? Math. Teacher (in press).

Osher, S. On systems of difference equations with wrong boundary values. Math. Computation 23, 567-72 (1969).

Osher, S. Stability of difference approximations of dissipative type for mixed initial-boundary value problems. I. Ibid. 335-40.

Osher, S. Systems of difference equations with general homogeneous boundary conditions. Trans. Amer. Math. Soc. 137, 177-201 (1969).

Peskin, A. Brooknet II. (A) Presented at AEC Computer Inform. Meet., Stanford, Calif., March 1969.

Pincus, J.D. Commutators and systems of singular integral equations. I. Acta Math. 121, 219-49 (1968).

Pincus, J.D. On Wiener-Hopf integral equations. Submitted to Amer. J. Math.

Pincus, J.D. And Rovnyak, J. A representation theorem for determining functions. Submitted to Proc. Amer. Math. Soc.

Robertson, J.E. An improved recursive method for finding multiplicative inverses, modulo $p$. Submitted to $J$. Ass. Comput. Machinery.

Rovnyak, J. - See Pincus, J.D.

Sirovich, L. - See Thurber, J.K.

TAINITER, M. Algebraic approach to stopping variable problems: Representation theory and applications. Submitted to Ann. Math. Statistics.

TAIniter, M. A characterization of idempotents. J. Combinatorial Theory 5, 370-3 (1968).

TAIniter, M. Generating functions on idempotent semigroups with application to combinatorial analysis. Ibid., 273-88.

TAIniter, $M$. Incidence algebras on generalized semigroups. I. Submitted to J. Combinatorial Theory.

Tang, K.T., Kleinman, B., and Karplus, M. A solvable quantum-mechanical model of three-body rearrangement scattering. J. Chem. Phys. 50, 1119-26 (1969).

TANG, K.T. - See also KLeInman, B.

Thurber, J.K. On limit points for roots of dispersion laws of model equations. In Rarefied Gas Dynamics (Proc. 6th Int. Symp., Cambridge, Mass., July 1968), Advan. Appl. Mech. Suppl. 5, Vol. 1, pp. 71-80, L. Trilling and H.Y. Wachman, Editors, Academic, New York, 1969.

Thurber, J.K. ANd Sirovich, L. Wave propagation and other spectral problems in kinetic theory. II. J. Math. Phys. 10, 239-49 (1969).

Thurber, J.K. - See also Dorning, J. J. (Applied Science).

VAN RYZIN, J. On strong consistency of density estimates. Submitted to Ann. Math. Statistics. 


\section{Department of Applied Science}

Adams, P.D. Concentration fluctuations in liquid binary alloys exhibiting a miscibility gap. (A) Presented at Conf. Physics and Liquids, Norwich, England, April 1969.

Adams, P.D. Model for the resistivity of liquid mercury alloys. Phys. Rev. Letters 21, 1324-7 (1968).

Adler, G. Radiation induced reactions in crystalline amides. (A) Acta Cryst. A25, Pt. S3, S225 (1969).

Adler, G. and Baysal, B. Some solid-state aspects of vinyl polymerization in organic crystals. Mol. Cryst. (in press).

Adler, G. - See also Baysal, B.; Faugitano, A.; PeROTTI, A.

Anderson, E.W. - See Mackenzie, D.R.

Aronson, S. and Salzano, F. J. Electrochemical study of the sodium-graphite reaction. (N) Nucl. Sci. Eng. (in press).

Aronson, S. and Salzano, F. J. The solid-state reaction of lithium hydride and aluminum. Inorg. Chem. 8, 15412 (1969).

Aronson, S., Salzano, F. J., and Bellafiore, D. Thermodynamic properties of the potassium-graphite lamellar compounds from solid-state emf measurements. $J$. Chem. Phys. 49, 434-9 (1968).

Aronson, S. - See also Auskern, A.B.; Salzano, F. J.

Ascarelli, P. Velocity of sound compressibility in liquid metals. Phys. Rev. 173, 271-4 (1968).

Attardo, M. J., Davidson, J.L., Galligan, J.M., and Jeannotte, D. Defect studies in metals. In Proc. Int. Conf. Strength of Metals and Alloys, Tokyo, Sept. 1967, Trans. Japan Inst. Metals 9 Suppl., 209-14 (1968).

Attardo, M. J. - See also Galligan, J.M.

Auskern, A.B. A model for the strength of cementpolymer and concrete-polymer systems. Informal Report BNL 13493.

Auskern, A.B. The strength of concrete-polymer systems. Informal Report BNL 12890.

Auskern, A.B. And Aronson, S. Electrical properties of thorium borides. J. Chem. Phys. 49, 172-6 (1968).

Auskern, A.B. ANd Aronson, S. Electrical properties of (Th, U)C thorium carbide-uranium carbide solid solutions. (L) J. Nucl. Mater. 29, 345-8 (1969).

Backstrom, J.E. - See Steinberg, M.

Ballantine, D.S. - See Metz, D. J.

Bargiuk, L. - See Galanter, L.

Bartlett, C. - See Emma, L.C.

Baysal, B. and Adler, G. Molecular weight distribution in the solid-state polymerization of acrylamide. $J$. Polymer Sci. (in press).

Baysal, B. - See also Adler, G.

Becker, W.W. - See Isaacs, H.S.; Susskind, H.

BEHL, W.K. Thermodynamic and transference number measurements in mixtures of molten $\mathrm{LiCl}-\mathrm{CdCl}_{2}$ by e.m.f. method. (A) Presented at Fused Salt Symposium, New York, May 1969.

Bellafiore, D. - See Aronson, S.

Beller, M. - See Powell, J.; Steinberg, M.

Berry, H.C. - See DWyer, O.E.

Bezler, P. - See Steinberg, M.

Bhat, M.R., Ghrien, R.E., Garber, D.I., and Wasson, O.A. Use of the HFBR fast-neutron chopper to mea- sure half-lives of isomeric states populated by neutron capture. (A) Bull. Amer. Phys. Soc. 13, 1422 (1968).

Bhat, M.R. - See also Beer, M. (Physics); Lone, M.A. (Physics); Price, D.L.; Wasson, O.A.

Bloch, F.W. and Mackenzie, D.R. Radiolysis of cyclic fluorocarbons. II. Perfluoroaromatics at elevated temperatures. J. Phys. Chem. 73, 552-7 (1969).

Bracker, J.M. - See Wilmshurst, J.K.

BRYNER, J.S. AND CHOW, J.G.Y. Fast-neutron irradiation effects on dislocation structures in iron strained at ambient temperature. Acta Met. (in press).

Castleman, A.W. JR., Horn, F.L., and Lindauer, G.C. On the behavior of aerosols under fast reactor accident conditions. (A) In Proc. Int. Congr. Diffusion of Fission Products, Saclay, France, Nov. 1969 (in press).

Gastleman, A.W. Jr. and Tang, I.N. Fission product vaporization from sodium systems. In Proc. Int. Conf. Sodium Technology and Large Fast Reactor Design, Argonne, Ill., Nov. 1968 (in press).

Castleman, A.W. JR. And TAng, I.N. Vaporization of fission products from irradiated uranium. II. Some observations on the chemical behavior of fission products iodine and cesium. Submitted to J. Inorg. $\mathrm{Nucl}$. Chem.

Castleman, A.W. Jr. - See also Horn, F. L.; Tang, I.N.

Chandramoleshwar, K. - See Price, G.A.

Chen, H.T. and Hill, F.B. Radiation-induced polymerization in continuous stirred tank reactors. Presented at Amer. Inst. Chem. Engrs. Meet., New Orleans, March 1969.

Chen, J.C. Incipient boiling superheats in liquid metals. J. Heat Transfer 90, 303-12 (1968).

Chen, J.C. and Kalish, S. An experimental investigation of two-phase pressure drop for potassium with and without net vaporization. (A) To be presented at 4th Int. Heat Transfer Conference, Paris, Aug. 31-Sept. 5, 1970.

Ghen, J.C., Kalish, S., and Schoener, G.A. Probe for detection of voids in liquid metals. Rev. Sci. Instr. 39, 1710-13 (1968).

CHEN, J.C. AND Yu, W.S. Entrance region and variable heat flux effects in turbulent heat transfer to liquid metals flowing in concentric annuli. Submitted to Int. J. Heat Mass Transfer.

CHow, J.G.Y. Effect of irradiation on mechanical properties of cobalt base alloys. In Proc. ASTM Symp. Effects of Radiation on Structural Metals, San Francisco, June 1968 (in press).

CHow, J.G.Y. Improvement of high temperature ductility of irradiated Fe-Ni-Cr alloys. (A) In Proc. IAEA Symp. Radiation Damage in Reactor Materials, Vienna, June 1969 (in press).

Chow, J.G.Y. - See also Bryner, J.S.

Clarke, J.T. AND Fox, B.R. The rate and heat of vaporization of graphite above $3000^{\circ} \mathrm{K}$. J. Chem. Phys. (in press).

Colombo, P., Fontana, J., and Steinberg, M. Radiation-induced copolymerization of ethylene and sulfur dioxide in the liquid and gas phases. J. Polymer Sci. 6A-1, 3201-15 (1968).

Colombo, P., Gabarain, R.V.A., Fontana, J., and Steinberg, $M$. A combined radiation and chemical catalyst 
system for improving polymerization rates. (A) Presented at Amer. Nucl. Soc. Meet. Radiation and Isotope Technology in Latin American Development, San Juan, Puerto Rico, May 1969.

Colombo, P. - See also Manowitz, B.; Steinberg, M. Conant, J.F. - See Palmedo, P.F.

Court, A. and Higinbotham, W.A. Survey of nuclear materials and possible measurement techniques. (A) Presented at Inst. Nucl. Mater. Management Meet., Las Vegas, April 1969.

Court, A. - See also Higinbotham, W.A.

Gullen, D.E. PløtfB (preliminary description). A code to list and plot ENDF/B data. In Informal Report BNL 13582.

Davidson, J.L. - See Attardo, M. J.

Davies, H.A. The absolute thermoelectric power of some dilute liquid sodium alloys. Phys. Chem. Liquids (in press).

Diamond, D. J. AND YIP, S. Use of time moments in the space-time slowing-down problem. (A) Trans. A mer. Nucl. Soc. 12, 211 (1969).

Dickey, J.M. And Paskin, A. Computer simulation of lattice dynamics in crystals. (A) Bull. Amer. Phys. Soc. 13, 1377 (1968).

Dickey, J.M. And Paskin, A. Phonon spectrum changes in small particles and their implications for superconductivity. Phys. Rev. Letters 21, 1441-3 (1968).

Dickey, J.M. - See also Paskin, A.

Dikeou, J.T. - See SteinberG, M.

Dolphin, D. - See Fajer, J.; Felton, R.H.

Dorning, J.J. and Nicolaenko, B. Time-dependence of finite, subcritical fast multiplying assemblies. (A) Trans. Amer. Nucl. Soc. 11, 577-8 (1968).

Dorning, J. J., Nicolaenko, B., and Thurber, J. The asymptotic behavior of the special function $\mu(x, \beta, \alpha)$. (A) Presented at Amer. Math. Soc. Meet., New York, April 1969.

Dorning, J. J., Nicolaenko, B., and Thurber, J.K. An integral identity due to Ramanujan which occurs in neutron transport theory. J. Math. Mech. (in press).

Dorning, J. J., Nicolaenko, B., and Thurber, J.K. Some comments on pseudo-mode solutions of the initialvalue problem obtained by analytic continuation: Thermal and fast systems. Presented at Conf. Transport Theory, Blacksburg, Va., Jan. 1969.

Dorning, J. J., Nicolaenko, B., And Thurber, J.K. Some mathematical considerations on Cauchy integrals related to pulsed neutron dispersion relations. Ibid.

Dorning, J. J., Nicolaenko, B., and Thurber, J.K. Unstable decay states of far subcritical fast assemblies. (A) Trans. Amer. Nucl. Soc. 12, 251-2 (1969).

Dorning, J. J. and Thurber, J.K. An explanation of the apparent violation of the maximum absorption theorem. (A) Ibid. 11, 579-80 (1968).

Dorning, J.J. and Thurber, J.K. Purely discrete neutron wave phenomena in the continuum region. (A) Ibid., 580-1.

Dorning, J. J. And Thurber, J.K. Some comments on observable modes and pseudo-modes imbedded in the continuous spectrum of the linear Boltzmann operator for the wave and poisoning problem. Presented at Conf. Transport Theory, Blacksburg, Va., Jan. 1969.
Drager, R.F., Emma, L.C., Fedelem, J. J., Hatch, L.P., Strickland, G., Tuthill, E. J., and Weth, G.G. Development of the Phosphate Glass Process for Ultimate Disposal of High-Level Radioactive Wastes. BNL 50130 (T-505), Jan. 1968.

DWYER, O.E. Heat transfer to liquid metals flowing inline through unbaffled rod bundles: A review. Nucl. Eng. \& Design (in press).

DWYER, O.E. Liquid metal boiling working group. (L) In Liquid Metal Boiling Working Group Progress Letters, 3rd issue, pp. 37-9, May 1969, EURATOM, Ispra, Italy. EUR/C-IS/473/69 e: IP/312/W.

DWYER, O.E. On incipient-boiling wall superheats in liquid metals. Int. J. Heat Mass Transfer (in press).

DWYER, O.E. AND BERRy, H.C. Effects of cladding thickness and thermal conductivity on heat transfer to liquid metals flowing in-line through bundles of closely spaced rods. To be presented at 4 th Int. Heat Transfer Conference, Paris, Aug. 31-Sept. 5, 1970.

Dwyer, O.E. and Berry, H.C. Slug-flow Nusselt numbers for in-line flow through unbaffled rod bundles. Submitted to Nucl. Sci. Eng.

Dwyer, O.E., Hlavac, P. J., and Helfant, M.A. Heat transfer to mercury flowing in-line through an unbaffled rod bundle: Effect of rod displacement on local surface temperature and heat flux. (A) To be presented at 4th Int. Heat Transfer Conference, Paris, Aug. 31Sept. 5, 1970.

Dwyer, O.E. - See also Hlavac, P. J

Egan, J. J. - See Heus, R. J.

Emma, L.C., Johnson, R., Bartlett, C., and Hatch, L.P. Disposal of Solid Wastes Generated in Fluidized-Bed Fluoride Volatility Fuel Reprocessing. BNL 50139 (T-511), Oct. 1968.

Emma, L.C. - See also Drager, R.F

Epstein, S.G. Electromigration and diffusion of cadmium and indium in liquid sodium. Phys. Chem. Liquids 1, 10919 (1968).

Epstein, S.G. Evidence for a correlation between electromigration and electrical resistivity. Trans. Met. Soc. AIME 242, 1771-4 (1968).

Epstein, S.G. and Weeks, J.R. Possible correlations between thermoelectric potentials, electromigration, and liquid metal corrosion. Submitted to J. Electrochem. Sac.

FaJER, J. The state of iron(III) chloride in nonaqueous solvents. (N) J. Inorg. Nucl. Chem. 30, 2259-62 (1968).

Fajer, J., Borg, D.C., Dolphin, D., and Felton, R.H. Oxidation of porphyrins: Cations and cation radicals. (A) Presented at Amer. Chem. Soc. Meet., Minneapolis, April 1969.

Fajer, J. - See also Felton, R.H.

Fallon, P.T. - See Hill, F.B.

Faucitano, A. And Adler, G. Annealing and recrystallization in the solid state polymerization in solid solutions of methacrylamide and isobutyramide. J. Macromol. Sci. (in press).

Faucitano, A., Perotti, A., And Adler, G. Reactions of gases with irradiated organic solids. II. Reactions of propionamide, $n$-butyramide, isobutyramide, and stearamide with oxygen and nitric oxide. Mol. Cryst. 9, 297 321 (1969).

Faucitano, A. - See also Perotti, A. 
Fedelem, J. J. - See Drager, R.F.

FÉlberbaum, J. - See Honeck, H.C.

Feldberg, S.W. Digital simulation: A general method for solving electrochemical diffusion-kinetic problems. In Electroanalytical Chemistry, Vol. 3, p. 199, A. J. Bard, Editor, Marcel Dekker, New York, 1969.

FeldberG, S.W. Theory of regenerative second-order mechanisms in chronoamperometry. The paradox of disproportionation. J. Phys. Chem. 73, 1238-43 (1969).

FELDBerG, S.W. Theory of relaxation of the diffuse double layer following coulostatic charge injection. Submitted to J. Phys. Chem.

Feldberg, S.W. - See also Kissel, G.; Nelson, R.F.

Felder, R.M. Classical differential cross sections for scattering from a repulsive inverse-power potential. (L) J. Chem. Phys. 49, 1438-9 (1968).

FELder, R.M. AND Hill, F.B. Mixing effects in chemical reactors. I. Reactant mixing in batch and flow systems. Chem. Eng. Sci. 24, 385-97 (1969); II. Chain reactions in batch and flow systems. Submitted to Ind. Eng. Chem. Fundamentals.

Felder, R.M. And Zucker, M.S. Code for calculating energy and angular spectra of beta particles emerging from a semi-infinite slab source. (A) Presented at Amer. Nucl. Soc. Meet., San Juan, Puerto Rico, May 1969.

Felder, R.M. - See also Zucker, M.S.

Felton, R.H., Dolphin, D., Borg, D.C., and Fajer, J. Cations and cation radicals of porphyrins and ethyl chlorophyllide a. (L) J. Amer. Chem. Soc. 91, 197-8 (1969).

Felton, R.H. - See also Fajer, J.

Ferrari, L.A. On the beam disruption in a plasma betatron. Phys. Fluids (in press).

Ferrari, L.A. and Zucker, M.S. Plasma betatron study. Informal Report BNL 13274.

FerRari, L.A. ANd Zucker, M.S. Stability of orbits in combined betatron and azimuthal magnetic fields. Phys. Fluids (in press).

Ferrari, L.A. ANd Zucker, M.S. Transient response of magnetic probes. Rev. Sci. Instr. 40, 925-6 (1969).

Ferrari, L.A. - See also Zucker, M.S.

Finkelstein, D. - See Powell, J.R.

Fleitman, A.H. The corrosion of high purity $\mathrm{Fe}, \mathrm{Cr}, \mathrm{Ni}$ and $\mathrm{Co}$ in $660^{\circ}-760^{\circ} \mathrm{C}$ liquid sodium. (A) Presented at 1969 Metals Congr. Meet., Philadelphia, Oct. 1969.

Fleitman, A.H., Romano, A. J., and Klamut, C. J. Corrosion by mercury and its inhibition in once-through 21/4 Cr-1 Mo steel boilers. Nucl. Appl. 3, 737-44 (1967).

Fleitman, A.H. and Sadofsky, J. Precipitation reactions in a cobalt base developmental super alloy containing titanium. (A) Presented at AIME Meet., Pittsburgh, Pa., May 1969.

Fleitman, A.H. - See also Sadofsky, J.

Fontana, J. - See Colombo, P.

Fox, B.R. - See Clarke, J.T.

Gabarain, R.V.A. - See Colombo, P.

Galanter, L. and Krishnamurthy, K. Fudge 4A- $A$ Computer Program for Gamma Dose Rate Distribution From Rectangular Sources. BNL 50126 (T-503), Aug. 1968.

Galanter, L., Rosen, S., Rizzo, F.X., and Bargiuk, L. Gird - A Computer Program for Design of Shuffle-Dwell Gamma Irradiators. BNL 50148 (T-519), March 1969. Galanter, L. - See also Manowitz, B.
Galligan, J.M. Field ion microscope studies of defects. In Vacancies and Interstitials in Metals, A. Seeger et al., Editors, North-Holland, Amsterdam (in press).

Galligan, J.M. On the saturation of radiation hardening. Phys. Letters 28A, 609 (1969).

Galligan, J.M. Some applications of field ion microscopy to point defect problems. (A) Presented at Int. Conf. Vacancies and Interstitials in Metals, Stuttgart, West Germany, Sept. 1968.

Galligan, J.M. and Attardo, M. J. Radiation hardening in platinum. Phys. Status Solidi 27, 383 (1968).

Galligan, J.M. and Inal, O.T. A measurement of radiation hardening and defects in irradiated platinum. (A) Bull. Amer. Phys. Soc. 14, 498 (1969).

Galligan, J.M. and Oku, T. Damage density and size distribution in neutron irradiated tungsten. (A) Presented at AIME Meet., Philadelphia, Oct. 1969.

Galligan, J.M., Schultz, H., and Lachenmann, R. Low temperature strain aging in deformed tantalum. Scripta Met. 3, 401-4 (1969).

Galligan, J.M. - See also Attardo, M.J.; Jeannotte, D.; OKU, T.; Soo, P.

Garcia, N. AND KaO, Y.H. Galvanomagnetic effects in single-crystal films of bismuth. (A) Bull. Amer. Phys. Soc. 14,97-8 (1969).

Garcia, N., Kao, Y.H., and Strongin, M. Anomalies in the resistivity of thin Bi films. (L) Phys. Letters (in press).

GiLAT, J. Algebraic approximation of the combinatorial calculation of nuclear level densities. Submitted to Phys. Rev.

Gilbert, T.W., Newman, L., and Klotz, P. Mixedmetal hydroxycarboxylic acid complexes. The chromium(III) inhibition of the solvent extraction of indium(III). Anal. Chem. 40, 2123-30 (1968).

GoldberG, M.D. The specification and characterization of neutron data. In Neutron Data Compilation - Report of a Panel Held in Brookhaven, 10-14 February 1969, Informal Report IAEA-III, pp. 107-25, 1969.

Goldberg, M.D. and HarveY, J.A. Neutrons. In American Institute of Physics Handbook, 3rd Ed., Chap. 8, McGraw-Hili, New York (in press).

Goldstein, R. Iterative solutions by means of trial operators. J. Math. Phys. 9, 1456-61 (1968).

GolDsteIn, R. Iterative solutions to reactor equations. In Proc. Int. Conf. Utilization of Research Reactors and Reactor Mathematics and Computation, Mexico City, May 1967, Vol. 1, p. 278, CNM-R-2, Reactor del Centro Nuclear de Mexico.

Goldstein, R. And Shotkin, L.M. Increasing the iterative time-step in fast-reactor calculations. (A) Trans. Amer. Nucl. Soc. 12, 148-9 (1969).

Goldstein, R. And Shotkin, L.M. Use of the promptjump approximation in fast-reactor kinetics. $\mathcal{N} u c l$. Sci. Eng. (in press).

Gotoh, Y. and Koyama, K.K. Time transient problem in a fast multiplying system. Submitted to Nucl. Sci. Eng. Grover, J.R. - See Hill.man, M.

Gurinsky, H.R. - See Schweitzer, D.G.

Hahne, R.M.A. - See Hillman, M.

Harvey, J.A. - See Goldberg, M.D.

Hasson, J. - See Liv, C.H. 
Hatch, L.P. - See Drager, R.F.; Emma, L.C.

Helfant, M.A. - See Dwyer, O.E.; Hlavac, P.J.

Hendrie, J.M., Hoffman, K.C., Kouts, H. J.C., Parsick, R. J., Phelps, J.P., Price, G.A., Reich, M., Takahash, H., AND Windsor, H.H. Brookhaven pulsed fast research reactor. Presented at Amer. Nucl. Soc. Meet., Albuquerque, Jan. 1969.

Hendrie, J.M. - See also Hoffman, K.C.

Heus, R. J., Tidwell, T., and Egan, J.J. Investigation of the capacity of the electrical double layer in molten chlorides using a dropping metal electrode. In Molten Salts: Characterization and Analysis, Amer. Chem. Soc. (in press).

Higinbotham, W.A. and Court, A. Survey of nuclear material and possible measurement techniques. Presented at Inst. Nucl. Mater. Management Meet., Las Vegas, April 1969.

Higinbotham, W.A. - See also Court, A.

Hill, F.B., Fallon, P.T., Muller, A.C., and Rothbart, M.A. The application of chemical reactor design principles to problems of dosimetry in flow systems. In Proc. Symp. Utilization of Large Radiation Sources and Accelerators in Industrial Processing, Munich, West Germany, Aug. 1969 (in press).

Hill, F.B., Reiss, N., and Shendalman, L.H. Nonuniform initiation of photoreactions. III. Reactant diffusion in single-step reactions. A.I.Ch.E. J. 14, 798-804 (1968).

Hill, F.B. - See also Chen, H.T.; Feider, R.M.; ShenDaLMaN, L.H.; YEMIN, L.

Hillman, M. and Grover, J.R. Shell-model combinatorial calculations of nuclear level densities. Phys. Rev. (in press).

Hillman, M., Kim, C.K., Shikata, E., and Weiss, A.J. Isotope effects in the Szilard-Chalmers reactions of hafnium and zirconium phthalocyanine. Radiochim. Acta 9, 212-8 (1968).

Hillman, M. and Shikata, E. Cross sections of some reactions of hafnium isotopes with $14.5-\mathrm{MeV}$ neutrons. J. Inorg. Nucl. Chem. 31, 909-13 (1969).

Hillman, M., Weiss, A.J., and Hahne, R.M.A. SzilardChalmers reactions of zirconocene dichloride and of hafnocene dichloride. Submitted to Radiochim. Acta.

Hillman, M. - See also Harbottle, G. (Chemistry); KIEFER, R.L.

Hlavac, P. J., Dwyer, O.E., and Helfant, M.A. Heat transfer to mercury flowing in-line through an unbaffled rod bundle: Experimental study of the effect of rod displacement. I. J. Heat Transfer (in press).

Hlavac, P. J. - See also Dwyer, O.E.

Hobdell, M.R. and Newman, L. The reaction of $\mathrm{HCl}$ with liquid sodium and a sodium-barium alloy. In Proc. Int. Conf. Sodium Technology and Large Fast Reactor Design, Argonne National Laboratory, Nov. 1968 (in press).

Hobdell, M.R. and Newman, L. Some observations on the kinetics of the liquid sodium-hydrogen reaction. (N) J. Inorg. Nucl. Chem. 31, 1843-7 (1969).

Hoffman, K.C. and Hendrie, J.M., Editors. Pulsed fast research reactor project: Management plan. Revision I. Informal Report BNL 13370.

Hoffman, K.G. and Hendrie, J.M., Editors. Pulsed fast research reactor project. Progress report for the period
May 1, 1968, to Oct. 31, 1968. Informal Report BNL 12750; Progress report for the period Nov. 1, 1968, to April 30, 1969. Informal Report BNL 13605.

Hoffman, K.C., Reilly, J.J., Wiswall, R.H., Sheehan, T.V., AND Winsche, W.E. Metal hydride energy storage systems. In IECEC' 68 Record, Vol. 1, pp. 981-5, IEEE, 1968

Hoffman, K.C. - See also Hendrie, J.M.; Winsche, W.E.

HonEcK, H.C. Retrieval subroutines for the ENDF/B system. In Informal Report BNL 13582.

Honeck, H.C. and Felberbaum, J. Checker - A program to check the data on an ENDF/B BCD card image tape; CRECT - A code to correct ENDF/B tapes; DAMMET - A program to delete, alter mode, and merge ENDF/B tapes. In Informal Report BNL 13582.

Horn, F.L. and Castleman, A.W. Jr. $\mathrm{PuO}_{2}-\mathrm{UO}_{2}-\mathrm{Na}$ aerosols produced by vaporization of fast reactor core materials. Presented at Symp. Operating and Developmental Experience in the Treatment of Airborne Radioactive Wastes, New York, IAEA, Aug. 1968.

Horn, F.L. - See also Castleman, A.W. JR.

Hsu, C.J. An exact analysis of low Peclet number thermal-entry-region heat transfer in transversely nonuniform velocity fields. Submitted to A.I.Ch.E. J.

Hsu, C. J. and Lindauer, G.C. Thermal-entry-region heat transfer in magnetohydrodynamic channel flow subject to the boundary condition of the third kind. (A) To be presented at 4th Int. Heat Transfer Conference, Paris, Aug. 31-Sept. 5, 1970.

Hsu, C. J. - See also Lindauer, G.C.

Huszagh, D.W. Human behavior considerations in control system design. Control Eng., p. 88, June 1968.

Inal, O.T. - See Galligan, J.M.

Ingraham, A. - See Salzano, F.J.

ISAACS, H.S. The corrosion of pure iron in sodium. (A) Presented at Metals Congr. Meet., Philadelphia, Oct. 1969.

IsAAGS, H.S. The determination of oxygen concentration and mass transfer coefficients in sodium using uranium. In Proc. Int. Conf. Sodium Technology and Large Fast Reactor Design, Argonne National Laboratory, Nov. 1968 (in press).

Isaacs, H.S., Minushin, B., and Salzano, F.J. A study of reactions between oxygen and impurities in sodium. Ibid.

IsaAcs, H.S., Singer, R.M., AND Becker, W.W. Segregation of metallic impurities in sodium. (A) Presented at Metals Congr. Meet., Philadelphia, Oct. 1969.

IsAacs, H.S. - See also Singer, R.M.

JAMES, S.D. Multilayer oxide films on anodized platinum. J. Electrochem. Soc. (in press).

Jeannotte, D. and Galligan, J.M. A study of radiation damage in tungsten. I. Submitted to Acta Met.

Jeannotte, D. - See also Attardo, M. J.

JefFERSON, L.R. - See SteInBerg, M.

Johnson, R. - See Emma, L.C.

Kalish, S. - See Chen, J.C.

Kammerer, O.F. - See Crow, J.E. (Physics); Strongin, M. (Physics); Thompson, R.S. (Physics).

KaO, Y.H. - See Garcia, N.

Katz, H. and Sears, J.T. Electric field phenomena in fluidized and fixed beds. Can. J. Chem. Eng. 47, 50-3 (1969). 
Katz, H. and Steinberg, M. Design of reformer for fossil-nuclear fueled MHD power cycle. Informal Report BNL 13285.

Kelsch, J. J. - See Steinberg, M.

KiEfer, R.L. AND Hillman, M. Relative yields of ${ }^{58}{ }^{\circ} \mathrm{Co}$, ${ }^{58 m} \mathrm{Co}$, and ${ }^{56} \mathrm{Co}$ produced by low-energy tritons. $J$. Inorg. Nucl. Chem. 31, 915-17 (1969).

Kim, G.K. - See Hillman, M.

Kissel, G. and Feldberg, S.W. Disproportionation of the technetate ion in aqueous alkaline media: An electrochemical study. J. Phys. Chem. (in press).

Klamut, C.J. - See Fleitman, A.H.; Romano, A.J.; WAGHTEL, S.J.

Klotz, P. - See Gilbert, T.W.

Koplik, B. - See ReICH, M.

Kopp, L.E. - See MEtz, D. J.

Kours, H. Systems studies for nuclear materials as safeguards. Presented at Inst. Nucl. Mater. Management Meet., Las Vegas, April 1969.

Kouts, H. - See also Hendrie, J.M.

Koyama, K.K. - See Gotoh, Y.

Krishnamurthy, K. - See Galanter, L.; Rizzo, F.X.

Kukacka, L. - See Manowitz, B.; Steinberg, M.

Lachenmann, R. - See Galligan, J.M.

LANCEFIELD, M.J. The overlapping group method. (A) Trans. Amer. Nucl. Soc. 12, 149-50 (1969).

LanCEFIELD, M. J. Space energy flux synthesis in transport theory. Nucl. Sci. Eng. (in press).

LeE, H. J. And Wilmshurst, J.K. The infrared and Raman spectra of phenyl acetate, phenyl- $d_{5}$ acetate and phenyl acetate- $\dot{d}_{3}$. Submitted to Austr. J. Chem.

LeE, H. J. - See also Sahweitzer, D.G.

Lellouche, G.S. A frequency criterion for Lagrange stability. Submitted to SIAM J. Contr.

Lellouche, G.S. Nøah. BNL 50157 (T-523), Jan. 1969.

Lellouche, G.S. On the location of higher spatial harmonic stability criteria. Informal Report BNL 13053.

Lellouche, G.S. Xenon instability in boiling water reactors. Submitted to Nucl. Sci. Eng.

Lelloughe, G.S. Xenon stability in boiling water reactors. (A) Trans. Amer. Nucl. Soc. 12, 298-9 (1969).

Lindauer, G.C. Thermal Conductivity and Viscosity of Helium at Low Temperatures. BNL 50138 (T-510), Sept. 1968.

Lindauer, G.C. AND Hsu, C. J. Unsteady forced-convection MHD heat transfer in a parallel plate channel. AIAA J. 6, 1973-9 (1968).

Lindauer, G.C. - See also Castleman, A.W. JR.; Hsu, C.J.

Liu, C.H., Newman, L., and Hasson, J. Potentiometric studies on tetrahalonickelate(II) ions in molten dimethyl sulfone. Inorg. Chem. 7, 1868-72 (1968).

Loeb, W. - See Manowitz, B.

Ludewig, H. A method for treating the neutron leakage in small exponential cores. Nucl. Sci. Eng. 35, 289-95 (1969).

Ludewig, H. - See also Price, G.A.

MacKenzie, D.R., Wilson, V.H., and Anderson, E.W. Perfluoro-1,1-dimethylcyclopentane from pyrolysis of perfluorobicyclohexyl. J. Chem. Soc. B 1968, 762-4.

MacKenzie, D.R. - See also Bloch, F.W.

Magurno, B.A. A compilation of neutron-induced threshold reactions. In Proc. USAEC Conf. Small Accelerators,
Oak Ridge, Tenn., April 1968, pp. 97-111, J.L. Duggan, Editor, CONF-680411, 1968.

Manowitz, B. The atmospheric sulfur pollutants program at Brookhaven National Laboratory. (A) Presented at Isotopes Conference, Gaithersburg, Tenn., April 1969.

Manowitz, B., Galanter, L., Loeb, W., Milau, J., and Rizzo, F. The production test irradiation of 30,000 pounds of bacon. Chem. Eng. Progr. Symp. Ser. 83 (Nucl. Eng. Pt. 19) 64, 49-62 (1968).

Manowitz, B., Steinberg, M., Kukacka, L., And CoLOMBO, P. The development of concrete-polymer materials. In Proc. Symp. Utilization of Large Radiation Sources and Accelerators in Industrial Processing, Munich, West Germany, Aug. 1969 (in press).

Manowitz, B. ANd Tucker, W. Determination of sulfur isotope ratios in the atmospheric diagnostics program at BNL. Presented at Amer. Nucl. Soc. Meet., San Francisco, Nov. 1969.

Manowitz, B. - See also Powell, J.; Steinberg, M.

McRiakaRd, S.B. The effects of neutron irradiation on the activation parameters for slip in iron. Acta Met. 16, 969-74 (1968).

McRickard, S.B. Saturation and exposure dependence of the yield stress of irradiated iron. Phil. Mag. 18, 915 (1968).

MEtz, D. J. Radiation-induced ionic polymerization. Advan. Chem. Ser. (in press).

Metz, D. J. and Ballantine, D.S. Gamma radiationinduced ionic vinyl polymerization. Ann. N.Y. Acad. Sci. 155, 468-81 (1969).

Metz, D. J. AND Kopp, L.E. Bibliography of Technical Publications and Reports of the Radiation Division, Brookhaven National Laboratory, July 1965-June 1968 Inclusive. BNL 50132 (L-407), Jan. 1969.

Michael, P. AND Schermer, R.I. Resource Letter REA1 on reactors. Am. J. Phys. 36, 1-10 (1968).

Milau, J. - See Manowitz, B.

Minushin, B. - See IsaAcs, H.S.

Moore, S.O. Optical Model Analysis of the Elastic Scattering of Neutrons by the Lead Isotopes and Bismuth at 0.5, 1.0, and $2.5 \mathrm{MeV}$. BNL $50151^{\circ}$ (T-520), Oct. 1968.

Mughabghab, S.F. and Chrien, R.E. Neutron cross sections and resonance parameters of $\mathrm{Yb}$ isotopes. Phys. Rev. 174, 1400-8 (1968).

Mughabghab, S.F. and Chrien, R.E. S-wave neutron strength functions of the Gd isotopes. Ibid. 180, 1131-8 (1969).

Mughabghab, S.F. and Ghrien, R.E. The thermal cross sections and paramagnetic scattering cross sections of the $\mathrm{Yb}$ isotopes. In Proc. 2nd Conf. Neutron Cross Sections and Technology, Washington, D.C., March 1968, p. 875, D.T. Goldman, Editor, Nat. Bur. Std. U.S. Spec. Publ. 299, Sept. 1968.

Mughabghab, S.F., Garber, D.I., Ghrien, R.E., and WASSON, O.A. Neutron capture $\gamma$ rays from $\operatorname{Pr}^{142}$. (A) Bull. Amer. Phys. Soc. 14, 514 (1969).

Mughabghab, S.F., Garber, D.I., Wasson, O.A., and Chrien, R.E. Resonant neutron capture in Te. (A) Ibid. 13, 1390 (1968).

Mughabghab, S.F. - See also Beer, M. (Physics).

Muller, A.C. - See Hill, F.B. 
Munkelwitz, H.R. An apparatus for measuring the diffusion of impurities in solids. Presented at 4th Symp. for Chemical Technicians, Minneapolis, April 1969. Munkelwitz, H.R. - See also TANG, I.N.

Nelson, R.F. and Feldberg, S.W. Ghronoamperometric determination of the rate of dimerization of some sub. stituted triphenylamine cation radicals. J. Phys. Chem. (in press).

Newman, L. - See Gilbert, T.W.; Hobdell, M.R.; LiU, C.H.

Nicolaenko, B. - See Dorning, J.J.

O'Brien, M. J. - See Richards, P.

Odette, R. - See Susskind, H.

Oku, T. and Galligan, J.M. Deformation studies in tungsten. (A) Presented at AIME Meet., Pittsburgh, Pa., May 1969.

OkU, T. And Galligan, J.M. Quantum mechanical tunneling of dislocations. Phys. Rev. Letters 22, 596-7 (1969).

OKu, T. And Galligan, J.M. Radiation hardening due to point defects and damage zones in tungsten crystals. (A) Presented at AIME Meet., Philadelphia, Oct. 1969.

OKu, T. - See also Galligan, J.M.

Oltmann, A. Brookhaven portable cesium development irradiator manual. Informal Report BNL 13506.

Palmedo, P.F. and Conant, J.F. Neutron diffusion in aluminum-water lattices: Measurements of anisotropy in the continuous eigenvalue region. Nucl. Sci. Eng. (in press).

Parsick, R. J. - See Hendrie, J.M.

PAskin, A. ANd Dickey, J.M. Surface phonons and the enhanced superconducting transition temperature in small particles. (A) Bull. Amer. Phys. Soc. 14, 129 (1969).

Paskin, A. - See also Bronk, B.V. (Physics); Dickey, J.M.; McInturfF, A.D. (Accelerator).

Pearlstein, S. Recent developments in the automated compilation and publication of neutron data. In Proc. 2nd Conf. Neutron Cross Sections and Technology, Washington, D.C., March 1968, p. 1041, D.T. Goldman, Editor, Nat. Bur. Std. U.S. Spec. Publ. 299, Sept. 1968.

Pearlstein, S. The role of the National Neutron Cross Section Center as link between measurer and user of neutron cross sections. (A) Presented at Conf. Computer Systems in Experimental Nuclear Physics, Skytop, Pa., March 1969.

Perotti, A., Faucitano, A., And Adler, G. Reactions of gases with irradiated organic solids. III. Reactions of propionamide, $n$-butyramide, isobutyramide, methacrylamide, valeramide, and stearamide with sulfur dioxide. Mol. Cryst. 9, 323-42 (1969); IV. Reactions of propionamide, $n$-butyramide, isobutyramide, valeramide, and stearamide with ethylene. Ibid., 361-82.

Perotti, A. - See also Faucitano, A.

Phelps, J.P. and Weinstock, E.V. Criticality measurements on nearly homogeneous enriched uraniumgraphite systems. Nucl. Sci. Eng. 34, 237-50 (1968).

Phelps, J.P. - See also Hendrie, J.M.

Popek, J. - See Stephenson, T.E.

Powell, J.R. And Finkelstein, D. Structure of ball lightning. Advan. Geophys. (in press).
Powell, J.R., Steinberg, M., Beller, M., and Manowitz, B. A study of $\mathrm{H}_{2}-\mathrm{O}_{2}$ combustion MHD cycles using combination fossil-nuclear energy input. (A) In Proc. 8th Annu. AIE-ARS Symp. Magnetohydrodynamics, Arnold Air Force Station, Tullahoma, Tenn., April 1968, pp. 14-15, Tullahoma Space Inst., Univ. of Tennessee, Tullahoma.

Price, G.A., Ludewig, H., Schiller, R., and GhandraMOLESHWAR, K. Studies of some plutonium exponential assemblies. (A) Trans. Amer. Nucl. Soc. 12, 244-5 (1969).

Price, G.A. - See also Hendrie, J.M.

Prince, A. Nuclear and physical properties of Cf ${ }^{252}$. In Proc. Symp. Californium-252, New York, Oct. 1968, pp. 23 130, James J. Barker, Editor, CONF-681032, 1969; BNL 50168 (T-530), April 1969.

Prince, A. Thermal neutron cross sections and resonance integrals for transuranium isotopes. In Proc. 2nd Conf. Neutron Cross Sections and Technology, Washington, D.C., March 1968, p. 951, D.T. Goldman, Editor, Nat. Bur. Std. U.S. Spec. Publ. 299, Sept. 1968.

Pruzansky, J. - See Steinberg, M.

Reich, M. AND Koplik, B. Analysis of multidimensional thermoelastic dynamic response of reactor fuel elements. Informal Report BNL 13311.

Reich, M. - See also Hendrie, J.M.

ReIlly, J.J. and Wiswall, R.H. The reaction of hydrogen with alloys of magnesium and nickel, and the formation of $\mathrm{Mg}_{2} \mathrm{NiH}_{4}$. Inorg. Chem. 7, 2254-6 (1968).

Reilly, J.J. - See also Hoffman, K.C.; Winsche, W.E. Reiss, N. - See Hill, F.B.

Richards, P. Technetium-99m - An inexpensive route to a versatile radiosotope. In Proc. Meet. Radiation and Isotope Technology in Latin American Development, San Juan, Puerto Rico, May 1969 (in press).

Richards, P. and Atkins, H.L. Technetium-99m labeled compounds. Jap. Nucl. Med. 7, 165-70 (1968).

Richards, P. AND O'BRIEN, M.J. Rapid determination of ${ }^{99}$ Mo in separated ${ }^{99 m}$ Tc. J. Nucl. Med. 10, 517 (1969).

Richards, P. - See also Atkins, H.L. (Medical); Hauser, W. (Medical).

RIzzo, F.X. Dosimeter selection criteria in radiation research and processing dosimetry. (A) In Proc. 9th Electrical Insulation Conference, Boston, Mass., Sept. 1969 (in press).

Rizzo, F.X., Editor. Proceedings of Third Dosimetry Workshop and Dosimetry Section of ASTM Subcommittee D9/D20. Informal Report BNL 12669.

Rizzo, F.X. and Krishnamurthy, K. Cellulose acetate butyrate dosimeter. (A) Trans. Amer. Nucl. Soc. 12, 61-2 (1969).

Rizzo, F.X. - See also Galanter, L.; Manowitz, B.

Romano, A. J., Wachtel, S. J., and Klamut, C. J. Preliminary corrosion results of Haynes 25 and 304 stainless steel with 4 and 12 ppm oxygen in sodium at temperatures up to $760^{\circ} \mathrm{C}$. In Proc. Int. Conf. Sodium Technology and Large Fast Reactor Design, Argonne National Laboratory, Nov. 1968 (in press).

Romano, A.J. - See also Fleitman, A.H.; Wachtel, S.J.; WEEKS, J.R.

Rosen, S. - See Galanter, L. 
Rothbart, M.A. - See Hill, F.B.

Rothenstein, W. ENDF/B effective resonance integrals. (A) Trans. Amer. Nucl. Soc. 12, 246-7 (1969).

Rubenstein, S. - See Steingerg, M.

Sabine, T.M. And Weinstock, E.V. The collimation of thermal neutrons. $J$. Appl. Cryst. (in press).

Sadofsky, J. and Fleitman, A.H. The correlations between microstructure and mechanical properties of Cobase superalloys. (A) In Proc. 22nd AEC Metallography Meet., San Diego, June 1968 (in press).

Sadofsky, J. - See also Fleitman, A.H.

Salzano, F. J. and Aronson, S. The reaction between cesium and carbon blacks. J. Inorg. Nucl. Chem. 30, 2317-29 (1968).

Salzano, F. J., Aronson, S., and Ingraham, A. Comment on compound formation in the lithium-carbon system. (N) J. Amer. Ceram. Soc. 51, 465 (1968).

Salzano, F. J. - See also Aronson, S.; IsaAcs, H.S.

SASTRE, C. The use of seals as a safeguards tool. Informal Report BNL 13480.

Sato, S. And Steinberg, M. Co $^{60}$ gamma oxidation of aerated aqueous ammonium solutions. Informal Report BNL 13693.

Sato, S. And Steinberg, M. Radiation chemical nitrogen fixation in air-water systems. Informal Report BNL 13692.

Schermer, R.I. - See Michael, P.

Schiller, R. - See Price, G.A.

Schoener, G.A. - See Ghen, J.C.

Schultz, H. - See Galligan, J.M.

Sch weitzer, D.G. Evidence for two types of hysteresis. Submitted to Phys. Rev.

Schweitzer, D.G. Hysteresis in superconductors. III. The effect of surface energies on the shielding and fieldretaining properties of hollow cylinders of type-I superconductors. Phys. Rev. 173, 461-72 (1968).

SchweItzer, D.G. New switching properties of membranes in air-flow and blood-flow systems. Submitted to Amer. J. Physiol.

SGHWEITZER, D.G. The origin of irreversibility from conventional equilibrium concepts. Phys. Letters 27 A, 402-4 (1968).

Schweitzer, D.G. The role of gravity in osmotic equilibria. Submitted to Amer. J. Phys.

Schweitzer, D.G. Superconducting surface current equilibria in applied fields. Phys. Letters 28A, 98-9 (1968).

Schweitzer, D.G., Lee, H. J., and Gurinsky, H.R. Macroscopic thermodynamics of irreversible phase transitions. Submitted to J. Math. Phys.

Schweitzer, D.G. And McInturf, A.D. Flux mobility properties associated with diamagnetism in hysteretic type-II superconductors. Solid State Commun. 6, 885-7 (1968).

SEARS, J.T. Abnormal Bubble Movement and Induced "Roll Cell" Formation in Fluidized Beds. BNL 50142 (T-514), June 1968.

SEARS, J.T. Effect of density and electron scavengers in nitrous oxide radiolysis. (N) J. Phys. Chem. 73, 1143-6 (1969).

Sears, J.T. - See also Katz, H.

Sheehan, T.V. - See Hoffman, K.G.; Winsche, W.E.
Shendalman, L.H. And Hill, F.B. Some limiting radical concentration profiles in a batch photoreactor. (L) Chem. Eng. Sci. 24, 909-11 (1969).

Shendalman, L.H. - See also Hill, F.B.

SHER, R. The $\mathrm{Mn}^{55}$ resonance activation integral. In Proc. 2nd Conf. Neutron Cross Sections and Technology, Washington, D.C., March 1968, p. 253, D.T. Goldman, Editor, Nat. Bur. Std. U.S. Spec. Publ. 299, Sept. 1968.

Shikata, E. - See Hillman, M.

Shotkin, L.M. Comparison of modal and iterative approaches to space and space-time nonlinear problems. Nucl. Sci. Eng. 36, 97-104 (1969).

SHOTkIN, L.M. Instability bounds in linearly stable systems. Ibid. 35, 211-19.

Shotкin, L. M. Some similarities between boiling and condensing flow. (N) Ibid., 154.

SHOtKIn, L.M. A technique for computing the nonlinear coupling of spatial modes. In Proc. Int. Conf. Utilization of Research Reactors and Reactor Mathematics and Computation, Mexico City, May 1967, Vol. 2, p. 983, CNM-R-2, Reactor del Centro Nuclear de Mexico.

Shotkin, L.M. - See also Goldstein, R.

Singer, R.M., Isaacs, H.S., and WeEks, J.R. Measurements of the solubility of $\mathrm{Fe}$ and $\mathrm{Cr}$ in sodium. (A) Presented at Metals Congr. Meet., Philadelphia, Oct. 1969.

Singer, R.M. AND WeEks, J.R. On the solubilities of Cu, $\mathrm{Ni}$, and $\mathrm{Fe}$ in liquid sodium. In Proc. Int. Conf. Sodium Technology and Large Fast Reactor Design, Argonne National Laboratory, Nov. 1968 (in press).

Singer, R.M. - See also IsaAcs, H.S.; Weeks, J.R.

Slater, L.M. Calibration of a polonium-210 alpha source. Informal Report BNL 11926.

StATER, L.M. Extraction of potassium, rubidium, strontium, and cerous polyiodides into nitrobenzene. J. Inorg. Nucl. Chem. 31, 851-4 (1969).

Soo, $\mathrm{P}$. On the rate-controlling slip process in neutronirradiated iron. (A) Presented at AIME Meet., Pittsburgh, Pa., May 1969.

Soo, $\mathrm{P}$. The recovery of neutron-irradiated niobium between $120^{\circ} \mathrm{C}$ and $160^{\circ} \mathrm{C}$. Submitted to Phys. Status Solidi.

Soo, $\mathrm{P}$. Strain aging of niobium in the temperature range 100 to $200^{\circ}$ C. Phys. Status Solidi 32, 815-21 (1969).

Soo, $\mathrm{P}$. A study of thermally activated slip in neutronirradiated iron by stress-relaxation techniques. Trans. Met. Soc. AIME (in press).

Soo, $\mathrm{P}$. Thermal activation of slip in prestrained neutronirradiated iron by flow stress and stress relaxation measurements. Trans. Met. Soc. AIME 245, 985-90 (1969).

Soo, P. and Galligan, J.M. On the temperature dependence of the flow stress in some body-centered cubic metals. Scripta Met. 3, 153-6 (1969).

Stang, L.G. JR. Radionuclide generators: Past, present, and future. Presented at 3rd Annu. Meet. Sociedad Mexicana de Medicina Nuclear, Puebla, Mexico, April 1969.

Steinberg, M. Value and prospects of recovery of uranium from U.S. coal deposits. Presented at 65 th Nat. Meet. Amer. Inst. Chem. Engrs., Cleveland, May 1969.

Steinberg, M. and Beller, M. Cost analysis of chemonuclear vs. electric discharge ozone production. Informal Report BNL 13691. 
Steinberg, M., Kukacka, L.E., Golombo, P., Kelsch, J.J., Manowitz, B., Dikeou, J.T., Backstrom, J.E., and Rubenstein, S. Concrete-Polymer Materials, First Topical Report. BNL 50134 (T-509) and USBR Gen. Rep. 41, Dec. 1968.

Steinberg, M., Pruzansky, J., Jefferson, L.R., and Manowitz, B. Removal of iron from acid mine drainage waste with the aid of high energy radiation. II and III. In Proc. Meet. Coal Industry Advisory Committee to ORSANCO (Ohio River Valley Water Sanit. Comm.), Pittsburgh, Pa., May 1968 (in press).

Steinberg, M., Tugker, W., Waide, C., Beller, M., Bezler, P., and Manowitz, B. Safety review of the Brookhaven chemonuclear in-pile research loop. Informal Report BNL 13001.

Steinberg, M. - See also Colombo, P.; Katz, H.; Manowitz, B.; Powell, J.; Sato, S.

Stephenson, T.E. The neutron cross section and resonance integrals of holmium. In Proc. 2nd Conf. Neutron Cross Sections and Technology, Washington, D.C., March 1968, p. 1031, D.T. Goldman, Editor, Nat. Bur. Std. U.S. Spec. Publ. 299, Sept. 1968.

Stephenson, T.E. The total neutron cross section of cobalt below $30 \mathrm{keV}$. (A) Bull. Amer. Phys. Soc. 14, 190 (1969).

Stephenson, T.E. and Popek, J. The total neutron cross section of iron below $100 \mathrm{keV}$. (A) Presented at Amer. Phys. Soc. Meet., Hawaii, Sept. 1969.

Strickland, G. - See Drager, R.F.; Tuthill, E. J.

Susskind, H., Odetre, R., And Becker, W. Controlled removal of spheres from a geometrically ordered packed bed by surface fluidization. In Fluidization (Proc. Symp., Los Angeles, Dec. 1968), A.I.Ch.E. Symp. Ser. (in press).

Susskind, H., Odette, R., And Becker, W. Modified jet pumping of solid spheres. Ind. Eng. Chem. Process Design Develop. 7, 565-72 (1968).

Sutheriand, J.W. - See Abrams, L. (Chemistry).

Takahashi, $H$. Coherent neutron scattering from polycrystalline graphite. Nucl. Sci. Eng. 37, 198-215 (1969).

Takahashi, H. Monte Carlo method for reactivity change due to geometrical perturbation. (A) Trans. Amer. Nucl. Soc. 11, 533-4 (1968).

TAKahashi, H. Screening and electron correlation effects on the electron-phonon interaction in monovalent metals. Phys. Rev. 172, 747-63 (1968).

TAKahashi, H. - See also Hendrie, J.M.

Tang, I.N., Gastleman, A.W. JR., and Munkelwitz, H.R. On the transport of fission-product cesium from sodium. Advan. Chem. (in press).

Tang, I.N. - See also Castleman, A.W. JR.

TAVEL, M.A. AND ZuGKer, M.R. Some quantitative results and new applications of the "phase-space time evolution" method. (A) Trans. Amer. Nucl. Soc. 12, 158-9 (1969).

Tidwell, T. - See Heus, R.J.

Tivers, R. J. - See Van Norman, J.D.

Tugker, W. - See Manowitz, B.; Steinberg, M.

Tuthill, E. J., Strickland, G., and Weth, G.G. Platinum for high temperature crucibles used in processing radioactive wastes. Ind. Eng. Chem. Process Design Develop. 8, 36-43 (1969).
Tuthill, E. J. - See also Drager, R.F.

Tveenrem, J.O. Pyrometry error on high-temperature graphite from soot formation. (L) J. Chem. Phys. 49, 2878-80 (1968).

Van Norman, J.D. and Tivers, R.J. Solutions of halogens in molten halides. In Molten Salts: Characterization and Analysis (Proc. Symp., Atlantic City, Sept. 1968), Amer. Chem. Soc., 1969 (in press).

Wachtel, S. J., Romano, A. J., and Klamut, C. J. Highflux induction heater for sodium corrosion testing. (A) Trans. Amer. Nucl. Soc. 12, 125-6 (1969).

Wachtel, S. J. - See also Romano, A. J.

Waide, C.H. - See Steinberg, M.; Winsche, W.E.

WEEKS, J.R. Estimation of impurity levels in LMFBR from solubility data, and comparison with impurity behavior in loops. In Proc. Int. Conf. Sodium Technology and Large Fast Reactor Design, Argonne National Laboratory, Nov. 1968 (in press).

WEEKS, J.R. Liquidus of the Na-Ag system: 0 to 6 at. \% Ag. (N) Trans. Amer. Soc. Metals 62, 304 (1969).

Weeks, J.R. and Romano, A.J. Liquidus curves and corrosion of $\mathrm{Fe}, \mathrm{Ti}, \mathrm{Zr}$, and $\mathrm{Cu}$ in liquid $\mathrm{Bi}-\mathrm{Pb}$ alloys. Corrosion 25, 130-6 (1969).

WeEks, J.R. AND Singer, R.M. On the solubilities of $\mathrm{Cu}$, $\mathrm{Ni}$, and $\mathrm{Fe}$ in liquid sodium. (A) In Proc. Int. Conf. Sodium Technology and Large Fast Reactor Design, Argonne National Laboratory, Nov. 1968 (in press).

Weeks, J.R. - See also Epstein, S.G.; Singer, R.M.

Weinstock, E.V. - See Phelps, J.P.; SAbine, T.M.

Weiss, A. J. - See Hillman, M.

Weth, G.G. - See Drager, R.F.; Tuthill, E. J.

Wilmshurst, J.K. AND Bracker, J.M. The investigation of fused salts by the inelastic scattering of cold neutrons. In Molten Salts: Characterization and Analysis (Proc. Symp., Atlantic City, Sept. 1968), Amer. Chem. Soc., 1969 (in press).

Wilmshurst, J.K. - See also LeE, H.

Wilson, V.H. - See MAcKenzie, D.R.

Windsor, H.H. - See HeNDrIE, J.M.

Winsche, W.E., Wiswall, R.H., Reilly, J.J., Sheehan, T.V., Waide, C.H., and Hoffman, K.C. Metal hydrides as a source of fuel for vehicular propulsion. Presented at Int. Automotive Eng. Congr., Detroit, Jan. 1969.

Winsche, W.E. - See also Hoffman, K.C.

Wiswall, R.H. - See Hoffman, K.C.; Reilly, J.J.; WINSCHE, W.E.

YeMin, L. AND Hill, F.B. The measurement of effects of mixing of chain centers in a nonuniformly initiated photopolymerization. Ind. Eng. Chem. Fundamentals 8, 210-15 (1969).

YIP, S. - See Diamond, D.J.

Yu, W.S. - See Chen, J.G.

ZuCKER, M.S. AND FELDER, R.M. Angular distribution of charged particles emitted from a semi-infinite slab source. (A) Presented at Amer. Nucl. Soc. Meet., San Juan, Puerto Rico, May 1969.

Zucker, M.S. AND FERRARI, L.A. A microwave cavity method of studying recombination following pulse radiolysis. Advan. Chem. Ser. 82, 539 (1968).

Zucker, M.S. - See also Felder, R.M.; Ferrari, L.A.; TAVEL, M.A 


\section{Biology Department}

Abata, J.J. and Baker, D.G. Liver RNA synthesis in continuously irradiated rats. (A) Radiat. Res. 35, 519 (1968).

Abraham, S., Ames, I.H., and Smith, H.H. Autoradiographic studies of DNA synthesis in the B chromosomes of Crepis capillaris. J. Heredity 59, 297-9 (1968).

Altszuler, N. - See Steele, R.

Ames, I.H. And Mitra, J. Distribution of heterochromatin in the chromosomes of Haplopappus gracilis. Can. J. Genet. Cytol. 10, 433-43 (1968).

Ames, I.H. And Mitra, J. DNA replication pattern in somatic chromosomes of Haplopappus gracilis. Bull. Torrey Botan. Club 95, 335-41 (1968).

Ames, I.H., Rice, T.B., and Smith, H.H. Inhibition of tumor induction by auxin in totally debudded Nicotiana glauca $\times \mathcal{N}$. Langsdorffi. Plant Physiol. 44, 305-7 (1969).

Ames, I.H. ANd Smith, H.H. Effects of mercaptoethanol on tumor induction in a Nicotiana amphiploid. Can. J. Botany 47, 921-4 (1969).

Ames, I.H. - See also Abraham, S.; Smith, H.H.

Artenstein, M.S. - See Gotschlich, E.C.

Baetcke, K.P., Sparrow, A.H., And Nauman, C.H. Correlations between nuclear parameters and radiosensitivities of woody and herbaceous plants. (A) Radiat. Res. 35, 526 (1968).

Baker, D.G. - See Abata, J. J.; Cohn, S.H. (Medical); DonNelly, G.M.; McF ADYEN, D.M.

Bankes, D.A. and Sparrow, A.H. Effect of acute gamma irradiation on the incidence of tumor-like structures and adventitious roots in lettuce plants. Radiat. Botany 9, 21-6 (1969).

Bankes, D.A., Sparrow, A.H., and Popham, R.A. Some effects of localized internode and entire shoot $x$-irradiation on survival and morphology of sunflower plants. (A) Presented at Ohio Acad. Sci. Meet., Columbus, April 1969.

BARr, D. - See Yourno, J.

Bartelt, D.C. - See Greene, J. J.

Bhatia, C.R. and Nilson, J.P. Isoenzyme changes accompanying germination of wheat seeds. Biochem. Genet. (in press).

Bjerknes, C. - See Steele, R.

Blackman, C.F. Jr. - See Cutler, R.G

Bodlaender, P. - See Feinstein, G.

Bostrack, J.M. and Sparrow, A.H. Effects of chronic gamma irradiation on the anatomy of vegetative tissues of Pinus rigida Mill. Radiat. Botany (in press).

Botkin, D.B. Prediction of net photosynthesis of trees from light intensity and temperature. Submitted to Ecology. Botkin, D.B. - See also Woodwel, G.M.

BRIGGS, R.W. Induction of endosperm mutations in maize with ethyl methanesulfonate. Maize Genetics $\mathcal{N}$ ews Letter (in press).

Briggs, R.W. - See also Klassen, W.

Broker, T.R. - See Ghosh, A.K.

BURton, A.J. AND WAKsmonski, C.A. The association of replicating forms of bacteriophage $\phi R$ DNA with distinct cell components. (A) Presented at 13th Annu. Meet. Biophys. Soc., Los Angeles, Feb. 1969.

Carty, R.P. and Hirs, C.H.W. Modification of bovine pancreatic ribonuclease A with 4-sulfonyloxy-2-nitro- fluorobenzene. Isolation and identification of modified proteins; Effect of $p \mathrm{H}$ and temperature on the rate of reaction. J. Biol. Chem. 243, 5244-65 (1968).

Chapman, D. J., Cole, W. J., and Siegelman, H.W. A comparative study of the phycoerythrin chromophore. Phytochemistry 7, 1831-5 (1968).

Chapman, D. J. - See also Cole, W. J.; Siegelman, H.W.

Chase, T. Jr. and Shaw, E. Comparison of the esterase activities of trypsin, plasmin, and thrombin on guanidinobenzoate esters. Titration of the enzymes. Biochemistry 8, 2212-24 (1969).

Chiu, C.C. - See Koenig, D.F.

Clayton, M.L. - See Thornber, J.P.

Cole, W. J., Chapman, D. J., and Siegelman, H.W. The structure and properties of phycocyanobilin and related bilitrienes. Biochemistry 7, 2929-35 (1968).

Cole, W. J. - See also Chapman, D. J.; Siegelman, H.W. Combatti, N.C. - See Smith, H.H.

Conger, A.D., Thompson, K., and Sparrow, A.H. Survival curve steepness for higher plants and animals. (A) Radiat. Res. (in press).

Conklin, M.E., Smith, H.H., and Keshishian, N.A. Effects of fast neutrons vs. $x$ rays on tumorous and normal growth in Nicotiana. (A) In Proc. 12th Int. Congr. Genetics, Tokyo, Aug. 1968, Vol. I (Abstracts), p. 105.

Conklin, M.E. - See also Smith, H.H.

Curtis, H.J. Aging in replicating cell systems in vivo. (A) Gerontologist 8, 14 (1968).

Curtis, H.J. Chromosomal recovery following irradiation in young and old mice. (A) Radiat. Res. (in press).

Curtis, H.J. The nature of the aging process. In The Biological Basis of Medicine, Vol. I, pp. 521-42, E.E. Bittar and N. Bittar, Editors, Academic, London, 1968.

CurTis, $H$. J. The role of mutations in the development of radiation induced aging. In Proc. 1st Eur. Symp. Late Effects of Radiation, Rome, April 1969 (in press).

Curtis, H. J. Role of somatic mutations in aging. (A) In Abstr. 8th Int. Congr. Gerontology, Washington, D.C., April 1969 (in press).

Curtis, H. J. The role of somatic mutations in carcinogenesis. In Proc. IAEA Symp. Radiation-Induced Cancer, Athens, April 28-May 2, 1969 (in press).

Curtis, H. J. - See also Gutler, R.G.; Ordy, J.M.; SamORAJSKI, T.

Cutler, R.G., Blagkman, G.F. Jr., Presowitz, B., and Hildebrandt, A. The physiological state of aging mice as represented by qualitative RNA measurements. (A) In Abstr. 8th Int. Congr. Gerontology, Washington, D.C., April 1969 (in press).

Gutrer, R.G. And Curtis, H. J. Synthesis of messenger RNA species as a function of age in mice. (A) Presented at 13th Annu. Meet. Biophys. Soc., Los Angeles, Feb. 1969.

Gutler, R.G., Medley, B. J., and Curtis, H. J. Information flow from DNA via RNA as a function of age in C57 BL6J mice. (A) Gerontologist 8, 11 (1968).

DALY, K. Effect of temperature on survival of gammairradiated Arabidopsis seed. Submitted to Radiat. Botany. deBodo, R.C. - See Steele, R.

Demerec, M. - See Gillespie, D.; Ino, I.

Donnelly, G.M., Sparrow, A.H., Nauman, C.H., and BAKER, D.G. Radiosensitivity of selected amphibians. (A) Radiat. Res. 35, 574 (1968). 
Dunne, F.T. - See Reinhold, V.N.

Ettinger, M.J. and Hirs, C.H.W. On the structure of 41-dinitrophenyl ribonuclease A. Solvent perturbation, thermal transition, optical rotatory dispersion, and binding studies. Biochemistry 7, 3374-80 (1968).

Feinstein, G., Bodlaender, P., and Shaw, E. The modification of essential carboxylic acid side chains of trypsin. Submitted to Biochemistry.

Ferreira, S.H. - Sce Greene, L.J.

Fox, L.R. and Hillman, W.S. Differences in photoresponse and phytochrome spectrophotometry between etiolated and de-etiolated pea stem tissue. Plant Physiol. 43, 1799-804 (1968).

Frydman, I. AND WhitTaker, R.H. Forest associations of Southeast Lublin Province, Poland. J. Ecol. 49, 896-908 (1968).

Ghosh, A.K., Broker, T.R., ANd Olson, J.M. A kinetic study of bacteriochlorophyll pheophytinization in the protein complex from a green photosynthetic bacterium. Biochim. Biophys. Acta 162, 402-13 (1968).

Ghosh, A.K. AND Olson, J.M. Effects of denaturants on the absorption spectrum of the bacteriochlorophyllprotein from the photosynthetic bacterium Chloropseudomonas ethylicum. Ibid., 135-48.

Gillespie, D., Demerec, M., and Itikawa, H. Appearance of double mutants in aged cultures of Salmonella typhimurium cysteine-requiring strains. Genetics 59, 43342 (1968).

Gilot, J. - See Moutschen, J.

Giordano, J.S. Jr. - See Greene, L. J.

Glick, D.M. Ligand-induced $p K$ changes in chymotrypsin. Biochemistry 7, 3391-6 (1968).

Glover, G., Wang, C.G., and Shaw, E. Selective inhibition of thrombin and plasmin by ester substrates. (A) Presented at Amer. Chem. Soc. Meet., New York, Sept. 1969.

Gotschlich, E.C., Liu, T.Y., and Artenstein, M.S. Human immunity to the meningococcus. III. Preparation and immunochemical properties of the group A, group B, and group C meningococcal polysaccharides. Submitted to J. Exp. Med.

Gotschlich, E.G. - See also Liv, T.Y.

Greene, L. J. Amino acid compositions of bovine pancreatic trypsin inhibitors. Ann. N.Y. Acad. Sci. 146, 386-7 (1968).

Greene, L. J. and Bartelt, D.C. The structure of the bovine pancreatic secretory trypsin inhibitor - Kazal's inhibitor. II. The order of the tryptic peptides. J. Biol. Chem. 244, 2646-57 (1969).

Greene, L. J. and Ferreira, S.H. Purification of peptides from bradykinin-potentiating factor. (A) In Proc. 4 th Int. Congr. Pharmacology, Basel, Switzerland, July 1969 (in press).

Greene, L. J. and Giordano, J.S. Jr. The structure of the bovine pancreatic secretory trypsin inhibitor - Kazal's inhibitor. I. The isolation and amino acid sequences of the tryptic peptides from reduced aminoethylated inhibitor. J. Biol. Chem. 244, 285-98 (1969).

Greene, L. J., Stewart, J.M., and Ferreira, S.H. Bradykinin-potentiating peptides from the venom of Bothrops jararaca. (A) In Abstr. Int. Symp. Cardiovascular and NeuroActions of Bradykinin and Related Kinins, Fiesole, Castel di Poggio, Italy, July 1969, pp. 159-60.
Greene, L. J. - See also Rigai, M.

HAmiLl, D.E. - See Smith, H.H.

Heath, R.L. AND Hind, G. The inhibition of water oxidation in isolated chloroplasts. (A) Presented at 53rd Annu. Meet. Fed. Amer. Soc. Exp. Biol., Atlantic City, April 1969.

Heath, R.L. AND Hind, G. The low temperature fluorescence emission of chloride-deficient chloroplasts. (A) Presented at 13th Annu. Meet. Biophys. Soc., Los Angeles, Feb. 1969.

Heath, R.L. and Hind, G. On the functional site of manganese in photosynthetic oxygen evolution. Submitted to Biochim. Biophys. Acta.

Heath, R.L. And Hind, G. The role of $\mathrm{Cl}^{-}$in photosynthesis. II. The effect of $\mathrm{Cl}^{-}$upon fluorescence. Biochim. Biophys. Acta 172, 290-99 (1969); IV. Studies on the low temperature fluorescence emission spectrum. $(\mathrm{N})$ Ibid. 180, 414-16.

Heath, R.L. - See also Hind, G.; Izawa, S.; Russell, G.K.; Yourno, J.

Heath, S. - See Yourno, J.

Hildebrandt, A. - See Cutler, R.G.

Hillman, W.S. Photomorphogenesis. In Penguin Science Survey 1968: Biology, pp. 93-108, A. Allison, Editor, Penguin Books, Middlesex, England, 1968.

Hillman, W.S. Phytochrome spectrum of Pisum leaves and stems. Biochim. Biophys. Acta 162, 464-6 (1968).

Hillman, W.S. - See also Fox, L.R.; Roux, S. J.; Sweet, H.C.

Hinckley, A.D. Concentration of the aphid, Myzocallis discolor, on irradiated white oaks. (A) Presented at Annu. Meet. Entomol. Soc. Amer., Chicago, Dec. 1969.

HinckLey, A.D. Gamma radiation as a tool in population ecology. (A) Presented at Ecol. Soc. Amer. Meet., AIBS, Burlington, Vt., Aug. 1969.

Hind, G., Nakatani, H.Y., and Heath, R.L. Chloride and manganese involvement in photosynthetic oxygen evolution. (A) In Proc. 11th Int. Botanical Congress, Seattle, Aug. 24-Sept. 2, 1969 (in press).

Hind, G., Nakatani, H.Y., and Izawa, S. The role of $\mathrm{Cl}^{-}$in photosynthesis. $\mathrm{I}$. The $\mathrm{Cl}^{-}$requirement of electron transport. Biochim. Biophys. Acta 172, 277-89 (1969).

Hind, G. - See also Heath, R.L.; Izawa, S.

Hirono, Y. and Smith, H.H. Mutations induced in Arabidopsis by DNA nucleoside analogs. Genetics 61,191 . 9 (1969)

Hirono, Y. And Smith, H.H. Mutations induced with a DNA base analog in a higher plant, Arabidopsis. (A) In Proc. 12th Int. Congr. Genetics, Tokyo, Aug. 1968, Vol. I (Abstracts), p. 117.

Hirono, Y., Smith, H.H., and Lyman, J.T. RBE of heavy ions in producing mutations, tumors and growth inhibition in Arabidopsis. (A) Radiat. Res. (in press).

Hirono, Y., Smith, H.H., and Lyman, J.T. Tumor induction by heavy ionizing particles and $\mathrm{x}$ rays in Arabidopsis. Radiat. Botany 8, 449-56 (1968).

Hirono, Y. - See also Smith, H.H.

Hirs, G.H.W., Jackson, R.L., and Kabasawa, I. The structure of porcine pancreatic ribonuclease, a glycoprotein. (A) Presented at Amer. Chem. Soc. Meet., New York, Sept. 1969.

Hirs, C.H.W. - See also Carty, R.P.; Ettinger, M. J.; JACKSON, R.L.; ReINHOLD, V.N. 
Holm, G. Predicted control by the genetic code of exchanges of polar and nonpolar amino acids in pointmutations. (A) In Proc. IAEA/FAO Symp. Nature, Induction and Utilization of Mutations in Plants, Pullman, Wash., July 1969 (in press).

Holm, G. and Sparrow, A.H. An improved method of predicting cellular radiosensitivity $\left(\mathrm{D}_{0}\right)$ from chromosome volume. (A) Radiat. Res. (in press).

Horrocks, L.A. - See Ordy, J.M.

ICHIKAWA, S. AND Sparrow, A.H. Analyses of radiationinduced loss of reproductive integrity in Tradescantia stamen hairs, an essentially single-meristematic-cell system. (A) In Proc. 12th Int. Congr. Genetics, Tokyo, Aug. 1969, Vol I (Abstracts), p. 330.

IchIKAWA, S. AND SPARROW, A.H. Radiation-induced loss of reproductive integrity, morphologically abnormal cells, and somatic mutations in Tradescantia stamen hairs. (A) Ibid., p. 108.

Ichikawa, S., Sparrow, A.H., and Thompson, K.H. Morphologically abnormal cells, somatic mutations and loss of reproductive integrity in irradiated Tradescantia stamen hairs. Radiat. Botany 9, 195-211 (1969).

INGLIS, A.S. AND LIU, T.Y. The stability of cystine and half-cystine in proteins during acid hydrolysis. Submitted to J. Biol. Chem.

Ino, I. And Demerec, M. Enteric hybrids. II. S. typhimurium-E. coli hybrids for the trp-cysB-pyrF region. Genetics 59, 167-76 (1968).

Itikawa, H. - See Gillespie, D.

Izawa, S., Heath, R.L., and Hind, G. The role of chloride ion in photosynthesis. III. The effect of artificial electron donors upon electron transport. Biochim. Biophys. Acta 180, 388-98 (1969).

Izawa, S. - See also Hind, G.

Jackson, R.L. and Hirs, C.H.W. On porcine ribonuclease: The presence of three polysaccharide side chains. (A) Fed. Proc. 28, No. 2, 344 (1969).

Jackson, R.L. - See also Hirs, C.H.W.

JAHN, A. - See Cohn, S.H. (Medical).

Jennings, W.H. - See Olson, R.A.

Jonsson, E.K. - See LiU, T.Y.

JoshuA, D.C. - See SMITH, H.H.

Kabasawa, I. - See Hirs, C.H.W.

Keshishian, N.A. - See Conklin, M.E.

Klassen, W., Norland, J.F., and Briggs, R.W. Sterilization of boll weevils with combinations of chemosterilants, $x$-rays or gamma rays, and fast neutrons. $J$. Econ. Entomol. (in press).

KLEIN, J.R. Heme and globin synthesis and related parameters in blood from the $\mathrm{x}$-irradiated duck. Radiat. Res. 57, 50-6 (1969).

KLEIN, J.R. Hemoglobin heterogeneity in the Pekin duck. Submitted to Proc. Soc. Exp. Biol. Med.

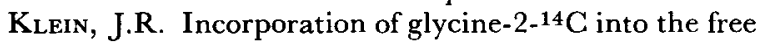
protoporphyrin of duck erythrocytes in vivo. Arch. Biochem. Biophys. 127, 666-71 (1968).

Koenig, D.F. A general program for $\Sigma 2$ phase generation and tangent refinement. (A) Presented at Amer. Cryst. Ass. Meet., Seattle, March 1969.

Koenig, D.F., Ghiu, C.C., Krebs, B., and Walter, R. Establishment of the stable geometric isomer of an $\alpha$ arylidenelactone: $\alpha$-(2-hydroxy-3,5-di-bromobenzylidene)- $\gamma$-butyrolactone. Acta Cryst. B25, 1211-14 (1969).
Koenig, D.F., Chiu, C.C., and Walter, R. Oxytocin: Structure of deamino-6-seleno-oxytocin. (A) In Proc. 8th Int. Congr. Crystallography, Buffalo and Stony Brook, N.Y., Aug. 1969 (in press).

Koenig, D.F. - See also Krebs, B.; Olson, J.M.

Kovacs, C. J. and Van't Hof, J. Synchronization of $G_{1}$ cells in cultured root meristems and evidence for a $G_{1}$ controlling site in the mitotic cycle. (A) Presented at 9th Annu. Meet. Amer. Soc. Cell Biologists, Detroit, Nov. 1969.

Krebs, B. And Koenig, D.F. Crystal and molecular structure of 4,5-dioxo-2-thioxo-1,3-dithiolan. Z. Naturforsch. 23b, 109-10 (1968).

Krebs, B. And Koenig, D.F. Crystal and molecular structure of 4,5-dioxo-2-thioxo-1,3-dithiolan ( $\beta$-modification). Acta Cryst. B25, 1020-30 (1969).

Krebs, B. - See also Koenig, D.F.

LACKs, S. Genetic regulation of maltosaccharide utilization in pneumococcus. Genetics 60, 685-706 (1968).

LAcks, S. A regulatory gene for the maltose utilization pathway of pneumococcus. (A) In Proc. 12th Int. Congr. Genetics, Tokyo, Aug. 1968, Vol. I (Abstracts), p. 21.

LACKs, S. Theoretical relationship between probability of marker integration and length of donor DNA in pneumococcal transformation. J. Mol. Biol. 37, 179-80 (1968).

Lacks, S. - See also Moutschen, J.

LeDBetTER, M.C. Vacuole. In 1969 rearbook of Science and Technology, McGraw-Hill, New York (in press).

Ledbetter, M.G. and Siegelman, H.W. Fine structure of leaf mesophyll in kidney beans during ten days of dark growth. (A) J. Cell Biol. 39, 78-79a (1968).

LedbetTer, M.C. - See also Olson, J.M.

Liu, T.Y., Jonsson, E., And Gotschlich, E.C. Chemical studies on the meningococcal cell wall polysaccharide. (A) Presented at Amer. Chem. Soc. Meet., New York, Sept. 1969.

Liu, T.Y., Nomura, N., Jonsson, E.K., and Wallace, B.G. Streptococcal proteinase catalyzed hydrolysis of some ester and amide substrates. Submitted to J. Biol. Chem.

Liv, T.Y. - See also Gotschlich, E.C.; Inglis, A.S.

Lyman, J.T. - See Hirono, Y.; Smith, H.H.

Maizel, J.V. Jr. - See Studier, F.W.

Marimuthu, K.M., Sparrow, A.H., and Schairer, L.A. The effects of space flight factors, radiation, vibration and clinostat on mortality and nuclear division in Tradescantia microspores. (A) In Proc. 11th Int. Botanical Congress, Seattle, Aug. 24-Sept. 2, 1969 (in press).

Marimuthu, K.M. - See also Schairer, L.A.; Sparrow, A.H.

Markland, F.S., Shaw, E., ANd Smith, E.L. Identification of histidine 64 in the active site of subtilisin. Proc. Nat. Acad. Sci. U.S. 61, 1440-7 (1968).

Marples, T.G. - See Woodwell, G.M.

McFadyen, D.M. AND BAKER, D.G. Alterations in the subcellular acid phosphatase and $\beta$-glucuronidase in rat mucosa after various doses of $\mathrm{x}$ irradiation. Gastroenterology (in press).

McMillan, B. - See Van't Hof, J.

Medley, B. J. - See Cutler, R.G.

Mitra, J. - See Ames, I.H.

Moutschen, J. AND LAGKs, S. Implications of pyrimidine nucleoside catabolism for cytological investigation of germinating seeds. Exp. Cell Res. 51, 462-72 (1968). 
Moutschen, J., Moutschen-Dahmen, M., Woodley, R., AND Gilot, J. The relative biological effectiveness of different kinds of radiations on chromosome aberrations in Nigella damascena seed. Int. J. Radiat. Biol. (in press).

Moutschen-Dahmen, M. - See Moutschen, J.

Nakatani, H.Y. - See Hind, G.

Nauman, C.H. - See Baetcke, K.P.; Donnelly, G.M.

Nilson, J.P. - See Bhatia, C.R.

Nomura, N. - See LiU, T.Y.

Norland, J.F. - See Klassen, W.

Odum, W.E., Woodwell, G.M., ANd Wurster, G.F. DDT residues absorbed from organic detritus by fiddler crabs. Science 164, 576-7 (1969).

OLson, J.M. Evolution of photosynthesis. Submitted to Science.

OLson, J.M. Evolution of photosystem II in blue-green algae. (A) In Proc. 11th Int. Botanical Congress, Seattle, Aug. 24-Sept. 2, 1969 (in press).

Olson, J.M., Koenig, D.F., and Ledbetter, M.C. A model of the bacteriochlorophyll-protein from green photosynthetic bacteria. Arch. Biochem. Biophys. 129, 42-8 (1969).

Olson, J.M. and Shaw, E.K. Cytochromes from the green photosynthetic bacterium Chloropseudomonas ethylicum. Photosynthetica (in press).

Olson, J.M. - See also Ghosh, A.K.; Ol.son, R.A.; THORNBER, J.P.

Olson, R.A., Jennings, W.H., And Olson, J.M. Chlorophyll orientation in crystals of bacteriochlorophyllprotein from green photosynthetic bacteria. Arch. Biochem. Biophys. 129, 30-41 (1969).

Olson, R.A. - See also Olson, J.M.

Ordy, J.M., SAMORajSki, T., Horrocks, L.A., Zeman, W., And Curtis, H. J. Changes in memory, electrophysiology, neurochemistry and neuronal ultra-structure after deuteron irradiation of the brain in $\mathrm{C} 57 \mathrm{Bl} / 10$ mice. J. Neurochem. 15, 1245-56 (1968).

Ordy, J.M. - See also SAMORAjSki, T.; Zeman, W.

Owens, R.A. - See Underbrink, A.G.

Pond, V. - See Underbrink, A.G.

Popham, R.A. - See Bankes, D.A.

Presowitz, B. - See Cutler, R.G.

Puglielli, L. - See Sparrow, A.H.

Rathgeb, I. - See Steele, R.

Reinhold, V.N., Dunne, F.T., Wriston, J.C., Schwarz, M., Sarda, L., and Hirs, C.H.W. The isolation of porcine ribonuclease, a glycoprotein, from pancreatic juice. J. Biol. Chem. 243, 6482-94 (1968).

Rice, T.B. - See Ames, I.H.

Rigbi, M. And Greene, L. J. Limited proteolysis of the bovine pancreatic secretory trypsin inhibitor at acid pH. J. Biol. Chem. 243, 5457-64 (1968).

Rogers, A.F. - See Sparrow, A.H.; Underbrink, A.G.

Rosenberg, A.H. ANd Studier, F.W. Intrinsic viscosity of native and single-stranded T7 DNA and its relationship to sedimentation coefficient. Biopolymers 7, 765-74 (1969).

Rossi, H.H. - See Smith, H.H.; Underbrink, A.G.

Roux, S. J. AND Hillman, W.S. The effects of glutaraldehyde and two monoaldehydes on phytochrome. Arch. Biochem. Biophys. 131, 423-9 (1969).

Ruscica, J. - See Shaw, E.
Russell, G.K., Lyman, H., and Heath, R.L. Absence of fluorescence quenching in a photosynthetic mutant of Euglena gracilis. Plant Physiol. 44, 929-31 (1969).

Samorajski, T., Ordy, J.M., Zeman, W., and Curtis, H.J. Head irradiation and aging. In Proc. Gerontol. Soc. Meet., Denver, Oct. 31-Nov. 2, 1969 (in press).

Samorajski, T. - See also Ordy, J.M.; Zeman, W.

Sand, S.A. and Smith, H.H. Somatic mutational transients. III. Response by two genes in a clone of Nicotiana to 24 roentgens gamma irradiation applied at different intensities. Submitted to Genetics.

SARDa, L. - See Reinhold, V.N.

Schairer, L.A., Sparrow, A.H., and Marimuthu, K.M. Radiobiological studies of plants orbited in Biosatellite II. In Proc. COSPAR Meet., Prague, May 1969 (in press).

Schairer, L.A. - See also Marimuthu, K.M.; Sparrow, A.H.

Schoenborn, B.P. Crystallographic studies of ligand binding. In Proc. 4th Johnson Found. Colloq. Structure and Function of Macromolecules and Membranes, Philadelphia, April 1969, B. Chance, Editor, Academic, New York (in press).

Schoenborn, B.P. A neutron diffraction study of myoglobin. (A) In Proc. 8th Int. Congr. Crystallography, Buffalo and Stony Brook, N.Y., Aug. 1969 (in press).

Schoenborn, B.P. Protein conformational changes studied by diffraction techniques. In Proc. 4th Johnson Found. Colloq. Structure and Function of Macromolecules and Membranes, Philadelphia, April 1969, B. Chance, Editor, Academic, New York (in press).

Schoengorn, B.P. The structure of alkaline met myoglobin xenon complex. J. Mol. Biol. (in press).

Schwarz, M. - See Reinhold, V.N.

Schwemmer, S.S. - See Sparrow, A.H.

Shaw, E. AND Ruscica, J. The essentiality of histidine in the catalytic action of subtilisin. Covalent modification by a specific reagent. J. Biol. Chem. 243, 6312-3 (1968).

Shaw, E. - See also Chase, T. Jr.; Feinstein, G.; Glover, G.; Markland, F.S.; SMith, R.L.

Shaw, E.K. - See Olson, J.M.

Siegelman, H.W. Photochemical control mechanisms of higher plants. In Handbook on Metabolism, pp. 491-2, P.L. Altman and D.S. Dittmer, Editors, Fed. Amer. Soc. Exp. Biol. (FASEB), Bethesda, Md., 1968.

Siegelman, H.W., Chapman, D. J., and Cole, W. J. The bile pigments of plants. In Porphyrin and Related Compounds, Biochem. Soc. Symp. 28, pp. 107-20, T.W. Goodwin, Editor, Academic, London, 1968.

Siegelman, H.W. - See also Chapman, D. J.; Cole, W. J.; LedBetTer, M.C.

Smith, E.L. - See Markland, F.S.

SMITH, H.H. Neutron irradiation of seeds as a tool in plant genetics and breeding. Jap. J. Genet. Suppl. 1 (in press).

Smiтh, H.H. Time in organic evolution. In The Physics and Biology of Time, A. Katchalsky and C.A. Tobias, Editors, Univ. of California Press, Berkeley (in press). Smith, H.H., Combatti, N.C., Hirono, Y., Conklin, M.E., and Lyman, J.T. Responses in mutation, cell lethality, growth inhibition, tumorization and isozyme multiplicity from exposing seeds to irradiations of different linear energy transfer. (A) In Proc. IAEA/FAO Symp. Nature, Induction and Utilization of Mutations in Plants, Pullman, Wash., July 1969 (in press). 
Smith, H.H., Combatti, N.C., and Rossi, H.H. A constant relation of neutron to $\mathrm{x}$-ray effects in Zea mays $\mathrm{L}$. (A) In Proc. 12th Int. Congr. Genetics, Tokyo, Aug. 1968, Vol. I (Abstracts), p. 105.

Smith, H.H., Combatti, N.C., and Rossi, H.H. Response of seeds to irradiation with $\mathrm{x}$ rays and neutrons over a wide range of doses. In Neutron Irradiation of Seeds II, IAEA Tech. Rep. Ser. 92, pp. 3-8, IAEA, Vienna, 1968.

Smith, H.H., Conklin, M.E., Hirono, Y., Ames, I., AND Lyman, J.T. Studies on plant tumors induced by irradiation. (A) In Proc. IAEA Int. Symp. Radiation-Induced Carcinogenesis, Athens, April 28-May 2, 1969 (in press).

Smith, H.H., Hamill, D.E., and Weaver, E.A. Isozyme patterns for peroxidases and esterases in species and polyploids of Nicotiana. (A) In Proc. 11th Int. Botanical Congress, Seattle, Aug. 24-Sept. 2, 1969 (in press).

Smith, H.H., Hirono, Y., Conklin, M.E., and Lyman, J.T. Responses in mutation, growth inhibition, tumorization and isozyme multiplicity from exposing seeds to irradiations of different linear energy transfer. In Proc. IAEA/FAO Symp. Nature, Induction and Utilization of Mutations in Plants, Pullman, Wash., July 1969 (in press).

Smith, H.H., Joshua, D.C., Combatti, N.C., ANd Thompson, K.H. Relative effects of fast neutrons vs. $x$ irradiation of seeds over a wide range of genotypes, doses and moisture levels. (A) In Proc. Symp. Neutrons in Radiobiology, Oak Ridge, Tenn., Nov. 1969 (in press).

Smith, H.H. - See also Abraham, S.; Ames, I.H.; Briggs, R.W.; Conklin, M.E.; Hirono, Y.; Sand, S.A.

Smith, R.L. and Shaw, E. Pseudotrypsin. A modified bovine trypsin produced by limited autodigestion. $J$. Biol. Chem. (in press).

Sparrow, A.H. Prediction of lethality and yield in vascular plants exposed to gamma radiation. (A) Presented at Annu. Meet. Health Phys. Soc., Pittsburgh, June 1969.

Sparrow, A.H. and Puglielli, L. Effects of simulated radioactive fallout decay on growth and yield of cabbage, maize, peas and radish. Radiat. Botany 9, 77-92 (1969).

Sparrow, A.H., Rogers, A.F., and Schwemmer, S.S. Acute gamma radiosensitivity of 28 woody plant species. (A) Radiat. Res. 35, 575 (1968).

Sparrow, A.H., Schairer, L.A., and Marimuthu, K.M. Radiobiological studies of Tradescantia plants orbited in Biosatellite II. In Results of Biosatellite II, Final Report, NASA Spec. Publ. (in press); (A) Radiat. Res. 35, 502 (1968).

Sparrow, A.H. - See also BaEtcke, K.P.; Bankes, D.A.; Bostrack, J.M.; Gonger, A.D.; Donnelly, G.M.; Holm, G.; Ichikawa, S.; Marimuthu, K.M.; Schairer, L.A.; UNDERBrinK, A.G.

Sparrow, R.C. - See Underbrink, A.G.

Steele, R., Altszuler, N., Rathgeb, I., And BJerknes, C. Effect of dibutyryl cyclic 3,5-AMP infusion on plasma glucose production and utilization in the normal dog. (A) Fed. Proc. 28, No. 2, 708 (1969).

Steele, R., Altszuler, N., Rathgeb, I., And Bjerknes, C. Increased sensitivity of the hypophysectomized dog to the elevation in glucose release induced by adenosine3',5'-mono-phosphate (CAMP). (A) Presented at 51st Annu. Meet. Endocrine Soc., New York, June 1969.
Steele, R., Altszuler, N., Rathgeb, I., and deBodo, R.C. Influence of growth hormone on glucose metabolism and plasma insulin levels in the dog. In Growth Hormone (Proc. Int. Symp., Milan, Italy, Sept. 1967), pp. 309-18, Excerpta Medica Foundation, Amsterdam, 1967.

Steele, R., Winkler, B., And Altszuler, N. Effect of bovine growth hormone ( $\mathrm{BGH}$ ) administration on free fatty acid (FFA) and glycerol turnover in the normal dog. (A) Fed. Proc. 28, No. 2, 558 (1969).

Steele, R., Winkler, B., And Altszuler, N. Effects of growth hormone administration on free fatty acid and glycerol turnover in the normal dog. Submitted to Endocrinology.

Steele, R., Winkler, B., and Altszuler, N. Relationship of glycerol uptake to plasma glycerol concentration in the normal dog. Amer. J. Physiol. 216, 191-6 (1969).

Stewart, J.M. - See Greene, L. J.

STUdiER, F.W. Conformational changes of single-stranded DNA. J. Mol. Biol. 41, 189-97 (1969).

Studier, F.W. Effects of the conformation of singlestranded DNA on renaturation and aggregation. Ibid., 199-209.

Studier, F.W. Factors affecting DNA strand combination. (A) Presented at 13th Annu. Meet. Biophys. Soc., Los Angeles, Feb. 1969.

Studier, F.W. The genetics and physiology of bacteriophage T7. Submitted to Virology.

Studier, F.W. and Maizel, J.V. Jr. T7-directed protein synthesis. Submitted to Virology.

Studier, F.W. - See also RosenberG, A.H.

Sweet, H.C. and Hillman, W.S. Response of pea stem tissues with different phytochrome contents to red light dosage. Plant Physiol. 44, 458-60 (1969).

Tanemura, S. - See Yourno, J.

Thompson, K.H. - See Conger, A.D.; Ichikawa, S.; SMITH, H.H.

Thornber, J.P. Chlorophyll-protein complexes and photochemical reaction centers. (A) In Proc. 11th Int. Botanical Congress, Seattle, Aug. 24-Sept. 2, 1969.

Thornber, J.P. Comparison of a chlorophyll $A$-protein complex isolated from a blue-green alga with chlorophyll-protein complexes obtained from green bacteria and higher plants. Biochim. Biophys. Acta 172, 230-41 (1969).

Thornber, J.P. and Olson, J.M. Isolation of the photochemical reaction center of Rhodopseudomonas viridis. (A) Presented at 13th Annu. Meet. Biophys. Soc., Los Angeles, Feb. 1969.

Thornber, J.P., Olson, J.M., Williams, D.M., and Clayton, M.L. Isolation of the reaction center of Rhodopseudomonas viridis. (N) Biochim. Biophys. Acta 172, 351-4 (1969).

Thornber, J.P. - See also Olson, J.M.

Underbrink, A.G. and Sparrow, A.H. Chromosomes and cellular radiosensitivity: Considerations of nucleic acid base ratios and fine structural damage. (A) Radiat. Res. 35, 531 (1968).

Underbrink, A.G. and Sparrow, A.H. Chromosomes and cellular radiosensitivity. III. Note on the inability to demonstrate a correlation between nucleic acid base ratios and radiation response at $D_{0}$ in several diverse organisms. Ibid., 311-17. 
Underbrink, A.G. AND Sparrow, A.H. The fine structure of the alga Brachiomonas submarina Bohlin. Botan. Gaz. 129, 259-66 (1968).

Underbrink, A.G., Sparrow, A.H., and Owens, R.A. The fine structure of the alga Brachiomonas submarina Bohlin after $\mathrm{x}$ - and $\gamma$-irradiation. Radiat. Botany 9, 24150 (1969).

Underbrink, A.G., Sparrow, A.H., Rogers, A.F., And Pond, V. Observations on the cytology and fine structure of mitosis in the fern Ophioglossum petiolatum Hook. Cytologia 32, 489-99 (1967).

Underbrink, A.G., Sparrow, R.C., Sparrow, A.H., and Rossi, H.H. Preliminary report on monoenergetic neutron experiments with Tradescantia. (A) Radiat. Res. (in press).

Underbrink, A.G., Sparrow, R.C., Sparrow, A.H., and Rossi, H.H. RBE's of $x$ rays and monoenergetic neutrons on somatic mutations and loss of reproductive integrity in Tradescantia stamen hairs. (A) In Proc. Symp. Neutrons in Radiobiology, Oak Ridge, Tenn., Nov. 1969 (in press).

VAN'T HoF, J. The action of IAA and kinetin in the mitotic cycle of proliferative and stationary phase excised root meristems. Exp. Cell Res. 51, 167-76 (1968).

VAN'T HoF, J. Aerobic respiration and the control of DNA replication in meristematic cells of cultured roots. (A) In Proc. 11th Int. Botanical Congress, Seattle, Aug. 24Sept. 2, 1969 (in press).

VAN'T HoF, J. Anaerobiosis and the recovery of $\mathrm{G}_{1}$ and $\mathrm{G}_{2}$ cells and DNA synthesis in cultured root meristems. Submitted to Radiat. Res.

VAN'T HoF, J. Control of cell progression through the mitotic cycle by carbohydrate provision. II. Macromolecules: Their amounts, synthesis and function in excised plant roots; III. Effect of anaerobiosis on the replication of DNA. Submitted to Exp. Cell Res.

VAN'T HoF, J. Experimental procedures for measuring cell population kinetic parameters in plant root meristems. In Methods in Cell Physiology, Vol. 3, pp. 95-117, D.M. Prescott, Editor, Academic, New York, 1968.

Van't Hof, J. and McMillan, B. Cell population kinetics in callus tissue of cultured pea root segments. Amer. J. Botany 56, 42-51 (1969).

Van't Hof, J. - See also Kovacs, C. J.; Webster, P.L.

Waksmonski, C.A. - See Burton, A. J.

Wallage, B.G. - See LiU, T.Y.

Walter, R. - See Koenig, D.F.

Wang, C.C. - See Glover, G.

Weaver, E.A. - See Smith, H.H.

Webster, P.L. and Van't Hof, J. Control of progression of cells through the mitotic cycle. Requirements for RNA and protein synthesis. (A) In Proc. 11th Int. Botanical Congress, Seattle, Aug. 24-Sept. 2, 1969 (in press).

Webster, P.L. and Van't Hof, J. Dependence on energy and aerobic metabolism of initiation of DNA synthesis and mitosis by $\mathbf{G}_{\mathbf{1}}$ and $\mathbf{G}_{\mathbf{2}}$ cells. Exp. Cell Res. 55, 88-94 (1969).

Webster, P.L. AND VAN'T Hof, J. Initiation of DNA synthesis and mitosis in stationary, transitional and proliferative phase meristems: Requirements for RNA and protein synthesis. Submitted to Amer. J. Botany.

Whittaker, R.H. and Woodwell, G.M. Structure, production and diversity of the oak-pine forest at Brookhaven, New York. J. Ecol. (in press).
Whittaker, R.H. - See also Frydman, I.

Williams, D.M. - See Thornber, J.P.

Winkler, B. - See Steele, R.

Woodley, R. - See Moutschen, J.

WoodWELL, G.M. Changes in the chemistry of the oceans: The pattern of effects. In Proc. AAAS Meetings WorldWide Pollution, Dallas, Dec. 1968 (in press).

Woodwell, G.M. Chemical pollution. (A) In Proc. Seminar, "Man is Destroying Himself - Can This Be Prevented?", Inst. on Man and Science, Rensselaerville, N.Y., Oct. 1967 (in press).

Woodwell, G.M. The DDT cycle in estuaries. (A) Presented at Amer. Chem. Soc. Meet., New York, Sept. 1969.

Woodwell, G.M. Prediction of the effects of pollution on the structure and comparative physiology of ecosystems. (A) In Proc. 11 the Int. Botanical Congress, Seattle, Aug. 24-Sept. 2, 1969 (in press).

Woodwell, G.M. AND Botkin, D.B. Metabolism of terrestrial ecosystems by gas exchange techniques: The Brookhaven approach. In Methods of Ecology, D. Reichle, Editor, Springer, New York (in press).

Woodwell, G.M. and Marples, T.G. The influence of chronic gamma irradiation on production and decay of litter and humus in an oak-pine forest. Ecology $\mathbf{4 9}$, 456-65 (1968).

WoodWell, G.M. - See also Odum, W.E.; WhitTaker, R.H.

Wriston, J.C. - See Reinhold, V.N.

Wurster, C.F. - See Odum, W.E.

Yourno, J.D. Amino acid replacements in histidinol dehydrogenase of Salmonella typhimurium double frameshift mutants. (A) Presented at Annu. Meet. Amer. Soc. Microbiol., Miami Beach, May 1969.

Yourno, J.D., BarR, D., AND Tanemura, S. An externally suppressible frameshift mutant of Salmonella typhimurium. Submitted to $J$. Bacteriol.

Yourno, J.D. And Heath, S. The nature of the hisD3018 frameshift mutation in Salmonella typhimurium. Submitted to J. Bacteriol.

Zeman, W. The effects of atomic radiations. In Pathology of the Nervous System, Vol. I, pp. 864-939, J. Minckler, Editor, McGraw-Hill, New York, 1968.

Zeman, W. Histologic events during the latent interval in radiation injury. In The Central Nervous System, Int. Acad. Pathol. Monogr. 9, Chap. 9, pp. 184-200, Williams \& Wilkins, Baltimore, 1968.

Zeman, W. Modification of radiation effects in the murine central nervous system by oxygen and actinomycin D. In Chemical Modification of Radiation Effects (Proc. San Francisco Cancer Symp., 1968), J. Vaeth, Editor, Karger, Basel, Switzerland (in press).

Zeman, W., Ordy, J.M., and Samorajski, T. Modification of acute radiation effect on cerebellar neurons of mice by actinomycin D. Exp. Neurol. 21, $52-7$ (1968).

Zeman, W. - See also Ordy, J.M.; Samorajski, T.

\section{Chemistry Department}

Abrams, L. and Allen, A.O. Radiolysis of ethanol adsorbed on silica. J. Phys. Chem. 73, 2741-5 (1969).

Abrams, L. and Sutherland, J.W. Infrared study of the radiolysis of $\mathrm{CO}_{2}, \mathrm{NH}_{3}$, and $\mathrm{H}_{2}$ adsorbed on silica. (N) J. Phys. Chem. 73, 3160-2 (1969). 
Ache, H.J., Christman, D.R., And Wolf, A.P. Mechanisms and isotope effects in the reactions of energetic carbon atoms with hydrocarbons and alkylfluorides. Submitted to Radiochim. Acta.

Ache, H. J. AND Wolf, A.P. Production and reactivity of carbon-11 in soljd compounds. (N) J. Phys. Chem. (in press).

Ache, H.J. And Wolf, A.P. Reactions of energetic carbon-11 atoms in fluorohydrocarbon systems. Radiochim. Acta 10, 41-3 (1968).

Ache, H.J. - See also Finn, R.D.

Al. Kital, R.A., Chan, L.H., and Sayre, E.V. Neutron activation analysis of pottery sherds from Hajar Bin Humeid and related areas. In Hajar Bin Humeid: Investigations at a Pre-Islamic Site in South Arabia, G.W. Van Beek et al., Appendix II, pp. 387-98, Johns Hopkins Press, Baltimore, 1969.

Allen, A.O. Radiolysis of adsorbed molecules and energy transfer mechanisms. (A) Presented at Conf. ESR in Radiation Chemistry, Chalk River, Canada, May 1969.

Allen, A.O. - See also Abrams, L.; Bielski, B.H.J.; Capellos, C.; Gebicki, J.M.; Schmidt, W.F.

Appleby, A. and Schwarz, H.A. Radical and molecular yields in water irradiated by $\gamma$-rays and heavy ions. J. Phys. Chem. 73, 1937-41 (1969).

Bachmann, K. Rare earth yields in the interaction of 28$\mathrm{GeV}$ protons with uranium, bismuth, and gold. Submitted to J. Inorg. Nucl. Chem.

Baker, E. - See Grover, J.R.

BAKER, W.A. JR. - See REIFF, W.M.

Banks, E. - See Kunnmann, W.

Bernal, I. - See Brennan, T.; Epstein, E.F.; Long, T.V. II.; Ricci, J.S.; Sequeira, A.

Bielski, B.H. J. And Allen, A.O. The radiolytic yield of reducing radicals in neutral aqueous solution. Int. $J$. Radiat. Phys. Chem. 1, 153-63 (1969).

Bielski, B.H. J. and Gebicki, J.M. Electron spin resonance studies of the mechanism of interaction between nitrogen dioxide and olefinic solvents. J. Phys. Chem. 73, 1402-6 (1969).

Bielski, B.H.J. and Schwarz, H.A. The absorption spectra and kinetics of hydrogen sesquioxide and the perhydroxyl radical. Ibid. 72, 3836-41 (1968).

Bigeleisen, J. and Wolfsberg, M. Comment on A.F. Grigor and W.A. Steele's paper, "Physical properties of fluid $\mathrm{CH}_{4}$ and $\mathrm{CD}_{4}$ : Theory." (N) J. Chem. Phys. 50, 561-2 (1969).

Bigeleisen, J. - See also Ishida, T.

Brennan, T. and Bernal, I. The crystal and molecular structure of tris(diethyldithiocarbamato)cobalt(III). $J$. Phys. Chem. 73, 443-5 (1969).

Brennan, T. and Bernal, I. The crystal and molecular structure of tris-(N,N-di- $n$-butyldithiocarbamato) Mo(NO). (A) In Proc. 8th Int. Union Crystallography Congress, Stony Brook, N.Y., Aug. 1969 (in press).

Butterworth, J.S. and Harbottle, G. Chemical effects of gamma irradiation on ${ }^{204} \mathrm{Tl}$ incorporated in $\mathrm{KCl}$ crystals. Radiochim. Acta 10, 57-64 (1968).

Byerrum, R.U. - See Dawson, R.F.

Capellos, C. and Allen, A.O. Ionization of liquids by radiation studied by the method of pulse radiolysis. I. Solutions of $\mathbf{N}, \mathbf{N}, \mathrm{N}^{\prime}, \mathrm{N}^{\prime}$-tetramethyl-p-phenylenedi- amine in hydrocarbons. J. Phys. Chem. 72, 4265-8 (1968); II. Solutions of triphenylmethyl chloride. Ibid. (in press); III. Solutions of galvinoxyl radical. Submitted to J. Phys. Chem.

Cefola, M. - See Erickson, N.E.; Kazimer, E.O.

Chan, L.H. - See Al Kital, R.A.; Sayre, E.V.

Chen, K., Friedlander, G., and Miller, J.M. The effects of using a velocity-dependent potential in a Monte Carlo simulation of intranuclear cascades. Phys. Rev. 176, 1208-10 (1968).

Christman, D.R. - See Ache, H. J.; Dawson, R.F.

Chu, Y.Y., Franz, E.-M., and Friedlander, G. Alpha branching ratio in the decay of ${ }^{149} \mathrm{~Tb}$. Phys. Rev. 175, 1523-4 (1968).

Chu, Y.Y., Franz, E.-M., and Friedlander, G. Isomerism in ${ }^{147} \mathrm{~Tb}$. Phys. Rev. (in press).

Ghu, Y.Y. and Hahn, R.L. Production cross-sections of some I, Te, $\mathrm{Ba}$ and $\mathrm{Cs}$ isotopes from the interaction of 1,2 , and $2.9-\mathrm{GeV}$ protons with uranium. J. Inorg. $\mathrm{Nucl}$. Chem. 30, 2561-9 (1968).

Conklin, D.P., Gentile, P.S., And Coppens, P. The crystal structure of trans-dicyanotriethylenetetramine cobalt(III) perchloráte. Submitted to Inorg. Chim. Acta.

Coppens, P. Diffraction studies of the chemical bond. In Organic Solid-State Chemistry, Gordon \& Breach, London (in press).

Coppens, P. Distribution of hydrogen isotopes in $\alpha$-oxalic acid dihydrate as determined by neutron diffraction. (A) Presented at Amer. Cryst. Ass. Meet., Seattle, March 1969.

Coppens, P. Errors in the calculated structure factors caused by the free-atom form factor model. Acta Cryst. A25, 180-6 (1969).

Coppens, P. Evidence for systematic errors in $\mathrm{x}$-ray temperature parameters resulting from bonding effects. Ibid. B24, 1272-4 (1968).

Coppens, $P$. Influence of bonding effects on thermal parameters as determined by $\mathrm{x}$-ray diffraction. In Molecular Dynamics and Structure of Solids (2nd Mater. Res. Symp., Gaithersburg, Md., Oct. 1967), pp. 385-8, R.S. Carter and J. J. Rush, Editors, Nat. Bur. Std. U.S. Spec. Publ. 301, June 1969.

Coppens, P. New perspectives in neutron diffraction analysis of small organic molecules. In Measurement and Interpretation of Accurate Neutron Structure Factors, B.T.M. Willis, Editor, Oxford Univ. Press (in press).

Coppens, P. and Hamilton, W.C. Anisotropic extinction corrections in the Zachariasen approximation. Acta Cryst. (in press).

Coppens, P., Sabine, T.M., Delaplane, R.G., and Ibers, J.A. An experimental determination of the asphericity of the atomic charge distribution in oxalic acid dihydrate. Ibid.

Coppens, P. - See also Conklin, D.P.

Corliss, L.M., Delapalme, A., Hastings, J.M., LaU, H., and Nathans, $R$. Critical magnetic scattering from $\mathrm{RbMnF}_{3}$. (A) J. Appl. Phys. 40, 1278 (1969).

Corliss, L.M. - See also Kunnmann, W.; Norvell, J.C.

Crespo, V.P., Cumming, J.B., and Poskanzer, A.M. Fission of $\mathrm{U}^{238}$ by $2.2-\mathrm{GeV}$ protons. Phys. Rev. 174, 1455-71 (1968).

Cumming, J.B. - See Crespo, V.P.; Remsberg, L.P.

D'Amico, J. - See McCorkell, R.H. 
Davies, G., Sutin, N., and Watkins, K.O. Redox reactions of cobalt(III) in acid perchlorate solution. (A) Presented at Amer. Chem. Soc. Meet., New York, Sept. 1969.

Davis, R. JR. Measurement of fast neutron fluxes underground. (A) Presented at Amer. Geophys. Union Meet., Washington, D.C., April 1969.

Davis, R. JR. Neutrinos from the sun. In 1969 rearbook of Science and Technology, pp. 230-3, McGraw-Hill, New York, 1969.

DAvis, R. JR. A search for neutrinos from the sun. In Proc. Discussion Meeting on Neutrino Physics, Moscow, Sept. 1968 (in press).

Davis, R. JR. The search for solar neutrinos. Umschau 5, $153(1969)$.

Davison, A. - See La Placa, S. J.

Dawson, R.F., Ghristman, D.R., and Byerrum, R.U. Biosynthesis of nicotinic acid in plants and microbes. Advan. Enzymol. (in press).

Delapalme, A. - See Corliss, L.M.

Delaplane, R.G. - See Coppens, P.

Denney, D.B. - See Seltzer, S.

De Paz, M., Leventhal, J. J., and Friedman, L. Experimental study of heat of proton solvation. J. Chem. Phys. 49, 5543-4 (1968).

De Paz, M., Leventhal, J.J., and Friedman, L. A tandem mass spectrometer study of $\mathrm{D}_{3} \mathrm{O}+$ and solvated derivatives. J. Chem. Phys. (in press).

Edmonds, J.W., Hamilton, W.C., And Tippe, A. Methyl group rotation and the low temperature transition in hexamethylbenzene: A neutron scattering study. (A) Presented at Amer. Cryst. Ass. Meet., Seattle, March 1969.

Edmonds, J.W. - See also Hamilton, W.C.

Ehrenson, S. An examination of hydrogen exchange in $\mathrm{CH}_{5}+$ by the CNDO method. Chem. Phys. Letters (in press).

EHRENSON, S. On damping in self-consistency cycling procedures. Theoret. Chim. Acta 5, 346 (1969).

Ehrenson, S. Semiempirical molecular orbital theory and molecular geometry. I. Analytic procedures for extended Hückel methods; II. Analytic procedures for charge redistribution methods. J. Amer. Chem. Soc. 91, 3693-709 (1969).

Ehrenson, S. and Seltzer, S. GNDO bond strength indices. (L) Submitted to J. Amer. Chem. Soc.

Ehrenson, S. - See also Wells, P.R.

Elias, H. ANd Wolf, A.P. The $\mathrm{CO}_{2} / \mathrm{CO}$ ratio as internal radiation dosimeter in recoil carbon atom studies. Radiochim. Acta 10, 111-12 (1968).

Elliott, N. - See Erickson, N.

Emery, G.T. - See Perlman, M.L.

Epstein, E.F. AND Bernal, I. The crystal structure of bis[cis-1,2-bis(trifluoromethyl)ethylene-1,2-dithiolato]iron triphenylarsine, $\left[\left(\mathrm{CF}_{3}\right)_{2} \mathrm{C}_{2} \mathrm{~S}_{2}\right]_{2} \mathrm{FeAs}\left(\phi_{3}\right)$. (A) Presented at Amer. Cryst. Ass. Meet., Seattle, March 1969.

Epstein, E.F., Bernal, I., Herlinger, A.W., and Long, T.V. II. An investigation of pentacoordination in $\mathrm{Co}\left(\mathrm{NH}_{3}\right)_{6} \mathrm{CuCl}_{5}, \mathrm{Co}\left(\mathrm{NH}_{3}\right)_{6} \mathrm{CdCl}_{5}$ and $\mathrm{Co}\left(\mathrm{NH}_{3}\right)_{6} \mathrm{ZnCl}_{5}$ using laser-Raman, infrared and $x$-ray diffraction techniques. (A) Presented at Amer. Chem. Soc. Meet., New York, Sept. 1969.
Epstein, E.F. - See also Long, T.V. II.

Erickson, N.E. Mössbauer studies of tin compounds with quadrupole splittings in externally applied magnetic fields. (A) Presented at Mid-Amer. Symp. Spectroscopy, Chicago, May 1969.

Erickson, N.E., Cefola, M., and Kazimer, E.O. Structural aspects of Mössbauer spectroscopy: Mössbauer studies of quadrupole split tin compounds in strong externally applied magnetic fields. (A) Presented at Amer. Chem. Soc. Meet., Minneapolis, April 1969.

Erickson, N.E. AND Elliott, N. Magnetic susceptibility and Mössbauer spectrum of $\mathrm{NH}_{4} \mathrm{Fe}^{3+} \mathrm{Fe}^{\mathrm{II}}(\mathrm{CN})_{6}$. Submitted to J. Phys. Chem. Solids.

Erickson, N.E. - See also Kazimer, E.O.; ReIfF, W.M.

Finn, R.D., Ache, H. J., ANd Wolf, A.P. Chemical effects following $\mathrm{N}^{14}(n, p) \mathrm{C}^{14}$ in magnesium nitride. J. Phys. Chem. (in press).

Fireman, E.L. - See McCorkell, R.H.

Franz, E.-M. - See Chu, Y.Y.

Friedlander, G. Chemists' use of multi-GeV accelerators at Brookhaven National Laboratory. (A) Presented at Amer. Chem. Soc. Meet., Minneapolis, April 1969.

Friedlander, G. - See also Chen, K.; Chu, Y.Y.

Friedman, L. Ion molecule reactions. Annu. Rev. Phys. Chem. 19, 273-300 (1968).

Friedman, L. - See also De Paz, M.; Leventhal, J. J.

Gebicki, J.M. ANd Allen, A.O. Relationship between critical micelle concentration and rate of radiolysis of aqueous sodium linoleate. (N) J. Phys. Chem. 73, 2443-5 (1969).

Gebicki, J.M. - See also Bielskı, B.H.J.

Gentile, P.S. - See Conklin, D.P.

Gilat, J. And Grover, J.R. Detailed evaporation calculations of heavy ion reactions in the $Z=30-35$ region. (A) Presented at Amer. Chem. Soc. Meet., New York, Sept. 1969.

Godel, J.B. - See Rudman, R.

Gold, V. Rule of the geometric mean: Its role in the treatment of thermodynamic and kinetic deuterium solvent isotope effects. Trans. Faraday Soc. 64, 2770-9 (1968).

Gopinathan, K.P. and Rubinson, W. Decay energies of the $e^{-}$capture nuclides $\mathrm{I}^{125}$ and $\mathrm{Cd}^{109}$ measured by internal bremsstrahlung spectra. (A) Bull. Amer. Phys. Soc. 13, 1452 (1968).

Groening, H. and Harbottle, G. Isotopic effects in recoil reactions of molybdenum isotopes in $\mathrm{Mo}(\mathrm{CO})_{6}$. (A) Presented at Meet. Chemical Effects of Nuclear Transformations, Cambridge, England, July 1969.

Grover, J.R. Molecular flow of gases of short-lived radioactive nuclides. J. Inorg. Nucl. Chem. (in press).

Grover, J.R., Lebowitz, E., and Baker, E. Prompt emanation of astatine and radon. Ibid.

Grover, J.R. - See also Gilat, J.

Guarino, A. and Wolf, A.P. The reactions of trans-2,3dimethylcyclopropyl methylene produced photolytically in the gas phase. (L) Tetrahedron Letters No. 8, 655-8 (1969).

Hagebø, E. - See Hudis, J.

HAHN, R.L. - See ChU, Y.Y.

HAlEvi, E.A. - See Shiner, V.J. JR.

Hamilton, W.G. Chemical applications of neutron diffraction. (A) Presented at Symp. Aims of Increased 
Accuracy in Diffraction Studies of Biological, Chemical, and Geological Problems, Buffalo, N.Y., March 1969.

Hamilton, W.C. Comment on the order-disorder transition in ferroelectric ammonium sulfate. (L) J. Chem. Phys. 50, 2275 (1969)

Hamilton, W.C. Comparison of $x$-ray and neutron diffraction structural results: A study in methods of error analysis. Acta Cryst. A25, 194-206 (1969)

Hamilton, W.C. Computers, chemists and crystallography. J. Comput. Phys. 2, 417-38 (1968).

Hamilton, W.C. Crystal structure analysis today. (A) Presented at Amer. Chem. Soc. Meet., New York, Sept. 1969.

Hamilton, W.C. Diffraction studies of molecular motion. In Organic Solid-State Chemistry, Gordon \& Breach, London (in press).

Hamilton, W.C. Structure and dynamics in molecular crystals: some comparisons. In Molecular Dynamics and Structure of Solids (2nd Mater. Res. Symp., Gaithersburg, Md., Oct. 1967), pp. 193-218, R.S. Carter and J. J. Rush, Editors, Nat. Bur. Std. U.S. Spec. Publ. 301, June 1969.

Hamilton, W.C., Edmonds, J.W., Tippe, A., ANo Rush, J.J. Methyl group rotation and the low temperature transition in hexamethylbenzene: a neutron diffraction study. Discussions Faraday Soc. (in press).

Hamilton W.C., Kamb, B., La Placa, S. J., and Prakash, A. Deuteron arrangements in high-pressure forms of ice. In Proc. Int. Symp. Physics of Ice, Munich, West Germany, Sept. 1968 (in press).

Hamilton, W.C., Klanderman, K.A., and Spratley, R. Geometry of the ethylene ligand in Zeise's salt: A neutron diffraction study. (A) In Proc. 8th Int. Union Crystallography Congress, Stony Brook, N.Y., Aug. 1969 (in press).

Hamilton, W.C. and La Placa, S. J. The crystal and molecular structure of an anti-leukemia drug: Methylglyoxal bisguanylhydrazone dihydrochloride monohydrate, $\mathrm{C}_{5} \mathrm{~N}_{8} \mathrm{H}_{12} \cdot 2 \mathrm{HCl} \cdot \mathrm{H}_{2} \mathrm{O}$. Neutron and $\mathrm{x}$-ray diffraction studies. Acta Cryst. B24, 1147-56 (1968).

Hamilton, W.C. - See also Coppens, P.; Edmonds, J.W.; Jonsson, P.G.; La Placa, S. J.; Ross, F.K.; Schlemper, E.O.; TIPPE, A.

Harbottle, G. The effect of analytical methodology on the interpretation of hot atom chemistry in crystals. (A) Presented at Amer. Chem. Soc. Meet., Minneapolis, April 1969.

HARBOTTLE, G. Neutron activation analysis of potsherds from Knossos and Mycenae. Submitted to Archaeometry.

HaRbottle, G. Some current problems in the chemical effects of nuclear transformations. Presented at 2 nd Inter-Amer. Conf. Radiochemistry, Mexico City, April 1968

Harbottle, G. and Hillman, M. Szilard-Chalmers processes for isotope production. Chapter in $I A E A$ Manual of Radioisotope Production (in press).

Harbottle, G. - See also Butterworth, J.S.; GroenING, $\mathrm{H}$.

Hastings, J.M. Research at the Brookhaven High Flux Beam Reactor. (A) Presented at Amer. Chem. Soc. Meet., Minneapolis, April 1969.

Hastings, J.M. - See also Corliss, L.M.; Kunnmann, W.; Norvell, J.C.

HAvens, W.W. JR. - See Leung, P.S

Herlinger, A.W. - See Epstein, E.F.; Long, T.V. II
Hudis, J., Hagebø, E., and Patzelt, P. ${ }^{43} \mathrm{Ar}$ and ${ }^{44} \mathrm{Ar}$ (A) Presented at Amer. Phys. Soc. Meet., Washington, D.C., April 1969.

Hudis, J. ANd Katcoff, S. High-energy-proton fission cross sections of $\mathrm{U}, \mathrm{Bi}, \mathrm{Au}$, and $\mathrm{Ag}$ measured with mica track detectors. Phys. Rev. 180, 1122-30 (1969).

Hunziker, H.E. Cadmium-photosensitized interconversion of cis-, $_{\text {trans-, }}$ and geminal-ethylene- $d_{2}$. J. Chem. Phys. 50, 1288-94 (1969).

Hunziker, H.E. Energy transfer by benzene in the cadmium-photosensitized isomerization of butene-2. Ibid., 1294-8.

Ibers, J.A. - See Coppens, P.; La Placa, S. J.

ISHIDA, T. AND BIGELEISEN, J. Vapor pressures of the isotopic ethylene. IV. Liquid ethylene- $d_{3}$ and $-d_{4} . J$. Chem. Phys. 49, 5498-509 (1968).

Jonsson, P.G. And Hamilton, W.C. Neutron and $\mathbf{x}$-ray diffraction studies of hydrazinium sulfate, $\mathrm{N}_{2} \mathrm{H}_{6} \mathrm{SO}_{4}$. Submitted to Acta Cryst.

KamB, B. - See Hamilton, W.C.

Katcoff, S. - See Hudis, J.

Kazimer, E.O., Gefola, M., and Erickson, N.E. Sign of the ${ }^{119} \mathrm{Sn}$ electric quadrupole field gradient in dimethyltin difluoride and origin of ${ }^{119}$ Sn electric field gradients in organotin halide compounds. (L) Submitted to J. Amer. Chem. Soc.

Kazimer, E.O. - See also Erickson, N.E.

Keenan, J.A. - See Sugihara, T.T.

Kelley, J.D. ANd Wolfsberg, M. Simple expression for "steric factor" in translational-vibrational energy transfer. (N) J. Chem. Phys. 50, 1894-6 (1969).

Kibby, C.L. And Weston, R.E. JR. Photolysis of hexafluoroacetone in the presence of $\mathrm{H}_{2}, \mathrm{D}_{2}$, and $\mathrm{HD}$. Kinetic isotope effects in the reaction of $\mathrm{CF}_{3}$ with molecular hydrogen. Ibid. 49, 4825-40 (1968).

Kirsten, T., Schaeffer, O.A., Norton, E., and StoenNER, R.W. Experimental evidence for the double beta decay of Te ${ }^{130}$. Phys. Rev. Letters 20, 1300-3 (1968).

Klanderman, K.A. - See Hamilton, W.C.

KRUTZIK, S. AND Wolf, A.P. The stereochemical course of hot halogen substitution reactions and the reactions of excited intermediates produced in these reactions. (A) Presented at 5th Int. Hot Atom Meeting, Cambridge, England, July 1969.

Kunnmann, W. Crystal growth by fused salt electrolysis. (A) Presented at ACCG Conf. Crystal Growth, Gaithersburg, Md., Aug. 1969.

KunNmann, W. A review of the preparation of single crystals by fused melt electrolysis and some general properties. In Preparation of Solid State Materials (in press).

Kunnmann, W., La Placa, S., Corliss, L.M., Hastings, J.M., AND BANKs, E. Magnetic structures of the ordered trirutiles $\mathrm{Cr}_{2} \mathrm{WO}_{6}, \mathrm{Cr}_{2} \mathrm{TeO}_{6}$ and $\mathrm{Fe}_{2} \mathrm{TeO}_{6}$. J. Phys. Chem. Solids 29, 1359-64 (1968).

La Placa, S. J., Hamilton, W.C., and Davison, A. The metal-hydrogen bond length in $\mathrm{Mn}(\mathrm{CO})_{5} \mathrm{H}$ : A neutron diffraction study. (A) Presented at Amer. Chem. Soc. Meet., Minneapolis, April 1969.

La Placa, S.J., Hamilton, W.C., Ibers, J.A., and Davison, A. Nature of the metal hydrogen bond in transition metal hydrogen complexes: Neutron and $\mathrm{x}$ ray diffraction studies of $\beta$-HMn $(\mathrm{CO})_{5}$. Inorg. Chem. 8, 1928-35 (1969). 
La Placa, S.J. and Post, B. The crystal structures of stoichiometric and defect $\mathrm{B}_{12} \mathbf{P}_{2}$. (A) Presented at Amer. Chem. Soc. Meet., New York, May 1969.

La Placa, S. J. - See also Hamilton, W.C.; Kunnmann, W.; SGHLEMPER, E.O.

LaU, H. - See Corliss, L.M.

Lebowitz, E. and Miller, J.M. The production of tritium from bombardment of $\mathrm{He}^{4}$ with $2.2-\mathrm{GeV}$ protons. Phys. Rev. 177, 1548-52 (1969).

Lebowitz, E. - See also Grover, J.R.

Lechtman, H.N. - See Sayre, E.V.

Leung, P.S., Taylor, T.I., and Havens, W.W. JR. Studies of phase transitions in ammonium salts and barriers to rotation of ammonium ions by neutronscattering cross sections as a function of temperature. J. Chem. Phys. 48, 4912-18 (1968).

Leventhal, J.J. and Friedman, L. Energy transfer in the de-excitation of $\left(\mathrm{H}_{3}{ }^{+}\right)^{*}$ by $\mathrm{H}_{2} . J$. Chem. Phys. 50, 2928-31 (1969).

Leventhal, J. J. and Friedman,L. Experimental determination of $\mathrm{D}_{3}+$ dissociation energy. Ibid. 49, 1974-5 (1968).

Leventhal, J.J - See also De Paz, M.

Long, T.V. II, Herlinger, A.W., Epstein, E.F., ANd BerNAL, I. The syntheses, structures, laser-Raman and infrared spectra of $\mathrm{Co}\left(\mathrm{NH}_{3}\right)_{6} \mathrm{CuCl}_{5},\left[\mathrm{Co}\left(\mathrm{NH}_{3}\right)_{5} \mathrm{OH}_{2}\right]$ $\mathrm{CuCl}_{5}, \mathrm{Co}\left(\mathrm{NH}_{3}\right)_{6} \mathrm{CdCl}_{5}, \mathrm{Co}\left(\mathrm{NH}_{3}\right)_{6} \mathrm{ZnCl}_{5}$ and $\mathrm{Co}-$ $\left(\mathrm{NH}_{3}\right)_{6} \mathrm{ZnCl}_{4}\left(\mathrm{NO}_{3}\right)$. Submitted to Inorg. Chem.

Long, T.V. II - See also Epstein, E.F.

Manojlovic-Muir, L.M. The O-H . . S hydrogen bond in barium thiosulphate monohydrate. (N) Submitted to Nature.

McCorkell, R.H., Fireman, E.L., D'Amico, J., and Thompson, S.O. Radioactive isotopes in Hoba West and other iron meteorites. Meteoritics 4, 113-22 (1968).

McEwen, W.E. - See Shen, K.W.

Miller, J.M. - See Chen, K.; Lebowitz, E.

Mylonakis, S.G. and Seltzer, S. The possibility of energy transfer through a hydrocarbon chain during thermal decomposition of an azo compound. J. Amer. Chem. Soc. 90, 5487-9 (1968).

Norton, E. - See Kirsten, T.

Norvell, J.C., Wolf, W.P., Corliss, L.M., Hastings, J.M., AND Nathans, R. Magnetic neutron scattering in dysprosium aluminum garnet. I. Long-range order; II. Short-range order and critical scattering. Phys. Rev. (in press).

Olin, J.S. And SAYre, E.V. The analysis of English and American pottery of the American colonial period. Presented at 1968 Int. Conf. Modern Trends in Activation Analysis, Gaithersburg, Md., Oct. 1968.

Olin, J.S. and SAYre, E.V. Compositional categories of some English and American pottery of the American colonial period. In Proc. Amer. Chem. Soc. Symp. Archaeological Chemistry, Atlantic City, Sept. 1968 (in press).

Orhanović, M., Po, H.N., and Sutin, N. The kinetics and mechanism of the oxidation of hexaaquovanadium(II) by the isothiocyanato- and thiocyanatopentaaquochromium(III) ions. J. Amer. Chem. Soc. 90, 7224-9 (1968).

Patzelt, P. - See Hudis, J.

Perlman, M.L. and Emery, G.T. Environmental effects on internal conversion rates. (A) Presented at Int. Conf. Radioactivity in Nuclear Spectroscopy, Nashville, Tenn., Aug. 1969.
Periman, M.L. - See also Remsberg, L.P.; Sugihara, T.T.

Plasil, F. - See Remsberg, L.P.

Po, H.N. - See Orhanović, M.

Poskanzer, A.M. - See Crespo, V.P.

Post, B. - See La Placa, S. J.

Prakash, A. - See Hamilton, W.C.

Rapp, M. - See Shiner, V. J. Jr.

ReEder, P.L. Excitation function for the $\mathrm{Mg}^{25}(p, 2 p) \mathrm{Na}^{24}$ reaction in the GeV energy region. Phys. Rev. 178, 1795801 (1969).

Reiff, W.M., Erickson, N.E., and Baker, W.A. JR. Mono(2,2',2"'-terpyridine) complexes of iron. Inorg. Chem. 8, 2019-21 (1969).

RemsBerG, L.P. Angular distributions of the recoil nuclei from the $\mathrm{Cu}^{65}(p, p n) \mathrm{Cu}^{64}$ reaction at $0.37,1.0$, and 2.8 GeV. Phys. Rev. 174, 1338-42 (1968).

Remsberg, L.P. Mechanism of the reactions $\mathrm{Cu}^{63}(p, n)$ $\mathrm{Zn}^{63}$ and $\mathrm{Cu}^{63}(p, 2 n) \mathrm{Zn}^{62}$ at $1.0 \mathrm{GeV}$. Phys. Rev. (in press).

Remsberg, L.P. Production of the $\Delta(1236)$ isobar in complex nuclei: Double differential cross sections for the recoil nuclei from the $\mathrm{Cu}^{65}\left(p, p \pi^{+}\right) \mathrm{Ni}^{65}$ reaction. Ibid.

Remseerg, L.P., Plasil, F., Gumming, J.B., and PerlMAN, M.L. Fragment energy and velocity measurements in fission of uranium by $2.9-\mathrm{GeV}$ protons. Ibid.

Ricci, J.S. And Bernal, I. The crystal structure of $\alpha$ bis( $p$-nitrophenyl) disulfide. J. Amer. Chem. Soc. 91, 4078-82 (1969).

Ricci, J.S. AND Bernal, I. The crystal structure of bis $(0-$ nitrophenyl) disulfide. Submitted to J. Chem. Soc. B.

Rigci, J.S. JR. AND BERnal, I. The crystal structures of ortho and para-dinitrophenyldisulfides. (A) In Proc. 8th Int. Union Crystallography Congress, Stony Brook, N.Y., Aug. 1969 (in press); presented at Amer. Chem. Soc. Meet., New York, Sept. 1969.

Ross, F.K. And Hamilton, W.C. Neutron diffraction study of the crystal structure of sodium nitroprusside. (A) Presented at Chem. Soc. Meet., Southampton, England, Sept. 1969.

Rowley, J.K. The use of Gran plots for determining end points in potentiometric redox titrations. (N) Submitted to Anal. Chem.

Rubinson, W. - See Gopinathan, K.P.

Rudman, R. Single-crystal alignment with a polaroid adapter for the Weissenberg goniometer. J. Appl. Cryst. 1, 126-7 (1968).

Rudman, R. and Godel, J.B. An automatic low-temperature apparatus for single crystal diffractometry. $J . A p p l$. Cryst. (in press).

Rush, J. J. - See Hamilton, W.C.

SAbine, T.M. - See Coppens, P.

SAITo, E. Electron spin resonance studies of gamma-irradiated ferrocene. J. Chem. Phys. 50, 3539-47 (1969).

Sayre, E.V. and Chan, L.H. High resolution gamma ray spectroscopic analyses of Mayan fine orange pottery. In Proc. Amer. Chem. Soc. Symp. Archaeological Chemistry, Atlantic City, Sept. 1969 (in press).

SaYre, E.V. and Lechtman, H.N. Neutron activation autoradiography of oil paintings. Stud. Consero. 13, 16185 (1968).

Sayre, E.V. - See also Al Kital, R.A.; Olin, J.S.

Schaeffer, O.A. - See Kirsten, T. 
Schlemper, E.O., Hamilton, W.C., and La Placa, S. J. A short, slightly asymmetrical intramolecular hydrogen bond: A neutron diffraction study. (A) In Proc. 8th Int. Union Crystallography Congress, Stony Brook, N. Y., Aug. 1969 (in press).

Schlemper, E.O., La Placa, S. J., and Hamilton, W.C. A short intramolecular hydrogen bond: The crystal structure of dichloro,bis(2-amino-2-methyl-3-butanone oximato)cobalt(III). (A) Presented at Amer. Cryst. Ass. Meet., Seattle, March 1969.

Schmidt, W.F. ANd Allen, A.O. Mobility of free electrons in dielectric liquids. (N) J. Chem. Phys. 50, 5037 (1969).

Schmidt, W.F. And Allen, A.O. Yield of free ions in irradiated liquids; determination by a clearing field. J. Phys. Chem. 72, 3730-6 (1968).

Schwarz, H.A. Applications of the spur diffusion model to the radiation chemistry of aqueous solutions. Ibid. 73, 1928-37 (1969).

Schwarz, H.A. - See also Appleby, A.; Bielski, B.H. J.

Seewald, D., Sutin, N., and Watkins, K.O. The reaction of chloropentaaquoruthenium(III) with chromium(II): Binuclear intermediates, reduction of perchlorate, and the effect of vanadium(II).J. Amer. Chem. Soc. (in press).

Seltzer, S. The mechanism of the Diels-Alder reaction. Advan. Alicyclic Chem. 2, 1-57 (1968).

Seltzer, S., Tsolis, A., and Denney, D.B. An isotope effect study of the acetolyses of some triphenylcarbalkoxymethylphosphonium salts. J. Amer. Chem. Soc. 91, 4236-43 (1969).

Seltzer, S. - See also Ehrenson, S.; Mylonakis, S.G.; SteVens, K.D.

Sequeira, A. And Bernal, I. The crystal structure of bistetraphenylarsonium tris-(cis-1,2-dicyano-1,2-ethylenedithiolato)Fe(IV). Submitted to J. Phys. Chem.

Sequeira, A. and Bernal, I. A neutron diffraction study of lithium perchlorate trihydrate. In Molecular Dynamics and Structure of Solids (2nd Mater. Res. Symp., Gaithersburg, Md., Oct. 1967), pp. 249-51, R.S. Carter and J. J. Rush, Editors, Nat. Bur. Std. U.S. Spec. Publ. 301, June 1969.

Shen, K.W., McEwen, W.E., And Wolf, A.P. On the possibility of phenyl group migration in 7-phenyl-1,3,5cycloheptatriene. (L) Tetrahedron Letters No. 10, 827-31 (1969).

Shen, K.W., McEwen, W.E., And Wolf, A.P. Photolysis and thermolysis of pentaphenylantimony in benzene. J. Amer. Chem. Soc. 91, 1283-8 (1969).

Shiner, V. J. Jr., Rapp, M., Halevi, E.A., and WolfsBERG, M. Solvolytic $\alpha$-deuterium effects for different leaving groups. (L) Ibid. 90, 7171-2 (1968).

Spratley, R. - See Hamilton, W.G.

Stevens, K.D. And Seltzer, S. Thiocyanate-catalyzed cis-trans isomerization of $c i s-\beta$-acetylacrylic acid. A model for maleylacetoacetic acid. J. Org. Chem. 33, 39228 (1968).

Stoenner, R.W. and Thompson, S.O. $\mathrm{Ar}^{37}, \mathrm{Ar}^{39}$ and $\mathrm{Cl}^{36}$ radioactivities in the iron meteorite Alandroal. (A) Presented at Amer. Geophys. Union Meet., Washington, D.C., April 1969.

Stoenner, R.W. - See also Kirsten, T.

Sugihara, T.T., Keenan, J.A., and Perlman, M.L. Gamma rays and transition multipolarities in the decay of 15.8-h 186 Ir. Phys. Rev. 181, 1650-64 (1969).
Sutin, N. Free energies, barriers, and reactivity patterns in oxidation-reduction reactions. Accounts Chem. Res. 1, 225-31 (1968).

Sutin, N. The mechanisms of some electron-transfer reactions in solution. Electrochim. Acta 13, 1175-80 (1968).

SuTin, N. Substitution-controlled oxidation-reduction reactions. (A) In Proc. 22nd Int. Congr. Pure and Applied Chemistry and 12th Int. Congr. Coordination Chemistry, Sydney, Australia, Aug. 1969 (in press).

SuTin, N. Substitution-controlled redox reactions involving metal complexes. (A) Presented at Amer. Ghem. Soc. Symp. Inorganic Kinetics, Washington, D.C., Feb. 1969.

Sutin, N. - See also Davies, G.; Orhanović, M.; SeeWALD, D.

TAFT, R.W. - See WELLS, P.R.

TAYLOR, T.I. - See Leung, P.S.

Thompson, S.O. - See McCorkell, R.H.; Stoenner, R.W.

Tippe, A. and Hamilton, W.C. Neutron diffraction study of decaborane. Inorg. Chem. 8, 464-70 (1969).

Tippe, A. - See also Edmonds, J.W.; Hamilton, W.C.

Tsolis, A. - See Seltzer, S.

Watkins, K.O. - See Davies, G.; Seewald, D.

Welch, M. J., Withnell, R., ANd Wolf, A.P. An automatic GLPG apparatus for the analysis of organic compounds labelled with short-lived radioisotopes. Chem. Instr. (in press).

WELCH, M. J. AND Wolf, A.P. The formation of excited intermediates in recoil carbon atom reactions. Pressure dependence studies in the gas phase. Submitted to $J$. Amer. Chem. Soc.

Wells, P.R., Ehrenson, S., and TAFt, R.W. Substituent effects in the naphthalene series. An analysis of polar and Pi delocalization effects. Progr. Phys. Org. Chem. 6, 147-322 (1968).

Weston, R.E. JR. High-temperature approximations for kinetic isotope effects. (N) J. Chem. Phys. (in press).

Weston, R.E. Jr. Interatomic Forces in Chemical Reactions, Brookhaven Lecture Series No. 76, July 10, 1968. BNL 50133 (T-507).

Weston, R.E. JR. - See also KibBy, C.L.

WIDMER, H.M. Ion association of strong acids in solvent extraction systems. (A) Presented at Amer. Chem. Soc. Meet., New York, Sept. 1969.

Withnell, R. - See Welch, M. J.

Wolf, A.P. - See Ache, H. J.; Elias, H.; FinN, R.D.; Guarino, A.; Krutzik, S.; Shen, K.W.; Welch, M. J.

Wolf, W.P. - See Norvell, J.C.

Wolfsberg, M. Correction to the effect of anharmonicity on isotopic exchange equilibria. Application to polyatomic molecules. (N) J. Chem. Phys. 50, 1484-6 (1969).

Wolfsberg, M. Isotope effects. Annu. Rev. Phys. Chem. (in press).

Wolfsberg, M. - See also Bigeleisen, J.; Kelley, J.D.; SHINER, V. J. JR.

\section{Instrumentation and Health Physics Department}

BaUM, J.W. Model for radiation induced cell inactivation and loss of repair process vs dose and linear energy transfers. (A) Presented at Radiat. Res. Soc. Meet., Cincinnati, May 1969. 
Baum, J.W., Kuehner, A.V., and Chase, R.L. Dose equivalent meter designs based on tissue equivalent proportional counters. Submitted to Health Phys. J.; (A) Health Phys. J. 15, 195 (1968).

Baum, J.W., Woodcock, R.C., and Kuehner, A.V. Factors affecting pulse size in sealed tissue-equivalent counters. (A) In Proc. 2nd Int. Conf. Accelerator Dosimetry and Experience, Stanford, Calif., Nov. 1969 (in press).

Baum, J.W. - See also Kemmoghi, M.; Wingate, C.L.

Brown, R.M. AND Stone, R.L. Some remarks on a saturable dye used for laser pulse shaping. Appl. Optics (in press).

Busch, N.E. - See Brown, R.M.; Singer, I.A.

Champagne, R. J. - See Phillips, L.F.

Chase, R.L. Flyback system for a constant velocity Mössbauer drive. Rev. Sci. Instr. 40, 85-8 (1969).

Chase, R.L. Pulse timing system for use with gamma rays on $\mathrm{Ge}(\mathrm{Li})$ detectors. Ibid. 39, 1318-26 (1968).

Chase, R.L. - See also Baum, J.W.; Chester, J.

Chester, J. The use of a differential voltage comparator as an alarm level control. In Proc. Mid-Year Topical Symposium Health Physics Society, Los Angeles, Jan. 1969 (in press).

Chester, J. and Chase, R. A compact counting system utilizing integrated circuits and a solid state detector. Submitted to Health Phys.

Colvett, R.D. - See Distenfeld, C.H.

Cowan, F.P. Policy and practice for accelerator radiation safety. Nucl. Safety (in press).

Dimmler, D.G. and Felberbaum, J. The use of a small computer as a communications controller for multiuser on-line systems. (A) In Proc. IEEE 1969 Nuclear Science Symposium, San Francisco, Oct. 1969 (in press).

Distenfeld, C.H., Colvett, R.D., and Omegna, R.L. Evaluation of accidental exposure to accelerator personnel. (A) Health Phys. 15, 179 (1968).

Felberbaum, J. - See Dimmler, D.G.

Fischer, J., Igo, G. J., And Orbeson, S.B. Digitized spark chamber with very high spatial resolution at high counting rates. (A) In Proc. Int. Symp. Nuclear Electronics, Versailles, France, Sept. 1968, Vol. 3, pp. 5-1 to 5-3, Documentation Française, Paris.

Fischer, J. AND Shibata, S. Electron attachment in noble gases with traces of $\mathrm{CCl}_{4}$ or Freon 12, using pulsed spark chambers as supersensitive electron indicators. (A) Presented at 9th Int. Conf. Phenomena in Ionized Gases, Bucharest, Sept. 1969.

Fischer, J. and Shibata, S. Proportional mode digital discharge chambers. (A) In Proc. Int. Symp. Nuclear Electronics, Versailles, France, Sept. 1968, Vol. 3, pp. 2-1 to 2-4, Documentation Française, Paris.

Fischer, J. and Shibata, S. Shower counting with transmission line type digital spark chambers. (A) Ibid., pp. 3-1 to 3-4.

Fischer, J., Shibata, S., and Roos, C.E. Digital sparkgrid-chamber for a superconducting magnet. (A) Ibid., pp. 4-1 to 4-3.

Fischer, J. - See also Carroll, A.S. (Physics).

Frizzola, J.A. The ascent of power plant plumes during various meteorological conditions. In Proc. American Power Conference, Vol. 31, 1969 (in press).

Frizzola, J.A. - See also Brown, R.M.; Singer, I.A. Gemmell, L. - See Pearsall, S.G.
Goodman, L. J. - See Hartin, W. J.

Hartin, W. J. and Goodman, L. J. An improved safety system. Presented at Annu. Meet. Health Phys. Soc., Pittsburgh, June 1969.

HAYES, J.V. - See RaYNor, G.S.

Hull, A.P. And SMIth, M.E. Environmental monitoring of ${ }^{131} \mathrm{I}$ as a verification of meteorological calculations of dispersion from a 100 meter stack. In Proc. 1st Int. Congr. International Radiation Protection Association, Rome, Sept. 1966, pp. 659-67, Pergamon, London, 1968.

Igo, G. J. - See Fischer, J.

Karlovac, N. - See Radeka, V.

Kemmochi, M. And Baum, J.W. Rem responding recombination chamber system. (A) Presented at Annu. Meet. At. Energy Soc. Japan, Tokyo, March 1969.

Kuehner, A.V. - See Baum, J.W.

Majeski, S. - See Pearsall, S.G.

Meinhold, C.B. Facility monitoring programs, techniques and problem solving. In Proc. Mid-Year Topical Symposium Health Physics Society, Los Angeles, Jan. 1969 (in press).

Miller, D.M. - See Palmer, J.P.

Nagle, C.M. - See Singer, I.A.

Ogden, E.C. - See Raynor, G.S.

Omegna, R.L. - See Distenfeld, C.H.

Ophir, D., Shepherd, B. J., ANd SpInrad, R. J. Threedimensional computer display. Commun. Ass. Computing Machinery 12, 309-10 (1969).

Orbeson, S.B. - See Fischer, J.

Palmer, J.P. And Miller, D.M. Dynamic modulus and internal friction instrumentation. Informal Report BNL 12772.

Pearsall, S.G., Majeski, S., and Gemmell, L. Design and testing of a shipping container for large quantities of radioactive waste. In Proc. 2 nd Int. Symp. Transportation and Packaging of Radioactive Materials, AEC, Gatlinburg, Tenn., Oct. 1968 (in press).

Phillips, L.F., Scalsky, E.D., and Champagne, R. J. Comparison of health physics radiation detectors in a reactor radiation environment. (A) Health Phys. 15, 193 (1968).

RADEKA, V. Field-effect transistor noise as a function of temperature and frequency. In Proc. Conf. Semiconductor Radiation Detectors and Circuits, Gatlinburg, Tenn., May 1967, Nat. Acad. Sci-Nat. Res. Council Publ. 1593, 393 (1968).

RAdEKA, V. $1 /|f|$ Noise in physical measurements. In Proc. Ispra Nuclear Electronics Symposium, Ispra, Italy, May 1969, pp. 1-19, Euratom, CID, Brussels.

RADEKA, V. Signal filtering - high counting rate problems. In Proc. Int. Symp. Nuclear Electronics, Versailles, France, Sept. 1968, Vol. 1, pp. 61-1 to 61-26, Documentation Française, Paris.

Radeka, V. Signal processing for high-resolution pulse amplitude spectrometry. In Proc. Conf. Semiconductor Radiation Detectors and Circuits, Gatlinburg, Tenn., May 1967, Nat. Acad. Sci.-Nat. Res. Council Publ. 1593, 511 (1968).

RADEKA, V. State of the art of low noise amplifiers for semiconductor radiation detectors. In Proc. Int. Symp. Nuclear Electronics, Versailles, France, Sept. 1968, Vol. 1, pp. 46-1 to 46-28, Documentation Française, Paris.

Radeka, V. and Karlovac, N. Time-variant filters for high-rate pulse amplitude spectrometry. In Proc. Conf. 
Semiconductor Radiation Detectors and Circuits, Gatlinburg, Tenn., May 1967, Nat. Acad. Sci--Nat. Res. Council Publ. 1593, 553 (1968).

RAYNOR, G.S. Variation in entrance efficiency of a filter sampler with air speed, flow rate, angle and particle size. Submitted to Amer. Ind. Hygiene Ass. J.

RAYNOR, G.S. Wind impaction efficiency of non-operating rotoslide samplers. Submitted to $J$. Allergy.

Raynor, G.S., Hayes, J.V., and Ogden, E.C. Areas within isopleths of ragweed pollen concentrations from local sources. Arch. Environ. Health 19, 92-8 (1969).

Rogers, E. J. Wide-range Van de Graaff pulser. IEEE Trans. Nucl. Sci. NS-16, No. 3, Pt. 1, 105-6 (1969).

Roos, G.E. - See Fischer, J.

Scalsky, E.D. - See Phillits, L.F.

Shepherd, B. J. - See Ophir, D.

Shibata, S. - See Fischer, J.

Singer, I.A., Busch, N.E., and Frizzola, J.A. The micrometeorology of the turbulent flow field in the atmospheric surface boundary layer. In Wind Effects on Buildings and Structures (Proc. Int. Res. Seminar, Ottawa, Sept. 1967), Vol. 1, pp. 557-94, Nat. Res. Council, Washington, D.C., 1968; Acta Polytech. Scand. Phys. Nucl. Ser. 59, 45 pp. (1968).

Singer, I.A. AND NAGLe, G.M. Steadiness of the wind within the United States. (A) Presented at World Meteorol. Org. Symp. Urban Climates, Brussels, Oct. 1968.

Singer, I.A. AND NAGLE, G.M. Variability of wind direction within the United States. Submitted to Nucl. Safety.

Singer, I.A. AND Smith, M.E. The adequacy of existing meteorological data for evaluating structural problems. In Proc. Meet. National Bureau of Standards, Washington, D.C., Jan. 1969 (in press).

Singer, I.A. AND SMith, M.E. A summary of the recommended guide for the prediction of the dispersion of airborne effluents (ASME). Presented at World Meteorol. Org. Symp. Urban Climates, Brussels, Oct. 1968.

SMITh, M.E. The influence of atmospheric dispersion on the exposure of plants to airborne pollutants. $J$. Amer. Phytopathol. Soc. 58, 1085-8 (1968).

Smith, M.E. - See also Hull, A.P.; Singer, I.A.

SPINRAD, R. J. The automated laboratory. Trans. N.Y. Acad. Sci. 30, 517 (1968).

Spinrad, R. J. - See also Ophir, D.

Stone, R.L. - See Brown, R.M.

Wingate, C.L. and Baum, J.W. Measured delta ray dose around a charged particle beam. (A) Presented at 2nd Int. Conf. Medical Physics, Boston, Aug. 1969.

Woodcock, R.G. - See Baum, J.W.

\section{Medical Department}

Abe, H. - See Shreeve, W.W.

Adamik, E.R. - See JoEL, D.D.

Alvi, Z.M. And LAX, L.C. A non-equilibrium mathematical model of body electrolytes. (A) Presented at 8th Int. Conf. Medical and Biological Engineering, Chicago, July 1969.

Amendola, F. - See Johnson, H.A.

Anderson, L., Hankes, L.V., Politzer, W.M., and TouSTER, O. Catabolism of inositol in animals: How, where and for what purpose? (A) Presented at Conf. Cyclitols and Phosphoinositides, New York, Sept. 1968. Anderson, L. - See also Hankes, L.V.

Aponte, G.E. - See Shellabarger, G. J.

ArchambeaU, J.O. The response of the skin of swine to increasing absorbed doses of the ${ }^{10} \mathrm{~B}(n, \alpha)^{7} \mathrm{Li}$ reaction. II. Histologic and cytologic changes. Submitted to Radiat. Res.

Archambeau, J.O., Fairchild, R.G., Brenneis, H., and STraub, R.F. The response of the skin of swine to increasing doses of the ${ }^{10} \mathrm{~B}(n, \alpha){ }^{7} \mathrm{Li}$ reaction. I. Gross observations. Submitted to Radiat. Res.

Archambeau, J.O., Mathieu, G.R., Brenneis, H. J., And Thompson, K.R. The response of the skin of swine to increasing multiple exposures of $x$-ray $(250 \mathrm{kVP})$. Radiat. Res. 37, 141-60 (1969).

Archambeau, J.O., Mathieu, G.R., Brenneis, H., Thompson, K., ANd FaIrchild, R.G. The response of the skin of swine to increasing single exposures of 250kVp x-rays. Ibid. 36, 299-326 (1968).

Archambeau, J.O., Stryckmanns, P., and Brenneis, H. The effect of food and water deprivation on the peripheral blood parameters of the mouse. Ibid., 396-409.

Aronson, R.B. - See Cotzias, G.C.; Popenoe, E.A.

Atkins, H.L. The medical use of californium-252. Isotop. Radiat. Technol. (in press).

Aткins, H.L. Radiotherapy with fast neutrons. In Proc. Nuclear Engineering Education Conference, Argonne National Laboratory, Feb. 1969 (in press).

Atkıns, H.L. Scintillation scanning with technetium99m. Proc. Acad. Sci. Kazakh SSR 288, No. 4, 59-64 (1969).

Atkıns, H.L. Use of californium-252 as an interstitial source of fast neutrons. In Proc. Transplutonium Program Committee Meeting, Germantown, Md., April 1968, CONF680420-1.

Atkins, H.L., Fairchild, R.G., and Drew, R.M. The effect of californium-252 neutrons on HeLa cells in culture. (A) Presented at Annu. Meet. Radiol. Soc. N. Amer., Chicago, Dec. 1968.

Atkins, H.L., Hauser, W., Nelson, K.G., AND Richards, P. Technetium-99m-DTPA: A new radiopharmaceutical for brain and kidney scanning. (A) Presented at Joint ASCP-CAP Interim Meet., Los Angeles, Feb. 1969.

Atkins, H.L., Hauser, W., and Richards, P. Factors affecting distribution of technetium-99m sulfur colloid. (A) Presented at Soc. Nucl. Med. Meet., New Orleans, June 1969.

Atrins, H.L., Hauser, W., and Richards, P. Reduction of respiratory artifact in scintillation scanning. Amer. J. Roentgenol. Radiat. Ther. Nucl. Med. 104, 682-5 (1968).

Atrins, H.L., Hauser, W., Richards, P., and RobertSon, J.S. Analysis of the optimum conditions for data display and image perception in photoscanning. In Medical Radioisotope Scintigraphy (Proc. Symp., Salbzurg, Austria, Aug. 1968), pp. 759-70, IAEA, Vienna, 1969.

Atkins, H.L. ANd Richards, P. Brain scanning with technetium-99m. In Clinical Use of Technetium-99m, Chap. 4, W.H. Green, St. Louis (in press).

Atkins, H.L. And Richards, P. Pertechnetate metabolism and its relationship to scanning of thyroid, salivary glands and stomach. Ibid., Chap. 3. 
Atkins, H.L., Richards, P., and Hauser, W. Five years' experience with $99 \mathrm{~m}$ Tc-S colloid. (A) Presented at Soc. Nucl. Med. Meet., New Orleans, June 1969.

Atkins, H.L., Richards, P., and Hauser, W. Technetium-sulfur colloid: Four-year experience. (A) Presented at Joint ASCP-CAP Interim Meet., Los Angeles, Feb. 1969.

Atkins, H.L. - See also Cohn, S.H.; Fairchild, R.G.; Hauser, W.; Richards, P. (Applied Science); SchifFER, L.M.; TAKETA, S.T.

Bateman, J.L. AND Bond, V.P. The relation of radiation quality and dose for causing lens opacification in the mouse. (A) Presented at 17 th Annu. Meet. Radiat. Res. Soc., Cincinnati, May 1969.

Bengtsson, G., Robertson, J.S., Goodman, L. J., and MARINo, S.A. Computer evaluation of monoenergetic neutron irradiations in radiobiology. Submitted to Radiat. Res.

Ben-Ishay, D. and Welner, A. Sensitivity to experimental hypertension and aggressive reactions in rats. Submitted to Proc. Soc. Exp. Biol. Med.

Ben-Ishay, D. - See also Welner, A.

Bond, V.P. The gastrointestinal syndrome. In Comparative Cellular and Species Radiosensitivity (Proc. Int. Seminar, Kyoto, Japan, May 1968), pp. 235-40, V.P. Bond and T. Sugahara, Editors, Igaku Shoin Ltd., Tokyo, 1969.

Bond, V.P. Radiation mortality in different mammalian species. Ibid., pp. 5-16.

Bond, V.P. Radiobiological bases for the understanding of the hematological consequences of radiation exposure. In Manual on Radiation Haematology, Chap. 6, IAEA and World Health Org. (in press).

Bond, V.P. Report of symposium, Comparative Cellular and Species Radiosensitivity in Animals. Submitted to Science.

Bond, V.P. - See also Bateman, J.L.; Carsten, A.L.; Feinendegen, L.E.; Jesseph, J.E.; Odartchenko, N.; Shellabarger, C. J.; TAketa, S.T.; Tisljar-Lentulis, G.M.

Borg, D.C. - See Fajer, J. (Applied Science); Felton, R.H. (Applied Science).

Borner, G. - See Vincent, P.C.

Brenneis, H. - See Archambeau, J.O.

BRONK, B.V. On radioactive labeling of proliferating cells: The graph of labeled mitosis. J. Theoret. Biol. 22, 468-92 (1969).

Brown, R.R. - See HaNkes, L.V.

Brysk, M.M., Corpe, W.A., AND Hankes, L.V. $\beta$-cyanoalanine formation by Chromobacterium violaceum. J. Bacteriol. 97, 322-7 (1969).

Burke, K. - See Mena, I.

Carsten, A.L. and Bond, V.P. Colony forming units in the bone marrow of partial body irradiated mice. In Proc. Teaching Symp. Normal and Malignant Cell Growth, Chicago, Feb. 1968, CONF-680206-2.

Carsten, A.L. and Bond, V.P. Effect of shielding on the CFU content of the mouse leg. (A) Radiat. Res. 35, 571 (1968).

Carsten, A.L. and Bond, V.P. Viability of stored bone marrow colony forming units. Nature 219, 1082 (1968).

Carsten, A.L. and Caveness, W.F. Alteration in photoevoked response following $x$-irradiation of the monkey cerebral cortex. (A) J. Electroencephalog. Clin. Neurophysiol. 24, 187-91 (1968).
Carsten, A.L., Caveness, W.F., Roizin, L., and Machex, J. Bilateral depression in photo-evoked response as a late effect of unilateral visual cortex $x$-irradiation (A) Presented at Alpine Joint Meet. European and American Encephalography Societies, Kitzbuchel, Austria, Jan. 1969.

Carsten, A.L., Caveness, W.F., Roizin, L., and Machek, J. Bilateral EEG depression as a late effect of unilateral cortex x-irradiation in the monkey. (A) In Proc. Meet. Radiation Research Society, Cincinnati, May 1969 (in press).

Carsten, A.L. - See also Caveness, W.F.; Graham, E.S.; Matsui, K.; Reincke, U.; Roizin, L.; Skougaard, M.R.; TAKETA, S.T.

Caveness, W.F., Roizen, L., Carsten, A.L., and Schade, J.P. The effects of $x$-irradiation on the cerebral cortex of the monkey. Trans. Amer. Neurol. Ass. 1967, 188-93.

Caveness, W.F. - See also Carsten, A.L.; Roizin, L.

Chanana, A.D., Cronkite, E.P., Joel, D.D., And SchifFER, L.M. Skin allograft survival in calves. Arch. Surg. 98, 803-6 (1969).

Ghanana, A.D., Gronkite, E.P., Joel, D.D., and SchifFER, L.M. Skin allograft survival in lymphocyte depleted calves. In Proc. 2nd Int. Congr. Transplantation Society, New York, Sept. 1968 (in press).

Chanana, A.D., Cronkite, E.P., Joel, D.D., Schiffer, L.M., And Schnappauf, H. Studies on lymphocytes. XII. The role of immunologically committed lymphocytes in rejecting skin allografts. Transplantation 7, 45967 (1969).

Chanana, A.D., Gronkite, E.P., Joel, D.D., and Sipe, C.R. Influence of thymectomy and extracorporeal irradiation of blood upon blood lymphocytes in goats. Fed. Proc. 28, 376 (1969).

Chanana, A.D. - See also Cronkite, E.P.; Joel, D.D.; Lowenhaupt, R.; Moon, H.W.; Schiffer, L.M.; Stoner, R.D.; STryckmans, P.A.; Vincent, P.C.

Chiv, C.C., Schwartz, I.L., and Walter, R. Oxytocin: Crystal data of a seleno analog. Science 163, 925-6 (1969).

Chiu, C.C. - See also Walter, R.

Cohn, S.H., Baker, D.G., Jahn, A., And Dombrowski, C. Influence of cold exposure on skeletal metabolism in rats. J. Appl. Physiol. 26, 524-9 (1969).

Cohn, S.H., Dombrowski, C.S., and Fairchild, R.G. In vivo neutron activation analysis of calcium in man. Submitted to Int. J. Appl. Radiat. Isotop.

Cohn, S.H., Dombrowski, C.S., Hauser, W., and Atkins, H.L. High calcium diet and the parameters of calcium metabolism in osteoporosis. Amer. J. Clin. Nutr. 21, 124653 (1968)

Cohn, S.H., Dombrowski, C.S., PAte, H.R., and Robertson, J.S. A whole-body counter with an invariant response to radionuclide distribution and body size. Phys. in Med. and Biol. (in press).

Cohn, S.H., Fairghild, R.G., and Dombrowski, C.S. Whole-body neutron activation analysis of calcium. (A) Presented at Congr. Progress and Problems in In Vivo Activation Analysis, Glasgow, April 1969.

Cohn, S.H. - See also Fairchild, R.G.; Scherrer, J.R.; TAKeta, S.T.

Colcock, B.P. - See Conard, R.A.

Conard, R.A. Acute and delayed effects of fallout radiation on man. (A) Radiat. Res. 35, 498 (1968). 
Conard, R.A. Etiology and treatment of hyperthyroidism in the adult. J. Clin. Endocrinol. Metab. 27, 1773-4 (1967).

Conard, R.A. Late effects of radioactive fallout on the thyroid gland in a Marshallese population. In Proc. $A A A S$, New York, Dec. 1967 (in press).

Conard, R.A. Quantitative study of radiation effects in phytochemagglutinin-stimulated leukocyte cultures. Int.J. Radiat. Biol. (in press).

Conard, R.A. and Demoise, C.F. Preparation and purification of tritiated phytohemagglutinin and studies of cellular localization in human leukocyte cultures. Submitted to Blood.

Conard, R.A., Sutow, W.W., Colcock, B.P., Dobyns, M.M., And Paglia, D.E. Thyroid nodules as a late effect of exposure to fallout. In Proc. IAEA Symp. Radiation-Induced Carcinogenesis, Athens, April 28-May 2, 1969 (in press).

Conard, R.A. - See also Sutow, W.W.

Corpe, W.A. - See BrysK, M.M

Cottier, H., Ruchti, C., Sordat, B., and Cronkite, E.P. Extracorporeal irradiation of circulating blood: Means of study of circulation and recirculation of lymphocytes in the calf. Nouv. Rev. Fr. Hematol. 8, 679-84 (1968).

Cottier, H. - See also Laissue, J.; Michalke, W.D.; Odartchenko, N.; Stoner, R.D.

Cotzias, G.C. Dihydroxyphenylalanine treatment of Parkinsonism. (L) J. Amer. Med. Ass. 205, 541 (1968).

Cotzias, G.C. Discrimination revisited. (L) New Engl.J. Med. (in press).

Cotzias, G.C. Dopa and Parkinsonism. (L) Brit. Med.J. $1967,497$.

CotZIAs, G.C. Importance of trace substances in environmental health as exemplified by manganese. In Proc. 1st Annu. Conf. Trace Substances in Environmental Health, Columbia, Mo.,July 1967, pp. 5-19, Univ. of Missouri, 1967.

Cotzias, G.C. Levodopa (L-dopa) treatment of Parkinsonism. (L) J. Amer. Med. Ass. 207, 1522 (1969).

Cotzias, G.G. Parkinsonism and dopa: An editorial. $J$. Chronic Diseases (in press).

Cotzias, G.C., Mena, I., and Papavasiliou, P.S. Amelioration of the dystonia of chronic manganese poisoning by L-dopa. (A) Presented at 21 st Annu. Meet. Amer. Acad. Neurol., Washington, D.C., April 1969.

Cotzias, G.C. and Papavasiliou, P.S. Autoimmunity in patients treated with levodopa. (L) J. Amer. Med. Ass. 207, 1353-4 (1969).

Cotzias, G.C. and Papavasiliou, P.S. Therapeutic studies of Parkinsonian patients: Long-term effects of D-L and L-dopa. In Progress in Neuro-Genetics (Proc. 2 nd Int. Congr., Montreal, Sept. 1967), Vol. I, pp. 358-66 (in press).

Cotzias, G.C., Papavasiliou, P.S., Fehling, C., KaufMAN, B., AND MENA, I. Similarities between neurological effects of L-dopa and of apomorphine. Submitted to New Engl.J. Med.

Cotzias, G.C., Papavasiliou, P.S., and Gellene, R. L-Dopa in Parkinson's syndrome. (L) Submitted to New Engl.J. Med.

Cotzias, G.C., Papavasiliou, P.S., and Gellene, R. Modification of Parkinsonism - Chronic treatment with L-dopa. New Engl.J. Med. 280, 337-45 (1969).

Cotzias, G.C., Papavasiliou, P.S., Gellene, R., and Aronson, R.B. Parkinsonism and dopa. Trans. Ass. Amer. Physicians 81, 171-83 (1968).
Cotzias, G.C., Papavasiliou, P.S., Gellene, R., Fehling, C., And Mena, I. L-Dopa in the treatment of Parkinson's syndrome and of cronic manganese poisoning. (A) In Proc. 8th Int. Congr. Gerontology, Washington, D.C., Aug. 1969 (in press).

Cotzias, G.C., Papavasiliou, P.S., and Mena, I. Parkinsonism, dopa and chronic manganese poisoning. (A) Presented at World Congress of Neurology, New York, Sept. 1969.

Cotzias, G.C. - See also Mena, I.; Papavasiliou, P.S. Court, J. - See Mena, I.

Cronkite, E.P. Acute leukemia: Is there a relationship between cell growth kinetics and response to chemotherapy? Presented at 6 th Nat. Cancer Conference, Denver, Sept. 1968.

Cronkite, E.P. Clinical aspects of radiation injury. In Manual on Radiation Haematology, Chap. 3, IAEA and World Health Org. (in press).

Cronkite, E.P. Effects of extracorporeal irradiation of the blood and lymph on lymphopoiesis. (A) Presented at 7 th Annu. Meet. Eur. Soc. Radiat. Biol., Ulm (Donau), West Germany, Sept. 1969.

Cronkite, E.P. Experimental application of extracorporeal irradiation of the blood and lymph for immunosuppression. (A) Presented at AEC Science Writers Seminar, Brookhaven National Laboratory, June 1969.

Cronkite, E.P. A historical account of development of knowledge about lymphocytic function. Nouv. Rev. Fr. Hematol. 8, 503-8 (1968).

CRONKITE, E.P. Lymphocyte depletion by extracorporeal irradiation of blood and lymph. Ibid., 643-56.

CRONKITE, E.P. Normal human granulocytopoiesis. In The Proliferation and Spread of Neoplastic Cells, pp. 281-94, Williams \& Wilkins, Baltimore, 1968.

Cronkite, E.P. The study of lymphopoiesis utilizing extracorporeal irradiation of the blood and lymph. In Progress in Clinical Cancer, Vol. 4, Chap. 9, I. Ariel, Editor, Grune \& Stratton, New York (in press).

Gronkite, E.P., Chanana, A.D., Joel, D.D., Ral, K.R., AND Schiffer, L. M. Influence of extracorporeal irradiation of the blood and lymph on lymphoiesis and immunity. In Effects of Radiation on Cellular Proliferation and Differentiation (Proc. Symp., Monaco, April 1968), pp. 307-26, IAEA, Vienna, 1968.

Cronkite, E.P. And Schiffer, L.M. Kinetics of normal lymphopoiesis and chronic lymphocytic leukemia. In Regulation of Hematopoiesis, A.S. Gordon, Editor, Appleton-Century-Crofts, New York (in press).

Cronkite, E.P. And Vincent, P.C. Granulocytopoiesis. Presented at Symp. Myeloproliferative Disorders of Animals and Man, Pacific Northwest Laboratory, Richland, Wash., May 1968.

Gronkite, E.P. - See also Chanana, A.D.; Cottier, H.; Joel, D.D.; Lowenhaupt, R.; Matsui, K.; Moon, H.W.; Reincke, U.; Schiffer, L.M.; Shellabarger, C. J.; Stoner, R.D.; Stryckmans, P.A.; Tonna, E.A.; VINCENT, P.C.

DAHL, L.K. Effects of endocrine glands, pregnancy and sodium. In Renal Hypertension, pp. 264-74, I.H. Page and J.W. McCubbin, Editors, Year Book Medical Publishers, Chicago, 1968.

DAHL, L.K. Response to annotation on "Salt and Blood Pressure." Lancet 1969-1, 622-3. 
Dahl, L.K. Salt in processed baby foods. Amer. J. Clin. Nutr. 21, 787-92 (1968).

Dahl, L.K., Heine, M., Leitl, G., and Tassinari, L. Experimental hypertension and death from chronic consumption of processed baby-foods. Submitted to Nature.

Dahl, L.K., Knudsen, K.D., And IwaI, J. Humoral transmission of hypertension: Evidence from parabiosis. Circulation Res. 24-25, Suppl. I, I-21 to I-33 (1969).

Dahl, L.K. - See also IWAi, J.; Welner, A.

Demoise, C.F. - See Gonard, R.A.

Dobyns, B.M. - See Conard, R.A.

Dombrowski, C.S. - See Cohn, S.H.; Fairchild, R.G.

Drew, R.M. - See Atrins, H.L.; Fairchild, R.G.; Feinendegen, L.E.

Dubois, B.M. - See Glass, J.D. Jr.; Overweg, N.I.A.; WALTER, R.

Eggena, P., Schwartz, I.L., and Walter, R. Effects of neurohypophyseal hormones, theophylline and nucleotides on the smooth muscle of the toad bladder. Life Sci. 7, 979-88 (1968).

Eggena, P., Schwartz, I.L., and Walter, R. A sensitive hydroosmotic toad bladder assay. Affinity and intrinsic activity of neurohypophyseal peptides. J. Gen. Physiol. 52, 465-81 (1968).

Eggena, P., Walter, R., and Schwartz, I.L. Relationship between hydro-osmotic flow and the inhibited response of the toad bladder to vasopressin. Life Sci. 7, 59-63 (1968).

Eggena, P., Walter, R., and Schwartz, I.L. Stimuluseffect relationship in the antidiuretic action of neurohypophyseal peptides and prostaglandin $E_{1}$. (A) Presented at 4 th Int. Congr. Nephrology, Stockholm, June 1969.

Eggena, P. - See also Walter, R.

Elias, J.M. Effects of temperature, poststaining rinses and ethanol-butanol dehydrating mixtures on methyl green-pyronin staining. Stain Technol. 44, $201-4$ (1969).

FaIRChild, R.G. Dosimetry of ${ }^{252}$ Cf. In Californium-252 (Proc. Symp., Amer. Nucl. Soc., New York, Oct. 1968), pp. 277-84, CONF-681032, Jan. 1969.

Fairchild, R.G., Dombrowski, C., Yamazaki, E., and Cohn, S.H. An evaluation of neutron sources for whole-body neutron activation analysis. (A) Presented at Annu. Meet. Radiol. Soc. N. Amer., Chicago, Dec. 1968.

Fairchild, R.G., Drew, R.M., and Atkins, H.L. The relative biological effect of ${ }^{252} \mathrm{Cf}$ radiation on $\mathrm{HeLa}$ cells in culture. Radiology (in press).

Fairchild, R.G., Tonna, E.A., Seibold, G.T., and STRAUB, R.F. Neutron autoradiographic determination of boron- 10 concentration and distribution in mammalian tissue. Radiat. Res. 36, 87-97 (1968).

Fairchild, R.G. - See also Archambeau, J.O.; Atkins, H.L.; CoHN, S.H.

Farrer, D.N. - See Graham, E.S.

Fehling, C. - See Cotzias, G.C.

Feinendegen, L.E., Drew, R.M., Bond, V.P., And Heiniger, H. J. Radiation-induced DNA labelling in $\mathrm{G}_{1}$ phase in HeLa-S $\mathrm{S}_{3}$ cells. In Effects of Radiation on Cellular Proliferation and Differentiation (Proc. Symp., Monaco, April 1968), pp. 39-55, IAEA, Vienna, 1968.

Funzalida, S. - See Mena, I.
Gellene, R. - See Cotzias, G.C.; Papavasiliou, P.S.

Glass, J.D. JR., Dubois, B.M., Walter, R., and Schwartz, I.L. Mechanism of the inactivation of neurohypophyseal hormones. (A) Presented at Annu. Meet. Endocrine Soc., New York, June 1969.

Glass, J.D. JR., Schwartz, I.L., and Walter, R. Enzymatic inactivation of peptide hormones possessing a C-terminal amide group. Submitted to Proc. Nat. Acad. Sci. U.S.

Glass, J.D. JR. - See also Walter, R.

Goodman, L. J. - See Bengtsson, G.

Gordon, W., Havran, R.T., Schwartz, I.L., and Walter, $R$. Conformational differences in neurohypophyseal peptides: A countercurrent distribution and partition chromatography study. Proc. Nat. Acad. Sci. U.S. 60, 1353-5 (1968).

Gordon, W. - See also Walter, R.

Graham, E.S., Farrer, D.N., and Carsten, A.L. Radiation induced blindness in the rhesus monkey (Macaca mulatta). (A) Presented at West. Psycholog. Ass. Meet., Vancouver, Canada, June 1969.

Greenberg, M.L. - See Schiffer, L.M.; Stryckmans, P.A.; Vincent, P.C.

Groen, J. J. - See Welner, A.

Gut, V., Rudinger, J., Walter, R., Herling, P.A., and Schwartz, I.L. Derivatives of 1-tosyl-3-amino-2piperidone: Preparation and conversion to derivatives and peptides of ornithine. Tetrahedron 24, 6351-63 (1968).

Hamilton, L.D. - See Kim, C.W.; Savel, H.

Hankes, L.V. And Brown, R.R. Metabolism of D- and L-kynurenine-keto- ${ }^{14} \mathrm{C}$ in rats and the effects of unlabeled enantiomers. Proc. Soc. Exp. Biol. Med. 129, 14453 (1968).

Hankes, L.V., Brown, R.R., Schmaeler, M., and LekLeM, J. Tryptophan metabolism in scleroderma studied with ${ }^{14} \mathrm{C}$ labeled intermediates. (A) Fed. Proc. 28, 856 (1969).

Hankes, L.V., Brown, R.R., Schmaeler, M., and SchifFER, L.M. Tryptophan metabolism in humans with various types of anemias. Blood 32, 649-61 (1968).

Hankes, L.V., Politzer, W.M., Touster, O., and AnDERSON, L. myo-Inositol catabolism in human pentosurics: The predominant role of the glucuronatexylulose-pentose phosphate pathway. Ann. N.Y. Acad. Sci. (in press).

Hankes, L.V. - See also Anderson, L.; Brysk, M.M.

Hauser, W., Atkins, H.L., Nelson, K.G., and Richards, P. Technetium-99m-DTPA: A new radiopharmaceutical for brain and kidney scanning. Submitted to Radiology.

Hauser, W., Atkins, H.L., and Richards, P. Lymph node scanning with ${ }^{99 m}$ Tc-sulfur colloid. Radiology 92 , 1369-71 (1969).

Hauser, W. - See also Atkins, H.L.; Cohn, S.H.

Havran, R.T., Schwartz, I.L., and Walter, R. Glutamine isomers of oxytocin and deamino-oxytocin: Synthesis and pharmacological properties of [4-N $\mathrm{N}^{4}$-methyl$\mathrm{L}$-asparagine]-oxytocin and [1- $\beta$-mercaptopropionic acid, 4-N ${ }^{4}$-methyl-L-asparagine]-oxytocin. J. Mol. Pharmacol. 5, 83-9 (1969).

Havran, R.T., Schwartz, I.L., And Walter, R. Oxytocin analogs with basic amino acid residues in positions 
4 and 5: Synthesis and pharmacological properties of [4-ornithine]- and [5-ornithine]-oxytocin.J. Amer. Chem. Soc. (in press).

Havran, R.T. - See also Gordon, W.; Meyers, C.

Heine, M. - See Dahl, L.K.; Iwal, J.

Heiniger, H. J. - See Feinendegen, L.E.

Herling, P.A. - See Gut, V.

Hess, M.W. - See Laissue, J.; Michalke, W.D.

Horiuchi, K. - See Mena, I.

Hoshi, M. and Shreeve, W.W. Chronic effects of mannoheptulose in hyperglycaemic-obese mice. Metabolism 18, 422-6 (1969).

Hoshi, M. and Shreeve, W.W. Hepatic lipogenesis from ${ }^{14} \mathrm{C}$-labeled sucrose, fructose, and glucose in rats in vivo. (A) Fed. Proc. 28, 861 (1969).

Hoshi, M. - See also Shreeve, W.W.

Iwai, J., Knudsen, K.D., Dahl, L.K., Heine, M., And LEITL, G. Genetic influence on the development of renal hypertension in parabiotic rats. Evidence for a humoral factor. J. Exp. Med. 129, 507-22 (1969).

IwaI, J., Knudsen, K.D., DahL, L.K., and Tassinari, L. Effect of adrenalectomy on blood pressure in salt-fed, hypertension-prone rats. Failure of hypertension to develop in absence of evidence of adrenal cortical tissue. Ibid., 663-78.

IwAI, J. - See also DAHL, L.K.

Jamuar, M.P. - See Kim, C.W.

Jesseph, J.E., Moore, W.H., Straub, R.F., TisljarLentulis, G.M., ANd Bond, V.P. The RBE of 2.2-BeV protons for 30-day lethality in mice. Radiat. Res. 36, 24253 (1968).

Joel, D.D., Adamik, E.R., Chanana, A.D., Cronkite, E.P., SChiffer, L.M., ANd SiPE, C.R. Separation of lymphocytes from blood of calves and goats. Amer.J. Vet. Res. 30, 1099-104 (1969).

Joel, D.D., Chanana, A.D., Gronkite, E.P., and SchifFER, L.M. Cytotoxic antibody response to skin allografts in calves: Effects of extracorporeal irradiation of lymph. Proc. Soc. Exp. Biol. Med. 129, 483-6 (1968).

Joel, D.D. - See also Chanana, A.D.; Cronkite, E.P.; Lowenhaupt, R.; Moon, H.W.; Stoner, R.D.; VinCENT, P.C.

Johnson, H.A. Cytoplasmic response to overwork. In Proc. Conf. Renal Hypertrophy, Galveston, Texas, Oct. 1968 (in press).

Johnson, H.A. Information Theory and Biology: A Critique and Resynthesis, Brookhaven Lecture Series No. 68, Sept. 20, 1967. BNL 50158 (T-524).

Johnson, H.A. Liver regeneration and the "critical mass" hypothesis. Amer. J. Pathol. (in press).

Johnson, H.A. Liver regeneration: Cell growth, mitosis, and the "critical mass" hypothesis. (A) Presented at Amer. Ass. Pathologists Bacteriologists Meet., San Francisco, March 1969.

Johnson, H.A. And Amendola, F. Mitochondrial proliferation in compensatory growth of the kidney. Amer. J. Pathol. 54, 35-45 (1969).

Johnson, H.A. And Amendola, F. Relative hypertrophy of the mouse's right kidney. Growth 32, 199-203 (1968).

Johnson, H.A. and Pavelec, M. A theory for the doubling dose rate of ionizing radiation. (A) Presented at 17th Annu. Meet. Radiat. Res. Soc., Cincinnati, May 1969.
Johnson, L.F., Schwartz, I.L., and Walter, R. Oxytocin and neurohypophyseal peptides: Spectral assignment and conformational analysis by $220-$ Mc nuclear magnetic resonance. Submitted to Proc. Nat. Acad. Sci. U.S.

Kaufman, B. - See Cotzias, G.C.

Kim, C.W., Jamuar, M.P., and Hamilton, L.D. Transformation of lymphocytes from animals sensitized to Trichinella spiralis. (A) Presented at Joint Meet. Amer. Soc. Parasitologists Amer. Soc. Trop. Med. Hyg., Washington, D.C., Nov. 1969.

Kim, C.W. - See also Savel, H.

Kirsten, C. - See Vincent, P.C.

KNudsen, K.D. - See DahL, L.K. ; IwaI, J.

Koenig, D.F. - See Walter, R.

Laissue, J., Hess, M.W., Stoner, R.D., Riedwyl, H., and Cottier, H. Regional disparity of germinal center development in neonatally thymectomized mice after stimulation with tetanus toxoid. In Proc. 2nd Conf. Germinal Centers in Immune Responses, Padua, Italy, June 1968 (in press).

Lamdin, E., Shreeve, W.W., Slavinski, R., and OJI, N. Biosynthesis of fatty acids in obese mice in vivo. II. Studies with DL-malate-2- ${ }^{3} \mathrm{H}\left(-3-{ }^{14} \mathrm{C}\right)$, succinate2,3-3 H (-2,3-14 C), and DL-isocitrate-2-3 H $\left(-5,6-{ }^{14} \mathrm{C}\right)$. Biochemistry 8, 3325 (1969).

Lax, L.C. - See Alvi, Z.M.

LEFEVRE, M.E. Calibration of Clark oxygen electrode for use in aqueous solutions. J. Appl. Physiol.26, 844-8 (1969).

Leitl, G. - See Dahl, L.K.; IWAi, J.

LekLem, J.E. - See Hankes, L.V.

Liu, J.C. - See Roizin, L.

Lowenhaupt, R., Chanana, A., Gronkite, E.P., Joel, D., AND Nathan, P. Platelet accumulation in renal allotransplants in dogs, goats and rats: An electron microscopic study. Exp. Hematol. 16, 3 (1968).

Lyman, H. - See Russell, G.K.

Machek, J. - See Garsten, A.L.; Roizin, L.

Marino, S.A. - See Bengtsson, G.

Mathieu, G.R. - See Archambeau, J.O.

Matsui, K., Garsten, A.L., and Cronkite, E.P. Changes in ratio of erythropoietic to other spleen colonies induced by storage and media. Exp. Hematol. 18, 6-8 (1969).

Matsui, K. - See also Rubini, J.R.

Mena, I., Court, J., Funzalida, S., Papavasiliou, P.S., AND Cotzias, G.C. Modification of chronic manganese poisoning: Treatment with L-dopa or 5-OH tryptophane. Submitted to New Engl.J. Med.

Mena, I., Horiughi, K., Burke, K., and Cotzias, G.C. Chronic manganese poisoning: Individual susceptibility and absorption of iron. Neurology (in press).

Mena, I. - See also Cotzias, G.C.

Meyers, C., Havran, R.T., Schwartz, I.L., and Walter, R. Some reactions of $\mathcal{N}$-hydroxysuccinimide esters of $o$-nitrophenylsulfenyl protected glutamine and asparagine. Chem. Ind. London 1969, 136-7.

Michalke, W.D., Hess, M.W., Riedwyl, H., Stoner, R.D., AND Cottier, H. Thymic lymphopoiesis and cell loss in newborn mice. Blood 33, 541-54 (1969).

Miller, S.T. - See Papavasiliou, P.S

Moon, H.W., Joel, D.D., Chanana, A.D., Cronkite, E.P., And Stoner, R.D. Response of lymphocyte-de- 
pleted calves to fluid tetanus toxoid. Amer. J. Vet. Res. 29, 1211-14 (1968).

Nathan, P. - See Lowenhaupt, R.

Nelson, K.G. - See Atkins, H.L.; Hauser, W.

Odartchenko, N., Cottier, H., And Bond, V.P. Studies on "ineffective erythropoiesis" in the dog. Brit. J. Haematol. (in press).

OJi, N. - See Lamdin, E.; Shreeve, W.W.

Okuyama, S. - See Schiffer, L.M.

Ornstein, L. - See Pande, C.S.

Overweg, N.I.A., Schwartz, I.L., Dubois, B.M., and WALTER, $R$. Inhibition of the action of oxytocin on the rat uterus by acyclic oxytocin intermediates. J. Pharmacol. Exp. Therap. 161, 342-7 (1968).

Paglia, D.E. - See Conard, R.A.

Pande, C.S., Rudick, J., Ornstein, L., Schwartz, I.L., AND Walter, R. Specific tritium labelling of a potent gastrin analog: Synthesis and pharmacological activities of C-terminal gastrin tetrapeptide analogs. $J$. Mol. Pharmacol. (in press).

Papavasiliou, P.S., Gellene, R., and Cotzias, G.C. Modification of Parkinsonism: Dyskinesias accompanying treatment with dopa. In Psychotropic Drugs and Dysfunctions of the Basal Ganglia (in press).

Papavasiliou, P.S., Miller, S.T., and Cotzias, G.C. Functional interactions between biogenic amines. $3^{\prime}, 5^{\prime}$ Cyclic AMP and manganese. Nature 220, 74-5 (1968).

Papavasiliou, P.S. - See also Cotzias, G.C.; Mena, I.

Pate, H.R. - See Cohn, S.H.

Pavelec, M. - See Johnson, H.A.; Severson, A.R.

Politzer, W.M. - See Anderson, L.; Hankes, L.V.

Popenoe, E.A., Aronson, R.B., and Van Slyke, D.D. The sulfhydryl nature of collagen proline hydroxylase. Arch. Biochem. Biophys. (in press).

Quadrifoglio, F. - See Urry, D.W.; Walter, R.

RaI, K.R. - See Cronkite, E.P.; Schiffer, L.M.

Rane, L. - See Rubini, J.R.

Reincke, U., Carsten, A.L., and Cronkite, E.P. Growth characteristics of hemopoietic spleen colonies. (A) Presented at Annu. Meet. Eur. Soc. Radiat. Biol., Ulm, West Germany, Sept.1969.

Reincke, U., Carsten, A.L., and Gronkite, E.P. Size distribution of early regenerative foci in the spleens of whole-body irradiated mice. (A) Presented at Annu. Meet. Radiat. Res. Soc., Gincinnati, May 1969.

Riedwyl, H. - See Laissue, J.; Michalke, W.D.

Robertson, J.S. Computer utilization in a medical research center. (A) Presented at Symp. Cross-Disciplinary Science in Biomedical Research, Johannesburg, Union of South Africa, April 1969.

RoBertSon, J.S. Information theory applications in radioisotope scanning. In Advances in Dynamic Radioactive Scanning (Proc. Symp., Pittsburgh, Oct. 1965), pp. 20-7, Y. Wang, Editor, Thomas, Springfield, Ill., 1968.

Robertson, J.S. The role of computers in processing scanning data. In Fundamental Problems in Scanning (Proc. Symp., Chicago, May 1965), Chap. 20, pp. 247-9, A. Gottschalk and R.N. Beck, Editors, Thomas, Springfield, Ill., 1968.

Robertson, J.S. - See also Atkins, H.L.; Bengtsson, G.; Cohn, S.H.; Tisljar-Lentulis, G.M.

Robinson, C.V. Analysis of the effect of individual fluctuations in radioresistance on the width of the animal dose-survival curve. (A) Presented at Annu. Meet. Radiat. Res. Soc., Cincinnati, May 1969.

Robinson, C.V. Analysis in terms of cellular parameters of interanimal variation of hemopoietic radioresistance. In Comparative Cellular and Species Radiosensitivity, pp. 21119, V.P. Bond and T. Sugahara, Editors, Igaku Shoin Ltd., Tokyo, March 1969.

Roizin, L., Garsten, A.L., Machek, J., LiU, J.C., and Caveness, W.F. The vasculo-circulatory factor in the central nervous system pathogenesis of the $\mathrm{x}$-ray postirradiation effects. Trans. Amer. Neurol. Ass. 93, 270-2(1968).

Roizin, L. - See also Carsten, A.L.; Caveness, W.F.

Rubini, J.R., Matsui, K., and Rane, L. Blood proliferocytes in human and animal malaria. Submitted to Acta Hematol.

Ruchti, C. - See Cottier, H.

Rudick, J. - See Pande, C.S.

Rudinger, J. - See Gut, V.

Russell, G.K. AND Lyman, H. Isolation of mutants of Euglena gracilis with impaired photosynthesis. Plant Physiol. 43, 1284-90 (1968).

Savel, H., Kim, C.W., and Hamilton, L.D. Synthesis of radioactive Trichinella spiralis larval antigen in vitro. Exp. Parasitol. 24, 171-5 (1969).

Schade, J.P. - See Caveness, W.F.

SCherRer, J.R. And Cohn, S.H. Formulation of a stochastic model of long-term strontium retention data using the likelihood function. Biometrics (in press).

SCHIFFER, L.M. Kinetics of chronic lymphocytic leukemia. Ser. Haematol. 1, 3-23 (1968).

Schiffer, L.M., Atkins, H.L., Chanana, A.D., Cronkite, E.P., Greenberg, M.L., Okuyama, S., Rai, K.R., Stryckmans, P.A., ANd Vincent, P.C. Extracorporeal irradiation of blood in man. IV. Treatment of chronic lymphocytic leukemia. Submitted to Ann. Internal Med.

Schiffer, L.M., Chanana, A.D., Cronkite, E.P., GreenBerg, M.L., Rai, K., Stryckmans, P., and Vincent, P. Extracorporeal irradiation of the blood in chronic lymphocytic leukemia. Nouv. Rev. Fr. Hematol. 8, 691-700 (1968).

Schiffer, L.M. - See also Chanana, A.D.; Cronkite, E.P.; Hankes, L.V.; Joel, D.D.; Stryckmans, P.A.; VinCENT, P.G.

Schlesinger, D.H. - See Walter, R.

Schmaeler, M. - See Hankes, L.V.

Schnappauf, H. - See Chanana, A.D.

Schwartz, I.L. - See Chiu, C.C.; Eggena, P.; Glass, J.D. Jr.; Gordon, W.; Gut, V.; Havran, R.T.; Johnson, L.F.; Meyers, C.; Overweg, N.I.A.; PANde, C.S.; URRY, D.W.; WALTER, R.

Seibold, C.T. - See Fairchild, R.G.

Severson, A.R., Tonna, E.A., And Pavelec, M. Localization and distribution of adenosine triphosphatase activity in the femurs of young mice. Anat. Record 161, 5768 (1968).

Shellabarger, C. J. Hypothyroidism and DMBA rat mammary carcinogenesis. (A) Presented at Amer. Ass. Cancer Res. Meet., San Francisco, March 1969.

Shellabarger, C. J. Radiation carcinogenesis in the rat mammary gland. (A) Presented at Seminar, Radiation Bio-Effects Program, National Center for Radiological Health, Rockville, Md., Jan. 1969. 
Shellabarger, C. J., Bond, V.P., Cronkite, E.P., and Aponte, G.E. The relationship of dose of total body ${ }^{60} \mathrm{Co}$ radiation to incidence of mammary neoplasia in female rats. In Proc. Symp. Radiation-Induced Carcinogenesis, Athens, April 28-May 2, 1969 (in press).

Shreeve, W.W., Hoshi, M., and OJI, N. Oxidation of glucose-1-14 $\mathrm{C},-1-{ }^{3} \mathrm{H}$ to ${ }^{14} \mathrm{CO}_{2}$ and ${ }^{3} \mathrm{HOH}$ after oral glucose load in obese patients. (A) Diabetes 18, Suppl. 1, 372 (1969).

Shreeve, W.W., Hoshi, M., OJi, N., Shigeta, Y., and ABE, $H$. Insulin and the utilization of carbohydrates in obesity. Amer. J. Clin. Nutr. 21, 1404-18 (1968).

Shreeve, W.W. - See also Hoshi, M.; Lamdin, E.

Sipe, G.R. - See Chanana, A.D.; Joel, D.D.; Stoner, R.D.

Skougaard, M.R. and Carsten, A.L. Collagen metabolism in skin and periodontal membrane of the rat. (A) Presented at Conf. Periodontal Research, Rochester, N.Y., Aug. 1969.

SLAVINSKI, R. - See Lamdin, E.

Sordat, B. - See Cottier, H.

Stoner, R.D., Cottier, H., Sipe, C.R., Chanana, A.D., Joel, D.D., and Cronkite, E.P. The effects of extracorporeal irradiation of circulating blood and thoracic duct lymph upon tetanus antitoxin responses in calves. Radiat. Res. 37, 539-50 (1969).

Stoner, R.D. - See also Laissue, J.; Michalke, W.D.; Moon, H.W.; Terres, G.

Straub, R.F. - See Archambeau, J.O.; Fairchild, R.G.; JESSEPH, J.E.

Stryckmans, P.A., Chanana, A.D., Gronkite, E.P., Greenberg, M.L., And Schiffer, L.M. Studies on lymphocytes. X. Influence of extracorporeal irradiation of the blood on lymphocytes in chronic lymphocytic leukemia: Apparent correlation with RNA turnover. Radiat. Res. 37, 118-30 (1969).

Strygkmans, P.A. - See also Schiffer, L.M.; Vincent, P.C.

Stryckmanns, P. - See Archambeau, J.O.

Sutow, W.W. and Conard, R.A. The effects of fallout radiation on Marshallese children. In Proc. 9th Annu. Hanford Biol. Symp. Radiation Biology of the Fetal and Juvenile Mammal, Richland, Wash., May 1969 (in press).

Sutow, W.W. - See also Conard, R.A.

Taketa, S.T., Carsten, A.L., and Bond, V.P. Comparison of the stem cell survival model in protonand gamma-irradiated monkeys. (A) Presented at 17th Annu. Meet. Radiat. Res. Soc., Cincinnati, May 1969.

Taketa, S.T., Carsten, A.L., Cohn, S.H., Atrins, H.L., AND Bond, V.P. Active bone marrow distribution in the monkey. Submitted to Science.

TAssinari, L. - See Dahl, L.K.; Iwai, J.

Terres, G. and Stoner, R.D. Antibody responses in normal and irradiated mice elicited with antigen and specific antiserum. Submitted to Science.

Thompson, K.R. - See Archambeau, J.O.

Tisljar-Lentulis, G.M., Bond, V.P., Robertson, J.S., AND Moore, W.H. JR. Counter telescope measurements of a beam of negative $\pi$ mesons. Submitted to Radiat. Res.

Tisljar-Lentulis, G.M. - See also Jesseph, J.E.
Tonna, E.A. And Cronkite, E.P. Skeletal cell labeling following continuous infusion with tritiated thymidine. Lab. Invest. 19, 510-15 (1968).

Tonna, E.A. - See also Fairchild, R.G.; Severson, A.R.

Touster, O. - See Anderson, L.; Hankes, L.V.

Urry, D.W., Quadrifoglio, F., Walter, R., aNd Schwartz, I.L. Conformational studies on neurohypophyseal hormones. The disulfide bridge of oxytocin. Proc. Nat. Acad. Sci. U.S. 60, 967 (1968).

URRY, D.W. - See also Walter, R.

VAn Slyke, D.D. - See Popenoe, E.A.

Vincent, P.C., Borner, G., Ghanana, A.D., Cronkite, E.P., GreenberG, M.L., Joel, D.D., Schiffer, L.M., and Stryckmans, P.A. Studies on lymphocytes. XIV. Measurement of DNA synthesis time in bovine thoracic duct lymphocytes by analysis of labeled mitoses and by double labeling, before and after extracorporeal irradiation of the lymph. Cell Tissue Kinetics 2, 235-47 (1969).

Vincent, P.C., Borner, G., Chanana, A.D., Cronkite, E.P., Joel, D.D., SGhiffer, L.M., ANd Stryckmans, P.A. Comparison of two methods of estimating DNA synthesis time in calf thoracic duct lymphocytes. (A) Clin. Res. 16, 316 (1968).

Vincent, P.C., Cronkite, E.P., GreenberG, M.L., Kirsten, C., Schiffer, L.M., and Stryckmans, P.A. Leukocyte kinetics in chronic myeloid leukemia. I. DNA synthesis time in blood and marrow myelocytes. Blood 33, 843-50 (1969).

Vincent, P.C. - See also Cronkite, E.P.; Schiffer, L.M.

WALter, R. Further studies with selenium-containing amino acids and peptides. In Proc. 1st American Peptide Symposium, New Haven, Conn., Aug. 1968 (in press).

Walter, R., Dubois, B.M., Eggena, P., and Schwartz, I.L. Comparison of the mode of action of oxytocin and lysine-vasopressin on the isolated rat uterus. Experientia 25, 33-4 (1969).

Walter, R., Dubois, B.M., and Schwartz, I.L. Biological significance of the amino acid residue in position 3 of neurohypophyseal hormones and the effect of magnesium on their uterotonic action. Endocrinology 83, 979-83 (1968).

Walter, R., Glass, J.D. JR., and Schwartz, I.L. Oxytocin and vasopressin inactivation: Substrate specificity of a "carboxamidopeptidase" from toad bladder. (A) Presented at 4th Int. Congr. Pharmacology, Basel, Switzerland, July 1969.

Walter, R., Gordon, W., Schwartz, I.L., QuadriFoglio, F., AND URRY, D.W. Conformation studies on the neurohypophyseal hormone oxytocin and its analogs. In Peptides 1968 (Proc. 9th Eur. Symp., Orsay, France, April 1968), pp. 50-5, North-Holland, Amsterdam, 1968.

Walter, R., Koenig, D.F., Chiv, G.G., and Schwartz, I.L. Contribution of deamino-oxytocin analogs to peptide hormone research. In Proc. Int. Symp. Rational Development and Application of Drugs, Univ. of Nijmegen, The Netherlands, July 1969 (in press).

Walter, R., Schlesinger, D.H., and Schwartz, I.L. The chromatographic separation of isologous sulfurand selenium-containing amino acids: Reductive scission of the selenium-selenium bond by mercaptans and selenols. Anal. Biochem. 27, 231 (1969). 
Walter, R. ANd Schwartz, I.L. Reaction of L-2-acetylamino-2-carbomethoxyethanesulfonyl chloride with ammonia. Z. Naturforsch. 22b, No. 1 (1967).

Walter, R. - See also Chiu, C.C.; Eggena, P.; Glass, J.D. JR.; Gordon, W.; Gut, V.; HaVran, R.T.; Johnson, L.F.; Meyers, C.; Overweg, N.I.A.; Pande, G.S.; URRY, D.W.

Welner, A., Ben-Ishay, D., Groen, J.J., and Dahl, L.K. Behavior patterns and sensitivity to experimental hypertension in rats. Proc. Soc. Exp. Biol. Med. 129, 88690 (1968).

WELNER, A. - See also Ben-ISHAY, D.

YAMAZAKI, E. - See FAIRchILd, R.G.

\section{Photography and Graphic Arts}

Huhphrey, D.F. Public relations photography - Moving away from the "standup single." Phot. Business Prod. News 5, 25-30 (Feb. 1969); Tech. Phot. 1, 15-20 (Dec. 1968-Jan. 1969).

Rosen, M. Executive portraiture - Face to face. Phot. Business Prod. News 5, 38-41 (May 1969).

SмITH, R.F. Unconventional applications of specialized photographic emulsions. Phot. Appl. Sci. Technol. Med. 11, 26-9, 48 (May 1969).

\section{Physics Department}

Abrams, K.L., Gonnolly, P.L., Crennell, K.M., Crennell, D. J., Culwick, B.B., Delaney, W.C., Frantz, E., Hough, P.V.C., Jessup, S. J., Nardi, J.A., Scarr, J.M., Skillicorn, I.O., Strand, R.C., and Thorndike, A.M. A ten experiment perspective view of an analysis system using road-guidance FSD's. In Proc. Int. Conf. Advanced Data Processing for Bubble and Spark Chambers, Argonne National Laboratory, Oct. 1968, PP. 120-9, R. J. Royston, Editor, ANL-7515, 1969.

Akyuz, R.O. - See Rorer, D.C.

Albright, J.R. - See Sims, W.H.

Alburger, D.E. and Harris, W.R. Decay scheme of $\mathrm{Mg}^{28}$. Phys. Rev. (in press).

Alburger, D.E., Warburton, E.K., Gallmann, A., And Wilkinson, D.H. Decay of the $6.18-\mathrm{MeV} J^{\pi}=0^{+}$ level of $\mathrm{Be}^{10}$. Phys. Reu. (in press).

Alburger, D.E. - See also Gallmann, A.; Harris, W.R.; Jones, K.W.; Warburton, E.K.; Wilkinson, D.H.

Alitti, J., Barnes, V.E., Grennell, D. J., Flaminio, E., Goldberg, M., Karshon, U., Lai, K.W., Metzger, W.J., O'Neall, J.S., Samios, N.P., Scarr, J.M., and Schumann, T.G. Evidence for the $K_{1}{ }^{0} K_{1}{ }^{0}$ enhancement near threshold produced by $K-\mathcal{N}$ interactions. Phys. Rev. Letters 21, 1705-8 (1968).

Alitti, J., Barnes, V.E., Flaminio, E., Metzger, W., Radojicic, D., Rau, R.R., Richardson, C.R., Samios, N.P., Bassano, D., Goldberg, M., and Leitner, J. Strangeness $S=-2$ baryon resonance. Ibid. 22, 79-82 (1969).

Alitti, J., Barnes, V.E., Flaminio, E., Metzger, W., Rau, R.R., Samios, N.P., Bassano, D., GoldberG, M., AND LEITNER, J. Observation of new $S=-2$ baryonic resonances in $K-p$ interactions. (A) Bull. Amer. Phys. Soc. 14, 40 (1969).
Alitti, J., Flaminio, E., Metzger, W., Radojicic, D., RaU, R.R., Richardson, C.R., Samios, N.P., Bassano, D., Goldberg, M., and Leitner, J. Confirmation of the $\bar{\Xi}(1815)$ and evidence for a new resonance with mass $2030 \mathrm{MeV}$. Presented at 14th Int. Conf. High Energy Physics, Vienna, Aug. 28-Sept. 5, 1968.

Alitti, J., Flaminio, E., Metzger, W., Radojicic, D., Rau, R.R., Richardson, C.R., Samios, N.P., SkilliCorn, I.O., Bassano, D., Goldberg, M., and Leitner, $\mathrm{J}$. Evidence for $\Xi^{*}$ resonance with mass $1930 \mathrm{MeV}$. Phys. Rev. Letters 21, 1119-23 (1968).

Alperin, H.A. - See McGuire, T.R.

Alvarez Rivas, J.L. and Levy, P.W. X-ray induced first and second stage $\mathrm{F}$-center coloring of $\mathrm{NaCl}$ between 78 and $300^{\circ} \mathrm{K}$. (A) Presented at Int. Symp. Color Centers in Alkali Halides, Rome, Sept. 1968.

Alvarez Rivas, J.L. And Levy, P.W. X-ray induced first stage coloring of $\mathrm{NaCl}$ at $78^{\circ} \mathrm{K}$. (A) Ibid.

Anderson, E.W., Bleser, E. J., Blieden, H.R., Collins, G.B., Garelick, D., Menes, J., Turkot, F., Birnbaum, D., Edelstein, R.M., Hien, N.C., McMahon, T.J., MucCI, J., AND Russ, J. Observations of $A_{1}{ }^{-}, A_{2}-$ and higher mass bosons produced near $180^{\circ}$ in $\pi^{-} p \rightarrow p$ (missing mass)- at $16 \mathrm{GeV} / c$. Phys. Rev. Letters 22, 1390-4 (1969).

Anderson, E.W., Bleser, E. J., Blieden, H.R., Collins, G.B., Garelick, D., Menes, J., Turkot, F., Birnbaum, D., Edelstein, R.M., Hien, N.G., McMahon, T. J., Mucci, J., AND Russ, J. Observations of a backward peak in $\pi^{-} p \rightarrow p \rho^{-}$at 8 and $16 \mathrm{GeV} / c$. Ibid., 102-5.

Anderson, E.W., Bleser, E.J., Blieden, H.R., Collins, G.B., Garel.ick, D., Menes, J., Turkot, F., Birnbaum, D., Edelstein, R.M., Hien, N.C., McMahon, T. J., Mucci, J., And Russ, J. Search for the $Z^{*}$ in $\pi^{-}+p \rightarrow$ $K^{-}+Z^{*}$ at 6 and $8 \mathrm{GeV} / c$. Phys. Rev. Letters 29B, 136-9 (1969).

Andrus, W.S., Muether, H.R., and Palevsky, H. Temperature dependence of quasi-elastic scattering of cold neutrons from liquid argon. In Neutron Inelastic Scattering (Proc. IAEA Symp., Copenhagen, May 1968), Vol. 1, pp. 457-62, IAEA, Vienna, 1968.

Arbab, F. Unitarity and the Veneziano representation. Phys. Rev. (in press).

Arbar, F. and Brower, R.C. Pomeranchuk exchange and low energy theorems in Compton scattering. Ibid. 181, 2124-9 (1969).

Armstrong, D.D. And Wegner, H.E. Supercollimation of negative ion beams with apertures. (A) Bull. Amer. Phys. Soc. 14, 532 (1969).

Arndt, R.A., Korn, A., and Damask, A.C. Photo Hall effect in anthracene. (A) Ibid. 13, 1453 (1968).

Arndt, R.A. - See also Damask, A.C.; Korn, A.I.; Ringel, H.; Whitten, W.B.

Ashmore, A. - See Orear, J.

Aslanides, E. - See Gallmann, A.

Auerbach, E.H., Hind, M., Martin, B.A., And Rogers, P.C. An operating system for the BNL low energy accelerator facility computer. (A) Presented at Conf. Computer Systems in Experimental Nuclear Physics, Sky Top, Pa., March 1969.

Auerbach, E.H., Kahana, S., Scott, C.K., and Weneser, J. Effect of residual interactions: Analogue state energies, neutron radii. Presented at Conf. Nuclear Isospin, Asilomar, Calif., March 1969. 
Axe, J.D. and Shirane, G. An investigation of the $\alpha-\beta$ quartz phase transformation by inelastic neutron scattering. (A) Bull. Amer. Phys. Soc. 14, 594 (1969).

Axe, J.D. and Shirane, G. A study of the $\alpha-\beta$ quartz phase transformation by inelastic neutron scattering. Submitted to Phys. Rev.

Axe, J.D., Shirane, G., and Müller, K.A. Zoneboundary phonon instability in cubic $\mathrm{LaAlO}_{3}$. Phys. Rev. (in press).

Bamberger, J.A., Mulholland, G.T., Prodell, A.G., Worwetz, H.A., and Whetstone, C.N. An 8-in.-bore 60-kG superconducting magnet test facility. Advan. Cryog. Eng. 13, 132-41 (1968).

Barnes, V.E., Borenstein, S.R., Kalbfleisch, G.R., Strand, R.C., Vander Burg, V., Chapman, J.W., Davidson, J., Kiang, R., Koschik, J., and Lys, J.E.A. A new study of $2.15-\mathrm{GeV} / c K-p \rightarrow \Lambda \pi^{+} \pi^{-} \gamma$. (A) Bull. Amer. Phys. Soc. 14, 637 (1969).

Barnes, V.E., Chung, S.U., Eisner, R.L., Flaminio, E., Guidoni, P., Kinson, J.B., and Samios, N.P. Evidence for an $\omega \pi \pi$ resonance in the $I=1$ state at a mass of 1695 MeV. Phys. Rev. Letters 23, 142-5 (1969).

Barnes, V.E., Ghung, S.U., Flaminio, E., Jaeger, K., Montanet, L., Samios, N.P., and Skillicorn, I.O. Production of the $r_{1}{ }^{*}(1690)$ in the reaction $K-p \rightarrow$ $\Lambda^{0} \pi^{+} \pi^{-}$at $3.9,4.6$, and $5.0 \mathrm{GeV} / c$. (A) Bull. Amer. Phys. Soc. 14, 40-1 (1969).

Barnes, V.E., Chung, S.U., Flaminio, E., Jaeger, K., Montanet, L., Samios, N.P., and Skillicorn, I.O. A study of the reaction $K-p \rightarrow \Sigma^{0} \pi^{+} \pi^{-}$at $3.9,4.6$, and $5.0 \mathrm{GeV} / c$. (A) Ibid., 40.

Barnes, V.E., Dornan, P., Guidoni, P., Samios, N., Goldberg, M., AND LeItNer, J. Recent evidence for a charged nonstrange meson at $\sim 975 \mathrm{MeV}$ produced in $K-p$ interactions at $4.6 \mathrm{GeV} / c$. Presented at 14 th Int. Conf. High Energy Physics, Vienna, Aug. 28-Sept. 5, 1968.

Barnes, V.E., Flaminio, E., Montanet, L., Samios, N.P., Skillicorn, I.O., Goldberg, M., ANd Jaeger, K. Production of $\Sigma$ hyperons in $K-p$ interactions. Phys. Rev. Letters 22, 479-82 (1969).

Barnes, V.E. - See also Alitti, J.; Chung, S.U.

Bartley, J. - See Sims, W.H.

Bartram, R.H. - See Kemmey, P.J.

Bassano, D. - See Alitti, J.

Bassel, R.H. AND WILkIN, C. High-energy proton scattering and the structure of light nuclei. Phys. Rev. 174, 1179-99 (1968).

Baumel, P. - See Crennell, D. J.; O'Neall, J.S.; SchuMANN, T.G.

Beckurts, K.H. and Brugger, R.M. Pulsed reactor applications to neutron inelastic scattering experiments. Presented at Amer. Nucl. Soc. Meet., Albuquerque, N.M., Jan. 1969.

BEER, M. Correlations and distributions of widths in resonance neutron capture. Phys. Rev. 181, 1422-7 (1969).

Beer, M. ANd Ghrien, R.E. Nuclear structure and the statistics of resonance $(n, \gamma)$ partial widths. (A) Bull. Amer. Phys. Soc. 14, 515 (1969).

Beer, M., Graves, R., Wasson, O.A., Bhat, M.R., Mughabghab, S.F., Rimawi, K., Garber, D.I., and Chrien, R.E. Statistics of neutron resonance partial widths. (A) Ibid. 13, 1421 (1968).
Beer, M. - See also Ghrien, R.E.; Lone, M.A.; Price, D.L.; Wasson, O.A.

Bellini, G. - See Bingham, H.H.

Bender, P.L. and Cohen, V.W. Collisional broadening of the $\mathbf{R b}^{87}$ hyperfine transition. In Proc. 6th Int. Conf. Physics of Electron and Atomic Collisions, Cambridge, Mass., July 1969 (in press)

BEnJAMIn, J.A. Selection of a vac-ion pump for a terminal ion source. IEEE Trans. Nucl. Sci. NS-16, No. 3, Pt. 1, 107-8 (1969).

Bennett, L.H. - See Swartzendruber, L. J.; Watson, R.E.; WolcotT, N.M.

Berger, E.L. Double-Regge-pole model analysis of $p p \rightarrow p n \pi^{+}$at $28.5 \mathrm{GeV} / c$; pion exchange and the 1400 $\mathrm{MeV} \pi \mathcal{N}$ enhancement. Phys. Rev. Letters 21, 701-4 (1968).

Berger, E.L. - See also Ellis, W.E.

Bertanza, L., Bigi, A., Carrara, R., Casali, R., Pazzi, R., Berley, D., Hart, E.L., Rahm, D.C., Willis, W. J., Yamamoto, S.S., and Wong, N.S. $K$ - $p$ interactions from 594 to $820 \mathrm{MeV} / c$. Phys. Rev. 177, 203647 (1969).

Bigi, A. - See Bertanza, L.

Bingham, H.H., Farwell, C.W., Fretter, W.B., Irwin, G.M., Lu, A., Moffeit, K., Huson, F.R., Louttit, R.I., Miller, D. J., O’Neall, J.S., Bellini, G., di Corato, M., Fiorini, E., Moriyasu, K., Muggia, E., Negri, P., Rollier, M., Daugeras, B., Fournier, D., Hennessy, J., Novak, M., Six, J., and Veillet, J.J. Study of coherent $K$-interactions on nuclei at 5.5 and $12.7 \mathrm{GeV} / c$. Presented at 14th Int. Conf. High Energy Physics, Vienna, Aug. 28-Sept. 5, 1968.

Birgeneau, R. J., Guggenheim, H. J., and Shirane, G. Magnetic phase transition in the two-dimensional antiferromagnet $\mathrm{Rb}_{2} \mathrm{MnF}_{4}$. (A) Bull. A mer. Phys. Soc. 14, 738 (1969).

Birgeneau, R. J., Guggenheim, H. J., and Shirane, G. Neutron scattering from $\mathrm{K}_{2} \mathrm{NiF}_{4}$ : A two dimensional Heisenberg antiferromagnet. Submitted to Phys. Rev. Letters.

Birgeneau, R. J. - See also Cox, D.E.; Hutchings, M.T. Birnbaum, D. - See Anderson, E.W.

Bleser, E. J. - See Anderson, E.W.

Blieden, H.R. - See Anderson, E.W.

Blume, M. Stochastic theory of line shape: Generalization of the Kubo-Anderson model. Phys. Rev. 174, 351-8 (1968).

Blume, M., Geschwind, S., and Yafet, Y. Generalized Kittel-Van Vleck relation between $g$ and $g^{\prime}$ : Validity for negative $g$ factors. Ibid. 181, 478-87 (1969).

Blume, M. And Hsieh, Y.Y. Biquadratic exchange and quadrupole ordering. (A) J. Appl. Phys. 40, 1249 (1969).

Blume, M. - See also Sternheimer, R.M.; Watson, R.E.

Blumenfield, B. J. and Kalbfleisch, G.R. Formation of the $r_{1}^{*}(1616)$ and $r_{1}^{*}(1700)$ in $K_{L}{ }^{*} p$ interactions. (L) Phys. Letters 29B, 58-61 (1969).

Borenstein, S.R. - See Barnes, V.E.

Borg, R. J. - See Mydosh, J.A.

Brand, W., WaUng, H.F., ANd Levy, P.W. Color center studies in $\mathrm{NaCl}$ crystals by positron annihilation. (A) Presented at Int. Symp. Color Centers in Alkali Halides, Rome, Sept. 1968.

Brandt, W. - See also Chasman, C.; Waung, H.F. 
Brayshaw, D. and Peierls, R.F. Connection between bound states or resonances of two-particle and threeparticle systems. Phys. Rev. 177, 2539-55 (1969).

Bronk, B. Spectra of randomly perturbed Hamiltonians. (A) Bull. Amer. Phys. Soc. 13, 1378 (1968).

Bronk, B.V., Dienes, G. J., AND Johnson, R.A. Kinetics of cellular cooperative control. (A) Presented at 3rd Int. Biophysics Congress, Cambridge, Mass., Aug. 29-Sept. 3, 1969.

Bronk, B.V., Dienes, G. J., and Paskin, A. The stochastic theory of cell proliferation. Biophys. J. 8, 1353-98 (1968).

Brower, R.C. - See Arbab, F.

Brown, D.P., Burgess, R.W., and Mulholland, G.T. The superconducting magnet for the Brookhaven National Laboratory 7-foot bubble chamber. In Proc. 1968 Summer Study on Superconducting Devices and Accelerators, Brookhaven National Laboratory, June 10-July 19, 1968, Pt. 3, pp. 794-814. BNL 50155 (C-55), 1969.

Brown, R.W., SHit, C.C., And Young, B.L. Regularization and Ward identity anomalies. Phys. Rev. (in press).

Brown, T. - See Kane, W.R.

Brucker, E.B. - See Sims, W.H.

Brugger, R.M. - See Beckurts, K.H.

Buck, B. - See Mariscotti, M.A. J.; Zuker, A.P.

Budnick, J.I. - See Burch, T. J.; Mydosh, J.A.

Bugg, W.M. - See Cinops, A.M.; Kenyon, I.R.; SKIlliCORN, I.O.

Burch, T. J., Budnick, J.I., And Kitchens, T.A. Spinecho measurements in ordered AuFe alloys. (A) Bull. Amer. Phys. Soc. 14, 540 (1969).

Burgess, R.W. - See Brown, D.P.

Burris, R.W. - See RaY, A.K.

Butterworth, J.S., Esser, P.D., and Levy, P.W. Formation and annealing of color centers induced in $\mathrm{KCl}$ :Tl by gamma-ray irradiation. (A) Presented at Int. Symp. Color Centers in Alkali Halides, Rome, Sept. 1968.

Canuto, V. - See Lee, H. J.

Garrara, R. - See Bertanza, L.

Carroll, A.S., Fischer, J., Lundby, A., Phillips, R.H., Wang, C.L., Lobkowicz, F., Melissinos, A.C., NagaShima, Y., Smith, C.A., and TewKsbury, S. $\kappa+p$ backward scattering in the region from 1.0 to 2.5 $\mathrm{GeV} / c$. Phys. Rev. Letters 21, 1282-5 (1968).

Casali, R. - See Bertanza, L.

Chan, Y.W. On the interaction of a charged particle with an electromagnetic plane wave. Submitted to Phys. Rev. Letters.

Chan, Y.W., Cohen, V.W., and Silsbee, H.B. Hyperfine structure and nuclear magnetic moment of $\mathrm{K}^{42}$. Phys. Rev. (in press).

Chapman, J.W. - See Barnes, V.E.

Chasman, C. Energy loss of low-energy germanium atoms to electrons in germanium. Presented at Amer. Phys. Soc. Meet., Washington, D.C., April 1969.

Chasman, C., Jones, K.W., Kraner, H.W., and Brandt, W. Band-gap effects in the stopping of $\mathrm{Ge}^{72}$ atoms in germanium. Phys. Rev. Letters 21, $1430-3$ (1968).

Ghasman, C., Jones, K.W., and Ristinen, R.A. Gamma rays from the $\operatorname{Sc}^{45}\left(p, p^{\prime}, \gamma\right)$ reaction. Phys. Rev. 173, 1072-7 (1968).

Ghiu, H.Y. - See Lee, H.J.

Chiuderi, C. - See Lee, H.J.
Chrien, R.E. Automated data acquisition at the HFBR. Presented at Conf. Computer Systems in Experimental Nuclear Physics, Sky Top, Pa., March 1969.

Chrien, R.E. Neutron resonance capture $\gamma$ rays. Presented at Amer. Phys. Soc. Meet., Albany, N.Y., Oct. 1968.

Chrien, R.E., Garber, D.I., Wasson, O.A., and Beer, M. Correlations between $(d, p)$ and $(n, \gamma)$ reaction widths. (A) Bull. Amer. Phys. Soc. 14, 105 (1969).

Chrien, R.E. - See also Beer, M.; Bhat, M.R.; Garber, D.I.; Lone, M.A.; Mughabghab, S.F. (Applied Science); Price, D.L.; Rimawi, K.; Wasson, O.A.

Chung, S.U., Barnes, V.E., Eisner, R.L., Luers, D., AND SKILlicorn, I.O. Study of the reaction $K-p \rightarrow K^{*} \pi p$ at 4.6 and $7.3 \mathrm{GeV} / c$. (A) Bull. Amer. Phys. Soc. 14, 41 (1969).

Chung, S.U., Eisner, R.L., Bali, N., and Luers, D. Double-Regge pole analysis of the reaction $K-p \rightarrow$ $K^{* 0}(890) \pi^{-p}$ at $7.3 \mathrm{GeV} / c$. Phys. Rev. (in press).

Chung, S.U. - See also Barnes, V.E.; Eisner, R.L.

Church, E.L. High energy internal-conversion coefficients. Phys. Letters 28B, 168-70 (1968).

Cnops, A.M., Hough, P.V.C., Huson, F.R., Kenyon, I.R., Scarr, J.M., Skillicorn, I.O., Cohn, H.O., McGulloch, R.D., Bugg, W.M., Condo, G.T., and Nussbaum, M.M. $A_{1}$ production in $8-\mathrm{GeV} / c \pi^{+} D$ interactions. Ibid. 29B, 45-7 (1969).

Cinops, A.M., Hough, P.V.C., Huson, F.R., Kenyon, I.R., Scarr, J.M., Skillicorn, I.O., Cohn, H.O., McCulloch, R.D., Bugg, W.M., Condo, G.T., and NussBAUM, M.M. Coherent production of $\pi^{+} \pi^{+} \pi^{-}$in deuterium by $8-\mathrm{GeV} / c \pi^{+}$. Phys. Rev. Letters $21,1609-12$ (1968).

Cnops, A.M., Hough, P.V.C., Huson, F.R., Kenyon, I.R., Scarr, J.M., Skillicorn, I.O., Cohn, H.O., McCulloch, R.D., Bugg, W.M., Condo, G.T., and NussBAUM, M.M. Enhancements in the 2- and 3-pion mass spectra near $1.7 \mathrm{GeV}$ observed in the reaction of $8-\mathrm{GeV} / c \pi^{+}$mesons with deuterium. Presented at 14 th Int. Conf. High Energy Physics, Vienna, Aug. 28-Sept. 5, 1968.

Chops, A.M., Huson, F.R., Louttit, R.L., Miller, D. J., aNd O'NeALL, J.S. Coherent production of $K^{0} \pi^{-} \pi^{0}$ and $K-\pi^{0} \pi^{0}$ on nuclei. (A) Bull. Amer. Phys. Soc. 14, 561 (1969).

Cnops, A.M. - See also Skiluicorn, I.O.

Conen, V.W. The electric dipole moment of the neutron. In Proc. Sommerfield Centennial Meeting, Munich, Sept. 1968 (in press).

Conen, V.W. Magnetic resonance and radio frequency spectroscopy. In Proc. 15th Colloque Ampère, Grenoble, France, Sept. 1969, pp. 42-60, E.P. Auerbach, Editor, North-Holland, Amsterdam, 1969.

Cohen, V.W., Nathans, R., Silsbee, H., Lipworth, E., and Ramsey, N.F. The electric dipole moment of the neutron. Phys. Rev. 177, 1942-5 (1969).

Cohen, V.W. - See also Bender, P.L.; Chan, Y.W.; Fuller, G.H.

Cohn, H.O. - See Cnops, A.M.; Kenyon, I.R.; SkilliCORN, I.O.

Collins, G.B. - See Anderson, E.W.

Collins, M.F., Minkiewicz, V.J., Nathans, R., Passell, L., and Shirane, G. Critical and spin-wave scattering of neutrons from iron. Phys. Rev. 179, 417-30 (1969). 
Collins, M.F., Minkiewicz, V.J., Nathans, R., Passell, L., and Shirane, G. High resolution studies of the critical scattering of neutrons from iron. J. Phys. Soc. Japan Suppl. 26, 169-73 (1969).

Collins, M.F., Minkiewicz, V.J., Nathans, R., Passell, L., and Shirane, G. Spin dynamics of iron and nickel near the critical temperature. (A) Presented at 6th Annu. Conf. Solid State Physics, Manchester, England, Jan. 1969.

Collins, M.F., Minkiewicz, V.J., Nathans, R., Passell, L., and Shirane, G. Study of spin dynamics in iron with slow neutrons. (A) J. Appl. Phys. 40, 1442 (1969).

Collins, M.F. - See also Minkiewicz, V.J.

Condo, G.T. - See Cnops, A.M.; Kenyon, I.R.; SkIlliCORN, I.O.

Connolly, P.L., Delaney, W.C., Hough, P.V.C., Howie, J.M., KINSEY, R.R., MorRIS, T.W., NARDI, J.A., AND WERnTz, T.L. The role of human guidance in a fast measurement system. In Proc. Int. Conf. Advanced Data Processing for Bubble and Spark Chambers, Argonne National Laboratory, Oct. 1968, pp. 194-201, R. J. Royston, Editor, ANL-7515, 1969.

Connolly, P.L., Kenyon, I.R., Kinsey, R.R., And Thorndike, A.M. The analysis of $28.5-\mathrm{GeV} / c p p$ interactions producing 6 or more charged particles. Presented at Int. Conf. Elementary Particles, Lund, Sweden, June 25-July 1, 1969.

Connolly, P.L. - See also Abrams, K.L.; Kenyon, I.R.; KINSEY, R.R.

Cooper, M.J. and Nathans, R. The resolution function in neutron diffractometry. II. The resolution function of a conventional two-crystal neutron diffractometer for elastic scattering. Acta Cryst. A24, 481 -4 (1968).

Corley, D.M. - See Friedes, J.L.

Cox, D.E. Recent magnetic structure and spin density investigations. (A) Presented at Amer. Chem. Soc. Meet., New York, May 1969.

Cox, D.E., Frazer, B.C., Newnham, R.E., and Santoro, R.P. Neutron diffraction investigation of the spiral magnetic structure in $\mathrm{Cr}_{2} \mathrm{BeO}_{4}$. J. Appl. Phys. 40, 11245 (1969).

Cox, D.E., Shirane, G., Birgeneau, R.J., and MaGChesney, J.B. Magnetic ordering in $\mathrm{Ca}_{2} \mathrm{MnO}_{4}$. (A) In Proc. 8th Int. Congr. Crystallography, Buffalo and Stony Brook, N.Y., Aug. 1969 (in press).

Cox, D.E., Shirane, G., Birgeneau, R. J., and MaGChesney, J.B. A neutron diffraction study of magnetic ordering in $\mathrm{Ca}_{2} \mathrm{MnO}_{4}$. Phys. Rev. (in press).

Cox, D.E. - See also KAy, M.I.; MenZinger, F.; WAnG, F.F.Y

Craig, P.P. Direct observation of stress-induced shifts in contact potentials. Phys. Rev. Letters 22, 700-3 (1969).

Craig, P.P. and Goldburg, W.I. Transport properties near magnetic critical points. J. Appl. Phys. 40, 964-71 (1969).

Craig, P.P., Kitchens, T.A., AND Taylor, R.D. Strongcoupled superconductors, the Mössbauer effect, and localized impurity modes. Phys. Rev. (in press).

Graig, P.P. - See also Kitchens, T.A.; TAylor, R.D.; Trousdale, W.

Crennell, D. J., Delaney, W.C., Flaminio, E., Karshon, U., Lal, K.W., Metzger, W. J., O'Neall, J.S. Scarr, J.M., Schumann, T.G., and Thorndike, A.M. Search for $I=3 / 2 \Xi$ resonances in $K-n$ interactions at
3.6 and $3.9 \mathrm{GeV} / c$. In Proc. 14th Int. Conf. High Energy Physics, Vienna, Aug. 28-Sept. 5, 1968 (in press); submitted to Phys. Rev. Letters.

Crennell, D. J., Delaney, W.C., Flaminio, E., Karshon, U., Lai, K.W., Metzger, W. J., O'Neall, J.S., Scarr, J.M., Thorndike, A.M., Baumel, P., Lea, R.M., Montwill, A., and Schumann, T.G. Existence of a $Y_{1}{ }^{*}$ resonance at $1616 \mathrm{MeV}$. Phys. Rev. Letters 21 , 648-50 (1968).

Crennell, D. J., Karshon, U., Lai, K.W., O'Neall, J.S., AND SCARR, J.M. " $A_{2}$ " meson production from $K-n$ interactions at $3.9 \mathrm{GeV} /$ c. Ibid. 22, 1327-30 (1969).

Crennell, D. J., Karshon, U., Lai, K.W., O'Neall, J.S., AND Scarr, J.M. Evidence for an isospin $-1 / 2 K \pi$ enhancement at a mass of $1160 \mathrm{MeV}$ produced in $K-n$ interactions at $3.9 \mathrm{GeV} / c$. Ibid., 487-9.

Crennell, D. J., Karshon, U., Lai, K.W., O'Neall, J.S., Scarr, J.M., Baumel, P., Lea, R.M., Schumann, T.G., AND URVATER, E.M. Further evidence for $Y_{1}{ }^{*}(1616)$ from 3.9-GeV/c $K-n$ interactions. Presented at Int. Conf. Elementary Particles, Lund, Sweden, June 25July 1, 1969.

Crennell, D. J., Karshon, U., Lai, K.W., O’Neall, J.S., Scarr, J.M., Baumel, P., Lea, R.M., Schumann, T.G., AND URVATER, E.M. Is there an $\eta(550) \pi$ resonance at 980 MeV? Phys. Rev. Letters 22, 1398-401 (1969).

Grennel. , D. J., Karshon, U., Lai, K.W., O'Neall, J.S., Scarr, J.M., Baumel, P., Lea, R.M., Schumann, T.G., AND URVATER, E.M. Study of the reaction $K-n \rightarrow \Lambda X^{-}$ at $3.9 \mathrm{GeV} / c$. (A) Bull. Amer. Phys. Soc. 14, 636 (1969).

Crennell, D. J., Karshon, U., Lai, K.W., O'Neall, J.S. Scarr, J.M., and Schumann, T.G. Search for $I=3 / 2 \Xi$ resonances produced in $K-n$ interactions at 3.6 and 3.9 $\mathrm{GeV} / c$. Submitted to Phys. Letters.

Grennell, D. J., Karshon, U., Lai, K.W., Scarr, J.M., AND SkIllicorn, I.O. Production of the $g(1640)$ meson in $\pi p$ interactions at $6 \mathrm{GeV} / c$. Phys. Letters 28B, 1369 (1968).

Crennell, D. J. - See also Abrams, K.L.; Alitti, J.; Karshon, U.; LaI, K.W.; O'Neall, J.S.; Schumann, T.G.; Strand, R.C.

Crennell, K.M. - See Abrams, K.L.

Crow, J.E., LeE, H. J., And Strongin, M. Effect of grain size on the phonon distribution and $T_{c}$ of superconductors. (A) Bull. Amer. Phys. Soc. 13, 1374 (1968).

Crow, J.E. and Strongin, M. The upper critical field of thin-film superconductors. (A) Ibid., $1741 ; J$. Appl. Phys. 40, 2162 (1969).

Crow, J.E., Strongin, M., Thompson, R.S., and KamMERER, O.F. The superconducting transition temperatures of disordered Nb, W, and Mo films. Phys. Letters A (in press).

Crow, J.E., Strongin, M., Thompson, R.S., and KaMMERER, O.F. Transition temperatures of disordered transition metal films. (A) Presented at Conf. Science of Superconductivity, Stanford, Calif., Aug. 1969.

Crow, J.E. - See also Strongin, M.; Thompson, R.S.

Culwick, B.B., Glazer, E., Hirst, D.V., and Meehan, T.C. A data terminal network with access to a large computer complex. In Proc. Int. Conf. Advanced Data Processing for Bubble and Spark Chambers, Argonne National Laboratory, Oct. 1968, pp. 184-93, R. J. Royston, Editor, ANL-7515, 1969. 
Culwick, B.B. - See also Abrams, K.L.

Damask, A.C. Radiation sintering. Radiat. Effects 1, 95-100 (1969).

Damask, A.C., Arndt, R.A., Spielberg, D.H., and LefkowITz, I. An investigation of the molecular dynamics of phenanthrene by cold-neutron inelastic scattering and dielectric measurements. (A) Bull. Amer. Phys. Soc. 13, 1394 (1968)

Damask, A.G., Dienes, G. J., Herman, H., and Katz, L.E. Enhanced diffusion in $\alpha$-brass during cyclic straining. Phil. Mag. 20,67-77 (1969).

Damask, A.C. - See also Arndt, R.A.; Korn, A.I.; Ringel, H.; Whitten, W.B.

Damerell, C. J.S. - See Orear, J.

Daniels, W.B. - See Leake, J.A.; Skalyo, J. Jr.

Daugeras, B. - See Bingham, H.H.

Davidson, J. - See Barnes, V.E.

Delaney, W.C., Guidoni, P., London, G.W., and MarTELLOTTI, G. Some results concerning $K-d$ interactions around $900 \mathrm{MeV} / c$. Submitted to Nuovo Cimento.

Delaney, W.C. - See also Abrams, K.L.; Connoliy, P.L.; CRennell, D. J.

DE Rafael, E. - See Lautrup, B.E.; Martin, B.R.

DER MAteosian, E. On-line small computer data handling in pulse height analysis and two-parameter multichannel coincidence data storage. Nucl. Instr. Methods (in press).

Der Mateosian, E. and Goldhaber, M. Study of the isobars $\mathrm{Cd}^{113 m} \mathrm{In}^{113 m}$. Phys. Rev. (in press).

der Mateosian, E. - See also Kane, W.R.

DiCarlo, J.A., Snead, C.L. Jr., and Goland, A.N. StageI interstitials in electron-irradiated tungsten. Phys. Rev. 178, 1059-72 (1969).

mi Corato, M. - See Bingham, H.H.

Dienes, G. J. "Off-center" ions in the alkali halides. (N) Comments Solid State Phys. 1, 81 (1968).

Dienes, G. J., Hatcher, R.D., Smoluchowski, R., AND Wilson, W.D. Interactions of an $\mathrm{H}$ center with substitutional impurities. (A) Bull. Amer. Phys. Soc. 13, 1474 (1968).

Dienes, G. J., Hatcher, R.D., Wilson, W.D., ANd Smoluchowski, R. Formation and stability of $\mathrm{H}^{\prime}$ centers. (A) Ibid. 14, 324 (1969).

Dienes, G. J. - See also Bronk, B.V.; Damask, A.C.; Hatcher, R.D.; Wilson, W.D.

Dodd, W.P., Joldersma, T., Palmer, R.B., and Samios, N.P. Results from $3.0-\mathrm{GeV} / c K+$ interactions in hydrogen. Phys. Rev. 177, 1991-2002 (1969).

Donorue, J.T. Spin and parity analysis for bosons decaying into spin-1/2 pairs. Ibid. 178, 2288-99.

Dornan, R. - See Barnes, V.E.

Douglass, D.H. Jr. - See Strongin, M.

DowD, R.M. - See Sims, W.H.

ECKer, B.M. - See RoRER, D.C.

Edelstein, R.M. - See Anderson, E.W.

EISNER, R.L. ANd ChUng, S.U. Double Regge pole analysis of the reaction $K-p \rightarrow K-\pi^{-} \Delta^{++}$at $7.3 \mathrm{GeV} / c$. (A) Bull. Amer. Phys. Soc. 14, 614 (1969).

Eisner, R.L. - See also Barnes, V.E.; Chung, S.U.

Ellis, W.E., Miller, D. J., Morris, T.W., Panvini, R.S., And Thorndike, A.M. Single-isobar production in proton-proton interactions at $28.5 \mathrm{GeV} / c$. Phys. Rev. Letters 21, 697-700 (1968).
Ellis, W.E., Mrller, D. J., Morris, T.W., Panvini, R.S., Thorndike, A.M., AND Berger, E.L. An investigation of low multiplicity final states in $p p$ interactions at 28.5 $\mathrm{GeV} / c$. Presented at 14th Int. Conf. High Energy Physics, Vienna, Aug. 28-Sept. 5, 1968.

Ellis, W.E., Morris, T.W., and Panvini, R.S. Momentum-transfer distributions in inelastic proton-proton scattering at $28.5 \mathrm{GeV} / c$. (A) Bull. Amer. Phys. Soc. 14, 636 (1969).

Ellis, W.E., Morris, T.W., Panvini, R.S., and ThornDIKE, A.M. A description of final states with three, four, and five particles in $p p$ interactions at $28.5 \mathrm{GeV} / c$. Presented at Int. Conf. Elementary Particles, Lund, Sweden, June 25-July 1, 1969.

Elis, W.E. - See also Panvini, R.S.; Strand, R.C.

EMery, G.T. - See KANE, W.R.

Emery, V. J. Dilute mixtures of $\mathrm{He}^{3}$ in liquid $\mathrm{He}^{4}$ at low temperatures. In Proc. Bat-Sheva Seminar on Quantum Fluids, Haifa, Israel, July 28-Aug. 16, 1968 (in press).

EMERY, V.J. Low temperature expansion of relaxation times in Fermi liquids. Ibid.

EMERY, V.J. Solution of Boltzmann equation for degenerate Fermi systems. Phys. Rev. 175, 251-6 (1968).

EsSER, P.D. AND LEvy, P.W. Structure of the optical absorption bands of $\mathrm{Tl}+$ substituted in KCl. (A) Bull. Amer. Phys. Soc. 14, 325 (1969).

Esser, P.D. AND Levy, P.W. Tl+ absorption bands in KCl: Experimental. (A) Presented at Int. Symp. Color Centers in Alkali Halides, Rome, Sept. 1968.

Esser, P.D. - See also Butterworth, J.S.

Falge, R.L. JR. - See Wolcott, N.M.

Farwell, C.W. - See Bingham, H.H.

Fine, H.L., Lipsicas, M., and Strongin, M. Knight shift in dirty superconducting aluminum films. (A) Bull. Amer. Phys. Soc. 14, 112 (1969).

Fine, H.L., Lipsicas, M., and Strongin, M. Vanishing Knight shift in dirty superconducting aluminum films. Phys. Letters (in press).

Frorini, E. - See Bingham, H.H.

Fischer, C.R. and Kemmey, P.J. Calculation of electronic states of $\mathrm{Li}_{2}{ }^{+}$as a free ion and in a point-ion lattice. Phys. Rev. (in press).

Fischer, C.R. AND Kemmey, P.J. Electronic states of $\mathrm{Li}_{2}+$ in LiF crystal lattice. (A) Bull. Amer. Phys. Soc. 14, $600(1969)$

Fischer, C.R. - See also Harrison, S.W.

FISK, H.E. - See RAY, A.K.

Flaminio, E. - See Alitti, J.; Barnes, V.E.; Grenneli, D. J.; Karshon, U.

Fieury, P.A. - See Hutchings, M.T.

Foley, K. J., Jones, R.S., Lindenbaum, S. J., Love, W.A., Ozaki, S., Platner, E.D., Quarles, C.A., and Willen, E.H. An experimental test of the pion-nucleon forward dispersion relations at high energies. Phys. Rev. 181, 1775-93 (1969).

Fossan, D.B., Henson, S.H., Paul, P., and Jones, K.W. Lifetime of the $3.86-\mathrm{MeV}$ state in ${ }^{13} \mathrm{C}$. (A) Bull. Amer. Phys. Soc. 13, 1387 (1968).

Fossan, D.B. - See also Jones, K.W.

Fournier, D. - See Bingham, H.H.

Frantz, E. - See Abrams, K.L.

Frazer, B.C. - See Cox, D.E.; Leake, J.A.; Meister, H.; Menzinger, F.; Skalyo, J. JR. 
Freeman, A. J. - See Watson, R.E.

Fretter, W.B. - See Bingham, H.H

Friedberg, S.A. - See Skalyo, J. Jr.

Friedes, J.L., Sutter, R., Palevsky, H., Bennett, G., Igo, G. J., Simpson, W.D., Phillips, G.C., Corley, D.M., Wall, N.S., and Stearns, R.L. Production of high momentum deuterons from nuclei bombarded by 1-BeV protons. In Proc. Int. Conf. Nuclear Structure, Tokyo, Sept. 1967, CONF-670910-2.

Frisken, W.R. - See Orear, J.

Fuller, G.H. and Cohen, V.W. Nuclear spins and moments. J. Nucl. Data 5A, 433-612 (1969).

Gallmann, A., Jundt, F., Aslanides, E., And Alburger, D.E. Beta decays of $\mathrm{C}^{15}, \mathrm{~F}^{17}$, and $\mathrm{F}^{20}$. Phys. Rev. 179, 921 (1969).

Gallmann, A. - See also Alburger, D.E.

Gambino, R. J. - See McGuire, T.R.

Garber, D.I., Rimawi, K., Ghrien, R.E., and Wasson, O.A. Resonance neutron capture $\gamma$-ray spectra from germanium. (A) Bull. Amer. Phys. Soc. 14, 104 (1969).

Garber, D.I. - See also Beer, M.; Bhat, M.R. (Applied Science); Chrien, R.E.; Mughabghab, S.F. (Applied Science); Rimawi, K.; Wasson, O.A.

GARDNER, D. - See Kane, W.R.

GARELICK, D. Highly inelastic $p p$ scattering and its interpretation. Phys. Rev. Letters 22, 674-7 (1969).

Garelick, D. - See also Anderson, E.W.

GARG, J.B. - See Rimawi, K.; Wasson, O.A.

Gelletly, W., Moragues, J.A., Mariscotti, M.A. J., AND KANE, W.R. The $\mathrm{Ba}^{135}(n, \gamma) \mathrm{Ba}^{136}$ reaction and level structure of $\mathrm{Ba}^{136}$. Phys. Rev. 181, 1682-96 (1969).

Gelletly, W., Moragues, J.A., Mariscotti, M.A. J., AND Kane, W.R. Level structure of $\mathrm{Ce}^{141}$ from the $\mathrm{Ce}^{140}(n, \gamma)$ reaction. (A) Bull. Amer. Phys. Soc. 14, 514 (1969).

Gelletly, W. - See also Kane, W.R.; Mariscotti, M.A. J.; Moragues, J.A.

Geschwind, S. - See Blume, M.

Giacomelli, G. Investigation of the reaction $K+p$ $\rightarrow K^{0} p \pi^{+} \pi^{+} \pi^{-}$at $2.95 \mathrm{GeV} / c$. Informal Report BNL 13554.

Glazer, E. - See Culwick, B.B.

Goland, A.N. The physics of track registration in solids. (A) In Proc. 26th Annu. Meet. Electron Microscopy Society of America, New Orleans, Sept. 1968 (in press).

Goland, A.N. and Keating, D.T. The influence of interstitial carbon in iron on the scattering of $x$-rays. In Proc. 2nd Julich Conf. Vacancies and Interstitials in Metals, Julich, West Germany, Sept. 1968, Vol. 2, p. 825, Sept. 1968.

Goland, A.N. - See also DiCarlo, J.A.; Keating, D.T.

Goldberg, M., Levy, P.W., ANd Herley, P. J. Coloring of crystalline sodium bromate by ultraviolet irradiation. (A) Bull. Amer. Phys. Soc. 14, 376 (1969).

Goldberg, M. - See also Alitti, J.; Barnes, V.E.

Goldburg, W.I. - See Craig, P.P.

Goldhaber, M. - See der Mateosian, E.

Graves, R. - See Beer, M.; Price, D.L.

Greene, A. - See Sims, W.H.

Greene, M.P., Lee, H. J., Quinn, J. J., And Rodriguez, S. Linear response theory for a degenerate electron gas in a strong magnetic field. Phys. Rev. 177, 1019-36 (1969).
Greene, M.P., Lee, H. J., Quinn, J. J., And Rodriguez, $S$. Linear response theory for an electron gas in a strong magnetic field. Presented at 11 th Int. Conf. Low Temperature Physics, St. Andrews, Scotland, Aug. 1968.

Guggenheim, H. J. - See Birgeneau, R. J.; Hutchings, M.T.

Guidoni, P. - See Barnes, V.E.; Delaney, W.C.

Harris, W.R., Nagatani, K., and Alburger, D. The beta decay of $\mathrm{Al}^{29}$. Phys. Rev. (in press); (A) Bull. Amer. Phys. Soc. 14, 18 (1969).

Harris, W.R. - See also Alburger, D.E.; Jones, K.W.; WARBURTON, E.K

Harrison, S.W. and Fischer, C.R. LCAO-MO-SCF calculations of interstitial He in LiH. (A) Bull. Amer. Phys. Soc. 14, 612 (1969).

Hart, E.L. - See Bertanza, L.

Hatcher, R.D., Wilson, W.D., SMoluchowski, R., AND Dienes, G. J. Mixed H centers in KCl. (A) Bull. Amer. Phys. Soc. 14, 324 (1969).

Hatcher, R.D. - See also Dienes, G. J.; Wilson, W.D.

Hausser, O. - See Rose, H. J.

Heller, P. - See Schulhof, M.P.

Hennessy, J. - See Bingham, H.H.

Henson, S.H. - See Fossan, D.B.

Herley, P.J. and Levy, P.W. Dependence of the thermal decomposition induction period on prior $\gamma$ - and $\mathrm{x}$-ray irradiation. Presented at Meet. Combustion Inst., Western States Section, China Lake, Calif., April 30May 2, 1969.

HeRLEY, P. J. AND LeVY, P.W. Effects of $x$ - and $\gamma$-ray radiation on the thermal decomposition of solid orthorhombic ammonium perchlorate. I. Experimental and results; II. Kinetics and discussion. J. Chem. Phys. 49, 1493-1509 (1968).

Herley, P. J. - See also Goldberg, M.; Levy, P.W.

Herling, G.H. - See Nagatani, K.

Herman, H. - See Damask, A.C.

Hien, N.C. - See Anderson, E.W.

Hill, D.G. and Palmer, R.B. A bubble chamber film measuring system employing high accuracy image plane digitizers. Rev. Sci. Instr. 40, 878-83 (1969).

HILl, D.G. - See also RAY, A.K.

Hind, M. - See Auerbach, E.H.

Hirakawa, K. - See Hutchings, M.T.

Hirst, D.V. - See Gulwick, B.B.

Hough, P.V.C. - See Abrams, K.L.; Cnops, A.M.; ConNOLLY, P.L.; SKILLIGORN, I.O.

Houk, T.L. Neutron cross-section difference of $\mathrm{C}^{13}$ and C12. (A) Bull. Amer. Phys. Soc. 14, 494 (1969).

Howie, J.M. - See Connoliy, P.L

Hsieh, Y.Y. - See Blume, M.

Huson, F.R., Miller, D. J., and O'Neall, J.S. Coherent production of the $p \pi^{+} \pi^{-}$enhancement at $1470 \mathrm{MeV}$ by 28-GeV/c protons on neon. Nucl. Phys. B8, 391-401 (1968).

Huson, F.R. - See also Bingham, H.H.; Chops, A.M.; Miller, D. J.; Skillicorn, I.O.

Hutchings, M.T., Samuelsen, E. J., Shirane, G., and Hirakawa, K. Neutron diffraction determination of the antiferromagnetic structure of $\mathrm{KCuF}_{3}$. Phys. Rev. (in press).

Hutchings, M.T., Thorpe, M.F., Birgeneau, R. J., Fleury, P.A., And Guggenheim, H. J. Neutron and 
optical investigation of magnons and magnon-magnon interactions in $\mathrm{NiF}_{2}$. (A) Bull. Amer. Phys. Soc. 14, 539 (1969).

Hutchings, M.T. - See also SAmuelsen, E. J.

Igo, G. J. - See Friedes, J.L.

Irwin, G.M. - See Bingham, H.H.

JAEger, K. - See Barnes, V.E.

Jensen, M.A. - See Strongin, M.

Jessup, S. J. - See Abrams, K.L.

Johnson, R.A. Effect of lattice structure on the calculation of stacking-fault and cohesive energies using longranged interatomic potentials. Submitted to J. Cryst. Lattice Defects.

Johnson, R.A. Kinetic study of vacancy clustering and annealing in metals. Phys. Rev. 174, 684-90 (1968).

Johnson, R.A. Sensitivity of lattice defect calculations for f.c.c. metals to empirically derived interatomic potentials. Radiat. Effects (in press).

Johnson, R.A. - See also Bronk, B.V.

Joldersma, T. - See Dodd, W.P.

Jones, K.W., Harris, W.R., McEllistrem, M.T., and Alburger, D.E. Beta decay of $\mathbf{B}^{13}$. Phys. Rev. (in press).

Jones, K.W., Schwarzschild, A.Z., Warburton, E.K., AND Fossan, D.B. Recoil-distance method for nuclear lifetime measurements: States of $\mathrm{Na}^{22}, \mathrm{Ne}^{22}$, and $\mathrm{F}^{19}$ Phys. Rev. 178, 1773-82 (1969).

Jones, K.W. - See also Chasman, G.; Fossan, D.B.; McELlisTrem, M.T.

Jones, R.S. - See Foley, K. J.

Jundt, F. - See Gallmann, A.

Kahana, S., Lee, H.C., and Scott, C.K. The $A=18 \mathrm{nu}$ clear reaction matrix. Phys. Rev. (in press).

Kahana, S., Lee, H.C., and Scott, C.K. Effect of WoodSaxon wave functions on the calculation of $A=18,206$, 210 spectra with a realistic interaction. Ibid.

Kahana, S. - See also Auerbach, E.H.

Kalbfleisch, G.R., Strand, R.C., and Vander Burg, V. An explanation of the $\bar{p} p$ total cross section enhancement at $1.32 \mathrm{GeV} / c$. Submitted to Phys. Rev. Letters.

Kalbfleisch, G.R., Strand, R., and Vander Burg, V. Formation of a mesonic state in the $\bar{p} p$ interaction at $1.32 \mathrm{GeV} / c$. Phys. Letters (in press).

Kalbfleisch, G.R. - See also Barnes, V.E.; BlumenFIELD, B. J.; VANDER BURG, V.

Kalbitzer, S. ANd Levy, P.W. First-stage F-center coloring in KCl. I. Growth at low dose rates; II. Kinetics of the thermal annealing above $22^{\circ} \mathrm{C}$; III. A low-intensity component unstable at $22^{\circ} \mathrm{C}$. Submitted to Phys. Rev.

Kane, W.R., Gardner, D., Brown, T., Kevey, A., der Mateosian, E., Emery, G.T., Gelletly, W., MariscotTI, M.A. J., AND SCHrödER, I. A neutron monochromator for studies of the $(n, \gamma)$ reaction. (A) Bull. Amer. Phys. Soc. 14, 514 (1969).

Kane, W.R. - See also Gelletly, W.; Mariscotti, M.A. J.; Moragues, J.A.

KANG, K. Field-current identities, broken symmetries, current-mixing model and the algebra of gauge fields. Submitted to Phys. Rev. Letters.

Karshon, U., Grennell, D. J., Flaminio, E., Lai, K.W., O'Neall, J.S., Scarr, J.M., and Schumann, T.G. Search for $I=1 / 2$ and $I=3 / 2 \Xi^{*}$ resonances in $K-n$ interactions at 3.6 and $3.9 \mathrm{GeV} / c$. (A) Bull. Amer. Phys. Soc. 13,1440 (1968).
Karshon, U. - See also Alitti, J.; Crennell, D. J.; LaI, K.W.; O'Neall, J.S.; Schumann, T.G.

Katz, L.E. - See DamasK, A.C.

Kawatra, M.P. - See Mydosh, J.A.

Kay, M.I., Okaya, Y., and CoX, D.E. Refinement of the room-temperature phase of phenanthrene, $\mathrm{C}_{14} \mathrm{H}_{10}$, a molecule with overcrowded hydrogen atoms. (A) In Proc. 8th Int. Congr. Crystallography, Buffalo and Stony Brook, N.Y., Aug. 1969 (in press).

Keating, D.T. Theory and measurement of the x-ray satellite reflections in holmium due to the aspherical $4 f$ charge density. Phys. Rev. 178, 732-42 (1969).

Keating, D.T. and Goland, A.N. Atomic displacements around dislocation loops. J. Appl. Phys. 39, 6018-22 (1968).

Keating, D.T. AND Goland, A.N. X-ray scattering from hcp structures containing prismatic dislocation loops. (A) Acta Cryst. (in press).

Keating, D.T. - See also Goland, A.N.; Mozer, B.

Kemmey, P. J., Mattern, P.L., ANd Bartram, R.H. Electron structure of hydrozoic acid $\left(\mathrm{HN}_{3}\right)$. (A) Bull. Amer. Phys. Soc. 14, 422 (1969).

Kemmey, P. J., Mattern, P.L., and Bartram, R.H. Ground state wave functions for the azide $\left(\mathrm{N}_{3}{ }^{-}\right)$ion. Submitted to J. Chem. Phys.

Kemmey, P. J. - See also Fischer, C.R.; Townsend, P.D.

KENNEDY, G.M. The absolute thermopower of nickel. Informal Report BNL 13340 .

Kenyon, I.R., Connolly, P.L., Kinsey, R.R., AND Thorndike, A.M. The analysis of 28.5-GeV/c pp interactions producing 6 or more charged particles. (A) Bull. Amer. Phys. Soc. 14, 561 (1969).

Kenyon, I.R., Kinson, J.B., Scarr, J.M., Skillicorn, I.O., Cohn, H.O., MGGulloch, R.D., BugG, W.M., Condo, G.T., and Nussbaum, M.M. Neutral $\pi \rho$ enhancements produced in $8-\mathrm{GeV} / \mathrm{c} \pi^{+} D$ interactions. Phys. Rev. Letters (in press).

Kenyon, I.R. - See also Gnops, A.M.; Connolly, P.L.; KINSEY, R.R.; SKILLIGORN, I.O.

Kevey, A. - See Kane, W.R.

Kiang, R. - See Barnes, V.E.

Kinsey, R.R., Connolly, P.L., Kenyon, I.R., AND ThornDIKE, A.M. A study of $p p$ interactions at $28.5 \mathrm{GeV} / c$ producing one or more observed strange particles. (A) Bull. Amer. Phys. Soc. 14, 614 (1969).

Kinsey, R.R. - See also Connolly, P.L.; Kenyon, I.R.

Kinson, J.B. - See Barnes, V.E.; Kenyon, I.R.; SkilliCORN, I.O.

Kitchens, T.A., Graig, P.P., and Taylor, R.D. Determination of zero point phonon parameters: Calibration of the second-order Doppler shift. In Mössbauer Effect Technology, Vol. 5, I. J. Gruverman, Editor, New England Nuclear Corp., Boston (in press).

Kitchens, T.A. and Trousdale, W.L. Magnetization distribution in magnetically diluted systems with application to iron-palladium. Phys. Rev. 174, 606-12 (1968).

Kitchens, T.A. - See also Bưrch, T. J.; Graig, P.P.; Minkiewicz, V.J.; Mydosh, J.A.

Koide, S. - See Watson, R.D.

Kolltveit, K. - See Nagatani, K.

Korn, A.I., ARndt, R.A., And Damask, A.C. Hall mobility of holes in anthracene. Phys. Rev. (in press).

Korn, A.I. - See also ARndT, R.A. 
Koschik, J. - See Barnes, V.E.

Kraemer, R.W. - See Ray, A.K.

Kraner, H.W. - See Chasman, C.

Krasner, S. ANd Levy, P.W. ESR of gamma-ray irradiated single crystals of sodium chlorate. (A) Presented at Amer. Phys. Soc. Meet., Hawaii, Sept. 1969.

KRMPOTí, F. AND TADić, D. $0^{-} \rightarrow 0^{+}$nuclear beta transitions. Phys. Rev. 178, 1804-14 (1969).

LAI, K.W. A guided tour of " $A_{2}$ ". Presented at Amer. Phys. Soc. Meet., New York, Feb. 1969.

LaI, K.W. The tale of two " $A_{2}$ " mesons. In Proc. Conf. Particle Physics, Boulder, Colo., June 1968 (in press).

Lai, K.W., Grennell, D. J., Karshon, U., and Scarr, J.M. Resonance production in the reactions $\pi^{-} p \rightarrow \Lambda+X$ and $\pi^{-} p \rightarrow K_{1}{ }^{0}+X$ at $6 \mathrm{GeV} / c$. (A) Bull. Amer. Phys. Soc. 13, 1441-2 (1968).

Lai, K.W. - See also Alitti, J.; Grennell, D. J.; Karshon, U.; O'Neall, J.S.; Schumann, T.G.

Lannutti, J.E. - See Sims, W.H.

Lautrup, B.E. ANd de Rafael, E. Calculation of the sixth-order contribution from the fourth-order vacuum polarization to the difference of the anomalous magnetic moment of muon and electron. Phys. Rev. 174, 1835-42 (1968).

Lea, R.M. - See Crennell, D. J.; O'Neall, J.S.; SchuMANN, T.G.

Leake, J.A., Daniels, W.B., Skalyo, J. Jr., Frazer, B.C., AND Shirane, G. Lattice dynamics of neon at two densities from coherent inelastic neutron scattering. Phys. Rev. 181, 1251-60 (1969).

Leake, J.A., Minkiewicz, V. J., and Shirane, G. Phonon dispersion relations for holmium. Solid State Commun. 7, 535-9 (1969).

LEE, H. J. Density fluctuations and ground state for a system of electrons in a strong magnetic field. Phys. Rev. 177, 786-9 (1969).

LEE, H. J. Nonuniform state for a degenerate Coulomb gas in a strong magnetic field. Ibid., 1083-8.

LEE, H. J. A nonuniform thermodynamic state for a system of electrons in the presence of a strong magnetic field. (A) Bull. Amer. Phys. Soc. 14, 367 (1969).

Lee, H. J., Canuto, V., Chiv, H.Y., and Chiuderi, C. New source of intense magnetic fields in neutron stars. Submitted to Nature.

Lee, H. J., Canuto, V., Chiu, H.Y., and Chiuderi, C. A new state of ferromagnetism in degenerate electron gas and magnetic fields in collapsed bodies. (L) Submitted to Phys. Rev. Letters.

LeE, H. J. - See also Crow, J.E.; Greene, M.P.; Kahana, S.; Scott, C.K.

Lefkowitz, I. - See Damask, A.C.

Leitner, J. - See Alitti, J.; Barnes, V.E.

Lemos, A.M. - See Stauber, M.C.

LeVY, P.W. AND Herley, P. J. Electronic mechanisms in solid-state photolysis. Mater. Sci. Res. 4, 156-71 (1969).

Levy, P.W. - See also Alvarez Rivas, J.L.; Brandt, W.; Butterworth, J.S.; Esser, P.D.; Goldberg, M.; Herley, P.J.; Kalbitzer, S.; Krasner, S.; Townsend, P.D.; WAUnG, H.F.; Zeller, E. J.

Lindenbaum, S.J. Asymptotic energies. In Proc. Int. Symp. Contemporary Physics, Trieste, June 1968 (in press).

Lindenbaum, S. J. Digitized spark chamber and on-line computer system for double vertex events. In Proc. Int.
Symp. Nuclear Electronics, Versailles, France, Sept. 1968, Vol. 3, pp. 50-1 to 50-25, 1968.

Lindenbaum, S. J. Forward dispersion relations. Their validity and predictions. In Pion-Nucleon Scattering (Proc. Conf., Irvine, Calif., Dec. 1967), G.L. Shaw and D.Y. Wong, Editors, Wiley, New York, 1969.

Lindenbaum, S. J. Large digitized spark chamber spectrometer and on-line computer systems. In Proc. Int. Conf. Advanced Data Processing for Bubble and Spark Chambers, Argonne National Laboratory, Oct. 1968, pp. 202-25, R. J. Royston, Editor, ANL-7515, 1969.

Lindenbaum, S. J. Some new detector systems. In Meson Spectroscopy - A Collection of Articles (Proc. Meet., Philadelphia, April 1968), pp. 419-41, C. Baltay and A.H. Rosenfeld, Editors, W.A. Benjamin, Inc., New York, 1968.

Lindenbaum, S. J. - See also Foley, K. J.

Linz, A. - See Y AMADA, Y.

Lipschultz, F.P. - See Minkiewicz, V.J.

Lipsicas, M. - See Fine, H.L.; Miller, C.E.

Lipworth, E. - See Cohen, V.W.

Lobkowicz, F. - See Garroll, A.S.

London, G.W. - See Delaney, W.G.

Lone, M.A., Ghrien, R.E., Wasson, O.A., Beer, M., Bhat, M.R., and Muether, H.R. Resonant and nonresonant capture of slow neutrons in $\operatorname{Tm}^{169}(n, \gamma) \mathrm{Tm}^{170}$. Phys. Rev. 174, 1512-24 (1968).

Lone, M.A. - See also Price, D.L.; Wasson, O.A.

Louttit, R.I. - See Bingham, H.H.; Cnops, A.M.

Love, W.A. The Brookhaven on-line data facility. In Proc. Conf. Computer Systems in Experimental Nuclear Physics, Sky Top, Pa., March 1969 (in press).

LOVE, W.A. - See also Foley, K. J.

Low, F.E. Regge Behavior and Multiperipheral Processes. BNL 50162 (T-527), Jan. 1969.

LowE, J. - See Wilkinson, D.H.

Lu, A. - See Bingham, H.H.

Luers, D. - See Chung, S.U.

Lundby, A. - See Carroll, A.S

Lys, J.E.A. - See Barnes, V.E.

MacChesney, J.B. - See Cox, D.E.

Mariscotti, M.A.J., Gelletly, W., Moragues, J.A., AND $\mathrm{K}_{\mathrm{ANE}}$, W.R. $\mathrm{Ba}^{137}(n, \gamma)$ reaction and level structure of $\mathrm{Ba}^{138}$. Phys. Rev. 174, 1485-93 (1968).

Mariscotti, M.A.J., Moragues, J.A., Gelletly, W., AND KANE, W.R. Correlations between $(n, \gamma)$ and $(d, p)$ reactions on $\mathcal{N}=82$ nuclei. Phys. Rev. Letters 22, 303-6 (1969).

Mariscotti, M.A.J., Scharff-Goldhaber, G., and Buck, B. Phenomenological analysis of ground-state bands in even-even nuclei. Phys. Rev. 178, 1864-87 (1969).

Mariscotti, M.A.J. - See also Gelletiy, W.; Kane, W.R.; Moragues, J.A.

Marshalek, E.R. and Weneser, J. Kelson criterion. (A) Bull. Amer. Phys. Soc. 13, 1364 (1968).

Marshalek, E.R. AND Weneser, J. Nuclear rotation and the random-phase approximation. Ann. Phys. N.Y. 53, 569-624 (1969).

Martellotti, G. - See Delaney, W.C.

Martin, B.A. - See Auerbach, E.H.

Martin, B.R. $K+p$ scattering and a possible $Z^{*}$ resonance. Phys. Rev. Letters 21, 1286-7 (1968).

Martin, B.R. $s$-Wave $K+p$ dynamics. Phys. Rev. 175, 2034-47 (1968). 
Martin, B.R. And de Rafael, E. Phenomenological description of $K_{S} \rightarrow 2 \gamma$ and $K_{L} \rightarrow 2 \gamma$ decays. Nucl. Phys. B8, $131-49$ (1968).

Martin, B.R. and Sakitt, M. Low-energy $\bar{K} \mathcal{N}$ and pionhyperon interactions. I. $K$-matrix analysis of $K-p$ reactions; II. Applications of the $K$-matrix analysis. Phys. Rev. 183, 1345, 1352 (1969).

Mattern, P.L. - See Kemmey, P. J.; Zeller, E. J.

MaCulloch, R.D. - See Cnops, A.M.; Kenyon, I.R.; Skillicorn, I.O.

McEllistrem, M.T., Jones, K.W., and Sheppard, D.M. Levels of ${ }^{53} \mathrm{Mn}$ from the ${ }^{53} \mathrm{Cr}(p, n \gamma)^{53} \mathrm{Mn}$ reaction. Submitted to Phys. Rev.; (A) Bull. Amer. Phys. Soc. 13, 1426 (1968).

McEllistrem, M.T. - See also Jones, K.W.

McGrory, J.B. - See Zuker, A.P.

McGuire, T.R., Gambino, R.J., Pickart, S.J., and Alperin, H.A. Magnetic structure and exchange interactions in cubic gadolinium compounds. (A) Presented at 14th Int. Conf. Magnetism and Magnetic Materials, New York, Nov. 1968.

McMahon, T. J. - See Anderson, E.W.

Meehan, T.C. - See Culwick, B.B.

Meister, H., Skalyo, J. Jr., Frazer, B.C., and Shirane, G. Lattice dynamical aspects of the antiferroelectric phase transition in $\mathrm{ND}_{4} \mathrm{D}_{2} \mathrm{PO}_{4}$. Phys. Rev. (in press).

Meister, H., Skalyo, J. Jr., Frazer, B.C., and Shirane, G. Lattice dynamics aspects of the antiferroelectric phase transition in deuterated ADP studied by neutron scattering. (A) Bull. Amer. Phys. Soc. 13, 1431 (1968).

Melissinos, A.C. - See Carroll, A.S.

Menes, J. - See Anderson, E.W.

Menzinger, F., Cox, D.E., Frazer, B.C., and UmebayaSHI, H. Polarized neutron study of the magnetic moment density in antiferromagnetic $\mathrm{CuSO}_{4}$. Phys. Rev. 181, 936-45 (1969).

Metzger, W. J. - See Alitti, J.; Crennell, D.J.

Miller, C.E. AND Lipsicas, M. Nuclear spin-lattice relaxation in very dilute solutions of orthohydrogen in parahydrogen. Phys. Rev. 176, 273-9 (1968).

Miller, D. J., Huson, F.R., AND O'Neall, J.S. Coherent production of the $1470 \mathrm{MeV} p \pi^{+} \pi^{-}$enhancement by $28-\mathrm{GeV} / c$ protons in a neon-hydrogen bubble chamber. (A) Presented at Phys. Soc. Meet. Elementary Particles, London, Sept. 1968.

Miller, D. J. - See also Bingham, H.H.; Cnops, A.M.; ElLIS, W.E.; Huson, F.R.

Minati, K.F. A high speed gas handling system for the Brookhaven National Laboratory three stage tandem Van de Graaff facility. IEEE Trans. Nucl. Sci. NS-16, No. 3, Pt. 1, 109-10 (1969).

Minkiewicz, V.J., Collins, M.F., Nathans, R., And Shirane, G. Critical and spin wave fluctuations in nickel by neutron scattering. Phys. Rev. 182, 624-31 (1969).

Minkiewicz, V.J., Kitchens, T.A., Lipschultz, F.P., Nathans, R., and Shirane, G. Phonon spectrum of hcp He4. Phys. Rev. 174, 267-75 (1968).

Minkiewicz, V.J. and Shirane, G. Soft phonon modes in $\mathrm{KMnF}_{3}$. J. Phys. Soc. Japan 26, 674-80 (1969).

Minkiewicz, V. J. - See also Collins, M.F.; Leake, J.A.

Misetich, A.A. AND Watson, R.E. Susceptibility of palladium metal. (A) J. Appl. Phys. 40, 1211 (1969).
Moffeit, K. - See Bingham, H.H.

Montanet, L. - See Barnes, V.E.

Montwill, A. - See Crennell, D. J.

Moragues, J.A., Gelletly, W., and Mariscotti, M.A.J. Internal conversion coefficients of high-energy gamma rays. Phys. Letters 27B, 441-2 (1968).

Moragues, J.A., Mariscotti, M.A. J., Gelletly, W., AND KANE, W.R. $\operatorname{Ba}^{138}(n, \gamma) \mathrm{Ba}^{139}$ reaction and evidence for direct capture. Phys. Rev. 180, 1105-13 (1969).

Moragues, J.A. - See also Gelletly, W.; Mariscotti, M.A.J.

Moriyasu, K. - See Bingham, H.H.

Morris, T.W. - See Connolly, P.L.; Ellis, W.E.; PanvinI, R.S.

Moss, S.C. - See Mozer, B.

Mozer, B., Keating, D.T., and Moss, S.C. Neutron measurement of clustering in the alloy CuNi. Phys. Rev. 175, 868-76 (1968).

Mucci, J. - See Anderson, E.W.

Mueller, A.H. 0(4), Off-shell $\mathcal{N} \overline{\mathcal{N}}$ scattering, and massless pions. Phys. Rev. 172, 1516-21 (1968).

Mueller, A.H. And Muzinich, I. J. Diagonalization of the integral equation for multiperipheral dynamics at vanishing values of the momentum transfer. Submitted to Ann. Phys. N.Y.

Mueller, J. - See Sims, W.H.

Muether, H.R. - See Andrus, W.S.; Lone, M.A.

Muggia, E. - See Bingham, H.H.

Mulholland, G.T. - See Bamberger, J.A.; Brown, D.P.

MÜlleR, K.A. - See AxE, J.D.

Muzinich, I. J. - See Mueller, A.H.

Myatт, G. Single pion production by neutrinos. Informal Report BNL 13648.

Mydosh, J.A., Kawatra, M.P., Budnick, J.I., Kitchens, T.A., AND Borg, R. J. A study of the temperature dependence of the electrical resistivity in concentrated AuFe alloys. Presented at Int. Conf. Low Temperature Physics, St. Andrews, Scotland, Aug. 1968.

Nagashima, Y. - See Carroll, A.S.

Nagatani, $K$. First order analysis of the reaction ${ }^{6} \mathrm{Li}(p, p \alpha) d$. Phys. Letters 29B, 468-72 (1969).

Nagatani, K. Nucleon-nucleon final state interactions. (A) Presented at Int. Conf. Three-Body Problem in Nuclear and Particle Physics, Birmingham, England, July 1969.

Nagatani, K., Herling, G.H., and Kolltveit, K. Comparative analyses of ${ }^{4} \mathrm{He}\left(p,{ }^{3} \mathrm{He}\right) p n,{ }^{4} \mathrm{He}\left(p,{ }^{3} \mathrm{H}\right) 2 p$, and ${ }^{4} \mathrm{He}\left(p_{,}{ }^{3} \mathrm{He}\right) d$ reactions. (A) Bull. Amer. Phys. Soc. 14, 511 (1969).

Nagatani, K. - See also Harris, W.R.

Nardi, J.A. - See Abrams, K.L.; Connoliy, P.L.

Nathans, R. - See,Cohen, V.W.; Collins, M.F.; Cooper, M. J.; Corliss, L.M. (Chemistry); Minkiewicz, V.J.; Norvell, J.C. (Chemistry); Schulhof, M.P.

Negri, P - See Bingham, H.H.

Newnham, R.E. - See Cox, D.E.

Novak, M. - See Bingham, H.H.

Nussbaum, M.M. - See Cnops, A.M.; Kenyon, I.R.; Skillicorn, I.O.

Okaya, Y. - See Kay, M.I.

Olness, J.W. Gamma-ray spectroscopy in light nuclei: $\mathrm{Na}^{22}$, an example. In Proc. 3rd Int. Symp. Structure of Low- 
Medium Mass Nuclei, Lawrence, Kans., April 1968, pp. 192229, J.P. Davidson, Editor, Univ. Press of Kansas, 1968. Olness, J.W. - See also Paul, P.

O’Neall, J.S., Crennell, D. J., Karshon, U., Lai, K.W., Scarr, J.M., Baumel, P., Lea, R.M., Schumann, T.G., AND URVATER, E.M. $K^{*}$ production in $K-n$ and $K-d$ interactions at $3.9 \mathrm{GeV} / c$. (A) Bull. Amer. Phys. Soc. 14, 561 (1969).

O'Neall, J.S. - See also Alitti, J.; Bingham, H.H.; Cnops, A.M.; Grennell, D.J.; Huson, F.R.; Karshon, U.; Miller, D. J.; Schumann, T.G.

Orear, J., Owen, D.P., Peterson, F.C., Read, A.L., Ryan, D.G., White, D.H., Ashmore, A., Damerell, C. J.S., Frisken, W.R., and Rubinstein, R. Elastic scattering of $\pi^{-}, K^{-}$and $\bar{p}$ from protons at 9.8 and 13.6 $\mathrm{GeV} /$ c. Phys. Letters 28B, $61-4$ (1968).

OWen, D.P. - See Orear, J.

Ozaki, S. - See Foley, K. J.

Palevsky, H. - See Andrus, W.S.; Friedes, J.L.

Palmer, R.B. - See Dodd, W.P.; Hill, D.G.

Panvini, R.S., Morris, T.W., and Ellis, W.E. Some general characteristics of one-, two-, and three-pion production in 28.5-GeV/c pp interactions. (A) Bull. Amer. Phys. Soc. 14, 636 (1969).

Panvini, R.S. -See also Ellis, W.E.

Parks, R.D. - See Strongin, M.

Passell, L. - See Gollins, M.F.; SGhermer, R.I.

Paul, P., Olness, J.W., and Warburton, E.K. Nuclear structure of $\mathrm{Na}^{22}$. V. Doppler-shift attenuation measurements. Phys. Rev. 173, 1063-71 (1968).

Paul, P. - See also Fossan, D.B.

Pazzi, R. - See Bertanza, L.

Peierls, R.F. - See Brayshaw, D.; Sternheimer, R.M.

Peterson, F.C. - See Orear, J.

Phillips, G.C. - See Friedes, J.L.

Phillips, R.H. - See Carroll, A.S.

Pickart, S. J. - See McGuire, T.R.

Platner, E.D. - See Foley, K. J.

Price, D.L., Chrien, R.E., Wasson, O.A., Bhat, M.R., Beer, M., Lone, M.A., and Graves, R. Neutron capture in ${ }^{238}$ U. Nucl. Phys. A121, 630-54 (1968).

Prodell, A.G. The 8-foot diameter 30 kilogauss superconducting magnet for the Brookhaven National Laboratory 7-foot bubble chamber. (A) Bull. Amer. Phys. Soc, 13, 1738 (1968).

Prodell, A.G. - See also Allinger, J. (Accelerator); Bamberger, J.A.

Quarles, C.A. - See Foley, K.J.

Quinn, J.J. - See Greene, M.P.

Radojicic, D. - See Alitti, J.

Rahm, D.C. - See Bertanza, L.; Yamamoto, S.S.

Ramsey, N.F. - See Cohen, V.W.

Rau, R.R. - See Alitri, J.

RAy, A.K., Burris, R.W., Fisk, H.E., Kraemer, R.W., Hill, D.G., AND SakitT, M. Resolution of the $T=0$ Fermi-Yang ambiguity in $K$ nucleon scattering. Phys. Rev. 183, 1183 (1969).

Read, A.L. - See Orear, J.

Richardson, C.R. - See Alitti, J.

Rimawi, K., Chrien, R.E., Wasson, O.A., and Garber, D.I. The direct capture mechanism in $\mathrm{Mn}^{55}(n, \gamma) \mathbf{M n}^{56}$. (A) Bull. Amer. Phys. Soc. 13, 1390 (1968).
Rimawi, K., Garber, D.I., Wasson, O.A., Garg, J.B., and Chrien, R.E. Neutron resonance capture in $\mathrm{Nb}^{93}$ and $\mathrm{Zr}^{91}$. (A) Ibid. 14, 104 (1969).

Rimawi, K. - See also BeEr, M.; Garber, D.I.

Ringel, H., Arndt, R.A., Whitten, W.B., and Damask, A.C. Color centers in $\gamma$-irradiated organic crystals. (A) Bull. Amer. Phys. Soc. 14, 376 (1969).

Ringel, H., Damask, A.C., and Arndt, R.A. Heatcapacity anomaly in chrysene. (N) Mol. Cryst. 5, 295-6 (1969).

Ringel, H. - See also Whitten, W.B.

Rodriguez, S. - See Greene, M.P.

Rogers, P.G. The data acquisition and analysis complex for the BNL low energy accelerator facility. IEEE Trans. Nucl. Sci. NS-16, No. 1, 142-3 (1969).

Rogers, P.C. - See also Auerbach, E.H.; Schwender, G.E.

Rollier, M. - See Bingham, H.H.

Rorer, D.C., Ecker, B.M., And Akyuz, R.O. Slow neutron cross sections for helium, neon, argon, and krypton. Nucl. Phys. (in press).

Rorer, D.C. - See also Schermer, R.I.

Rose, H.J., Hausser, O., and Warburton, E.K. Evidence for a nuclear tensor force from mass-14 beta- and gamma-ray data. Rev. Mod. Phys. 40, 591-610 (1968).

Rubinstein, R. - See Orear, J.

Russ, J. - See ANDERson, E.W.

Ryan, D.G. - See Orear, J

SAILOR, V.L. Oriented targets in neutron spectrometry. Informal Report BNL 13624.

Sakitt, M. - See Martin, B.R.; Ray, A.K.

SAmios, N.P. Status of $\eta^{\prime}$ and $\delta$ bosons. In Meson Spectroscopy - A Collection of Articles (Proc. Meet., Philadelphia, April 1968), pp. 121-35, C. Baltay and A.H. Rosenfeld, Editors, W.A. Benjamin, Inc., New York, 1968.

Samios, N.P. - See also Alitti, J.; Barnes, V.E.; Dodd, W.P.

Samulelen, E. J., Hutchings, M.T., and Shirane, G. Inelastic neutron scattering investigation of spin waves and magnetic interactions in $\mathrm{Cr}_{2} \mathrm{O}_{3}$. Solid State Commun. (in press); (A) Bull. Amer. Phys. Soc. 14, 539 (1969).

Samuelsen, E. J. - See also Hutchings, M.T.

Santoro, R.P. - See Cox, D.E.

Scarr, J.M. - See Abrams, K.L.; Alitti, J.; Cnops, A.M.; Crennell, D. J.; Karshon, U.; Kenyon, I.R.; LaI, K.W.; O'Neall, J.S.; Schumann, T.G.; SkilliCORN, I.O.

Scharff-Goldhaber, G. Elasticity of nuclei. (A) Presented at Symp. Nuclear Structure Research, Amer. Chem. Soc., New York, Sept. 1969.

Scharff-Goldhaber, G. - See also Mariscotti, M.A.J.

Schermer, R.I., Passell, L., and Rorer, D.C. Phase separation in $\mathrm{He}^{3}-\mathrm{He}^{4}$ mixtures observed with slow neutrons. Phys. Rev. 173, 277-84 (1968).

Schneeberger, M. - See Sims, W.H.

Schneps, J. - See Sims, W.H.

SchröDER, I. - See Kane, W.R.

Schulhof, M.P., Heller, P., and Nathans, R. Critical magnetic scattering from $\mathbf{M n F}_{2}$. (A) Bull. Amer. Phys. Soc. 14, 738 (1969).

Schumann, T.G., Baumel, P., Lea, R.M., Urvater, E.M., Crennell, D.J., Karshon, U., Lai, K.W., 
O'Neall, J.S., and Scarr, J.M. $\Upsilon^{*}$ production in $K-n$ interactions at $3.9 \mathrm{GeV} / c$. (A) Ibid., 561 .

Schumann, T.G. - See also Alitti, J.; Crennell, D. J.; Karshon, U.; O'Neall, J.S.

Schwarzsghild, A.Z. and Warburton, E.K. The measurement of short nuclear lifetimes. Annu. Rev. Nucl. Sci. 18, 265-90 (1968).

Schwarzschild, A.Z. - See also Jones, K.W.

Schwender, G.E. ANd Rogers, P.C. The data collection interface used by the BNL data acquisition and analysis complex. IEEE Trans. Nucl. Sci. NS-16, No. 1, 162-4 (1969).

Scott, C.K. And Lee, H.C. Recurrence relations for Talmi coefficients and shell model radial integrals. Informal Report BNL 12741.

Scott, C.K. - See also Auerbach, E.H.; Kahana, S.

Sheppard, D.M. - See McEllistrem, M.T.

Sнiн, C.C. Regge-pole analysis for $\pi^{-}$production of $\rho^{-}$ meson near the backward direction. Phys. Rev. Letters 22, 105-8 (1969).

Shir, C.C. AND TUNG, W.K. Forward photoproduction of $\pi^{+}$and $K^{+}$mesons in the Regge-pole model. Phys. Rev. 180, 1446-8 (1969).

Shin, C.C. and Tung, W.K. A study of the kinematic constraints and factorization in the Regge-pole model for photoproduction processes. Phys. Rev. (in press).

Shih, C.C. - See also Brown, R.W.

Shirane, G. Neutron inelastic scattering study on soft modes. In Proc. Int. Meet. Ferroelectricity, Kyoto, Japan, Sept. 1969 (in press).

ShIRANE, G. AND Yamada, Y. Lattice-dynamical study of the $110^{\circ} \mathrm{K}$ phase transition in $\mathrm{SrTlO}_{3}$. Phys. Rev. 177, 858-63 (1969).

Shirane, G. - See also Axe, J.D.; Birgeneau, R. J.; Collins, M.F.; Cox, D.E.; Hutchings, M.T.; LEAKE, J.A.; Meister, H.; Minkiewicz, V.J.; Samuelsen, E.J.; SKalyo, J.JR.; Yamada, Y.

Shuld, C.G. Observation of Pendellosung fringe structure in neutron diffraction. Phys. Rev. Letters 21, 1585 (1968).

Shull, C.G. Single slit diffraction of neutrons. Phys. Rev. Letters (in press).

Silsbee, H.B . - See Chan, Y.W.; Cohen, V.W.

Simpson, W.D. - See Friedes, J.L.

Sims, W.H., Albright, J.R., Brucker, E.B., Lannutti, J.E., Greene, A., Bartley, J., Dowd, R.M., Schneps, J., Mueller, J., Schneeberger, M., And Wolf, S.E. A discussion of lambda-nucleon interactions observed in $\Lambda p \pi^{-}, \Lambda n \pi^{-} \pi^{+}$, and $\Lambda p \pi^{-} \pi^{0}$ final states. (A) Bull. Amer. Phys. Soc. 13, 1442 (1968).

Six, J. - See Bingham, H.H.

Skalyo, J. Jr., Frazer, B.C., Shirane, G., and Daniels, W.B. Pressure dependence of the transition temperature in KDP and ADP. J. Phys. Chem. Solids 30, 2045-51 (1969); (A) Bull. Amer. Phys. Soc. 13, 1432 (1968).

Skalyo, J. Jr., Shirane, G., and FriedberG, S.A. Twodimensional antiferromagnetism in $\mathrm{Mn}(\mathrm{HCOO})_{2} \cdot 2 \mathrm{H}_{2} \mathrm{O}$. Phys. Rev. (in press).

Skalyo, J. Jr. - See also Leake, J.A.; Meister, H.

Skillicorn, I.O., Cnops, A.M., Hough, P.V.C., Huson, F.R., Kenyon, I.R., Kinson, J.B., Scarr, J.M., Cohn, H.O., McCulloch, R.D., Bugg, W.M., Condo, G.T., and Nussbaum, M.M. An analysis of the neutral $3 \pi$ system produced by $8-\mathrm{GeV} / c \pi^{+}$mesons on deuterium. (A) Bull. Amer. Phys. Soc. 14, 561 (1969).

Skillicorn, I.O. - See also Abrams, K.L.; Alitti, J.; Barnes, V.E.; Chung, S.U.; Chops, A.M.; Crennell, D. J.; Kenyon, I.R.; Strand, R.C.

Smoluchowski, R. - See Dienes, G. J.; Hatcher, R.D.; WILSON, W.D.

SNEAD, C.L. JR. - See DiCarlo, J.A.

SokolofF, J.B. Inelastic neutron scattering from itinerant ferromagnetic and antiferromagnetic metals. (A) Bull. Amer. Phys. Soc. 13, 1432 (1968).

Sokoloff, J.B. A multiband theory of inelastic neutron scattering by ferromagnetic metals at low temperatures. Phys. Rev. 180, 613-21 (1969); (A) Bull. Amer. Phys. Soc. 14, 79 (1969).

SoKoloff, J.B. Polar spin waves in ferromagnetic metals. Phys. Rev. 173, 617-30 (1968).

SokolofF, J.B. Theory of inelastic neutron scattering in the itinerant model of antiferromagnetic metals. Phys. $R e v$. (in press).

Sokoloff, J.B. Theory of longitudinal spin fluctuations and the antiferromagnetic phase transition in chromium metal. Ibid.

Sondericker, J.H. The bubble chamber technique for photographing interactions of high energy particles. Presented at Conf. Society of Motion Picture and Television Engineers, Washington, D.C., Nov. 1968.

Spielberg, D.H. - See Damask, A.C.

Stauber, M.C. ANd Lemos, A.M. Tl+ absorption bands in $\mathrm{KCl}$ : Theoretical. (A) Presented at Int. Symp. Color Centers in Alkali Halides, Rome, Sept. 1968.

Stearns, R.L. - See Friedes, J.L.

Sternheimer, R.M. Electronic polarizabilities of the alkali atoms. I. (A) Bull. Amer. Phys. Soc. 14, 82-3 (1969); II, Phys. Rev. (in press).

Sternheimer, R.M. Wave functions for crystal field calculations. Amer. Doc. Inst. Auxiliary Publ. Proj. Document No. 10047, Library of Congress, Washington, D.C.

Sternheimer, R.M. Wave functions for the electronic polarizabilities of the alkali atoms. NAPS Document No. 00457, ASIS National Auxiliary Publications Service, New York.

Sternheimer, R.M., Blume, M., and Peierls, R.F. Shielding of crystal fields at rare-earth ions. Phys. Rev. 173, 376-89 (1968).

Strand, R.C., Grennell, D. J., Ellis, W.E., and SkilliCORN, I.O. Use of FSD bubble density measurements for three BNL Bubble Chamber Group 80-inch chamber experiments. In Proc. Int. Conf. Advanced Data Processing for Bubble and Spark Chambers, Argonne National Laboratory, Oct. 1968, pp. 362-70, R. J. Royston, Editor, ANL-7515, 1969.

Strand, R.C. - See also Abrams, K.L.; Barnes, V.E.; Kalbfleisch, G.; Vander Burg, V.

Strongin, M. Superconductivity in thin films and particles. (A) In Proc. Conf. Science of Superconductivity, Stanford, Calif., Aug. 1969 (in press).

Strongin, M., Kammerer, O.F., and Crow, J.E. Destruction of superconductivity in disordered near monolayer metallic films. (A) Bull. Amer. Phys. Soc. 14, 112 (1969). 
Strongin, M., Kammerer, O.F., and Grow, J.E. High $T_{c}$ 's in ultra thin films. (A) Bull. Amer. Phys. Soc. 13, 1741 (1968); J. Appl. Phys. 40, 2161 (1969).

Strongin, M., Kammerer, O.F., Crow, J.E., Parks, R.D., Douglass, D.H. JR., and Jensen, M.A. Enhanced superconductivity in layered metallic films. Phys. Rev. Letters 21, 1320-3 (1968).

Strongin, M., Kammerer, O.F., Thompson, R.S., And CRow, J.E. The transition in weak superconductors. (A) Bull. Amer. Phys. Soc. 14, 437 (1969).

Strongin, M. - See also Crow, J.E.; Fine, H.L.; Garcia, N. (Applied Science); Thompson, R.S.

Sunyar, A.W. Anomalous $\gamma$-band intraband transition rates in $\mathrm{Er}^{166}$. Phys. Rev. Letters (in press).

Sutter, R. J. - See Friedes, J.L.

Swartzendruber, L. J., Bennett, L.H., and Watson, R.E. Dilute ${ }^{57} \mathrm{Fe}$ Mössbauer studies in $\mathrm{Cu}-\mathrm{Ni}$ alloys. J. Appl. Phys. 40, 1489-90 (1969).

Swartzendruber, L. J., Bennett, L.H., and Watson, R.E. Weak magnetism in the Cu-rich $\mathrm{Cu}-\mathrm{Ni}-\mathrm{Fe}$ alloy system. (A) Bull. Amer. Phys. Soc. 14, 577 (1969).

TADIĆ, D. Weak parity-nonconserving potentials. Phys. Rev. 174, 1694-1703 (1968).

TADIć, D. - See also Krmpotić, F.

TAYlor, R.D. and CRaig, P.P. Correlation between Mössbauer resonance strength and second order Doppler shift: Estimate of zero point velocity. Submitted to Phys. Rev.

TAYlor, R.D. and GRaig, P.P. Use of Mössbauer techniques to directly determine the zero point velocity of impurities: Calibration of the second order Doppler shift. (A) In Proc. 5th Annu. Symp. Mössbauer Methodology, New York, Jan. 1969 (in press).

Taylor, R.D. - See also Graig, P.P.; Kitchens, T.A.

Tewrshury, S. - See Garroll, A.S.

Thompson, R.S. Fluctuation resistivity of dirty superconductors. (A) Bull. Amer. Phys. Soc. 14, 128 (1969).

Thompson, R.S. The influence of magnetic fields on the paraconductivity due to fluctuations in thin films. (A) In Proc. Conf. Science of Superconductivity, Stanford, Calif., Aug. 1969 (in press).

Thompson, R.S. The microwave, flux flow, and fluctuation resistance of dirty type II superconductors. Phys. $R e v$. (in press).

Thompson, R.S., Strongin, M., Kammerer, O.F., And CROW, J.E. The resistive transition in weakly coupled superconductors. Phys. Letters 29A, 194-5 (1969).

Thompson, R.S. - See also Crow, J.E.; Strongin, M.

Thorndike, A.M. - See Abrams, K.L.; Connolly, P.L.; Crennell, D. J.; Ellis, W.E.; Kenyon, I.R.; Kinsey, R.R.

ThORPE, M.F. Anisotropy in ferromagnets: An exact result. Submitted to Phys. Rev.

Thorpe, M.F. Magnon-magnon interactions in doped antiferromagnets. Phys. Rev. Letters (in press).

Thorpe, M.F. Model of a three-dimensional order-disorder ferroelectric. (A) Bull. Amer. Phys. Soc. 14, 322 (1969).

Thorpe, M.F. Two magnon Raman scattering and infrared absorption in $\mathrm{MnF}_{2}$. (A) Presented at 15th Annu. Conf. Magnetism and Magnetic Materials, Philadelphia, Nov. 1969.

Thorpe, M.F. - See also Hutchings, M.T.
Townsend, P.D., Kemmey, P. J., And Levy, P.W. Gammaray, $x$-ray and ultraviolet-light induced low temperature thermoluminescence of $\mathrm{KN}_{3}$ and $\mathrm{NaN}_{3}$. Submitted to Radiat. Effects.

Trousdale, W. and Craig, P.P. Limitation on relaxation rates of ${ }^{57} \mathrm{Fe}$ in $\mathrm{CoO}$. Phys. Letters 27A, 552-3 (1968).

Trousdale, W.L. - See also Kitchens, T.A.

Trueman, T.L. Kinematic singularities and constraints of helicity amplitudes. Phys. Rev. 173, 1684-94 (1968).

Trueman, T.L. Kinematic structure of vertex functions. Phys. Rev. 182, 1469-74 (1969).

Tung, W.K. - See Shiн, C.C.

Turkot, F. - See Anderson, E.W.

Umebayashi, H. - See Menzinger, F.

Urvater, E.M. - See Crennell, D. J.; O’Neall, J.S.; Schumann, T.G.

Vander Burg, V., Strand, R.C., and Kalbfleisch, G.R. Spin-parity studies of the $\pi(2190)$ and $\omega(2190)$. (A) Bull. Amer. Phys. Soc. 14, 499 (1969).

VAnder Burg, V. - See also Barnes, V.E.; KalbFLEISCH, G.

Veillet, J.J. - See Bingham, H.H.

Vineyard, G.H. - See Watson, R.E.

WALl, N.S. - See Friedes, J.L.

WANG, F.F.Y. AND Cox, D.E. The coherent neutron scattering amplitude of $\mathrm{Rb}$ : A neutron diffraction study of RbCl. Submitted to Acta Cryst.

Warburton, E.K., Harris, W.R., and Alburger, D.E. Beta decay of $\mathrm{N}^{16}$ to the $6.05-\mathrm{MeV}$ state of $\mathrm{O}^{16}$. Phys. Rev. 175, 1275-82 (1968).

Warburton, E.K. - See also Alburger, D.E.; Jones, K.W.; Paul, P.; Rose, H. J.; Schwarzschild, A.Z.

Wasson, O.A., Chrien, R.E., Bhat, M.R., Lone, M.A., AND BeER, M. The $\operatorname{Au}^{197}(n, \gamma) \mathrm{Au}^{198}$ reaction mechanism. Phys. Rev. 173, 1170-84 (1968).

Wasson, O.A., Ghrien, R.E., Bhat, M.R., Lone, M.A., AND BeER, M. Direct neutron capture in $\mathrm{Co}^{59}(n, \gamma) \mathrm{Co}^{60}$. Ibid. 176, 1314-22.

Wasson, O.A., Chrien, R.E., and Garber, D.I. Resonance neutron capture in $\mathrm{La}^{138}(n, \gamma) \mathrm{La}^{139}$ and $\mathrm{La}^{139}(n, \gamma) \mathrm{La}^{140}$. (A) Bull. Amer. Phys. Soc. 14, 513 (1969).

Wasson, O.A., Chrien, R.E., Lone, M.A., Bhat, M.R., AND BEER, M. Resonance neutron capture in ${ }^{181} \mathrm{Ta}(n, \gamma){ }^{182} \mathrm{Ta}$. Nucl. Phys. A132, 161-76 (1969).

Wasson, O.A., Garg, J.B., Chrien, R.E., and Bhat, M.R. Gamma rays following neutron capture in iron, sodium, and thorium. In Proc. 2nd Conf. Neutron Cross Sections and Technology, Washington, D.C., March 1968, Vol. 1, pp. 675-86, D.T. Goldman, Editor, Nat. Bur. Std. U.S. Spec. Publ. 299, Sept. 1968.

Wasson, O.A. - See also Beer, M.; Bhat, M.R.; Chrien, R.E.; Garber, D.I.; Lone, M.A.; Mughabghab, S.F. (Applied Science); Price, D.L.; Rimawi, K.

Watson, R.E., Bennett, L.H., and Freeman, A. J. Comments on Origin of Solvent Knight Shifts in Alloys. Phys. Rev. 179, 590-2 (1969).

Watson, R.E., Blume, M., and Vineyard, G.H. Spin motions in a classical ferromagnet. Ibid. 181, 811-23.

Watson, R.E. and Freeman, A.J. Exchange coupling and conduction-electron polarization in metals. II. Ibid. 178, 725-31. 
Watson, R.E., Freeman, A. J., AND KoIde, S. Role of interband mixing in exchange coupling and conductionelectron polarization in metals. Phys. Rev. (in press).

Watson, R.E. - See also Misetich, A.A.; SwartzenDRUBER, L. J.; WolcotT, N.M.

WaUng, H.F., BRandt, W., AND Levy, P.W. Color-center studies in $\mathrm{NaCl}$ crystals by positron annihilation. (A) Bull. Amer. Phys. Soc. 13, 1473 (1968).

WAUng, H.F. - See also Brandt, W.

Wegner, H.E. Tandem accelerators. IEEE Trans. Nucl. Sci. NS-16, No. 3, Pt. 1, 81-9 (1969).

Wegner, H.F. The 30-MeV three-stage Van de Graaff facility and its associated research program at Brookhaven National Laboratory. In Proc. 19th Annu. Int. Conf. Nuclear Spectroscopy and Structure of the Atomic Nucleus, Yerevan, USSR, Jan. 27-Feb. 4, 1969 (in press).

Wegner, H.E. - See also Armstrong, D.D.

Weneser, J. - See Auerbach, E.H.; Marshalek, E.R.

Werntz, T.L. - See Connolly, P.L.

Whetstone, G.N. - See Bamberger, J.A.

White, D.H. - See Orear, J.

Whitten, W.B., Arndt, R.A., and Damask, A.C. Triplet exciton decay in phenanthrene single crystals. Mol. Cryst. 9, 239-48 (1969).

Whitten, W.B., Ringel, H., and Damask, A.C. Carrier lifetimes in $\gamma$-irradiated anthracene. (A) Bull. Amer. Phys. Soc. 13, 1394 (1968).

Whitten, W.B. - See also Ringel, H.

Wilkin, C. - See BAsSel, R.H.

Wilkinson, D.H., Alburger, D.E., AND Lowe, J. Gammaray decay of the 6.92-, 7.12-, 8.88-, and $13.10-\mathrm{MeV}$ states in $\mathrm{O}^{16}$. Phys. Rev. 173, 995-1001 (1968).

Wilkinson, D.H. - See also Alburger, D.E.

Willen, E.H. - See Foley, K.J.

Willis, W. J. - See Bertanza, L.

Wilson, W.D., Hatcher, R.D., Dienes, G. J., AND Smoluchowski, $\mathrm{R}$. $\mathrm{Cu}^{+}$and $\mathrm{Ag}^{+}$centers in alkali halides. Phys. Rev. (in press); (A) Bull. Amer. Phys. Soc. 14, 325 (1969).

Wilson, W.D. - See also Dienes, G. J.; Hatcher, R.D.

Wolgott, N.M., FAlge, R.L. JR., Bennett, L.H., AND WAtson, R.E. Nuclear magnetic resonance studies in paramagnetic and ferromagnetic $\mathrm{CrBe}_{12}$. Phys. Rev. Letters 21, 546-9 (1968).

Wolf, S.E. - See Sims, W.H.

Wong, N.S. - See Bertanza, L.

WORWETZ, H.A. - See BAMBERger, J.A.

YAFET, Y. - See Blume, M.

Yamada, Y. ANd Shirane, G. Neutron scattering and nature of the soft optical phonon in $\mathrm{SrTiO}_{3} . J$. Phys. Soc. Japan 26, 396-403 (1969).
Yamada, Y., Shirane, G., And Linz, A. Study of critical fluctuations in $\mathrm{BaTiO}_{3}$ by neutron scattering. Phys. Rev. 177, 848-57 (1969)

Yamada, Y. - See also Shirane, G.

Yamamoto, S.S. And RaHM, D.C. Two-prong interactions by $\pi^{+}+p$ collisions at $2.77 \mathrm{BeV} / c$. Ibid. 173, 13026 (1968).

Yamamoto, S.S. - See also Bertanza, L.

Young, B.L. Hard-meson calculations and the AdlerDashen-Gell-Mann-Fubini sum rule. Phys. Rev. 181, 1878-83 (1969).

Young, B.L. - See also Brown, R.W.

Yuan, L.C.L. - See Prunster, S. (Accelerator).

Zeller, E. J., Levy, P.W., ANd Mattern, P.L. Geologic dating by electron spin resonance. In Proc. IAEA Symp. Radioactive Dating and Methods of Low-Level Counting, Monaco, March 1967, pp. 531-40, IAEA, Vienna, 1967.

ZuKer, A.P., Buck, B., AND McGrory, J.B. Shell-model calculations in F18. (A) Bull. Amer. Phys. Soc. 14, 35 (1969).

Zuker, A.P., Buck, B., ANd McGrory, J.B. Shell model calculations in $\mathrm{N}^{13}$. (A) Ibid.

Zuker, A.P., Buck, B., AND McGrory, J.B. Shell model calculations in $\mathrm{O}^{17}$ and a puzzle. (A) Presented at Conf. Properties of Nuclear States, Montreal, Aug. 1969.

\section{Plant Engineering and Planning Division}

Hansteen, H.B. Total Electric Service Feasibility Study. BNL 50173 (T-532) (in press).

Hansteen, H.B. and Krikawa, J.F. The fossilectric ratio. Presented at Winter Meet. ASME, New York, Dec. 1968.

Krikawa, J.F. - See Hansteen, H.B.

\section{Reactor Division}

Powell, R.W. Reactor Operations Monthly Reports (For Internal Use Only):

July 1968 BNL 50125 (T-502)

Aug. 1968 BNL 50128 (T-504)

Sept. 1968 BNL 50135 (T-508)

Oct. 1968 BNL $50144(\mathrm{~T}-515)$

Nov. 1968 BNL 50152 (T-521)

Dec. 1968 BNL 50156 (T-522)

Jan. 1969 BNL 50160 (T-525)

Feb. 1969 BNL 50165 (T-528)

Mar. 1969 BNL 50167 (T-529)

Apr. 1969 BNL 50172 (T-531)

May 1969 BNL 50178 (T-535)

June 1969 BNL 50182 (T-537) 


\title{
Appendix B
}

\section{OFFICERS AND SCIENTIFIC AND PROFESSIONAL STAFF}

\author{
Maurice Goldhaber, Director \\ George H. Vineyard, Deputy Director \\ Victor P. Bond, Associate Director \\ Rodney L. Cool, Associate Director \\ R. Christian Anderson, Assistant Director \\ William J. Catacosinos, Assistant Director \\ Vincent R. O'Leary, Assistant Director \\ Samuel M. Tucker, Assistant Director \\ G. Kenneth Green, Chairman, Accelerator Department \\ Yoshio Shimamoto, Chairman, Applied Mathematics Department \\ Warren E. Winsche, Chairman, Department of Applied Science \\ C.H.W. Hirs, Chairman, Biology Department \\ Gerhart Friedlander, Chairman, Chemistry Department \\ Horner Kuper, Chairman, Instrumentation and Health Physics Department \\ Eugene P. Cronkite, Chairman, Medical Department \\ R. Ronald Rau, Chairman, Physics Department \\ Irving J. Polk, Head, Mechanical Engineering Division \\ Robert W. Powell, Head, Reactor Division \\ Lewis R. Burchill, Controller \\ Dennis Puleston, Information Officer \\ Charles F. Dunbar, Legal Counsel \\ N. Peter Rathvon, Jr., Associate Counsel \\ Joseph S. Washburne, Personnel Manager \\ Maxwell M. Small, Manager, Plant Engineering and Planning \\ Norbert J. Dernbach, Public Relations Officer \\ Edward J. Burke, Security Officer
}

\section{Accelerator Department}

G. Kenneth Green, Chairman

Roger R. Adams

Christopher Agritellis

Theodore Alper

Charles Ankenbrandt

(postdoctoral appointment)

Gregory P. Bagley

David A. Barge

Mark Q. Barton

Kenneth Batchelor

Gerald W. Bennett

(on leave to

Deutsches Elektronen-Synchrotron,

Hamburg, West Germany)

Arnold Benton

*Terminated before July 1, 1969
David Berley

Richard A. Beth

John W. Bittner

John P. Blewett

Leroy N. Blumberg

Richard B. Britton

Hugh N. Brown

Theodore Carides

Charles A. Casey*

Renate W. Chasman

Peter J. Clarke

Johannes Claus

Jack A. Cockrill

George W. Cornish, Jr.

James G. Cottingham

Joseph A. Curtiss

Per Fridtjof Dahl

Gordon T. Danby

Donald A. Davis
Edward W. Dexter

Emory G. Egler

Stephen E. Eiseman

Arnold V. Feltman

Norman M. Fewell

Horst W. J. Foelsche

Eric B. Forsyth*

John D. Fox

Robert S. Frankel

Martin Fruitman

John J. Gabusi

William Gefers

Salvatore T. Giordano

Joseph W. Glenn, III

Eileen C. Gran

David L. Greenberg

Harald Hahn

Henry J. Halama

Joseph P. Hannwacker* 
William E. Harrison, Jr.

John C. Herrera

Garrett A. Hughes

Robert S. Hulliger

John W. Jackson

David D. Jacobus

Kurt Jellett

Henry Kasha

Ralph R. Kassner

John T. Keane

Vincent J. Kovarik

John H. Lancaster

Ronald F. Lankshear

Robert A. Larson

Martin J. Lee*

Lawrence B. Leipuner

Robert J. Lenuzza

Gerald S. Levine

Su Tang Lin

Isador J. Livant

Walter Livant

Robert E. Lockey

Vincent S. Lo Grasso

Robert A. Loper

Edward A. MacDougall

Harvey McChesney, Jr.

Austin J. McGeary

Alfred D. McInturff

Lowell McLean

Andrew J. McNerney

Walter W. Merkle

(deceased January 2, 1969)

Stephen J. Merlan

(graduate siudent from Yale Univ.)

Peter A. Montemurro

Melvin Month

William H. Moore, Jr.

Gerry H. Morgan

Ronald J. Nawrocky

Ernest Okress

Leslie W. Oleksiuk*

Jean M. Oostens*

Arthur N. Otis, Jr.

George Parzen

Jens C. Pettersen

Martin Plotkin

Sepp J. Prunster*

(on leave from Physikalisches Staatsinstitut,

Institut fur Experimentalphysik,

Hamburg, West Germany)

Icarus E. Pyros

Eugene C. Raka

George Rakowsky

George J. Reichenbacher*

Raymond H. Rheaume

Everett J. Rutan*

William B. Sampson

Ralph T. Sanders

James R. Sanford*

Albert J. Schoenemann*

*Terminated before July 1, 1969.
Joseph F. Sheehan, Jr.

Theodorus J.M. Sluyters

Lyle W. Smith

Anastasios Soukas

Joel W. Spinner

Julius Spiro

Raymond J. Stefanski

(graduate student from rale Univ.)

Colin D. Stewart

Robert C. Talsma

Timothy E. Toohig

Arthur Tranis

Clarence M. Turner

Arie van Steenbergen

Uriel Vogel

Peter J. Wanderer, Jr.

(graduate student from Yale Univ.)

Ching Lin Wang

Robert J. Warkentien

Arnold R. Watts

Irwin Weitman

George W. Wheeler

Richard L. Witkover

Samuel P. Yamin

(postdoctoral appointment)

\section{Applied Mathematics Department}

Yoshio Shimamoto, Chairman

Barry S. Arbeit

George A. Baker, Jr.

Lydia Bargiuk

Steven Bender*

Carmen M. Benkowitz

Harold C. Berry

Kenneth J. Brightman

Ludvik Burgar

Graham H. Campbell

Hong-Bo Chiang

John E. Denes

John E. de Pillis*

(on leave from Univ. of California)

Karl P. Duerre

(on leave from Technische Hochschule,

Hanover, West Germany)

Jerry M. Friedman

Kurt Fuchel

Bernard D. Garfinkel

David S. Gaunt

(on leave from

King's College, Univ. of London, England)

Michael D. Goldfarb

Charles I. Goldstein

Frederick J. Hamilton

Arthur D.S. Harris

Heinrich Heesch

(on leave from Technische Hochschule,

Hanover, West Germany)

Sidney Heller

Margaret F. Hind

Richard S. Horwitz

Joseph Kahane

Anatoly Kandiew
Minato Kawaguti

Burton S. Kleinman

Joshua K. Kopp

Leslie L. Lawrence

Raymond E. Lawrence

Alvin A. Legerlotz*

Marc Leopold

Irwin Lewis

Dale W. Lick*

(on leave from Univ. of Tennessee)

Robert B. Marr

Bruce A. Martin

Gerald M. Nonte*

Stanley J. Osher

Lorraine J. Osterer

Larry S. Padwa

Ilse H. Perlman

Arnold M. Peskin

Joel D. Pincus*

Clarence G. Pittenger

Dysart A. Ravenhall

Stuart S. Rideout

Robert N. Salerno

Alan M. Saltzman*

Niels F. Schumburg

Arthur B. Scott

Susan M. Sevian

Morris Skibinsky*

Sharon Spark

Morris M. Strongson

Melvin Tainiter

James K. Thurber

Ralph A. Trondle

Carol Wawrzusin

Department of Applied Science

Warren E. Winsche, Chairman

Peter D. Adams*

Anthony Adin

George Adler

Arnold L. Aronson

Seymour Aronson*

Clements Auerbach

Allan Auskern

David S. Ballantine

(on leave to US AEC, Washington, D.C.)

Charles B. Bartlett

(on leave to US AEC, Washington, D.C.)

Helen B. Bauman

Mendel Beer

Morris Beller

Mulki R. Bhat

Fritz Bloch

John S. Bookless

William Bornstein

Joseph S. Bryner

William J. Cahill

Albert W. Castleman, Jr.

Krishnamurthi Chandramoleshwar

(assigned from Bhabba A tomic

Research Centre, Trombay, India)

John Chen 
Jack Chernick

Joe G.Y. Chow

John T. Glarke

(deceased March 14, 1969)

Peter Colombo

John F. Conant

Helen R. Connell

Anita J. Court

Dermott E. Cullen

John H. Cusack

David J. Diamond

Joan M. Dickey

(postdoctoral appointment)

Russell N. Dietz

Robert F. Doering

Roy F. Domish

John J. Dorning, Jr.

Kenneth W. Downes

Raymond F. Drager

Marvin K. Drake

Orrington E. Dwyer

James J. Egan

Leonard G. Emma

Lester G. Epel

Seymour G. Epstein*

Jack Fajer

Peter T. Fallon

Stephen W. Feldberg

Richard M. Felder*

Albert H. Fleitman

Jack J. Fontana

Joseph Forrest

Bernard R. Fox

Roberto V.A. Gabarain

(IAEA Fellow on leave from

Comisión Nacional de Energía Atómica,

Buenos Aires, Argentina)

Leonard Galanter

James M. Galligan

Meyer Garber

Jacob Gilat

(on leave from

Israel A tomic Energy Commission, Yaune)

Althea Glines

Norman N. Gold

Murrey D. Goldberg

Rubin Goldstein

Robert W. Goodrich

Barry M. Gordon

Yorio Gotoh*

(assigned from

Japan Atomic Energy Research Inst.,

Tokai-mura)

Leon Green

(on leave to IAEA, Vienna, Austria)

Margaret W. Greene

David H. Gurinsky

Loranus P. Hatch

Michael A. Helfant

Joseph M. Hendrie

*Terminated before July 1, 1969.
Raymond J. Heus

William A. Higinbotham

Frank B. Hill

Manny Hillman

Peter J. Hlavac

Michael Hobdell

(postdoctoral appointment)

Frederick L. Horn

Chia-Jung Hsu

Hugh S. Isaacs

Mahmut Isildar

(Assigned from

Middle East Technical Univ.,

Ankara, Turkey)

Robert J. Isler

Carl L. Johnson

Richard Johnson

Sheldon Kalish

Otto F. Kammerer

Mary T. Kinsley

George Kissel

Carl J. Klamut

Paul J. Klotz

Herbert J.C. Kouts

Theodore J. Kreiger

William H. Kropp

Lawrence E. Kukacka, Jr.

Michael J. Lancefield

(postdoctoral appointment)

Daniel F. Leahy

Elliot Lebowitz

Gerald S. Lellouche

Melvin M. Levine

Steven Lewkowitz*

George C. Lindauer

Hans Ludewig

Donald R. MacKenzie

Benjamin Magurno

Bernard Manowitz

Victoria L. May

James J. McNicholas

George E. Meinken

Donald J. Metz

Paul A. Michael

Julius Milau

Francis T. Miles

Bertram Minushkin

Sophie O. Moore

M. Luis Muga*

(on leave from Univ. of Florida)

Said F. Mughabghab

Anil K. Mukherji

(one leave from

Drexel Inst. of Technology)

Albert C. Muller

Leonard Newman

Basil Nicolaenko*

(postdoctoral appointment)

Tatsuo Oku

(assigned from

Japan A tomic Energy Research Inst.,

Tokai-mura)
Eric H. Ottewitte

(assigned from

Atomics International Division,

North American Rockwell Corp.)

Odelli Ozer

Philip F. Palmedo

Raymond J. Parsick

Arthur Paskin*

Sol Pearlstein

James P. Phelps

James R. Powell, Jr.

Thomas F. Prach

Glenn A. Price

Augustus Prince

Jacob Pruzansky

Surendra N. Purohit

Chad J. Raseman

James J. Reilly, Jr.

Powell Richards

Francis X. Rizzo

Anthony Romano

Michael A. Rothbart*

Wolfgang Rothenstein (on leave from Technion,

Israel Inst. of Technology, Haifa)

Alexander Sabosto

Jerome Sadofsky

Francis J. Salzano

Saurabh Sanatani

(assigned from IAEA, Vienna, Austria)

César A. Sastre

Shoichi Sato*

(assigned from

Japan Atomic Energy Research Inst.,

Tokai-mura)

Clifford H. Scarlett

Thomas E. Shea

(graduate student from

Rensselaer Polytechnic Inst.)

Richard Schiller*

Ursula M.R. Schulze

Donald G. Schweitzer

John T. Sears*

Thomas V. Sheehan

Takanori Shimooke*

(assigned from

Japan Atomic Energy Research Inst.,

Tokai-mura)

Louis M. Shotkin

Robert M. Singer

Peter Soo

Louis G. Stang, Jr.

John R. Stehn

Meyer Steinberg

Thomas E. Stephenson

Gerald Strickland

Nils L.G. Stromberg

(on leave from

Research Inst. of National Defense,

Stockholm, Sweden)

Sylvester C. Suda

Herbert Susskind 
James W. Sutherland

Hiroshi Takahashi

Ignatius Ning-Bang Tang

Silvio J. Tassinari

Richard S. Thompson

Richard J. Tivers

Ping-Shiu Tu*

Walter D. Tucker

William J. Tunney

Edwin J. Tuthill

James O. Tveekrem*

John D. Van Norman

Stephen J. Wachtel

Jean I. Wagner

Charles H. Waide

John R. Weeks

Eugene V. Weinstock

Allen J. Weiss

Jerome Weiss

George G. Weth

John K. Wilmshurst

Robert G. Wilson

Virginia H. Wilson

Henry $\mathrm{H}$. Windsor

Edward Wirsing, Jr.

Richard H. Wiswall, Jr.

John J. Woods

(assigned from Babcock \&Wilcox,

Lynchburg, Va.)

Wen-Shi Yu

Martin S. Zucker

\section{Biology Department}

C.H.W. Hirs, Chaiman

Joseph J. Abata*

(postdoctoral appointment)

Nicholas Alonzo

Ira H. Ames*

(postdoctoral appointment)

Diana C. Bartelt

Shabtai T. Bauer

(postdoctoral appointment)

Arnold M. Becker

Robert R. Becker

(on leave from Oregon State Univ.)

Clara A. Bjerknes

Carl F. Blackman, Jr.

(AUI Postdoctoral Fellow,

National Institutes of Health Fellowship)

Peter Bodlaender*

(postdoctoral appointment)

Robert J. Bores

(graduate student from Rutgers Univ.)

Paul J. Bottino

(postdoctoral appointment)

Robert W. Briggs

Dennis R. Burholt

(National Institutes of Health Fellow)

Alice J. Burton

Yu-Hua Chang

*Terminated before July 1, 1969.
Theodore Chase, Jr.*

(postdoctoral appointment)

Nicholas C. Combatti

Howard J. Curtis

Richard G. Cutler*

(postdoctoral appointment)

Rosemarie Dearing

Florence T. Dunne

Daniel Dupourque (postdoctoral appointment)

Frieda M. Englberger

Edward Flaccus

(on leave from Bennington College)

L. Raymond Fox*

(postdoctoral appointment)

Walter J. Geisbusch

Joseph S. Giordano, Jr.*

George I. Glover

(National Institutes of Health Fellow)

Bill Greenberg

Lewis J. Greene

Eleanor Grist

Kenneth L. Grist

D. Elizabeth Hamill*

Robert L. Heath

(postdoctoral appointment)

Susan S. Heath*

William S. Hillman

Alden D. Hinckley

(on leave from

Coconut Rhinoceros Beetle Project,

Apia, West Samoa)

Geoffrey Hind

Gerhard Holm

(postdoctoral appointment)

Alan D. Horrill

(postdoctoral appointment)

Richard A. Houghton, III

Adam S. Inglis*

(assigned from Commonwealth Scientific

and Industrial Research Organization,

Melbourne, Australia)

Richard L. Jackson

(postdoctoral appointment)

Alexandra H. Jahn

Elsy K. Jonsson*

Devanayagom C. Joshua

(IAEA Fellow on leave from Bhabba

Atomic Research Centre, Trombay, India)

Izumi Kabasawa

(postdoctoral appointment)

Marita D. Kane

Helen J. Kelly

Julie Klee*

J. Raymond Klein

Donald Koenig

Tadahiko Kohno

Alexander A. Kortt

(postdoctoral appointment)

Charles J. Kovacs

(National Institutes of Health Fellow)

Jadwiga H. Kycia
Sanford A. Lacks

H. George Latham, Jr.

Myron C. Ledbetter

Teh-Yung Liu

Robert A. Love, Jr.*

Timothy Macdonald

(postdoctoral appointment)

Gareth A. Mair

(postdoctoral appointment)

Kodumudi M. Marimuthu

(on leave from Univ. of Michigan)

Dorothy M. McFadyen

W. Arthur McKee

Barbara McMillan

Dorothy P. Meyer

Barbara C. Michelsen

David L. Mulcahy*

(on leave from Univ. of Georgia)

J. Hurley Myers

(AUI Postdoctoral Fellow,

National Institutes of Health Fellowship)

Herbert Y. Nakatani

Anne F. Nauman

Charles H. Nauman*

Marta Nawrocky

Leslie F. Nims

Noboru Nomura*

(postdoctoral appointment)

Joel S. O'Connor

John M. Olson

Theresa F. Petty*

James J. Phelan*

(postdoctoral appointment)

Thomas S. Platt*

Virginia Pond

Bonnie J. Presowitz

Merton H. Pubols

(on leave from Washington State Univ.)

LeAnne Puglielli*

Marjorie L. Rudert

Richard N. Ruffing

John J. Ruscica

Lloyd A. Schairer

Benno P. Schoenborn

Susan S. Schwemmer

Roslyn Shapanka

Elizabeth K. Shaw

Elliott N. Shaw

Michiko Shibata

Harold W. Siegelman

Mohinder P. Singh*

(on leave from Indian Agricultural

Research Inst., New Delhi, India)

Harold H. Smith

Miriam K. Sokoloff

Arnold H. Sparrow

Rhoda C. Sparrow

Sylvia S. Springhorn

Robert Steele

William J. Stegeman*

Herbert I. Student

F. William Studier 
Haven C. Sweet*

(postdoctoral appointment)

Shigeji Tanemura

Keith H. Thompson

J. Philip Thornber

(postdoctoral appointment)

John J. Tilley, Jr.

Alan G. Underbrink

(assigned from Columbia Univ.)

Jack Van't Hof

Anna-Maria Vasquez

Derinda A. Walker

Chih-Cheng Wang

(postdoctoral appointment)

Tung-Chia Wang

Elizabeth A. Weaver*

Peter L. Webster

(postdoctoral appointment)

Robert G. Woodley

George M. Woodwell

Ruth V. Wright

Jeanne R. Wysocki

Shizuko Yagi

Joseph D. Yourno

\section{Chemistry Department}

Gerhart Friedlander, Chairman

Lloyd Abrams*

(postdoctoral appointment)

Augustine $O$. Allen

Elizabeth W. Baker

Ivan Bernal

Benon H. Bielski

Jacob Bigeleisen*

Christos S. Capellos*

Lui-Heung Chan*

(postdoctoral appointment)

David R. Christman

Yung Yee Chu

Philip Coppens*

Lester M. Corliss

James B. Cumming

Geoffrey Davies

(postdoctoral appointment)

Raymond Davis, Jr.

Mario DePas

(postdoctoral appointment)

Richard W. Dodson

James W. Edmonds

(postdoctoral appointment)

Stanton Ehrenson

Norman Elliott

Earl F. Epstein

(postdoctoral appointment)

Bruce R. Erdal

(postdoctoral appointment)

Nils E. Erickson

Juan M. Figuera*

(postdoctoral appointment)

Joanna Fowler

(postdoctoral appointment)

*Terminated before July 1, 1969.
Eena-Mai Franz

Lewis Friedman

Salomon Fuks*

(postdoctoral appointment)

Naomichi Furukawa

(postdoctoral appointment)

Alessandro Gandini*

(postdoctoral appointment)

Bernard Gatty

(postdoctoral appointment)

Kodasseri P. Gopinathan*

(postdoctoral appointment)

James R. Grover

Walter C. Hamilton

Garman Harbottle

George D. Harp

Julius M. Hastings

Michael G. Holliday

(postdoctoral appointment)

Robert M. Hoyte

Jerome Hudis

Liaquat Husain

(postdoctoral appointment)

Adolph P. Irsa

Per-Gunnar Jönsson*

(on leave from Univ. of Uppsala, Sweden)

Miltiades Karayannis*

(assigned from Inst. of Fine Arts, New York Univ.)

Paul J. Karol*

(postdoctoral appointment)

Seymour Katcoff

Edward O. Kazimer*

(graduate student from Fordham Univ.)

F. Michael Kiely

(postdoctoral appointment)

Siegfried R. Krutzik*

(postdoctoral appointment)

Walter Kunnmann

Ernest Y.Y. Lam

(postdoctoral appointment)

Richard M. Lambrecht

(postdoctoral appointment)

Sam J. LaPlaca

Hon-Yen Lau

(postdoctoral appointment)

Jacob B. Leventhal*

Louis Lindner

(on leave from

Institute voor Kernphysisch Onderzoek,

Amsterdam, The Netherlands)

Warren J. Lyman

Ljubica Manojlovic-Muir*

(postdoctoral appointment)

Harold R. Martin* (postdoctoral appointment)

Louis J. Massa* (postdoctoral appointment)

Francis J. Menes*

(assigned from

Commissariat à lÉnergie Atomique,

Saclay, France)
Pieter Meyers

(postdoctoral appointment)

Stamatios G. Mylonakis*

Marjorie T. Nieh

Beverly J. Nine

Elinor F. Norton

Catherine T. Paul*

Morris L. Perlman

Henry N. Po*

(postdoctoral appointment)

Laura T. Ramer

Carol S. Redvanly

Louis P. Remsberg, Jr.

Frederick K. Ross

(postdoctoral appointment)

J. Keith Rowley

William Rubinson

Robert A. Sack

(graduate student from

New York Medical College)

Cielo Maria Santiago*

Edward V. Sayre

Werner F. Schmidt

(postdoctoral appointment)

Harold A. Schwarz

David Seewald*

(postdoctoral appointment)

Stanley Seltzer

Hagai Shaked

(on leave from Nuclear Research Center,

Negev Beer Sheva, Israel)

Jacob S. Shapiro*

(postdoctoral appointment)

Kelvin K.-W. Shen*

(graduate student from

Univ. of Massachusetts)

Philip B. Shevlin

(postdoctoral appointment)

Frederick J. Silkworth, Jr.

Raymond W. Stoenner

Norman Sutin

Robert Thomas

Sydney O. Thompson

Armin K. Tippe*

(assigned from Fritz Haber Institut der Max

Planck Gesellschaft, Berlin, West Germany)

Alexandros K. Tsolis

(postdoctoral appointment)

Aldo E. Tucciarone

(postdoctoral appointment)

Bjorn Warnquist

(postdoctoral appointment)

Kay O. Watkins*

(on leave from Adams State College)

Ralph E. Weston, Jr.

Hans Michael Widmer

(postdoctoral appointment)

Ronald Withnell

Alfred P. Wolf

Max Wolfsberg*

Pak Kuen Wong

(postdoctoral appointment) 
Instrumentation and Health Physics Department

Horner Kuper, Chairman

John W. Baum

William H. Bishop, Jr.

Robert M. Brown

Michael J. Butler

Robert L. Chase

Lester A. Cohen

Robert D. Colvett*

Frederick P. Cowan

John B. Deitz

D. Gerd Dimmler

Carl H. Distenfeld

Joan F. Felberbaum

Joachim Fischer

Charles W. Flood, Jr.

Charles F. Foelix

John A. Frizzola

Lee Gemmell

Norwood E. Greenlaw

William J. Hartin

Andrew P. Hull

John F. Jacobs*

Mary Anne Kelley

John J. Kelsch

Kazuo Kemmochi*

(on leave from Japan Defense Agency,

Tokyo)

Hobart W. Kraner

Alan V. Kuehner

Young Bok Lee

Charles B. Meinhold

William Michaelson

Constance M. Nagle

Anthony Nappi

Casimir Z. Nawrocki

Michael J. O'Brien

David Ophir*

James P. Palmer

Smith G. Pearsall

Leigh F. Phillips

David W. Potter

Veljko Radeka

Seymour Rankowitz

Gilbert S. Raynor

Edwin J. Rogers

Martin J. Rosenblum

Jesús I. Sánchez*

(on leave from Junta de Energía Nuclear, Madrid, Spain)

Shinkicki Shibata

(on leave from Univ. of Tokyo)

Stanley I. Silverman

Irving A. Singer

Maynard E. Smith

Raymond W. Stong

Sanford E. Wagner

Gaylord N. Wall

*Terminated before July 1, 1969.
Catharine L. Wingate

Stanley Wood

Robert W. Young

\section{Mechanical Engineering Division}

Irving J. Polk, Head

John R. Aggus

Richard W. Aichroth

Richard C. Albert

Joseph E. Allinger

Richard C. Amari

Frank H. Atkinson

Robert D. Baldwin, Jr.

Joseph A. Bamberger

Alden J. Banslaben

Alfred A. Bertsche

Paul Bezler

Thomas J. Blair

Donald P. Brown

Thomas W. Brown

Robert H. Browne

Vernon J. Buchanan

Ronald W. Burgess*

Leonard N. Chimienti

Thomas M. Coyle

Rudolph Damm

Jack E. Detweiler

Carmine DiLullo

Basil DeVito

Arthur C. Dick

Santo J. DiNaro, Jr.

Bernard J. Durman

Carlo Ferraro, Jr.

Carl R. Flatau

Edward H. Foster

John J. Fuhrmann

Donald W. Gardner

Robert J. Gibbs

Donald M. Gilzinger

Jules B. Godel

Anthony T. Gody

Carl L. Goodzeit

Charles L. Gould

Pierre Grand

Melvin E. Griffing

John J. Grisoli

Eugene E. Halik

Rudolph S. Hodor

Kenneth C. Hoffman

Daniel Hoober

John N. Hopping, Jr.

Hank C.H. Hsieh

Donald W. Huszagh

Eugene Jablonski

Jack E. Jensen

Marshall G. Jones

David A. Kassner

Andrew Kevey

John T. Koehler

Calman Lasky

Paul LeDoux

Nathan Levenson
Boris M. Lomonosoff

Kenow H. Lou*

(assigned from Lawrence Radiation

Laboratory, Berkeley, Calif.)

Joseph Lypecky

Stanley J. Majeski

John G. Marinuzzi*

Raven B. McKenzie-Wilson

Thomas F. McKinley

Hugh T. McTeague

Anthony P. Meade

Kurt F. Minati

Mordechai Montag

William J. Museler

Adolph Oltmann

Frederick O. Pallas

Nicholas J. Parrinello

Francis C. Pechar

Paul A. Pion*

Carl J. Pozgay

Arno Raag

Clive E. Reed

Morris Reich

Louis E. Repeta

Wesley G. Ripperger

Eugene W. Rogers

Albert P. Schlafke, Jr.

William J. Schneider

Joseph C. Schuchman

Anthony Semplicino

Stewart Senator

Gerard E. Tanguay

Charles O. Theisen

Harvey J. Thomas

Reese D. Thomas

Helmuth Thorwarth

Dietrich Trocha

Vincent Troisi, Jr.

Stanley L. Ulc

John C. Walker

William G. Walker

Irving J. Winters, Jr.*

Donald $\mathrm{H}$. Wright

Arieh Zahavi

\section{Medical Department}

Eugene P. Cronkite, Chairman

Emil R. Adamik

Robert B. Aronson

Harold L. Atkins

John L. Bateman

Gunnar L. Bengtsson

(assigned from Columbia Univ.)

Donald C. Borg

Arland L. Carsten

Arjun Dev Chanana

Vivien A. Chen

Celia C. Chiu

(assigned from Mount Sinai Medical and

Graduate Schools, New York)

Stanton H. Cohn

Spencer L. Commerford 
Robert A. Conard, Jr. George C. Cotzias Lewis K. Dahl

Nicholas Delihas

Charles F. Demoise (AUI Postdoctoral Fellow, National Institutes of Health Fellowship)

Ruth M. Drew

Patrick Eggena

(assigned from Mount Sinai Medical and Graduate Schools, New York)

Ralph G. Fairchild

Clas Fehling (on leave from Univ. of Goteborg, Sweden)

William A. Fingal (on leave from Texas College)

Arthur Forman (medical associate)

Rosemary A. Gellene*

John D. Glass, Jr. (assigned from Mount Sinai Medical and Graduate Schools, New York)

Frank W. Gonzalez (assigned from Columbia Univ.)

Leon J. Goodman (assigned from Columbia Univ.)

Leonard D. Hamilton

Lawrence V. Hankes

Wolfgang Hauser

Mitsuru Hoshi (on leave from Osaka Univ., Japan)

Robert J. Iorio (AEC Postdoctoral Fellow)

Junichi Iwai (on leave from Univ. of Tokyo, Japan)

Mahendra P. Jamuar (medical associate)

Darrel D. Joel

Horton A. Johnson

Stanley L. Johnson*

Barry M. Kaufman

Charles W. Kim

Knud D. Knudsen

Kazuo Kubo*

(medical associate)

Marian E. LeFevre

(assigned from Mount Sinai School of

Medicine, New York)

Anthony F. Lo Monte

Robert A. Love

Kengo Matsui

(James Picker Foundation Fellow)

Shin-Ichi Okuyama

(Eastern Leukemia Association Fellow)

Chandra S. Pande

(assigned from Mount Sinai Medical and Graduate Schools, New Tork)

Paul S. Papavasiliou

Howard R. Pate

Mildred Pavelec

*Terminated before July 1, 1969
Nina B. Pluss*

Edwin A. Popenoe

Kanti R. Rai

Paul N. Reilly*

Ursula Reincke

(Damon Runyon Fellow)

James S. Robertson

Charles V. Robinson

Albert J. Rotermund, Jr.

(AUI Postdoctoral Fellow,

National Institutes of Health Fellowship)

Jean R. Scherrer

(on leave from

Clinique Médicale and Institut de Physique,

Université de Genève, Switzerland)

Lewis M. Schiffer*

Max A. Schmaeler

John J. Schmidt

(AUI Postdoctoral Fellow,

National Institutes of Health Fellowship)

Claire J. Shellabarger

Walton W. Shreeve

Myron S. Silverman

(assigned from U.S. Naval Radiological

Defense Laboratory, San Francisco)

Glyde R. Sipe

Yolante E. Skrzywan

(medical associate)

Jerry B. Stevens

(on leave from Univ. of Minnesota)

Richard D. Stoner

Rita F. Straub

Geoffrey P. Tauro

(National Leukemia Association Fellow)

Donald D. Van Slyke

Roderich W. Walter

(assigned from Mount Sinai Medical and

Graduate Schools, New York)

Clyde Zalut*

\section{Physics Department}

R. Ronald Rau, Chairman

Robert J. Abrams

Sabine Ahrens

David E. Alburger

Jean Allitti*

(on leave from

Commissariat à l'Energie Atomique,

Essone, France)

Eric W. Anderson

Farzam Arbab

(postdoctoral appointment)

Richard Arndt

Stephen Arnold

(graduate student from

Queens College, City Univ. of New York)

Elliot H. Auerbach

John D. Axe, Jr.

(assigned from

Thomas J. Watson Research Center, International Business Machines Corp.)
Charles P. Baker

Virgil E. Barnes

Daniel A. Bassano

(graduate student from Syracuse Univ.)

Karl H. Beckurts*

(assigned from Institut für Angewandte

Kernphysik, Gesellschaft für Kernforschung,

Karlsruhe, West Germany)

John A. Benjamin

Darlene Bentz*

Robert H. Bergoffen

Charles C. Blank

Harry R. Blieden*

Martin Blume

Robert W. Brown

(postdoctoral appointment)

Gunter Brunhart

Brian Buck

Allan S. Carroll

Lai-Him Chan*

(postdoctoral appointment)

Yau W. Chan

Ashoka Chandra

(postdoctoral appointment)

Chellis Chasman

David Cheng

Dunson K. Cheng (graduate student from

State Univ. of New York at Stony Brook)

Robert E. Chrien

James H. Christenson

(assigned from Columbia Univ.)

Suh Urk Chung

Eugene L. Church

(assigned from Frankford Arsenal)

Anne-Marie Cnops

Victor W. Cohen

George B. Collins

Malcolm F. Collins* (assigned from

United Kingdom Atomic Energy Authority,

Harevell, England)

Philip L. Connolly

Rolland R. Conte*

(assigned from

Commissariat à l'Énergie Atomique,

Fontenay-aux-Roses, France)

Ernest D. Courant

David E. Cox

Paul P. Craig

David J. Crennell

Kathleen M. Crennell

Jack E. Crow

Bernard B. Culwick

Arthur C. Damask

William C. Delaney*

Alain Delapalme*

(assigned from Centre d'Études Nucléaires de Grenoble, France)

Theodore A. Dellin

(graduate student from

Queens College, City Univ. of New Tork) 
Claude M. Delorme* (assigned from Univ. of Rochester)

Eduardo de Rafael* (postdoctoral appointment)

Edward der Mateosian

George J. Dienes

Robert P. DiNardo

(graduate student from

Stevens Inst. of Technology)

Jean V. Domish

John T. Donohue

(postdoctoral appointment)

Robert L. Eisner

(postdoctoral appointment)

S. Thomas Emerson

(postdoctoral appointment)

Victor J. Emery

John R. Ficenec

(assigned from Virginia Polytechnic Inst.)

Vincenzo Flaminio

(on leave from

CERN, Geneva, Switzerland)

Kenneth J. Foley

Hugie L. Foote, Jr.

William T. Ford

(assigned from Princeton Univ.)

William B. Fowler

B. Chalmers Frazer

Joseph L. Friedes

Donald I. Garber

David A. Garelick

William Gelletly

G. Norris Glasoe

(retired June 30, 1969)

Bruno Gobbi

(assigned from Univ. of Rochester)

Allen N. Goland

Conrad S. Goldberg

(graduate siudent from

Queen College, City Univ. of New York)

Malcolm Goldberg

Gertrude S. Goldhaber

Samuel A. Goudsmit

John J. Gould

Robert G. Graves

(graduate student from

State Univ. of New York at Stony Brook)

William J. Hakel

(graduate student from Univ. of Rochester)

Jimpei Harada

William R. Harris

(postdoctoral appointment)

James B. Healy

Patrick J. Herley

(assigned from Picatinny Arsenal)

Benjamin H. Hertzendorf*

George S. Hicks

(graduate student from Columbia Univ.)

David G. Hill

\footnotetext{
*Terminated before July 1, 1969.
}

Donald V. Hirst

John Hornbostel *

(deceased March 8, 1969)

Paul V.C. Hough

Theodore L. Houk

(postdoctoral appointment)

James M. Howie*

(assigned from

CERN, Geneva, Switzerland)

Arthur H. Huffman

(postdoctoral appointment)

James J. Hurst, Jr.

Frederick R. Huson

Michael T. Hutchings

Richard T. Imossi

Shelby P. Jessup

Robert A. Johnson

Keith W. Jones

Sidney H. Kahana

George R. Kalbfleisch

Walter R. Kane

David T. Keating

Peter J. Kemmey

(assigned from Picatinny Arsenal)

Ian R. Kenyon

Robert R. Kinsey

John B. Kinson

Ottmar C. Kistner

Thomas A. Kitchens, Jr.

Michael J. Koczak

(graduate student from

Univ. of Pennsylvania)

Harold W. Koenigsberg

Thaddeus F. Kycia

Kwan Wu Lai

Franklin T. Langdon

James D. Larson

Benny E. Lautrup

(postdoctoral appointment)

Otto W. Lazareth, Jr.

Barbara Lebowitz*

Hyung J. Lee

Paul Hoong-Chien Lee*

(graduate student from

McGill Univ., Montreal, Canada)

Kurt Lengweiler

Boran A. Leontic*

Michael J. LeVine

(postdoctoral appointment)

Paul W. Levy

Kelvin K. Y. Li

Peter J. Limon

(assigned from Columbia Univ.)

Seymour J. Lindenbaum

Georges W. London

Robert I. Louttit

William A. Love

Mario A. J. Mariscotti

Brian R. Martin*

(postdoctoral appointment)

Peter L. Mattern

Michael McKeown
Herbert Meister*

(assigned from Euratom, Ispra, Italy)

Jack Menes

Wesley J. Metzger*

(postdoctoral appointment)

Daniel N. Michael

(postdoctoral appointment)

David J. Miller*

(postdoctoral appointment)

Vincent J. Minkiewicz

Paul M. Mockett

Patricia A. Moll

Jaime B.A. Moragues*

('assigned from

Comisión Nacional de Energía Atómica,

Buenos Aires, Argentina)

Thomas W. Morris

Alfred H. Mueller

George T. Mulholland

Ivan J. Muzinich

Gerald Myatt

(on leave from

CERN, Geneva, Switzerland)

Yorikiyo Nagashima

(assigned from Univ. of Rochester)

Kunio Nagatani

Joseph A. Nardi, Jr.

Robert Nathans*

Howard W. Nicholson, Jr.

(graduate student from

California Inst. of Technology)

David M.M. North

(postdoctoral appointment)

Anthony C. Nunes

(postdoctoral appointment)

John W. Olness

John S. O'Neall

(postdoctoral appointment)

E. Boyd Osgood

(postdoctoral appointment)

Tom Oversluizen

Satoshi Ozaki

Harry Palevsky

Robert B. Palmer

Robert S. Panvini

Laurence Passell

Simon Pasternack

(Editor of The Physical Review)

Anthony J. Pawlicki

(graduate student from Cornell Univ.)

Ronald F. Peierls

Robert H. Phillips

Edward D. Platner

Bernard G. Pope

(graduate student from Columbia Univ.)

Albert G. Prodell

David C. Rahm

Ronald S. Remmel (graduate student from Princeton Univ.)

Karim Rimawi

(graduate student from

State Univ. of New York, Stony Brook) 
Herbert Ringel (graduate student from Queens College, City Univ. of New York)

Paul C. Rogers

David C. Rorer

Roy Rubinstein

Vance L. Sailor

Mark Sakitt

Morris S. Samberg

(graduate student from

Queens College, City Univ. of New York)

Arthur W.C. Sambles

(assigned from

CERN, Geneva, Switzerland)

Nicholas P. Samios

Emil J. Samuelson

(assigned from

Institutt for Atomenergi, Kjeller, Norway)

Alfred C. Saulys

James M. Scarr

Rober I. Schermer

Michael P. Schulhof

(graduate student from Brandeis Univ.)

Arthur Z. Schwarzchild

George E. Schwender

C. Keith Scott*

(graduate student from

McGill Univ., Montreal, Canada)

Chia-Chang Shih

(postdoctoral appointment)

Gen Shirane

Ralph P. Shutt

William H. Sims

(postdoctoral appointment)

W. Dwain Simpson

John Skalyo, Jr.

Ian O. Skillicorn*

A. J. Stewart Smith

(assigned from Princeton Univ.)

Charles A. Smith

(graduate student from Univ. of Rochester)

Gary A. Smith

Joseph E. Smith

C. Lewis Snead

Jeffrey B. Sokoloff

(postdoctoral appointment)

John H. Sondericker

Paul A. Souder

(graduate student from Princeton Univ.)

Carmelo A. Spirio

*Terminated before July 1, 1969.
Rudolph Sternheimer

Richard C. Strand

Myron Strongin

Andrew W. Sunyar

Richard J. Sutter

Dubravko Tadic*

(on leave from

Univ. of Zagreb, Yugoslavia)

Stuart K. Tewksbury

(graduate student from Univ. of Rochester)

Royal E. Thern

(graduate student from

Massachusetts Inst. of Technology)

Pedro A. Thieberger

Alan Thorndike

Michael F. Thorpe

(postdoctoral appointment)

Jayashree R. Toraskar

(graduate student from Columbia Univ.)

George L. Trigg

(Editor, Physical Review Letters)

T. Laurence Trueman

Frank Turkot

William A. Tuttle

Ronald P. Uhlig*

Vance D. Vander Burg

Ludo R. Vanneste

(assigned from Univ. de Louvain, Belgium)

Ernest K. Warburton

(on leave to Nuclear Physics Laboratory,

Oxford, England)

Oren A. Wasson

Richard E. Watson

Harvey E. Wegner

Joseph Weneser

Theodore L. Werntz

William B. Whitten

Gian Carlo Wick

(on leave to Columbia Univ.)

Erich H. Willen

Ernest Windschauer

Estarose Wolfson*

John K. Yoh

(graduate studen from

California Inst. of Technology)

Bing-Lin Young

(postdoctoral appointment)

Yan Pong $\mathrm{Yu}$

Luke C.L. Yuan

Emilio Zavattini

(assigned from

CERN, Geneva, Switzerland)
Andres P. Zuker

(postdoctoral appointment)

\section{Plant Engineering and Planning Department}

Maxwell M. Small, Manager

Gordon K. Ahlers

Frederick E. Baumfalk

Stephen C. Biemer

Robert E. Doerr

Harold Euler

Darnley Eversley

Henry B. Hansteen

(retired June 30, 1969)

Louis A. Harson

John J. Hennessy

Edgar J. Hunter

Robert J. Hupper

John F. Iulo

Joseph F. Krikawa

John F. Lorenc

Donald A. MacCornack

Alfred Mahlmann

Walter Milian

Paul V. Mohn

M. Joseph Rose

John W. Schluter

Abe Seifert

Edward E. Shelton

Robert C. Terwilliger

Paul L. Walser

Paul H. Weitzel

\section{Reactor Division}

Robert W. Powell, Head

Michael H. Brooks

William J. Brynda

Paul Colsmann

Francis A. Dugan*

John J. Floyd

John R. Halpin

Gerald C. Kinne

Paul E. Mamola

Charles L. Osborne

John A. Penney

Jack E. Phillips

dePuyster G. Pitcher

Seymour R. Protter

Paul R. Tichler 
LIST OF PHOTOGRAPHS

\begin{tabular}{|c|c|c|c|}
\hline Page No. & Negative No. & Page No. & Negative No. \\
\hline vii & $5-112-69$ & 29 & CN4-393-69 \\
\hline $\mathrm{x}$ & & 31 & \\
\hline$T o p$ & $1-765-69$ & Top & $2-805-69$ \\
\hline Bottom & $3-787-67$ & Bottom & $10-740-68$ \\
\hline $\mathbf{x i}$ & & 43 & $1-829-69$ \\
\hline$T_{o p}$ & 6-698-69 & 55 & $2-1827-69$ \\
\hline Bottom & $1-1078-69$ & 66 & $1-849-69$ \\
\hline xii & & 71 & $3-1648-69 A$ \\
\hline Top & $5-14-69$ & 78 & $1-803-68$ \\
\hline Bottom & CN7-316-69 & 84 & $2-1844-69$ \\
\hline xiv & & 88 & $1-1078-69$ \\
\hline$T o p$ & $11-1948-68$ & 92 & $4-554-69$ \\
\hline Bottom & $4-575-69$ & 108 & CN10-194-69 \\
\hline xv & & 109 & CN10-485-66 \\
\hline$T_{o p}$ & $5-670-69$ & 110 & CN2-9-69 \\
\hline Bottom & $5-667-69$ & 111 & \\
\hline xvii & & $T_{o p}$ & $3-1284-69$ \\
\hline$T o p$ & $6-1099-69$ & Bottom & $3-1281-69$ \\
\hline Bottom & $12-640-68$ & 115 & $4-969-69$ \\
\hline xviii & $6-596-69$ & 127 & \\
\hline xix & & Bottom left & none \\
\hline Top left & $4-1056-69$ & 128 & $12-498-68$ \\
\hline Top right & none & 129 & $11-115-67$ \\
\hline Bottom & none & 137 & $10-773-68$ \\
\hline xxi & $5-1631-69$ & 138 & CN11-1705-68 \\
\hline 9 & $9-224-69$ & 141 & $5-218-67$ \\
\hline 10 & CN3-714-69 & 148 & \\
\hline 11 & $8-1249-68$ & $T o p$ & $10-13-68$ \\
\hline 15 & CN1-66-69 & Bottom & $10-14-68$ \\
\hline 25 & none & 173 & $6-1728-69$ \\
\hline \multicolumn{4}{|l|}{28} \\
\hline Top & $3-990-69$ & & \\
\hline Bottom & $11-1854-68$ & & \\
\hline
\end{tabular}

